GENERAL SCIENCE

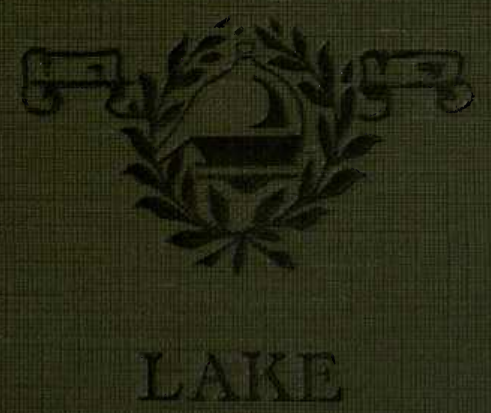




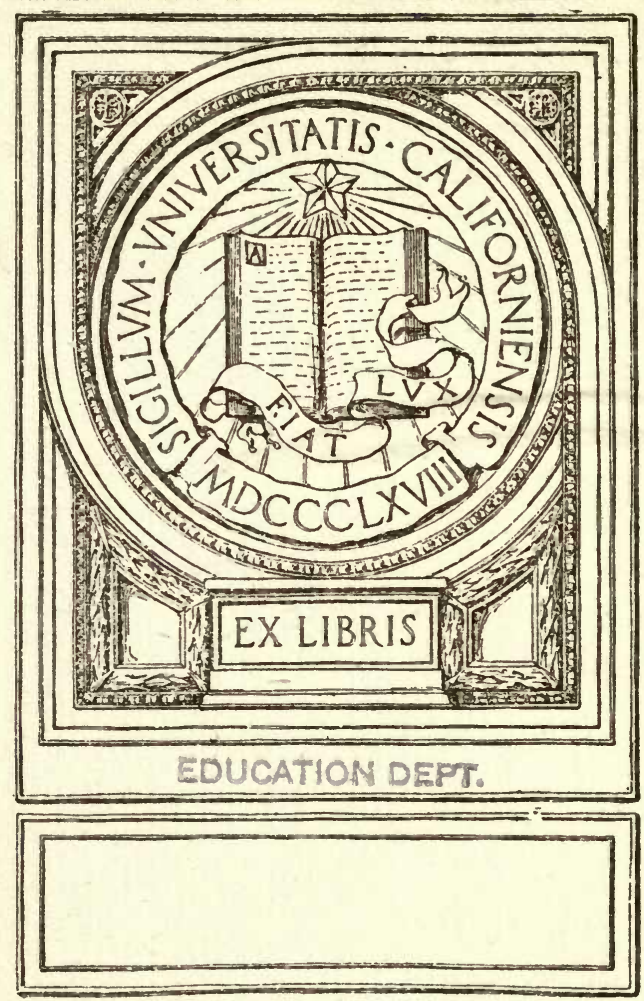







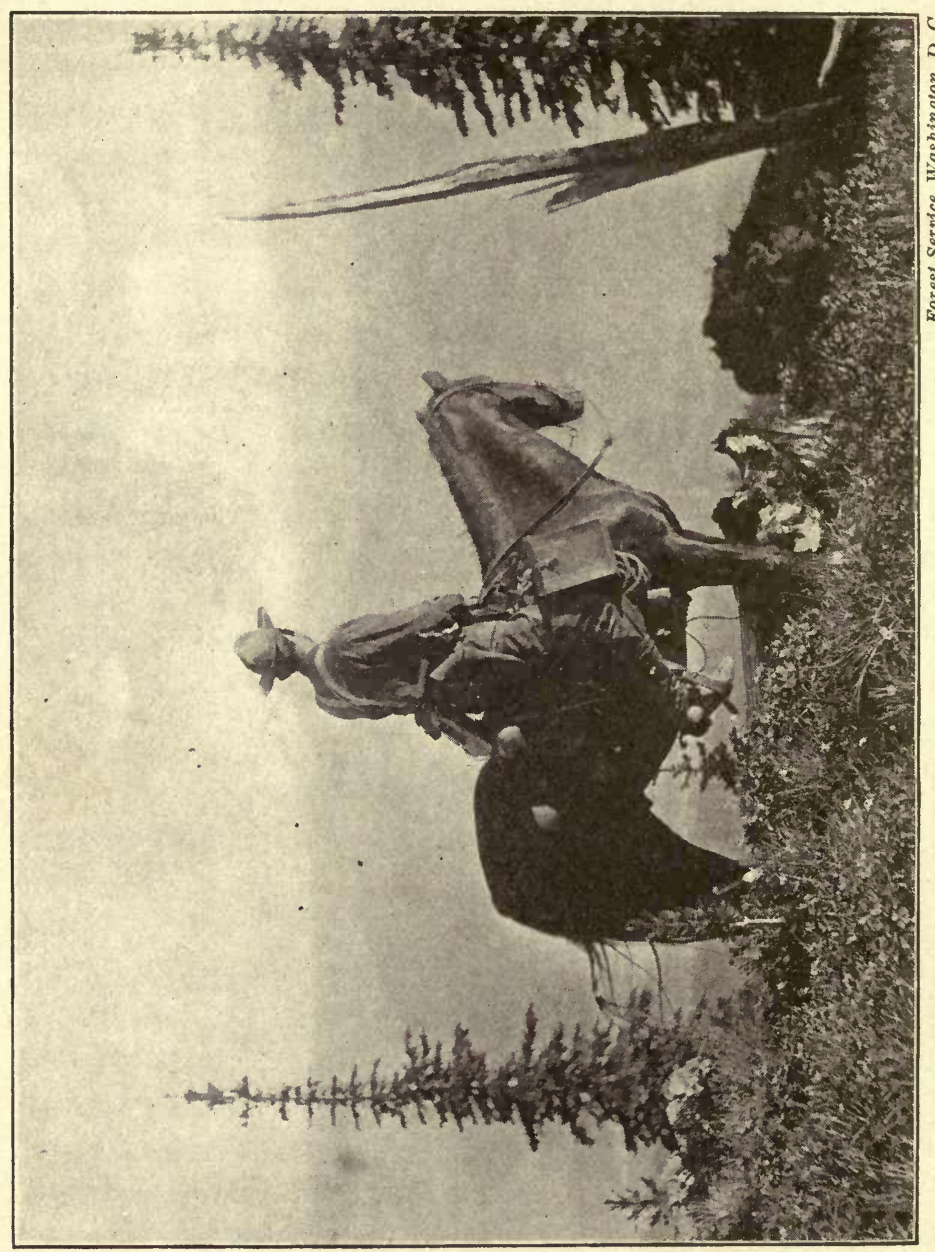

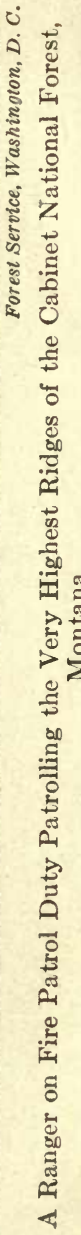




\section{GENERAL SCIENCE}

BY

CHARLES H. LAKE, M.A.

PRINCIPAL OF EAST TECHXICAL HIGII SCHOOL

CLEVELANI), OHIO

(8)

SILVER, BURDETT AND COMPANY 


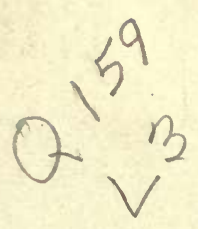

Copyright, 1917,

BY SILVER, BURDETT AND COMPANY

EOUCATION DEDh. 


\section{PREFACE}

THE question of what science shall be taught in the first year of the regular high school, or in the eighth or ninth years of the Junior High School, is becoming one of steadily increasing importance. The logical course for this period of the child's school life seems to be a general course in those elements of science which will form a foundation for the sciences which usually come later in the high school courses of study, and which will give the best training to those who may withdraw from school at the end of the ninth year. For a number of years physical geography was the science commonly taught in the ninth year. Teachers of this subject found that in order to do their best work, it was necessary to give a considerable portion of their time to teaching the elements of related sciences such as physics, chemistry, and botany. The same thing was true of the teachers of the biological sciences. This fact furnishes an excellent argument for the substitution of a course in general science for the sciences which were formerly taught in the first year of our high schools.

First year science is intended for immature minds. It must be presented and illustrated in such a way that the pupil will not be confused with terms which are too technical nor with treatments of topics which are too advanced for him. The course should be so organized that the pupil, while dealing with some known facts, will be con- 


\section{PREFACE}

tinuously relating them to phenomena about him which are of vital interest to him and also will be gaining the correct ideas of science study. He will be studying those phenomena which relate his daily existence to the fundamental principles of science.

As science the treatments in this book are not intended to be exhaustive. They are however intended to be scientifically correct as far as they go and there are no facts which will need to be unlearned as the pupil progresses in his scientific study. The subjects treated are those which are vitally connected with our daily life and which are also worthy of study as organized scientific material. The questions asked are not intended to be exhaustive. They are merely suggestive and should be supplemented with many others by the teacher.

The object in such a course of study as in all other courses of study should be to teach the pupil to live, regardless of whether he withdraws from school at an early date or whether he continues his education through the high school and college. We should always be teaching a pupil "what he needs and what he can use". and a general science course well taught fulfils this ideal admirably.

There should not be a great deal of laboratory work done by beginning students in science. Some of the suggested experiments in the text should be performed by the teacher while others are quite simple and may well be performed by the pupils at home. By so doing they will develop self-reliance and the varied experiences of the members of the class will bring out many practical relationships between the experiments and the life around them. Experiments are not ends in themselves and no experiments should be performed unless some emphasis is placed on the use of the principles involved. 
The author desires to make grateful acknowledgment to the following individuals, manufacturers, and departments of the United States Government, who have so materially assisted in collecting the illustrative material : Professor McAdie, Blue Hill Observatory, Readville, Mass.; H. A. Hutchins ; Dr. J. A. Bownocker; The Philadelphia Museums; The Warner and Swasey Company; Prest-o-Lite Company; Cutler Hammer Manufacturing Company; Niagara Falls Power Company; H. Koppers Company ; Landers-Frary Company ; Davey Tree Expert Company; Chicago, Milwaukee and St. Paul Rail Road; A. I. Root Company; Wright-Martin Company; Swift and Company; U. S. Navy Department; Bureau of Standards, Washington, D. C. ; U.S. Geological Survey; U. S. Weather Bureau; U. S. Department of Agriculture; U. S. Reclamation Service ; Forest Service, Washington, D. C. ; Ohio Agricultural Experiment Station.

C. H. L. 



\section{CONTENTS}

\section{CHAPTER I}

The Earth

The Earth and its Relation to the Universe. - The Planets. - Satellites or Moons. - Planetoids and Comets. - Stars. - Constellations. - The North Star. - The Moon. - Phases of the Moon. - The Sun. - Shape of the Earth. - Cause of the Shape of the Earth. - Size of the Earth. - Consequences of the Shape and Size of the Earth. - Structure of the Earth. - Motions of the Earth. Rotation. - Directions. - The Celestial Meridian. Meridians and Parallels. - Latitude and Longitude. Local Time. - Standard Time. - Revolution of the Earth. - Change of Seasons.

\section{CHAPTER II}

Matter and Its Properties . . . . . . .

Constitution of Matter. - Properties of Matter. - Measurement. - The Metric System. - Metric Tables. - The Measurement of Length. - The Measurement of Volume. The Measurement of Mass. - Density and Specific Gravity. - Densities of Liquids and Solids in Grams per Cubic Centimeter.

\section{- chaPTER III}

ENergy AND Force

Gravity. - Weight. - Center of Gravity. - Effect of Air on Falling Bodies. - Energy. - Inertia and Force. Centrifugal Force. - Force of Expanding Gases. - Molecular Forces. - Elasticity. - Cohesion and Adhesion. Shape of a Free Liquid. - Capillarity. - Diffusion. Osmosis. 


\section{CHAPTER IV}

Machines .

The Evolution of Machines. - The Principle of Work. The Lever. - Classes of Levers. - Pulleys. - Wheel and Axle. - The Inclined Plane. - The Screw. - The Wedge. Mechanical Advantage. - Efficiency in Machines. - Power. - Unit of Power. - Water Power. - The Overshot Wheel. - The Undershot Wheel. - The Water Turbine. - Wind Power. - The Aëroplane. - The Steam Engine. - The Steam Turbine. - Gasoline Engines.

\section{CHAPTER V}

\section{The Atmosiphere}

Weight of the Atmosphere. - Air Pressure. - Changes in Atmospheric Pressure Due to Elevation. - The Common Pump. - The Force Pump. - The Centrifugal Pump. The Siphon. - Compressed Air. - Oxygen. - Oxygen and Life. - Nitrogen. - Air, a Mixture. - Respiration. - The Lungs. - The Air Passages. - Mechanism of Breathing. Mouth Breathing. - Adenoids. - Colds.

\section{CHAPTER VI}

\section{IVATER}

Electrolysis of Water. - Water by Synthesis. - Physical and Chemical Changes. - Preparation of Hydrogen. Properties of Hydrogen. - Three States of Water. Freezing of Water. - Steam. - Solutions. - Properties of Solutions. - Water of Crystallization. - Evaporation. Evaporation, a Cooling Process.

\section{CHAPTER VII}

\section{USES OF WATER}

Manufacture of Ice. - Cold Storage. - Occurrence of Water in Nature. - Water Pressure. - Water Pressure on the Sides of a Tank. - Transmission of Pressure by Liquids. - The Hydraulic Press. - Artesian Wells. Archimedes' Principle. - Submarines. - Density of a Solid 
Heavier Than Water. - Density of Solids Lighter Than Water. - Common Uses of Water. - Hardness of Water. - Purification of Water. - City Water Supply.

PAGE

\section{CHAPTEIR VIII}

Heat .

Sources of Heat. - Heat by Friction. - Heat by Compression. - Heat from Chemical Action. - The Sun. Measurement of Temperature. - Effects of Heat. Expansion. - Expansion of Liquids. - Expansion of Gases. - Fusion. - Vaporization. - Boiling. - Relation of Boiling Point to Pressure. - Laws of Ebullition.

\section{CHAPTER IX}

Quantity of Heat and Transmission of Heat

The Calorie. - Heat Capacity. - Latent Heat. - Transference of Heat. - Conductivity of the Earth. - Conductivity and Sensation. - The Fireless Cooker. - The Thermos Bottle. - The Davy Safety Lamp. - Convection in Liquids. - Convection in Gases. - Radiation. - Heating and Ventilating of Buildings. - Hot-air Heating. - Hot-water Heating. - The Thermostat. - Ventilation. - Need of Moisture in the Air.

\section{CHAPTER X}

WEATHER

Meaning of the Term "Weather." - Functions of the Air. - Colors of the Atmosphere. - Air Density. - Isobars. Atmospheric Temperature. - The Thermograph. - Isotherms. - Change of Temperature with the Seasons. Movements of the Atmosphere. - Terrestrial or Planetary Winds. - Trade Winds. - Prevailing Westerlies. - Cyclonic Winds. - Hurricanes. - Winds with Special Names. - Monsoons. - Humidity and Precipitation. - The Dew Point. - Dew and Frost. - Clouds. - Cumulus Clouds. Cirrus Clouds. - Stratus Clouds. - Rainfall. - Thunderstorms. - Weather Changes. - The Weather Bureau. 


\section{CHAPTER XI}

Magnetism and Electricity .

Magnets. - Law of Magnetic Poles. - Induced Magnetism. - Nature of Magnetism. - Magnetic Condition of the Earth. - The Dipping Needle. - Electrification by Friction. - Two Kinds of Electricity. - Conductors and Insulators. - Theory of Electricity. - Charging a Body by Induction. - Storing a Charge of Electricity. - Condensers. - The Leyden Jar. - The Electrophorus. - Atmospheric Electricity. - Current Electricity. - Kinds of Cells for Producing Electricity. - Effects of Electric Currents. Heating Effects. - Table of Resistances. - Electric Lighting. - Magnetic Effects of Currents. - The Electromagnet. - The Electric Bell. - The Telegraph. - The Telephone. - Chemical Effects of Electricity. - Electrolysis. - Electroplating, - The Dynamo. - Electric Motors.

\section{CHAPTER XII}

Sound

What Causes Sound. - Echoes. - Musical Tones and Noises. - Pitch. - Vibrating Strings. - The Voice. - The Hearing. - How We Hear.

\section{CHAPTER XIII}

LIGHT

Light and its Properties. - Sources of Light. - Luminous Bodies. - Transparent, Translucent, and Opaque Bodies. - Shadows. - Light as Energy. - How Light is Measured. - The Bunsen Photometer. - Reflection of Light. - The Reflection of Light Compared with the Reflection of Sound. - Diffused or Scattered Light. - Refraction of Light. - Lenses. - Uses of the Lens. - The Prism and the Composition of White Light. - Length of Light Waves. - Absorption of Light and Color Phenomena. - The Sight.

- Protection of the Eyes. - Structure of the Eyeball. How the Eye Does its Work. - Light in the House. How Glass is Made. - Artificial Lighting. - The Kerosene 
Lamp. - Gases for Lighting. - Natural Gas. - Acetylene.

- Electric Lighting. - Lighting Fixtures.

\section{CHAPTER XIV}

Elements, Compounds, and Mixtures.

Interrelation of the Sciences. - How Matter is Changed. Oxidation. - Elements, Compounds, and Mixtures. - The Common Elements. - Partial List of Elements. - Metals. - Iron. - Copper. - Mercury. - Sodium. - Silver. Gold. - Chlorine. - Sulphur. - Carbon.

\section{CHAPTER XV}

\section{Fuels and Carbon Compounds}

Fuels. - Wood. - Coal. - Hydrocarbons. - Petroleum. - Flash Test. - Alcohols. - Sources of Fire. - Apparatus for Utilizing Fuels. - Stoves. - Carbon Dioxide. - Preparation of Carbon Dioxide. - Properties and Uses of Carbon Dioxide. - Fermentation. - Carbonates.

\section{CHAPTER XVI}

Common Compounds of Other Elements

Classes of Compounds. - Oxides. - Acids. - Uses of Acids. - Alkalies and Bases. - Salts. - Uses of Salts. Electrolytes. - Analysis of Chemicals. - Iron. - Copper. - Sodium. - Sulphates. - Chlorides.

\section{CHAPTER XVII}

SoILs

The Crust of the Earth. - Weathering. - How Soil is Made. - Glaciated Soil. - Composition of Soils. - Table Showing Mechanical Analysis of Soils. - Names of Soils. Importance of the Size of Soil Particles. - Size of Soil Particles in Relation to Temperature and Crops. - Conservation of the Soil. - Measure of Soil Values. - Fertilizers. - Nitrogen as a Fertilizer. - Potassium as a Ferti- 
lizer. - Phosphorus as a Fertilizer. - Lime as a Fertilizer.

- How to Experiment with Fertilizer. - Why We Cultivate.

— Dry Farming.

\section{CHAPTER XVIII}

Surface Water, Drainage, and Irrigation

What Becomes of the Rainfall. - Ground Water. Work of Ground Water. - River Formation. - Lakes and Inland Seas. - Work of Rivers. - Erosion. - Deposition. - Soil Water. - Regulation of the Amount of Soil Water. Artificial Drainage. - Irrigation.

\section{CHAPTER XIX}

Plants

Properties of Living Matter. - The Living Plant. - Cells. - Tissues. - Organs. - Multiplication of Cells. - Flowers. - Pollination. - Fertilization. - Dispersal of Seeds. Germination of Seeds. - Roots. - Stems. - Leaves. Starch Making by Leaves. - Digestion in Plants. - Flowerless Plants. - Algæ. - Fungi. - Mosses and Ferns. Distribution of Plants.

\section{CHAPTER XX}

Plants from an Economic Standpoint .

The Value of Trees. - Trees as a Protective Covering for the Earth. - Uses of Wood. - Other Uses of Trees. - Food Plants. - Textile Plants. - Weeds. - Plant Diseases. Wheat Rust. - Brown Rot. - Pear Blight. - Mildews. Potato Scah. - Chestnut Canker. - Molds. - Smuts. Black Knot. - Peach Leaf Curl. - Yeast.

\section{CHAPTER XXI}

\section{Animal Life}

Relation of Plants to Animals. - One-celled Animals, Amœba. - Division of Labor. - Hydra. - Worms. Insects. - Bees and Ants. - Fishes. - Amphibians. Reptiles. - Birds. - Mammals. - Animals Used as Food. 
- Animal Products Used for Clothing. - Animals which Aid Man. - Animal Pests. - Scale Insects. - The San Jose Scale. - The Codling Moth. - The Chinch Bug. The Hessian Fly. - Poisons for Crop Pests. - Stomachic Poisons. - Contact Poisons.

\section{CHAPTER XXII}

Man's Place in Nature

Man's Place among the Animals. - Language. - Man's Tools and Weapons. - The Home.

\section{CHAPTER XXIII}

\section{Foods and Nutrition}

Need of Food. - Bodily Energy. - Measurement of Food Values. - Source of Food. - Food Preservation as Related to Food Supply. - Transportation as Related to Food Supply. - The Manufacturer's Place in Food Supply. - Nature's Food Factories. - Kinds of Foods. - Proteins. - Nitric Acid Test for Protein. - Biuret Test for Protein. Carbohydrates. - Starch. - Glucoses. - Fehling's Solution Test. - Sugars. - Fats. - Vitamines. - Ash Constituents. - Water. - Beverages. - Al'cohol. - Effects of Alcohol.

- How Alcohol is Made. - Patent Medicines. - Tobacco. - Purchase of Food. - The Dietary. - Table of Food Values. - Principles of Cooking.

\section{CHAPTER XXIV}

Community Sanitation

Sanitation. - The Growth of Cities. - Bacteria. Germs. - Source of Germs. - Conditions Favorable to Growth of Germs. - Resisting Power of the Body. Food and Disease. - Danger in Milk. - Preservatives. Danger from Water. - Public Drinking Cups, Towels, Etc. - Street Cleaning. - Garbage, Ashes, and Rubbish. - Flies. - Sewage Disposal and Public Health. - Typhoid Fever. Colds. - Diphtheria. - Pneumonia. - Tuberculosis. - Scarlet Fever. - Measles. - Smallpox. - Diseases Carried by Insects. - Malaria. - Yellow Fever. - Quarantine. - Disinfectants. - Fumigation. 


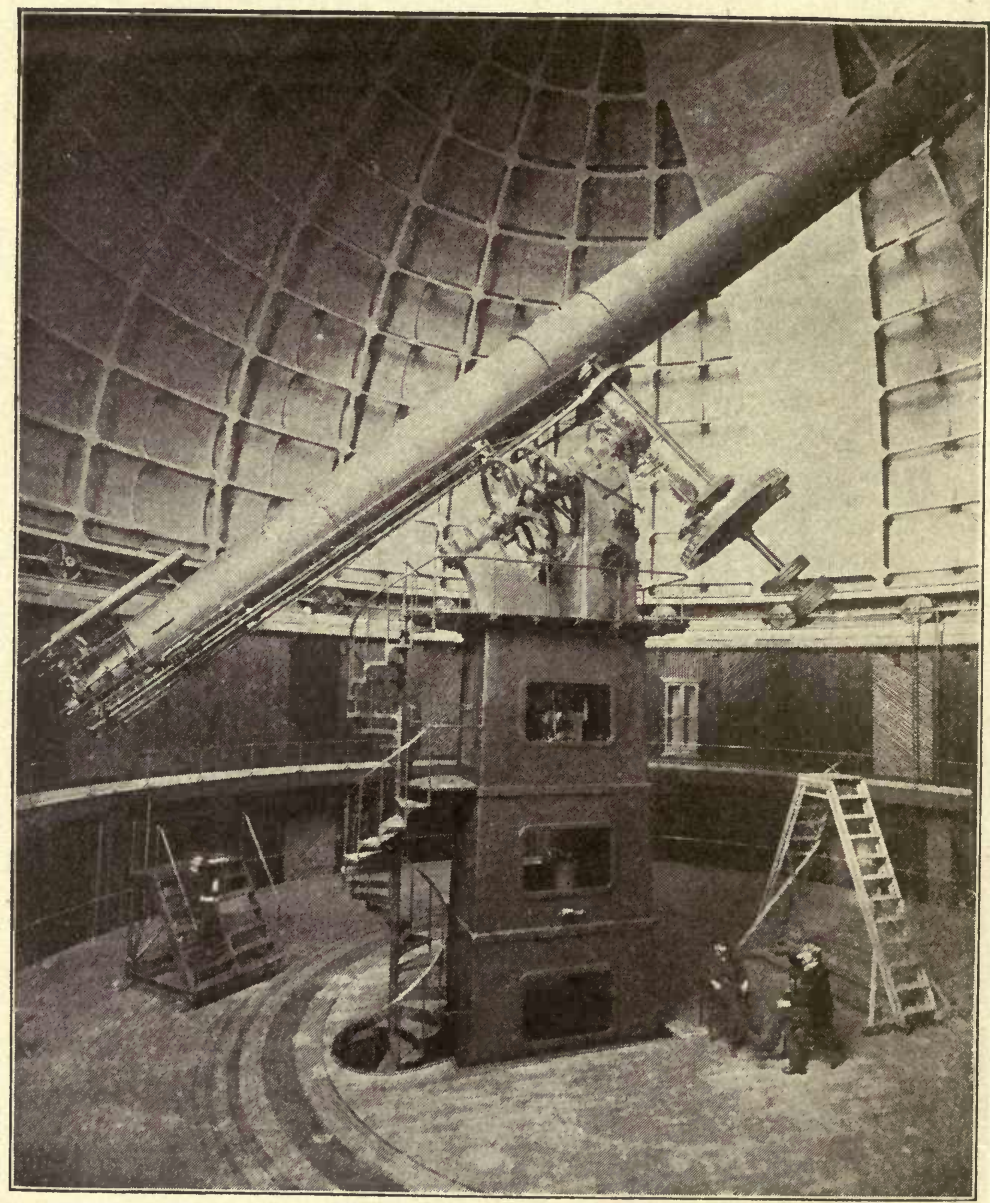

The 36-inch Telescope of Lick Observatory. 


\section{GENERAL SCIENCE}

\section{CHAPTER I}

\section{THE EARTH}

The Earth's Relation to the Universe. - The ancients regarded the earth as the center of the universe and their conception of it was a very natural one, both because of its importance to them and because the heavenly bodies actually appeared to revolve about the earth as a center. Men saw the sun rise in the east and set in the west every day, and the stars appear in nearly the same positions every night. Hence they concluded that the sun and stars all moved around the earth once in twenty-four hours.

They made careful observations, mapped out the heavens, and recorded the positions of the stars. Gradually they discovered that many of their ideas were wrong, and little by little through hundreds of years the real facts have become known concerning the vast organism which we call the universe.

From these facts we find that the earth is but one of a number of similar bodies called planets and is not at all conspicuous among them. It is neither the largest nor the smallest; the farthest from nor nearest to the sun. If we were to view it from some distant point, we should be surprised at its comparative unimportance. 
The universe of which we are so small a part is made up of many systems of heavenly bodies. These systems probably consist, each of a central body like our sun, around which revolve planets with their satellites, and comets. Many of these systems are no doubt much larger than our solar system, great and complex though it seems to us.

The Solar System - so called from the Latin word sol meaning sun - consists of the sun at the center, the planets and their satellites, the planetoids or asteroids, and some comets. There are seven other planets besides the earth revolving around the sun. They have no light of their own like the true stars, but the light that comes to us from them is a reflection of the light of the sun. If we carefully observe the bright points of light in the sky at night, we shall see that the true stars twinkle, while the planets, when they are visible, give a steady light like that of the moon.

The Planets. - The names of the planets comprising the solar system, beginning with the one nearest the sun, are: Mercury, Venus, Earth, Mars, Jupiter, Saturn, Uranus, and Neptune (Figure 1). Because of their motion around the sun the planets are continually changing their positions in relation to the other stars, whereas the positions of the true stars do not appear to change relatively to one another. It is due to this change of position that they are called planets - from the Greek word meaning wanderer. The amount of time required by the several planets to make a revolution about the sun of course varies to a considerable degree, due to the great difference in distance which they have to travel. This difference in time of revolution accounts for their change of position in relation to each other. 
The largest of the planets is Jupiter, which is more than three hundred and seventeen times as large as the earth. Saturn is next smaller, and then follow in order Neptune, Uranus, Earth, Venus, Mars, and Mercury.

The brightest of the planets are Venus, Mars, Jupiter, and Saturn, and they are plainly visible at certain periods.

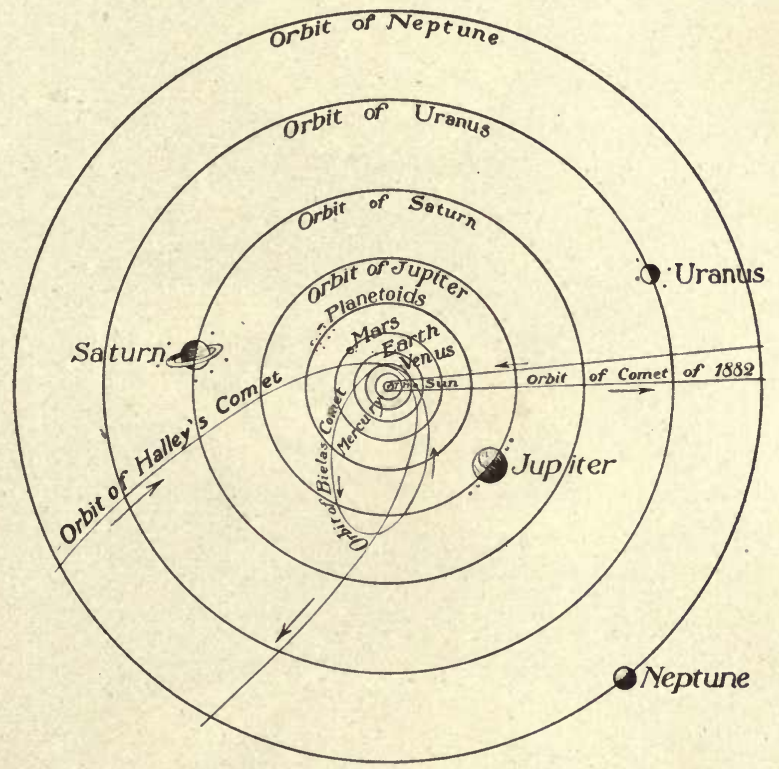

Fig. 1. - The Sun and Planets.

It is very seldom that all four are seen at the same time in any evening, but. two are often in the sky together and sometimes three. Part of the time they are morning stars and at other times evening stars. The other three planets - Mercury; Uranus, and Neptune - cannot be seen easily without a telescope.

Satellites or Moons. - Not only do the planets revolve around the sun, but each of them, except Mercury and 


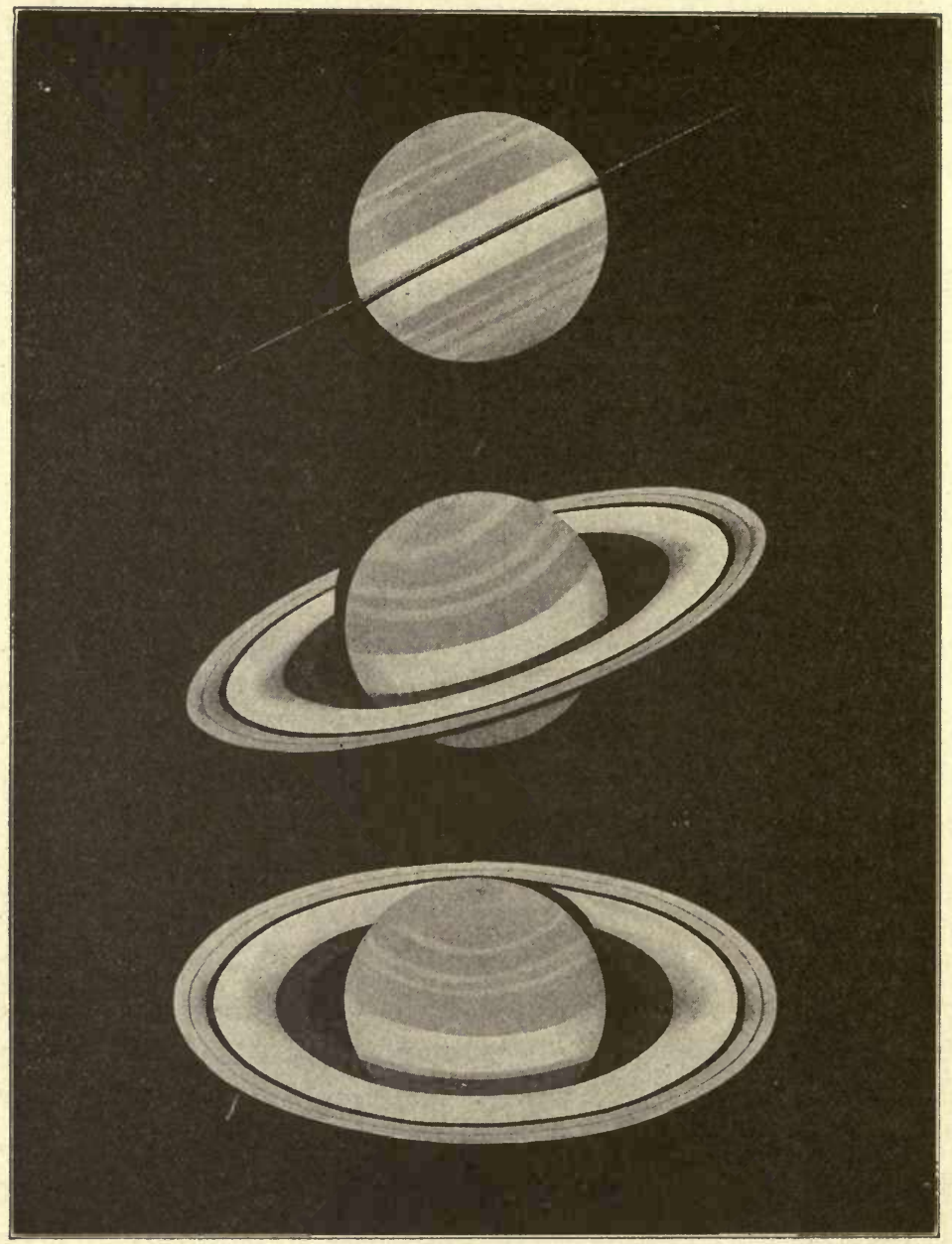

FIG. 2. - The Planet Saturn and Its Rings. 
Venus, has one or more smaller bodies revolving around it. These bodies are called satellites or moons. The earth has one of these moons, and Saturn has the greatest number, ten in all. In addition to its ten satellites Saturn has several concentric, bright rings surrounding it and revolving about it (Figure 2). When Saturn is situated so as to show the broad side of the rings, -

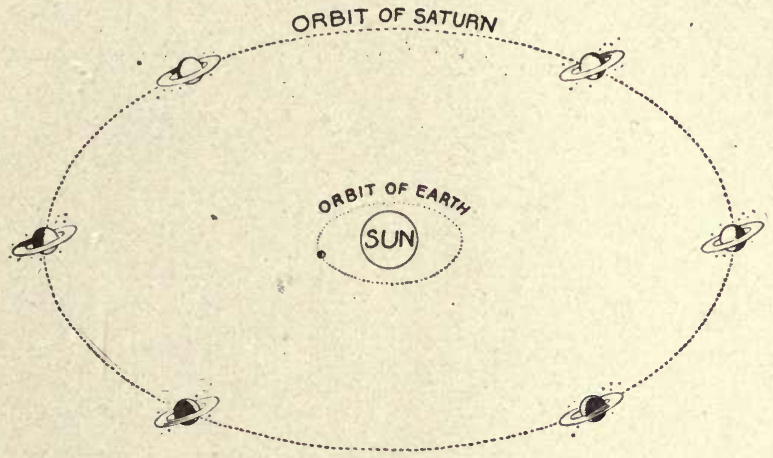

FIG. 3.

once in fifteen years, - it is in its brightest phase and is a wonderful sight through the telescope (Figure 3).

Planetoids and Comets. - Besides the planets and their satellites there are in the solar system about six hundred planetoids or asteroids. These are solid bodies much smaller than the planets, revolving in orbits between Mars and Jupiter. Within the limits of the solar system are also comets - heavenly bodies consisting of a head, with a very bright spot gradually shading into a less luminous portion, and a tail or streamer (Figure 4). Some of the comets seem to have regular paths about the sun, making their appearance at regular intervals, and must be considered a part of the solar system; others appear as occasional visitors, never to return. 


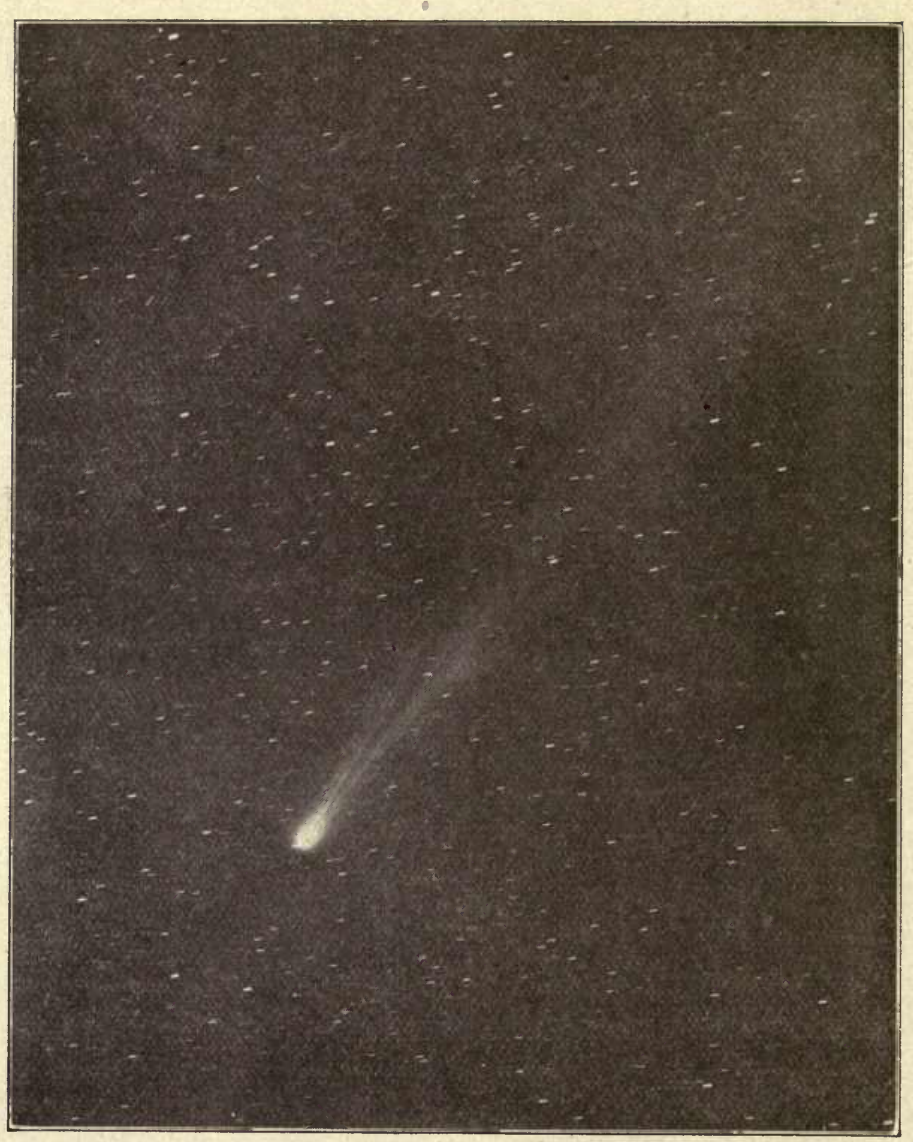

Fig. 4. - A Comet.

Stars. - When we observe the heavens at night, the stars seem to be innumerable, but it is not strictly true to say that the number actually visible to us is countless. There are only about 7000 stars bright enough to be seen with the naked eye under perfect conditions, that is, on a clear, moonless night with the atmosphere free from dust 


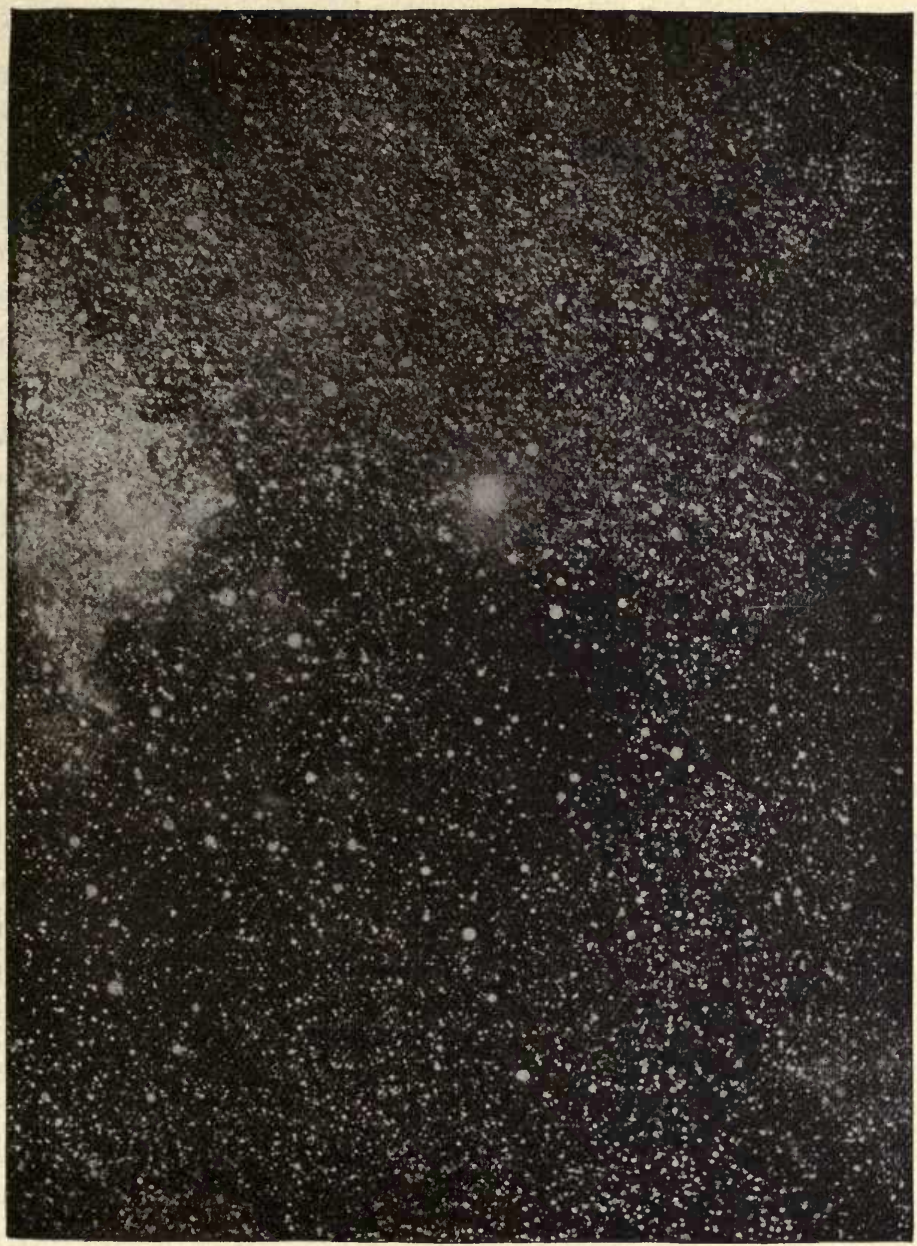

FIG. 5. - The Milky Way.

and vapor. Owing to the presence of dust and vapor, however, the number of stars in the whole sky that can readily be seen is only about 2500 . With the largest telescope about one hundred million may be seen (Figure 5). 
As we have said before, the true stars shine with a twinkling light as distinguished from the steady light of the planets; most of the fixed stars are suns like ours - many of them much larger - with their own systems of planets. We can readily understand how very large and luminous they must be when we learn that, although the nearest fixed star is millions of millions of miles away, yet we can see it and observe its brilliance, even though it takes three and one half years for its light to reach us, traveling at the enormous speed of 186,000 miles a second.

Constellations. - Certain groups of bright stars near together are known as constellations. Most of them were traced and given their names long ago by the common people - shepherds and sailors, who lived much out of doors with nothing to aid them in their study of the heavens but their own eyes and fancy.

Of the ancient constellations there are about fortyeight. All are outlined by stars that may be seen with the naked eye, for they were discovered long before the invention of the telescope. Their names have been given from some fancied resemblance to an ancient hero, an animal, or an object. Most of them do not much resemble the thing for which they are named and in this respect prove rather disappointing. On the whole they are mere abstractions bounded by certain imaginary and by no means definite lines. Many of them overlap each other.

The North Star. - If we look in the northern part of the sky, we may see a group of seven stars which outline the form of a long-handled dipper. This group is known as the "Big Dipper" and is a part of a larger constellation called the Great Bear (Ursa Major). The two 
stars on the side of the bowl farther from the handle are called the "pointers" because an imaginary line drawn through them and extended for five times the distance between them will end almost exactly at the North Star or Pole Star. The Pole Star (Polaris) is so called because the imaginary axis of the earth, if extended from the north pole, would pierce the sky very near this star (Figure 6).

The circle of stars in the northern sky seems to revolve about this Pole Star, just as all the lands of the terrestrial globe seem to revolve around the north pole as the globe is rotated. The stars in this northern circle complete their small round once in twentyfour hours, moving in

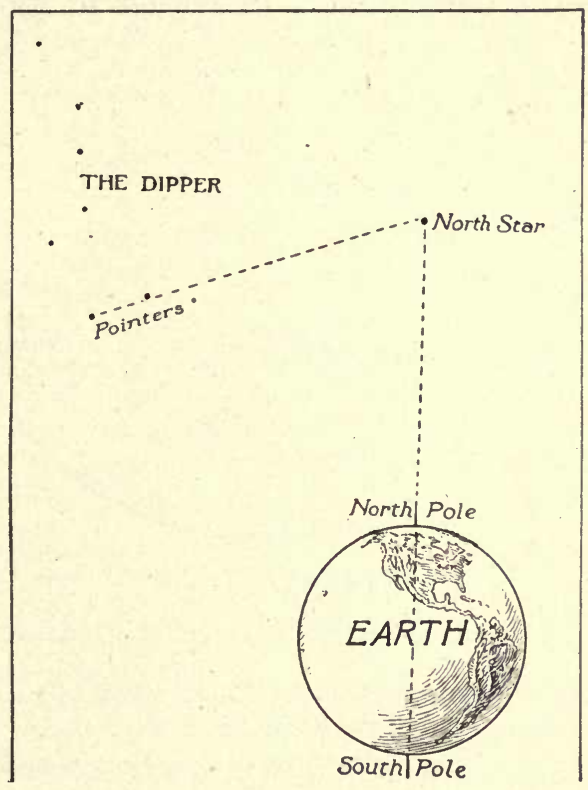

a direction opposite Fig. 6.-Diagram showing the Position of the to the hands of a North Star in the Sky.

clock (Figure 7). As the path of the northern circle of stars lies entirely above the horizon, they never rise or set, but are only obscured by the light of day. They are visible on any clear night in the year from all points in the northern hemisphere.

Six well-defined constellations are included in this northern circle, and they are always above the horizon 
in our latitude. They are: The Big Dipper, the Little Dipper or Little Bear (Ursa Minor), of which the North Star forms the end of the handle or tail, Draco or the dragon, Cassiopeia (in her chair), Cepheus, the royal husband of Cassiopeia, and Perseus (Figure 8).

Next below the northern circle comes a line or belt of stars describing a larger circle around the pole. These

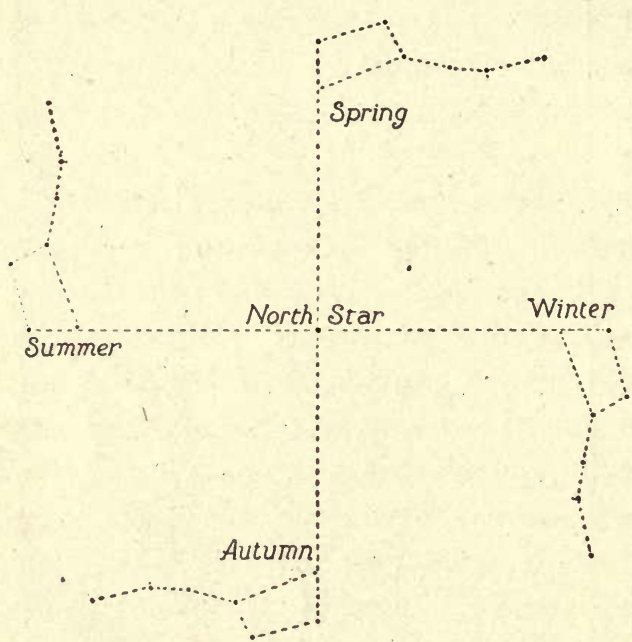

Fig. 7.- Positions of the Dipper in Relation to the North Star.

Seen in the early evening at the seasons named. stars lie below the horizon for a longer or shorter time, depending on their position in relation to the pole. They rise in the northeast, make a long slow sweep of the sky, and set in the northwest, finishing their circle below the horizon. There is another belt of stars that rises somewhere near the exact eastern point of the horizon, crosses the heavens to the western point in about twelve hours, and remains below the horizon for another twelve hours. The stars in those circles which lie farther and farther south of us never rise very high in the heavens, only very small portions of these circles being visible to us.

Some of the most important constellations in these latter belts of stars are: Orion, Hercules, Taurus, Scorpius, and Canis Major. In the last-named constellation. 
is Sirius the "Dog Star," which when visible is the brightest star in the whole sky.

The change in the position of stars which can be observed from hour to hour is caused by the rotation of the earth on its axis, and the change in position apparent from month to month is caused by the revolution of the earth around the sun. The apparent motion of the stars is always westward, because the earth's motion on its axis and around the sun is from west to east.

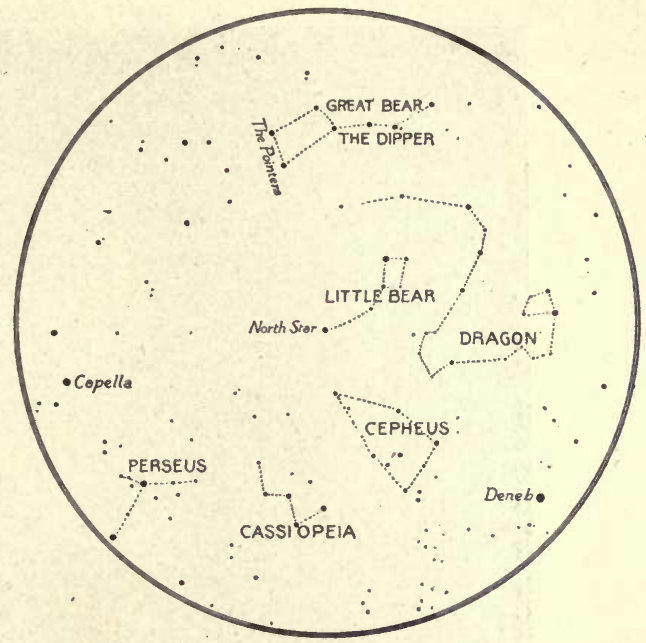

Fig. 8. - Constellations in the Northern Sky.

The Moon. - The earth has one satellite, the moon, which revolves around it once a month (27.32 days) and accompanies it through space in its journey around the sun. The diameter of the moon is about 2163 miles and its distance from the earth is about 240,000 miles. Although we see the moon as a very bright object at night for a part of every month, yet it has no light of its own but shines entirely by reflected light from the sun (Figure 9). It has a rough, barren, rocky surface and as far as is known has no air or water upon it. While the moon makes one journey around the earth, it rotates only once on its axis." Because the moon's period of rotation is the same as 
its period of revolution, it always turns the same side toward the earth.

Phases of the Moon. - When the moon is in that part of its orbit nearest the sun, it is almost between

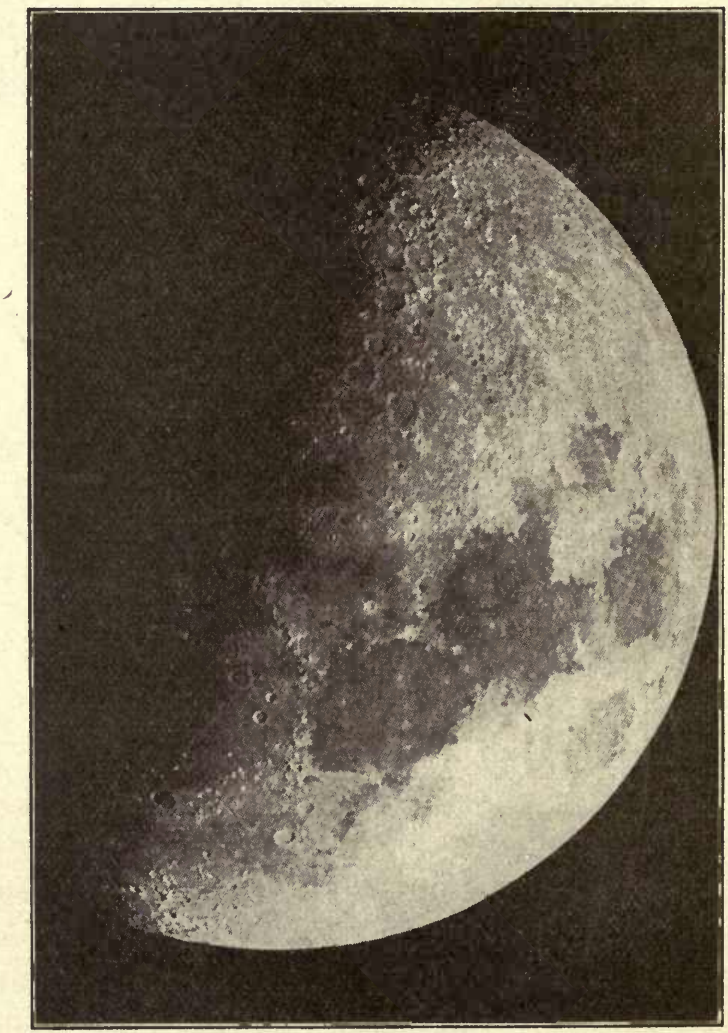

Fig. 9. - The Moon.

Photographed at the Lick Observatory.

the earth and the sun. The side illuminated is then turned away from the earth and we see but a mere fringe of illumination, a thin crescent. We call this phase the 
"new moon" (Figure 10). When it has completed a fourth of its journey, we see one half of its illuminated surface or one fourth of its total surface; this is the "first quarter." When it has completed half its circuit and is on the opposite side of the earth from the sun, we see half of its total surface or its whole illuminated surface ; it is then "full moon." When it is at the third

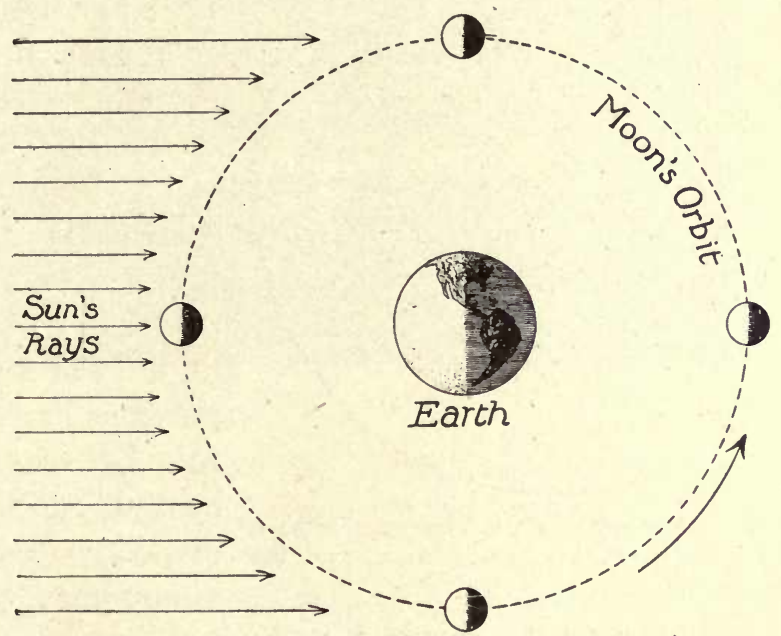

FIG. 10. - Phases of the Moon.

quarter, we again see one fourth of its total surface or one half its illuminated surface.

The moon rises about fifty minutes later each day than on the previous day. It has moved eastward from the place where it was the day before, and so the earth must turn a little farther on its axis before the moon comes into view at the horizon.

The Sun. - The sun is the center of, and by far the largest and most important body in, the solar system. All the planets of the system revolve about it and receive 
heat and light from it. It has a diameter of 866,000 miles and is much larger than all the other planets combined. If the earth were placed at its center, there would be room for the moon to revolve in its regular orbit,

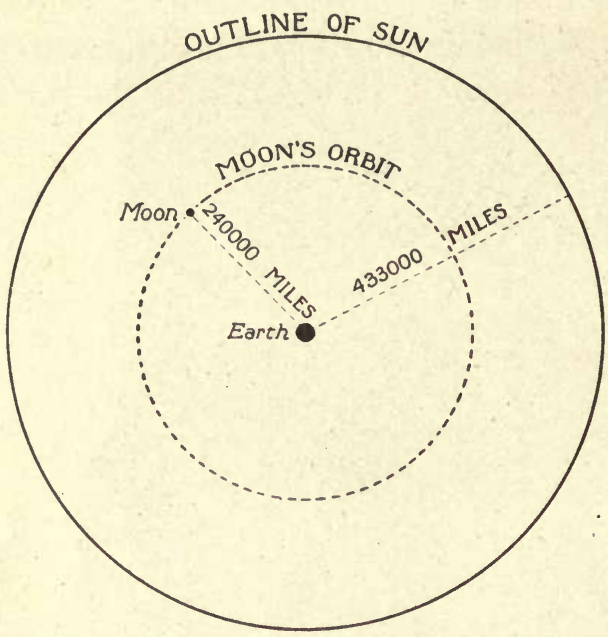

Frg. 11. - Diagram Showing Comparative Sizes of the Earth, the Orbit of the Moon, and the Sun's Disk. which would still be 200,000 miles from the circumference of the sun (Figure 11).

The reason for the extremely hot condition of the sun is not known. Its interior is thought to be composed of a dense white-hot liquid, and the outer portions are known to be intensely heated gases (Figure 12). The sun is composed of the same elements as the earth, but its condition is so hot that these elements can be recognized only by means of the spectroscope, an instrument used in the study of light.

Shape of the Earth. - Children, if they are concerned with the question at all, imagine that the earth is a flat place bounded by a few hills or a quantity of water, according to where they live; they are concerned with no people other than those in their immediate locality and those that they see in their very limited travels. In the childhood of the race, people in general held the same ideas. They imagined the earth as flat, and in sailing their crude vessels were careful not to venture 
too far from land for fear of encountering strange monsters or falling over the " edge " of the earth.

The great Greek philosopher, Aristotle, who lived about the middle of the fourth century B.c., observed

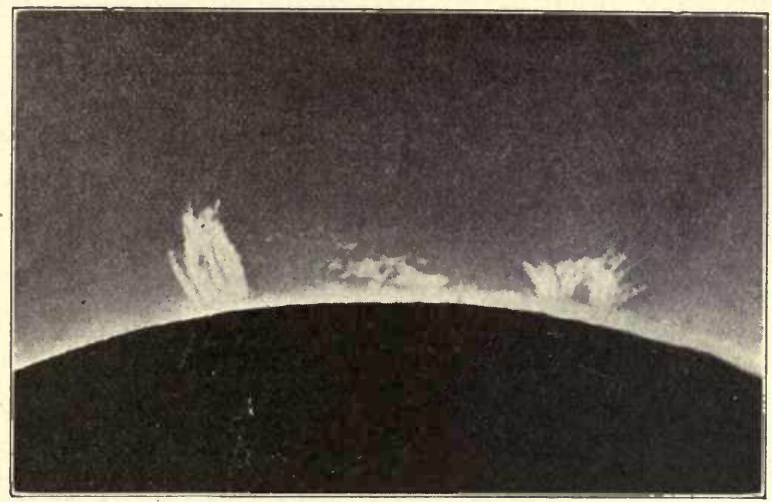

FIG. 12. - Flames on the Edge of the Sun.

the curved outline of the earth's shadow on the moon at the time of an eclipse and concluded from this observation that the earth must have a curved surface like a globe. About the beginning of the Christian Era, writers began to refer to the argument for the curvature of the earth based on the disappearance of the lower part of a vessel when sailing out to sea (Figure 13); that fact

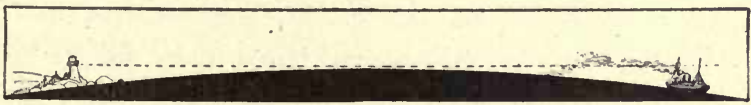

Fig. 13. - Showing the Curvature of the Earth.

had evidently been noted by many for some time previous.

The knowledge thus gained by the wise men of the ancient Mediterranean countries concerning the shape 
of the earth was unknown to the rest of the world and afterwards forgotten. Not until about the time of Columbus was it regained, and since that time many people have sailed around the earth. Wonderful discoveries have been made concerning the earth, and almost every part of its surface has been explored.

Men who have made careful measurements of the shape of the earth tell us that it is an oblate spheroid,

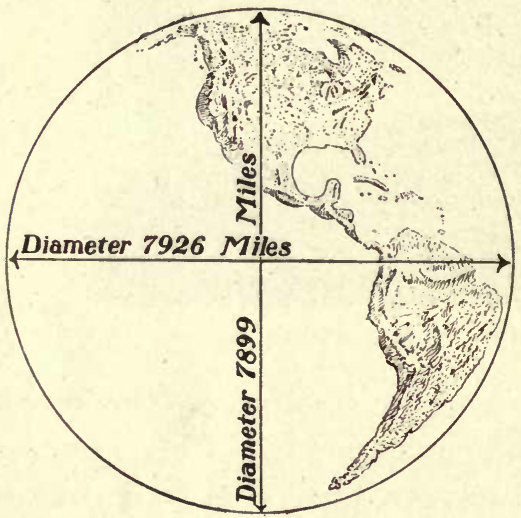

Fig. 14. - The Earth is an Oblate Spheroid. that is, a sphere which is somewhat flattened at two opposite points. An orange is an oblate spheroid, but the flattening of an orange is much greater in proportion to its diameter than is that of the earth (Figure 14). The polar diameter of the earth is only twentyseven miles shorter than the equatorial diameter, and twenty-seven miles is a very small amount when compared to the average diameter of the earth, which is about 8000 miles.

When we look at the very high mountains on the surface of the earth and think of the depressions: on the floor of the ocean, which are more than five miles deep, we wonder how a body with such great irregularities can be called a sphere at all. However, we must remember that this notion is due to our nearness of view, and that, when compared to the great size of the whole earth, these irregularities are less, in proportion, than the slight ridges on the surface of the orange. 
Cause of the Shape of the Earth. - Gravitation tends to make a mass spherical in shape. This result is accomplished more readily if the mass is plastic and elastic, which the earth is to a slight extent, although it seems very firm and rigid to us. In addition to the effect which gravitation has, there is also the effect of rotation, which causes the slight oblateness or flatness at the poles. The particles of a rotating body tend to fly off in straight lines tangent to the direction in which the

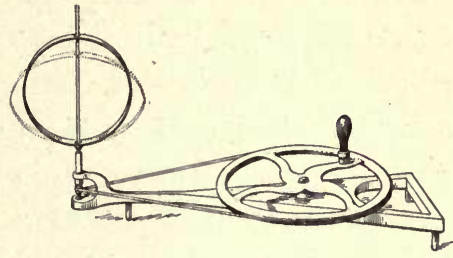

FIg. 15. body is rotating, and this tendency would cause an accumulation of the earth's material at the equator and the loss of it at the poles.

If a metal hoop which is free to move along its axis is rotated on a whirling table, this effect of centrifugal force will be well illustrated (Figure 15).

Size of the Earth. - The earliest recorded estimate of the size of the earth was made by a Greek philosopher in the third century B.c. He reckoned it to be about 8111 miles in diameter, arriving at this conclusion through measurements taken on the surface of the earth. The actual polar diameter as discovered later is 7899 miles, and the equatorial diameter is 7926 miles.

Consequences of the Shape and Size of the Earth. The great size of the earth and the irregularities of its surface have affected the distribution and growth of peoples, animals, and plants to a remarkable degree. The great distances between tribes, and the almost insurmountable barriers in the form of mountains, oceans, and deserts; for centuries hindered the mingling of tribes 
and caused marked differences in their customs and languages. The same barriers confined animals and plants to certain localities, thus fostering and developing their peculiar characteristics.

The advancement of science, the invention of various means of travel and communication, and the progress of civilization have done much toward overcoming these natural barriers, so that now the earth may be considered a relatively small body, with almost every portion of its surface accessible to the active traveler.

Structure of the Earth. - For convenience of study, the earth is divided into several parts or spheres: (1) the outer, gaseous envelope, or atmosphere; (2) the liquid envelope, the water or hydrosphere; (3) the solid rocky part, the lithosphere; (4) the center, the nucleus or centrosphere.

Motions of the Earth. - The earth has three motions, two of which greatly influence all things living upon it. The first is a daily motion, or rotation on its axis; the second is a yearly motion, or revolution about the sun; the third, which is usually disregarded because of its lack of noticeable effects, consists of an onward motion through space which the earth has, together with the other parts of the solar system.

Rotation. - The earth turns once on its axis every twenty-four hours (Figure 16). As this rotation takes place, the sun is shining upon one half of the earth's surface, leaving the other half in darkness. The effect to us is that of the sun rising at one point in the horizon, moving over the sky, and setting at some point in the horizon nearly opposite. In reality we are being turned into and out of the sunlight. The same effect is noticed when riding on a swiftly moving train. The train seems 
to be standing still while the objects along the track whirl by.

The succession of day and night, which is caused by the rotation of the earth, has given man and many animals the habit of working in the light and resting in the darkness. Rotation is also one of the factors along with others in producing tides, influencing certain belts of winds and calms, affecting the direction of ocean currents, and causing the flattening of the earth at the poles.

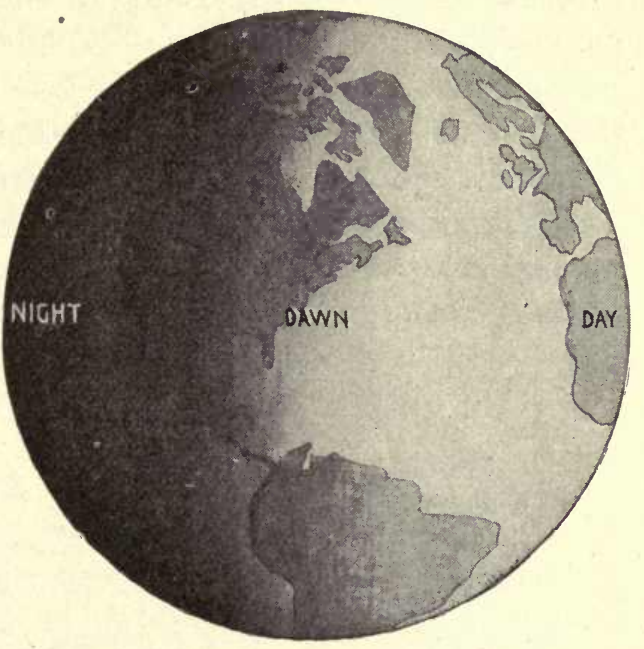

Fig. 16. - The Succession of Day and Night Caused by the Rotation of the Earth.

Directions. - We say doun when we mean toward the center of the earth and up for the opposite direction. In addition to these the rotation of the earth suggests a natural system of directions by which the relative positions of different places may be indicated. Toward the North Pole on the surface of the earth is north; toward the South Pole is south. The direction in which the sun "rises" we call east; that in which it "sets" is west. However, the true east and west line, at any place, is always at right angles to the north and south line.

The Celestial Meridian. - An imaginary line called the celestial meridian is also an important factor in the 
location of places and the reckoning of time. This line is a circle which passes through the north point of the horizon, the zenith - the portion of the sky directly over one's head - the south point on the horizon, and extends around the other side of the earth to the north point again.

It divides the sky into an east and a west half. When the sun or moon crosses the meridian (mid-day line), it has made half its daily journey from rising to setting. The abbreviations A.M. and P.M. refer to the time when the sun crosses the meridian, being taken from the Latin phrases, ante meridiem and post meridiem. Since this

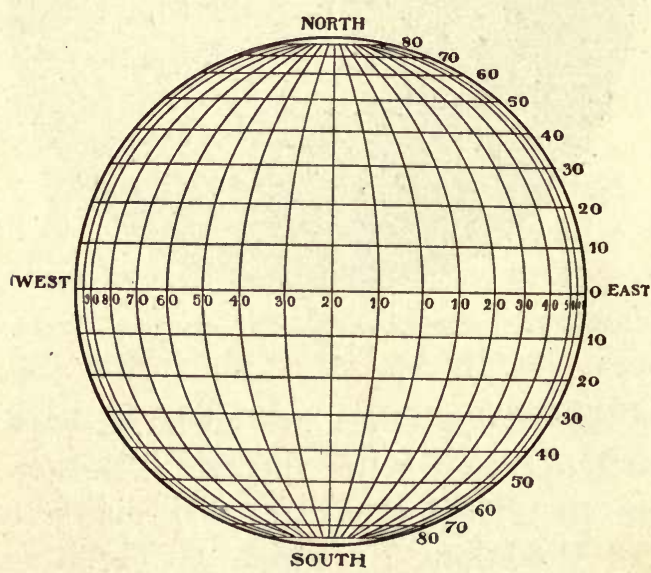

Fig. 17. - Parallels and Meridians. imaginary circle passes through the zenith, there is a celestial meridian for every observer.

Meridians and Parallels. - For purposes of calculating distance and time, imaginary lines are drawn on the earth's surface extending from pole to pole. These lines are at certain intervals and lie on the earth directly under the celestial meridian of the places through which they run. They are numbered east and west, so many degrees from the prime meridian, or the meridian of $0^{\circ}$. This meridian passes through Greenwich, England (Figure 17).

Running at right angles to the meridians are the 
parallels, or imaginary lines running around the earth parallel to the equator, which is the largest of these circles and is considered as $0^{\circ}$. The other parallels are numbered so many degrees north or south of the equator.

Latitude and Longitude. - The distance north or south of the equator is called latitude and is measured in degrees on the meridians by the parallels. Distance east and west of the prime meridian is called longitude and is measured in degrees on the parallels by the meridians.

Local Time. - The period of the earth's rotation furnishes a natural unit of time, the day, easily recognized and everywhere constant. Before there. were any clocks, people told the time of day by the sundial (Figure 18). This consisted of a vertical rod, the shadow of which fell on a horizontal plane. From noon or the time when the sun cast the shortest shadow on one day until it cast the shortest shadow on the next day was considered a day's time or a solar day, and was divided into twenty-four hours. Before the advent of railroads and the telegraph, each community used the mean solar time of its own meridian.

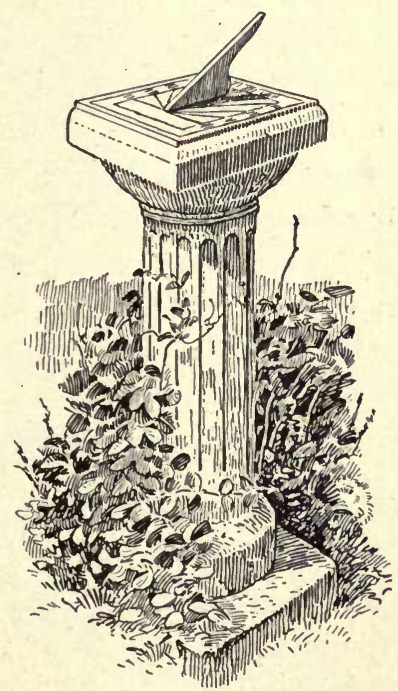

FIG. 18. - Sundial.

Standard Time. - When railways extending east and west became numerous in the United States, it became very inconvenient to use local time, for the traveler 
found his timepiece always too fast or too slow according to the direction in which he journeyed. To avoid this confusion the American Railway Association in 1883 persuaded the government to adopt standard time.

By this plan the United States is divided into four time sections, or belts. Certain meridians $15^{\circ}$ apart in longitude and one hour apart in time are taken as

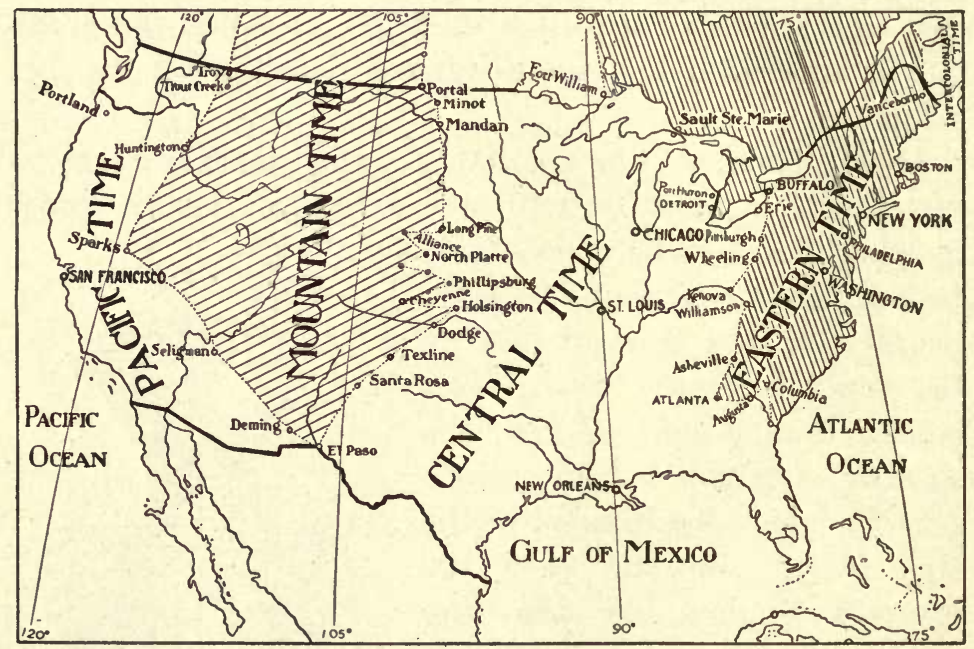

Fig. 19. - Standard Time Belts in the United States.

standard. Each belt uses the mean solar time of its standard meridian. Only the middle of the belt has the true time by the sun; other parts of the belt differ from the sun's time in periods of from one to thirty minutes.

The names of the four time belts are the Eastern, Central, Mountain, and Pacific, each extending for approximately $7 \frac{1}{2}^{\circ}$ on each side of the meridians numbered respectively $75^{\circ}, 90^{\circ}, 105^{\circ}$, and $120^{\circ}$ west of Greenwich 
(Figure 19). Everywhere in a given belt the clocks are an hour ahead of those in the next belt west and an hour behind those in the next belt east. When it is noon at New York, it is 11 A.M. in Chicage, 10 A.M. in Denver, and 9 A.M. at San Francisco. The accurate standard time is sent once a day at twelve o'clock, by telegraph, from the Naval Observatory at Washington to all cities in the United States. By electric connection many clocks are thus set exactly right each day.

Revolution of the Earth. - The earth moves once around the sun in $365 \frac{1}{4}$ days. The path in which it travels is elliptical in shape and is called its orbit. The revolution of the earth around the sun gives us our measure of time called a year and causes the sun to appear to shift its position in the heavens from day to day.

The axis of the earth constantly points in the same direction whatever its position in its orbit. The axis is inclined to the plane of that orbit $66 \frac{1}{2}^{\circ}$ or $23 \frac{1}{2}^{\circ}$ from the perpendicular. This inclination of the axis causes the earth to assume quite different positions with reference to the sun at different times of the year. To this is due the varying lengths of day and night and the change of seasons.

Change of Seasons. - From spring to autumn when the north pole is inclined toward the sun (Figure 20), the sun's rays are received vertically at places in the torrid zone north of the equator and obliquely at places within a distance of $90^{\circ}$ north of the torrid zone. During this time the sun's rays cover the north pole continuously, causing the very long days in the frigid zone. It is then summer in the northern hemisphere, the days being longer and warmer there than in the southern hemisphere, because the rays of the sun are more nearly 
vertical over that whole portion of the globe. On the twenty-first of June the rays of the sun fall vertically on the Tropic of Cancer, $23 \frac{1}{2}^{\circ}$ north of the equator. The sun has now reached its farthest point north, the days being longest and nights shortest at this time in the frigid and temperate zones.

From autumn to spring the north pole is turned away from the sun. It is then winter in the northern latitudes,

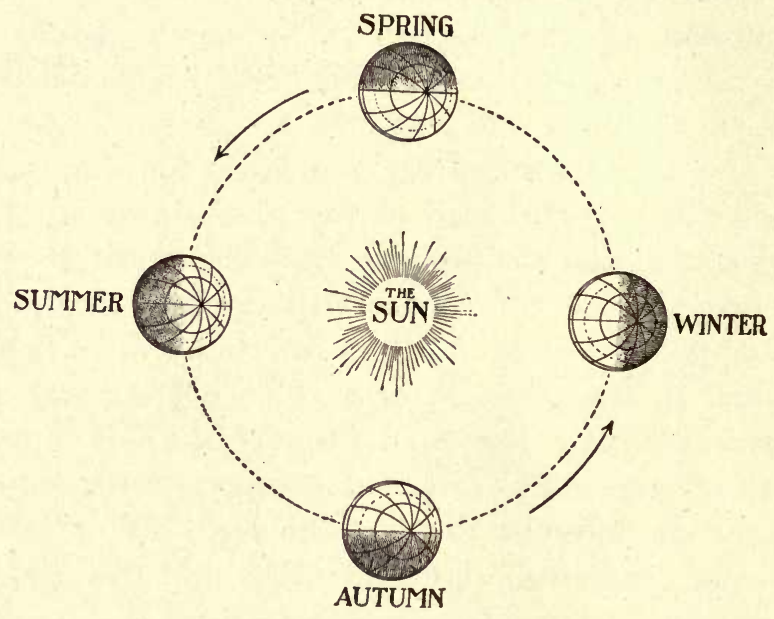

Fig. 20. - Change of Seasons.

and the days are shorter and colder than in the southern latitudes. On December twenty-first the sun reaches its most southern point, the direct rays falling on the Tropic of Capricorn, $23 \frac{1}{2}^{\circ}$ south of the equator. In its apparent moving from the Tropic of Cancer to the Tropic of Capricorn and back, the sun seems to cross the equator twice, the vertical rays falling on that imaginary line about March twenty-first and September twenty-third. 


\section{QUESTIONS}

1. Why do we see no stars in the day time? How may planets be distinguished from true stars?

2. What planets are visible to the naked eye? What planets have no satellites?

3. How often does Halley's Comet make its appearance? When did it last appear?

4. Name two constellations that are visible on any clear night in the northern hemisphere. Why is the Pole Star so called?

5. How many rotations does the earth make while the moon is making one?

6. Does the moon have day and night? What is the length of a "day" on the moon?

7. What simple proofs are there that the earth is round?

8. What proportion of the earth's diameter is the height of the highest mountain?

9. If a man left Havana, Cuba, about June twenty-first and traveled slowly to Rio Janeiro, reaching there about December twenty-first, what changes of season would he experience?

10. What part of the earth's surface is illuminated by the sun at one time? Why does the lighted space move to the westward? How many degrees per hour does it move?

11. If the sun rises at 7 A.M. at places on the 75 th meridian west longitude, at what meridian will it rise an hour later?

12. When it is eight o'clock at Philadelphia, what time is it in St. Louis? Pittsburg? Los Angeles? Detroit? Cleveland? 


\section{CHAPTER II}

\section{MATTER AND ITS PROPERTIES}

OuR previous study of geography has given us some ideas of the material or matter of which the world is composed. Matter is defined as anything which occupies space. A substance is any particular kind of matter. The earth is composed of many different substances rocks and soils, water, air, and various forms of animal life that use the soil, the water, and the air to sustain their lives.

Matter exists in three different forms, namely: solids, liquids, and gases, and all the substances of the earth exist in one or more of these forms. Many solids may be changed to liquids by the application of heat. Metals are the best examples of this phenomenon. Water exists in all three states, solid, liquid, and gaseous ; as ice, water, and steam.

A solid has a definite volume and a definite shape, as a stone or a piece of wood. A liquid has definite volume, but the shape is that of the containing vessel. A gas has neither definite volume nor definite shape, but expands indefinitely as the pressure on it decreases.

Constitution of Matter. - To know that a certain substance is called wood, and that one article is made of glass while another is made of iron, is not enough to satisfy the inquiring mind. We want to know what these substances are composed of. 
While man has succeeded in combining a number of elements, or fundamental substances, in supposedly new ways to form many new compounds, yet all these substances that man has been working with have existed in some form since the very beginning of time. We have found a number of elemental things on the earth, but the number is not so large as might be supposed, for while we have several hundred thousand different compound substances they are all composed of combinations of about eighty elements.

A few of these fundamental substances are well known to us, such as iron, lead, zinc, copper, tin, aluminum, mercury, nickel, silver, gold, and platinum among the metals ; oxygen, hydrogen, and nitrogen among the gases, and carbon, which does not belong with either the metals or the gases. Our bodies are composed chiefly of carbon and three of these gases - oxygen, hydrogen, and nitrogen.

We might think at first that if we knew the properties of each of the elements, it would be quite easy to predict the properties of each of the compounds of these same elements. This, however, is far from being the case. As the elements combine, they lose their individuality entirely. For example, if we combine oxygen and hydrogen, two gases, we have water, very different in appearance and different in all its properties (cf. p. 94).

Just how the elements differ from one another is hard to tell. All the elements are composed of extremely small particles, called atoms. Atoms are so very small that they cannot be seen with the most powerful microscopes that have been made. There are just as many different kinds of atoms as there are elemental substances. We use a different term to designate the particles formed when two or more of these atoms unite. We do not 
speak of an atom of water, for the smallest particle of water that can exist is composed of one atom of oxygen combined with two atoms of hydrogen. This combination of atoms we call a molecule of water. A molecule is the smallest particle of water that can possibly exist; for if the atoms of oxygen and hydrogen are separated, we no longer have water but the two elements in the form of gases.

Molecules are usually composed of atoms of different kinds; the atoms may be all of one kind, however. The molecules of some substances are composed of a large number of atoms of different kinds. The molecule of alum is composed of at least one hundred atoms. In our work on electricity we shall have something more to say about atoms and the way they are held together.

Properties of Matter. - With so many different people in the world it would seem at first impossible to distinguish the individuals. However, our experience has shown us that with all the millions of human beings there are always individual characteristics or properties which enable us to tell one from another. It is the same way with the substances which go to make up the earth.

Those characteristics or properties which are held in common by all substances are called general properties, and those properties which belong only to certain kinds of matter and enable us to distinguish one substance from another are called special properties.

All matter occupies space to the exclusion of all other matter from that same space.

Experiment r. - Fit a two-hole rubber stopper to a pint bottle (Figure 21). Through one hole of the stopper pass a funnel tube and through the other pass a delivery tube leading to a bowl of water. Pour water into the funnel tube. What causes the 
bubbles in the bowl of water? Now close the delivery tube and pour more water into the funnel tube. Does the water go into the bottle as before? Is air matter? In this respect air is like all other matter. Air, however, has other properties which enable us to distinguish it from other gases, such as hydrogen or carbon dioxide.

Other examples of general properties of matter are weight, inertia, and porosity.

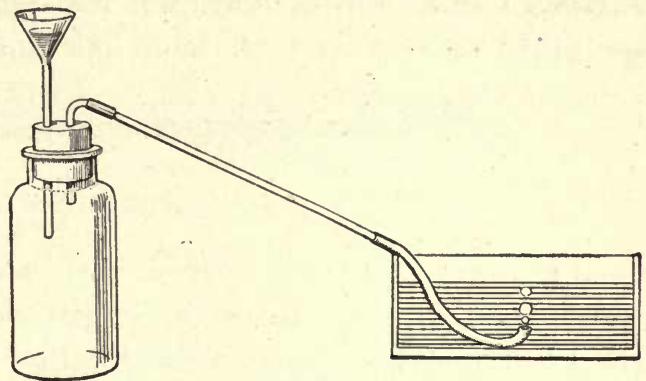

FIg. 21.

We can easily tell salt from sugar by the taste. If we examine a piece of chalk and a piece of iron, we find that the chalk is more brittle than the iron. The iron is also quite heavy and will scratch a stone, while the chalk is light and will leave a white mark on the stone. Glass and wood have quite different properties. Glass is heavier than water, transparent, brittle, and very hard. Wood on the other hand is lighter than water, opaque, not so brittle as glass, and so soft that it may be cut easily with a knife. These properties are special properties, and we might make a long list of them, for there are enough to enable us to distinguish all the thousands of substances that are known. Many of these properties are relative, as we may have observed from the illustrations given above. We say that a substance is harder than another, or that it is more elastic, or that its color is a deeper shade of red, and thus by comparison distinguish one substance from another.

Measurement. - It is necessary in our discussion of 
matter and space to agree on certain standards by which they shall be measured. There have been such standards used by all the civilized nations, but they have been of various values. For example, the unit of length corresponding to the English foot has had different lengths

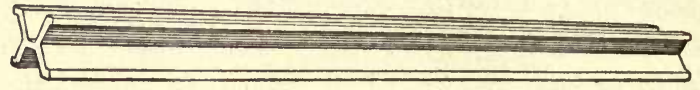

Fig. 22. - Standard Yard Bar.

in different countries. This unit was usually derived from the supposed average length of the human foot. In England, the yard was finally established as the standard unit of length and was supposed to represent the length of the arm of Henry the First, but later the standard was more definitely fixed and is now the length of a metal bar kept in London (Figure 22): The United States uses the same standard, a copy of the standard

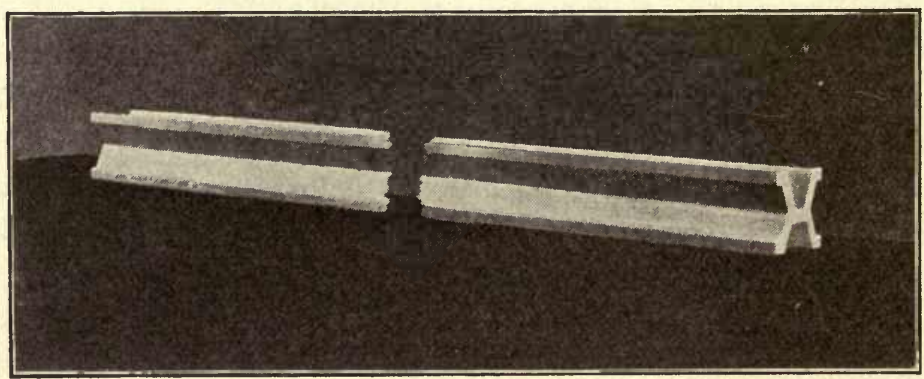

Courtesy of Bureau of Standards, Washington, D.C.

Fig. 23. - United States National Prototype Meter Bar.

yard being kept at our Capitol. The foot was arbitrarily taken as one third of the yard, and the other units were derived in the same way. There are 12 inches in a foot, 3 feet in a yard, $5 \frac{1}{2}$ yards or $16 \frac{1}{2}$ feet in a rod, and 320 rods in a mile. 
It will be seen that there is very little relation between these different numbers and that some of them are difficult to use as multipliers. This led the people of Europe to adopt a different system of measurements known as the metric system.

The Metric System. - At the time of the French Revolution a commission was appointed in France to

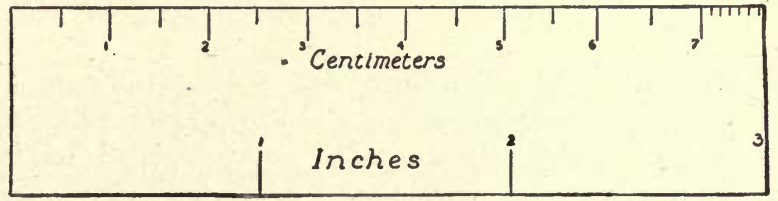

F'1G. 24. - Comparison of the Centimeter with the Inch.

devise a system of measurements which would do away with the existing confusion arising from the use of so many different standards in different localities. The system was adopted in France in 1793 and has since been adopted by the governments of nearly all the large nations except England and the United States. In science, however, it is used even by FIG. 25. - Relative Size of Kilogram and these nations.

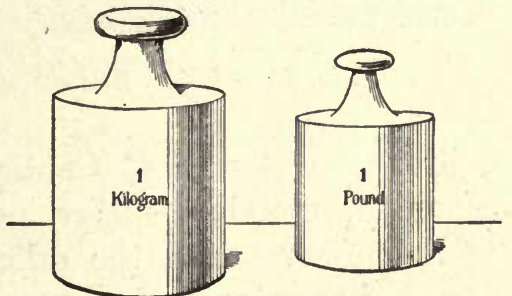

The meter is the standard unit of length of the metric system. It is the distance between two transverse lines ruled on a bar of platinum which is kept in the palace of the Archives in Paris (Figure 23). The Government of France has made a number of copies of this bar and distributed them among the principal governments of the world. Two of them are kept in the Capitol of the United States at 
Washington. The length of the meter in terms of the English system is 39.37 inches (Figure 24).

The standard unit of volume of the metric system is the liter. It is the volume of a cube each edge of which is one tenth of a meter. The liter is a little larger than a quart, being equivalent to 1.057 quarts.

The standard unit of weight of the metric system is the kilogram (Figures 25-26). The kilogram is the weight of

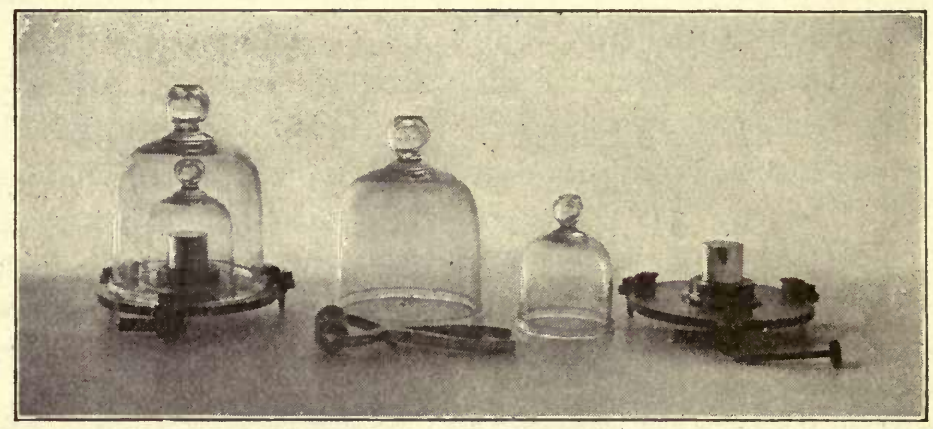

Courtesy of Bureau of Standards, Washington, D.C.

Fig. 26. - United States National Prototype Kilogram.

a liter of water of $4^{\circ}$ Centigrade or $39.20^{\circ}$ Fahrenheit. Since the standard meter has been measured in terms of light waves and the other standards are defined in terms of the standard meter, it would now be possible to make new standards if those which now exist should be destroyed.

\section{Metric Tables}

LENGTH

10 millimeters $(\mathrm{mm})=$.1 centimeter

10 centimeters $(\mathrm{cm})=$.1 decimeter

10 decimeters $(\mathrm{dm})=$.1 meter

1000 meters $(\mathrm{m})=$.1 kilometer 
Surface Measurement

100 square millimeters $=1$ square centimeter

100 square centimeters $=1$ square decimeter

100 square decimeters $=1$ square meter

100 square meters $\quad=1$ are

Cubic Measure

1000 cubic millimeters (c.mm.) $=1$ cubic centimeter 1000 cubic centimeters (c.c.) $=1$ cubic decimeter 1000 cubic decimeters (c.dm.) $=1$ cubic meter

\section{WeIGHT}

10 milligrams (mg.) $=1$ centigram

10 centigrams (cg.) $=1$ decigram

10 decigrams (dg.) $=1$ gram

1000 grams (g.) $=1$ kilogram

1000 kilograms $(\mathrm{kg}) .=1$ metric ton

\section{Table of English Equivalents \\ 1 meter $=39.37$ inches, or 3.28 feet \\ 1 liter $\quad=1.057$ quarts. \\ 1 kilogram $=2.2046$ pounds \\ 1 metric ton $=2204.6$ pounds}

A little study will convince us that the metric system has some advantages over other systems. It is a decimal system throughout and can be used just as our decimal system of coinage is used. For example, we may write five dollars, three dimes, and five cents as $\$ 5.35$; five meters, two decimeters, one centimeter, and three millimeters may be written 5.213 meters.

The Measurement of Length. - All measurements of length are simply the comparison of the length of the object with some standard. If the measurement is to be taken in the metric system, the comparison is with the standard meter bar ; if by the English system, the comparison is with the standard yard at London. 
The Measurement of Volume. - All bodies have three dimensions, and if the shape of the body to be measured is regular, it will be easy to measure the length, breadth, and thickness and compute the volume. If the solid is irregular, it is necessary to use other means in determining the volume of space it occupies. When a solid is immersed in a liquid, it displaces its own volume of the liquid, and this means may be used in determining the volume of such solids as cannot be accurately measured. Can you devise a method of determining the volume of a solid which floats in water?

Experiment 2. - Determine the volume of a cylinder of wood by first measuring it with a ruler and then by the displacement method. How do the two determinations compare?

Measurement of Mass. - Mass must not be confused with volume. The measurement of mass simply means

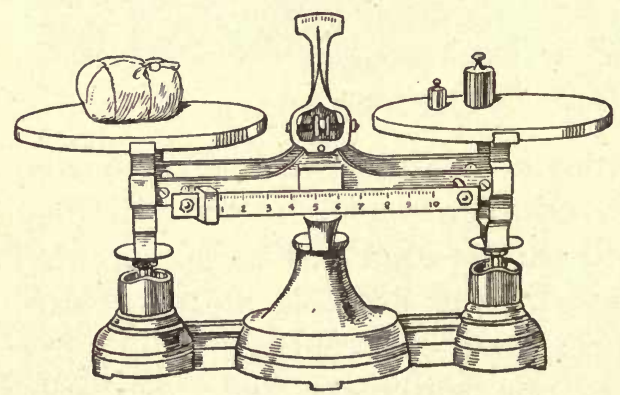

Fig. 27. - Common Tip Scales or Balances. that we shall determine the weight of an object by comparing it with some standard. We are able to detect differences in weight by our muscular sense if the differences are great enough, but we should not care to rely upon the judgment of the grocer who sold his sugar by weight as estimated by his muscles. For this work a balance is used (Figure 27). If the arms of the balance are of equal length, the weights used must be as heavy as the object to be weighed. The object to be weighed is then placed on one side and the weights 
are placed on the other side. The sum of the weights necessary to balance the beam is the weight of the object. For heavier masses a system of levers is used, as in a platform scale where the weights used are fractional multiples of the reading on the scale beam.

\section{Density and Specific Gravity. -} Since equal volumes of different substances such as iron, wood, lead, aluminum, and granite do not have the same mass or weight, we use the term density to indicate the differences in the weights of definite volumes of these various substances. Before we are very old we learn that some substances are heavier than others. A piece of iron is heavier than a piece of wood of the same size, and we say that the density of iron is greater than that of wood. To get the mass of a body we weigh it and to get the density we divide
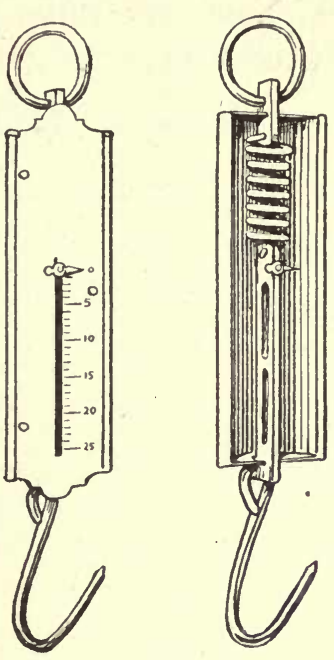

FIG. 28. - The Spring

Balance.

Quite commonly used in the determination of small weights. its weight in grams by its volume in cubic centimeters. Density determined by this procedure also gives the numerical value of the specific gravity of the substance. Specific gravity is the ratio of the weight of a substance to the weight of an equal volume of water.

$$
\text { Density }=\frac{\text { mass in grams }}{\text { volume in c.c. }}
$$

Specific gravity $=\frac{\text { weight of body }}{\text { weight of equal volume of water }}$

Density in grams per cubic centimeter is specific gravity, but density in terms of any other system of weights 
and measures will not correspond numerically to the specific gravity.

A cubic foot of water weighs 62.3 pounds or about 1000 ounces, while by definition a cubic centimeter of water weighs one gram.

\section{Densities of Liquids and Solids in Grams per Cubic Centimeter}

\section{Liquids}

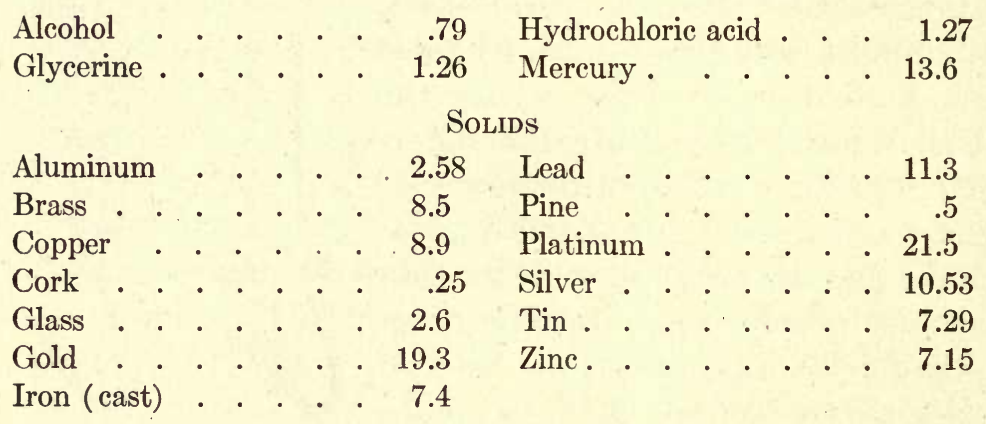

\section{QUESTIONS AND PROBLEMS}

1. A tank is 2 feet deep, 3 feet wide, and 5 feet long. What weight in pounds of water can it hold?

2. A rectangular block of iron is $10 \mathrm{~cm}$. long, $4 \mathrm{~cm}$. deep, and $3 \mathrm{~cm}$. wide and weighs 888 grams. What is the density of the iron?

3 . Find the weight of 5 cubic centimeters of glycerine.

4. How does a molecule differ from an atom?

5. Name ten special properties of matter.

6. In what respects does a liquid differ from a gas?

7. Make a list of properties of the following substances: chalk, glass, coal, lead, and salt.

8. Why should a barrel have a vent hole in it?

9. How does iron differ from copper?

10. Do you see any advantages of the metric system over the English system?

11. What difficulties would there be in changing from our system to the metric system for all measurements? 


\section{CHAPTER III}

\section{ENERGY AND FORCE}

ENERgY appears in so many different forms and its changes from one form to another are made so rapidly, that it is sometimes hard for us to believe that we are dealing with varied forms of the same thing.

The usual definition of energy is capacity for doing work, and we say that work is done when force acts through space. The'words energy and force are often misused for each other in ordinary speech, but in science these two words have distinctive meanings. Force is any cause which alters a body's state of rest or of uniform motion in a straight line. It requires force to stop a moving body just as it requires force to set it in motion when it is in a state of rest. Considerable force must be exerted by a team of horses to start in motion a loaded wagon. After it is started, however, just as much force must be exerted to stop it, but the force necessary to stop it will not all need to come from the horses. There is another force at work which causes the wagon to rub very hard on the surface over which it passes. This force is called gravity or the force of gravitation.

Gravity. - Sir Isaac Newton (1642-1727) first announced the law which we now call the law of gravitation, although it is now quite certain that the much abused Galileo had a very definite knowledge concerning this force. The law states that every body in the universe attracts every other body with a force which varies 
inversely as the square of the distance between the two bodies and which varies directly as the product of the masses of the two bodies. Newton formulated the law in order to account for the fact that the earth pulls bodies toward it and also to account for the maintenance of the planets and their satellites in their respective orbits.

We know that objects which are free to fall do fall to the earth, and probably most of us would be willing

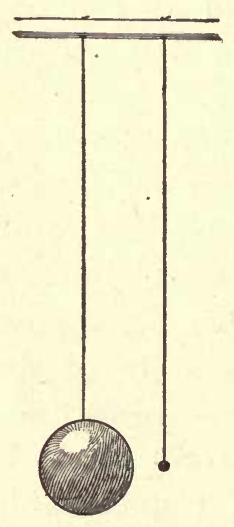

FIG. 29. to accept this without question, because we become so accustomed to this force that the question "why" might never occur to us. It is not easy to imagine this great force. It acts through space, but there are no attachments between the bodies attracted. The apple on the tree is exerting a pull on the earth just as the earth is exerting a pull on the apple. Whenever the force which holds the stem of the apple to the tree is less than the pull of the earth, the apple falls to the ground. Why does not the earth seem to fall to the apple?

If two balls, one quite large and the other small, are hung side by side (Figure 29) the small ball will be drawn toward the large one and the supporting cord will no longer hang plumb.

When we say that the law of gravitation is universal, we simply mean that it applies to all the matter of the universe. It exists between the earth, sun, moon, and stars as well as between the objects which are so near us.

Weight. - Weight is simply the measure of the force of gravitation on any particular mass or quantity of matter. The quantity of matter in a body is the mass of the body. In the last chapter we learned that the 
gram is the unit of mass in the study of science, although we are accustomed to think of mass in terms of pounds of the English system. The attraction, or pull, that the earth exerts on a given mass is irrespective of the kind of matter in the body. The pull on a pound of feathers is the same as the pull on a pound of lead.

Center of Gravity. - A large body such as a block of stone is made up of countless small particles of matter. Now according to our law of gravitation, each little particle of matter attracts and will be attracted by the others and by the earth. In other words there

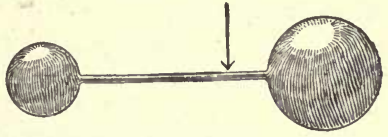

Fig. 30. - Center of Gravity or Center of Mass of a Complex Form. will be a countless number of little pulls between these particles of matter in the stone and the earth, and it is evident that the sum of these little pulls between the particles and the earth will equal the total pull of the earth on that

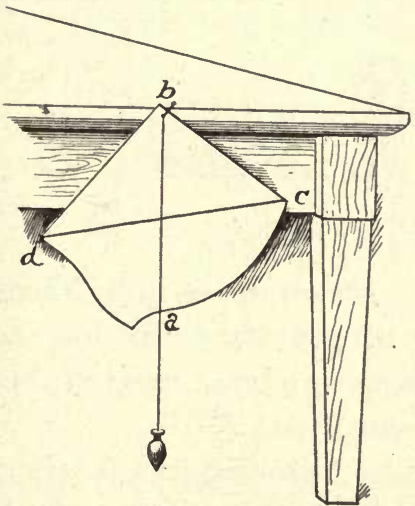

Fig. 31. - Determining the Center of Gravity. body. The point in a body at which a single force equal in magnitude to the weight of the body and directed upward can be applied so that the body will remain at rest in whatever position it is placed is called the center of gravity. It is the place at which all the weight may be said to be. To do this mechanically we simply find a place at which the body will balance (Figure 30).

Experiment 3. - The center of gravity of a piece of uniformly rolled metal may be found in the following manner. Support it from one 
corner by a pin stuck through a hole near its edge. Hang a plumb line from the pin and draw a line $b a$ (Figure 31) parallel to and directly under the plumb line. Now hang the piece of metal from another point $c$ and draw another line $c d$ in the same way that the line $a b$ was drawn. The intersection of these two lines will be the center of gravity of the piece of metal. It should balance at this point, and if a needle is passed through a hole made at this point, the metal should remain at rest in whatever position it is placed.

The center of gravity of the earth is somewhere near the geometrical center of it. The pull of the earth for mathematical purposes may be considered to be at that point. Now an object at the poles is about thirteen miles nearer to the center of the earth than an object at the equator, and as a consequence an object will weigh more at the poles, the difference being about one part in 590. What will be the effect on the weight of objects of taking them up in the air several miles?

Objects are said to be in "stable" or " unstable" equilibrium according to the position of the center of

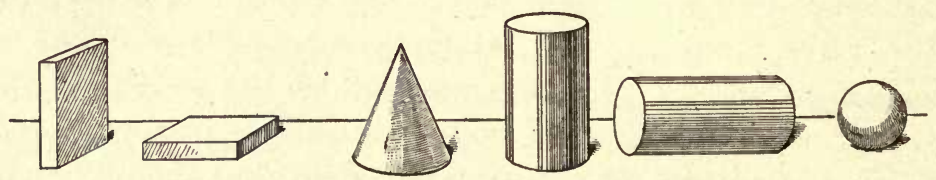

Fig. 32. - Stable and Unstable Equilibrium.

gravity of the mass (Figure 32). An object is in its most stable position when its center of gravity is as low as possible. In what position will a sphere be in most stable equilibrium? A cylinder? A cone?

Effect of Air on Falling Bodies. - A stone and a feather, if dropped from the top of a tall building, will not reach the earth in the same time. The reason is that the air resists being pushed out of the way, and the heavier object more easily overcomes this resistance. If the air 
is exhausted from a tube arranged for the purpose, it will be found that the feather will fall as rapidly as the stone, because it is not influenced by the resistance of the air (Figure 33).

Energy. - It is quite easy for us to realize that energy may be transferred from one body to another.' A moving billiard ball strikes another ball "full," and apparently all of its energy is immediately transferred to the second ball, while the first ball stops. In fact, however, not quite all the energy of the first ball was transferred to the second. Part of it went into other forms of energy, as heat and sound. The billiard ball in motion possesses energy of motion. The name which scientists have given this form of energy is kinetic

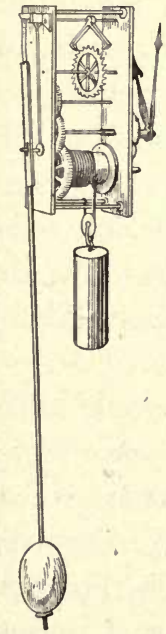

FIG. 34. energy, a name derived from the Greek word kineo, moving. In recent years we have had a new word from this FIg. 33. root, kinematograph or cinematograph. Kinetic energy is one of the several distinct forms of energy which we shall learn about in our work in science.

We may pull a stone away from the earth, and the earth pulls it back again. If, however, we pull the stone away from the earth and support it there, the stone will have the power of doing work when it is freed. This form of energy is called potential energy. There are numerous instances of such energy. The weights of a clock when lifted possess potential energy (Figure 34). Confined steam furnishes another example. It is the energy of strain or deformation as well as the energy due to position. Potential 
energy until released is valueless. It is simply because it can readily be transformed into kinetic energy that we think of it as energy. Although there are several distinct forms of energy, all energy is either kinetic or potential. The capacity for doing work can be possessed only by a body already in motion or by one held under some sort of strain. Other forms of energy are chemical energy, light energy, heat energy, and electrical energy.

Inertia and Force. - All matter has a tendency to continue in its state of rest or motion. If it is at rest,
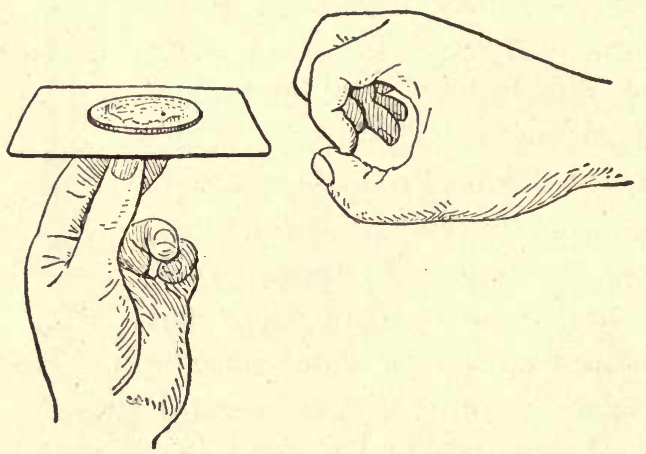

Fig. 35. - Illustration of Inertia. The inertia of the coin causes it to remain on the fingers when the card is snapped away. force is required to put it in motion, and when it is in motion, force is required to bring it to rest again or even to change its rate of motion. This tendency of matter to continue in a state of rest or motion we call inertia (Figure 35). We have often noticed this tendency, but we have never heard its name. As the street car starts, the people who are standing are thrown backward and as it stops suddenly they are thrown forward. This is on account of the inertia of their bodies. A loaded wagon requires more force to start it in motion than to keep it in motion when once started, and if it is moving on a level pavement, it requires considerable force in addition to that exerted by gravity to bring it to a stop. The direction of the motion is always in a straight line or simply a 
continuation of motion at any particular instant. When mud is thrown from a rapidly rotating carriage wheel, it does not follow the curve of the wheel, but flies off in a straight line with the direction in which it was going when it left the wheel. The same phenomenon is noticed when a sling is rotated (Figure 36) and suddenly released. The weighted sling goes in a straight line which is tangent to the circle in which it was rotating.

Centrifugal Force. - The force which caused the sling to pull so hard on the hand is centrifugal force or center fleeing force. It is the same force which causes the water to stay in a rotating

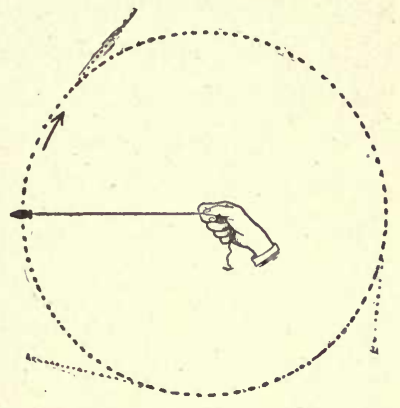

Fig. 36. - Centrifugal Force. bucket. It is not a new force but a name given to this particular manifestation of inertia. It is the force which sometimes causes emery wheels to burst, which enables us to separate the milk from the cream in the separator by causing the milk to move farther out than the lighter cream, and which makes the equatorial diameter of the earth greater than the polar diameter.

At the beginning of this chapter we said it would be necessary to think of force and energy as having distinct meanings. There are a number of forces with which we are in almost daily contact : gravitational force; muscular force, such as that exerted by man and beasts of burden; the force of the wind; the force of expanding gases; and others which are not so common.

Muscular force is a force which will always be used. Even if beasts of burden should no longer be needed, muscular force exerted by man will always be a neces- 
sity. Our very existence depends on it. Our health requires it.

It is necessary to take into account the element of time in calculating the amount of work done by a force. If one horse can do a piece of work in one hour and it requires three hours for another to do the same work, there is a great difference in their rate of doing work. This rate of doing work is called power. James Watt (17361819), who invented the steam engine, thought that the average horse could do 33,000 foot pounds of work per minute; that is, raise 33,000 pounds one foot in one minute or 550 pounds one foot in one second. While this number is probably too high, it has been taken as the unit of power in English-speaking countries and has been named the horse pouer (H.P.). Steam engines and motors are usually rated in horse power.

Force of Expanding Gases. - There are a number of ways in which the force of expanding gas does work. The steam engine is simply a device for utilizing the energy of steam. When steam is produced under high pressure and confined, it is potential energy, but when it is allowed to expand in a cylinder, the energy is used to produce motion which may be employed in various ways (Figure 37).

In the gasoline engine, the energy is set free by the explosion of gasoline vapor and air. This explosion produces a large volume of gases, the expansion of which causes the piston to move, as in a steam engine.

The efficiency of modern war engines, some of which throw immense shells to a distance of twenty-five miles, is due to the production of compounds which explode with the evolution of large quantities of gases. With these explosives it is necessary that the energy should 


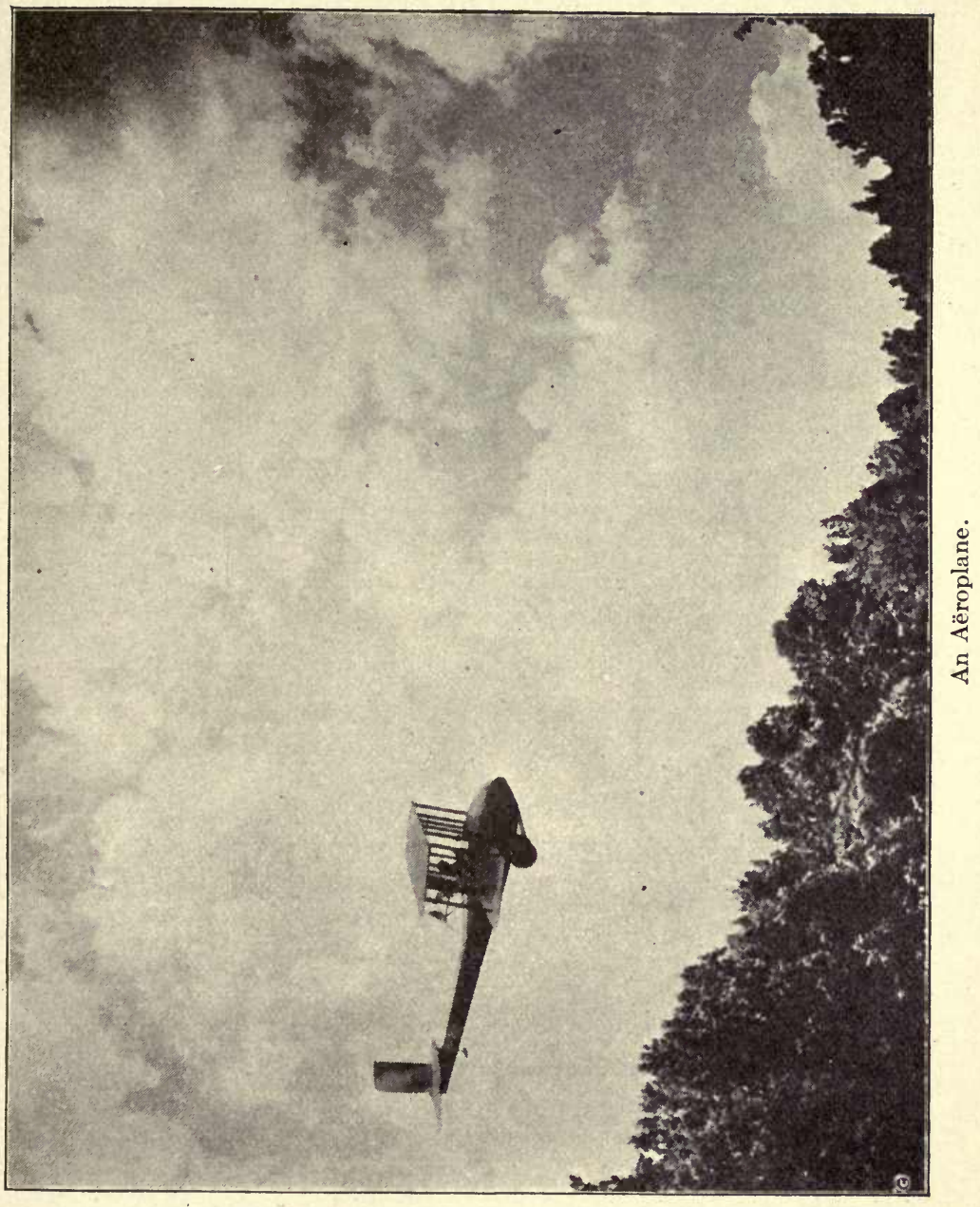



be released quickly. Many of the most violent explosives possess less stored-up energy than the same weight of other materials, but in the case of the explosive the energy is all released in an instant, while with a substance such as coal considerable time is required to release its energy. When powder explodes, a gas is formed which would occupy at ordinary pressure several hundred times as

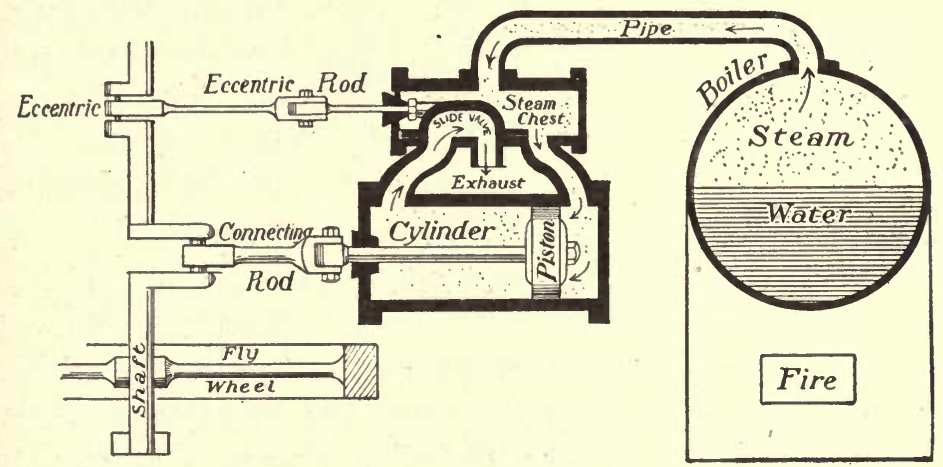

Fig. 37. - Diagram Showing Action of Steam in a Common Steam Engine of the Reciprocating Type.

much space as the original powder. In the gun the gas is under great pressure on account of the limited space, and as it expands it drives the bullet or projectile rapidly before it.

Molecular Forces. - In the case of gases, the molecules seem to have little attraction for each other. With liquids, however, the molecules resist being pulled apart, and with solids this force is very great, as is shown by the weight required to break a small steel wire.

Elasticity. - The tendency of a substance to return to its original form and volume after having been stretched or distorted is called elasticity. Gases have perfect elasticity of rolume, but they have no shape. The elas- 
ticity of air is the property which makes it so valuable as a shock absorber in pneumatic tires. If you strike the handle of a bicycle pump when the outlet is closed, the handle will fly back to its first position.

Almost all solids are elastic to some extent. You may test the elasticity of rubber, steel, marble, and

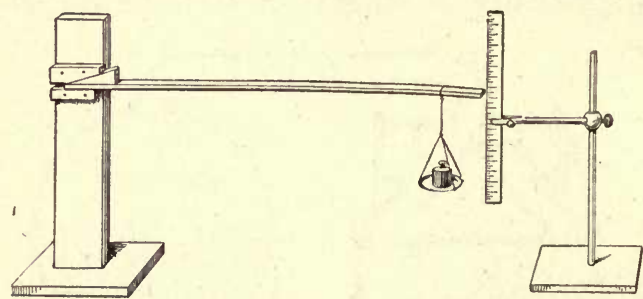

Fig. 38. - A Simple Apparatus for Testing the Elastivity of a Wooden Bar. wood by dropping balls of these substances on a slab of iron or stone and noting the height of the rebound.

Experiment 4. Test the bending elasticity of a wooden yardstick (Figure 38 ) by adding weights to the scale pan and reading the amount of the bend from the scale. Is there any'relation between the amount of the bending and the weights? Give six uses of elasticity in a commercial way.

Cohesion and Adhesion. - The attractive force which binds molecules of the same kind together is called cohesion, and the force which binds together molecules of unlike kind is called adhesion. It is cohesion which produces the rigidity of solids, and it is adhesion which enables us to glue together two pieces of wood. This distinction between cohesion and adhesion is one made simply for convenience and not because the forces are essentially different. We have no reason for supposing that the force holding particles of wood to molecules of glue is different from that holding molecules of glue together.

If a piece of plate glass is held flat on the surface of water, it will require some force to remove it (Figure 39). 
If we examine the glass after it has been removed, we shall find it wet, showing that when we lifted the glass plate, we separated water molecules from water molecules and not water from glass. This force may be measured easily by arranging a balance, as shown in Figure 40, and determining the weight that is required to lift the plate of glass and break the cohesive force. The cohesive force will be this weight less the actual weight of the plate of glass. If the size of the plate is known, the force per square inch may be easily determined.

Shape of Free Liquid. - Small

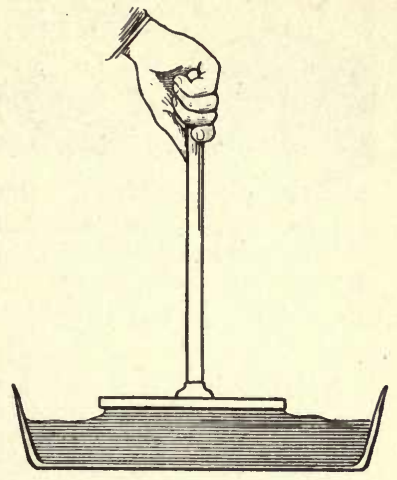

Fig. 39. - The glass plate adheres to the water. drops of a liquid are spherical in shape, for the surface of a sphere is the minimum surface for a given mass, and the cohesive forces, acting between the molecules of a

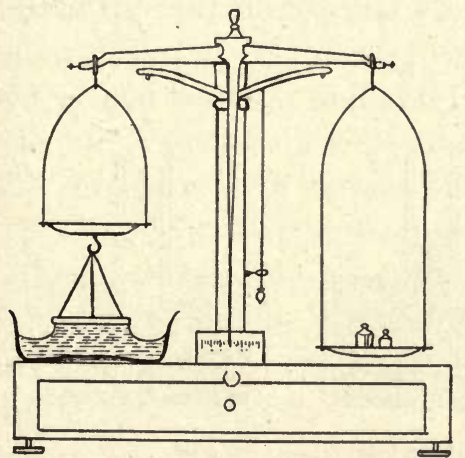

Fig. 40.- Measuring Cohesive Force. given mass of liquid, tend to reduce it to the volume having the smallest possible surface. In larger quantities of liquids, the force of gravitation is large enough to be more of a factor in determining the shape than the cohesive forces, hence the drops flatten out.

On the surface of a liquid the attractive forces between the molecules are sufficiently strong to form a film. A needle which is much heavier than water may be floated 
on its surface. When the needle breaks through the film, it sinks quickly to the bottom.
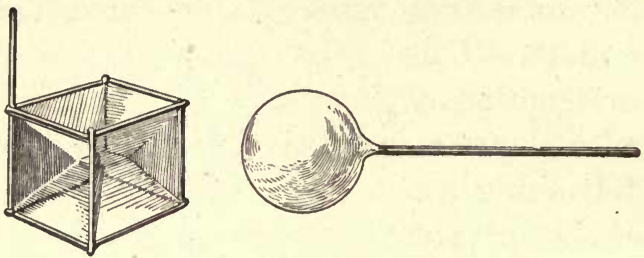

FIG. 41.- Interesting film forms may be obtained by using wire frames of different shapes.

Experiment 5 . Make a wire frame in the form of a cube similar to the one shown in Figure 41 and dip it into a strong solution of soapsuds. Note the tendency of the films to contract to the shortest lines between the different points of the cube.

Experiment 6. - If, after blowing a soap bubble, one discontinues before the bubble breaks from the tube, the bubble will slowly decrease in size as the contraction of the film forces the air out.

How is shot made?

Capillarity. - The tendency of liquids to rise in hairlike tubes is capillarity or capillary attraction. Capillarity takes place in all fibrous materials as well as in tubes. The rise of sap in trees depends largely on this property.

Experiment 7. - Heat some soft glass tubing and draw it out into smaller tubes. Break these tubes into pieces of four or five
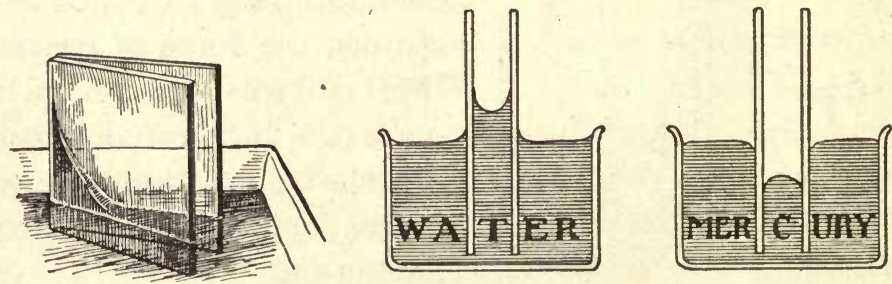

Fig. 42.- Capillarity.

The water is lifted where it touches glass, while the mercury is depressed.

inches in length. Hold them vertically and lower them into a glass of water. Note the sudden rise of the water in the tubes 
(Figure 42). In which tube does the water rise the highest? Try mercury instead of water. What happens?

Touch a lump of sugar to the surface of a glass of water and watch the rise of the water. Mention other examples of capillarity.

Capillary action is due to two forces, cohesion in the water or liquid and adhesion between the water and the tube. If a glass tube of large diameter is used, the water is raised only at the edges, since the elastic surface of the water cannot exert enough force to raise all the water of the tube; but if the tube is small, the weight of the water in the tube is small and the whole column of water is carried up the tube by the force of the contraction of the elastic surface. We noticed that the height of the water was different in tubes of different diam-

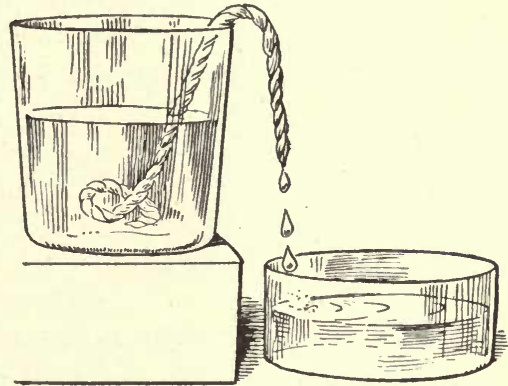

Fig. 43.-An Example of Capillarity.

eter. The water is raised in each case until its weight is just equal to the force of the elastic surface of the water. Will the water ever run out of the top of the tube? Why?

If a wick is wet and allowed to hang over the side of a dish of water (Figure 43), the water will rise in the wick and flow over the side of the dish. The flame of the kerosene lamp is fed by the oil that is carried to it by capillarity.

Mention three uses of capillarity.

Diffusion. - Diffusion is the close intermingling of gases or liquids which takes place independently of gravity or of any currents in the substances themselves. 
Experiment 8. - Introduce a solution of copper sulphate into the bottom of a hydrometer jar or any tall jar filled with water. This may be done by pouring the solution through a funnel

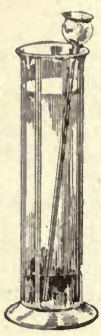

Fig. 44.-The eopper sulphate solution slowly diffuses through the entire jar, as is shown by the change in color. tube which reaches to the bottom of the jar. Allow the jar to stand for a few days, noting the change from day to day (Figure 44).

Experiment 9. - Into a small jar put a few drops of strong ammonia water and cover it with a piece of paper. Into a second jar put a few drops of concentrated hydrochloric acid and invert on the first jar. Now withdraw the paper. The chemical action which follows is evidence of the rapid diffusion of the gases. Although these gases are not visible, their presence may be determined by their odors. As they diffuse, a new substance is formed.

Some few liquids will not diffuse. Devise an experiment to show that oil and water will not diffuse. Can you put oil, alcohol, and water into the same bottle so that they will not mix? How?

Osmosis. - Osmosis is the diffusion of liquids or gases through porous walls or membranes.

Experiment 10. - Tie a piece of parchment or bladder over the mouth of a large funnel tube, and after having filled it to the depth of about three inches with a saturated solution of copper sulphate, lower the funnel end into a jar of water until the level of the solution in the tube is the same as that of the water in the jar. Support it in this manner and allow it to stand for several hours, when it will be found that the blue solution is several inches higher than the level of the water in the jar. This is due to the fact that although the water and the solution pass through the membrane in opposite directions, the water passes through at a much more rapid rate, thereby diluting and increasing the volume of the solution in the funnel tube (Figure 45). The water in the jar gradually acquires a blue tinge, proving that some of the solution of copper sulphate has passed through the membrane into the jar. If the water and the solution had diffused at the same rate, 
there would have been no change in the level of the liquids, but osmosis would have taken place just the same, as indicated by the color of the liquids. The same experiment may be performed with a solution of sugar with excellent results. By tasting the water in the jar, the presence of sugar may be detected.

The essential for an experiment illustrating osmosis is to have two solutions of different densities separated by a semipermeable partition. Water will pass through a membrane several times as fast as a strong salt solution, and hydrogen will diffuse four times as rapidly as air.

Osmosis is important in the distribution of food to the different parts of plants and animals. That part of digestion known as

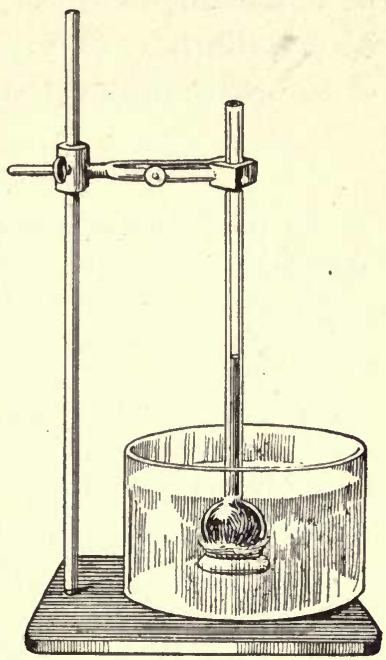

Fig. 45.-Osmosis.

Note the height of the liquid in the tube. absorption is largely osmotic action, the food passing through the walls of the stomach and the intestines. Since only substances known as crystalloids pass through animal membranes, starch, which is an amorphous substance and a very important article of food, is changed to sugar before it is absorbed. This particular kind of osmosis is called dialysis.

Plants get most of their nourishment from the' soil, and osmosis seems to furnish a logical explanation of the absorption of food by the roots of plants. By the process of osmosis the roots take in food in the form of dilute solutions which contain the plant food, while the denser solution of cell sap inside the roots does not pass readily 
out again. Each little fiber of the root is really an osmotic apparatus, and the myriads of them are able to take in sufficient food for the entire plant. Then, assisted by capillarity, these solutions reach the different parts of the plant where the food is assimilated.

\section{QUESTIONS}

1. Why does blotting paper absorb ink better than writing paper?

2. What is the function of a lamp wick?

3. How do we prove that air is matter?

4. What property of matter enables us to blow soap bubbles?

5. What are the laws of capillarity?

6. Why do feathers fall slowly?

7. What force causes water to flow in streams and rivers?

8. If the earth rotated much faster than it does, what effect would it have upon the weight of objects at the equator? At the poles?

9. What force causes a postage stamp to cling to an envelope?

10. Why does thick molasses flow more slowly than water?

11. If some oil, water, and mercury were placed in a rotating vessel, how would they arrange themselves with reference to distance from the center of the vessel?

12. How does air affect the flight of a thrown ball?

13. Are projectiles and rifle balls affected by the wind? How?

14. What kind of energy is possessed by the water behind a dam?

15. What makes a pendulum move back and forth? Why does a short pendulum move faster than a longer one?

16. What is the reason an automobile skids? Why are chains used on automobile wheels?

17. Which is greater, the cohesive force between water particles or the adhesive force between water and glass? How may you determine the truth of your answer?

18. Why is a golf ball so elastic? 


\section{CHAPTER IV}

\section{MACHINES}

THE adage "Necessity is the Mother of Invention" is especially applicable in respect to the uses to which man has put machines. Work is often accomplished more easily by the use of some simple machine than in any other way. Sometimes it is quite impossible to accomplish a certain piece of work without the aid of a machine. For example, the man who desires to lift a weight of 1000 pounds finds that it is beyond his power. With the aid of a lever the task is easily performed. And so it is with a multitude of applications of the mechanical principles. If materials are to be raised to the top of a building, a rope and pulley are used. If heavy logs are to be loaded on trucks, the inclined plane is used. If a building is to be lifted from its foundations, the screw is used.

The Evolution of Machines. - Machines have been the greatest of civilizing agencies. Primitive man knew nothing concerning even the simplest machines. $\mathrm{He}$ did not realize that he had any use for such devices. 'The sling was probably the first mechanical device used by man. With it he could throw a stone with sufficient force to make it a valuable weapon for protection and for securing food. Since that time all ages have had their inventors. The sling was followed by the lever and the sharp bone or sharp stone, used as a cutting 
instrument. These simple devices enabled man to live better and started him on the road to civilization. His thinking resulted in better places to live, in better protection from his enemies, and in better food. He learned that useful plants would thrive better if they were cared for, and thus began the simplest forms of agriculture. From this time on the development has been very rapid. Man has learned to harness the various forces of nature and set them to work doing the countless tasks that his fancy and needs have set for them. Each generation has had the enormous advantage of the cumulative results of the thinking of all previous generations in the extent of its inventions. At present our daily life is so intimately related to the mechanical world through its devices for producing, collecting, and distributing food, for transportation, and for communication, that the very existence of many people depends upon it.

The six simple machines are: the lever, pulley, wheel and axle, inclined plane, wedge, and screw. It is sometimes difficult to believe that the modern machines use no more than these six mechanical principles in the complicated motions they make and in doing the wonderful things they do, but a careful analysis will show that these machines are made up entirely of combinations. of the six simple machines.

The Principle of Work. - A machine cannot create any energy nor can it do work unless work is done upon it. A machine is simply an apparatus which enables us to apply force advantageously.

When a force moves a body on which it acts, we say that work has been done upon that body. The amount of work done is always equal to the force multiplied by the distance through which it moves the body. Thus 
if a one-pound weight is lifted two feet, we say that the work done is equal to two foot pounds.

Experiment II. - Pass a cord over a pulley as shown in Figure 46. Attach a spring balance $F$ to the end of the cord and a weight $W$ to the other end. What force is required to support the weight? Weigh the weight $W$. The force and weight in this case will be found to be the same. If $F$ moves a certain distance, $W$ will be moved up an equal distance. The force multiplied by the distance through which it moves will just equal the weight multiplied by the distance through which it moves. If $d$ is the distance $F$ moves and $d^{\prime}$ the distance $W$ moves, then

$$
F d=W d^{\prime}
$$

This is the general law of all machines. The power or force multiplied by the distance through which it acts equals the resistance or weight multiplied by the distance through which it acts.

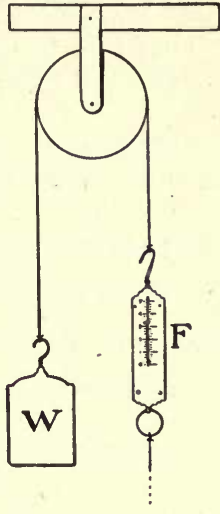

Fig. 46. - The advantage of a fixed pulley is simply one of direction.

The Lever. - This is the most common of all the simple machines. It is a bar of any kind arranged to turn on a rest or pivot, called its fulcrum. The power is the

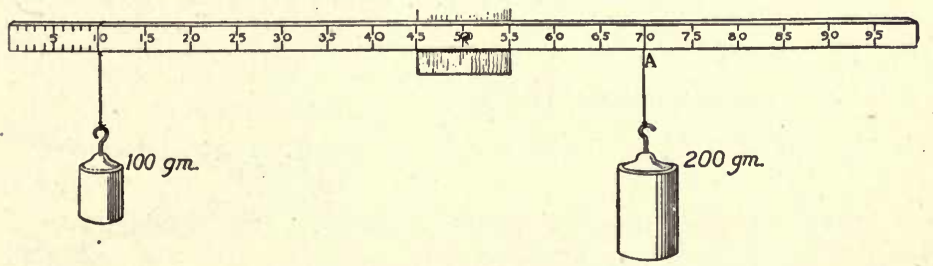

FIg. 47.

force applied to any part of the lever and the weight is the body to be moved or balanced by the application of power. 
Experiment 12. - Pierce a meter stick in the center and balance it on a nail as shown in Figure 47. By means of a thread suspend a 200 -g. weight at $A, 20 \mathrm{~cm}$. from the point of support, the fulcrum. Hang a 100-g. weight on the other arm of the lever and move it until the bar just balances. It will be found that the $100 \mathrm{~g}$. weight is $40 \mathrm{~cm}$. from the fulcrum.

$$
\begin{gathered}
W \times d=F \times d^{\prime} \\
200 \times 20=100 \times 40
\end{gathered}
$$

Perform the experiment with other weights at different distances from the fulcrum. Does the law hold in every case?

Classes of Levers. - There are three classes of levers depending upon the relative location of the power, the fulcrum, and the weight. In the lever of the first class (Figure 48), the fulcrum is placed between the weight and
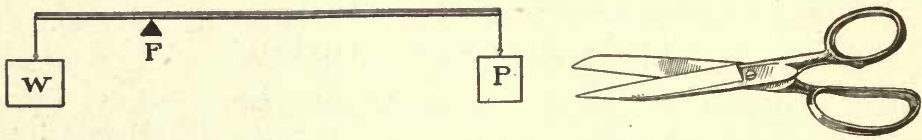

Fig. 48. - Lever of the First Class.

the power. Scissors, weighing balances, pump handles, and crowbars are examples of this class of lever.

In the lever of the second class (Figure 49) the weight is between the fulcrum and the power. The nutcracker
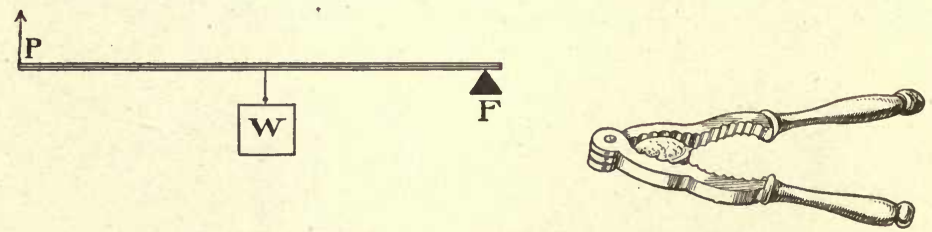

Fig. 49. - Lever of the Second Class.

and the wheelbarrow are levers of the second class. In the nutcracker the hinge is the fulcrum, the nut is the weight, and the force applied to the handles is the power. 
In the lever of the third class (Figure 50), the power is between the fulcrum and the weight, as in sugar tongs, sheep shears, and the treadle on a sewing machine.

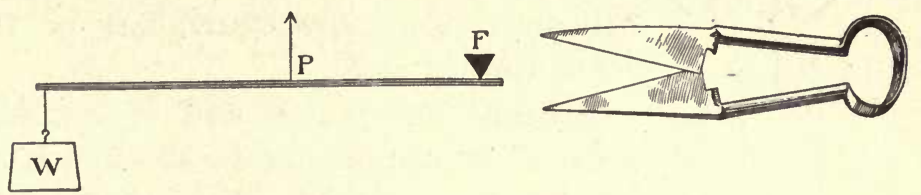

FIG. 50. - Lever of the Third Class.

\section{EXERCISES}

1. What kind of lever is a claw hammer when used to pull a nail? The oar of a boat? Wire cutters?

2. A man places a fulcrum 1 foot from the end of a 6 -foot crowbar. If he presses down on the other end with a force equal to 150 pounds, what weight can he raise?

3 . With the same power and bar, what weight can he raise if the fulcrum is placed 6 inches from the end?

4. On a balanced meter bar a weight of $300 \mathrm{~g}$. is placed 30 $\mathrm{cm}$. from the point of support. Where must a weight of $450 \mathrm{~g}$. be placed to balance it?

5. Where is the fulcrum in a wheelbarrow? The weight? The power?

6. What kind of lever is the forearm? Why is it better than another class of lever?

7. What advantage is gained in a lever when the power is quite close to the fulcrum, as in the case of a boat oar? book?

8. Name three uses of the lever not mentioned in this

9. A 200-pound weight is placed 1 foot from the axle of a wheelbarrow. How much force must be exerted on the handles 5 feet from the axle to lift the weight? (The weight of the wheelbarrow is not considered in this problem.)

10. An oar is $7 \frac{1}{2}$ feet long and the oarlock is $1 \frac{1}{2}$ feet from the handle. If a man pulls on the oar with a force of 100 pounds, what force is exerted on the water? 
Pulleys. - Figure 51 represents the use of a single fixed pulley. In this pulley the only advantage gained

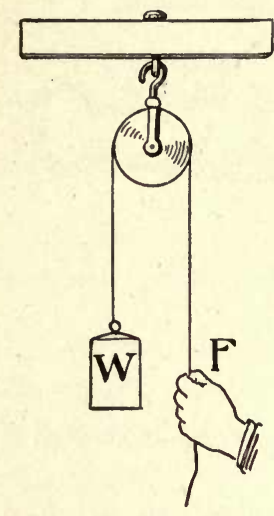

FIG. 51.- Single Fixed Pulley. is direction. By pulling down we may lift a body vertically, but we cannot lift more than the equivalent of the force applied at $F$.

Arrange the pulley and spring balance as shown in Figure 52, so that a single movable pulley is attached to the weight $W$. It is quite evident that the weight is supported equally by each strand of the rope. This may be verified by the spring balance. It should be understood, however, that the weight at $W$ includes the weight of the pulley.

Make a test to see how far the force must be moved to move the weight a distance of 1 foot. Does the law of machines hold in this case of the pulley?

Other arrangements of pulleys are shown in Figure 53. In each case there are four strands of rope supporting the weight. Then the force necessary to support $W$ is one fourth of the total weight, or the weight equals the force multiplied by the number of supporting strands of rope.

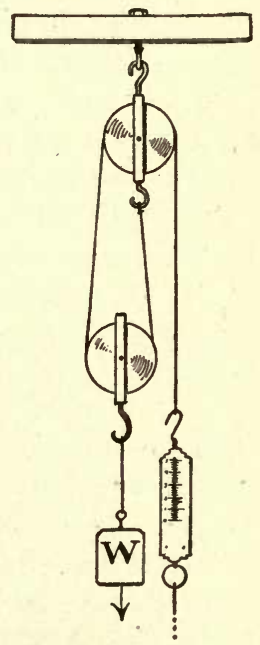

Fig. 52.-Measuring the Mechanical Advantage of a Single Movable Pulley.

$$
W=F n \text {. }
$$

With most arrangements of pulleys there is so much friction that it may seem that the law does not hold. 
The best way to measure the force is to take the mean of the force which will just cause the weight to ascend

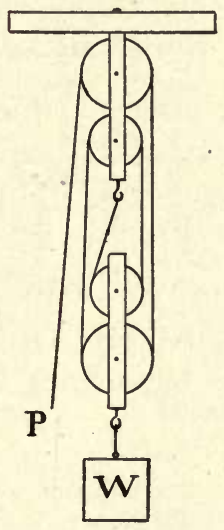

A.

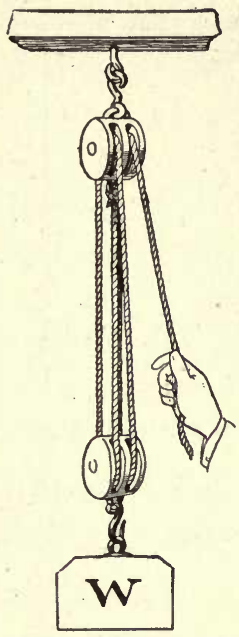

B. Block and tackle.

Fig. 53. - A Common Way of Arranging Pulleys for Lifting Heavy Weights.

slowly and that which will just cause it to move down slowly.

\section{EXERCISES}

1. Draw a diagram of pulleys arranged so that 50 pounds will support 100 pounds.

2. Hay is sometimes carried from the wagon to the mow. Can you arrange a set of four pulleys to do this?

Wheel and Axle. - Figure 54 shows a diagram of a simple form of the wheel and axle. If the radius $O A$ of the wheel is four times the radius $O C$ of the axle, then one pound of force will support four pounds of weight on the axle. Of course if the radius of the wheel is four 


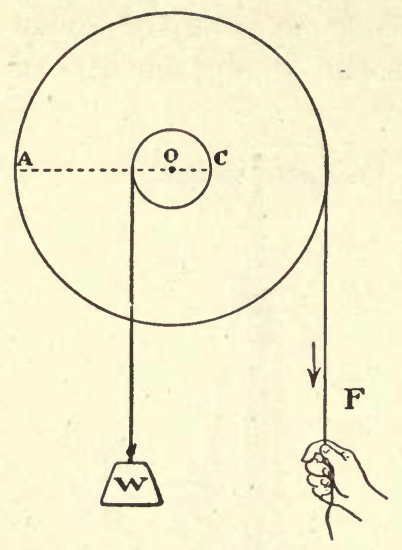

Fig. 54. - Wheel and Axle.

times the circumference of the axle, the force $F$ will move four times as far as the weight $W$, or Weight $\times$ distance through which it moves

$$
\begin{aligned}
& =\text { Force } \times \text { distance through } \\
& \text { which it moves. }
\end{aligned}
$$

In the windlass, which is a common form of the wheel and axle, a crank takes the place of the wheel (Figure 55). The principle, however, is exactly the same. Figure 56 shows how two machines of the wheel-and-axle type may be combined to lift enormous weights.

\section{EXERCISES}

1. Name three uses of the wheel and axle.

2. The radius of a wheel is 2 feet and that of the axle 4 inches. What force will be required to overcome a weight of 600 pounds?

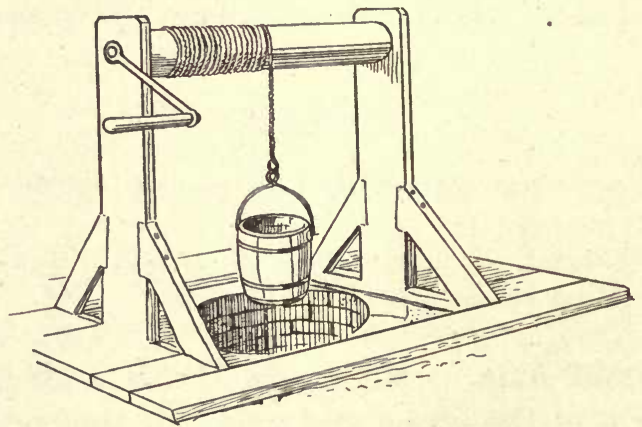

FIG. 55. - The Windlass.

3. What kind of machine is a capstan? A coffee grinder? A bicycle pedal? 
4. If a bicycle pedal is 7 inches long and the radius of the sprocket wheel is $3 \frac{1}{2}$ inches, what pull will be exerted on the sprocket wheel by a force of 50 pounds on the pedal?

The Inclined Plane. - Arrange a board as shown in Figure 57, so that it makes an angle of 20 or 30 degrees

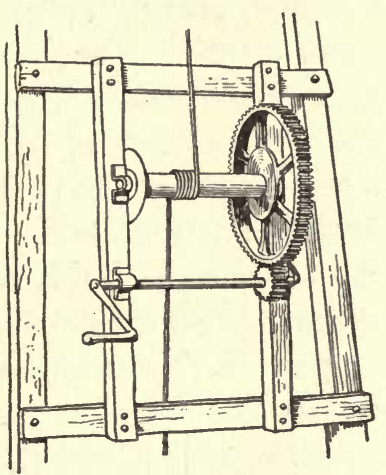

Frg. 56. - A Combination of Wheels and Axles to Lift Heavy Weights. with the table. In the absence of a carriage, a roller skate may be used. Find the mean of the force at $F$ that will just cause the carriage to move up the incline slowly, and that will just cause it to move down the incline slowly. Then

$$
\begin{aligned}
F & \times \text { length of incline }(l) \\
& =W \times \text { height of plane }(h)
\end{aligned}
$$

The inclined plane is used in loading all sorts of heavy materials on cars and trucks. When the objects to be loaded are round, such as barrels, they are simply rolled on the plane. Such objects as pianos

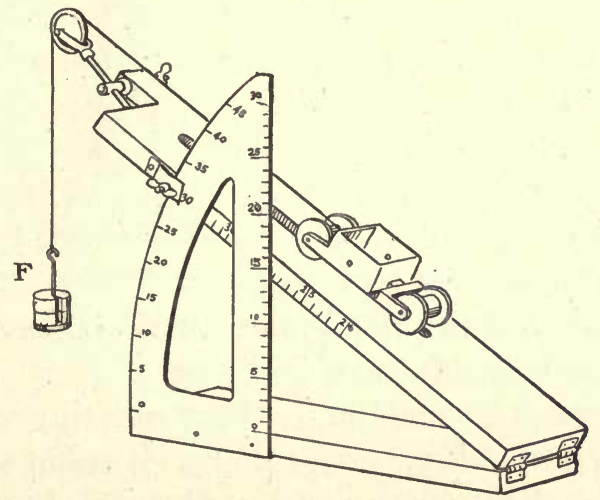

Fig. 57.- Inclined Plane and Truck. 
are put on low-wheeled carriages and moved up the plane.

The Screw. - Cut a piece of paper in the form of a right-angled triangle (Figure 58); the side $C$ is an in-

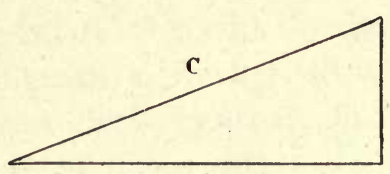
clined plane. Wind the paper around a pencil, and it has the appearance of a screw. Can you follow the inclined plane from the bottom to the top of the pencil? The elevation made in one turn is called a thread.

A stairway is a common form of inclined plane. Stairways are often arranged in towers and

lighthouses in the form of a Fı. 58. - Showing the Principle Involved in the Screw. spiral, the inclined plane turning round and round the inside of the tower. The reason the screw is so powerful is that the power moves through such a long distance while the screw moves a very small distance. In the jackscrew in Figure 59, suppose the handle to be 4 feet long and the pitch of the screw one half an inch. Then as the end of the handle moves around the circle 25 feet $(4 \times 2 \times 3.1416)$ in circumference, the screw is moved up one half an inch. A force of one pound exerted at the end

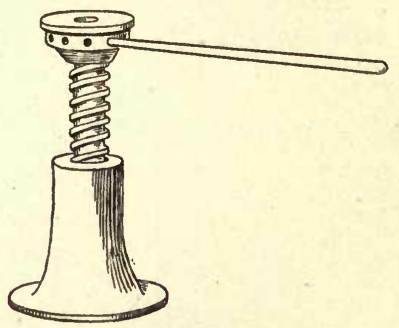

FIG. 59. - Lifting Jack. of the handle will lift 600 pounds at $W$, since one half an inch is contained 600 times in 25 feet.

The wedge is a machine used for splitting and forcing materials apart where great powier is required. Figure 60 illustrates the use of a wedge. If the wedge is 12 inches 
long and 2 inches thick at the thickest part, how far will the force have moved when the wedge has been driven into the block? How does the amount of work done compare with the force applied?

The wedge may be considered as an inclined plane which is forced by blows between two resistances in such a way as to separate them.

Mechanical Advantage. - It is often possible with the aid of a machine to overcome a certain resisting force by applying a

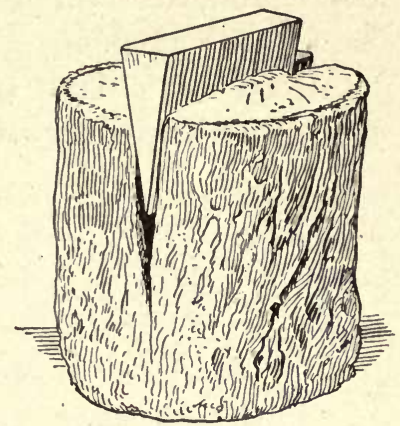

Fig. 60. - The Wedge. much smaller force. The ratio of the resistance overcome to the force applied is called the mechanical advantage of the machine. Thus if both arms of a lever of the first class are of the same length, the mechanical advantage will be 1 . If the force arm is two times as long as the resistance arm the mechanical advantage will be 2 , while if the force arm is one half as long as the resistance arm, the mechanical advantage will be one half.

Suppose the force arm of a lever of the first class to be four feet long and the resistance arm to be one foot long, then a force of one pound will overcome a resistance of four pounds; but it will be noticed that the acting force moves four times as far as the resisting force and also four times as fast. We can sacrifice speed and distance to gain force or we can sacrifice force to gain speed or distance.

Efficiency in Machines. - Not all the force applied to a machine is effective in doing useful work. Some work must be done in overcoming friction in the machine and in moving parts of the machine itself. 
If three fourths of the force applied to a machine is available for useful work, while one fourth is used in overcoming friction in the machine and moving its parts, we say the efficiency of the machine is 75 per cent.

Many ways have been devised for reducing friction and thus increasing the efficiency of machines. Roller

A

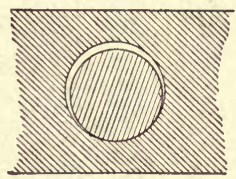

$B$

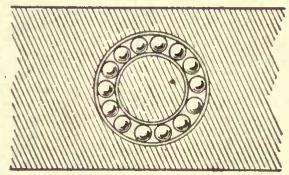

$C$

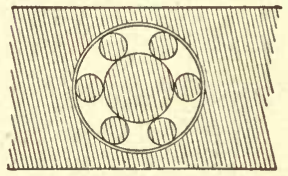

Fig. 61. $-A$, Common Bearing; $B$, Ball Bearing; $C$, Roller Bearing.

and ball bearings greatly reduce friction by substituting rolling friction for sliding friction (Figure 61).

Experiment 13. - Determine the force necessary to slide a mass of 500 grams over a level table top. Now determine the force necessary to move a similar mass on wheels. Which is greater, the sliding friction or the rolling friction? What effect has lubricating oil on sliding friction?

\section{EXERCISES}

1. What power must be exerted to roll a barrel weighing 300 pounds up a plank 10 feet long into a wagon 3 feet high?

2. Mention three uses of the inclined plane.

3 . The diameter of the wheel on a letter press is 16 inches. The pitch of the screw is one half inch. What pressure will be produced by a force of 100 pounds applied to the wheel?

4. How may the pitch of a screw be determined?

5 . Why is it difficult to walk on highly polished floors?

6. Has friction any value?

7. Mention four uses of rollers to reduce friction.

8. What causes bearings to become hot?

9. How long must an inclined plane be so that a force of 100 pounds will roll a barrel weighing 400 pounds into a wagon 3 feet high? 
10. The nuts on one side of a wagon have right-handed threads and on the other side left-handed threads. Why?

11. State the general law of machines.

12. What is the advantage of using iron rails on a railroad?

13. Why do wide-tired wagon wheels make hauling over soft fields easier?

14. Why do we scatter sawdust on icy pavements?

Power. - The term power is one which is used quite generally to designate sources of energy, but power in a specific sense means the rate of doing work. Time is not a factor in the determination of work. The same amount of work will be done in moving a ton of coal into the basement whether the work be done in two hours or ten hours, but the rate at which energy is consumed will be much greater when the work is done in two hours. An engine which can do a certain piece of work in an hour has twice as much power as one which can do but half the work in an hour. The first engine liberates energy faster than the second.

Unit of Power. - The unit of work in the English system is the foot pound, the unit of time is the second, and the unit of power is a foot pound of work in a second, or

$$
\text { Power }=\frac{\text { Work }}{\text { Time }} \text { written } P=\frac{W}{T}
$$

If $W$ and $T$ are unity, then $P$ equals one foot pound per second.

It is quite common now to speak of the rating of an engine or motor in terms of horse power. Cf. page 44 .

Man has learned to use the energy that is stored in nature to aid him in the operation of his machines. The energy of the wind, the water, the sun, and that stored in materials used as fuels and foods is utilized in this way. 
Water Power. - The power of water is very great. As we watch the water in a small stream trickling over the pebbles, we can hardly appreciate the vast power that results when the stream is dammed and a considerable head of water maintained. The force of the waves

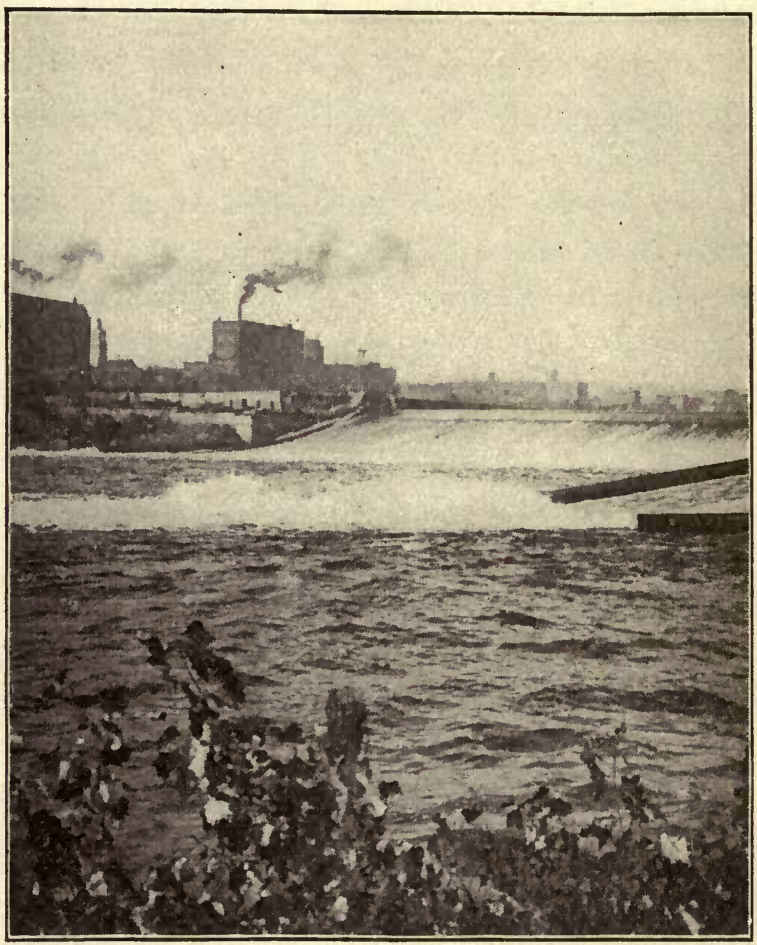

Frg. 62. - Flour Mills on the Mississippi River.

and of flood waters often does immense damage to shipping, to crops, and to the works of man which have been built in their paths.

It is much more difficult to row a boat or to swim against the current of a stream than with it, since water exerts 


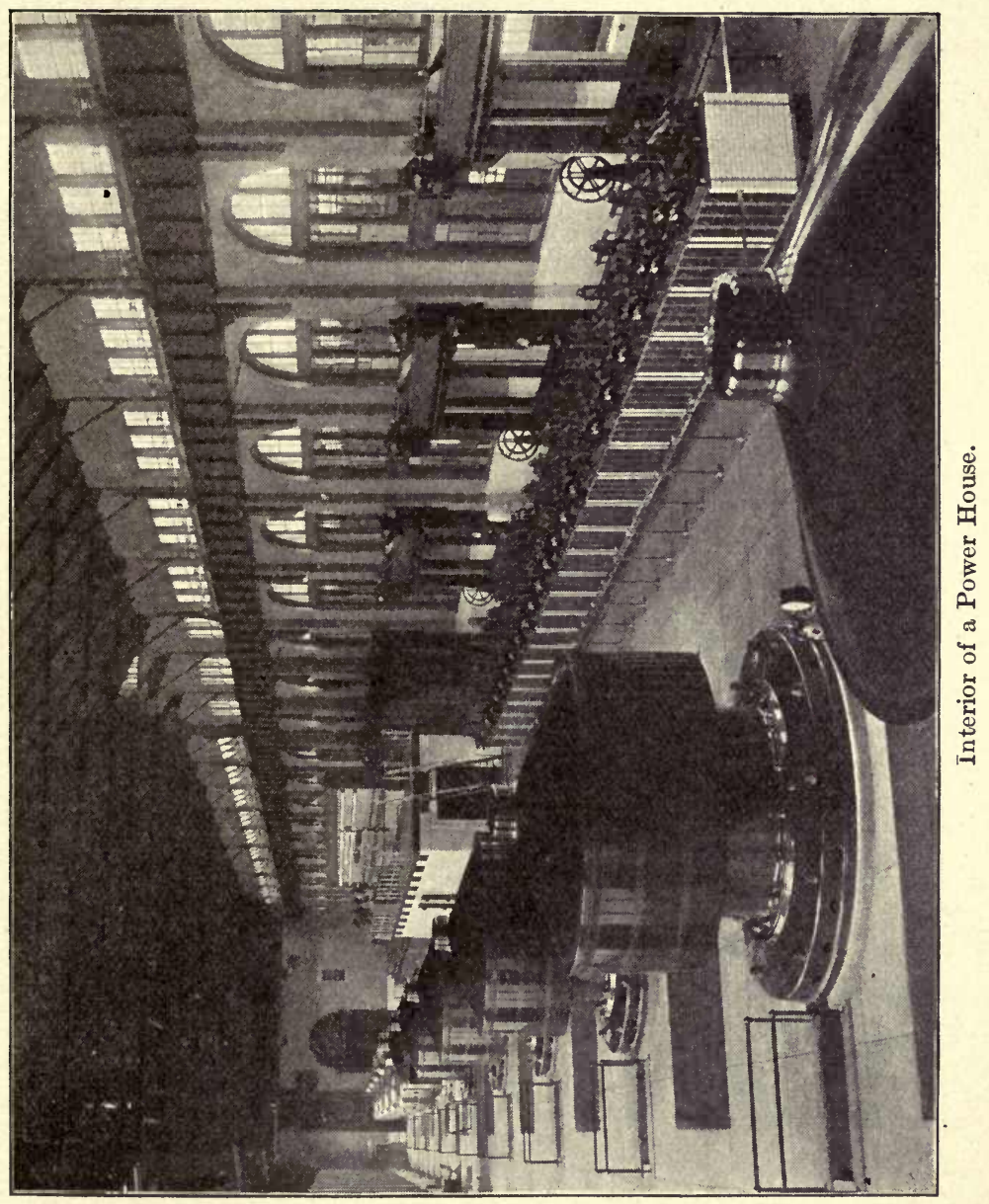



a force in the direction in which it is flowing. The power of many streams can be used in factories, in mills, and for making electricity, which can be utilized in numerous ways.

Some of the power of the Niagara River is used to turn great dynamos, which develop the electricity used in the near-by cities of the United States and Canada. The mills of many New England cities are run by water power. The largest flour mills in the world are run by the water of the Mississippi River (Fig. 62).

Several types of water wheels are used to transform the poten-

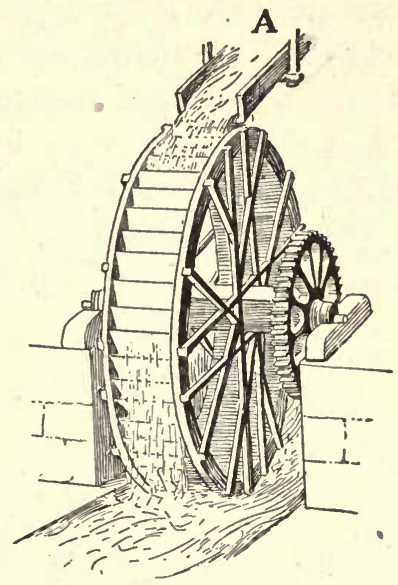

Frg. 63. - Overshot Water Wheel. tial energy of the water above into mechanical energy.

The Overshot Wheel. - This type of wheel (Figure 63)

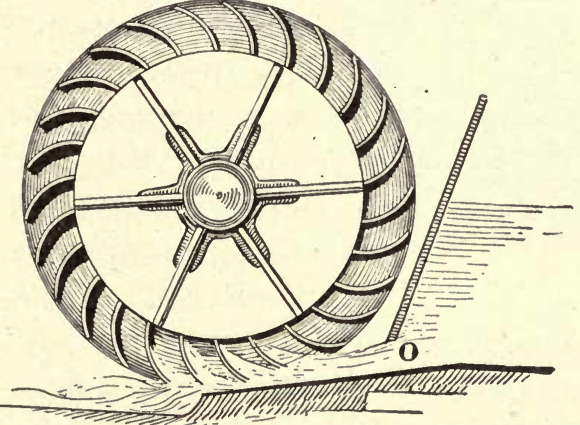

Fig. 64. - Undershot Water Wheel. utilizes the weight of the water at $A$. The work expended on the wheel in a second is the product of the weight of the water which falls upon it in a second and the distance through which it falls. This is a very efficient type of water wheel and is the common type in hilly regions where the streams are small and have considerable fall. 
The Undershot Wheel. - This type of wheel (Figure 64 ) is much less efficient than the overshot wheel and is used in more level regions where there is an abundance of water and little fall. It utilizes the kinetic energy of the water as it runs through the opening $O$. Such a wheel seldom develops more than 25 per cent of the potential energy of the water above the dam.

The Water Turbine. - This form of water wheel is now used more than any other. Figure $65^{\circ}$ shows the instal-
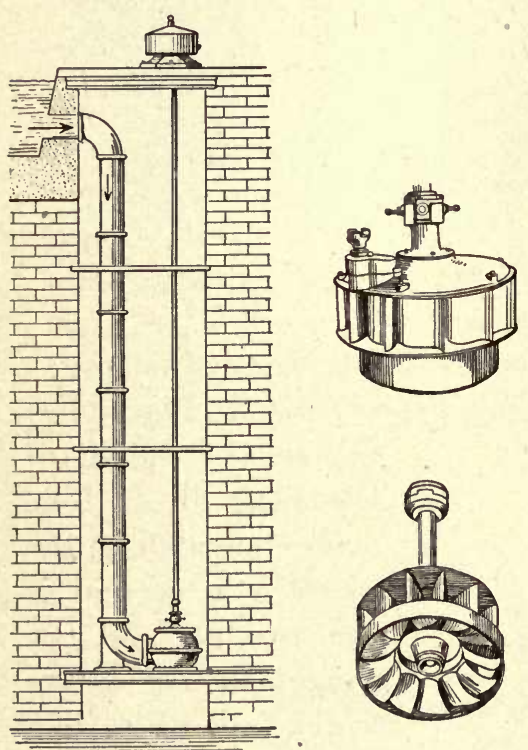

Fig. 65. - Diagram of Water Turbine.

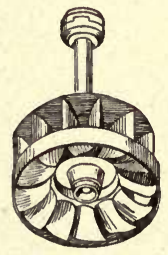
lation of such a wheel. It rotates in a horizontal plane and stands at the bottom of a turbine pit. The power developed depends upon the depth of the pit and the amount of water which passes through the wheel. The efficiency of the turbine is often as high as 90 per cent. It is used exclusively in the power plants at Niagara, where the pits are about 135 feet deep and where individual turbines develop as high as 5000 horse power.

Wind Power. - Wind power like water power is to be commended for its cheapness. The principle of the windmill is exactly the same as that of the water wheel. Moving air strikes the blades of the windmill and causes the wheel to rotate (Figure 66). This mechanical energy 
is used to pump water, grind corn, and do other. kinds of work which the farmer has to do. In Holland the water is pumped from the lowlands (Figure 67) by numerous windmills. The sailboat is a contrivance for utilizing the power of the wind for travel and transportation on water. The sail of the boat merely provides a large area of resistance to the wind.

The Aëroplane. - There are a number of craft to which the term airship may properly be applied, but the aëroplanes only are properly called flying machines. They are maintained in their positions by the resistance of the air. When the motor is working, the aëroplane is driven rapidly

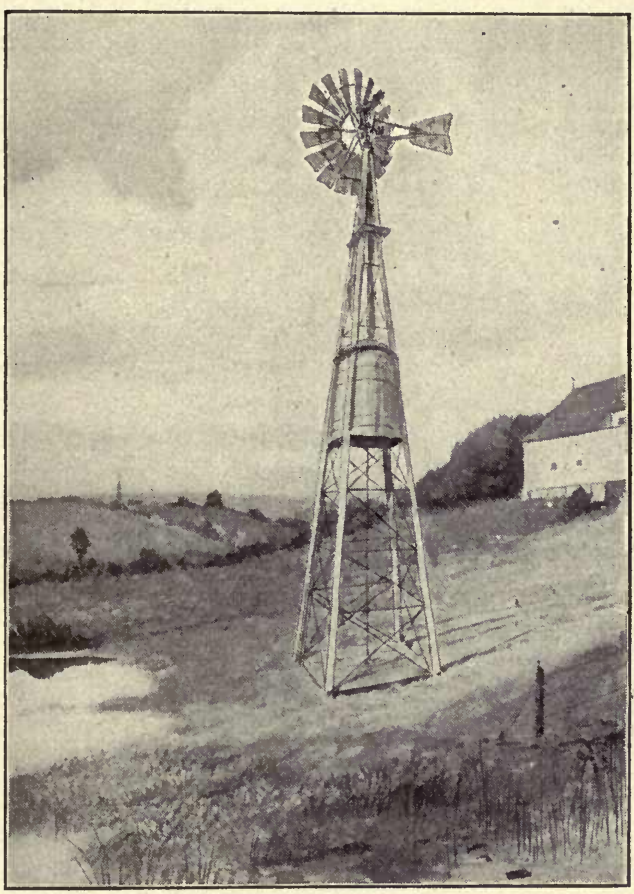

FIG. 66. - Windmill. forward by the action of the propeller against the resisting air. Then when the plane or planes of the airship are tilted upward, the resistance which the air offers to their forward movement causes the machine to move upward. The aëroplane is heavier than air and must be in rapid forward motion in order to maintain its position in the air. 
The Steam Engine. - When water changes to steam, its volume increases about 1600 times. That is, one liter of water will make 1600 liters of steam at standard pressure. Steam is a gas, and the molecules of all gases move about rapidly. The higher the temperature the

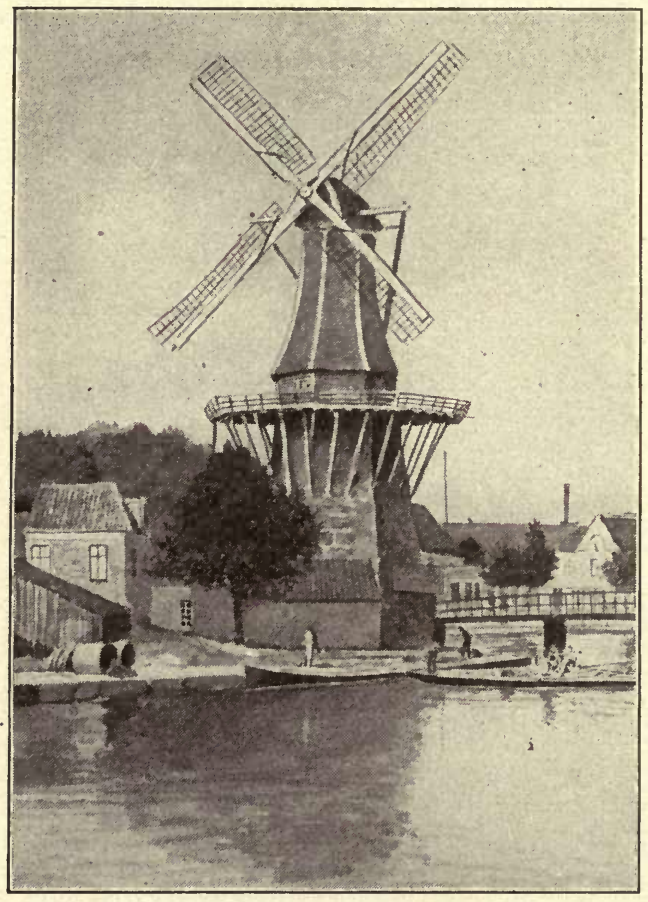

Fig. 67. - A Windmill in Holland. more rapidly they move and the harder they strike the walls of any containing vessel. Since this is true, the molecules of compressed steam, as of any other compressed gas, will rush rapidly through any passage that is offered for them from a chamber which incloses them. This stream of gas has the power of doing work. Figure 37 shows the essential parts of a double-acting steam engine. The steam is produced under high pressure in the boiler and is allowed to expand in the cylinder, first on one side of the piston and then on the other, thus forcing the piston rapidly to and fro. This motion is converted into the type of motion desired, by a shaft and cogwheels 
and pulleys. The eccentric controls the exhaust of the used steam.

The Steam Turbine. - This is a form of the steam engine which converts the energy of steam into mechanical energy in much the same way that the water turbine converts the energy of falling water into mechanical energy (Figure 68). Steam is directed by nozzles against the blades of the turbine wheel, which is caused to revolve at a very high rate of speed. The steam can be used several times by arranging the turbines in series. Such engines are used on the great ocean liners and where tremendous power is needed.

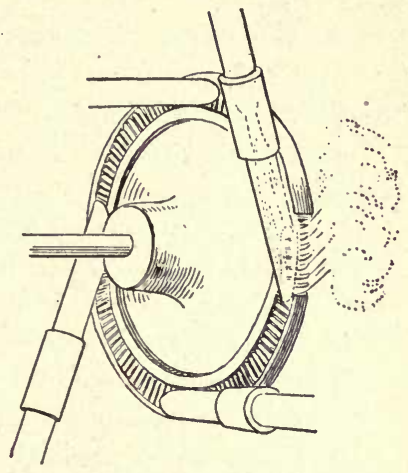

Gasoline Engines. - The force in the gasoline engine is that of expanding gases formed by the explosion of gasoline vapor and air.

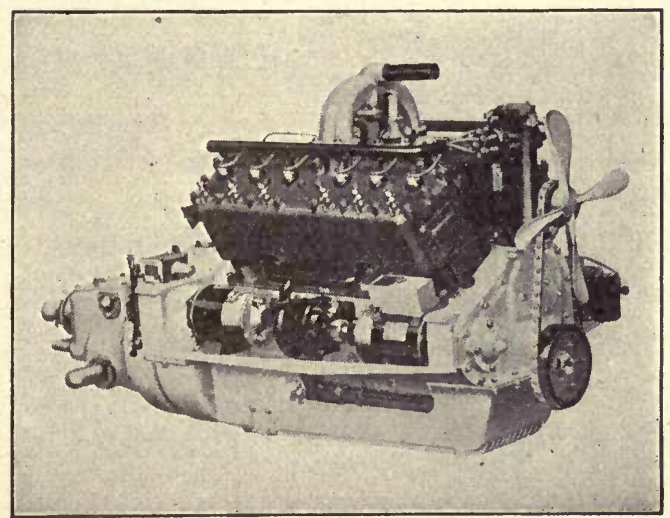

A Gasoline Engine. 


\section{QUESTIONS}

1. What important inventions did James Watt make?

2. How many foot pounds a minute is a horse power?

3. Name one disastrous flood and tell something of the extent of the damage.

4. Where are the largest flour mills in the world?

5. Explain how we may travel in a sailboat in other directions than with the wind.

6. Why do windmills not work at all times when the wind is blowing?

7. Of what use is a keel on a boat?

8. What influence did the development of the gas engine have upon the invention of the aëroplane?

9. What is a stationary engine?

10. Name six uses of the steam engine.

11. What is the principal use of the gas engine?

12. From where does the energy of the steam and gas engines really come?

13. What mechanical principles are involved in the construction of the ordinary farm wagon?

14. Name six uses of the pulley.

15. What force is utilized by the wheel brake on a wagon? On an automobile?' On a railway car? How is this force applied in each case? 


\section{CHAPTER V}

\section{THE ATMOSPHERE}

THE atmosphere is a light, transparent mixture of gases. It rests upon the land and the sea, forming the outermost part of the earth. The atmosphere becomes less dense as the distance from the earth's crust increases, one half of it being within four miles of the solid earth. The change in density, however, is very gradual. Just how deep the atmosphere is we cannot tell, but there is probably very little of it beyond a distance of fifty miles from the crust of the earth, although it has been estimated that there is some atmosphere at a distance of two hundred miles.

The principal gases of the atmosphere are nitrogen and oxygen mixed with a small amount of argon and carbon dioxide. Below is a table showing the approximate percentage of each gas in the atmosphere, although the amounts vary slightly.

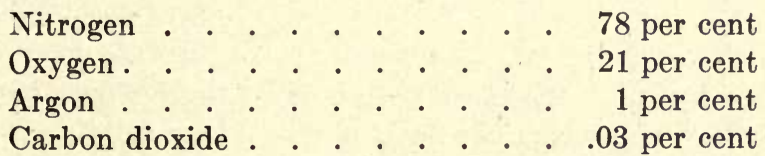

A number of experiments may be devised to prove that air is matter. We cannot see air, but we are concerned with it every moment of our lives, and we have only to stand out of doors on a windy day to be convinced of its reality. 
Experiment 14. - Fit a bottle with a one-hole rubber stopper and attempt to pour water into it through a thistle tube (Figure

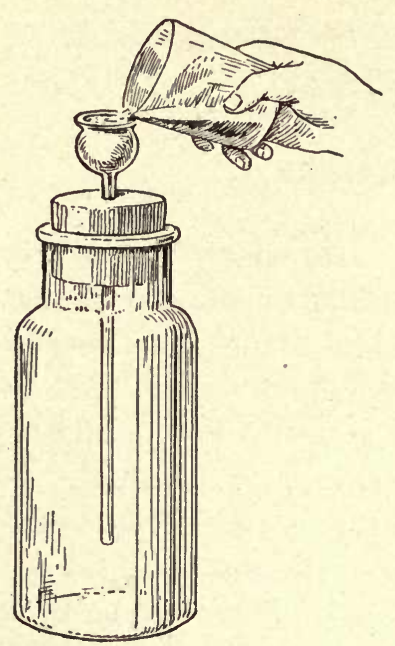

FIG. 69. - Impenetrability is one of the general properties of matter. 69). What property of matter is demonstrated by this experiment? Experiment 15. - Place a cork with a small piece of lighted candle on it in a jar of water. Invert a small jar over the cork and force it down into the water in the jar.

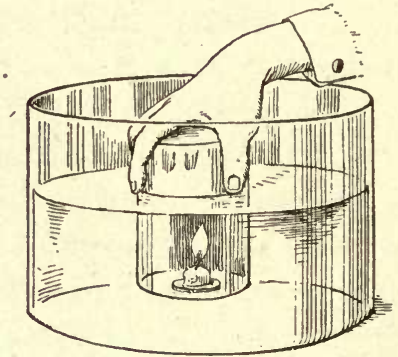

Fig. 70.

The candle may be seen burning below the level of the water in the first jar (Figure 70).

Weight of the Atmosphere. - The atmosphere is drawn toward the center of the earth as all other substances are, and since weight is the measure of this force, air has weight. .

Experiment 16. - Exhaust the air from the hollow globe of a Florence flask and weigh the flask carefully on a suitable balance. Then admit air to the flask and weigh again (Figure 71). What is the result? A cubic foot of air weighs a little more than one and one quarter ounces.

Air Pressure. - Since air has weight, it must exert pressure upon all bodies immersed in it. If a small hand glass is placed on the receiver of an air pump and 
some of the air exhausted after placing one hand over the glass, some idea may be gained of the extent of this pressure. The experiment may be performed in a slightly

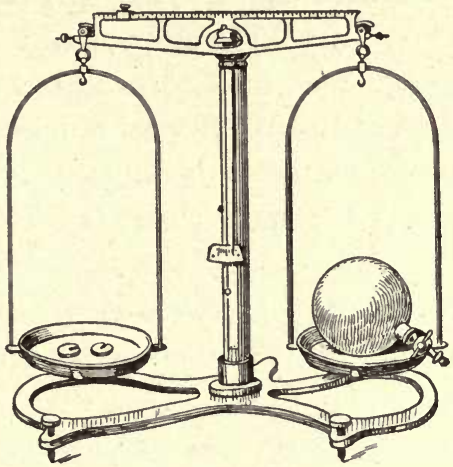

Frg. 71. - Weighing Air.

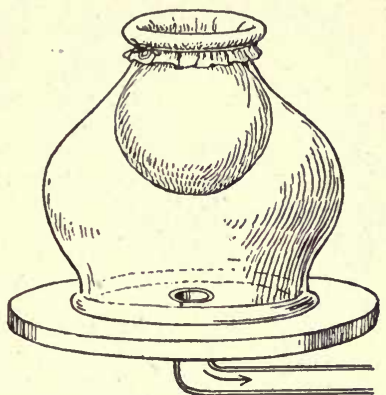

FIG. 72. - The pressure of the air forces the rubber diaphragm into the bell-jar.

different way by tying a piece of sheet rubber over the hand glass and then exhausting the air (Figure 72).

The pressure of the atmosphere is usually measured by the barometer, the most accurate kind being the mercurial barometer (Figure 73), which consists of a strong glass tube about thirty-one inches long and closed at one end. The tube is filled with mercury and inverted in a cup of mercury. At or near the sea level the mercury in the tube will stand at a height of about twenty-nine or thirty inches, leaving a vacuum at the top of the tube. Since this column of mercury is held up by the pressure of the atmosphere on the mercury in the

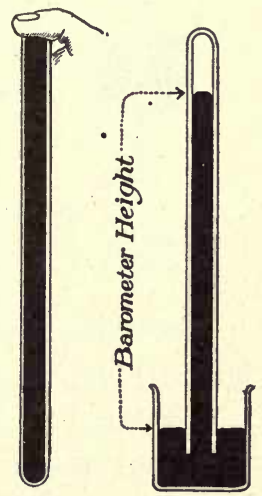

FIG. 73.-This type of barometer may be easily and quickly made. 
cup, it must weigh the same as a column of air of equal cross section and extending as high as there is any atmosphere (Figure 74).

Torricelli first proved that atmosphere has pressure. He noticed that an ordinary suction pump could not lift water more than thirty-two feet and formulated the

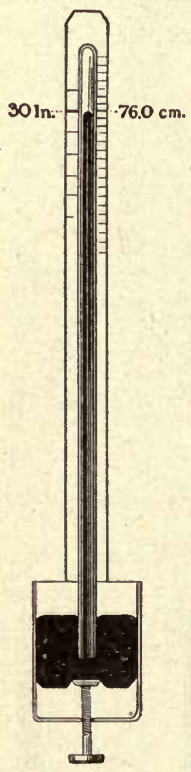

Fig. 74. - Commercial Mercurial Barometer. theory that the atmosphere was not heavy enough to push water to a higher level. Since mercury is thirteen times as heavy as water, he reasoned that mercury could only be pumped one thirteenth as high. Upon testing out his conclusions he found them to be correct.

Torricelli's apparatus was simply a mercurial barometer similar to the one described in this chapter.

In calculating the atmospheric pressure on one square inch of the earth's surface, it is necessary simply to consider the column of mercury in the barometer as having a cross section of one square inch. If the mercury' in the tube stands at twenty-nine and one half inches, it would have a volume of twenty-nine and one half cubic inches. Such an amount of mercury weighs about 14.6 pounds, which is approximately the pressure of the atmosphere on each square inch of the earth's surface.

Changes in Atmospheric Pressure due to Elevation. If a barometer is carried up the side of a mountain, the height of the mercury column will decrease. This is because it is only the atmosphere above which exerts pressure, and as we go up the side of a mountain much 
of the atmosphere is below us. An ascent of one thousand feet causes a lowering of about one inch in the barometer column. Barometers may thus be used to measure the height of the mountains or other elevations. For convenience in handling, a different type of barometer is much used for this work. It is called an aneroid barometer and consists of a small, flat, metal box from which the air has been exhausted (Figure 75). A variation in the pressure of the atmosphere causes a slight change in the shape of the little

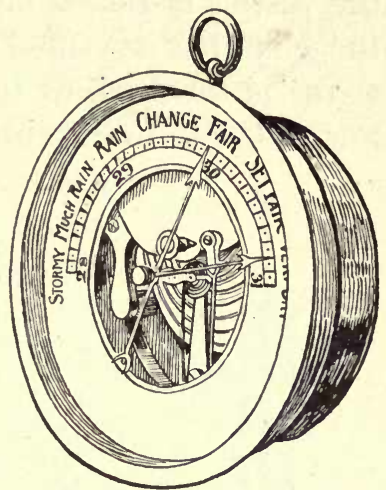

Fig. 75. - Aneroid Barometer. box. This change is read on a dial as atmospheric pressure, in terms of a column of mercury.

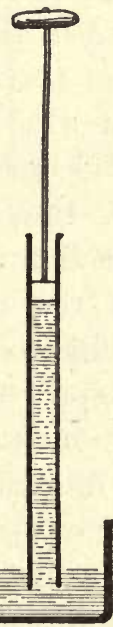

FIG. 76.-Diagram Showing Action of Piston. One half of the atmosphere is within a distance of less than four miles of the earth's surface, and the barometer reading at such a height is about fifteen inches.

Experiment 17. - Into a glass tube three fourths of an inch in diameter fit a plunger (Figure 76). Place the bottom of the outer tube in water and raise the plunger. Why does the water rise in the tube? In case a common glass pump is available-it may be used for this experiment.

Pumps. - The common pump (Figure 77) removes the air from the pump stock and also lifts the water above the level of the spout. The weight of the water 
causes it to flow through the spout. Notice the location and working of the two valves in such a pump. When the piston is raised and a partial vacuum is created in the chamber of the pump, the lower valve opens and water is forced into the chamber. When the piston is lowered, the lower valve is closed by the weight of the

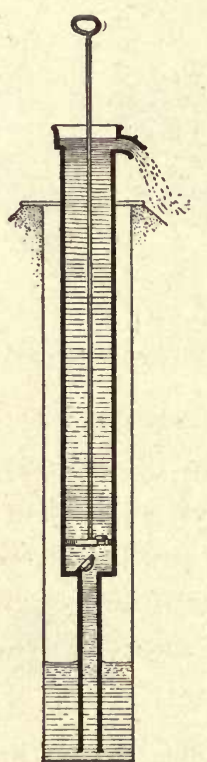

FIG. 77.-Common

Suction Pump. water on it and the valve in the piston opens and allows the water to pass through into the chamber above. As the piston is again raised the piston valve closes and the water above it is raised to the level of the spout, where it escapes, while the lower valve is again opened and more water enters the chamber as before.

In the force pump (Figure 78) the piston has no valve. When the piston is raised, a partial vacuum is created in the chamber and the water rises in the chamber as it does in the case of the common pump. When the piston is lowered, the valve at the bottom of the chamber closes and the water is forced out through the discharge valve into the discharge pipe and into the air chamber.' The pressure of the water in the discharge pipe compresses the air in the air chamber and this pressure in turn causes the water to flow from the discharge pipe in a continuous stream. On such a pump the discharge nozzle must be smaller than the supply tube in order to insure sufficient pressure to keep the flow of water continuous.

The centrifugal pump is a valveless pump which is especially valuable where a pump is needed which will 
not be clogged by sand and dirt. Such a pump is used for pumping out the water from coffer dams and swamps, and is also much used in city water systems. The pump consists of wheel blades similar to those of a turbine wheel. As the wheel is turned rapidly by some power, the air is removed from the intake pipe and the water is forced

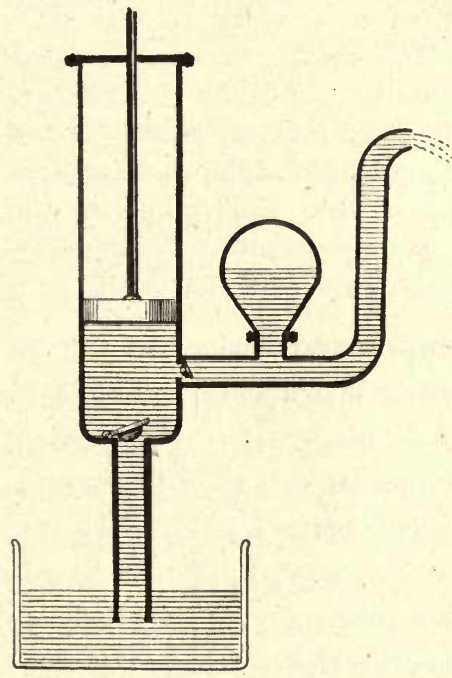

Fig. 78. - Force Pump.

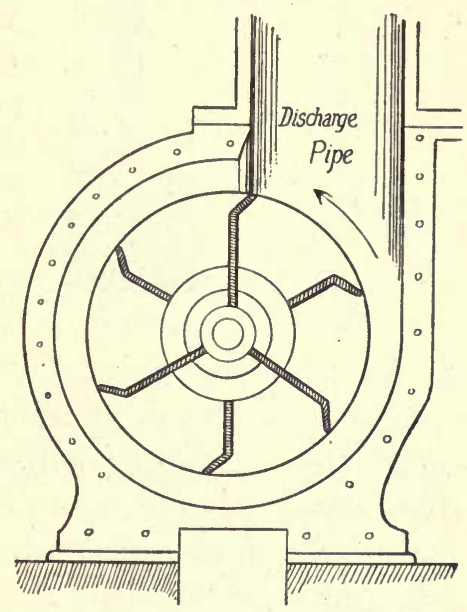

Fig, 79. - Section of Centrifugal Pump.

up by atmospheric pressure into the housing of the wheel. The blades of the wheel then force the water out through the discharge pipe (Figure 79).

The Siphon. - Take a short glass tube about twelve inches long and bend it into the shape of the letter $\mathrm{U}$, with one arm of the tube slightly longer than the other. Fill the tube with water, and then, holding the finger over the long end of the tube, insert the other end in a jar of water (Figure 80), letting the long end hang over 


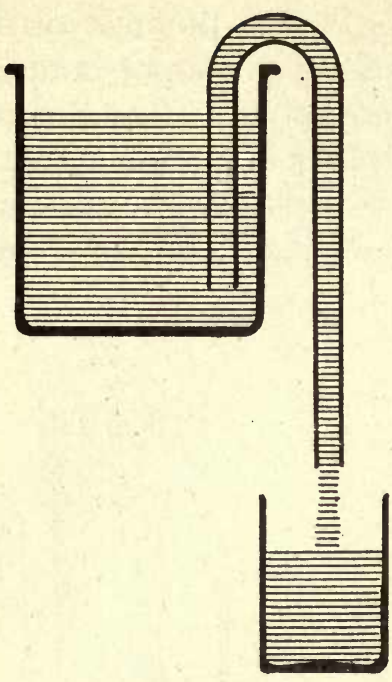

Frg. 80. - Siphon.

the side of the tumbler. Can you explain the action of the water in this experiment?

Experiment 18. - Fit a flask with a two-hole rubber stopper and arrange glass tubing as shown in Figure 81 . The shorter tube should terminate in a much smaller bore inside the flask, while the longer tube should just reach the stopper. A little water must be placed in the flask to start the siphon. How does this differ from the siphon in the previous experiment? What determines the force of water in the jet?

Compressed Air. - In laying the foundations of piers and docks below the water level, it is quite common for the laborers to work in a large steel chamber called a caisson. The water is kept out of the caisson by air, which is forced into it by pumps with sufficient pressure to overcome the pressure of the water outside. In the more complex caissons, the entrance and exit of the workmen without allowing the air to escape from the caisson is made possible by means of air locks which work on the same principle as the water locks of a canal. Compressed air can do work in various ways. The sand blast, the air brake, the pneumatic hammer, and the diving bell (Figure 82) are illustrations of many uses.

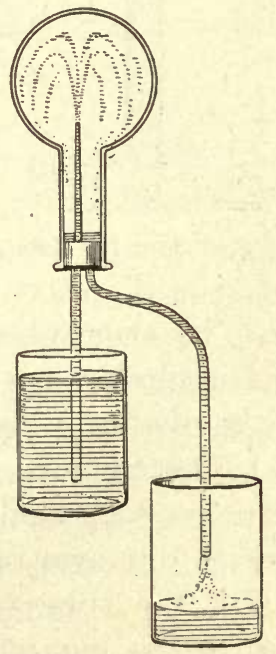

FIG. 81. - Another Form of Siphon. 
Oxygen. - Carl Wilhelm Scheele, a Swedish chemist, first prepared oxygen in 1771 by heating mercuric oxide and also by other methods. On August 1, 1774, Joseph Priestley, an English experimenter, without knowing anything of Scheele's work, obtained oxygen by heating mercuric oxide. Priestley's apparatus consisted of a bottle of mercury inverted in a bath of the same liquid. A little mercuric oxide was floated on top of the mercury in the bottle. He then focused sunlight on the oxide by means of a burning lens. The heat from the sunlight caused the oxide to disappear and a colorless gas appeared in its place. When he introduced a glowing splinter into the gas, the spark burst into flames. He later put a live mouse into the gas and it continued to live. Scheele called the gas, "Fire air" and Priestley called it "Good air."

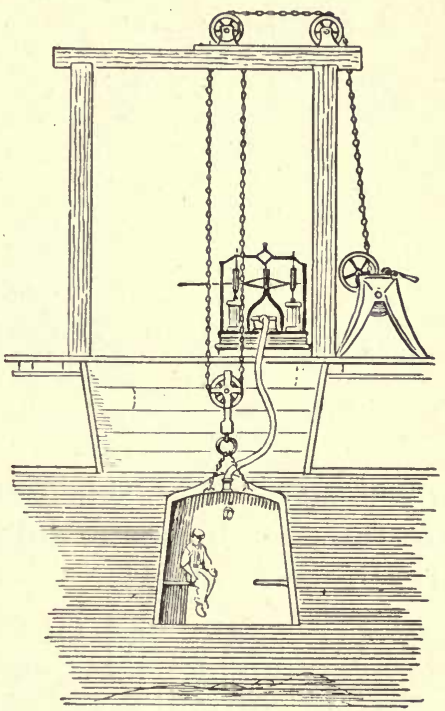

FIg. 82. - Diving Bell. Both of these are appropriate names, as we shall soon see.

Although oxygen is one of the chief constituents of the air, we have to use some means of entrapping it before we can obtain it in its pure state. Oxygen unites with many metals, and the compounds formed are the oxides of the metals. We may obtain oxygen from many of its compounds, but of these potassium chlorate is probably the best for laboratory purposes. Potassium chlorate 
is a white crystalline solid which gives off its oxygen when sufficiently heated. If manganese dioxide or iron rust is mixed with potassium chlorate, it gives off its oxygen more uniformly and at a lower temperature.

Figure 83 shows the arrangement of apparatus for preparing and collecting oxygen. A test tube contains

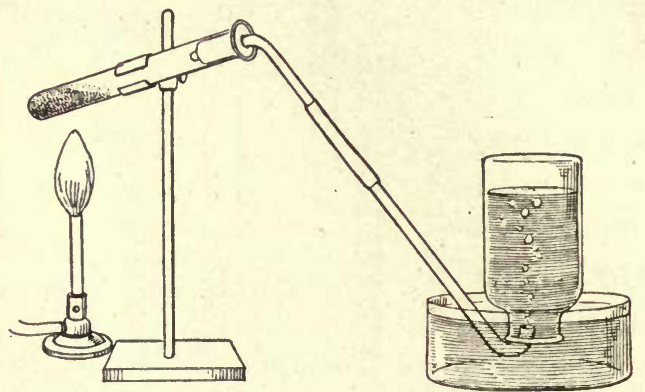

Fra. 83. - Preparation of Oxygen. the mixture of potassium chlorate and manganese dioxide. A delivery tube leads from the test tube to a pneumatic trough, where several bottles have previously been filled with water and inverted ready for collecting the gas. Heat is then applied to the test tube and the escaping gas is collected in the bottles over water.

Oxygen may also be prepared by allowing a solution of hydrogen peroxide to drop into a flask containing some crystals of potassium permanganate covered with a dilute solution of sulphuric acid.

Having collected several bottles of oxygen, we may test its properties.

Experiment 19. - Ignite a pine splinter and extinguish the flame. While it is still glowing introduce it into one of the bottles of oxygen. This was the test used by Priestley.

Experiment 20. - By means of a piece of wire hold a small piece of charcoal in the flame until it is aglow and then introduce it into a bottle of oxygen. It should burn vigorously, giving off a brilliant light. The product is the colorless gas, carbon dioxide. If limewater is put into the bottle in which the charcoal has been 
burned, the limewater will look milky, due to the insoluble solid which is formed by the union of the limewater and the carbon dioxide. Test the limewater with pure oxygen and air.

Experiment 21. - Iron may be burned in oxygen in the following manner (Figure 84). Put enough sand into one of the bottles of oxygen to cover the bottom well so that the bottle may not be broken. Take a piece of picture wire, tip it with sulphur; and light the sulphur. Then put it into the bottle of oxygen. The burning sulphur heats the wire to its kindling point, after which it will burn rapidly, giving off a very brilliant light. Globules of iron oxide are formed and fall to the bottom of the bottle.

Experiment 22. - The burning of sulphur in oxygen may also be tested. Place the sulphur in a deflagrating or combustion

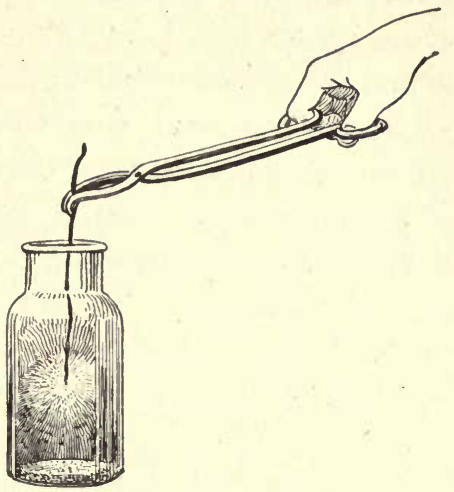

FtG. 84.-Burning Iron Wire in Oxygen. spoon, and after lighting it put it into the oxygen. Sulphur burns with a blue flame in air, but in oxygen it burns with a bright violet flame.

The nature of oxidation has probably occurred to the student by this time. It is simply the chemical union of a substance with oxygen, usually the oxygen of the air. The uniting of a substance with oxygen is called the oxidation of the substance, and the substance is said to be oxidized. If the oxidation is so rapid that light and heat are evolved, it is called burning or combustion. It was Lavoisier, a French scientist, who first gave to us the explanation of combustion. This was in the same year and shortly after the discovery of oxygen by Priestley. A large number of metals unite with the oxygen of the air to give their oxides. When iron rusts, it oxidizes. 
Melted lead yields a yellow powder called lead oxide. Zinc and magnesium burn brightly with the formation of their oxides.

When a substance burns with no flame, there is no gas evolved, since a flame is burning gas. Hard coal burns with much less flame than soft coal, indicating the absence of gaseous materials in the hard coal. Coke and charcoal are coal and wood respectively without their gases, which have been driven off by heat.

Experiment 23. - Place a lighted candle in a shallow basin and then lower a tall slender lamp chimney over the candle, supporting

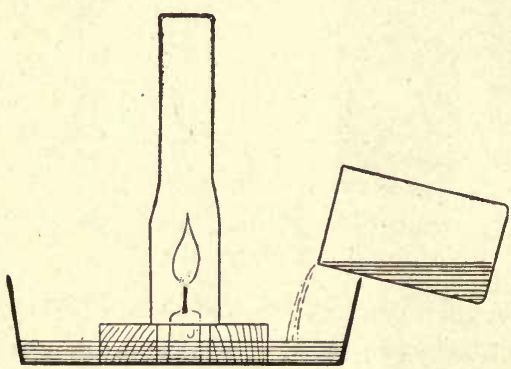

FIG. 85. - The candle flame will be extinguished when the supply of air is cut off. the chimney by two pieces of wood so that the free passage of air at the bottom of the chimney may not be obstructed. Now pour water in the basin until the bottom of the chimney is covered and note the action of the flame (Figure 85).

\section{Oxygen and Life.-} Oxygen is the life-giving principle in the animal and vegetable kingdoms, and an abundant supply of it is necessary for the sustenance of life. Neither plants nor animals can live without it. Plants take oxygen from the air through their leaves, while the animals usually have special organs for the purpose of separating the oxygen from the air. The process of obtaining oxygen from the air by plants and animals is called respiration, which consists both of breathing and oxidation in the tissues. In the higher animals the oxygen is taken directly into the lungs, where by osmosis it reaches the blood and is then carried to all parts of the body by 
the corpuscles of the blood. The purpose of respiration is to oxidize the materials of the body that are constantly wearing out into a form of waste that may be removed from the body more readily.

The results of oxidation in the body are: energy, which is created in the form of heat; carbon dioxide, formed as a result of the union of the carbon in the waste products with oxygen; and waste matter, in forms which may be easily'disposed of. Part of the heat energy is used in the body and part of it escapes as heat. This oxidation of the body is constantly changing it.

Nitrogen. - Air is approximately one fifth oxygen and four fifths nitrogen. Oxygen cannot be taken directly from the air. Nitrogen, however, may be obtained from the air by burning out the oxygen from a limited quantity of it.

Experiment 24. - Place a small quantity of red phosphorus on a slice of cork. Support the cork by a wire held upright by sticking it into a large rubber stopper. Place in a pan and surround by two inches of water (Figure 86).

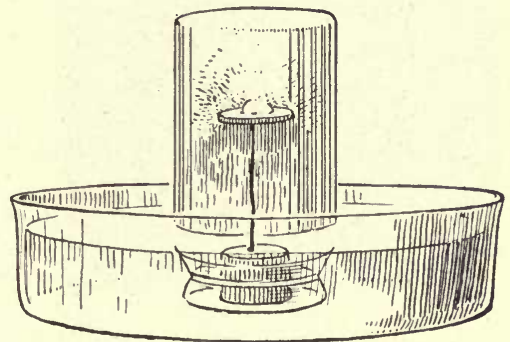

Fig. 86. Light the phosphorus and place over it a quart jar. Water will rise in the jar as the oxygen is used in the combustion of the phosphorus. When the white smoke, phosphorus oxide, has dissolved in the water, slip a piece of glass under the jar and set it upright on the table. Test the nitrogen in a number of ways in which oxygen was tested.

Nitrogen does not readily unite with other elements. Its principal value in the air is to dilute the oxygen, which would otherwise be too active for many of its uses. Nitro- 
gen has neither color, odor, nor taste. Due to the fact that it unites so reluctantly with other elements, it is used in all of our common explosives, such as gunpowder, dynamite, and nitroglycerine.

Nitrogen has a very important work to do in the manufacture of proteins by the plants, but just how this takes place is not well known. The plant seems to add

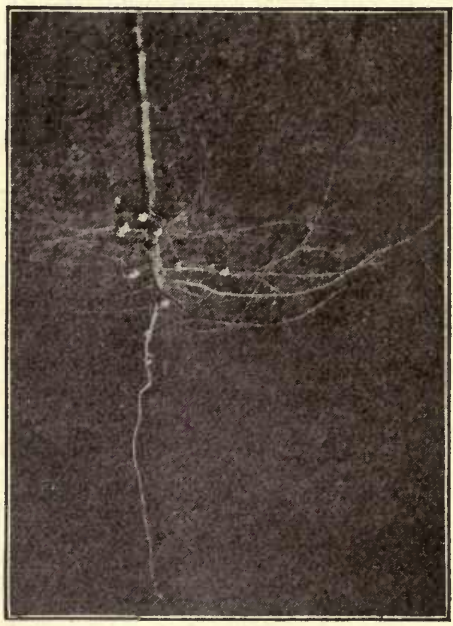

Fig. 87.- Roots showing Nitrogen Tubercles. nitrogen and other elements to the carbohydrates. These foods are taken into the plants in the form of solutions in ground water, but there are a few plants that seem to take up nitrogen directly from the air. These are such plants as beans, peas, alfalfa, and clover. This power is due to certain bacteria or very small plants which attach themselves to the roots (Figure 87) and, taking the nitrogen from the air, build it into certain albuminous foods which the plant can use. The growing of crops of peas, beans, clover, and alfalfa is very beneficial to the soil, since these little bacteria take more nitrogen from the air than the plants to which they are attached need, and therefore, when the plant dies, an excess of valuable nitrogen is left in the soil for other plants to use. Farmers often grow such crops simply for the purpose of enriching soil which has become impoverished through the raising of other crops. Artificial fertilizers which contain nitrogen compounds are also 
much used for this purpose. The greatest supply of available nitrogen for use in commercial fertilizers and explosives is found in Chile in the form of Chile saltpeter, which is mined in great quantities and exported to all parts of the world.

-Protein is one of the necessary foods of animals, and since they cannot make their own protein, the animals must get it from the plants. Plants make protein from nitrogen and other elements such as carbon and oxygen, and this in turn becomes natural food for animals.

Air, a Mixture. - We are now able to answer the question as to whether air is a chemical compound of nitrogen and oxygen or simply a mixture of the two gases. We can do this by comparing the properties of nitrogen and oxygen with those of air. The properties of air are simply those of oxygen modified by those of nitrogen. For example, air supports the combustion of other substances in the same way that oxygen does but much less energetically, because the nitrogen interferes by diluting the oxygen. We have already compared the burning of sulphur in air with its burning in pure oxygen. Since only about one fifth of air is oxygen, a substance burned in air is supplied with oxygen only about one fifth as fast as when burned in pure oxygen. If air were a chemical compound, its properties would in all probability be very different from those of the two gases which compose it. Another proof is that the weight of the air may be calculated by calculating the weight of each gas separately in the proportion of four of nitrogen to one of oxygen. In 100 liters of air there are 79 liters of nitrogen which will weigh : 
The weight of the oxygen will be :

$$
\begin{aligned}
1.43 \times 21 & =30 \text { grams } \\
99.3+30 & =129.3 \text { grams, the weight of } 100 \text { liters of air. }
\end{aligned}
$$

This result agrees with that obtained by actually weighing the air. All four results indicate that air is simply a mixture of these two gases.

Respiration. - Respiration serves two purposes: that of bringing oxygen to the blood and removing carbon dioxide from the blood. As the blood exchanges food and oxygen for the wastes of the tissues, it gradually becomes laden with these waste products which must be removed by the different organs of excretion. Some

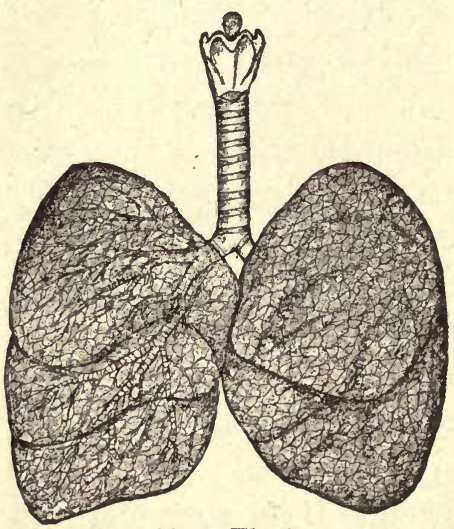

FIG. 88. - The Lungs.

of them are removed by the kidneys, some by the skin, while the carbon dioxide is removed by the lungs. The chief organs of breathing or respiration are the lungs and air passages.

The Lungs. - The lungs are two pinkish gray organs of light, spongy appearance (Figure 88). They are located in the chest cavity. Each lung is surrounded with two layers of an elastic, serous membrane, called the pleura. One layer closely covers the lung, while the other is attached to the wall of the chest in such a way as to form a closed sac. As the lungs change in size these two layers glide on each other with little friction. Pleurisy is an inflammation of this membrane due to the cesssation of the lubricating serum. 
The lungs are composed of combinations of air sacs or air cells which are grouped around the numerous small subdivisions of the bronchial tubes. These air sacs are composed of a thin elastic outer layer of connective tissue and a lining of mucous membrane. Between these two layers the minute thin-walled capillaries are located. The blood here is separated from the air only by thin walls of the capillaries and the mucous membrane lining the air sacs.

The Air Passages. - Air enters the body through the mouth or nose and passes through the pharynx and larynx into the windpipe or trachea. The trachea is a tube about three fourths of an inch in diameter and about four inches long. Its walls are strengthened by rings of cartilage. The trachea divides at its lower end into two branches called the bronchial tubes. These subdivide and finally terminate in the tiny air sacs. The tubes and lungs are lined throughout with a mucous membrane. The entrance to the larynx and trachea is guarded by the epiglottis, which stands open to admit air. As food is forced back in the act of swallowing it strikes the epiglottis and closes it over the trachea, forming a bridge over which the food passes into the gullet.

Mechanism of Breathing. - Breathing involves two processes, inhaling or inspiration and exhaling or expiration. Inhaling consists of drawing the air through the various parts of the lungs to the air sacs. Exhaling is simply the reverse of this process. The diaphragm forms a movable floor to the chest cavity and draws air into the lungs. The air is really forced into the lungs by the air pressure, to relieve the partial vacuum that has been created. When the diaphragm relaxes, it returns 
to its normal position and forces air from the lungs by reducing the size of the chest cavity.

A conscious effort should be made occasionally to take an increased amount of air into the lungs, " deep breath,"

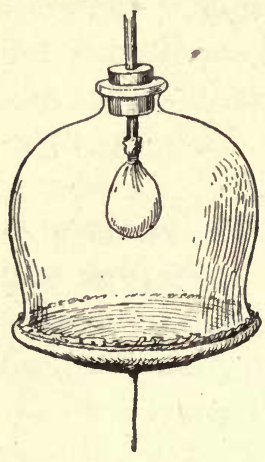

FIG. 89: since by so doing a larger portion of the lungs will be used.

Experiment 25. - Tie a rubber bag partially filled with air to one end of a glass tube and arrange the tube in a bell jar as shown in Figure 89. A diaphragm for the bell jar may be made by gluing a piece of leather with a string through it to a piece of sheet rubber. As the diaphragm is pulled down, the rubber bag inside the bell jar will increase in size. Why? Does it return to its former size when the diaphragm is allowed to go back to its normal position?

Mouth Breathing. - The proper passage for the air to the lungs in breathing is through the nose. When air is inhaled through the nose, it is warmed before it reaches the delicate tissues of the throat and lungs. The moistened hairs of the nose also remove dust particles and disease germs before they reach places where serious injury may be done.

The habit of mouth breathing is a bad one for a number of reasons. (a) The dust and germs are not removed in the mouth as in the nose by the hairs and folds of mucous membrane. (b) The air reaches the lungs before it is properly warmed. (c) Mouth breathing hinders the normal development of the upper jaw and destroys the natural beauty of the face.

With practice we can acquire the habit of nose breathing even when exercising violently. Trainers of athletes are careful to cultivate this habit, since mouth breathing 
always places a contestant for athletic honors at a great disadvantage.

Adenoids. - Occasionally small bulbous growths partially fill the nasal passages leading to the throat. These growths are called adenoids. A good surgeon can readily remove them. This should always be done, since mouth breathing is such a serious menace to health.

Colds are inflammations of the membranes lining the air passages. If the area affected is limited to the nasal cavities, we call the inflammation a cold in the head; if the pharynx is affected, a sore throat is the result; if the trachea and bronchial tubes are affected, we call it a chest cold. When the inflammation extends to the lining of the air sacs, pneumonia results.

Diphtheria, croup, and tuberculosis are all germ diseases which are produced by special kinds of bacteria.

\section{QUESTIONS}

1. Who was Torricelli?

2. Why is the air less dense on the top of a mountain than that at its base?

3. What would be some of the effects of increasing the amount of oxygen in the air?

4. How can you show that exhaled breath contains carbon dioxide?

5. How does paint prevent iron from rusting?

6. What is the working principle of the ordinary commercial fire extinguisher?

7. Why does a good draft make a fire burn better?

8. Why do we use kindling in starting a coal fire?

9. Why does a blanket thrown over a fire put it out?

10. What causes wood to rot?

11. What is the value of nitrogen in the air?

12. Give some proofs that air has "inertia."

13. Of what value are breathing exercises? 
14. Do babies breathe through the nose or mouth?

15. What should be done when children are found to have the habit of mouth breathing?

16. How does dust affect the normal functioning of the lungs?

17. Why are mouth breathers more susceptible to contagious diseases?

18. Is breathing voluntary or involuntary?

19. In what respects does exhaled air differ from inhaled air? 


\section{CHAPTER VI}

\section{WATER}

WATER is not an element like oxygen, nor is it a mixture such as we have found air to be, but a compound of two elements, hydrogen and oxygen. Water as it occurs in nature usually has a number of other substances, such as limestone, different salts, and carbon dioxide, dissolved in it; but pure water is composed of only the two substances named above.

It is quite easy to separate water into the elements which compose it by electrolysis, which means "breaking apart" or analysis by the aid of electricity.

Electrolysis of Water. - The commercial piece of apparatus sold for this experiment is called a Hoffmann apparatus (Figure 90). However, if such an apparatus is not available, the experiment may be performed very well by such an apparatus as shown in Figure 91. The water used should be slightly acidulated

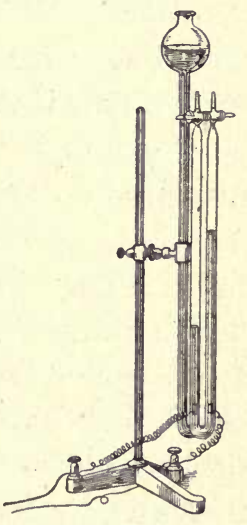
with sulphuric acid, and the wires leading from the battery should terminate in platinum foil. The ends of the wires are called poles or electrodes. The test tubes are filled with water and suspended over the electrodes so that the mouths of the test tubes shall be below the level of the water in the pan. The amount of electricity needed 
will be at least as much as that furnished by four dry cells, and six or eight cells will be better to hasten the action. It requires much energy to electrolyze water, since the union of the two gases is a strong one. When the batteries are attached, a current will pass through

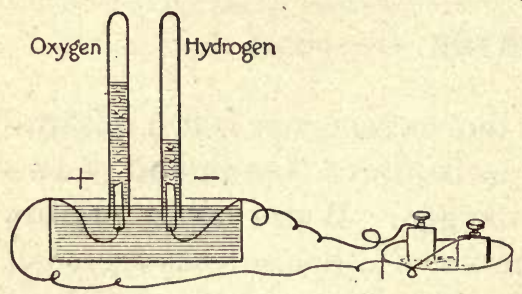

FIG. 91.- An Easily Constructed Apparatus for Showing Electrolysis.

the wires and the water, and little bubbles' of gas will be seen arising from each electrode. The sulphuric acid furnishes the passageway for the current between the electrodes. It will be noticed that the gas is evolved from one electrode much faster than from the other. Test with a glowing splinter the gas in the tube which has the least gas in it. What gas that you have studied does it resemble? Test the gas in the other tube with a flame. This gas is hydrogen, which we shall soon study more fully. The other gas is of course oxygen. What is the approximate proportion of the volumes of the two gases? Hydrogen is so named because it helps to form water, the Greek word for water being Hydor. Mention some other English words in which this name appears.

Water by Synthesis. - If the gases secured in the preceding experiment are mixed in the same proportion in a tube over mercury and exploded by an electric spark, the result will be water. The proportion of oxygen to hydrogen should be one volume of oxygen to two of hydrogen. Simply mixing these gases, however, will not produce water. The temperature must be high enough $\left(620^{\circ} \mathrm{C}\right.$.) to cause action between them. A slight explosion is the evidence of such chemical action. 
Physical and Chemical Changes. - If we throw some water into the air, it will fall to the ground, but the composition of the water is not changed. It is still water. The water of the ocean is changed to vapor and carried long distances by winds, to fall again as water in the form of rain or snow, but it is-water during the entire change of location. These changes are called physical changes. In the experiment we have just performed, however, we have a different kind of change. The result was no longer water but two substances of entirely different character: one of them a strong supporter of burning or combustion, and the other a substance which will burn. How different from water which is so much used to extinguish flames! The electrolysis of water is a chemical change. If we place a piece of paraffin in an evaporating dish and apply heat to it, a change will take place in the paraffin. It melts and becomes a liquid, but the substance is still paraffin and possesses the same properties as it did before, with the addition of the ordinary properties of liquids. A change which does not alter the nature or properties of a substance, but only the form or appearance, is called a physical change. A chemical change is one in which the properties are changed and an entirely new substance is formed. When ice is changed to water or water to ice, is there any change in the real nature of the substance? What kind of change is vaporization? Burn a piece of wood. What kind of change is demonstrated here?

Experiment 26. - Into a test tube pour a little solution of ordinary table salt and in another test tube a similar amount of silver nitrate solution. Note the appearance of each. Now pour the contents of one tube into the other. The solid that slowly falls to the bottom of the tube is called a precipitate. What kind of change is this? 
If a piece of iron is exposed to the weather, it rusts and a reddish substance is formed. The iron is slowly oxidized. What kind of change is this?

Preparation of Hydrogen. - The electrolysis of water taught us that water is composed of two gases - oxygen and hydrogen. One of these gases we have studied so that we are quite well acquainted with its properties. It now remains for us to study the properties of the other gas in which we are so vitally interested. Hydrogen is another element which as a component of water is found

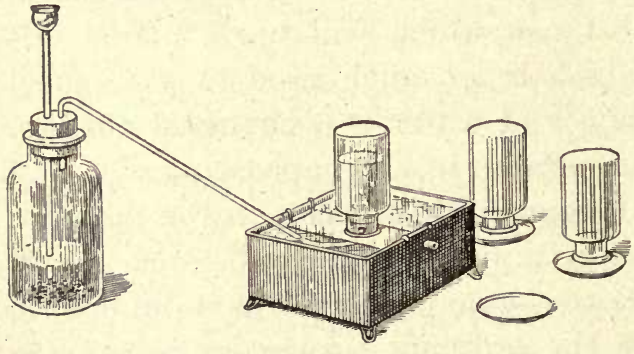

- Fig. 92. - Collecting Hydrogen. in all the tissues of the body and therefore is absolutely essential to life.

Hydrogen may be easily prepared by bringing together certain acids and metals. All acids contain hydrogen, but the best acids for our purpose are dilute sulphuric acid and dilute hydrochloric acid. The apparatus needed for the preparation of hydrogen is shown in Figure 92.

Experiment 27. - Into a wide-mouthed pint bottle intróduce a handful of zinc. Use a two-hole rubber stopper in this bottle and through one hole pass a thistle tube reaching nearly to the bottom of the bottle. Through the other pass a delivery tube leading to a pneumatic trough. Pour the dilute acid through the thistle tube and collect the gas formed over water as in the case of oxygen. The first gas evolved is always mixed with air and should not be used.

To determine whether the gas is nearly pure, collect some of it in a small test tube and carry it mouth downward to a flame a 
few feet away. When the hydrogen in the test tube does not explode but burns slowly up the tube, the gas is pure enough to be collected.

Properties of Hydrogen. - Hydrogen is the lightest known substance, weighing only one sixteenth as much as oxygen. A liter of hydrogen weighs .09 gram. Like oxygen and nitrogen, hydrogen is a colorless, odorless, and tasteless gas. In testing the gas for its purity, it was noticed that the less the quantity of air mixed with it the less violent the explosion; and we learned also that hydrogen would burn. However, if we desire to burn hydrogen in a jet, we must exercise some care in lighting it in order to avoid the possibility of a dangerous explosion. Arrange the apparatus as shown in the dia-

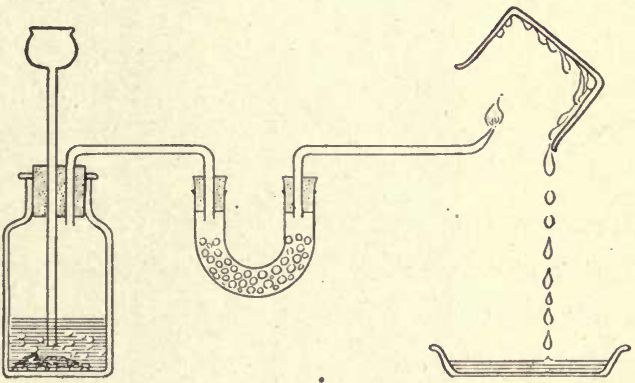
Fig. 93. - Burning Hydrogen.

gram (Figure 93). In lighting the gas it is well to use a test tube in the following manner. Hold the tube over the jet for a minute and then carry it mouth downward to a flame several feet away. When the gas in the test tube burns slowly enough that we may carry it back and light the jet with it, the hydrogen is pure enough to be safe.

Hydrogen burns with a very hot, colorless flame, with the formation of water. To test this, hold a dry beaker or evaporating dish over the flame and note the result. The same result may have been noticed when the hydrogen was exploded in the test tube. 
Experiment 28. - Bring a bottle of pure hydrogen over a lighted candle. Notice that the gas takes fire at the mouth of
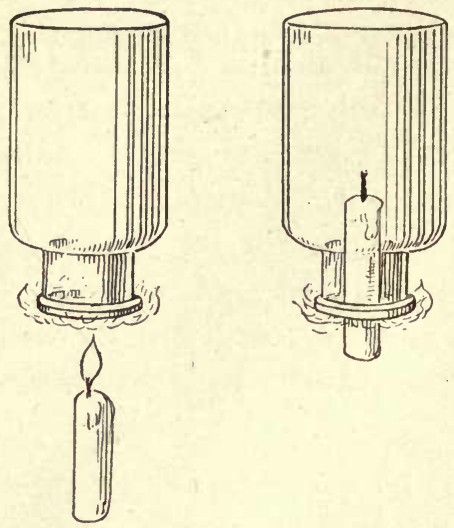

Fig. 94. - Hydrogen burns but does not support combustion. the bottle and burns quietly, while the flame on the candle is extinguished as the bottle is further lowered. Remove the bottle slowly and the candle will be relighted as the wick passes through the burning hydrogen (Figure 94).

The most important property of hydrogen is its combustibility. The flame is exceedingly hot, but not very luminous. When it burns in pure oxygen, it is the hottest of all flames, burning at a temperature of nearly $4000^{\circ} \mathrm{C}$. It is much used in the melting of certain metals and the cutting of steel. A sixinch shaft of solid steel has been cut in less than forty seconds with such a flame (Figure 95).

Three States of Water. - Water occurs quite commonly in all three states of matter - solid, liquid, and gaseous. The liquid form is most common and the name "water" is usually applied to this state; however, when water is vaporized or when it freezes and becomes a solid, it does not cease to be water. The water that is contained in the air is sometimes called "water vapor" or steam, while the solid form is called ice.

Freezing of Water. - The temperature at which water freezes is definite and under ordinary circumstances is always the same. On the Centigrade thermometer the freezing point of water is taken as the starting point in making the scale and is called zero. On the Fahrenheit 
thermometer the freezing point is taken as thirty-two degrees.

If an apparatus is arranged such as is shown in Figure 96 , it will be found that as the water in the flask is heated

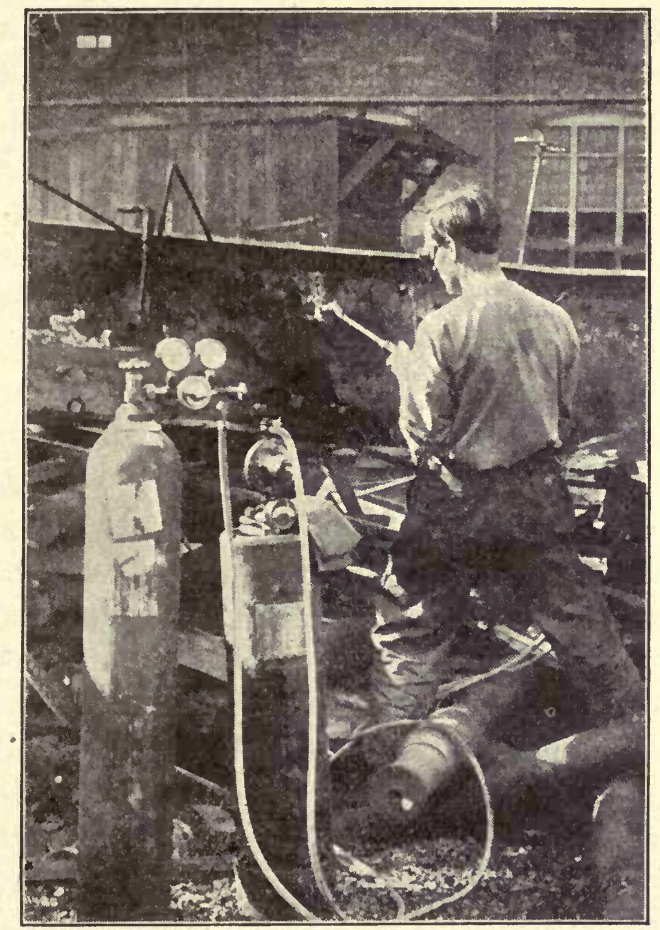

Fig. 95. - Cutting Heavy Steel Plate with Oxy-acetylene Flame.

the water in the tube will move up, due to the expansion of the water. If on the other hand the water in the flask be cooled, the water in the tube will move down, showing that the water in the flask is contracting. As the water is further cooled the contraction oontinues 
until a temperature of $4^{\circ} \mathrm{C}$. is reached, when the water

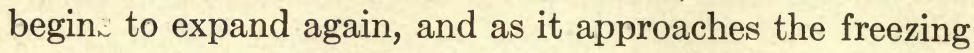
point becomes much lighter than water at higher temperatures. As water is cooled after it becomes ice it contracts like ordinary solids, but it never contracts enough so that its volume is as small as when it was a

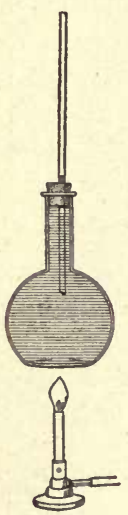

FIG. 96. liquid. Since ice is lighter than water, it floats on water and forms a covering which prevents the water beneath it from freezing, thus keeping it habitable for fish and other sea animals, even in the coldest regions.

If water is cooled until the temperature reaches zero, ice will begin to form. As cold is further applied, no change in temperature will be noticed until the whole mixture is frozen, when the temperature will again begin to fall. The freezing point of water may be lowered by dissolving salts of various sorts in it. The water of the ocean freezes at a lower temperature than the still water of our inland lakes. Can you think of a reason why this is so?

Steam. - When water is sufficiently heated, it changes to steam. The steam rises through the water in bubbles and escapes at the surface into the air. When the formation of these bubbles occurs rapidly, the water is said to boil. The temperature at which boiling begins is called the boiling point. On the Centigrade scale this point is $100^{\circ}$. On the Fahrenheit it is $212^{\circ}$. However, this point varies with the air pressure. As the pressure on the surface of the liquid is decreased, the temperature of the boiling point is lowered. This change is readily noticed in ascending mountains. On the top of Mont Blanc the boiling point of water is $84^{\circ} \mathrm{C}$. Water in Denver 
boils at $95^{\circ}$, in Quito at $90^{\circ}$. It would be posisible fo $^{\circ}$ tell the height of elevátions by a determination made from the boiling of water. What would be the effect of high altitudes on the cooking of such foods as are prepared by boiling?

We have stated that liquids have a definite boiling point under ordinary conditions. However, water will pass into vapor at any temperature. Even ice and snow evaporate or change to vapor directly, without passing through the intermediate stage, water. This vapor exerts a definite pressure which may be measured, but it is very small at the lower temperatures. As the temperature of the water rises, the pressure of the vapor or steam increases until, at the boiling point, it has a pressure equal to the pressure of the atmosphere, which we have studied in terms of a column of mercury. If the steam cannot then escape and the heating of the water is continued, the pressure increases enormously until in large volumes it is able to do a vast amount of work, such as pulling a heavily loaded train of many cars.

When water changes to steam, it increases enormously in volume and of course becomes correspondingly lighter. Steam is colorless, but when it escapes into the air there is always a white cloud which is sometimes called steam. This cloud is not steam, but is produced by little particles of water caused by the condensation which takes place when the hot steam comes in contact with the colder atmosphere.

Solutions. - Salt dissolves in water. That is, when salt is thrown into water, it disappears slowly, if left to itself in the water, and more rapidly if the water is shaken. A solid which behaves in this way when thrown into a liquid is said to be soluble in that liquid. The 
Hiquid is called the solvent and the solid the solute. Of course the salt did not really disappear. Invisible particles of it are held in the water, as is shown by the fact that the water tastes salty. The salt water obtained is called a solution. If the solid is some other color than white, the water solution of it will be colored; but the solution will always be clear even if colored. An insoluble substance like starch, powder, lime, or clay can be scattered through the water by shaking, but the mixture will be turbid. Such a mixture of a solid and a liquid is called a suspension. If allowed to stand long enough, the suspended material will settle to the bottom, leaving the liquid clear. In the case of a solution, however, there is no settling of the solute.

If we continue to add salt to a definite volume of water, there will come a time when the salt is no longer dissolved. The solution is then said to be saturated, and if more salt is added, it simply falls to the bottom. 100 grams of water at $0^{\circ} \mathrm{C}$. will dissolve 35.5 grams of salt and no more. If the water is heated, the amount of salt that it will dissolve is increased slightly. In the case of some solids, the effect of heating the water is more marked than with salt.

Tinctures are solutions in which alcohol is the solvent.

Properties of Solutions. - As we have stated before, the taste and color of the solutes are imparted to the solution. A small bit of potassium permanganate will be sufficient to color a large quantity of water. As a usual thing it will be found that as water dissolves salts, the volume will increase, and it will be found that the water has been otherwise changed. It boils at a higher temperature than it did before, and freezes at a lower temperature; a saturated solution of common salt 


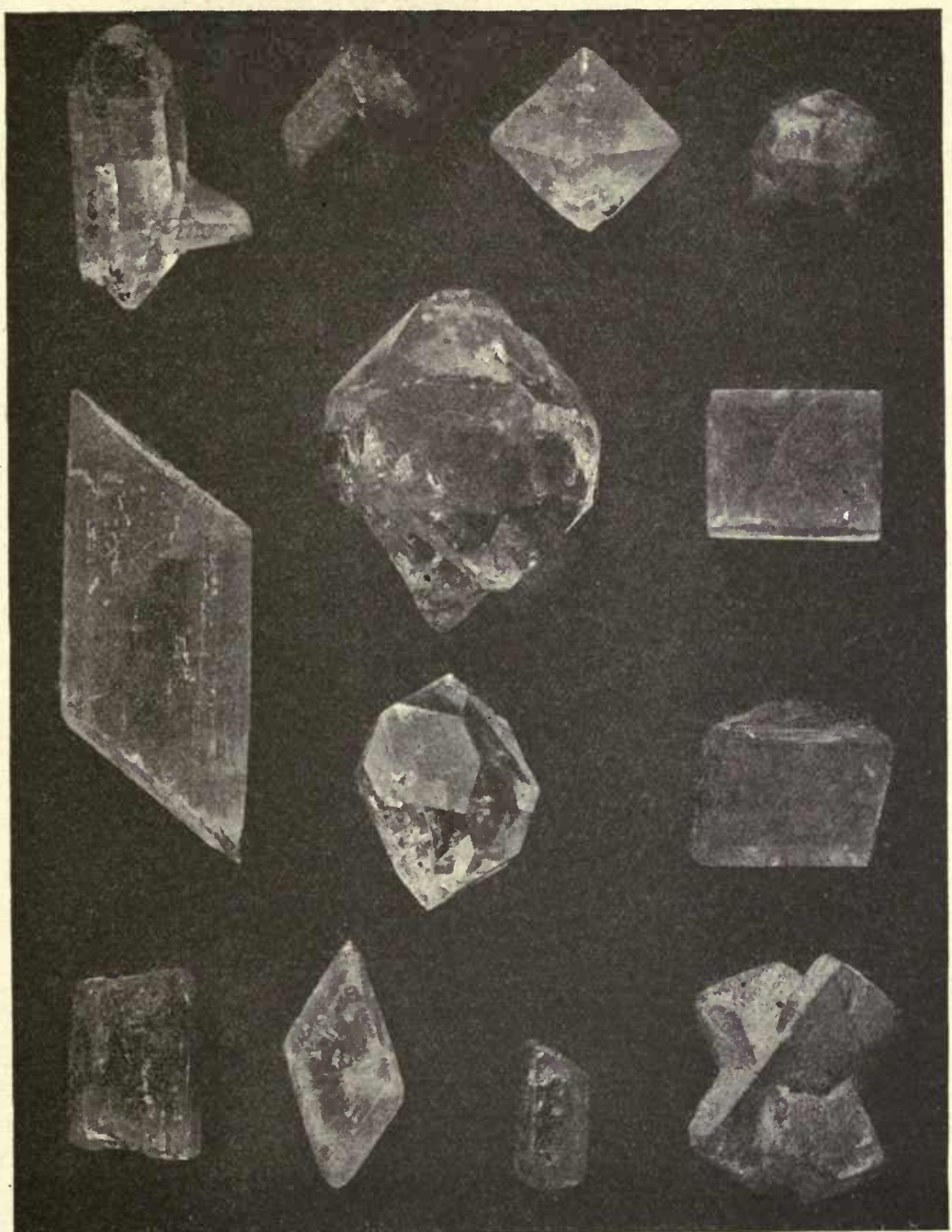

Fig. 97. - Some Forms of Crystals.

The mineral species are named from left to right for each of the four rows:1. Quartz.

2. Zircon.

3. Fluorite.

4. Garnet.

5. Quartz. 6. Rock salt.

$\begin{array}{lll}\text { 7. Gypsum. 8. Quartz. 9. Calcite. } & \text { 9. }\end{array}$
10. Emerald.
11. Gypsum.
12. Topaz.
13. Staurolite. 
boiling at about $108^{\circ} \mathrm{C}$. and freezing at $-21^{\circ} \mathrm{C}$. The density of a salt solution should be tested to determine whether it is different from that of pure water.

Experiment 29. - Into a vessel containing some pure water at about the temperature of the room, place a thermometer and note the exact temperature. Next add some ammonium nitrate and again read the temperature from the thermometer.

This is the usual effect of dissolving solids in water.

After salt has been' dissolved in water, how may it be recovered?

Water of Crystallization. - When a substance crystallizes, it takes up water which is known as water of crystallization. If we place a few crystals of copper sulphate in an evaporating dish and heat them, they will change to a grayish blue powder. This substance may be recrystallized by dissolving it in water and allowing the water to evaporate slowly. If sodium sulphate is placed in a test tube and heated, it will be dissolved in its own water of crystallization.

Each substance has its own crystalline form. For example, salt crystals are cubical in shape, while other
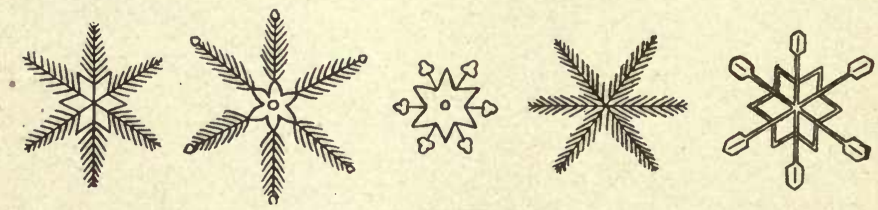

Fig. 98. - Snow Crystals.

crystals are shaped like those shown in Figure 97. Some of the most beautiful crystals are those of snow, made by the freezing of moisture. The different flakes have varied patterns, but they all have six sides or points (Figure 98). 
Substances which do not crystallize are called amorphous, or without form.

Evaporation. - Water will evaporate even when the temperature is far below the boiling point. Even a cake of ice will decrease in size through evaporation without melting. Wet clothes hung out of doors on a cold day will often remain frozen until they are dry.

The rate of evaporation depends upon the following factors :

1. The area of the surface exposed;

2. Temperature of the liquid;

3. Pressure of the atmosphere;

4. Dampness (humidity) of the atmosphere;

5. Rate of renewal of fresh air.

Most of us have noticed the influence of some of these conditions. 1. If we desire to evaporate a pint of water, we can do so more quickly if we put the water in a shallow pan than if we put it into a deep cup. Evaporation takes place only from the surface of liquids, so of course the greater the surface exposed the faster will be the rate of evaporation.

2. Evaporation simply means that some of the molecules of water enter the air, but molecules of water vapor in the air also reënter the water in the exposed vessel. Now if any real decrease in the volume of the water occurs, it must mean that molecules of water from the vessel enter the air faster than those in the air enter the water in the vessel. The warmer the water the faster the molecules move and the more rapidly they pass into the air.

3. The greater the pressure of the atmosphere the greater will be the force which is tending to prevent the escape of the little molecules of water from its surface 
into the air, and so as the pressure is reduced the rising molecules of vapor encounter less resistance and escape more easily. Sometimes the pressure over evaporating liquids is reduced by having vacuum pans, which are pans with air-tight covers connected with a pump. When air is partially exhausted from these pans, evaporation is greatly hastened.

4. There is a limit to the amount of water that the air can hold; and so, if the atmosphere is already saturated with moisture in the form of water vapor, no more evaporation can take place from the liquids under it. This simply means that molecules of water are entering the surface of the liquid from the air as fast as they are entering the air from the liquid.

5. If there were no movement of air over an evaporating liquid, the air would soon become saturated with water vapor and evaporation would cease. The oftener the air is renewed the more rapid will be the rate of evaporation.

Some liquids evaporate much more rapidly than others under the same conditions. Alcohol, gasoline, and ether are examples of such liquids. Liquids which evaporate rapidly are said to be volatile, while others like heavy oils are said to be non-volatile.

Evaporation, a Cooling Process. - Place a little alcohol on the back of the hand and blow on it. Fasten some cotton around the bulb of a thermometer, noting the temperature. Now wet the cotton with ether and take the reading of the thermometer again. What is the effect in each of these cases?

Evaporation is a cooling process, because heat is used in the change. Evaporation involves a change of state, and energy in the form of heat is necessary for such a 
change. As the heat is used, of course, the temperature must fall in those objects from which the heat was abstracted.

\section{QUESTIONS}

1. Why will clothes dry better on a windy day than on a still one?

2. Why are large shallow pans used in making maple sirup?

3. What is the temperature of deep water? Does it change much in summer and winter?

4. If ice is 0.92 as heavy as water, what fractional part of the ice will float out of water?

5 . Is the freezing of water a physical or chemical change?

6. Why is it difficult to cook vegetables at Quito?

7. Why are vessels often broken by freezing water?

8. If ice were heavier than water, would there be any disastrous results?

9. What are the essential differences between oxygen and hydrogen?

10. Name three chemical changes. Six physical changes.

11. When a pan is over a gas stove with the flame burning low, water forms on the surface of the pan next the flame. Why?

12. Can you see steam? What is it we see and call steam?

13. Is the ocean water more dense than the water of Lake Erie? Why?

14. When we heat copper sulphate crystals, we may hear little cracking sounds. What causes these sounds? 


\section{CHAPTER VII}

\section{USES OF WATER}

When pure water is cooled to $0^{\circ}$ Centigrade, or $32^{\circ}$ Fahrenheit, it freezes, and ice is formed. With the present stage of civilization, ice, which was once a luxury, has become almost a necessity. Large quantities of ice are cut from our lakes and ponds and stored for summer use, but much larger quantities are manufactured in our modern ice plants.

The artificial ice has several advantages: it is usually purer than the natural ice; it is free from air spaces and melts more slowly; and since nearly every municipality has its own ice plant, the distribution is a much simpler problem than when the ice must be shipped in carload lots to remote inland points.

Manufacture of Ice. - In nearly all of the modern ice plants, the low temperature required for freezing the water is produced by the evaporation of liquid ammonia. We have already learned that evaporation is a cooling process. It requires heat to evaporate liquids, and the heat is taken from the surrounding objects, thus reducing their temperature. The cooling resulting from evaporation will be in proportion to the rate of evaporation. The evaporation of liquid ammonia is very rapid, and in the ice plant sufficient heat is used in the change to cool the surrounding objects to a temperature below the freezing point of water. At ordinary temperatures ammonia is a gas. The temperature above which 
ammonia gas cannot be liquefied is $130^{\circ}$ Centigrade (the Critical Temperature). At temperatures below this point it may be liquefied by subjecting it to great pressure. At $80^{\circ}$ Fahrenheit a pressure of 155 pounds per square inch will produce its liquefaction.

Figure 99 is a diagram of the essentials of a modern ice plant.

The condensing pump forces the ammonia gas into the condensing pipes at a pressure of 155 pounds to the

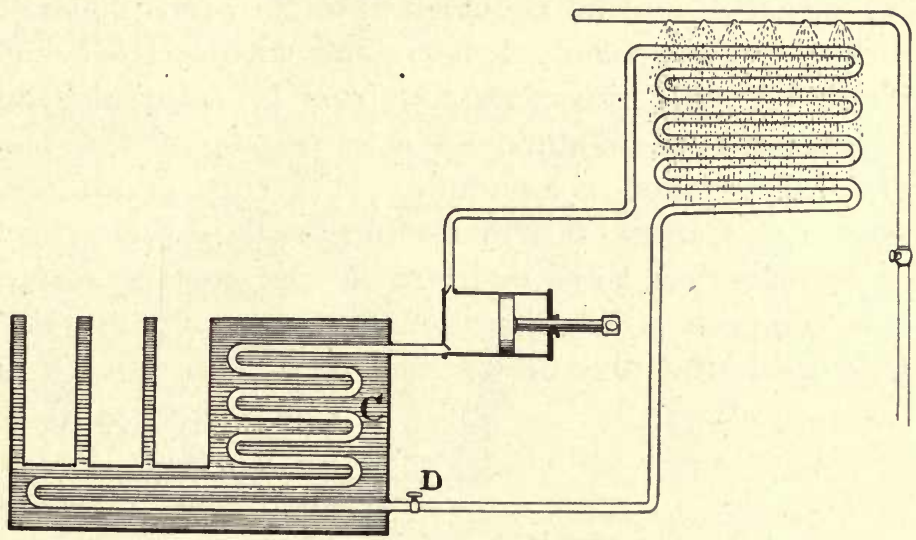

Fig. 99. - A Modern Ice Plant.

square inch of surface. The heat of the condensation of ammonia is carried off by the cold water which is kept in contact with the condensing coils. From the condenser the liquid ammonia is allowed to pass slowly through the expansion valve at $D$ into the coils of the evaporator $C$ from which the evaporated ammonia is pumped, so that the pressure in the evaporating pipes is maintained at about the pressure of two atmospheres. The pump shown in the diagram acts both as a compressor for the ammonia in the condensing coils and an exhaust for the 
gas in the evaporating pipes. As the liquid ammonia passes through the valve at $D$, it evaporates so rapidly that enough heat is taken from the surrounding pipes and brine to cool them below the freezing point of water. This brine then circulates around the cans containing the water to be frozen. It is possible to use the same ammonia gas over and over again.

Cold Storage. - The same kind of apparatus is now used in the artificial cooling of cold storage rooms, except that the cooled brine is forced through pipes placed in the rooms to be cooled. It is possible with such a system to have the temperature ranging from $16^{\circ}$ Fahrenheit up.

Occurrence of Water. - Water is one of the most widely distributed compounds. It occurs in all three states of matter and is very useful in each of these states. Besides covering three quarters of the earth's surface, water composes a large part of the earth and the plants and animals that live on it. Our own bodies are 70 per cent water, and the

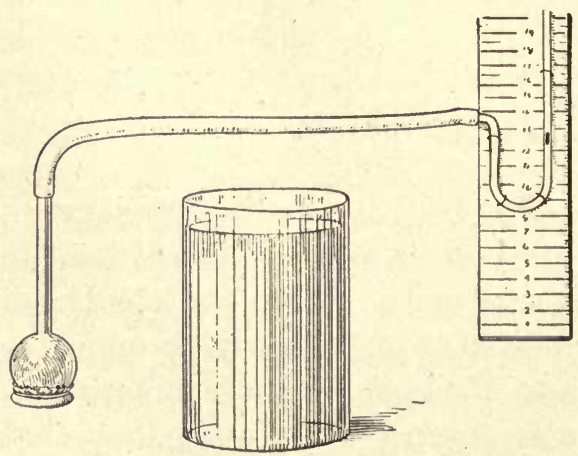

FIG. 100. - The pressure in liquids varies with the depth. foods which we eat are largely composed of it.

\section{Water Pressure. -} We are all aware of the pressure that is exerted by water. If a cork is held under water and then released, it will rise to the surface. This is proof of the upward pressure of the liquid. It is this. pressure which causes boats to float. 
Experiment 30. - A very simple experiment may be arranged to determine whether the pressure changes as the depth of the water is increased. Stretch a rubber diaphragm over the mouth of a thistle tube and attach the tube to a pressure gauge (Figure 100). The working of the apparatus may be tested by pressing on the diaphragm with the finger. The drop of ink moves out as the pressure is increased and returns to its former position when the finger is removed. Test the pressure at different depths and also at the same depth with the tube in a number of different positions. What is the effect of doubling the depth? Trebling the depth? What is the effect of changing the direction of the pressure with the depth remaining the same?

The upward force on any horizontal surface in a liquid is equal to the weight of a column of the liquid whose base is the given surface beneath the free surface of the liquid and whose height is equal to the depth of the liquid.

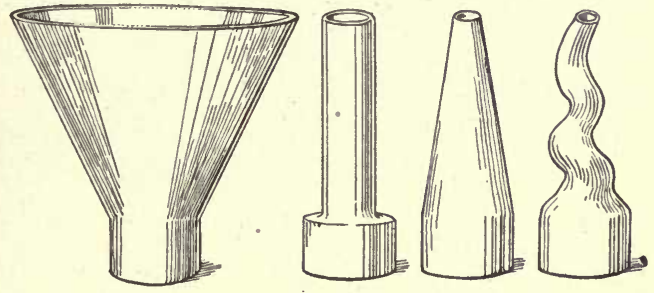

Since the above Fig. 101. - Irregular Shapad Vessels having Equal statement is true Bases.

and since of course the downward force on such a surface is exactly equal to the upward force, it will be seen that these forces are not dependent on the shape of the vessel but simply upon the area of the surface considered and the depth and density of the liquid. For example, if the four vessels of Figure 101 have bases of the same area and are filled with the same liquid to the same depths, the downward pressure on the bases will be exactly the same in each case. This conclusion seems unreasonable at first, since it means that the pressure in some cases is much more than the total weight of the water in the vessel; 
however; it can be proved true. To calculate the pressure in terms of some unit we have only to take area $X$ depth $\times$ weight of unit volume. At a given depth a liquid presses in every direction with exactly the same force.

Pressure on the Sides of a Tank. - It is quite easy to calculate the pressure on the side of a tank, since the pressure at any point of unit area is equal to the weight of the column of water which has for its area this same unit and for its height the depth of the water. Since the pressure increases in direct proportion to the depth below the free surface of the liquid, the total pressure on the side of the tank will be found by multiplying the area of the side of the tank by the average depth of all the units of area below the free surface of the liquid (area $X$ average depth $\times$ weight of unit volume). The pressure against one square centimeter of the wall of a tank containing water, with the upper edge of the square centimeter 10 centimeters below the surface of the water, will be $1 \times 10.5 \times 1$ gram. What will be the pressure if inches and pounds be substituted for centimeters and grams?

\section{1 cubic foot of water weighs 62.5 pounds \\ 1 cubic centimeter of water weighs 1 gram}

Transmission of Pressure by Liquids. - We have learned that pressure in a free liquid depends simply upon the depth and density of the liquid. From this Pascal (1623-1662), a French scientist and philosopher, deduced a very surprising and important conclusion which is now known as Pascal's Law.

In the city water system the pressure in one part of the system is transmitted to all parts of the city. This pressure is often obtained by means of a reservoir located on some high spot of land and into which the water is 
pumped. The pressure may be obtained, however, directly from the pumps. In either case it is often transmitted through miles of underground pipes to thousands

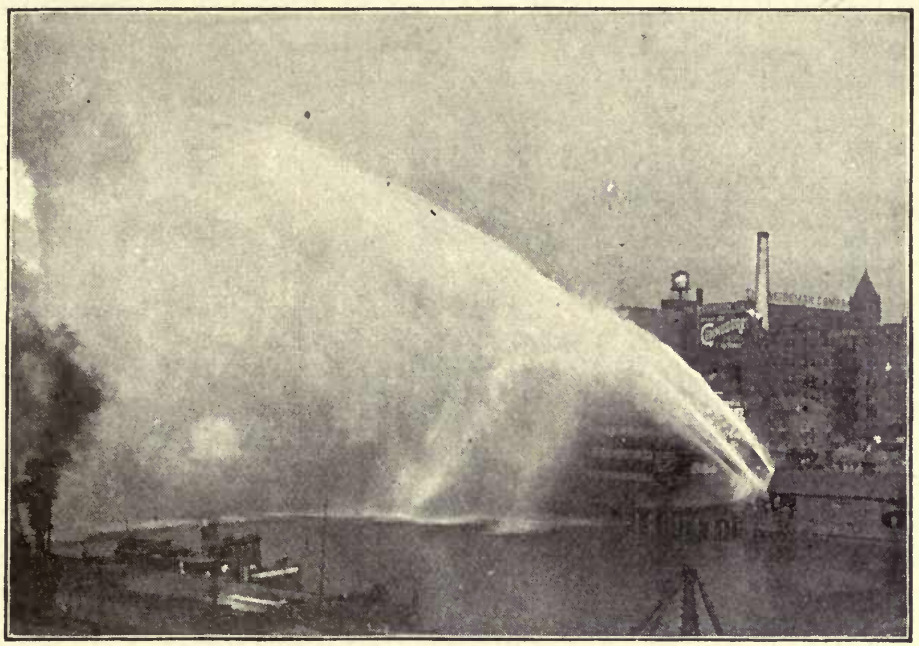

Fig. 102. - High Pressure Fire Service Streams.

The four pumps are discharging 12,000 gallons per minute. The streams are 600 feet long.

of faucets. The fire protection of a large number of our cities is dependent upon the water pressure of such a system (Figure 102).

Pascal discovered that pressure applied anywhere to a body of confined liquid is transmitted by the liquid so as to act with undiminished force on every unit of area of the containing vessel.

Suppose a vessel such as shown in Figure 103 to be filled with water to the level $a b$. Suppose the area of the upper part of the vessel is one square inch. Now if an ounce of water be poured into the tube, an extra ounce of pressure will

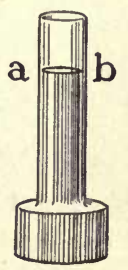

Fig. 103. 
be added to every square inch of surface in the vessel. If the inside area of the vessel be 16 square inches, the extra force exerted will be one pound.

The Hydraulic Press. - It is obvious that a piston may be used instead of water in the above illustration,

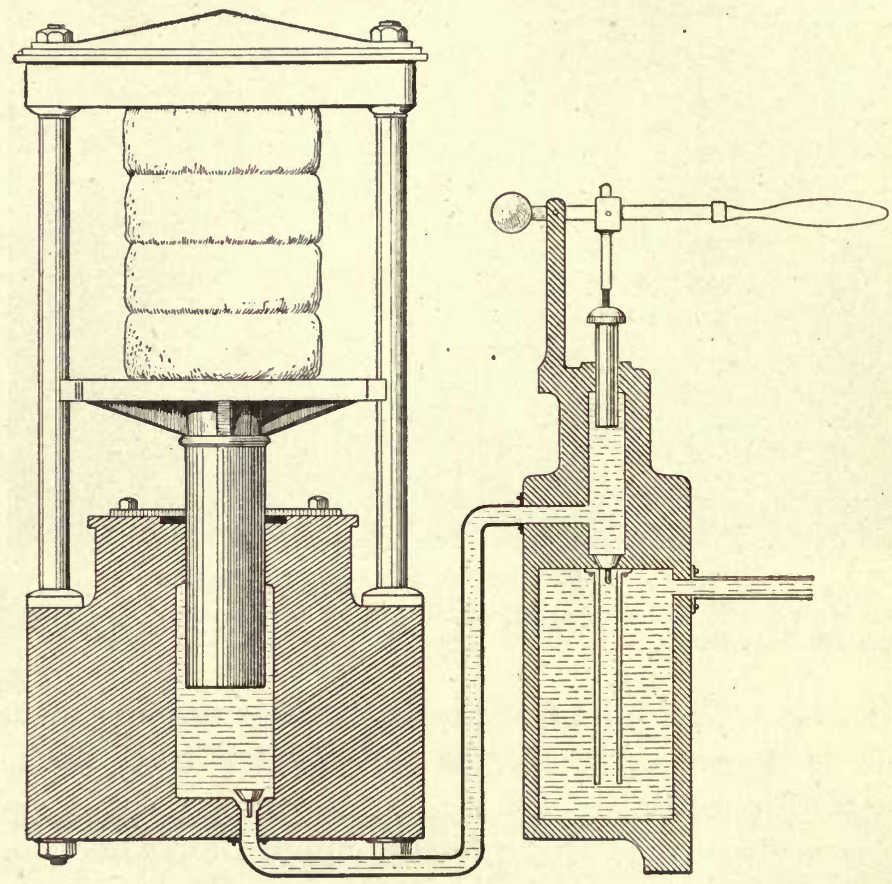

FIG. 104.-- Hydraulic Press.

and this means is commonly used to secure the application of great pressure at a given place. Such a machine is called the hydraulic press. This machine consists of two communicating cylinders each fitted with a piston. One cylinder is usually much larger than the other (Figure 104). If the areas of the pistons are in the ratio of one 


$$
\text { d. }
$$



hundred to one, according to Pascal's Law a pressure of one pound on the smaller piston will transmit a force of one hundred pounds to the larger piston. By increasing the ratio of the larger piston to the smaller piston, we can obtain enormous pressures. Hydraulic presses are used in many places where a great force is needed; as in lifting jacks, cotton presses, iron testing machines, and many others.

It will be observed that while the hydraulic press exerts a very great force it acts quite slowly through a given space. For example, if the ratio of the areas of the pistons is 100 to 1 and the smaller piston moves one inch, the larger piston will move but $\frac{1}{100}$ of an inch, so that the products of the force times the distance which the respective pistons move are always equal.

Artesian Wells are dependent on the principle of transmission of pressure by liquids. Water percolates through

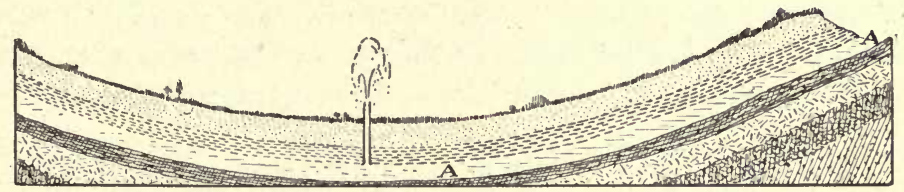

Frg. 105.-Artesian Basin, Showing Arrangement of Rock Strata.

the soil and finally becomes entrapped under an impervious stratum of rock through which it finds no outlet. If a well is bored through this stratum, water gushes up often to a considerable height. Figure 105 shows a diagram of what the geologists call an artesian basin. Water enters the stratum $A$ at the outcropping ends. The impervious strata hold it entrapped until a boring is made. Probably the most famous well is at Grenelle near Paris. It is $\mathbf{1 7 4 0}$ feet deep and delivers over 500 gallons of water a minute at a temperature of $84^{\circ}$ Fahrenheit. The 
deepest artesian well is near Berlin. It is 4200 feet deep. Near Kissengen, Germany, is an artesian well which is 1800 feet deep and throws a stream of water 58 feet high. A large number of artesian wells exist in the United States.

\section{QUESTIONS}

1. Calculate the pressure on an area 2 centimeters square on the side of a vessel, the top of the area being 12 inches below the surface of the water in the vessel.

2. Find the force in pounds acting on the bottom of a box 10 inches long, 4 inches wide, and 5 inches deep, filled with water.

3. A vessel in the shape of a cone is 10 inches high and has a base of 12 square inches. What is the pressure on the base when filled with water?

4. In the above is the pressure on the base greater than the force required to lift the cone? Why?

5. In the second problem what would be the pressure if alcohol were used instead of water, considering alcohol to be .8 as heavy as water?

6. If the large piston of a hydraulic press has a diameter of 20 inches and the smaller piston a diameter of ${ }^{\circ} 1$ inch, what force will be exerted by the large piston when a force of 5 pounds is applied to the small piston?

7. Why is artificial ice ordinarily purer than natural ice?

Archimedes' Principle. - Archimedes, a great scientist and mathematician who lived in Syracuse, Sicily (287-212 B.c.), was the first person to announce the discovery that a floating body displaces its own weight of the fluid and that a body immersed in a fluid is buoyed up with a force equal to the weight of the liquid displaced.

The story is told that Hiero, the tyrant of Syracuse, suspecting that the crown that he had had made was not pure gold as specified, ordered Archimedes to discover whether or not this were true. It was a great problem for Archimedes to find a way to do this without destroy- 
ing the crown. While in his bath one day, he happened to notice the loss of weight of his own body, and the whole principle suddenly occurred to him that a body immersed in water must lose a weight equal to the weight of the water displaced. This discovery made his problem quite simple, since gold has a volume which in proportion to its weight is smaller than that of any other metal except platinum. Archimedes determined, therefore, that the crown was made of pure gold, since an equal volume of any other metal would lose a greater proportion of its weight when immersed in water.

Most of us are acquainted with this principle long before any statement of it is made. We know that many objects float, and it is quite evident that if an object such as a piece of wood or a boat floats, the upward force of the water must be equal to the weight of the body. A piece of iron will sink in water, but if mercury is used, the iron will float as cork on water. An iron vessel will float on water because the vessel is so shaped that it displaces more water than the volume of the metal used in its construction. As a vessel is loaded, it sinks deeper and deeper into the water, but only sinks deep enough to displace an amount of water, equal in weight to the weight of the ship and its cargo.

Submarines. - A submarine boat is so constructed that no water can enter it even if it is completely submerged, excepting as permitted to enter by the occupants of the boat. If the boat is to dive, water is admitted into special compartments until the weight of the boat slightly exceeds the weight of the water it displaces. If the weight of the boat exactly equals the weight of the water it displaces, it will remain stationary at any depth below the surface. When the boat is to rise to the surface, water 
is forced out of the special compartments and air takes its place, making the boat lighter.

Density of a Solid Heavier than Water. - It is quite easy. to find the density of a regular-shaped solid by measurement, the density of a body being its mass divided by its volume. With irregular solids, however, other means must be employed; since, while it is always possible to find the mass of a body by weighing, it is sometimes quite difficult to find the volume of it by measuring. Archimedes' principle, however, furnishes an easy and accurate method for determining the volume of any solid, regular or irregular. Water has unit density, since a cubic centimeter of water weighs a gram. Now since a solid immersed in water is buoyed up with a force equal to the weight of the water it displaces, the loss in weight of the solid when weighed in water will just equal the weight of an equal volume of water.

The statement, the density $=\frac{\text { mass }}{\text { volume }}$, becomes numerically the same as density $=\frac{\text { mass }}{\text { loss of weight in water. }}$

Density of Solids Lighter than Water. - The density of solids lighter than water may be found in a similar way by using a heavy sinker to hold the lighter solid under water. Arrange the apparatus as shown in Figure 106. Find the weight with the body in the air and the sinker in water, then weigh both under water. The difference in the two weights will be the buoyant force on the body alone and will be equal to the weight of the displaced water and numerically equal to the volume of the body. The density may then be obtained by the use of the same equation:

$$
\text { density }=\frac{\text { mass }}{\text { volume or loss of weight in water }}
$$


The density of liquids may be found by a comparison of the weights of equal volumes of water and the liquid whose density is to be determined. Special bottles, commercially called "Specific Gravity" bottles, are used for

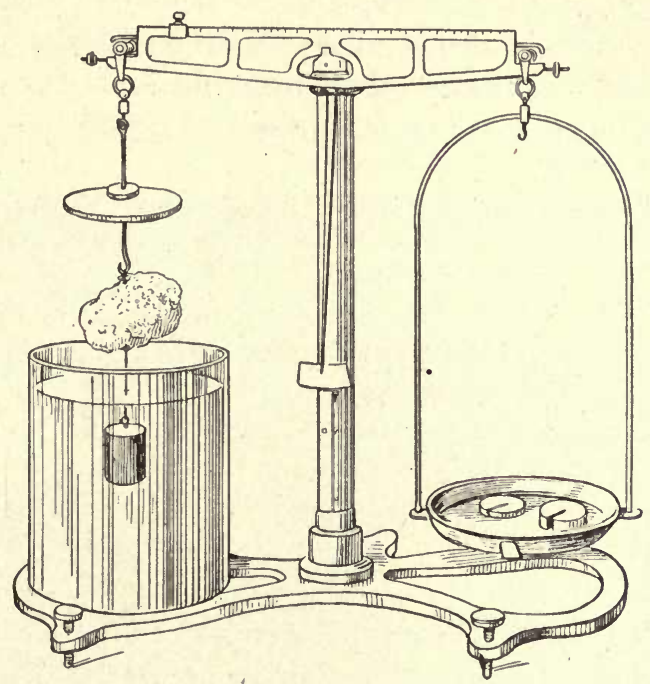

Fig. 106. - Apparatus for Determining the Density of Insoluble Substances Which are Lighter than Water.

this purpose. After the bottle has been dried, weigh it filled with the liquid whose density is undetermined. The density of several liquids should be determined.

See table of densities on page 36 .

\section{QUESTIONS}

1. Which is denser, cream or milk?

2. Will gold float or sink in mercury?

3. Why is it easier to swim in the water of the ocean than in the water of Lake Erie?

4. As a vessel passes from the river into the ocean, will its water line rise or fall? 
5. A block of wood 8 inches high sinks 4 inches in water. What is the density of the wood?

6. Suppose the above block of wood sinks 5 inches in alcohol, what is the density of the alcohol?

7. A stone weighs, $\mathbf{1 5}$ pounds in air and 10 pounds in water. What is the density of the stone?

8. Why does a stone seem lighter under water?

9. Explain the principle of the hydraulic press.

10. Would a piece of iron float in melted copper?

11. What solids sink in mercury?

12. A solid weighs 25 grams in air and 20 grams in water. What does an equal volume of water weigh? What is the density of the solid?

13. How much water does a floating piece of wood displace?

14. An iron weight weighs 10 pounds in air and 140 ounces in water. What is its density?

15. A fresh egg sinks in pure water. Why? Why does it not sink in strong brine? How can you find the density of an egg?

16. How may you determine the density of a piece of butter?

17. How could Archimedes tell whether or not Hiero's crown was pure gold?

Common Uses of Water. - Great quantities of water are used daily in our large cities, and the problem of supplying pure and wholesome water for the people of a great city like New York is by no means a small one. Each city has a different problem, depending upon its location, the nature of the underlying soil and rock, and the amount of water needed. Many cities pump the water from deep wells near by ; others carry the water in immense tubes for miles from some source which has a higher altitude than the city to be supplied; other cities like Chicago or Cleveland take the water from the lakes near by; still others use the rivers (Figure 107).

Hardness of Water. - Water is usually spoken of as hard or soft. Hardness of water may be measured by its soap-consuming power. The hardness of water depends 
upon the amount of mineral matter that it has dissolved from the ground that it has passed through. Usually this mineral matter is calcium carbonate (lime) and magnesium carbonate, but often-other minerals are found. The ocean is said to contain traces of all the soluble elements, together with large quantities of the substances we have named above, and common salt. Well water is always hard, but of varying degrees of hardness. The

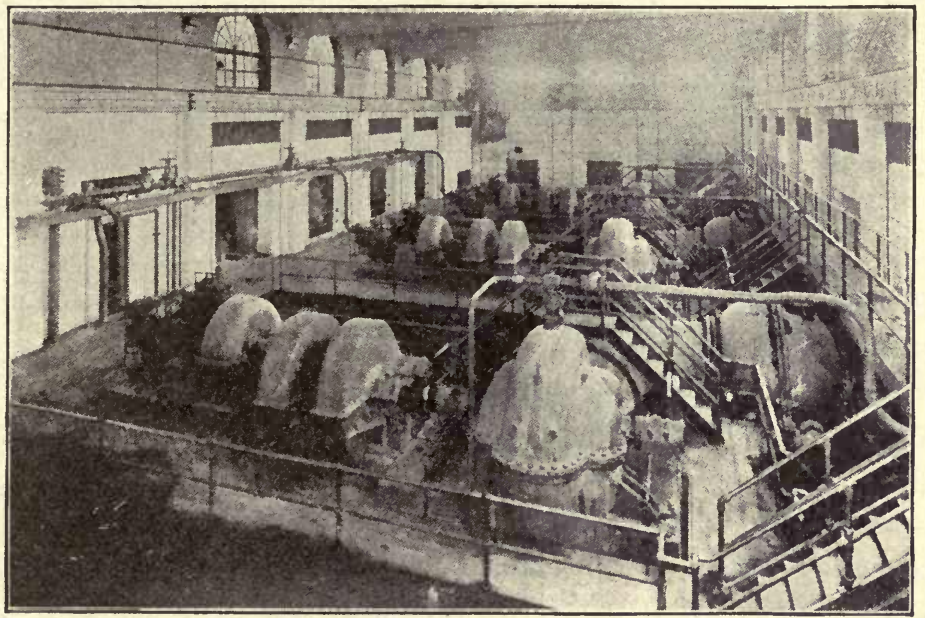

Fig. 107. - Low Lift Pump Room, Cleveland, Ohio.

This is the largest low lift pumping station in the United States.

water of the streams is usually quite soft, increasing in hardness as it gets farther away from its source and comes in contact with more soil from which it dissolves small quantities of solid matter. If hard water is boiled for a short time, a white deposit will be noticed in the vessel in which it was boiled. This deposit is mostly lime. Is there any deposit when soft water is boiled?

Spring water is simply rain water that has percolated 
through the-soil. In doing so the water takes into solution various salts. Sometimes these salts have certain medicinal properties, and the water containing them is sold for medicinal purposes. There now exist a number of health resorts which base their claims on the peculiar properties of the water of their springs.

Purification of Water. - In many cities the water is so impure that some means must be taken to free it from impurities. Some cities have established immense filtration plants to remove the solid matter from the water supply. Columbus, Ohio, has such a plant which has been in successful operation for a number of years, with excellent results from both the standpoint of health and of suitability for commercial uses. In this plant much of the lime in solution is removed by the use of chemicals. Ordinarily a city filtration plant consists simply of large areas of sand, gravel, and sometimes charcoal through which the water is allowed to percolate. After one filter has been used for a while the water is turned into another filter to permit the first to be purified by the action of the air and sun. Such filters remove most organic matter, but of course they do not remove the soluble substances.

A filter of sand and charcoal may be used for removing the solid particles from water, and such filters are quite common both on a small and large scale. However, such filters soon become clogged with small particles of clay and other foreign substances and must be renewed if their efficiency is to be maintained.

To separate all the solid matter in the form of solutions from water, distillation must be resorted to. This is merely a process of driving the water off in steam and catching and condensing the steam again. For home use the water may be rendered safe by boiling, since all 
dangerous disease germs are killed by the continuous application of heat. To kill the germ of typhoid fever, water should be boiled at least twenty minutes. It is usually quite easy to take this precaution when the purity of the water is in question, and by doing so manv lives may be saved.

City Water Supply. - Civilization requires an abun- dance of water. A water famine is more serious than a

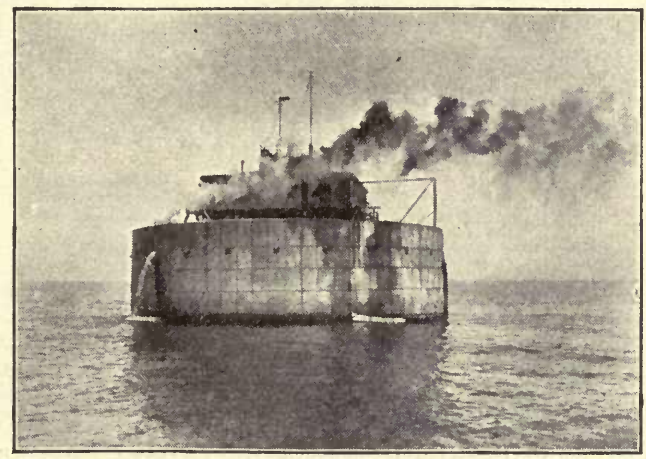

Fig. 108. - A Steel Intake Crib about Five Miles from Shore.

food famine. The community utilizes water for the removal of sewage and for fire protection. The various manufacturing plants use vast quantities of water, and in the homes it is a constant necessity for drinking purposes, for cooking, for the cleansing of soiled clothes and dishes, and for bathing.

The water supply of any city always furnishes a real community problem. The Romans brought water from the Apennine Mountains to Rome in great aqueducts. The cities on the Great Lakes obtain their water supply from the lakes, but it is necessary to take the water from "cribs" considerable distances from the shore to avoid the impurities from the sewage which is poured into the 
lake (Figure 108). Chicago no longer pours its sewage ințo Lake Michigan but into the Mississippi River by way of the Chicago "Drainage Canal" and the Illinois River. Many small inland cities rely upon large wells for their water supply, while the large inland cities take

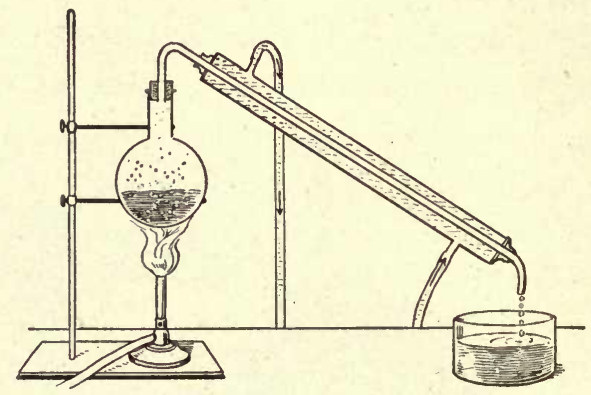

FIG. 109. - Pure Water may be Obtained by Distillation.

the water from near-by streams. Such water is usually quite impure and should be filtered and in many cases boiled before it is used for drinking purposes.

Experiment 31. - Arrange a Liebig condenser as shown in Figure 109. Fill the flask with water that has been discolored with foreign matter, such as coal dust, dirt, or ink. As the water passes into steam and is again condensed it will be found to be as

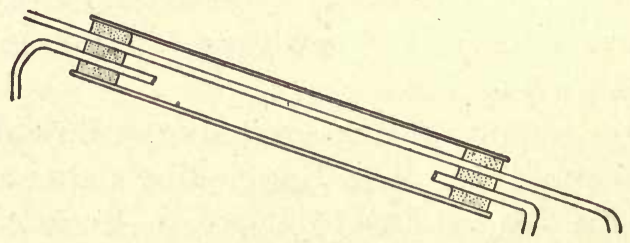

Fig. 110. - Home Made Condenser.

clear as water can be. This water is also free from solid matter. In the absence of a Liebig condenser a condenser may be made by arranging a large glass tube as shown in Figure 110. 


\section{QUESTIONS}

1. What is the source of the water supply of your city?

2. Has there ever been an epidemic of typhoid fever in your city? If so, what was the cause?

3. Is any method to purify the water supply used in your city?

4. Should rain water be used for drinking purposes? Why?

5. Where do the five largest cities of the United States get their water supply?

6. What does the term "plumbing" include?

7. What is the use of "traps" in plumbing systems?

8. Why does the water pressure vary at different points in a city water system? 


\section{CHAPTER VIII}

\section{HEAT}

HEAT is a form of energy with which we are well acquainted, since there are so many effects of heat that are very important in our daily lives. Heat was supposed to be a fluid until the beginning of the nineteenth century. This fluid was called " caloric" and its mysterious passage from one body to another was supposed to result in changes of temperature.

Heat results from the motion of molecules. As we have learned, matter is made up of minute particles which, although closely crowded together, still have spaces between them. These particles are in constant motion, striking each other at every turn. The velocity with which these little particles move to and fro in their short excursions determines the temperature of the body the faster the motion the higher the temperature. When the activity of the particles is lessened, the body becomes cooler. If the vibrations are sufficiently rapid, both heat and light are produced.

Sources of Heat. - There are a number of sources of heat, but the principal sources are friction, compression, chemical action, the sun, body heat, and electrical resistance.

Friction. - The resistance offered by the sliding of one body on another is friction, and it is a well-known fact that friction produces heat. Most of us have observed this in numerous instances. The journals of cars sometimes 
become so hot that the packing in the boxing becomes ignited. In machinery, oil is used in many places to reduce the friction and prevent excessive heating.

When a bullet is stopped by a steel plate, it becomes hot. The motion has been changed to another form of energy, namely, heat. It is the same with friction. Friction retards the motion, and this loss in motion shows up as heat. The greater the loss in motion or the greater the friction, the greater the amount of heat developed. It is said that man formerly obtained his fire by rubbing two sticks together. We still obtain our fire by friction, but the process has been very much simplified by the substitution of other substances for one of the sticks, as in the modern match.

Compression. - In the chapter which treated of the making of artificial ice it was noted that the expanding ammonia gas produced a temperature cold enough to freeze water. If the gas is compressed, an opposite effect is produced, the gas becoming much warmer. This may be tested with a common bicycle pump by closing the tube leading from the pump and repeatedly compressing the air. Note the temperature of the barrel of the pump.

Chemical Action. - For many years man has used fire, and in so doing he uses some of the energy that has been stored up by the plants. The chief fuels are wood, coal, natural gas, petroleum, and alcohol. When man causes these fuels to unite with oxygen and burn, he is using some of the energy of the sun which was stored up by the plants, and in some cases by the animals.

Sun. - Practically all the heat of the earth's surface comes from the sun. When we realize that we receive only one two-billionth of the sun's heat and still have enough to make the earth a very pleasant place in which 
to live, we can get some vague idea of the very great amount of heat in the sun.

Measurement of Temperature. - While we have no difficulty in deciding whether we are too warm or too cold, we cannot rely on our sense of feeling to determine temperature except within a very narrow range; and then the result is only a comparative one. For example, it is a well-known fact that if a person puts one hand into hot water and the other into cold water for a time, and then puts both hands into warm water it will feel cold to the hand which has been in hot water, and hot to the hand
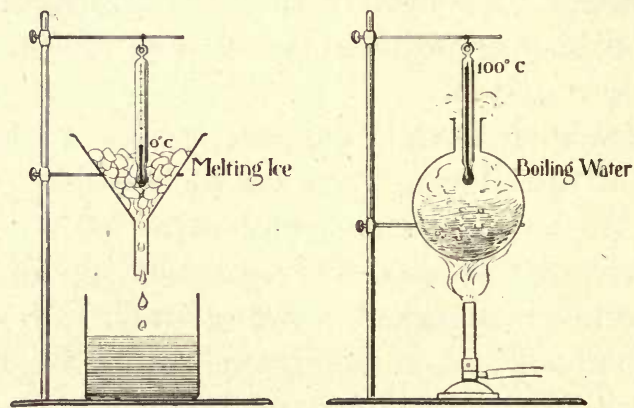

FIG. 111. - Determination of the Fixed Points on a Thermometer.

which has been in cold water. By comparison the sensations are correct, but as a test of temperature they are quite unreliable. The instrument used for measuring temperatures is called a thermometer. The ordinary commercial thermometer consists of a capillary tube with a bulb at the end. The tube is partly filled with mercury, and after the air from the remaining part has been removed the tube is sealed at the top.

The freezing and boiling points of water enable us to graduate the thermometer easily. The sealed tube is placed in melting ice, and the point at which the mercury 
stands is marked as the freezing point. This point is called zero on the Centigrade scale and thirty-two degrees on the Fahrenheit scale (Figure 111). To locate the boiling point of water, place the tube in boiling water, or better, the steam immediately over boiling water. The point at which the mercury now stands is marked 100 on the Centigrade scale and 212 on the Fahrenheit. The space between these two fixed points is divided into one hundred equal parts for the Centigrade thermometer and 180 equal parts for the Fahrenheit scale. In scientific work the Centigrade scale has come into almost universal use and in many countries it is the only thermometer used. It is unfortunate that the Fahrenheit thermometer ever came into use in our country as a weather bureau instrument, since its scale is so cumbersome. It must be remembered that the two thermometers differ only in their scales (Figure 112).

The difference between the freezing point and the boiling point on

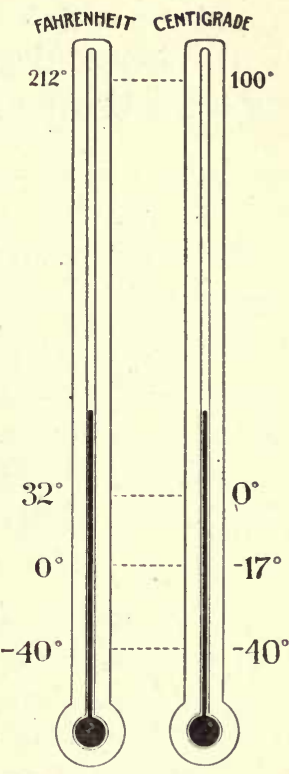

Fig. 112. - Comparison of the Fahrenheit and Centigrade Thermometer Scales. the Centigrade scale is 100 degrees and on the Fahrenheit scale 180 degrees.

$180^{\circ} \mathrm{F}$. equal $100^{\circ} \mathrm{C}$.
$18^{\circ} \mathrm{F}$. equal $10^{\circ} \mathrm{C}$.
$1^{\circ} \mathrm{F}$. equal $\frac{5}{9}^{\circ} \mathrm{C}$.
or $\quad 1^{\circ} \mathrm{C}$. equal $\frac{9}{5}^{\circ} \mathrm{F}$.

Were it not for the fact that the freezing point is marked 32 on the Fahrenheit scale, it would only be necessary 
ro multiply by one or the other of the above factors in changing from one reading to the other. As it is, to change degrees Centigrade to degrees Fahrenheit multiply by $\frac{9}{5}$ and add 32 .

$$
\mathrm{C} \times \frac{9}{5}+32=\mathrm{F}
$$

To change degrees Fahrenheit to degrees Centigrade, subtract 32 from the number of degrees and multiply by $\frac{5}{9}$.

$$
\frac{5}{9}(\mathrm{~F}-32)=\mathrm{C}
$$

Mercury is quite generally used in ordinary thermometers, but since mercury freezes at about $-40^{\circ}$ Centigrade and boils at about $350^{\circ}$ Centigrade, mercury thermometers cannot be used for extreme temperatures. Alcohol thermometers are commonly used in cold climates. For temperatures above the boiling point of mercury, other kinds of thermometers are used.

\section{EXERCISES}

1. Reduce $60^{\circ} \mathrm{F}$. to Centigrade degrees.

2. Reduce $-20^{\circ} \mathrm{F}$. to Centigrade reading.

3. Reduce $-40^{\circ}$ C. to Fahrenheit.

4. Mercury boils at $350^{\circ} \mathrm{C}$. What will this temperature be in Fahrenheit scale?

5. Absolute zero is $-273^{\circ} \mathrm{C}$. Reduce this to Fahrenheit.

Effects of Heat. - The three main physical effects of heat are expansion, fusion, and vaporization. There are other effects, but they are mainly physiological and chemical effects.

Expansion. - The first effect of heat on a body is to cause its molecules to move faster. As they move faster all the molecules strike against their neighbors with greater force, pushing them farther and farther apart. This 
causes the body as a whole to become larger, or we say expansion has taken place.

Many materials expand irregularly when heated, but rubber is the only material which contracts when heated.
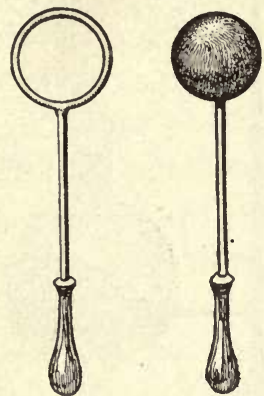

Fig. 113. - The Ball and Ring Experiment.

Experiment 32. - The ball and ring experiment is a classical one (Figure 113). Given an iron ring and an iron ball that will just pass through it at ordinary temperature. Heat the ball and see if it will now pass through the ring. In what direction has the ball expanded? Now heat the ring and see if the ball will pass through. Cubical expansion is expansion in every direction. Linear expansion means simply an increase in length.

Experiment 33. - Arrange an iron rod as shown in Figure 114 so that one end rests on an ordinary knitting needle attached to a pointer. As the rod is heated it increases in length. This expansion is shown by the movement of the pointer.

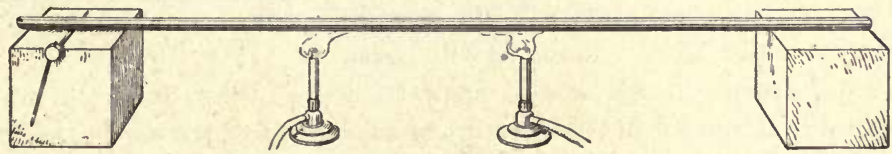

FIG. 114. - Expansion of Solids.

As the rod expands or contracts the pointer moves correspondingly.

Expansion of Liquids. - Liquids expand as we have already seen in the mercury and alcohol thermometers.

Experiment 34. - Take a flask and fit it with a two-hole rubber stopper. Pass a glass tube about sixteen inches long through one hole of the stopper and a thermometer through the other hole. Fill the flask with water and place the stopper in the flask so that the water rises a fraction of an inch above the base of the stopper. Now heat the water slowly, noting temperatures and heights of the water in the tube. A scale may be improvised from an ordinary meter bar as shown in Figure 115. Draw a curve of the expan- 
sion of water, plotting scale readings on the horizontal axis and thermometer readings on the vertical axis.

We have already spoken of the great importance of the irregular expansion of water. Review it here.

Expansion of Gases. - Gases are no exception to the rule that heat expands matter. The effect of heat on gases is very marked. Since the molecules are farther separated in gases than in other forms of matter, the application of heat greatly increases their speed and their expansive force.

Experiment 35. - Fit a Florence flask with a one-hole rubber stopper and a glass tube twenty inches long (Figure 116). Invert this apparatus in a vessel of water and apply heat to the flask.

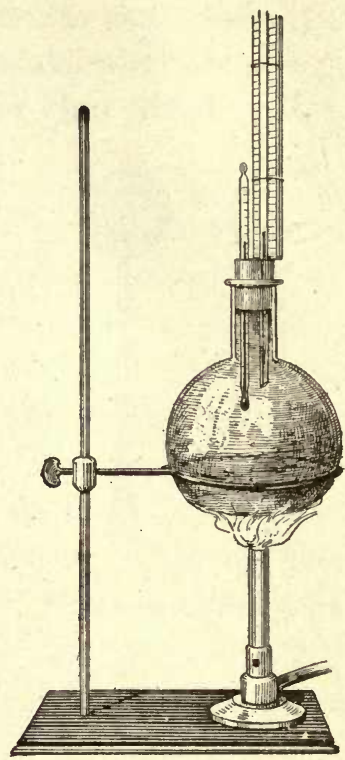

FIG. 115.-Expansion of Liquids. What happens? Allow the flask to cool. Why does the water ascend? How could the amount of expansion of the air in the flask be determined?

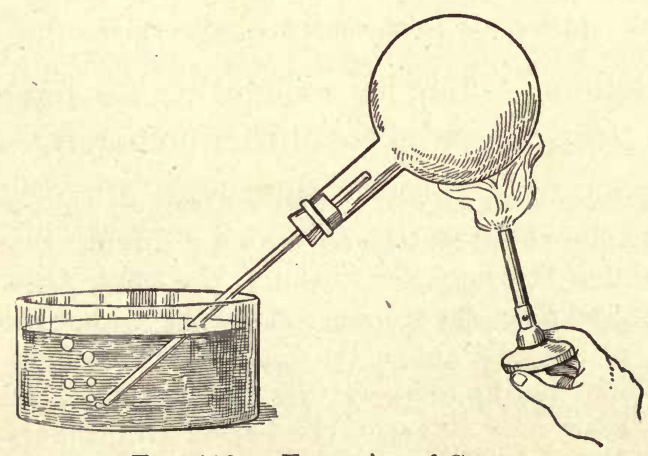

Fig. 116.- Expansion of Gases.

If several gases are tested, it will be found that they expand about the same amount for each degree of temperature and not irregularly as do solids and liquids. A gas expands $\frac{1}{273}$ of its 
volume at zero for each degree Centigrade of rise in temperature. This number is called the coefficient of expansion of gases. If a gas is cooled from zero, it contracts at the same rate; however, before it reaches $-273^{\circ}$ it becomes a liquid and so no longer obeys the law stated above. All gases have been liquefied by low temperature and great pressure.

Fusion. - Changing a body from a solid to a liquid form is called fusion. Other terms which have the same meaning are liquefaction and melting. The reverse process is called freezing or solidification. It is a wellknown fact that the melting point of ice and the freezing point of pure water is the same, $32^{\circ} \mathrm{F}$. or $0^{\circ} \mathrm{C}$. Other substances have different melting points, but in every case the melting point and the solidifying point of a substance is the same.

\section{Table of Melting Points}

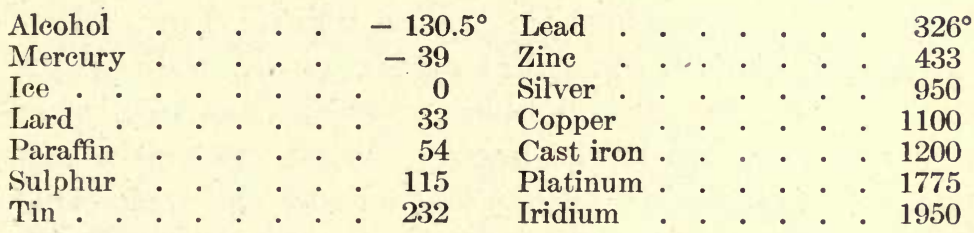

Experiment 36. - Place some pieces of ice in a beaker and heat slowly over a small flame. Stir the ice and water constantly with a thermometer and note the temperature from time to time.

Most liquids decrease in volume when they solidify, but the opposite is true of water, liquid iron, and a few other substances in which there is a sudden marked increase in volume at the moment of solidification. Water increases about nine per cent on freezing, and the force exerted is great enough to burst the usual containers such as water pipes and pitchers and other household utensils. 
Experiment 37. - Fill an ordinary large-mouthed bottle with water and fit it with a one-hole rubber stopper. Insert a glass tube in the stopper and crowd the stopper down until the water fills the tube. Now place the bottle in a freezing mixture of ice and salt and watch the contraction and expansion of the water. What is the first change noticed? At what temperature does water cease to contract and begin to expand? Watch the experiment until the whole mass of water in the bottle is frozen.

Vaporization. - The changing of a liquid to a gas is called vaporization. Slow vaporization is called evaporation. It takes place at all temperatures below the boiling point. Ebullition or boiling takes place at a definite temperature, and if the pressure on the liquid does not change, the temperature will remain constant until the liquid is completely vaporized.

Boiling. - While evaporation takes place only at the surface of the liquid, boiling takes place throughout the entire mass. It is interesting to watch the water in a beaker as it approaches the boiling point. From where do the air bubbles come that gather on the walls of the beaker? Do the first bubbles of steam get smaller or larger as they rise in the liquid? What causes the agitation of the liquid? What becomes of the water that disappears? If a thermometer is placed in the liquid it will probably read less than $100^{\circ}$. Why?

Determine the boiling point of alcohol.

\section{Boiling Points at Standard Pressure}

Ether Chloroform . . . . . . 60 Alcohol . . . . . . 78 Benzine.

Water

Mercury .

Sulphur

Zine . $100^{\circ}$

350 1050

Relation of Boiling Point to Pressure. - Of course the atmosphere is exerting a pressure on the surface of a 
liquid at all times. The boiling point of a liquid is the temperature at which the vapor pressure of the liquid

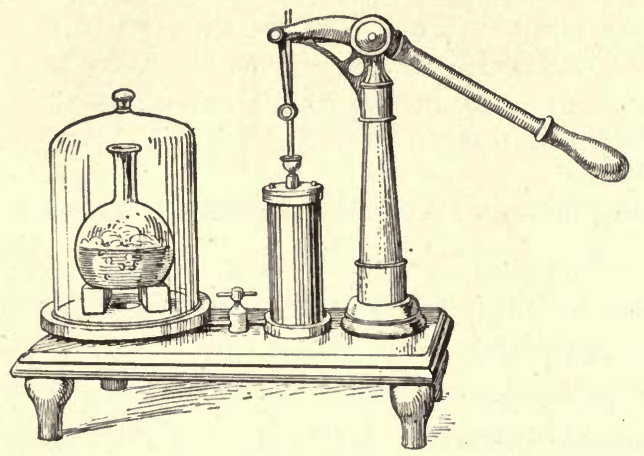

FIG. 117. - Boiling Water at a Temperature below $100^{\circ}$ Centigrade by Reducing the Air Pressure with the Aid of an Air Pump. becomes greater than the atmospheric pressure on the liquid. Since the atmospheric pressure becomes less and less as we ascend, the boiling point for any certain liquid will be lowered as we ascend. Water boils at a much lower temperature on the tops of our high mountains than at sea level.

Experiment 38. - Boil some water in a flask and then remove it from the fire and place it under the receiver of an air pump (Figure 117). Exhaust some of the air and note the result. How do you explain the fact that the water again begins to boil violently when it is evidently much colder than when it was boiling over the fire?

Experiment 39. Fill a Florence flask half full of water and boil the water vigorously for a minute. Now close the flask

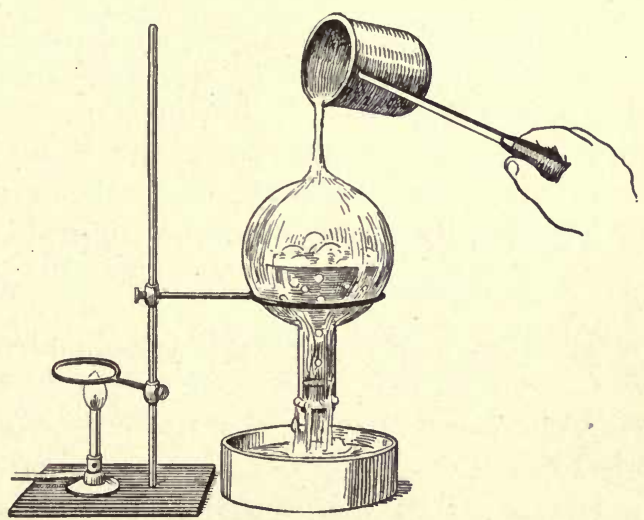

FIG. 118. - Boiling Water at a Temperature below $100^{\circ}$ Centigrade. 
with a rubber stopper and invert it on a ring stand (Figure 118) and pour cold water over it. The water in the flask will boil vigorously. When the boiling point is reduced as low as possible, remove the stopper and take the temperature. Why do we need to boil the water some time before starting the experiment? Why is it difficult to cook eggs or potatoes by boiling on high mountains?

\section{Laws of Ebullition.}

1. Under constant pressure every liquid has a definite boiling point.

2. While the liquid is boiling the temperature remains constant until all is vaporized.

3. The boiling point varies with the pressure, the greater the pressure the higher the boiling point and vice versa.

\section{EXERCISES AND QUESTIONS}

1. Why do telegraph wires sag more in summer than in winter?

2. What temperature Fahrenheit is equal to $35^{\circ}$ Centigrade?

3. A rod 40 inches long expands $\frac{1}{10}$ of an inch when heated $50^{\circ}$. How much would it expand if heated $1^{\circ}$ ?

4. Why are railroad tracks not laid so that the ends meet when laid in winter?

5. How could you make an air thermometer?

6. To what temperature must a quart of air at $0^{\circ} \mathrm{C}$. be heated to double its volume?

7. Why does freezing often burst water pipes?

8. Will roads dry faster on a still or windy day?

9. Why does sweet oil evaporate so slowly?

10. How do salts in solution affect the boiling point of water? Test.

11. How may we separate alcohol and water? 


\section{CHAPTER IX}

\section{QUANTITY OF HEAT AND TRANSMISSION OF HEAT}

Temperature and quantity of heat must not be considered the same thing. Temperature or degree of heat indicates how hot or cold a body is, and depends upon the rapidity with which the molecules are moving and not upon the number of molecules. Heat, or quantity of heat, which a body possesses depends upon the speed of the molecules, the number of molecules affected, and also upon the kind of molecules of which a body is composed.

The Calorie. - The metric unit used in measuring the quantity of heat in a body is called the calorie. It is the amount of heat required to warm one gram of water through one degree Centigrade. It is also the amount of heat given off by one gram of water when its temperature falls one degree Centigrade. To warm one gram of water from $0^{\circ} \mathrm{C}$. to $100^{\circ} \mathrm{C}$. requires one hundred calories. The same amount of heat is required to heat four grams of water from $0^{\circ} \mathrm{C}$. to $25^{\circ} \mathrm{C}$., or ten grams of water from $0^{\circ} \mathrm{C}$. to $10^{\circ} \mathrm{C}$.

Heat Capacity. - Different substances have different capacities for taking heat. If equal amounts of water and mercury be subjected to the same heat, the mercury will become hot much quicker than the water. The quantity of heat that is required to raise the temperature of a gram of water from $0^{\circ} \mathrm{C}$. to $1^{\circ} \mathrm{C}$. will raise the temperature of thirty grams of mercury from $0^{\circ} \mathrm{C}$. to $1^{\circ} \mathrm{C}$. ; or it requires thirty times as much heat to raise a given 
mass of water through a number of degrees as to raise the same mass of mercury the same number of degrees. Water has a greater capacity for heat than mercury. The ratio of the heat capacity of any substance to the heat capacity of water is called its specific heat. For example, the specific heat of iron is about $\frac{1}{9}$, or in other words a pound of water in cooling from $100^{\circ}$ to $0^{\circ}$ will give out as much heat as a pound of iron in cooling from $900^{\circ}$ to $0^{\circ}$.

Experiment 40. - In one beaker put 200 grams of water at $15^{\circ}$ and in another beaker put 200 grams of water at $35^{\circ}$. Now pour the two together and take the temperature. What temperature would you expect? Substitute 200 grams of lead shot for the water in the second beaker and repeat the experiment.

\section{Table of Specific Heats}

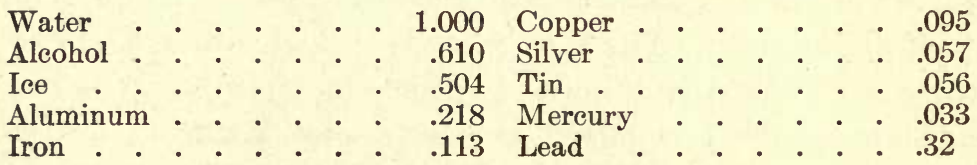

Latent Heat. - It was noticed that when heat was continuously applied to a mixture of melting ice and water the temperature did not change but remained near $0^{\circ}$ until all the ice was melted. What becomes of the heat? Evidently it represents the work which has been done in effecting the change of state from a solid to a liquid. Eighty calories of heat are required to change a gram of ice at $0^{\circ}$ to water at the same temperature. Thus water is said to have a latent heat of eighty calories. Since this heat disappears when ice or other substances melt and reappears when they solidify, it has been called latent or hidden heat. Although the name is not appropriate it is the one which is commonly used. Heat of fusion is a better name. 
To change a gram of water at $100^{\circ}$ to steam at the same temperature requires 536 calories of heat, steam being said to have a heat of vaporization of 536 .

Experiment 4I. - Place 200 grams of finely cracked ice in a tin cup. Upon this pour 200 grams of water at $80^{\circ}$ and note the resulting temperature. Why is the resulting temperature not $40^{\circ}$ ?

Experiment 42. - Pass steam through a steam trap (Figure 119) into a vessel containing 300 grams of water at about $15^{\circ}$ ? Take

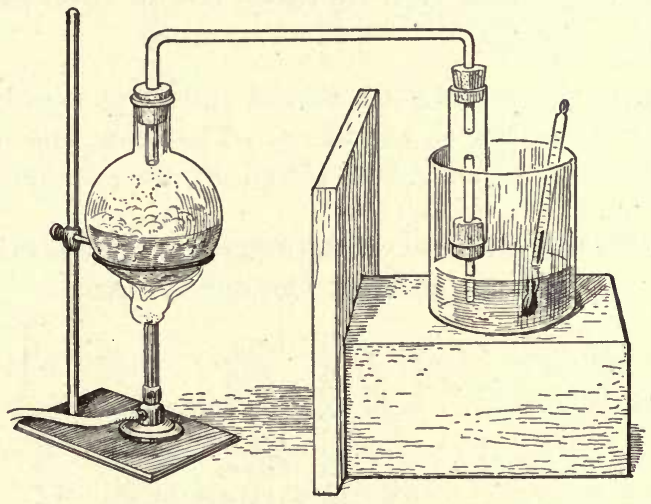

FIG. 119. - A Steam Trap.

the temperature of the water and then weigh carefully. From the data calculate the heat of vaporization.

$$
\frac{300(t-15)}{W-300}=H\left(100^{\circ}-t\right)
$$

$t=$ final temperature, $w=$ final weight, $H=$ heat of vaporization.

The condensation of steam is a great source of heat and is much used in systems of heating for buildings. The condensation point is the point at which the most heat is given off. For example, a gram of water gives up 536 calories of heat in simply changing from steam at $100^{\circ}$ to water at $100^{\circ}$.

We are now better able to understand why evaporation is a cooling process. It is simply that, since only the 
molecules which move very rapidly are able to get away from the surface of the liquid, it is the slow-moving molecules that are left. Temperature depends upon the speed of the molecules in a body. If the swiftly moving molecules are removed, the temperature falls.

Transference of Heat. - Iron is a better conductor of heat than glass. In fact all metallic solids are better conductors of heat than non-metallic solids such as glass and wood.

Experiment 43. - Hold a glass rod and an iron rod so that the end of each rod will be in the flame. The other end of the metal rod soon becomes hot, while with the glass rod no change is observed.

Some Common Substances Arranged in the Order of their Relative Heat Conductivities

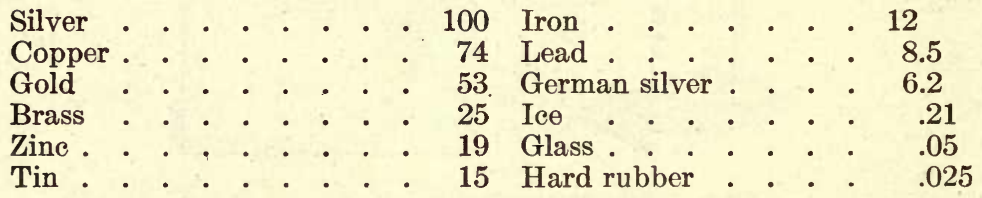

Experiment 44. - Arrange four wires, one of copper, one of brass, one of iron, and one of German silver, on a piece of cardboard as shown in Figure 120. Hold the ends in the flame of a Bunsen

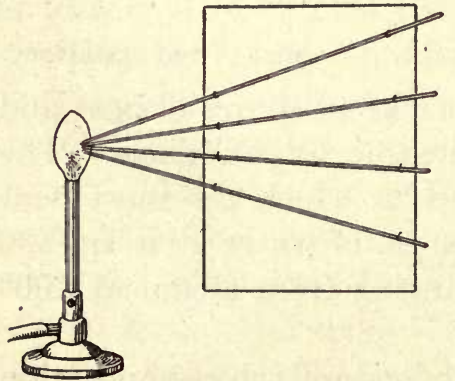

Fig. 120. - Metals vary in their conduction of heat. burner. Determine their relative conductivities by touching a match to them at equal distances from the flame.

Liquids and gases are poor conductors of heat. That water is a poor conductor may be shown in the following way : Weight a small piece of ice so that it will rest at the bottom of a test tube full 
of cold water. Heat the upper part of the tube with a Bunsen burner as shown in Figure 121. The water at

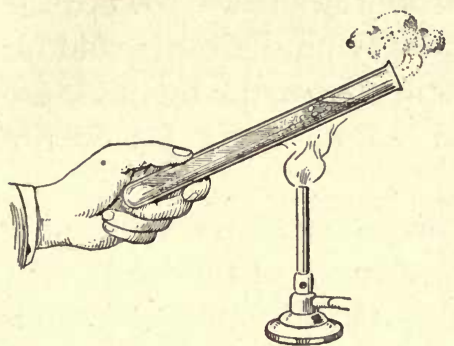

Fig. 121. - Water is a poor conductor of heat, as is shown by the experiment. the upper part of the tube may be boiled for some time without melting the ice.

Experiment 45. - Place the bulb of an air thermometer a fraction of an inch below the surface of the water in a funnel arranged as in Figure 122. Now pour some ether on the water and set it on fire. Hardly any change in temperature will be indicated by the air thermometer. The conductivity of water is about $\frac{1}{1200}$ that of silver.

Gases are even poorer conductors than liquids. Dry air has almost no conductivity. The warmth of fur and woolen garments is due to the fact that they have so many minute spaces containing non-conducting air. There are many uses of non-conducting air. It is on account of this that snow is such an efficient protection to wheat and other vegetation. Ice houses have double walls with the space between filled with sawdust. Many houses now have double windows. Loosely woven cloth is warmer than the same weight of closely woven ma-

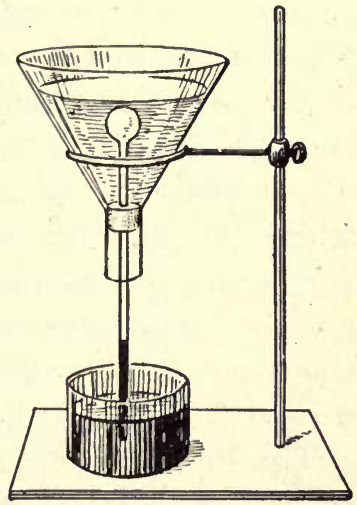

FIG. 122. terial. Loose and fibrous materials are always poor conductors on account of the air entrapped between their fibers. 
Conductivity of the Earth. - Although the interior of the earth is highly heated, the surface of the earth is very little affected by it. As we go from the surface toward the center of the earth we find that the ground is quite cold for a few hundred feet. As we go still deeper the temperature increases at the rate of about $1^{\circ} \mathrm{C}$. for every 110 feet of descent.

Water pipes and drain pipes are placed a few feet under ground so that they will be out of reach of the frost.

Conductivity and Sensation. - On a cold day metals feel much colder than a piece of wood, although the temperature of the wood be the same as the temperature of the metal. On the other hand, if both bodies have been lying in the hot sun the metal will seem much hotter to the hand than the wood. The explanation is found in the fact that the metal, being a much better conductor of heat than wood, conveys the heat away from the hand much more rapidly when it is cold, and conveys the heat to the hand much more rapidly when it is hot, than does the wood. If the metal is warmer than the body, it will feel hotter than the wood; if it is colder than the body, it will feel colder than the wood. We speak of "warm blankets," when the blankets are evidently no warmer than the surrounding objects. We simply mean that the blankets are poor conductors of heat, and so they feel warmer than the other objects. So also the rug feels much warmer than a tiled floor or an oilcloth.

The Fireless Cooker. - This is merely an arrangement of non-conducting substances to prevent the escape of heat from heated foods placed in it (Figure 123). The two walls of the fireless cooker are separated by a thick layer of non-conducting materials such as sawdust, felt, or cork shavings. These inclose a considerable amount of air, 
which is a very poor conductor of heat. The packing prevents convection currents and retards radiation and

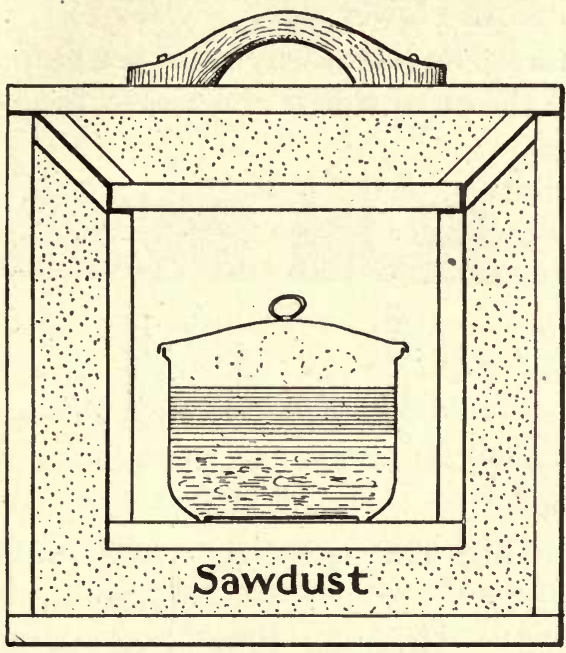

FIG. 123. - A Homemade Fireless Cooker. conduction. Food to be cooked is first heated and then placed in the cooker where the cooking continues, since the heat cannot escape.

It is quite easy for anyone who is at all handy with carpenter tools to make a very good fireless cooker after the plan of the one shown in the cut.

The Thermos Bottle. - The thermos bottle is similar to the fireless cooker. It is made of two glass bottles which are sealed together at the top after the air in the space between them has been exhausted. This prevents the loss of heat from the inside bottle by convection and conduction. The outside bottle is lined on the inside with an excellent reflecting surface which prevents a loss of heat from the inside bottle by radiation (Figure 124).

The Davy Safety Lamp. - If a piece of wire gauze be held above an open gas jet and a match applied below the gauze, the flame will burn below the gauze as in Figure $125(b)$, but it will not pass

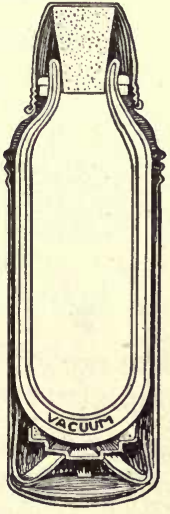

Fig. 124. - Cross Section of Thermos Bottle. 
through to the upper side. If it is ignited above the gauze, the flame will burn as shown in Figure 125 (a), but it will not pass through to the lower side.

The metal gauze conducts the heat away from the flame so rapidly that the gas on the other side is not heated to the ignition point. It is on this principle that the Davy Safety Lamp, much used in mines, depends. The distinctive feature of the lamp is that the flame is completely inclosed by
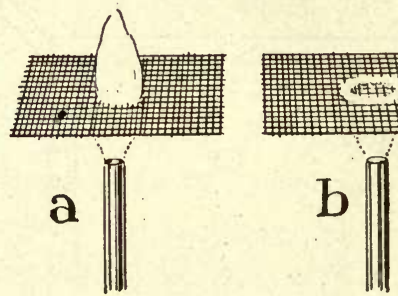

a wire gauze chimney, so Fig. 125. - Effect of Wire Gauze on a that if the mine is full of Flame. inflammable gases, they are not ignited by the lamp burning inside the gauze.

Convection in Liquids. - Although liquids are poor conductors of heat, they may be readily heated by con-

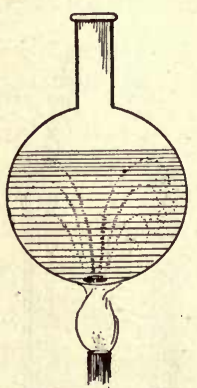

Fig. 126. - The whole mass of water is evenly heated because of the convection currents. vection. In conduction there was no movement of the molecules from one end of the metal rod to the other, but only vibrations of the molecules in a limited space. In convection, however, the molecules move from place to place. If the heat had been applied to the bottom of the test tube in Figure 121, the ice would have melted quickly, and the whole tube of water would have heated evenly. This shows that heat is transferred much more rapidly from the bottom of the tube toward the top than from the top toward the bottom.

Experiment 46. - Fill a Florence flask two thirds full of water and drop into it a crystal of potassium permanganate. Heat the 
bottom of the flask with the tip of a Bunsen burner flame. The coloring matter will show the direction of the convection currents (Figure 126). The water nearest the flame becomes heated and expands, thus becoming less dense than the surrounding water. This lighter water is then forced up by the denser water which comes in from the sides to take its place.

Convection in Gases. - The winds are convection currents in the atmosphere caused by the unequal heating of the earth by the sun. This principle easily explains the land and sea breezes near the coasts of large bodies of water. During the daytime the land is heated more rapidly than the water, the specific heat of water being much greater than that of the earth. The hot air over the land, being lighter, is forced up by the cooler air from the ocean. This is the sea breeze which blows during the daytime and reaches its maximum strength usually late in the afternoon. At night the earth cools more rapidly than the sea, and in a short time the sea is warmer than the land and the current of air is reversed. This is the land breeze which blows during the night and reaches its maximum toward morning. These winds are more noticeable in the tropics, since the change in temperature from day to night is greatest there.

Radiation. - When we stand before a fireplace, it is evident that we are receiving heat that comes to us neither by conduction nor convection. It cannot be due to conduction, because the conductivity of air is very small. It cannot be due to convection, because the currents of air are moving toward the fire instead of away from it. There must therefore be some way in which heat travels across space other than by conduction or convection. This third method of heat transference in which the heat emanates in straight lines from a source inde- 
pendently of air currents or any conducting matter is called radiation.

It will be well for us to note some of the differences between conduction, convection, and radiation.

Conduction and convection are comparatively slow, while radiation is rapid. The sun's heat comes to us with the enormous speed of light, 186,000 miles a second. The heat which comes to us by radiation comes in straight lines, while conducted or convected heat may come by the most roundabout paths. A screen placed between the source of radiant heat and a body will cut off the heat from the body.

Radiant heat will pass through certain media without heating them. The heat from the sun will pass through ordinary window glass and leave it much colder than the objects it falls upon inside the room. Also the upper regions of the atmosphere are very cold even in the hottest time of the year.

\section{QUESTIONS AND PROBLEMS}

1. Tubs of water are sometimes put in cellars in cold weather that the freezing of the water may prevent the freezing of the vegetables. Explain this phenomenon.

2. Why is a quart of heated water a better foot-warmer than an equal volume of heated lead?

3. How did "latent heat" get its name?

4. 100 grams of water at $10^{\circ}$ C. are mixed with 200 grams of water at $50^{\circ} \mathrm{C}$. What is the resulting temperature?

5. If 20 grams of ice at $0^{\circ}$ are mixed with 100 grams of water at $80^{\circ}$ what will be the temperature?

6. Why do we put salt on ice in an ice-cream freezer?

7. What is the temperature of a mixture of ice and water?

8. What must be the temperature of the water if when equal weights of water and ice at $0^{\circ}$ are mixed the result is water at $0^{\circ}$ ?

9. Water boils at $90^{\circ} \mathrm{C}$. at Quito. How do you account for the low boiling point? 


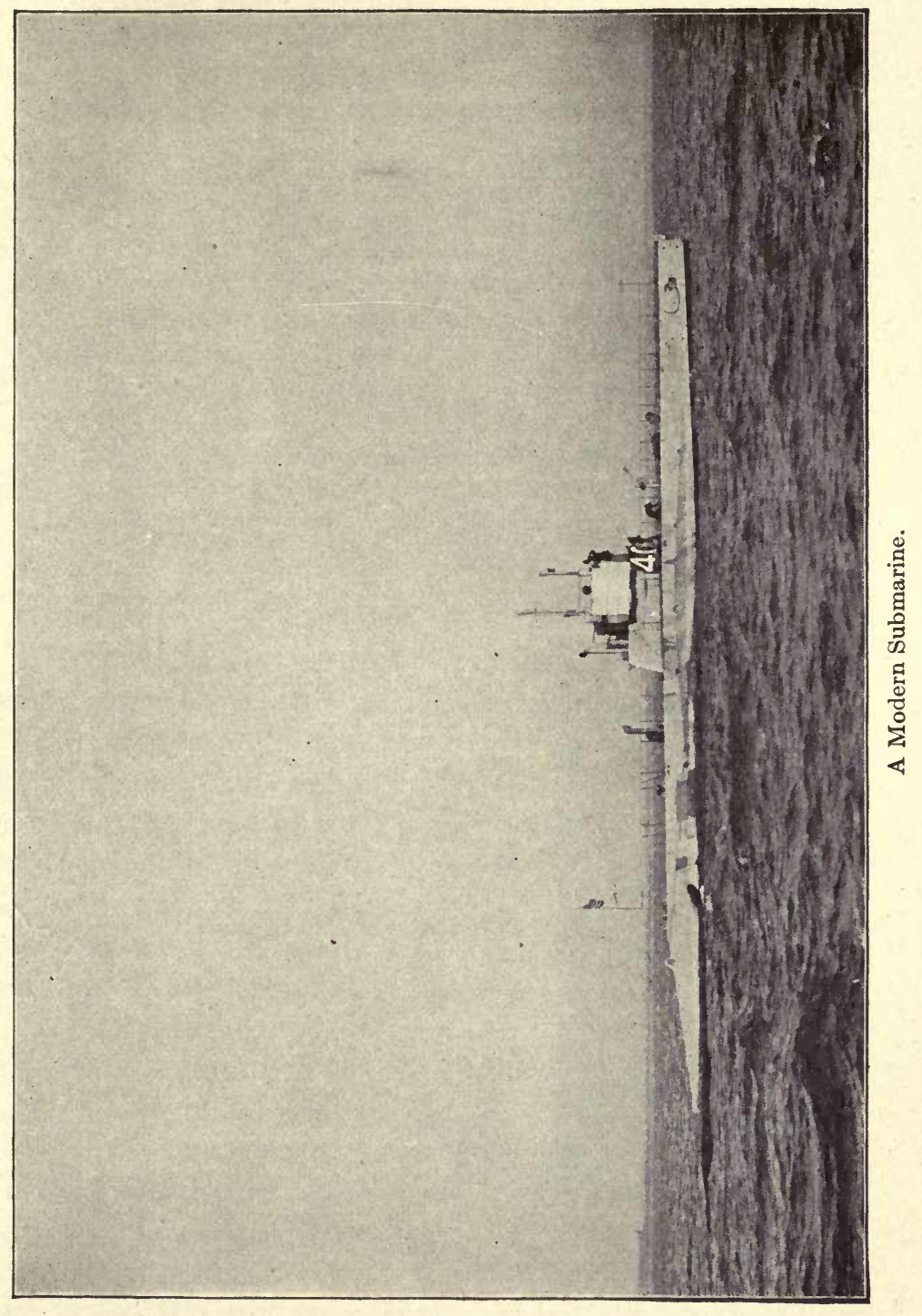



10. Why do small bubbles rise in a vessel of water that is being heated long before the boiling point is reached?

11. Why is scalding by steam more serious than scalding by water at the same temperature?

12. How do freezing and thawing break up rocks?

13. If 10 grams of steam at $100^{\circ}$ are mixed with 80 grams of water at $0^{\circ}$, what will be the resulting temperature?

14. Why are stove irons commonly made with wooden handles?

15. Why do we wrap ice in blankets to keep it from melting?

16. Why is woolen cloth warmer than linen?

17. How may we prove that air goes up over a hot stove?

18. What is the principle involved in the fireless cooker?

19. Why is the air inside a hothouse warmer than the air outside, even if it is not heated artificially?

20. How may we prove that radiant heat and light travel at the same speed?

21. Why is there no loss of heat by convection in a thermos bottle?

Heating and Ventilating of Buildings. - All three modes of heat transference are used in the heating and ventilating of our homes and other buildings, but convection is by far the most important principle involved. Hot-air and hot-water systems are both applications of this principle. Hot-air Heating.- - This system is in quite common use in small buildings. It consists of a furnace which heats the air in a chamber (Figure 127) surrounding the fire

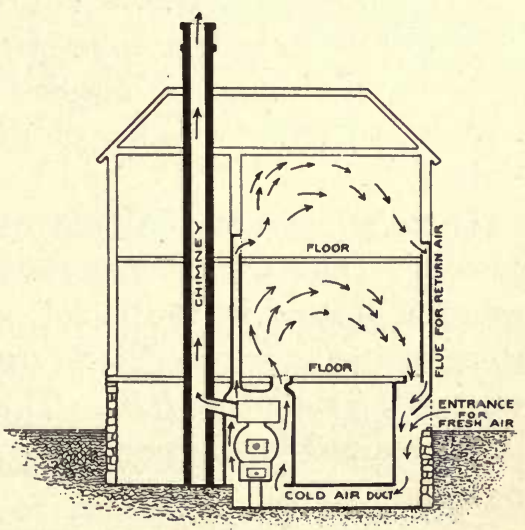

Fig. 127.

Diagram of a Hot-air Heating System. box. As the air is heated, convection currents are produced in the pipes leading to the different rooms 
of the house. After losing some of its heat the air returns to the furnace through the cold-air duct, where it is mixed with fresh air from the outside and reheated. Many furnaces have no fresh-air inlet. The air in houses having this sort of furnace is almost

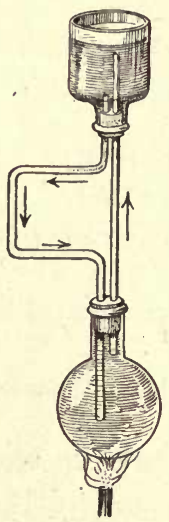

Fig. 128. - Convection Currents in a Liquid. certain to have many impurities in it, since the only sources of fresh air are the cracks around the doors and windows. The air which feeds the fire does not reach the rooms, but passes out the chimney, as indicated by the arrows. After the fire is well started the damper should be closed, since a great deal of heat is lost by way of the chimney.

Hot-water Heating. - Figure 128 shows an arrangement which illustrates the principle of hot-water heating. The whole apparatus is filled with water, the water in the upper vessel being colored. When heat is applied to the lower vessel, convection currents will be produced, as indicated by the arrows.

There are several different ways of arranging hot-water systems. One arrangement is shown in Figure 129. The water is heated in the jacket around the furnace $A$ and rises to the reservoir $R$, returning through pipes $p p^{\prime}$ by way of the radiators $B B^{\prime}$. The circulation in this system is maintained in the same way as in the apparatus shown in Figure 128.

In modern buildings a system known as the " directindirect" is now much used. In this system fresh-air ducts lead to coils heated by steam or hot water. These coils heat the air, which by convection is carried to the 
different rooms. In large systems fans are used to supplement the convection currents, thus insuring a more even distribution of heat in the building.

The Thermostat. - The thermostat is an instrument used to regulate the temperature of rooms and buildings. Figure 130 shows the working principle of a simple form of thermostat. The vertical bar is a part of two electric

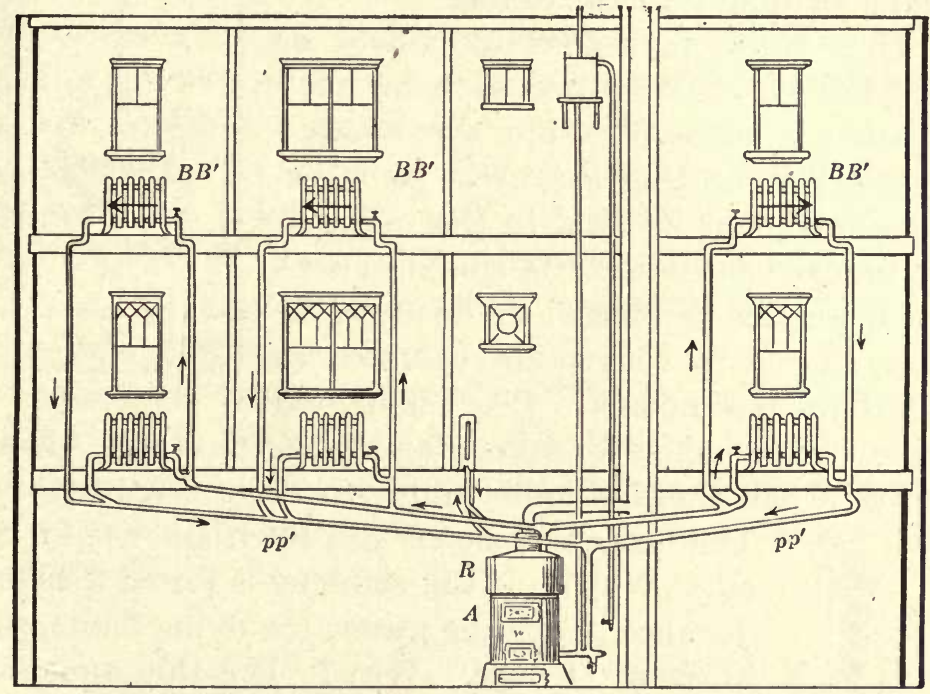

FrG. 129. - Hot-water System for House Heating.

circuits. The uneven expansion and contraction of the two elements of the compound bar throws the vertical rod against $A$ when the room becomes too hot and against $B$ when it becomes too cold. Electromagnets in the two circuits control the supply of steam, hot water, or hot air to the different rooms and thus regulate the temperature.

Ventilation. - Since the lungs are constantly exchang-. ing large quantities of air laden with carbon dioxide for oxygen, it is necessary that the air in the rooms in which 
we live be renewed frequently. All modern schoolrooms and public halls are now provided with systems of ventilation or apparatus to force in fresh air and remove foul air. The basis of all ventilation methods is found in the fact that cold air is heavier than warm air. The air in a room is usually colder near the floor than near the ceiling.

If a house is heated by stoves or fireplaces, no special provision for ventilation is necessary; but where other systems of heating are used, some attention should be paid to the question of proper ventilation. A simple experiment may be performed to illustrate the way in which convection operates to ventilate our houses. Place a lighted

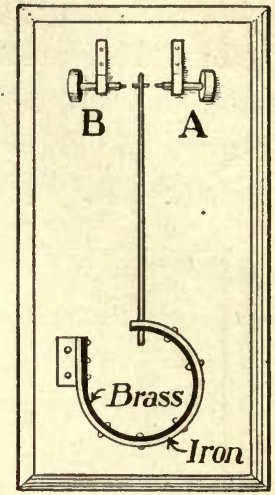

Fig. 130. - Thermostat whose Action Depends upon, the Unequal Expansion of Two Metals. candle in a shallow vessel containing a. little water. Now bring a tall lamp chimney down over it (Figure 131).

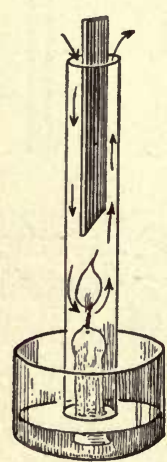

FIG. 131. The flame will slowly die down and finally go out. Why? If the chimney is raised a slight distance above the water, the dying flame will brighten at once. Why? If a thin metal or cardboard partition is lowered in the chimney almost to the flame, the latter will burn continuously although the bottom of the chimney be under water. The reason will be evident if a piece of burning torch paper is held near the chimney.

In order to secure satisfactory ventilation it is estimated that a room should be supplied with a minimum of thirty cubic feet of fresh air per minute for each person. If the speed at which the fans drive 
the air in a school building is three hundred feet a minute, the area of the opening required for each person is about fifteen square inches.

Need of Moisture in the Air. - It is quite important that the air in our homes, schoolrooms, and other such buildings have the proper amount of moisture in it: When the humidity of the air is less than 40 , it takes up the perspiration from our bodies too rapidly, and the skin becomes dry. When the humidity is more than 60 , the perspiration does not evaporate rapidly enough, and the air seems oppressive or " muggy."

As cold air enters a heating system and is heated, its capacity for holding moisture is greatly increased, while the amount of moisture in it has not been changed. As a result the amount of moisture present in the air of our homes is usually too small.

In hot-air furnaces care should be taken to keep the water pan in the air box full of water. If other systems of heating are used, water should be kept in the rooms in open dishes, or small buckets of water may be hung on small wires in the registers.

\section{QUESTIONS}

1. How is your building heated?

2. What is the objection to forcing air into a room faster than 350 feet a minute?

3. Explain why the old-fashioned fireplace furnished good ventilation.

4. Why do we need more air during sleep?

5. How are most ordinary dwelling houses ventilated? 


\section{CHAPTER $\mathrm{X}$}

\section{WEATHER}

Meaning of Term "Weather." - The term "weather" is a comprehensive one. It includes all the many conditions of the earth's atmosphere, such as its temperature, its pressure, and the amount of moisture it contains, the variations of the wind, the amount of rainfall, and the appearance of the sky. We have made quite a careful study of the two substances with which we are concerned in. the study of weather. These two substances are air and water - air or atmosphere, as the outer portion of the earth; and water in its many forms: as water vapor in the atmosphere, clouds, fogs floating in the atmosphere; and rain, snow, hail, dew, and frost as it leaves the atmosphere again. The weather is almost constantly changing. We say "It is cold to-day" or "It is sultry to-day" according as the conditions of the atmosphere are such as to produce the one effect or the other. The average of the weather conditions for a given place is the climate of the place.

Functions of the Air. - The atmosphere is of vital importance and serves us in a number of important functions : (1) It supports life in various ways ; (2) it conducts sound; (3) it transfers and distributes heat, and also acts as a non-conductor of heat under certain conditions ; (4) it diffuses light; (5) it supports combustion; (6) it drives wind-power machines; (7) it exerts a buoyant force on all objects immersed in it, thus enabling some 
animals to fly and balloons to rise from the surface of the earth; (8) it distributes moisture; (9) it produces waves and ocean currents ; (10) it is an important factor in weathering and has many other functions.

The atmosphere as we consider it in studying weather includes much more than simply the mixture of the gases, nitrogen and oxygen. It includes all the various substances floating in it, such as dust particles and minute organisms in the form of bacteria, microbes, and decayed plant and animal tissue. The dust of the air is thought to be important in the precipitation of moisture. The minute dust particle, by virtue of the law of gravitation, becomes the nucleus of condensation. This little particle of moisture increases in size until it forms a raindrop or snowflake and then falls to the ground.

Colors of the Atmosphere. - On clear days the sky has a beautiful blue color. This color is due to the breaking up of the sunlight by the countless number of small particles floating in the air. If the particles are quite large, other colors are produced. The smoke from the lighted end of a cigar is blue because the particles in the smoke are very minute. The smoke particles from the other end of the cigar, however, are increased in size by the moisture from the smoker's mouth, and a dull grayish color is the result. Red and yellow colors predominate in the sky near sunrise and sunset, because the sunlight passes obliquely through a great thickness of atmosphere near the earth's surface and the other colors are sifted out by the large particles of dust which float near the ground.

The twilight arch, a rosy arch of lighted air, may be seen in the east after a clear sunset as the sun sinks below the horizon. As the sun sinks lower and lower this arch rises until it vanishes in the darkening shadow of night. 
Air Density. - If the earth's surface had the same temperature at every point, the air near this surface would have the same density. With the varying degrees of temperature of the surface, the air becomes heated in certain places and then being less dense is forced up by the heavier cold air around it, just as a cork released at the bottom of a basin of water is forced up by the heavier substance surrounding it. Air also becomes lighter as it absorbs moisture or water vapor, since steam has a lower density than air.

For measuring the pressure of the atmosphere, the barometer is used. This instrument has been described in Chapter V. It should be observed that the barometer does nothing more than indicate the air pressure, and this with other facts enables the forecaster to predict changes in the weather.

Isobars. - In order to forecast weather conditions, it is necessary to compare barometric readings from many different parts of the country. Since barometric readings vary with the elevation and the temperature, in order to make intelligent comparisons it is necessary to make corrections for these differences in elevation and temperature. By the aid of prepared tables this is readily done, all readings being reduced to the corresponding reading at the elevation of sea level and the temperature of the freezing point of water. After the corrections are made lines are drawn connecting points having equal atmospheric pressure. These lines are called isobars. A map. showing lines of equal pressure is called an isobaric map. On the daily weather map isobars (continuous black lines) are drawn representing variations in pressure of one tenth of an inch. See Figure 132.

An examination of several daily weather maps will 


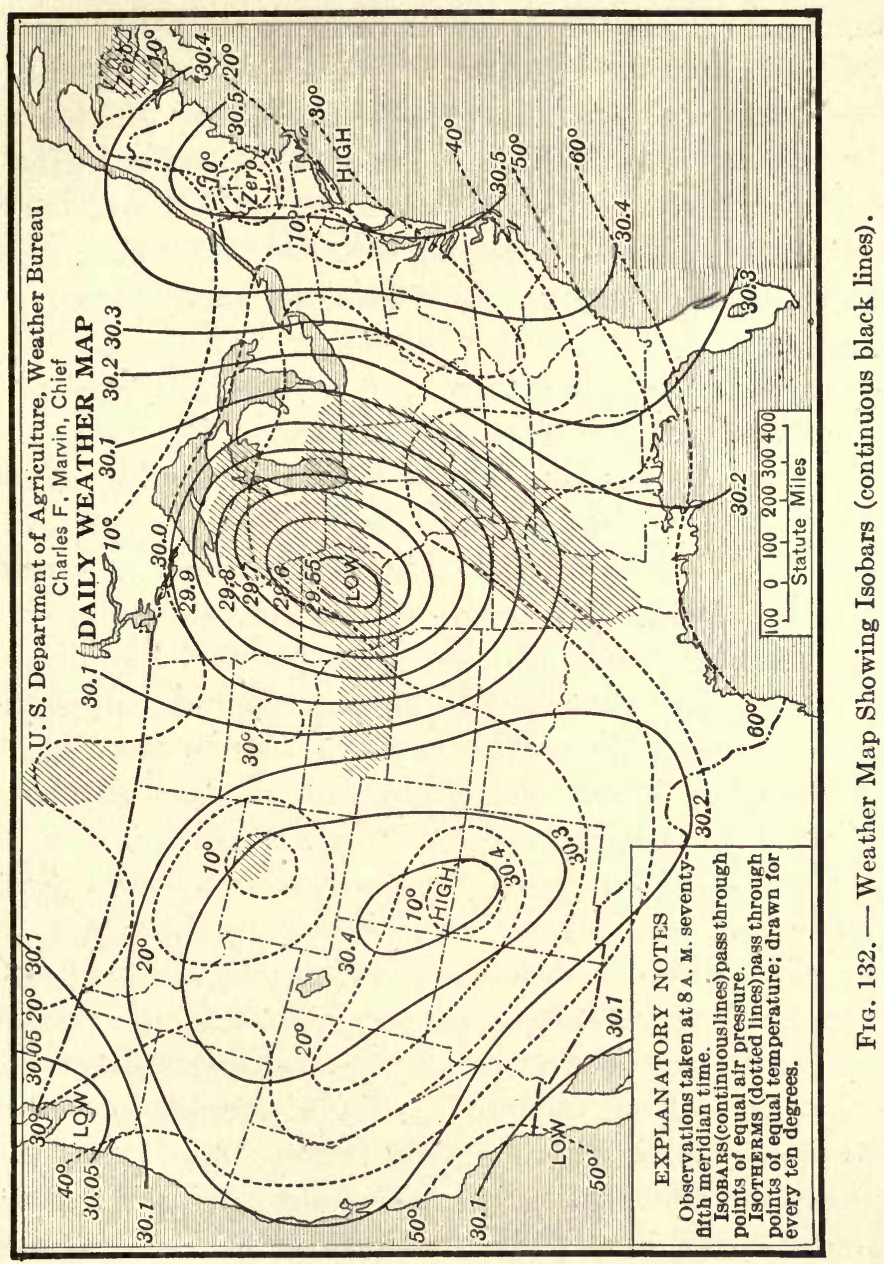


show that the isobars usually run in concentric lines around areas marked in some cases high pressure, and in other cases low pressure. The atmosphere tends to move from high to low pressure, and the greater the difference between the high and the low pressure the faster will be the movement of the air or the higher will be the wind velocity. This may be tested by comparing the weather maps on a windy day with the weather maps on a calm day.

Atmospheric Temperature. - A very small amount of heat comes from the stars, but the sun is the real source of nearly all the heat of the earth's surface. The atmosphere absorbs a small part of the heat of the sun's rays as they first pass through it, while the remainder is intercepted by the earth. Here it is partly absorbed and partly reflected back into the atmosphere. The proportion of the rays absorbed to those reflected varies with different parts of the earth's surface. Much more heat is reflected from water than from soil, and an increasing proportion is reflected as the angle of incidence of the rays increases. When the rays are vertical, the greatest percentage of the incident radiant heat is absorbed; this gradually decreases until at sunset nearly all the heat is reflected. The reflected radiant heat aids in warming the air, but most of the heat of the atmosphere is received by conduction from the earth. The lower portions of the atmosphere absorb heat mùch better than the higher portions for the reasons that the air near the earth is more dense and is also filled with impurities, such as dust, heavy gases, and water vapor, which absorb heat readily. Each little particle of dust in the atmosphere becomes a secondary source of heat and heats the air around it. As the lower layer of the atmosphere becomes heated, 
convection currents are produced which distribute the heated portions throughout the whole atmosphere.

The air is also heated by compression and by precipitation. We have learned that air becomes heated when compressed, as in the chamber of a bicycle pump. As air descends from the higher altitudes, as from the mountain sides to the valleys below, it is compressed and warmed. As it ascends it expands and becomes colder, about $1^{\circ} \mathrm{C}$. for every 550 feet of ascent. This heating of the air by

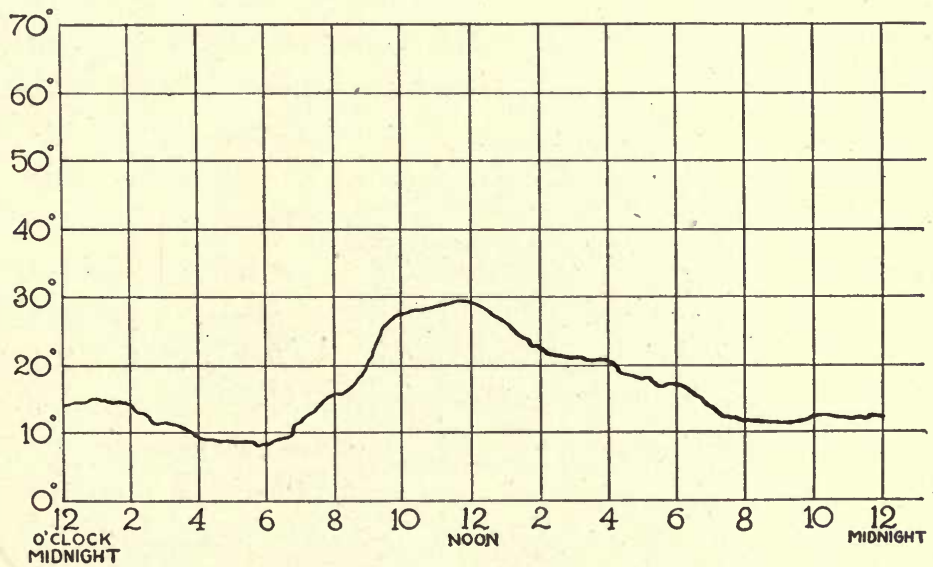

FIG. 133. - Temperature Curve Showing Variation in Temperature.

compression and cooling by expansion is known as adiabatic heating and cooling.

Air is being warmed in areas of high pressure and cooled in areas of low pressure, and it may seem at first as though the air should be warmer in areas of high pressure than in areas of low pressure. This is not the case. The reason for the low pressure is that the area is warmer; hence the air is lighter. Although the expansion of air is a cooling process, the effect is more than counteracted by the heat 
it receives by conduction from the earth. The energy required to change water to vapor appears again in the form of heat when the vapor changes back to water and falls as rain. This heat is considerable, 536 calories for each gram of water. With snow the effect is still more noticeable. Why?

Not all the heat which reaches the earth is retained. A large part of that which is reflected and radiated by the earth passes back through the atmosphere into the endless space beyond.

Thermograph. - Figure 133 shows a temperature curve or a record of the variation in temperature of a given

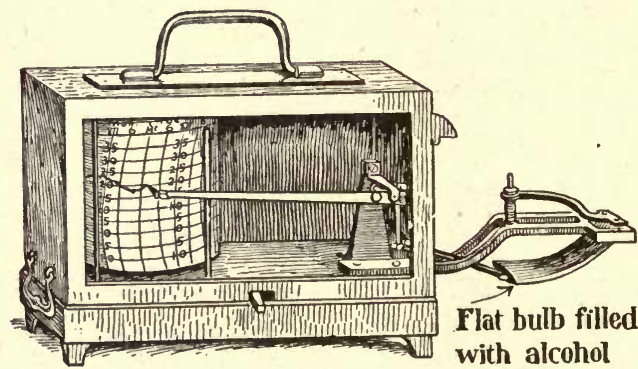

Fig. 134. - Thermograph.

This instrument is so constructed that the temperature is recorded automatically.

place for a given length of time. Such a curve may be produced automatically with great accuracy by a thermograph (Figure 134).

Experiment 47. - Take four or more thermometer readings at the same time each day for one week and plot the temperature curve.

Isotherms. - Lines which are drawn on the weather maps connecting points having the same temperature are called isotherms. There is considerable variation in 
the position of isotherms from day to day, as will be seen by consulting the daily weather maps. If these lines are averaged for a certain number of days, the result will be the mean temperature for that period of time. Figure 135 shows an isothermal map for July, Figure 136 one for January, and Figure 137 shows a chart of the mean annual temperatures for the year. On consulting these charts it will be noticed that the isotherms of 70 degrees lie at some distance on either side of the equator, but that the distances for July and January are not the same. These isotherms form the boundaries of the hot zone through which runs the heat equator or the line of highest temperature. It lies north of the true equator in July and south of it in January. The temperate zone is the belt inclosed between the isotherms of 70 degrees and 30 degrees. Beyond 30 degrees lie the frigid zones. These zones follow the movements of the heat equator and are not fixed belts.

Change of Temperature with the Seasons. - As the earth moves in its orbit around the sun, there are six months in each year in which the northern hemisphere is inclined toward the sun and six months in which it is inclined away from the sun. In the months in which it is inclined toward the sun it gains more heat by absorption during the long days than it loses by radiation during the short nights, and the temperature rises above the average temperature for the year. The opposite is true of the southern hemisphere during this period. During the other six months of the year the northern hemisphere is inclined away from the sun and the southern hemisphere toward the sun, with the result that the southern hemisphere is then the summer hemisphere and the northern the winter hemisphere. 


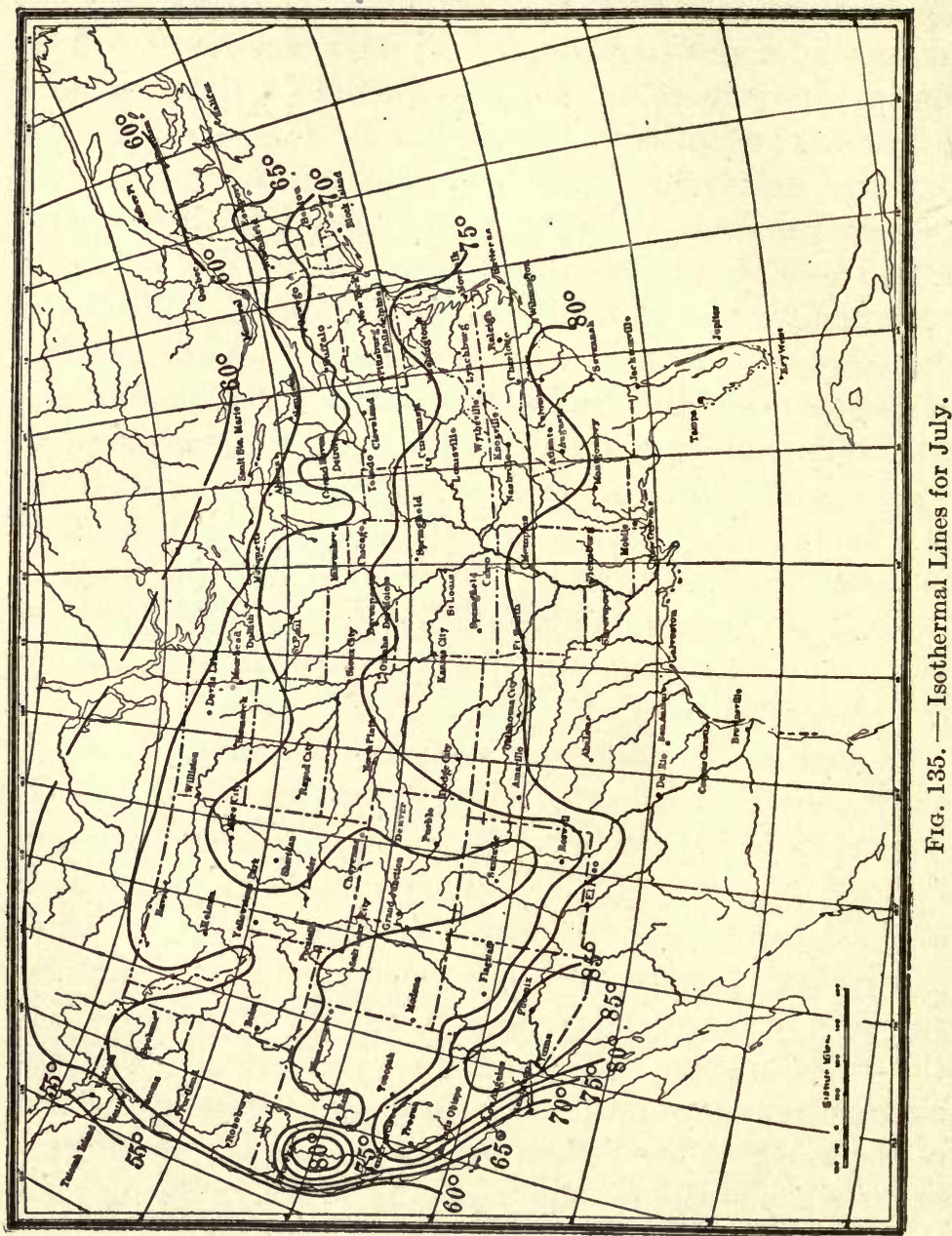




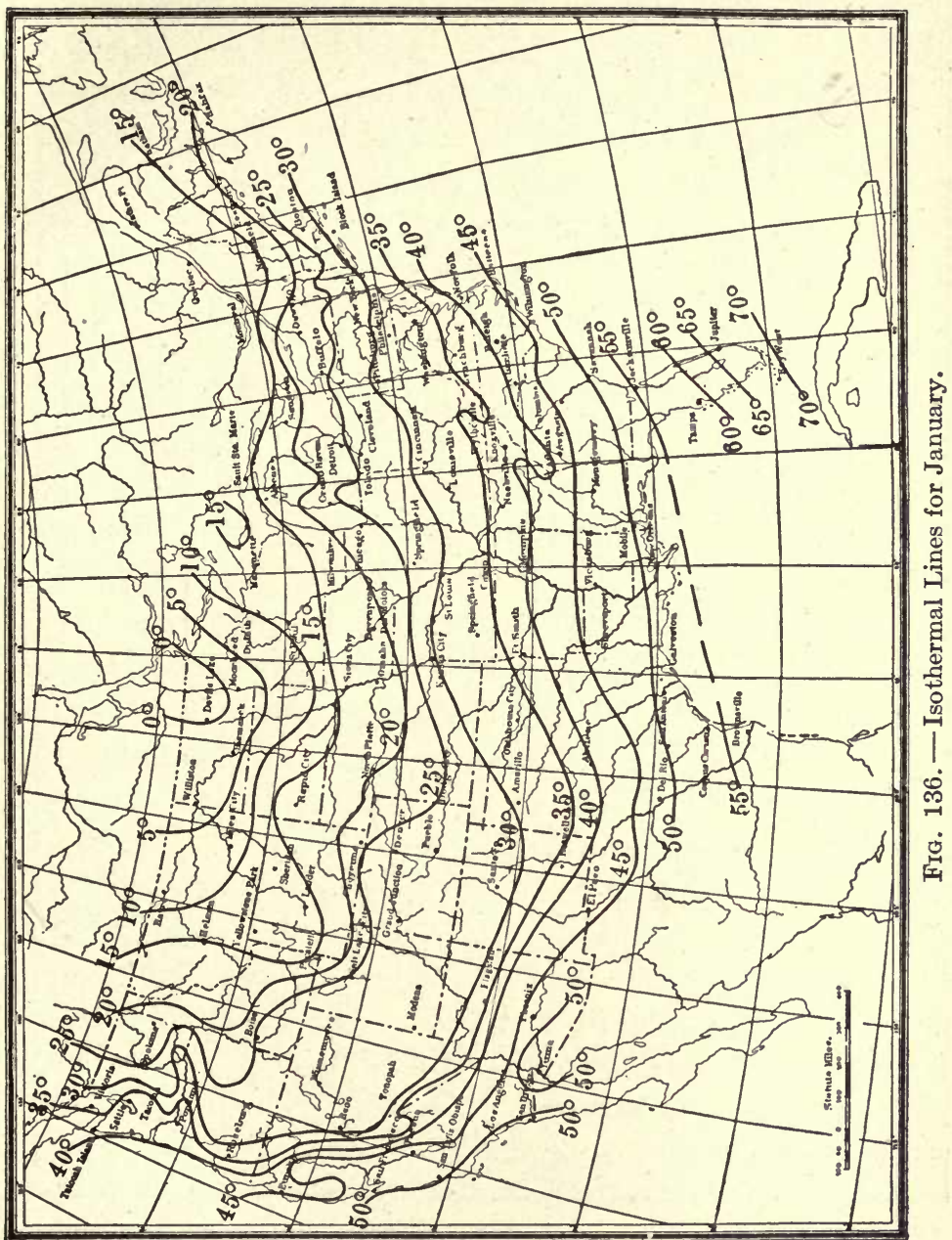




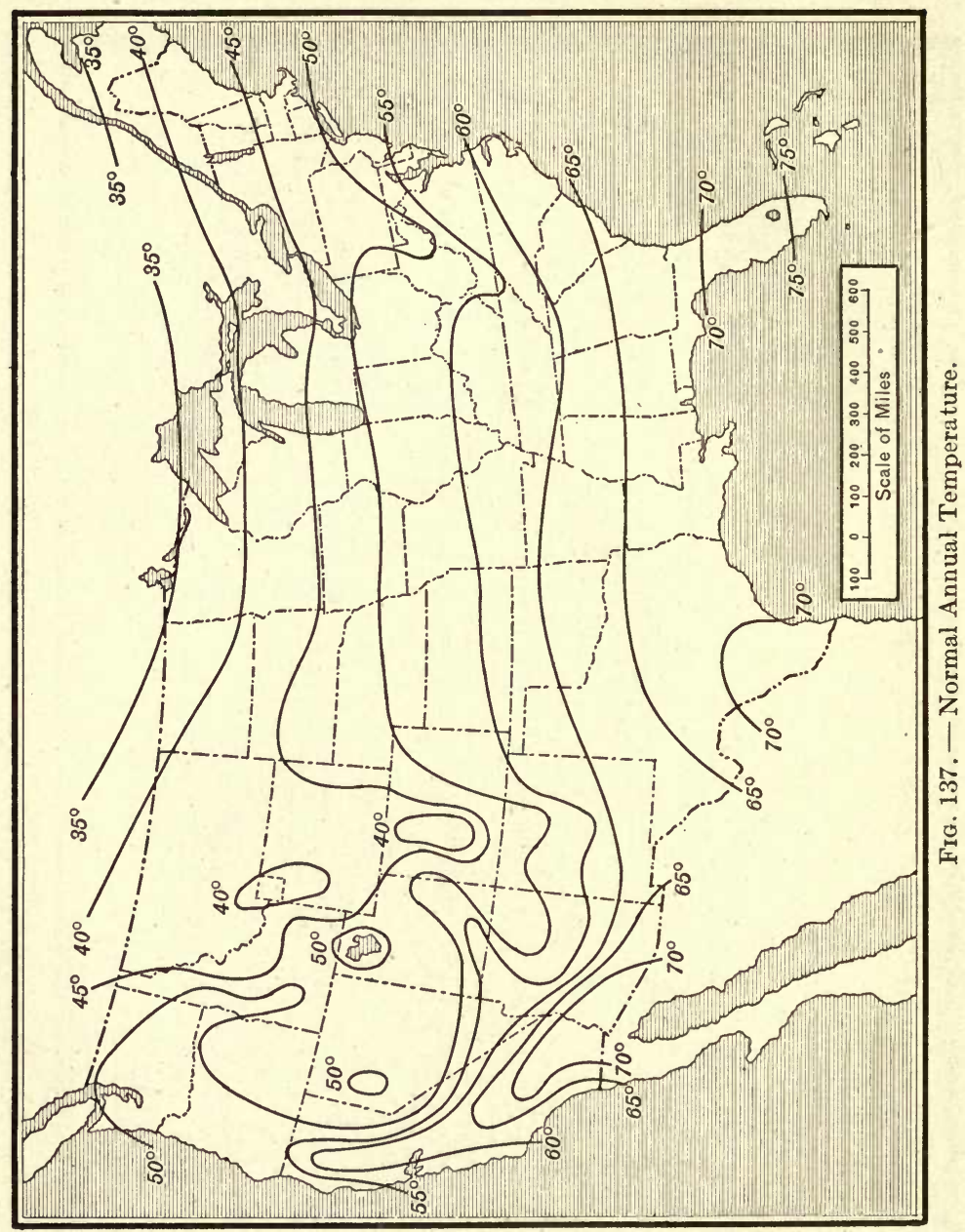


Movements of the Atmosphere. - Movements of the air are caused by the force of gravity wherever differences in atmospheric pressure exist. These differences are due to the differences in the temperature of the air, at any area of low pressure the heavier air from all sides forcing up the lighter air. A simple experiment may be performed to show the origin of the winds.

Experiment 48. - Cut a piece of paper in the form of a spiral (Figure 138) and hold it over a lighted Bunsen burner. The rapid upward current of the air will cause the spiral to turn, while colder air will be moving toward the flame, as shown in the diagram.

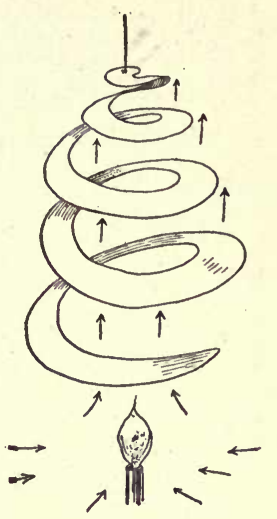

FIG. 138.

The horizontal movements of the air are called winds, when the movements are strong enough to be perceptible. The vertical movements are called calms. To summarize :

1. Winds are caused by the force of gravity;

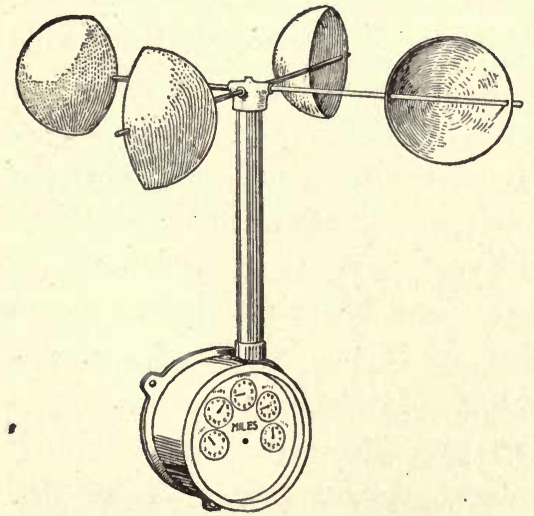

Frg. 139. - The Anemometer, an Instrument for Determining the Velocity of the Wind.

2. Winds blow from regions of high barometric pressure to regions of low pressure.

3. The velocity of the wind depends upon the barometric gradient. The steeper the grade, or the greater the difference in pressure between two areas the greater will be the velocity of the wind.

Figure 139 shows an instrument which is used 
to measure the velocity of the wind, the anemometer. The speed with which it revolves is registered as a certain number of miles per hour.

For purposes of classification winds may be grouped into terrestrial, cyclonic, and continental winds.

Terrestrial or Planetary Winds. - On all the planets which have an atmosphere, there are winds which are due to the fact that the planet rotates and that it is heated from some external source. On the earth the strength of these winds and the boundaries of the wind belts vary with the seasons. The air is heated in the region of the heat equator and moves up, thus producing a low pressure belt at the equator and a high pressure belt near the tropics (Figure 140).

The low pressure belt caused by the ascending air is known as the equatorial calms or the doldrums, and the high pressure bèlts caused by the descending air are known as horse latitudes.

The winds which blow from the regions of the horse latitudes or tropical calms toward the equator are called trade winds, so named because of the regularity with which they blow. In the days of sailing vessels they were important factors in navigation. The trade winds do not blow directly north and south but are deflected toward the west by the rotation of the earth.

The anti-trade winds are those currents of air which are high above the trade winds and blow in the opposite direction. It is the descent of these winds which produces the tropical winds or horse latitudes.

The prevailing westerlies are the winds which blow from the horse latitudes toward the poles. Like the trade winds their course is affected by the shape and rotation of the earth; they move northeast in the north- 

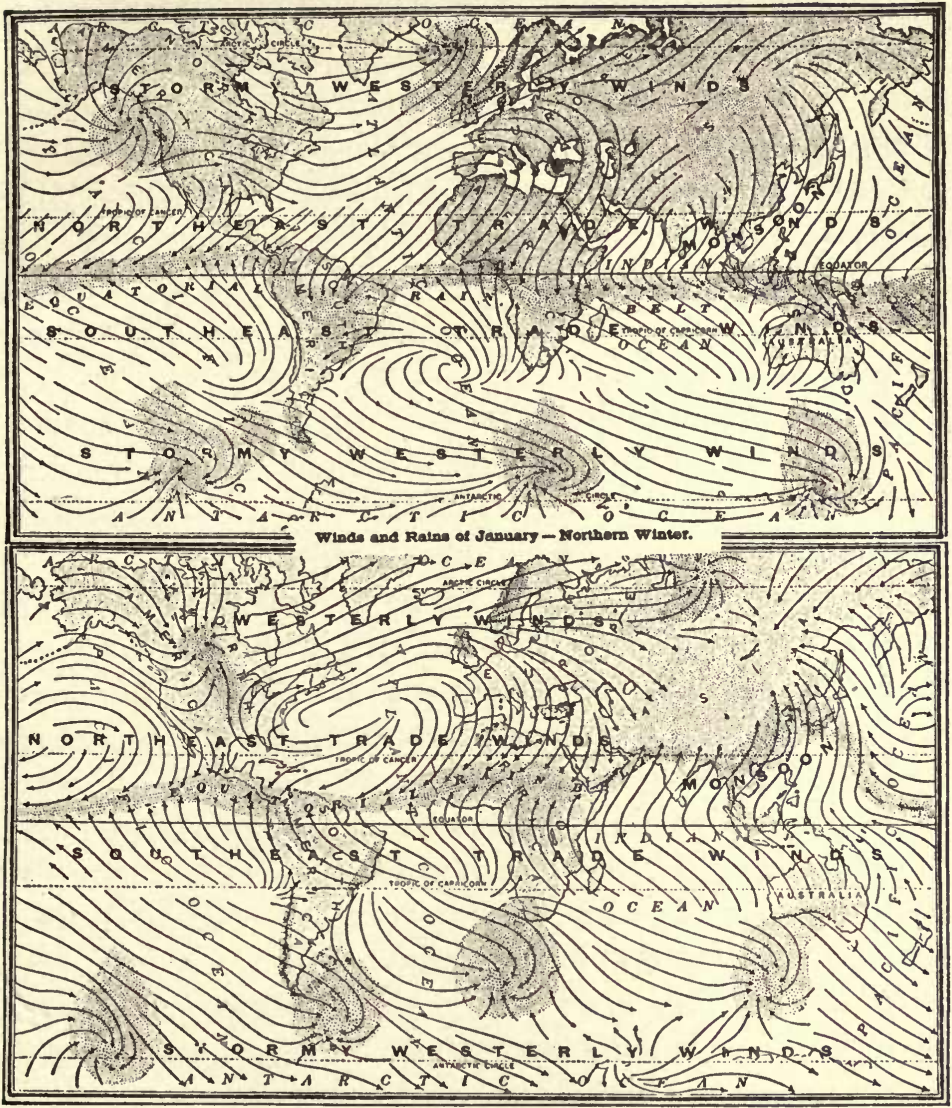

Winds and Kains of July - Northern summer.

FIG. 140. - Terrestrial Wind Belts in Summer and Winter. The Heat Equator Lies in the Midst of the Equatorial Rain Belt.

ern hemisphere and southeast in the southern hemisphere. These winds are usually a part of the great whirls known as cyclones and anti-cyclones and are subject to frequent changes in direction.

Cyclonic Winds. - Because of the rotation and shape of the earth, air flows toward a low pressure area by a 
curved route. The winds coming from all sides from similarly curved routes set the air at the center in a whirl. These cyclonic whirls cover large areas, being frequently 1000 miles or more in diameter. They should not be confused with the violent whirling storms which are so commonly called cyclones. Such storms are properly called tornadoes (see United States weather map showing cyclonic (low) and anti-cyclonic (high) areas) (Figure 141).

In the northern hemisphere the direction of the rotation of the air in a cyclonic whirl is opposite to that of the hands of a clock. In the southern hemisphere the direction is the same as that of the hands of a clock.

The general direction in which the whole cyclonic area moves is from west toward the east in the United States. Sometimes cyclones enter the United States from southwestern Canada and travel southeast to the Mississippi valley and then northeast to the Atlantic Ocean. Sometimes they move almost due east across the continent and at other times they develop in the southwest and move in a northeasterly direction. They travel at varying rates of speed, averaging 600 to 700 miles a day.

An anti-cyclone is an area of high pressure. It is the opposite of a cyclone, the winds blowing away from the center instead of toward the center.

Hurricanes. - Very violent storms, known as hurricanes in the Atlantic Ocean and typhoons in the Pacific Ocean, occasionally develop in the doldrums. They grow to be several hundred miles in diameter and are accompanied by violent winds, which whirl in great spirals around an area of low pressure and an enormous fall of rain. They usually come when the heat equator is farthest from the geographical equator. They occur in the Atlantic 


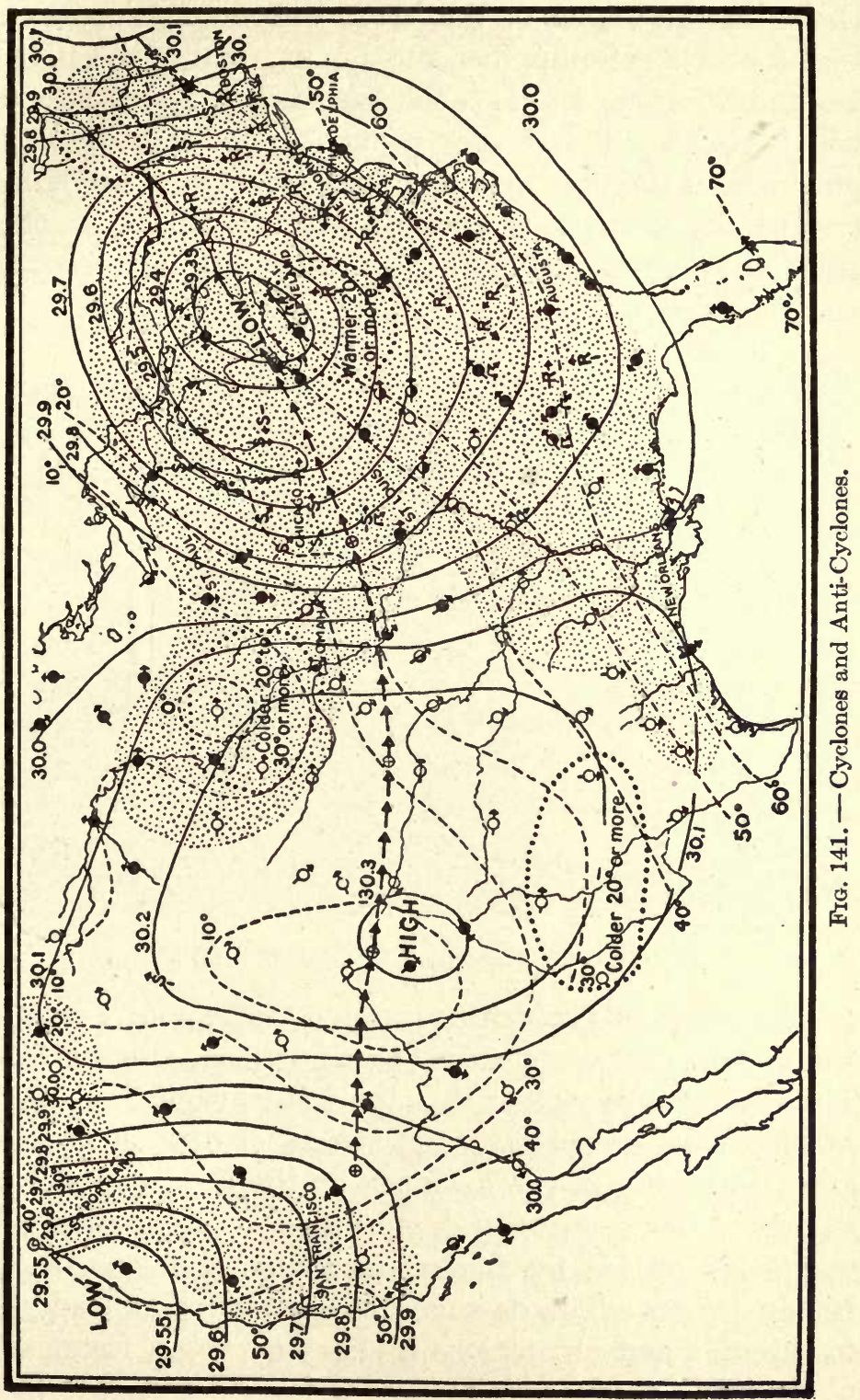


Ocean in the region of the West Indies; in the Pacific Ocean near the Philippines and Japan; and in the Indian Ocean near India and also the Island of Madagascar. It will be observed that they occur in relatively the same positions in the two oceans. They travel in a curve first toward the northwest, gradually turning until, as they enter the temperate zone, the direction is northeast.

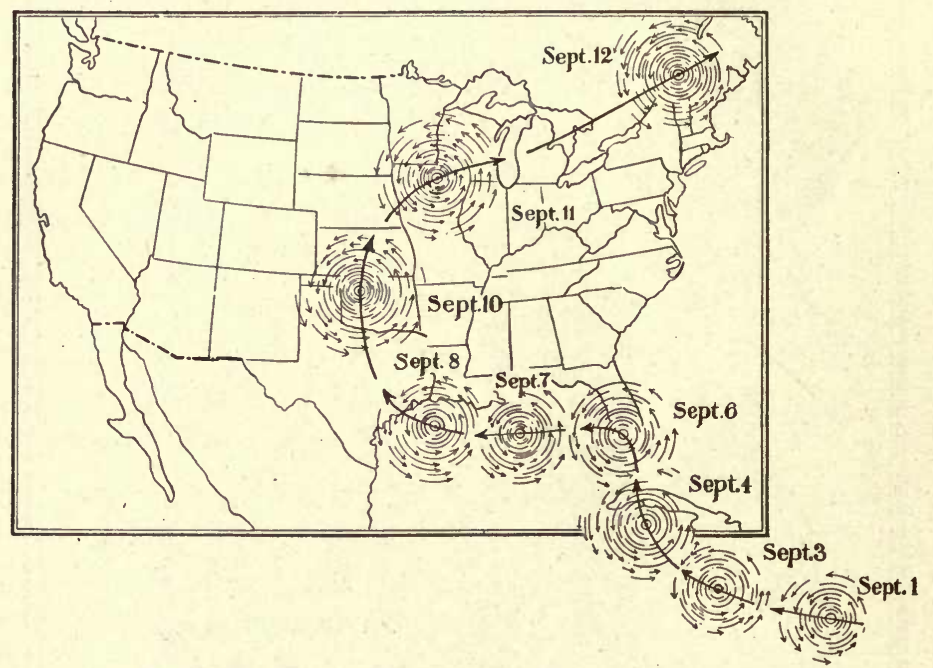

Fig. 142. - The Path of the Galveston Hurricane.

The. sea floods which devastated Galveston, Texas, in September, 1900, were caused by a hurricane (Figure 142). The wind was so violent that the waters from the Gulf of Mexico were forced into the streets of the city, doing great damage. In the lowlands at the head of the Bay of Bengal thousands of people have lost their lives in the sea floods caused by the oft-recurring typhoons of the Indian Ocean. The damage done by the wind and rain to shipping and plantations is also very great, frequently 
driving vessels ashore and stripping plantations of every vestige of usable plant growth.

Tornadoes differ from hurricanes and typhoons in duration and area affected. They originate in the region of the prevailing westerlies and are quite common in the plains in the western Mississippi valley. Their diameter varies from a few feet to a mile or even more, but the violent, destructive effects are usually confined to a path

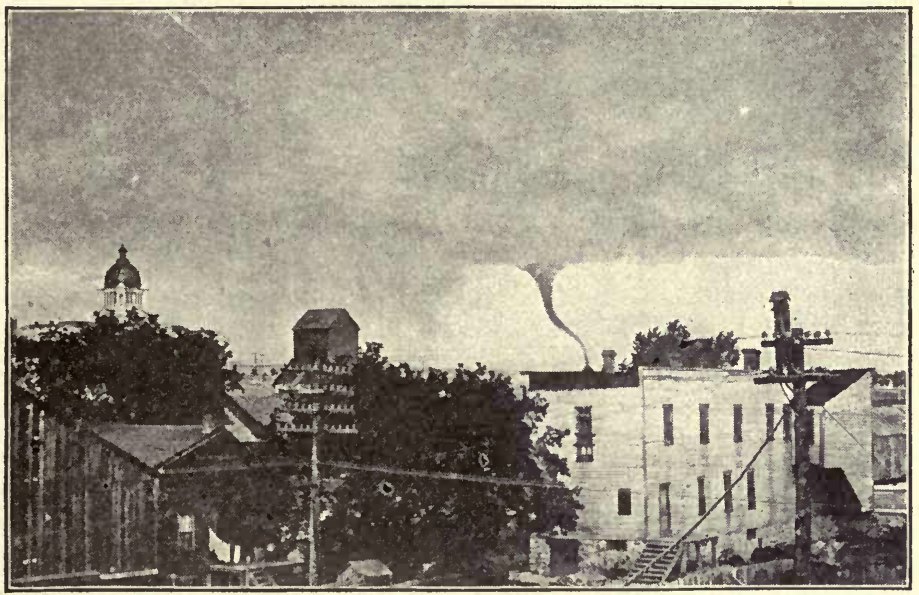

U. S. Weather Bureau.

Fig. 143. - A Tornado Cloud as Seen from Pierre, South Dakota, in July, 1910.

a few rods wide. In the summer season they are often associated with thunderstorms. As they approach they have the appearance of a funnel-shaped cloud hanging from the black mass of thunder cloud above (Figure 143). They are the most violent storms which occur in the United States. As a tornado approaches, a low roar which increases momentarily in intensity is heard. Thunder, lightning, rain, and hail blend to make the effect more terrible. Buildings that lie in the path of such a storm are scattered 
in all directions (Figure 144). Trees are uprooted and whirled about like feathers. Some of the effects, such as driving straws through pine boards and removing the feathers from chickens, are almost incredible. Such storms move forward at the average rate of about thirty miles

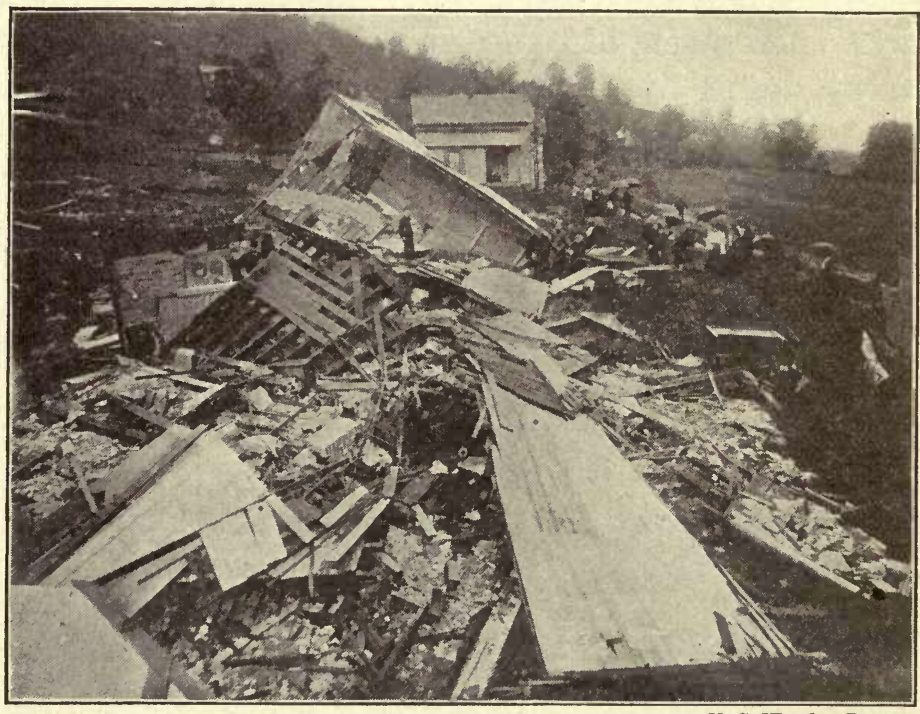

U. S. Weather Bureau.

Fig. 144. - A House Wrecked by a Tornado at Binghamton, N. Y.

an hour, but the rotary speed of the air in the whirls may reach a velocity of 500 miles an hour.

The centrifugal force of the rapidly whirling mass is sufficient to produce an area of very low pressure. As a tornado passes over a lake or the ocean, a column known as a water spout sometimes forms in the vortex (Figure 145). Some scientists hold the opinion that the water spout is formed by water which is drawn up from the ocean into the vortex of the whirl, while others think that the water comes from the clouds. 
Winds with Special Names. - The inflowing winds of cyclonic storms are sometimes given special names in different parts of the world. The hot, scorching winds which blow from the African desert across Italy are called siroccos, and the name is now often applied to any south winds which are very hot and dry. In southern Australia such winds are called brickfielders because they bake the

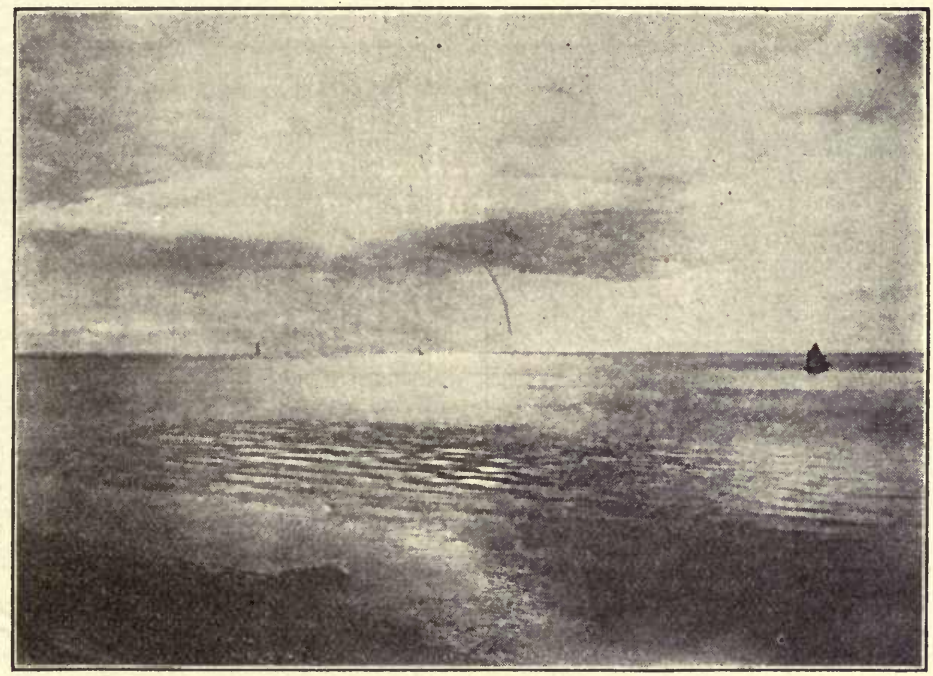

U. S. Weather Bureau.

FIG. 145. - Waterspout at Vineyard Sound, August 19, 1896.

fields until they are like bricks. The chinook is the wind that blows from the Rocky Mountains toward the Mississippi valley. It has lost its moisture on the western slope of the Rocky Mountains, and as it descends and is warmed its capacity for holding moisture is greatly increased, with the result that it takes up the moisture from the fields instead of bringing moisture to them. The blizzard of the western plains is an especially cold wind accom- 
panied by snow. The norther of Texas and the buran of Siberia are simply local names for cold winds.

Monsoons. - If land is located with reference to the belt over which the equatorial calms move so that the heat equator is over the land for a part of the year and over the adjacent water the remainder of the year, monsoons are developed. That is, the trade winds will blow from the sea toward the land for a part of the year
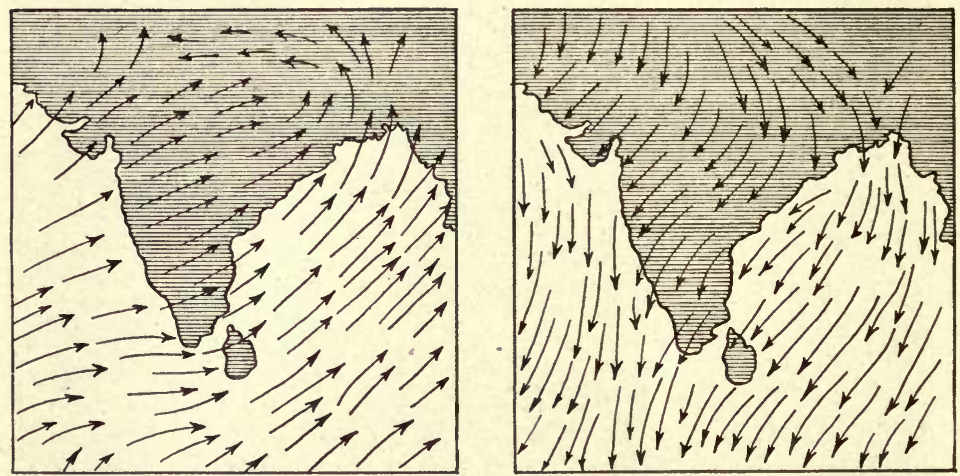

FIg. 146. - Southern Asia, Showing the Monsoon Region and the Region of Heavy Rainfall.

The arrows indicat? the direction of the wind during July and August (the lefthand illustration) and January and February (the right-hand illustration).

and from the land toward the sea the remainder of the year. The best-known monsoon winds are in the region of India, where the sea breeze in summer is so strong that it reaches the southern slopes of the Himalayas (Figure 146). As the wind passes over the warm seas it becomes laden with moisture, which is precipitated as the air is cooled on the mountain sides. The rainfall here is enormous, in some places amounting to as much as 500 inches a year. As the heat equator moves south, the direction of these winds is reversed. Since they are warmed as they descend they 
take up moisture instead of precipitating it, thus producing a period of drouth in the land. Thousands of lives have been lost in the famines following the winds.

Land and sea breezes occur along the coast of large bodies of water. During the daytime the land is heated more rapidly than the water, the specific heat of water being much greater than that of the earth. The hot air over the land expands and becomes lighter, and the heavier air from the ocean forces it up and takes its place. This is the sea breeze which blows during the daytime and reaches its maximum strength usually late in the afternoon. At night the earth cools more rapidly than the sea, and in a short time the sea is warmer than the land and the current of air is reversed. This is the land breeze which blows during the night and reaches its maximum toward morning. These winds are more noticeable in the tropics, since the change in temperature is greatest there.

Humidity and Precipitation. - Water vapor is always present in the atmosphere; the amount, however, varies greatly. The relative humidity, or degree of saturation, of the atmosphere is defined as the ratio between the amount of moisture actually present in a given volume of air and the amount which would be present if the air were saturated. When air contains all the moisture that it can hold at a given temperature, it is said to be saturated. Air over the ocean is usually quite near a state of saturation. As a usual thing we can tell something of the humidity of the atmosphere without the aid of scientific instruments. When the air contains a large amount of water vapor and feels damp, we say the humidity is high. When it contains little vapor and feels dry, the humidity is low. Dry air is more invigorating than damp air, since it produces active evaporation of the perspiration of the 
body. If the air is warm and damp we say it is " close" or sultry. Cold, damp air is penetrating and "raw."

As air is warmed, its capacity for retaining moisture is increased; and the reverse is true if it be cooled. If we cool air having a relative humidity of 80 , it will soon reach a temperature at which it is saturated or has a relative humidity of 100 per cent. If it be cooled further, some of its moisture will be precipitated as dew, rain, or some other form of precipitation. If we heat air, the relative humidity will be reduced. If we cool it, the relative humidity will be increased:

Dew Point. - The temperature of air at its point of saturation is the dew point.

Experiment 49. - Fill a polished metal cup with water and stir it with a thermometer as ice is added slowly. The temperature at which a film of moisture forms on the outside of the cup is the dew point of the air surrounding the cup.

We have often noticed the "sweating" of pitchers containing ice water. This simply means that the air surrounding the pitcher has been cooled below its dew point, and some of its moisture must be deposited.

The dew point apparatus may be used to determine the relative humidity of the atmosphere, since the ratio between the amount of moisture present in the air and the amount which would be present if the air were saturated is the same as the ratio between the pressure which the water vapor in the air exerts and the pressure which it would exert if the air were in a saturated condition. For example, let us suppose that the dew point of the air in a room be $12^{\circ}$ and the temperature of the air in the room be $25^{\circ}$. The amount of moisture in the air is enough to saturate it at the temperature of $12^{\circ}$. By referring to the Appendix, Table I, we find that the pressure of water 
vapor at $12^{\circ} \mathrm{C}$. is 10.5 millimeters. Hence the air contains $10.5 / 23.5$ or .447 as much water vapor as it is capable of holding. The relative humidity is 44.7 per cent.

Humidity determinations have a practical value in that they enable us better to predict weather conditions and to have proper conditions in public buildings, schools, and homes. For the most healthful conditions the relative humidity should be from 50 per cent to 60 per cent.

Dew and Frost. - Dew is a deposit of moisture from the air upon the ground, grass, stones, and other objects near the ground. After sunset these objects radiate their heat very rapidly and soon become quite cold. The air which comes in contact with them is cooled below the dew point and some of its moisture is deposited. If their temperature is below the freezing point of water, frost is formed instead of dew. Dew and frost are formed most rapidly on the surfaces of substances which are the best radiators of heat.

Clouds are not favorable to the formation of dew, since they act as a blanket over the earth and prevent the radiation of its heat. Some of the rural weather prophets consider the absence of dew as a "sign" of rain. It is an indication that there were clouds during the night or a relatively high humidity of the atmosphere. Strong winds also are unfavorable to the formation of dew, since the air does not remain long enough in contact with the cool objects to be cooled to its dew point and deposit moisture.

Clouds. - Clouds are formed by the condensation of the moisture in the air. If a cloud meets the surface of the earth, it is called a fog, and other names are applied to it according to the manner of its formation, its shape, its color and appearance, and its elevation. The more common forms are the cumulus, the cirrus, the stratus, 
and the nimbus. In the daytime of fair summer weather ascending convection currents are produced in the atmosphere. The air expands and cools as it rises. If the cooling continues until the dew point is reached, some of the vapor is condensed, forming great masses of fleecy clouds which appear like the lightest wool in the sunshine. These clouds are very irregular in shape, and their tops are constantly changing. Their bases are more regular

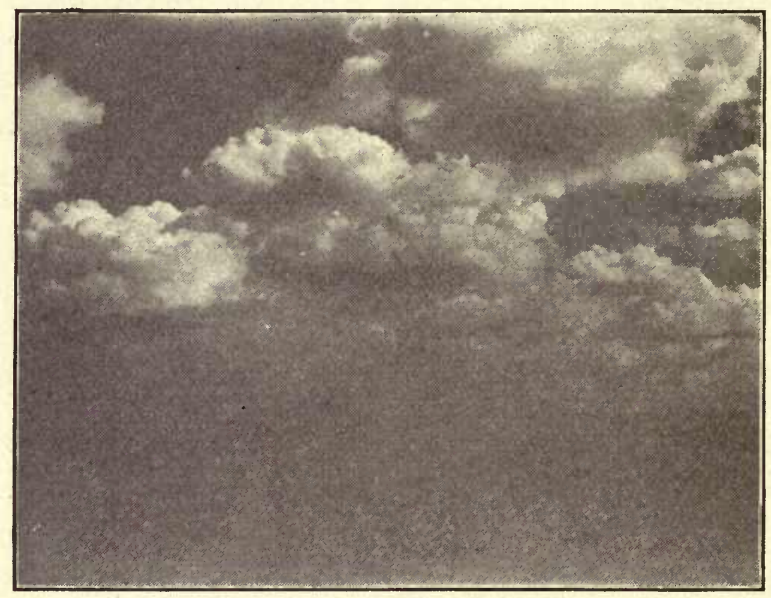

Fig. 147. - Cumulus Clouds.

and float in the air at the height of 1000 feet to 2500 feet. These clouds are called cumulus clouds (Figure 147). They are usually seen from the middle of the forenoon to the middle of the afternoon, when they dissolve and disappear.

Cirrus Clouds. - Quite commonly an advancing cyclone is preceded by plumelike strips of pale, whitish cloud (Figure 148), often five or ten miles high. These clouds are formed in the currents of air that flow out and forward from the upper part of the storm and they move quite 
steadily forward with the area of low pressure. On account of their great height they consist of minute ice crystals. If the cloud spreads out in a thin sheet, it is called a cirrostratus. When we look at the sun or moon through such a cloud, we have the familiar " ring around the moon " or " ring around the sun " phenomenon. Such a ring is called a halo and is formed by the refraction of the light in passing through the ice crystals.

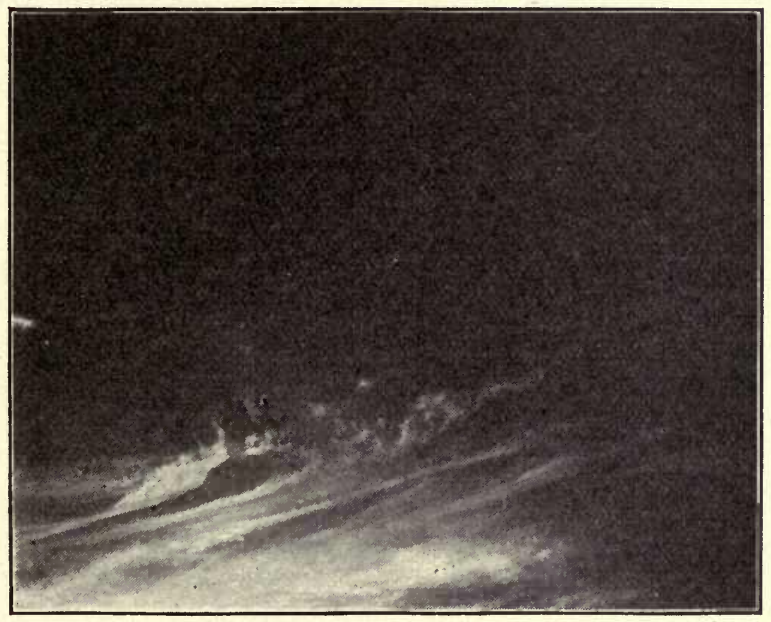

Fig. 148. - Cirrus Clouds.

Stratus clouds are frequently seen near the horizon in the late afternoon or early morning. They occur in long narrow bands (Figure 149) or strata. Nimbus is the name applied to the dark gray rain cloud. These clouds usually occupy the whole central portion of a cyclonic storm and often have an area of many thousand square miles. Their height above the surface of the earth varies with conditions, but they are often but a few hundred feet above our heads. In winter they are especially large and dense 
and sometimes remain over a certain area for several days. Nimbus clouds have no especial form, and the term is sometimes applied to any storm cloud.

Rainfall. - The term "rainfall" includes all forms of precipitation - rain, snow, hail, and sleet. Rain occurs when the moisture condenses in drops large enough to fall to the earth. Snow is formed when the moisture of the air is condensed at temperatures below the freezing

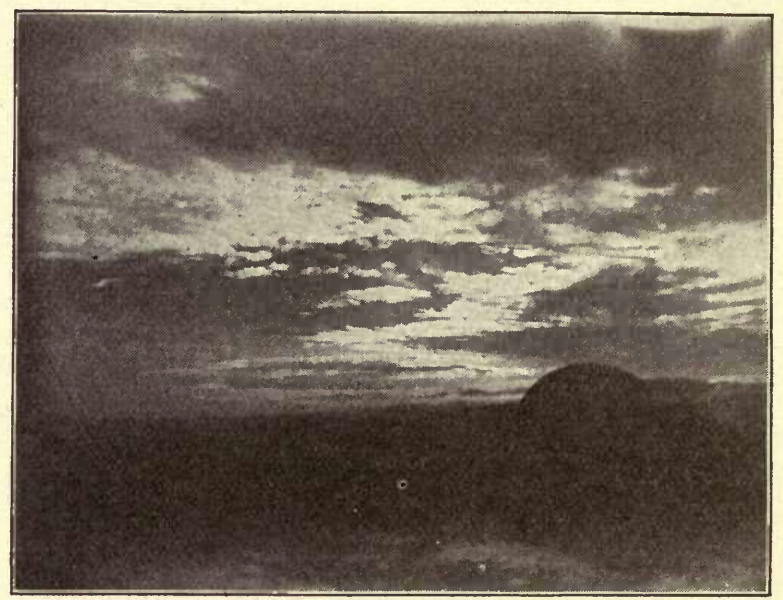

FIG. 149. - Stratus Clouds.

point, $0^{\circ} \mathrm{C}$. Sometimes moisture condenses as snow in the higher atmosphere and then melts as it falis through the warmer, lower atmosphere, reaching the earth as rain. Hail occurs chiefly during thunderstorms in summer, when the raindrops are frozen on their way to the earth. There are several theories to account for the formation of hailstones in concentric layers, but it is quite probable that the ascending air currents of high thunderstorm clouds carry the raindrops up into a region of quite low temperature and that as they fall they pass through 


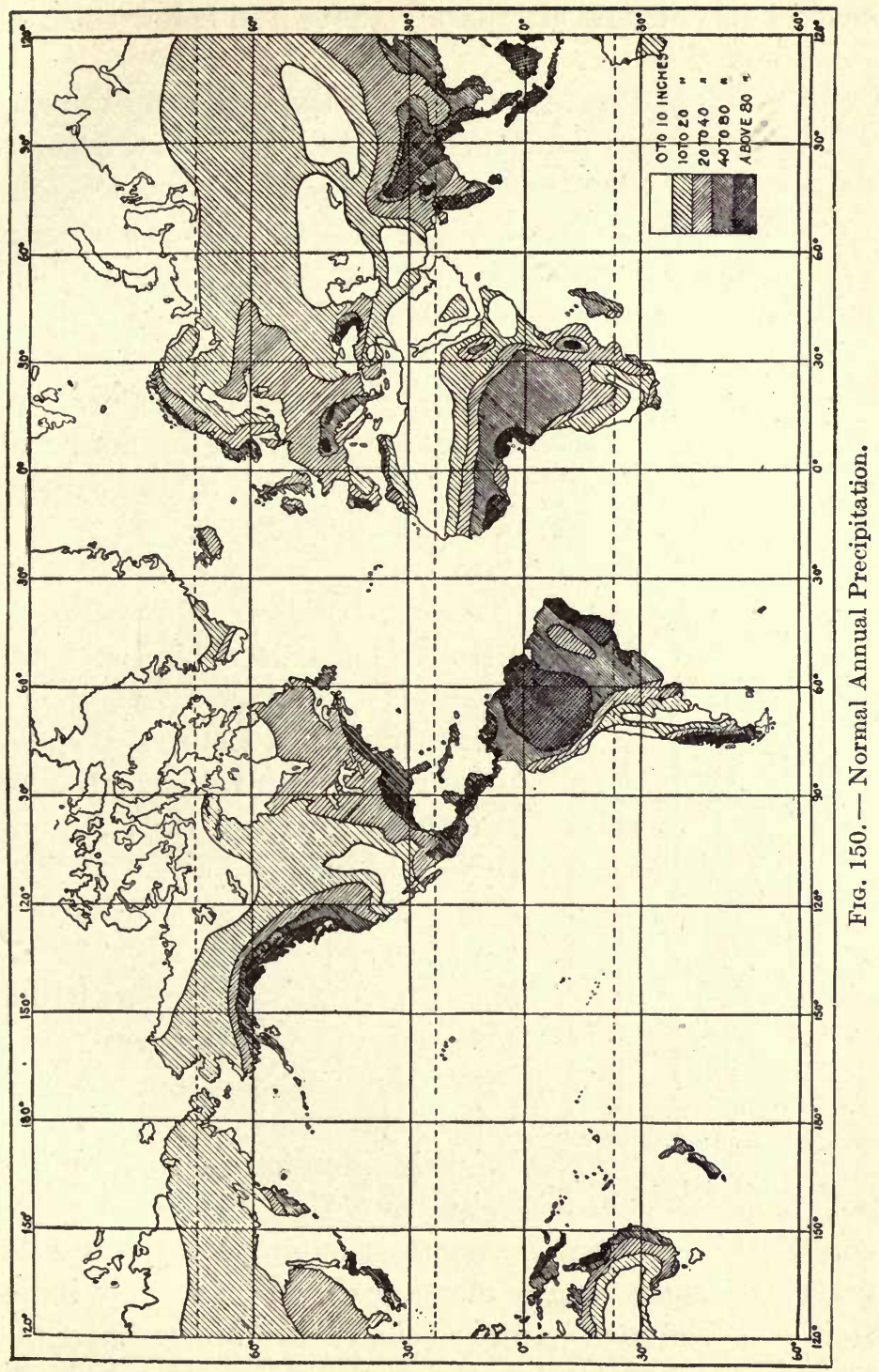


several air currents alternately above and below the freezing point of water.

The distribution of rainfall over the surface of the earth (Figure 150) shows that there is more rainfall in the tropical belts and in regions where the trade winds and prevailing westerlies ascend the mountain slopes. The desert regions are the lowlands which are crossed by the trade winds, or interior regions which are crossed only by winds that have previously lost most of their moisture. Such a region is found just east of the Rocky Mountains. The heaviest rainfall in the world occurs on the land bordering the Bay of Bengal and the southwest coast of India, where the average annual rainfall is about 30 feet.

Rainfall is measured in an instrument called a rain gauge. Figure 151 shows the
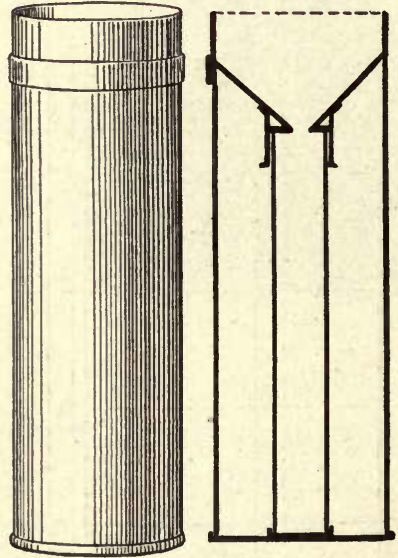

Fig. 151.- Rain Gauge. rain gauge used by the United States Weather Bureau. An inch of rainfall means that enough rain has fallen to cover the area considered to a depth of one inch of water. Snow and hail are melted before the measurements are taken.

Perform the experiment to determine how many inches of snow are equivalent to one inch of rainfall.

Thunderstorms are frequent in the United States. They commonly occur on the warmest summer days, and during the warmest parts of those days. The first indication of a thunderstorm is usually the appearance of a heavy cumulus cloud in the west. The original cloud is 


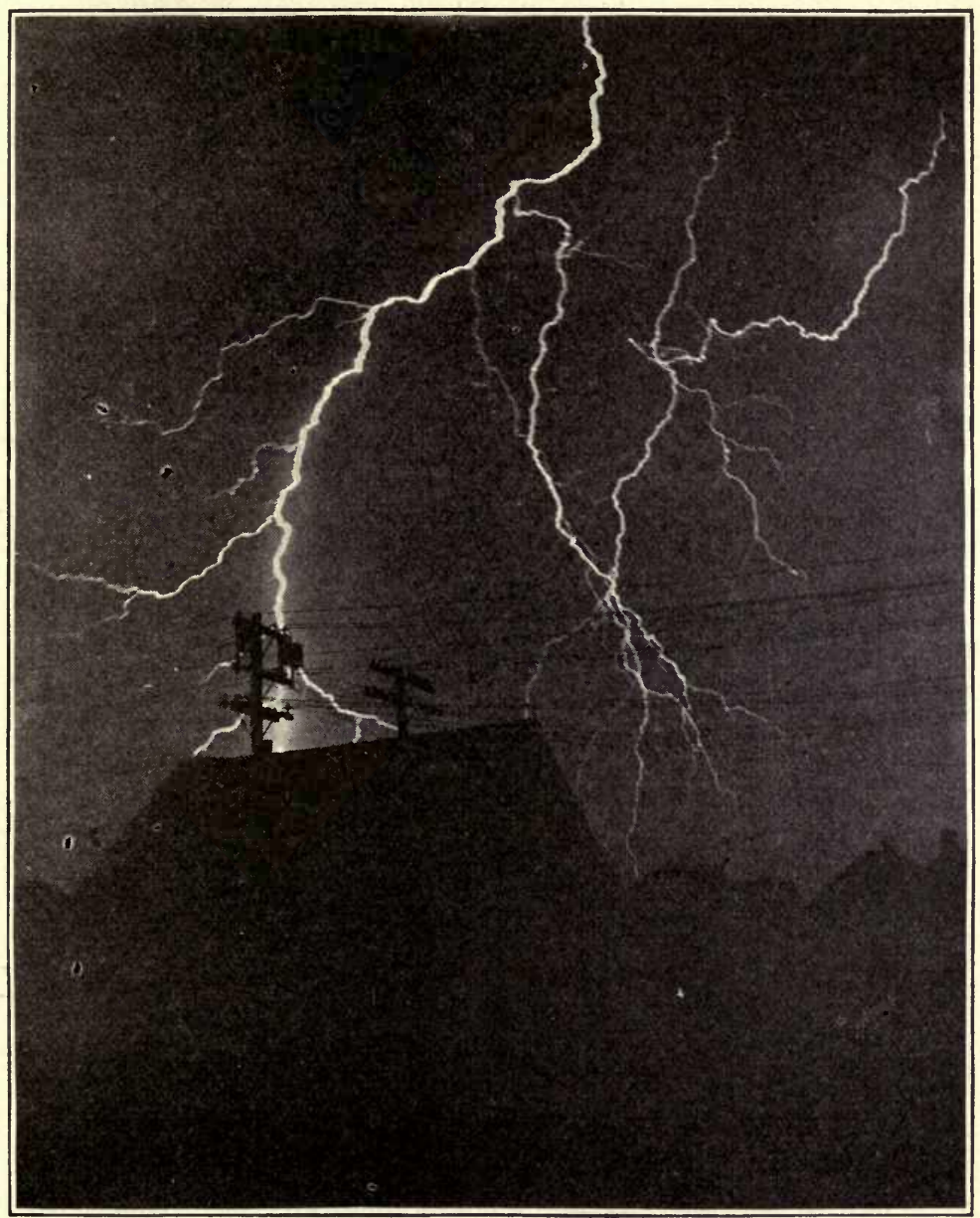

Lightning.

U. S. Weather Bureau. 
formed in the ordinary way; then as other ascending currents of warm air strike the lower part of the cloud they are deflected along its surface and cooled so that there is usually a heavy downpour of rain. The duration of the storm is often but a few minutes, though sometimes it continues for several hours.

When water is condensed rapidly in the air, electricity

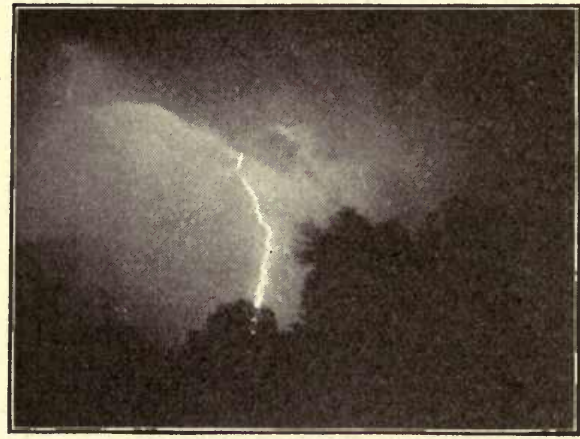

FIG. 152. - Lightning. is generated, and each particle of water becomes charged with electricity. Lightning is due to the passage of a large electric spark from one cloud to another or from the cloud to the ground (Figure 152). Thunder which follows the flash of lightning is due to the vibrations in the air along the path of the discharge. It is possible to estimate the distance of a flash of lightning by observing the time in seconds that elapses between a flash and its thunder and multiplying by the speed of sound in air. The velocity of light is so great that the time consumed by the flash in traveling from its source to the observer may be neglected. Heat lightning is lightning at such a great distance that the thunder accompanying it cannot be heard. The flash shows merely as light on the surrounding clouds.

At the heat equator heavy rains usually accompanied by thunder and lightning occur almost daily. These storms give the name " rainy season" to the time of year in which they occur. 
Weather Changes. - We have learned that "weather" is a very inclusive term. It includes all the atmospheric conditions that can be observed, such as temperature, the amount and kind of precipitation, the humidity of the air, the condition of the sky with reference to the clouds, and the direction and velocity of winds.

In the torrid zone the weather is quite regular. In the temperate zones the weather changes in the summer season are moderate, but in winter the changes occur more often and cover nearly every variety of "weather," due to the occurrence of numerous cyclonic storms. In the frigid latitudes the changes are not quite so marked, the temperature being very cold during the winter season and slightly warmer during the summer.

Weather Bureau. - A large number of observing stations have been established in the United States and adjacent countries for the purpose of making systematic observations of weather conditions. At the same time each morning and evening at the different stations a record is made of the barometric pressure, temperature, relative humidity, the direction and velocity of the wind; the appearance of the sky, and the amount of rainfall. This information is telegraphed to the Weather Bureau at Washington. From these reports, weather maps showing the weather conditions over the entire United States are printed. The tabulated information is sent back to the stations in the larger cities, and a smaller map is printed and mailed throughout the section in which the substation is located. In an almost incredibly short time the whole country has been given the benefit of the information collected. From the map it is quite easy for the novice to forecast the weather for the next twenty-four hours, while the trained observer will be able to forecast the weather for a 
week or even more. It should be noted, however, that the forecasts are made for large areas and not likely to prove correct in every detail for every locality. The movement of areas of low and high pressure in the belt of prevailing westerlies can only be estimated. Sometimes they increase in speed and sometimes decrease.

The information given out by the Weather Bureau is of great value to farmers, those interested in shipping, in fact all those whose business or pleasure in any degree depends upon the weather. The farmer may be able to save his grain by having knowledge of an approaching storm or his crop of peaches by learning of a coming frost. Hurricanes and other storms often do much damage to shipping and would do much more if it were not for advance information which enables ships to move out of the path of the storm to seek some safe harbor. News of a cold wave enables railroad companies and commission merchants to save their patrons a great deal, since many articles of food such as fruit and vegetables are spoiled by freezing and cannot be shipped without danger of loss in cold weather. Flood warnings are also the means of saving much property along the larger rivers.

It can usually be arranged to have several copies of the weather maps sent to the school by applying to the nearest Weather Bureau office and giving assurance that the maps will be properly used.

\section{QUESTIONS}

1. Why does low barometer usually indicate the approach of a storm?

2. How does air distribute heat?

3. Why do balloons float lower at night than in the daytime?

4. Why does a rise in temperature usually follow the beginning of a snowstorm? 
5. Name the ways in which the air serves us.

6. What becomes of the fog as it disappears on a foggy morning?

7. Why is it cold on the mountain tops even in tropical countries?

8. Why does air laden with dust become heated more rapidly than clean air?

9. Why should fruit trees be planted on the higher ground rather than in the valleys?

10. How is air heated?

11. How does the shape of the earth affect the distribution of temperature?

12. How do clouds prevent the formation of dew and frost?

13. Why does covering a plant with paper or cloth prevent it from being frostbitten?

14. What kind of soil is best for absorbing heat?

15. What is the effect of large bodies of water on the climate of a region?

16. What is the relation of trade winds to rainfall?

17. Will the pressure in an ascending column of air be low or high?

18. Does moisture in the air make the air heavier? Why?

19. What is relative humidity? How may it be determined?

20. Is it correct to say that snow is frozen rain?

21. What is the difference between a fog and a cloud?

22. Define meteorology.

23. How does humidity vary with the temperature?

24. Where is the heaviest rainfall in the United States? 


\section{CHAPTER XI}

\section{MAGNETISM AND ELECTRICITY}

Magnets. - Many years ago it was observed that an occasional piece of ore possessed the property of attracting small bits of iron and steel. It was also observed that the pieces of iron and steel which were rubbed with this ore acquired the same property. This strange ore is known as magnetite and probably derived its name from Magnesia, a province of Asia Minor in which the ore is quite abundant. The ore is also found in Norway, Sweden, and in the American continent. Pieces of ore which possess this property of attraction are called lodestones or natural magnets. Pieces of steel which have acquired the property of attracting other pieces of iron and steel, by being stroked with natural magnets, are called artificial magnets. Artificial magnets may also be made by passing an electric current around bars of steel. More will be said about this method later.

Experiment 50. - Rub a knife blade or small piece of steel against a bar magnet. Now bring the knife blade near some iron filings. Has it acquired the power to attract bits of iron? What kind of magnet is it?

Law of Magnetic Poles. - If iron filings are sifted over a magnet, it will be observed that the filings cling in a large bunch at each end of the magnet, but in the middle of the bar scarcely any filings will cling. These centers of attraction are called the poles of the magnet. The 
end that points north when the magnet is free to turn is called the north pole and the other end the south pole.

Experiment 51.- - Suspend a bar magnet in such a way that it is free to move (Figure 153) and determine its north pole and south pole. In the same way determine the poles of another similar magnet. Now bring the North $(N)$ Pole of the first magnet near the $N$ pole of the second and note the result. Test the attraction of the two South $(S)$ Poles for each other. Now test the attraction of the $N$ and $S$ Poles for each other. In the absence of bar magnets, knitting needles may be magnetized and used in the above experiment.

From this experiment the law of magnetic poles may be formulated: Like poles repel each other and unlike poles attract.

Induced Magnetism. - A piece of iron becomes a magnet by induction when it touches or is

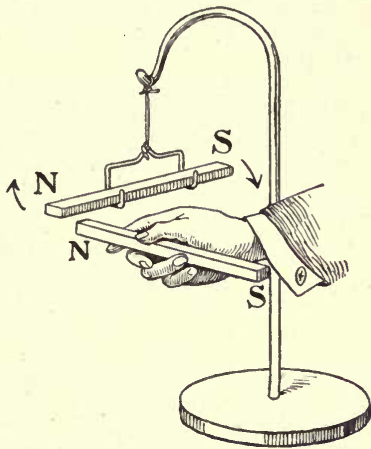

Fig. 153. - Like poles of magnets repel each other; unlike poles attract each other. brought near the pole of a magnet. Suspend a nail from the end of a strong bar magnet. Another nail may be suspended from the first, a third from the second, and so on. 'Now hold the first nail firmly and remove the bar magnet by sliding so that the nails will not be jarred. The instant that the bar magnet leaves the first nail the others will drop apart, showing that the nails were strong magnets only so long as they were in contact with the bar magnet.

Experiment 52. - Magnetic Fields. - Lay a bar magnet on the table and cover it with a paper, over the surface of which fine iron filings are strewn. When the paper is gently tapped, the filings 'will arrange themselves in lines reaching from one pole to the 
other (Figure 154). These lines are parallel to the lines of force of the magnet and may be said to represent them. Blueprint paper may be used for this experiment, and if held in the sunlight while the filings are in place, will

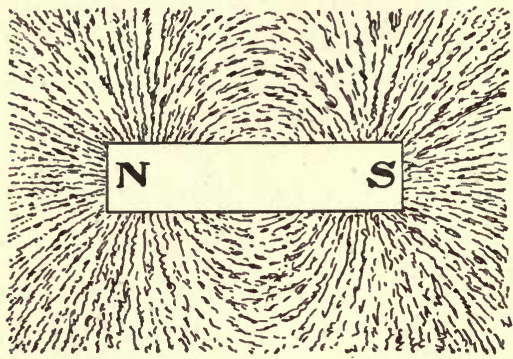

FIG. 154. - Direction of the Lines of Force Around a Bar Magnet. give a permanent record of their position, when developed.

Perform the experiment using two magnets with like poles near each other (Figure 155).

The magnetic field is the region about a magnet in which magnetic forces can be detected. The above experiments show the direction of these forces at every point in the plane cut by the sheet of paper. Each little particle becomes a magnet by induction and arranges itself so that it lies lengthwise in the direction of the lines of force at that point.

Nature of Magnetism. - Make a small magnet by rubbing a small piece of watch spring on a bar magnet. Test its strength on some filings or small nails. Heat the piece of watch spring and again test its

magnetism. Make another such magnet and hammer it. Its magnetism will be found to be much diminished.

Fill a small test tube with iron filings and stroke the 
tube with a magnet. The tube of filings will now act as a single magnet. If the tube is shaken, the magnetism is lost.
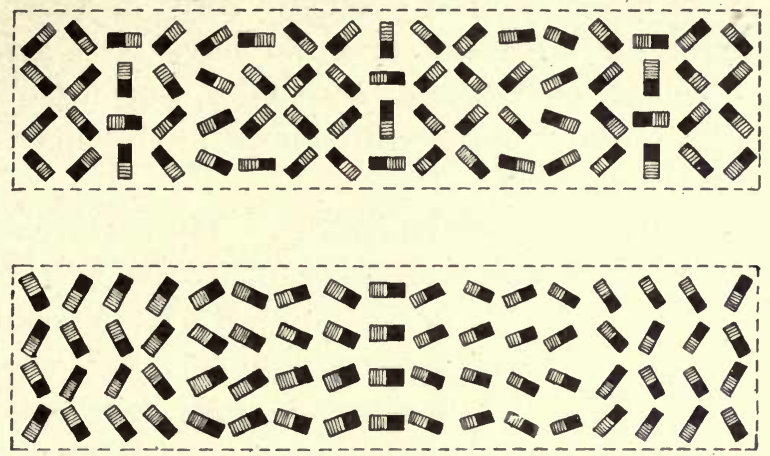

Fig. 156. - When the molecules of a bar magnet are disarranged, the bar loses its magnetism.

These experiments lead to the conclusion that at all times every molecule in a piece of iron or steel is a magnet, but that the bar as a whole becomes a magnet only when

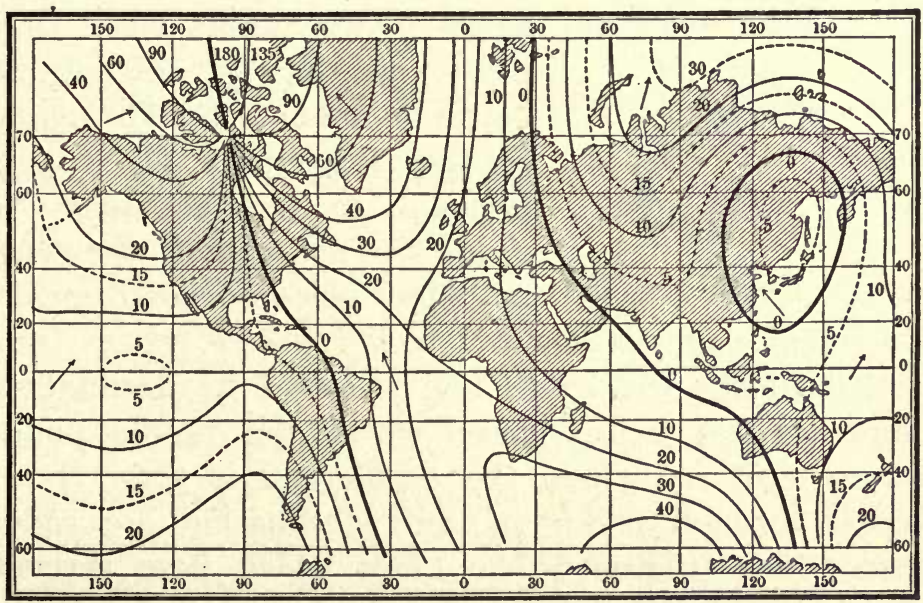

FIg. 157. - The Earth's Agonic and Isogonic Lines.

Heavy black lines are agonic lines; light black, isogonic lines. 
some force causes the molecules to arrange themselves in a certain order. The force which we have used in our experiments is simply the magnetic force in other magnets. After the molecules in a bar have been arranged so that the bar itself acts as a magnet, any force which disarranges the molecules will cause a loss of magnetism in the bar (Figure 156).

Magnetic Condition of the Earth. - The earth is a great magnet with its $S$ pole somewhere near the geographical

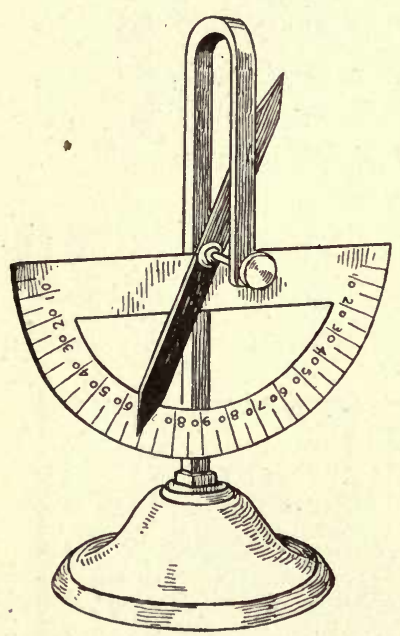

FrG. 158. - A Dipping Needle. north pole and its $N$ pole near the geographical south pole. Dr. William Gilbert first suggested that the earth was a magnet and offered it as an explanation of the behavior of the compass. All of us have read of the troubles of Columbus on his first voyage, and especially those due to the alarm of his sailors when they discovered that the compass no longer pointed north. The reason for the variation of the compass is found in the fact that the magnetic poles and the geographical poles do not coincide. The number of degrees which the needle varies from the true north and south line is called its declination. There are not many places where the needle points exactly north or where the declination is zero. Lines passing through points of zero declination are called agonic lines (Figure 157). Lines which pass through points having the same declination are called isogonic lines. 
The Dipping Needle. - Just as the iron filings arranged themselves so that they were lying in the direction of the lines of magnetic force at that point, so a compass needle which is balanced and free to move in a vertical plane will arrange itself parallel with the earth's magnetic lines of force at that place (Figure 158).

Experiment 53. - Pass two unmagnetized knitting needles through a cork near the center and at right angles to each other (Figure 159). With a pin (a) the system may be adjusted so that it will be perfectly bal-

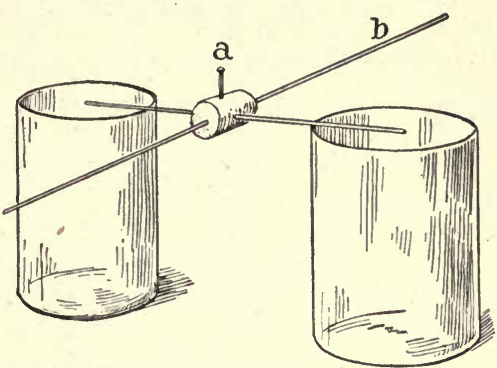

Fig. 159. - A Homemade Dipping Needle. anced. Now magnetize the neeble $b$, being careful not to change its position in the cork. Replace the needle on the supports so that the $N$ pole will point north. Does the needle balance as before?

\section{QUESTIONS}

1. What is the difference between permanent magnetism and induced magnetism?

2. Name four ways by which the magnetism of a magnet may be destroyed.

3. What is meant by the magnetic field?

4. How would an explorer know when he had reached the north or south magnetic pole?

5. How should magnets be stored in the laboratory so that they will not lose their magnetism so rapidly?

6. Will two horseshoe magnets of equal strength cling to each other? Explain.

7. Why does soft iron lose its magnetism so quickly?

Electrification by Friction. - If a stick of sealing wax or a piece of hard rubber is rubbed with a woolen cloth or a piece of cat's fur, it will attract small pieces of paper, pith balls, cork, and other light bodies. A glass rod 
rubbed with a piece of silk also acquires this power of attraction. It is thought that a Greek philosopher named Thales discovered this property in a substance called amber, and for many years amber was supposed to be the only substance possessing this property. Since amber in common with a few metals was called " electron" by the Greeks, Dr. Gilbert of England many years later gave the name "electric" to these phenomena.

Place some bits of paper under a pane of glass which is supported by two books (Figure 160). Rub the upper

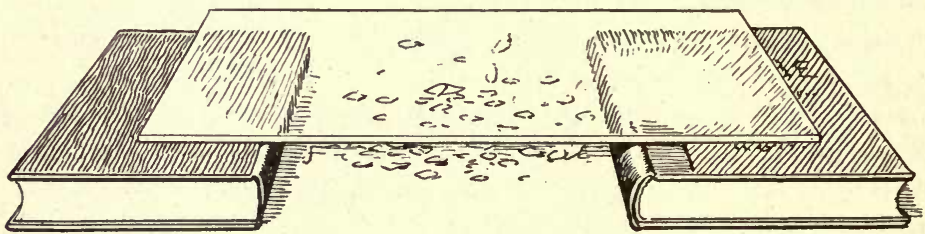

FIG. 160.

surface of the glass with a piece of silk and notice how the bits of paper are disturbed.

Two Kinds of Electricity. - The electricity which is produced upon glass by rubbing it with silk is called positive electricity and that produced upon the rubber is called negative electricity. Electricity is usually produced when two unlike substances are rubbed together, and the kind may be determined by comparing it with that formed on glass or rubber. Thus all electrified bodies which act with respect to other bodies like a glass rod rubbed with silk are said to be positively electrified, while those that act like a piece of hard rubber that has been rubbed with the woolen cloth are said to be negatively electrified. 
Experiment 54. - Suspend a pith ball with a silk thread as shown in Figure 161. When an electrified glass rod is brought near, the pith ball is attracted to the rod. It clings to the rod for a moment and then springs away from it. The ball has received a positive charge from the positive charged rod. Now as the rod is brought near the ball it moves away as if pushed by some invisible force. Next rub a hard rubber or ebonite rod with flannel or cat's fur and bring it near the pith ball. It will be found that the ball is no longer repelled as with the glass rod, but that it is strongly attracted. To discharge the pith ball, hold it in the finger for a moment.

Hang two pith balls from the same point of suspension and charge both balls with the same kind of electricity. The balls now repel each other (Figure 162).

It is evident from these experiments that electrical charges of like kind repel

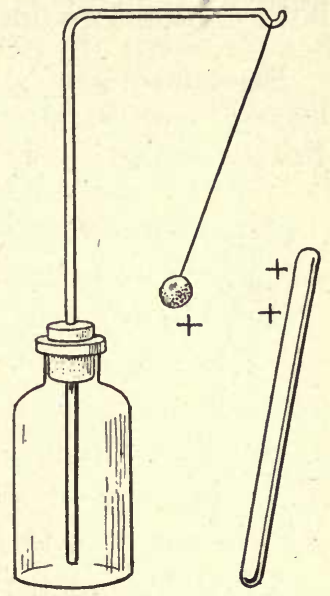

Fig. 161. - A Pith Ball Electroscope. each other and charges of unlike kind attract each other.

Conductors and Insulators. - A

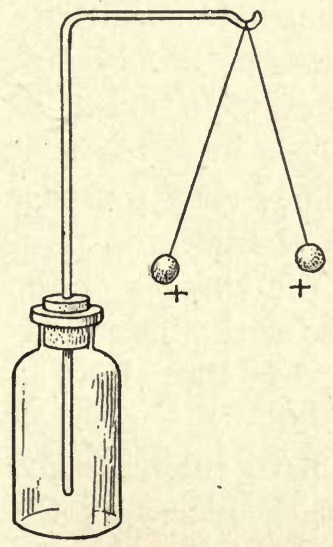

FIG. 162. substance which conducts or transmits electricity readily is called a conductor, while a substance that does not is called an insulator, or non-conductor. All metals and solutions of salts and acids in water are conductors of electricity, while glass, rubber, shellac, dry air, wood, silk, and oils are good insulators. When a metal rod is held in the bare hand and rubbed with some substance such as a woolen cloth, a charge does not appear upon the rod, because the 
body conducts the electricity away as fast as it is made. With non-conductors such as glass, the charge remains where it is developed. In the commercial use of electricity both conductors and insulators are of great value.

Experiment 55. - Support one end of a metal rod on an electroscope and the other end on an insulating stand (Figure 163). Bring a charged body near the end of the rod. The divergence

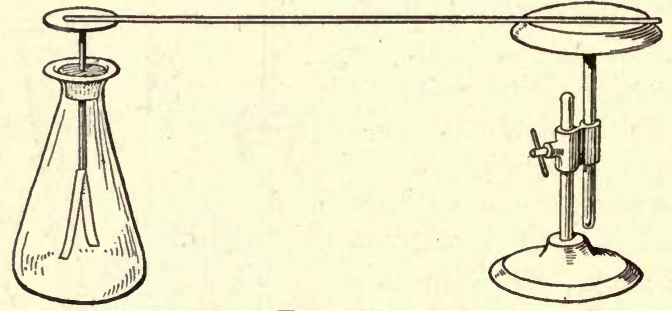

Frg. 163. of the leaves of the electroscope shows that the charge has been carried by the rod to the electroscope. The rod is a conductor of electricity. Test the conductivity of other substances.

Theory of Electricity. - To study the theory of electricity we must introduce the reader to a new and very interesting physical character, the electron. All the atoms of all substances are known to contain both positive and negative electricity. The negative electricity exists in the form of electrons or particles of negative electricity. The positive electricity probably exists as the center or nucleus around which these little particles of negative electricity are grouped. They cling together by reason of having opposite or unlike charges, the positive charge just equaling the sum of the charges of the little electrons. We are most concerned with these negative particles or electrons at present, for they have a very important part in our everyday life. If a piece of woolen cloth is rubbed on a piece of hard rubber, some of these electrons are brushed from their places on the atoms of the piece of cloth and collect on the piece of 
rubber, and we say the rubber rod has a negative charge. We simply mean that some of the electrons which belong on the cloth have been removed to the rod and that for the present the atoms of the rubber rod are overcrowded with electrons. Now, since the electrons are little particles of negative electricity, of course the rod will exhibit a negative charge. We must remember that the little electrons have like charges and repel each other; so whenever we have this overcrowding of electrons, we find them pushing each other in all directions as if to make their condition less crowded. In fact, if any other object is brought near, a number of them will be pushed over on it. As the number of these electrons on a given insulated area is increased, the crowding becomes so great that finally they are forced across great air gaps with a disruptive charge; as is the case when an electrical machine is operated or as in the case of lightning.

If glass is rubbed with silk, we say the glass receives a positive charge, but what really happens is that the electrons let go of the glass rod and collect on the silk. There is a lack of negative particles on the glass, and we say it has a positive charge.

An unelectrified body is composed of atoms in which the strength of the charge on the positive nucleus is exactly counterbalanced by the sum of the negative charges or electrons.

Charging a Body by Induction. - Suspend two eggshells which have been covered with tin foil by silk threads, so that they touch each other (Figure 164). Bring a positively charged rod near the shell $A$. While the rod is in this position both shells will be electrified. $A$ will have a negative charge and $B$ a positive charge; the reason being that some of the electrons (negative 
particles of electricity) of the shells are attracted by the positively charged rod, thus producing an excess of electrons on $A$ and a lack of electrons on $B$. If the rod is taken away, the electrons which have been drawn to $A$

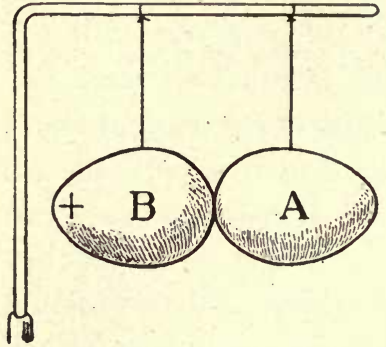

Fig. 164. will flow back to their places and neither shell will show any charge. If, however, the shells are separated while the rod is near the shell $A$, each shell will be permanently electrified, $A$ having a negative charge and $B$ a positive charge. The charges on the shells may be tested in the usual way.

A similar experiment may be performed with one foilcovered shell. When the positively charged rod is brought near one end of the shell (Figure 165), the electrons are drawn to that end, producing a lack of electrons at the other end. Now while the rod is still in this position, touch the opposite end with the finger. Electrons will flow

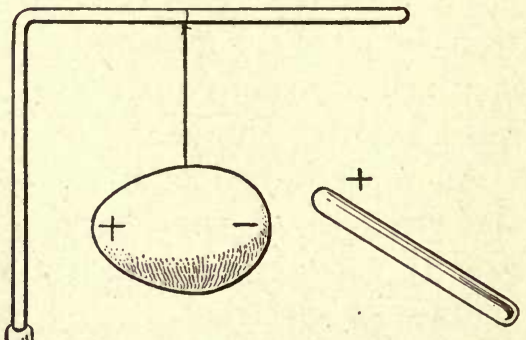
from the finger to the Fig. 165. - Electrons are drawn toward shell to make up the defithe positively charged glass rod.

ciency of electrons on that end. When the rod is taken away, the shell will be found to be negatively electrified.

Charging a body in this way is called charging by induction. 
Storing a Charge: Condensers. - If the finger or some other conductor is brought near a charged body, sparks will pass. The amount of the charge which may be stored on an object is limited by the size and shape of the object and its proximity to conductors. A charge escapes more readily from points than from evenly curved surfaces. Usually before the charge on an object is very great it escapes by way of the air or some other conductor. A greater charge can be stored on a body on a dry day than on a damp day, because moisture in the air makes it a better conductor of electricity. To store a large amount of electricity on an object, it is simply necessary to insulate it properly and bring it near another conductor which is attached to the earth. This is the principle of the condenser.

Experiment 56. - Place some tin foil on both sides of a common windowpane as shown in Figure 166 and connect one side with the earth. Now if the foil on the side $A$ be connected with some source of positive electricity, electrons will be attracted from the earth to plate $B$ in sufficient numbers to balance the positive charge on $A$. A very large charge thus may be stored on the insulated plates. The plates. may be discharged by touching the end of a wire to one side, $B$, and bringing the other end near $A$.

Explain what would happen if plate $A$ be connected with a source of negative charges instead of positive charges.

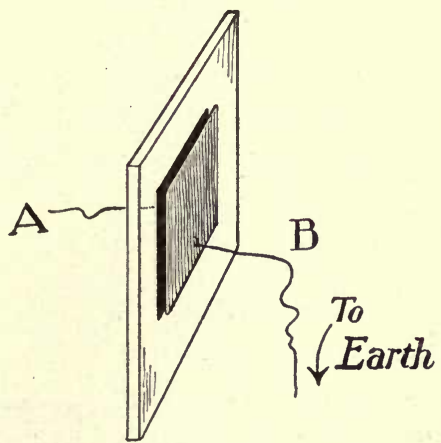

FIG. 166.

The Leyden Jar was one of the first forms of condensers. It was first used in 1745. It consists of a glass jar, coated inside and outside to about two thirds 
of its height with tin foil (Figure 167). The inside coating is connected by a chain and rod to a knob, and the outside covering is connected to the earth. A charge may
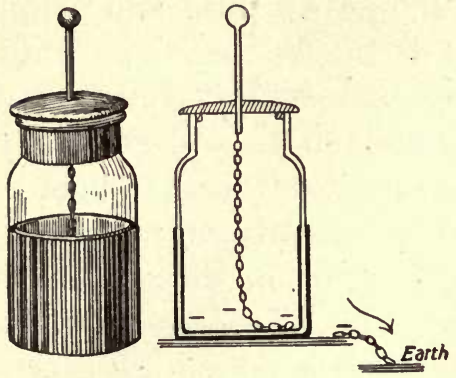

FIG. 167. - A Leyden Jar. be passed by way of the knob and rod to the inside coating of the jar, the action being the same as that described above.

The Electrophorus. - The charges which we are able to develop on glass or ebonite rods are small. We may produce larger charges by taking advantage of the principle of induction. Figure 168 represents a sectional view of the electrophorus. $A$ consists of a plate of ebonite or a shallow pan filled with sealing wax or resin; $B$ is a metal disk (tin or brass), fastened to an insulating handle of ebonite or glass. Rub the plate $A$ with some cat's fur or woolen cloth. Place the metal disk $B$ on the plate and touch it with the finger while it is in this position. Now lift the disk and test its charge. Lift the disk without touching it and test its charge. Lift the disk before re-

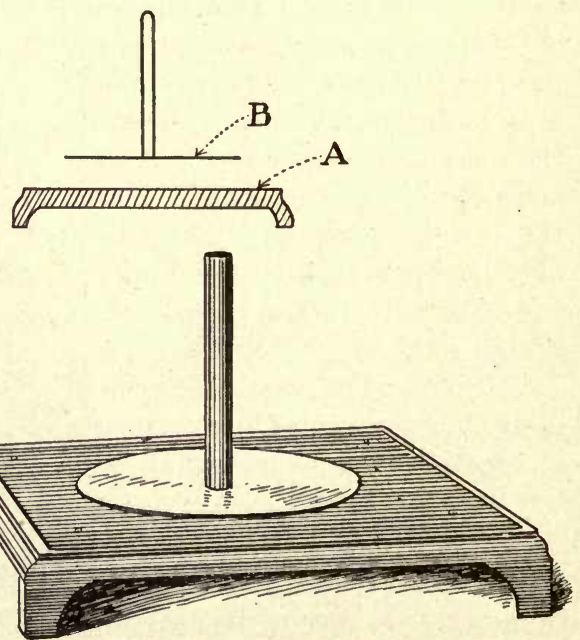

FIG. 168. - The Electrophorus. 
moving the finger from the disk and test its charge. What kind of charge should be on the disk? What kind of charge passes through the body to the earth when the finger is placed on the disk?

When the charged disk is brought near an object, a spark will pass with a slight cracking noise. When the spark passes, the air is heated and its sudden expansion and contraction causes the noise.

Atmospheric Electricity. - It was Benjamin Franklin who first demonstrated that lightning is simply a great spark of frictional electricity. On the approach of a thunderstorm he sent up a kite having at its top a pointed metal rod. The kite string was insulated from the earth. As soon as this string became moistened by the rain electric sparks were drawn from a key attached to the string. These sparks proved to be the same as those obtained by rubbing a glass rod with silk (Figure 169).

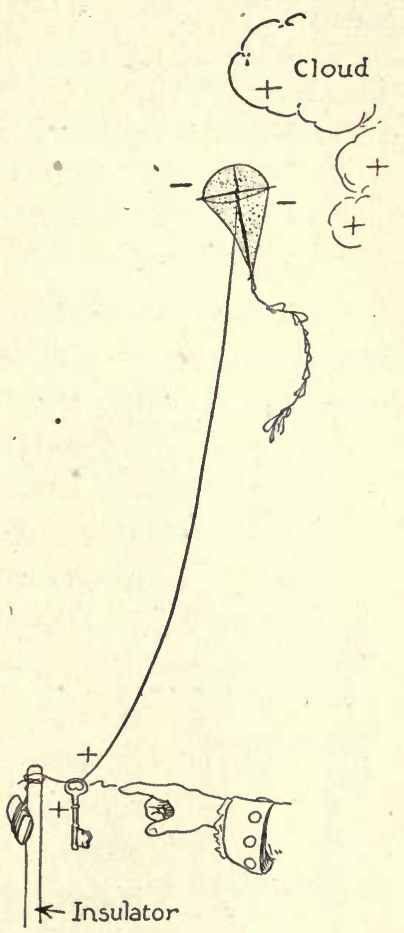

FIG. 169. - Franklin's Experiment to Test the Identity of Lightning.

The air between two charged clouds or between a cloud and the earth acts like the insulating substance in a condenser. The rapid formation of raindrops generates large quantities of electricity and finally the charge on the cloud becomes so great that it bursts through the intervening air to the neighboring cloud or to the earth. 


\section{QUESTIONS}

1. Name some uses of insulators. Of conductors.

2. How may a metal rod be electrified?

3. Why do we connect the outer coating of a Leyden jar to the earth?

4. How do lightning rods protect buildings? Why are lightning rods pointed?

-5. Explain how the " electrophorus" is charged.

6. What do we mean when we say lightning "strikes"?

7. Experiments with frictional electricity are performed more easily on dry days. Why?

Current Electricity. - If a wire is connected with the outer coating of a charged Leyden jar and then brought near the knob at the top, a spark will pass and the two coatings will be brought to the same electrical potential. A current passes through the. wire, but only for a very

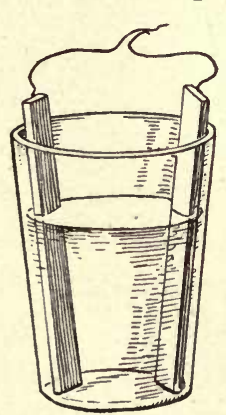

Fig. 17)._A Simple Voltaic Cell. short time. About the beginning of the nineteenth century two men, Galvani (1786) and Volta (1792), working independently, discovered and studied a method of maintaining a constant difference of electrical potential between two substances and thus a means of producing a continuous electric current. If two different metals such as copper and zinc are placed in dilute sulphuric acid and connected by a wire (Figure 170), a continuous current of electricity will pass from the copper through the wire to the zinc. Such an arrangement is called a voltaic cell. In commercial cells carbon is commonly used instead of copper.

Kinds of Cells. - There are a number of different kinds of cells, but in every case they depend upon chemical action of some sort. In the voltaic cell, zinc is slowly decomposed with the formation of hydrogen and zinc 
sulphate in addition to the current produced. This form of cell is of little practical value, however, because the little bubbles of hydrogen collect in such numbers on the carbon or copper that they weaken and finally stop the passage of the current. A cell in such a condition is said to be polarized. If bichromate of potassium is dissolved in the sulphuric acid, it will unite with the hydrogen as fast as it forms at the zinc and thus prevent it from reaching the carbon or copper and stopping the current.

Another form of cell in which polarization is completely avoided is the gravity or crowfoot type of the Daniell cell. This cell is commonly used on telegraph lines. A group of copper plates is placed in the bottom of the jar and surrounded with crystals of copper sulphate (Figure 171). A zinc plate is hung near the top, and the jar is then filled with a very dilute solution of sulphuric acid. Hydrogen is formed at the zinc plate, but on its way to the copper it is

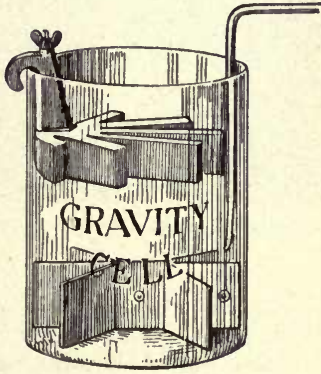

FIG. 171. used up, and copper from the copper sulphate is deposited on the copper plate in its stead. The copper sulphate solution, being heavier than the acid solution, remains near the bottom of the jar.

One of the most common and convenient types of cells is the dry cell. In this cell the zinc serves a double purpose. It forms one plate of the cell and also is the container for the chemicals which fill the space between the carbon center and the zinc. These chemicals are sal ammoniac, manganese dioxide, and charcoal, mixed with water enough to make a paste. The cell is sealed to prevent the evaporation of the water. 
Effects of Electric Currents : Heating Effects. - Connect two or three dry cells in series with a short piece of fine iron or German silver wire. The wire will become red hot and may be burned. Try this experiment with different lengths of fine iron and copper wire with different-sized batteries.

A current in passing through a wire must overcome the resistance of the wire. This resistance differs with the kind of material of which the wire is made, the size

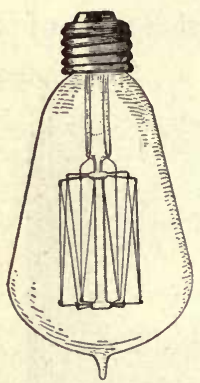

Fig. 172. - A Commercial Tungsten Lamp. of the wire, and its length. Some substances are much better conductors of an electric current than others. For example; copper is a better conductor than iron, and silver is a slightly better conductor than copper. For a given kind of material the resistance varies with the size and length of the wire. The smaller the wire the greater the resistance, and the longer the wire of a given size the greater the resistance.

\section{Table of Resistances of a Few Common Metals}

Silver • $\quad 1.00$ Iron • .6 .00 German silver . 15.00
Copper . . 1.11 Platinum . 7.20 Spring steel . 13.5

German silver wire is commonly used in electric heaters, electric toasters, electric stoves, electric flatirons, and similar appliances. The size of the wire is carefully graded for the current on which it is to be used in order that the temperature will be right for the different uses.

Electric Lighting. - The same principle as that involved in electric heating is involved in the electric light bulb. A fine wire or filament of carbon is inclosed in a 
bulb from which air has been almost exhausted. When the current passes through the filament, it is heated to the point of incandescence. It does not burn because of lack of oxygen (Figure 172).

At the present time tungsten and tantalum filaments have largely re-

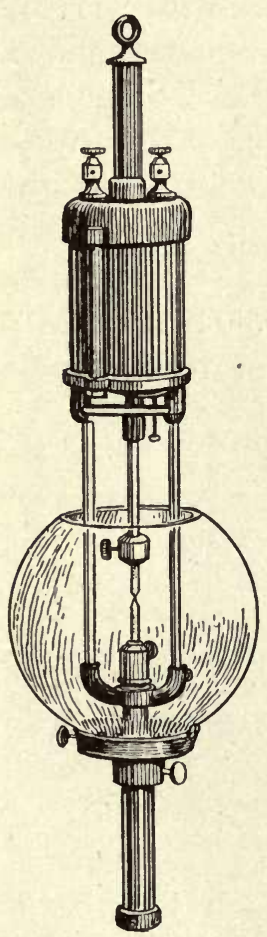

FIg. 174.-The Electric Arc Lamp. placed the carbon for use in incandescent lamps, since they are nearly three times as efficient as the carbon lamps.

The electric "arc" light is produced by placing two carbon rods end to end in a strong

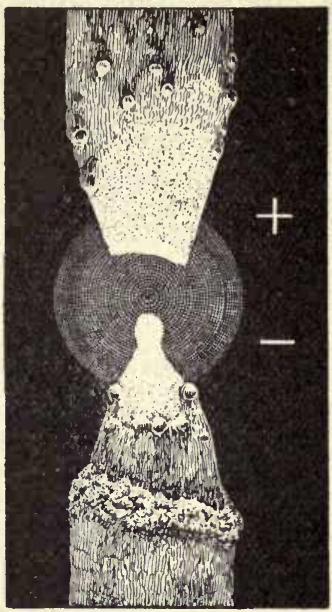

FIG. 173. - The Electric Arc. electric current. If the carbons are separated slightly after the ends are heated red hot, the current will continue to flow. The conducting layer of incandescent vapor between the ends of the carbon is called the electric arc (Figures 173-174). The temperature in this arc is the hottest that man has been able to produce. It will vaporize all known substances. When the electric arc is inclosed in a box (Figure 175) made of high heat-resisting materials, it is called an electric furnace. Magnetic Effects of Currents. - If we hold a wire carrying a current near a compass, the needle will be deflected. If the current is moving from south to north 
in a wire held directly over the compass, the $N$ pole of the needle will move to the west (Figure 176). If the wire is held under the compass, the $N$ pole will move to the

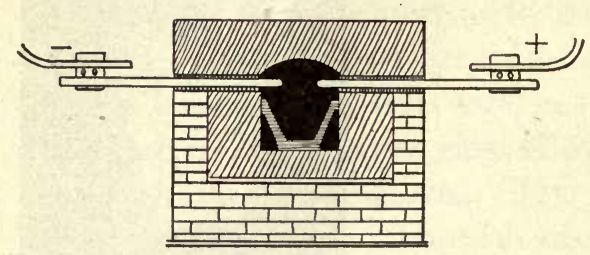

Fig. 175. - An Electric Furnace. east. If the current is reversed, the direction of the needle will be reversed.

Pass a wire carrying a current through a sheet of cardboard which is held in a horizontal position (Figure 177). With the aid of a compass test the magnetic field about the wire. As the compass is moved it will be found that the position of the needle changes in such a way as to form a right angle with a line drawn from the wire to the middle of the needle. The needle of the compass

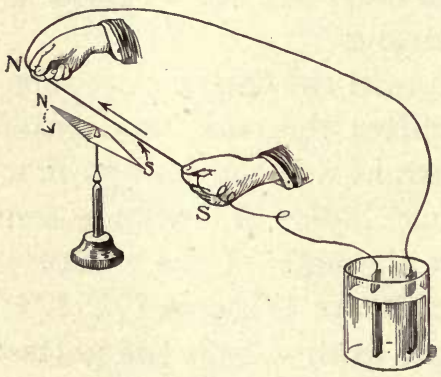

Fig. 176. - Effect of Electric Current on Magnetic Needle.

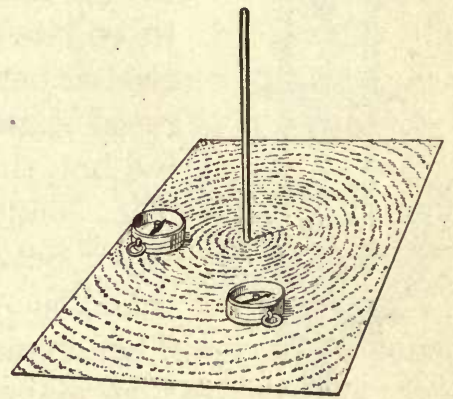

FIG. 177 .

will be tangent to a circle drawn through that point with the wire as the center of the circle.

From these experiments it will readily be seen that a wire carrying a current is surrounded by a magnetic field. The magnetic lines of this field lie in circles about the wire in such a way that if the wire is grasped with the 


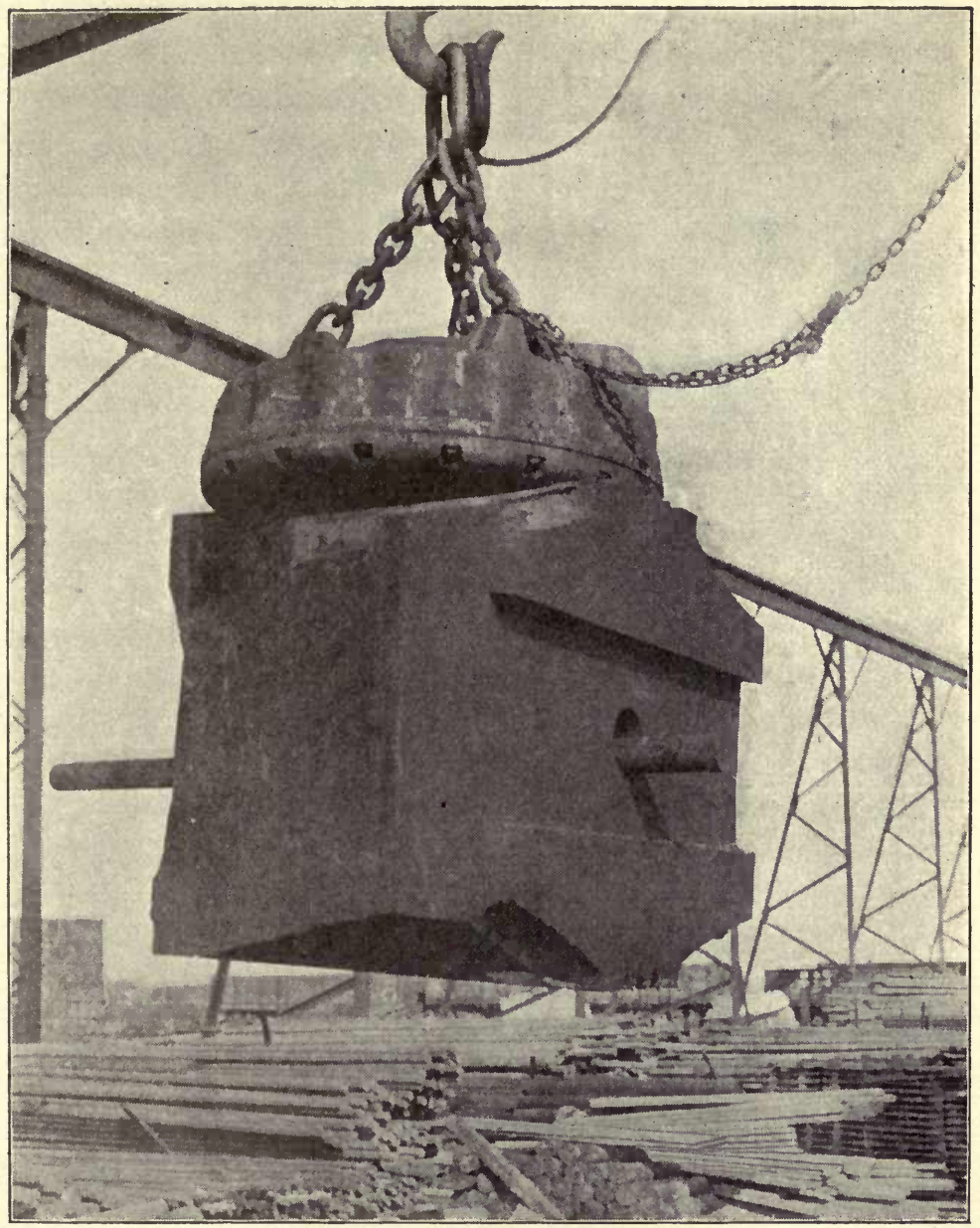

A Lifting Magnet Lifting a Large Casting. 

right hand so that the thumb points in the direction in which the current is flowing, the fingers will encircle the wire in the same direction as do the magnetic lines.

The Electromagnet. The magnetic effect of an electric current is of great practical value in its many applications. We have learned that a wire carrying a current is surrounded by a magnetic field, and we should expect that if we have a number of wires in a given

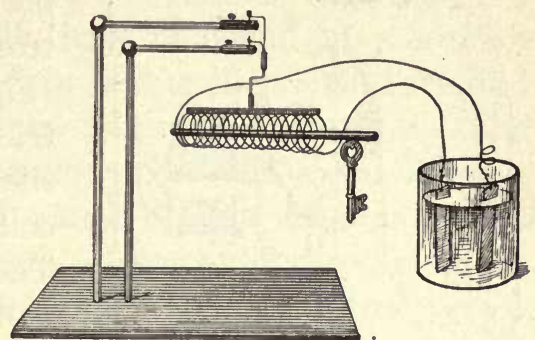

FIG. 178. - If the helix is free to turn, it will arrange itself in a north and south position.

space, all carrying currents in the same direction, the magnetic field would be strengthened. The same effect may be obtained by winding a single wire in the form of a helix (Figure 178). The wire should be insulated to

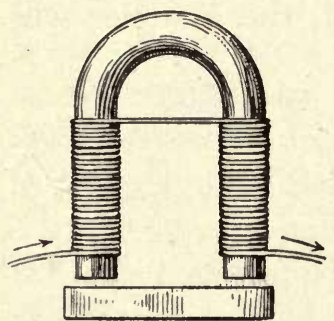

Fig. 179. - An Electromagnet of the Horseshoe Form. avoid the short-circuiting of some of the turns should they come in contact with each other. If this helix is free to turn, it will behave. like a magnetic needle in taking a north and south position. A bar of soft iron placed in the coil becomes a temporary magnet. Such a magnet is called an electromagnet. The greater the number of turns of the wire in the coil the stronger will be the magnetic field and consequently the stronger will be the electromagnet.

Experiment 57. - Make an electromagnet by winding a bar of soft iron in the shape of a horseshoe with insulated wire (Figure $179)$ and test its lifting power. 
The electromagnet is used in electric bells, telegraph instruments, the electric crane, and many other appliances.

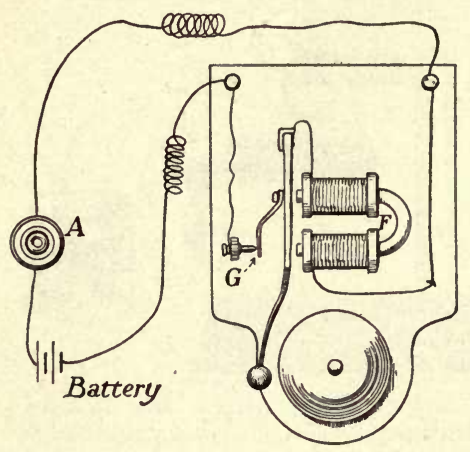

Fig. 180. - Diagram of the Electric Bell.

The Electric Bell. - A simple application of the electromagnet is the electric bell (Figure 180). When the electric circuit is closed at $A$, the current flows through the coils of the electromagnet $F$. The magnet attracts the hammer or armature, causing it to strike the bell. At the same time the circuit is broken at $G$ and the magnet is demagnetized. The hammer is thrown back by the spring which supports it. This closes the circuit again and the operation is repeated. So rapidly does this opening and closing of the circuit take place that if the bell is removed, the hammer will produce a buzzing noise.

\section{The Telegraph. -} In this instrument, which is another application of the electromagnet, the earth is used instead of a

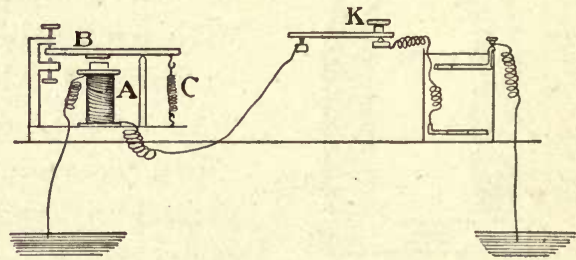

Fig. 181. - Diagram of Telegraph Circuit.

second wire. When the key $K$ (Figure 181) is closed, the circuit is complete, and the electromagnet $A$ attracts the sounder $B$ and holds it as long as the key is closed. When the circuit is broken at $K$, the sounder is pulled away from the magnet by a spring at $C$. A short closing of the key is called a dot, a longer time a dash. 
Telegraph operators have learned to read these messages by interpreting the clicks, representing the dots and dashes as letters of the alphabet.

The Telephone. - In 1875 Alexander Graham Bell demonstrated to the world that the sound of a human voice could be transmitted by electricity. He invented what we still use and know as the Bell receiver. The essential parts of this receiver are a permanent magnet wound with fine wire and a disk of thin sheet iron held in place by a hard rubber case. Figure 182 shows a simple arrangement of the telephone parts and how they work. At each end of the line is a bar magnet, surrounded by

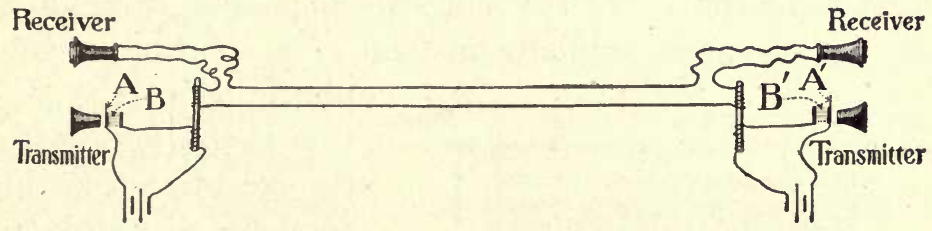

Fig. 182. - Telephone System and Battery Circuit.

a coil of fine wire and a thin iron disk. The disk $A$ is set in vibration by the sound waves whenever such waves are produced in front of it. As $A$ moves back and forth in the magnetic field, currents are induced in the coil $B$. These currents are transmitted to the similar coil $B^{\prime}$ at the other end of the line and there produce changes in the magnetic field similar to the changes that produced the current at $B$. The disk $A^{\prime}$ is set in motion and vibrates in exactly the same way as $A$, with the result that the sounds which caused the vibration at $A$ are reproduced at $A^{\prime}$.

The modern telephone uses a transmitter and a receiver, and also a second wire as shown in the above diagram. This second wire is necessary because of the 
noise and numerous electrical disturbances due to the many uses to which electricity is put in the modern city.

Chemical Effects - Electrolysis. - In the chapter on water we used the electric current to separate water into the two gases of which it is composed. In this experiment the gas was formed at each terminal, but it was formed at the terminal at which the current left the liquid twice as fast as at the other terminal. The larger volume of gas was hydrogen and the smaller volume oxygen. Solutions of a number of compounds may be decomposed in this way.

Electroplating. - If the current is passed through a solution of copper sulphate instead of a solution of sul-

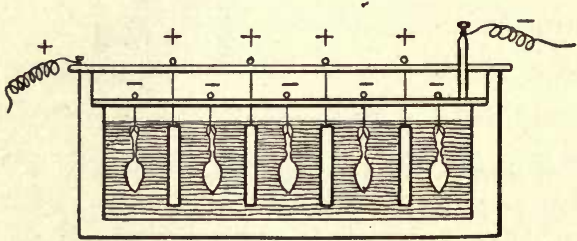

Fig. 183. - An Electroplating Bath. phuric acid, the action is the same with the exception that the copper is deposited at the negative pole instead of hydrogen. If the positive pole is made of copper, it slowly wastes away and is deposited on the other pole. This is the method used in commercial electroplating. The positive pole is made of the material with which the other pole is to be plated. In Figure 183 the positive pole (anode) is pure silver, while the negative pole (cathode) is the spoon to be plated. These are both placed in a solution of a silver compound. When the current passes, silver is deposited on the spoon.

The Dynamo. - We learned that, when a bar of iron was put in the magnetic field of a coil carrying a current, the bar was magnetized. The reverse of this is also 
true. When the magnet is placed in or removed from a coil of wire, a current is produced in the wire.

Experiment 58. - Connect a coil of several hundred turns of insulated wire with a galvanometer (Figure 184). Now bring the pole of a bar or horseshoe magnet suddenly into the coil and notice the deflection of the pointer of the galvanometer. When the pointer comes to rest, remove the magnet quickly; the pointer will again be deflected, but in the opposite direction.

Experiments will show that it makes no difference whether the coil be held sta-

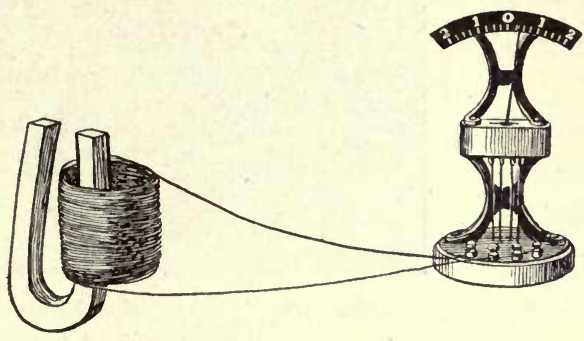

Fig. 184. - When the permanent magnet is placed in the coil a current of electricity is induced in the coil as is shown by the deflection of the galvanometer needle.

tionary and the magnet placed in it, or the magnet held stationary and the coil moved over it. The current in the coil of wire is made when it moves through a magnetic field. This is the principle of the dynamo (Figure

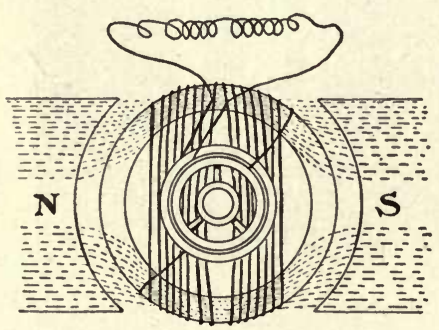

Fig. 185. - Diagram of a Dynamo.

185). A dynamo consists of a coil of wire revolving in a magnetic field. The magnet may be a permanent magnet or an electromagnet. In the commercial instrument the armature is usually made up of a large number of separate coils of insulated wire. The dynamo is used to produce electricity wherever it is used in very large quantities. Through its agency almost any kind of power may be changed to electric power (Figure 186). The Falls of Niagara furnish power to run many dynamos 


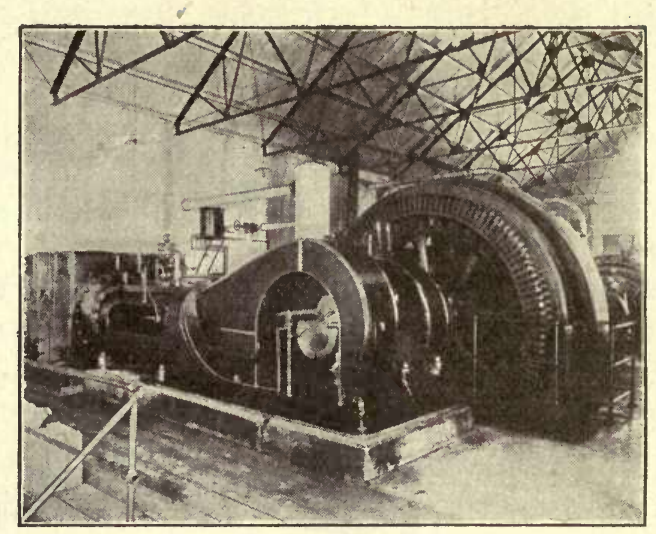

Frg. 186. - A Gasoline Engine Operating a Westinghouse Generator.

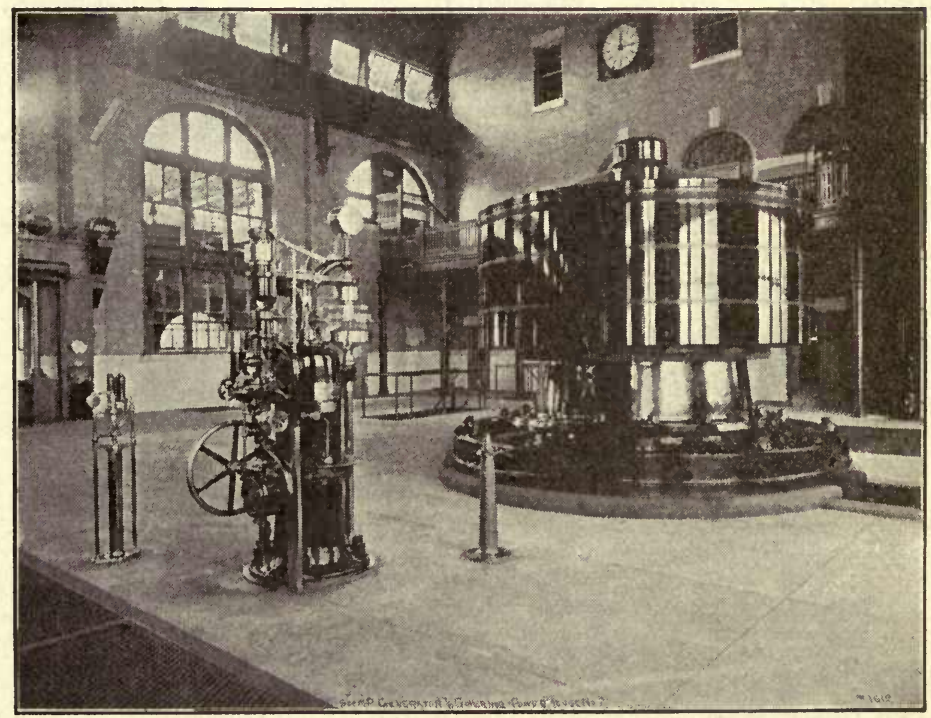

FIg. 187. - A 5000-Horse Power Generator and Governor. 
whose electricity is, in turn, used for lighting, for propelling street cars, for heating, for factory power, and in various other ways. Electricity may be cheaply made by water power and conducted great distances by wires to cities and mills where it is needed (Figure 187).

Electric Motors. - The appearance of the electric motor is similar to that of the dynamo, but its action is reversed. We revolve the armature of a dynamo in a magnetic field to produce a current, but in the electric motor the current is passed through coils of the armature, causing it to revolve. The dynamo is a machine for producing electric current, while the motor is a machine for utilizing electric current and may be used to run machinery wherever power is needed.

\section{QUESTIONS}

1. Explain how polarization stops the current.

2. Why are gravity cells called by that name?

3. Why is copper used in electric wiring?

4. What do we mean by " earthing " a wire?

5. Name four uses of the electromagnet.

6. Draw a diagram of an electric bell, showing how it works.

7. Why do we use soft iron in an electromagnet?

8. Why are fuse plugs used on electric circuits?

9. How does a current given by a cell differ from that given by a Leyden jar?

10. Learn how to make a sal ammoniac cell. 


\section{CHAPTER XII}

SOUND

What Causes Sound. - When the string of a guitar is plucked, it gives forth a continuous sound. If a light piece of metal is held near the string, it will be struck a number of tiny blows by the string in its movements.

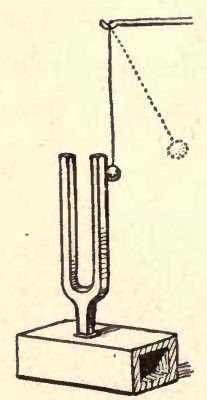

FIG. 188.

Experiment 59. - Attach a pith ball or a light glass ball to a string and hold it near a tuning fork that has just been struck (Figure 188). The ball is set in motion by the vibration of the fork.

If we hold a piece of metal near a large bell that has just been struck, we find that the metal in the bell is in violent vibration. In short we find that where a sound is produced, matter has been set in motion. It may be the falling of a tree, the collision of two bodies, the firing of a shot, or the blowing of a whistle; but an examination will show in every case that vibrating matter of some sort has caused the sound.

Experiment 60.- Sound Waves. - Suspend a small electric bell in the receiver of a vacuum pump (Figure 189). If we set the bell to ringing and pump out the air, we find that the sound becomes fainter as the vacuum becomes greater. Now admit the air slowly and note the increase in sound.

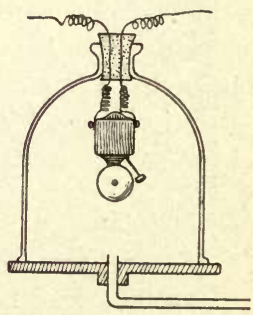

FIG. 189.

From this experiment it will seem a logical conclusion that sound will not pass through a vacuum and that air 
carries sound to the ears. This is correct; but air is not the only substance that will carry sound. Solids, liquids, and other gases will also carry sound. If the ear is held near the steel rails of a railroad, a train approaching at considerable distance may be heard. It is also quite a familiar fact that if the ear is held under water, the noise made by hitting two stones together some distance away may be heard distinctly. The speed of sound in air at $0^{\circ} \mathrm{C}$. is 1087 feet a second. In water the speed is about 4600 feet a second and in iron 16,700 feet. The speed of sound in air increases with the temperature.

When two boards are brought together suddenly, air is driven from between them with considerable force and the surrounding air is pushed back in all directions. This motion is communicated to the next layer of air and to succeeding layers as wave motion. As the wave moves farther and farther from its source, a larger volume of air is affected and the intensity of the wave decreases. If an ear is within the range of these waves, the sensation of sound is produced. Sounds may be heard in all directions, which is evidence that the wave advances in the form of the surface of a sphere.

When a stone is dropped in still water, waves are produced. These waves move in circles outward from the source. That the water in these waves does not move out with the wave may be proved by scattering some light material on the surface of the water. Sound waves are similar to water waves. Water waves advance as the circumference of a circle, while sound waves in the air advance in the form of the surface of a sphere. In such a wave the air does not actually move forward. Air is very elastic. When an impulse is given to the air by some sound-producing source, the adjacent layer of air is 
compressed. As it expands it compresses a second layer and so on, so that the volume of the air affected is made

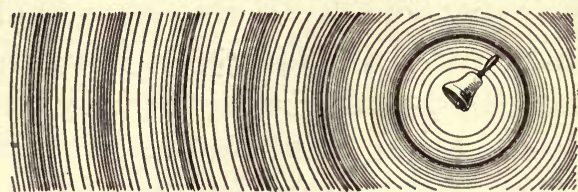

Fig. 190. - Diagram Illustrating the Way Sound Travels. up of layers of compressed and rarefied air (Figure 190).

Waves pass over fields of standing grain ; but the grain simply bends under the pressure of the wind and rises again. Wave motion may be shown by the following experiment.

Experiment 6r. - Attach one end of a small rope about 25 feet long to some solid object such as the wall. Hold the other end of the rope in the hand and cause waves to run along it by quick movements of the hand. The waves pass from one end of the rope to the other, but the particles of the rope do not move forward.

Echoes are due to the reflection of sound. When we speak, the sound waves often strike some reflecting surface and are returned to the source. If the reflecting surface is near, as in the case of the walls of a small room, the echo will not be noticed; but in large halls the echoes may be so strong as seriously to inconvenience a speaker. Such effects may be remedied by substituting light, porous materials for the hard surfaces of reflecting walls and by hanging curtains in certain parts of the hall to destroy returning sound waves. In hilly districts echoes are sometimes heard several seconds after the original sound.

Musical Tones and Noises. - The human ear quite readily distinguishes between pleasing sounds and those that are not. Pleasing sounds are musical and are produced when the vibrations of a sounding body follow each other at precisely equal intervals of time. If the vibra- 
tions do not follow each other in equal intervals of time, the result is noise.

Pitch. - If the vibrations which produce tones were all of the same frequency, music would be impossible, since we would have but one tone. Happily this is not the case. As the number of vibrations in a second increases, the tone becomes higher. By pitch we mean the highness or lowness of a sound. Middle $\mathrm{C}$ on the piano is produced by a string vibrating 256 times a second. Any other string vibrating the same number of times a second will produce a tone of the same pitch. If the number of vibrations a second be doubled, the result will be another $\mathrm{C}$ an octave higher; and if the number be decreased to 128 a second, the result is $\mathrm{C}$ an octave lower than middle $\mathrm{C}$.

Vibrating Strings. - A large number of musical, instruments employ vibrating strings or wires. Variations in pitch are obtained by varying the length, tension, and

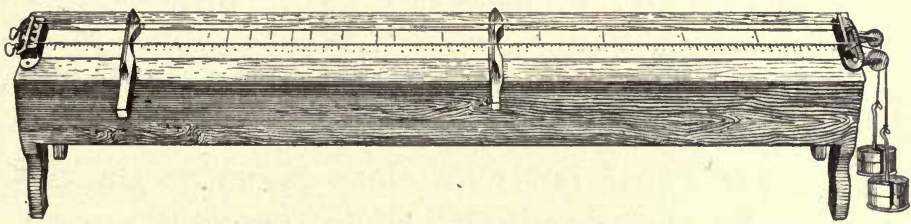

FIG. 191. - A Sonometer.

mass of the strings. For example, if a string 20 inches long produces a tone of a certain pitch, the pitch will be an octave higher if the length of the string be changed to 10 inches. The shorter the string the higher the pitch. Or we may say the vibration frequencies of strings are inversely proportional to their length.

If we increase the tension on a string, the pitch will be higher; and if we decrease the size of the string and keep 
the length and tension the same, the pitch will be higher. The wires of a piano show the application of these laws of strings. They vary from strings of small diameter and a few inches in length to quite large strings several feet in length. The high notes are made by the short strings, the low ones by the long strings. Test the truth of these statements by experiments with a sonometer. (Fig. 191.)

The Voice. - The larynx or voice is just below the throat or pharynx at the top of the trachea. It is composed of cartilages bound together by ligaments and surrounding muscles. In the larynx are the vocal cords, which are the chief organs of the voice. These cords are folds of connective tissue placed in such a way that they may be stretched at will across the opening. The passage of air causes them to vibrate, and sounds are produced.

If we cut a small slit in a piece of sheet rubber and tie it over one end of a tube, we will have a mechanism similar to that which produces voice. When we blow through the tube, a sound is produced which will become higher in pitch as the tension on the rubber is increased. Many animals have voice, but man alone possesses the power to express his thoughts in articulate sounds or speech.

The Hearing. - Sound is transmitted from a vibrating object to the ear by waves in the air. The ear is the organ of hearing. For convenience in description it is divided into three parts, the external ear, the middle ear, and the inner ear. (Figure 192.)

The external ear is an irregularly folded cartilage covered with skin. Its shape is especially well adapted for catching sound waves and directing them through the auditory canal to the middle ear. At the inner end of the 
auditory canal is a thin layer of very flexible skin, the tympanic membrane, which also forms the external covering of the middle ear.

The middle ear is a small cavity lined with mucous membrane and connected with the pharynx by the Eustachian tube. It contains a chain of three.bones named from their shapes the hammer (malleus), the anvil

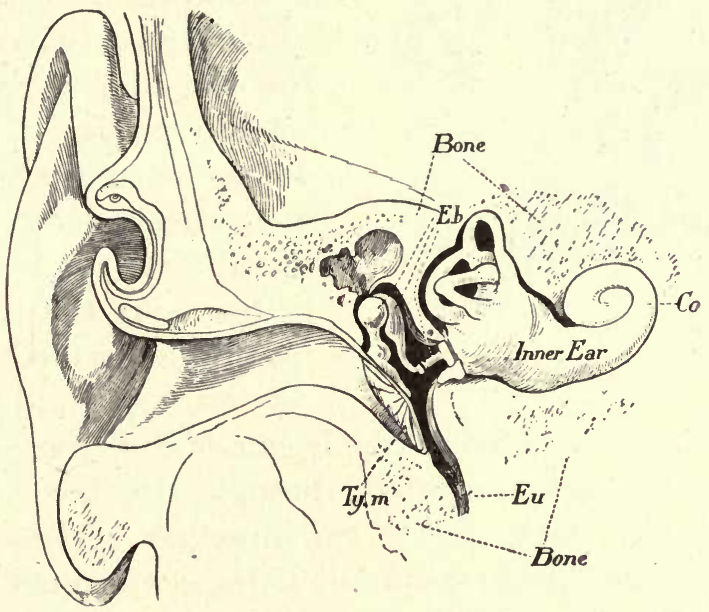

Fig. 192. - The Human Ear Shown in Section. Co, Cochlea (location of real hearing organ); E.b, Ear Bones; Eu, Eustachian Tube; Ty.m, Tympanic Membrane.

(incus), and the stirrup (stapes) (Figure 193). These bones extend from the tympanic membrane to the membrane which closes the opening, fenestra ovalis, into the inner ear.

The inner ear is located in an irregular cavity in the temporal bone. Its essential part is composed of a membranous sac which is filled with a liquid called endolymph. In this liquid are tiny stones, otoliths. The first part of the inner ear is called the vestibule. To the vestibule on one side is attached the cochlea in which 
the fibers of the auditory nerve terminate and where the auditory impulses originate. To the other side of the vestibule the three semicircular canals are attached.

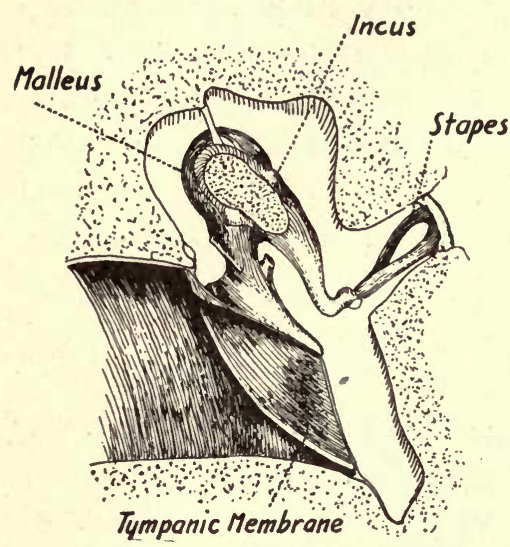

Fig. 193. - The Ear Bones. These canals seem to have something to do with balancing the body.

How We Hear. - When a vibrating body produces sound waves which reach the ear, the sensation of sound is produced. As the waves reach the ear they are collected by the external ear and concentrated upon the tympanic membrane, which is thus set into sympathetic vibration with the object which caused the waves. This vibration is communicated through the bones of the middle ear to the liquids of the inner ear. These liquids distribute the vibrations to all parts of the inner ear and finally to the fibers of the auditory nerve (fibers of Corti) which originate the proper impulses to be conveyed to the brain as sound sensations.

\section{QUESTIONS}

1. What is meant by wave length?

2. If the speed of sound increases two feet for each degree Centigrade, what will be the speed at $23^{\circ} \mathrm{C}$.?

3 . If the velocity of sound on a certain day is 1120 feet per second and a sounding body makes 560 vibrations per second, what is the wave length?

4. Three seconds elapse between a flash of lightning and its attendant thunder. How far away was the lightning? 
5. What is the form of a sound wave?

6. Two strings have the same length and tension, but one is larger than the other. In what respect will their tones differ?

7. Can we hear sounds farther on a foggy or a clear day?

8. What is meant by "pitch" of sound? By quality?

9. Why does a cold sometimes cause deafness?

10. What is the function of the ear wax?

11. What is sound?

12. Why should a drum head be well stretched? 


\section{CHAPTER XIII}

\section{LIGHT}

Light and its Properties. - We have just learned that sound waves are caused by the vibrations of the material bodies about us, and we are now to learn that light is due to waves produced in a similar way. Light waves are not waves in the air, but in the ether, a medium which fills all space but which possesses few of the properties of ordinary matter. These waves in the ether are produced by the vibrations of the same little electrons which produce the charges of electricity.

While studying sound we learned that the rate of vibration of the shorter and smaller strings was much greater than the rate of vibration of the longer and larger ones. The electron is very much smaller than the smallest string, and its rate of vibration is very much greater.

Heat and light are very closely associated. Not all hot objects give off light; but if the heating continues the temperature is finally reached at which light is given off, and we say the body is incandescent. Heat is due to the motion of the molecules. As the temperature increases the molecules strike each other harder and finally cause the electrons to vibrate rapidly enough to produce the sensation of light.

The velocity of light is so great that for many years it was supposed to pass instantly from its source to any distance. In 1676 a Danish astronomer by the name of Roemer determined the speed to be 186,000 miles a second. Other experiments have verified his results. 
Light travels in straight lines through a transparent medium of uniform density.

Sources of Light. - Much of the light we enjoy comes from the sun; but since the sun is hidden from our view for one half the time of each year, man has devised numerous means of producing artificial light. Whaleoil lamps, pine knots, candles, kerosene lamps, gas lamps, and electricity have been used for the purpose of giving light. The light of flames is due to little particles of carbon which have been heated to incandescence by the combustion of gases from heated oil or wax. Most light sources are quite hot.

Luminous Bodies. - We cannot see light itself, but we can see the bodies which emit the light. Such bodies, as the sun, the lighted candle, the arc light, are called luminous bodies. Every point in a luminous body sends out rays of light in all directions. Most of the objects we see are non-luminous. We see them by the light which they receive from some other source and then reflect to our eye. If a non-luminous object reflects no light, it is invisible. 'The moon is a non-luminous body and receives its light from the sun.

Transparent, Translucent, and Opaque Bodies.From observation we have learned that light passes through many substances, such as air, glass, water, and mica. Substances through which objects may be distinctly seen are said to be transparent. Substances which allow some of the light to pass, but through which objects cannot be seen distinctly are called translucent. Parchment, oiled paper, and ground glass are examples of translucent substances. Substances such as stone and wood through which light does not pass are called opaque substances. 
Experiment 62. - Shadows. - Place an opaque cylinder, two or three inches in diameter, in a dark room. Place two lighted can-

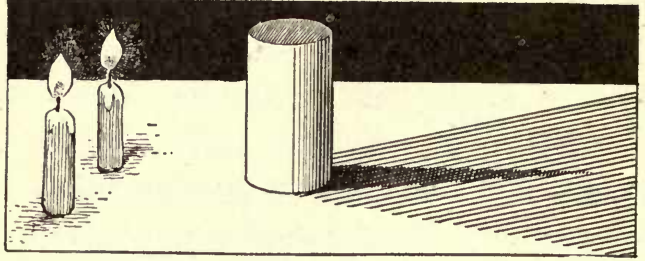

FIG. 194. dles about six inches away from the cylinder, making the distance between the candles equal to the diameter of the cylinder (Figure 194). Is there any space behind the cylinder that does not receive light from either candle? Why are there two light shadows? Could we have used one large source of light in place of the two candles? Explain.

The space that does not receive light from either candle is called the umbra. The region that receives light from one candle only is called the penumbra.

Figure 195 illustrates a total eclipse of the moon. When the moon passes into the umbra, the shadow of the earth, a total eclipse of the moon occurs. If the moon passes so that it is part in the umbra and

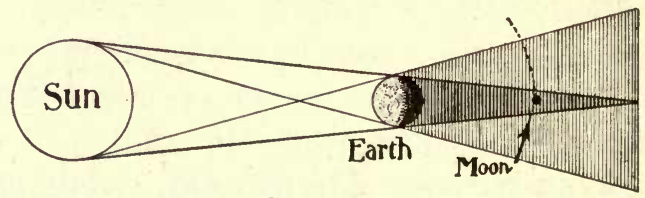

Fig. 195. part in the penumbra, a partial eclipse occurs.

Light as Energy. - Light does work so quietly that we may not think of it as a form of energy. Light has much to do with our food supply. Plants containing chlorophyll, the green coloring matter of our vegetation, are able to produce starch, sugar, and other complex organic substances, when stimulated by sunlight. The change which takes place is a chemical change, and the energy required is supplied by the sun as light. These 
substances are later used as food by animals, and the energy reappears as heat energy or muscular energy. As we continue our scientific studies we will observe that energy is never lost or destroyed. It is often changed in form, but it never disappears.

When a photographic plate is exposed to light, a chemical change takes place. New substances are formed on the plate in such a way that when properly developed a picture may be produced on sensitized paper. It is this same light energy which affects the various sensitized papers used in photographic and blueprint work.

Experiment 63.-Intensity of Light. - Figure 196 furnishes an illustration of the way the intensity of light is affected by distance. Let $L$ be a small source of light and $A$ a screen one foot square, placed at a distance of four feet from $L$. Since light travels in straight lines, the area of the shadow

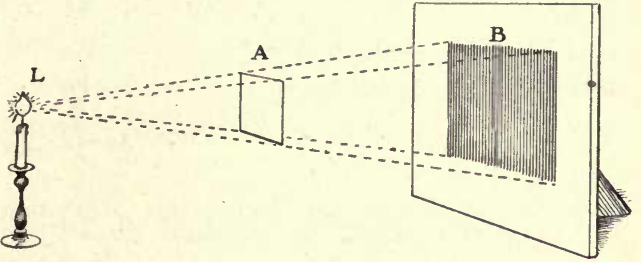

FIG. 196. of $A$ on $B$ placed eight feet from $L$ will be four square feet.

From this experiment it is evident that the light which is received by a surface having an area of one square foot is scattered over an area of four square feet when the distance from the light source is doubled, and nine square feet when the distance is trebled. A pupil who sits twice as far from a lamp as another pupil, receives but $\frac{1}{4}$ as much light.

How Light is Measured. - The amount of light given by a lamp is commonly designated as a certain number of candle power. A candle power is the amount of light given off by a sperm candle known as " sixes," meaning 
six candles to the pound. A photometer is an apparatus for measuring the candle power of light sources.

Bunsen Photometer. - Place a drop of oil on a sheet of unglazed white paper. When we hold the paper between the eye and the window, the spot appears light. When held so that the eye is between the paper and the window, the spot appears dark. By experimenting we learn that when the paper is viewed from the side of the greater illumination the oiled spot appears dark but when viewed from the other side it appears light.

Experiment 64. - Place a lighted candle $A$ at a distance of two decimeters from a screen $S$ having in its center a square of oiled

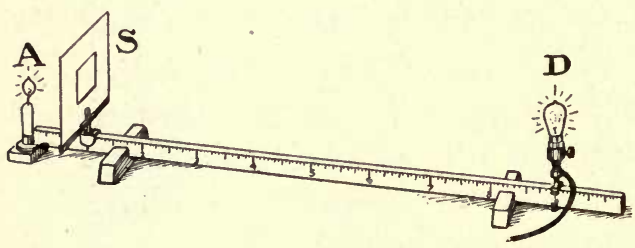

Fig. 197. - A Simple Arrangement for Measuring the Intensity of Light.

paper (Figure 197). On the other side of the screen place the light $D$ to be measured, the light in this case being that of an electric bulb. Move $D$ until a position is found at which the oiled paper appears the same from either side. The square of the distance from $S$ to $D$ divided by the square of the distance from $A$ to $S$ will give the candle power of the light D. Suppose the distance from $S$ to $D$ to be eight decimeters. Then the candle. power of $D$ is 16 , since 64 divided by 4 is 16 . The room must be free from light sources excepting those used in the experiment.

Reflection of Light. - An ordinary piece of glass both reflects and transmits light. Many of us have noticed the reflection of the open fire by the window-panes, making it appear as if there were another fire outside. On the outside of the window the fire may be seen by transmitted light.

Hold a mirror so that the sun's rays falling upon it will appear as a spot of light on the wall or ceiling of the 
room. The spot of light has been reflected by the bright surface of the mirror. The rays of light which fall upon the mirror are called incident rays, while the rays which the mirror sends off to the wall are called reflected rays.

Experiment 65. - From stiff cardboard make a semicircle having a radius of twelve inches (Figure 198). Support the cardboard so that the line marked $0^{\circ}$ is perpendicular to a plane mirror on which it rests. The mirror should not be wider than one half inch. Thrust a bright-headed pin through the semicircle near the end of one of the lines. Move the eye along the other side of the semicircle until a position is found where the pin may be seen in the mirror. How does

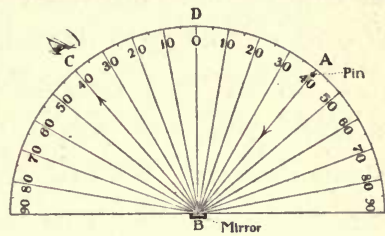

Fig. 198. - Angles of Incidence and Reflection. the angle of incidence $A B D$ compare with the angle of reflection $C B D$ ? Make readings with the pin in a number of different positions along the circumference. Can you formulate the law for the reflection of light?

\section{The Reflection of Light as Exhibited in the Mirror.}

The bouncing of balls from the pavement or walls and

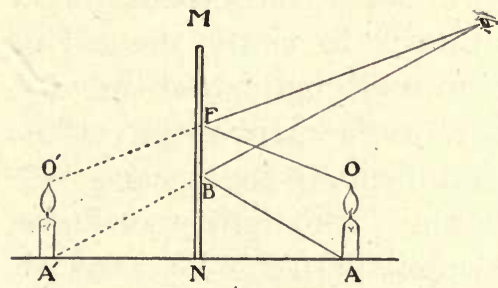

FIG. 199. - Diagram Showing the Apparent Position of an Object when Viewed in a Mirror. the action of billiard balls on striking the cushion of the billiard table are all examples of reflection similar to the reflection of light in which the angle of incidence is equal to the angle of reflection.

An object viewed in a mirror appears to be behind the mirror a distance equal to the distance of the object in front of it. Figure 199 shows the position of such an object. $M N$ represents a vertical mirror. Rays of light 
from the tip of the flame strike the mirror at all points, but only the ray which strikes the mirror at $F$ is reflected to the eye. Why? It is the same with the rays of light from other points on the object $O A$; for example, only the ray from $A$ which strikes the mirror at $B$ will reach the eye. The eye sees the image of $O$ at $O^{\prime}$, since the line $O^{\prime} F$ is a continuation of the direction from which the ray from $O$ entered the eye.

It is to be noted that as you face the mirror the left side of the face appears as the right side in the mirror. It is difficult to tell the time of day by looking at the image of the clock in the mirror for this reason. Writing to be read in the mirror must be written backwards.

Diffused or Scattered Light. - The snow reflects the sunlight in countless directions. This sort of reflection is called diffusion. The "glare" of the sun upon the snow, which is so painful to weak eyes, is due to the fact that no matter which way we turn we encounter strong reflected light. Most objects do not have a mirror-like

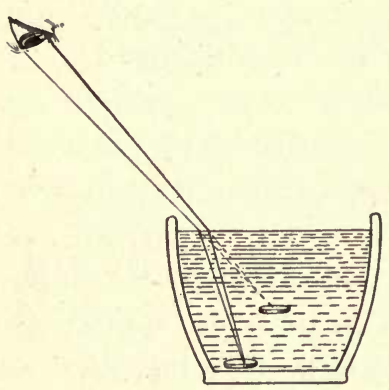

FIg. 200. surface and hence diffuse the light which strikes them in all directions. It is by the aid of this diffused light that we see these objects. Mirrors are sometimes difficult to see because the reflection of the light upon them is so nearly perfect. Rooms with mirrored walls may seem to be very large rooms.

Refraction of Light. - A number of familiar phenomena are due to the refraction of light or the bending of a ray of light as it passes from one transparent medium to another. If a pencil be placed in a tumbler of water, 
it will seem to be bent where it enters the water. Place a coin in a cup with opaque sides so that it is just out of view (Figure 200). Keep the eye fixed on the same place and fill the cup with water. The coin will now be visible.

Figure 201 represents the bending of a beam of light as it enters and leaves the water. The reason that it is bent is that light travels slower in water than in air. The lower side of the beam enters the water first and is retarded so that the upper part of the beam gets ahead, thus bending it toward a perpendicular as shown in the figure. As the beam leaves the water, the lower part reaches the air first and immediately increases its speed, while the upper part of the beam is still in the water. Thus it is

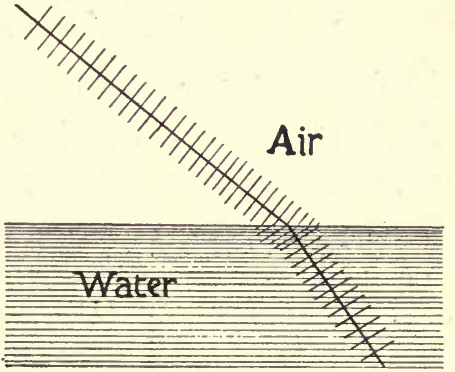

Fig. 201. - As light passes from the air into the water at an angle other than a perpendicular it is bent or refracted.

bent again but in the opposite direction. If the beam strikes the surface of the water at right angles, it will not be bent in either direction.

Light is bent or refracted when it passes obliquely from one transparent medium to another of different density. This principle is of great importance, since it is the one involved in the eye and in nearly all of our important optical instruments, including the microscope, the telescope, camera, and stereopticon.

Lenses. - There are a great many practical uses made of lenses. They are used in cameras, microscopes, telescopes, spectacles, field and opera glasses, stereopticons, and other similar instruments.

A lens is usually made of glass and has two curved sur- 
faces or one curved and one plane surface. The curved surfaces are usually spherical and may have any of the forms shown in Figure 202. There are two general classes
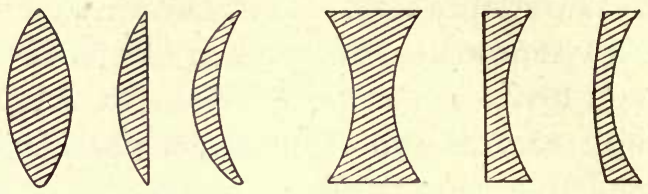

Fig. 202,- Lenses of Different Forms.

of lenses, convex lenses which are thicker at the middle than at the edge and concave lenses which are thicker at the edge.

Experiment 66. - Hold a double convex lens in the sun's rays. Scatter some crayon dust under the lens and notice how the refracted rays converge to one point. This point is called the focus or " fireplace." Hold a piece of tissue paper at this point. What is the effect? View some print through this lens. Does the lens magnify or not?

Experiment 67. - Draw the blinds on all the windows in the room except one. Hold a convex lens near the wall opposite the window, and then move it until a clear image of the window is thrown on the wall. The distance from the lens to the wall is called the focal length of the lens.

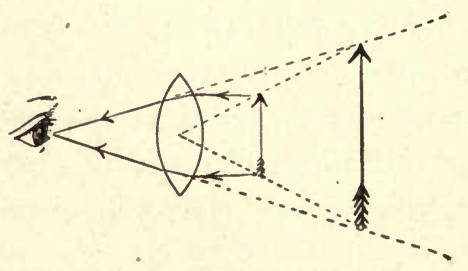

Fig. 203. - A Convex Lens Used as a Magnifying Glass.

When rays of light pass through a lens, they are bent toward the thickest part of the lens.

Uses of the Lens. - The simple microscope is nothing more than a convex lens as shown in Figure 203. An object viewed through such a lens appears in its natural position, but larger. Spectacles are often but a pair of convex lenses of small magnifying power. 
The compound microscope consists of a convex lens $O$ (Figure 204) of short focal length, called the objective, and a larger convex lens $L$ called the eyepiece. When the object $M N$ is viewed, an image is formed at $m n$. This image is further magnified by the eyepiece so that it appears as $M^{\prime} N^{\prime}$.

The telescope is like the compound microscope with this difference - the object lens is made large for collecting a large amount of light (Figure 205).

The camera is a light-proof box fitted with convex lenses. Light enters the camera through the lens, which produces on a ground glass at the rear of the box an inverted image of the objects within the range of the lens (Figure 206). If a sensitized plate or film is substituted for the ground glass, a picture will be pro-

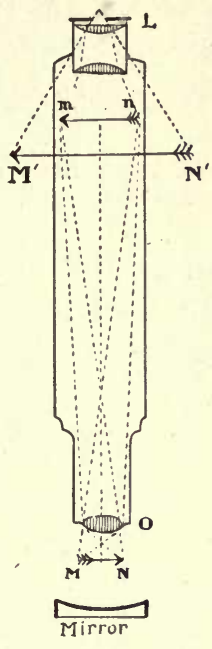

Frg. 204. - A Compound Microscops.

duced by the chemical action of the light on the plate or film. This plate is now treated with a developing fluid, which brings out a visible and permanent image

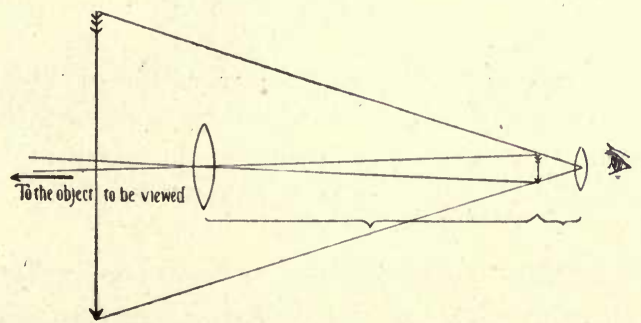

Frg. 205. - Telescope Lenses.

of the objects taken in the picture. The plate is now called a negative, because the light and shade are 


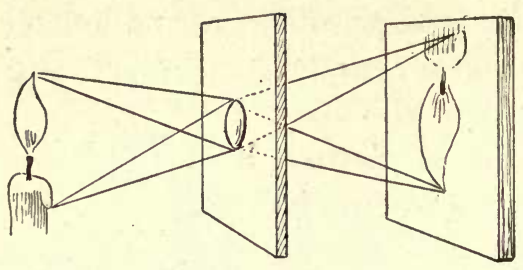

Frg. 206. - Showing the Inversion of the Image in a Pin-hole Camera.

reversed in the picture upon it. This negative may be used to produce any number of positive photographs on sensitized paper (Figure 207).

The eye is essentially a small camera. Light enters the eye through the pupil, which corresponds to the shutter of a camera. It then passes through the crystalline lens (convex lens) into the dark space and

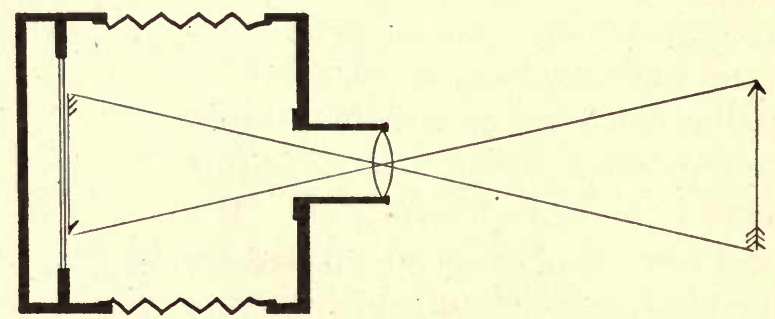

Fig. 207.- Diagram of a Camera.

finally forms an image on the retina at the back of the eye.

Experiment 68. - Look obliquely through a block of glass (Figure 208) and set four pins, two on each side of the block, so that they form a straight line. Remove the block and draw lines connecting the pins. Are they in a straight line?

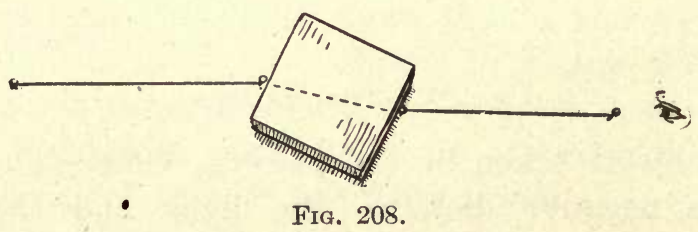


The Prism and Composition of White Light. - If sunlight be allowed to fall on a glass prism (Figure 209) and the refracted rays be caught on a white screen, a beautiful band of colors will be seen. These colors in their order are violet, indigo, blue, green, yellow, orange, and

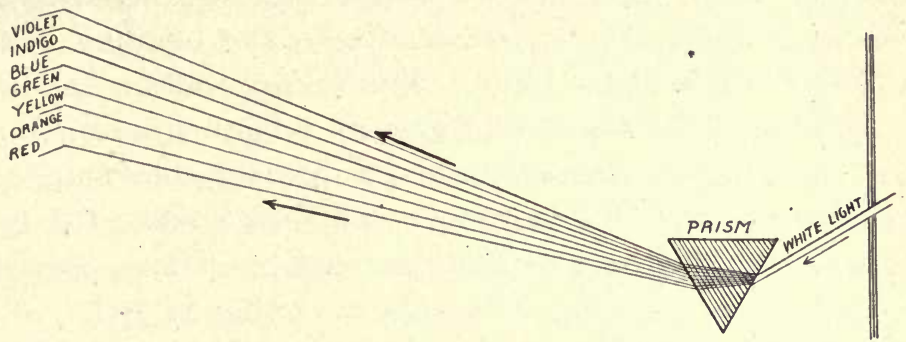

FIG. 209. - The prism separates white light into its constituent colors, which are arranged as shown.

red. The violet color is refracted or bent from its original path the most ; the red color is refracted the least. From this experiment it is evident that the white light from the sun is composed of these several colors. The entire band of colors is called the solar spectrum, and the process

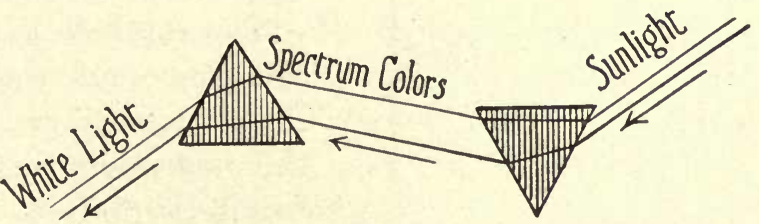

Fig. 210. - A piece of paper placed between the prisms will show the separated colors.

of separating colors by refraction is called dispersion. If the solar spectrum made by a prism is passed through a similar prism in a reversed position (Figure 210), the several colors will be reunited to form a single band of white light. 


\section{Length of Light Waves}

Violet . . . $.000397 \mathrm{~mm}$. Yellow . . . $.000589 \mathrm{~mm}$.

Indigo . . . $.000430 \mathrm{~mm}$. Orange . . . $.000656 \mathrm{~mm}$. Blue . . . $.000480 \mathrm{~mm}$. Red . . . $.000689 \mathrm{~mm}$. Green . . . $.000527 \mathrm{~mm}$.

In the solar spectrum we notice that the bending increases as the wave length decreases, the bending being greater for the violet light. The reason for the bending of a ray as it strikes a transparent medium obliquely is that its speed is changed, and the greater the change in speed the greater the bending. Therefore, since the violet light was bent more than the red light, the speed of violet light in glass must be less than that of red.

Red light waves are the longest and have the least frequency of light waves producing visible rays. When a piece of iron is heated to incandescence, it first gives off red light. As the heating continues the activity of the molecules and electrons increases, producing shorter

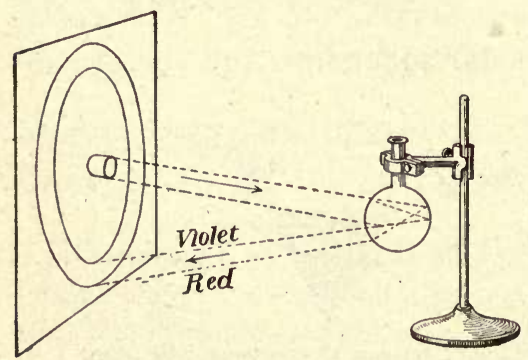

Fig. 211. - An Artificial Rainbow. light waves and consequently a change in color to almost a pure white.

The rainbow is a solar spectrum on a large scale. Rainbows are caused by the dispersion of sunlight by drops of rain. They may be seen only in the morning and late afternoon, since the sun must not be higher than $42^{\circ}$ from the horizon. In order to see a rainbow it is necessary to look at falling raindrops upon which the sun is shining from a position behind you. Miniature rainbows may be seen in the spray from fountains or lawn sprinklers (Figure 211). 
Absorption of Light and Color Phenomena. - Color depends upon the wave length of light. Only wave lengths within a certain limit affect the nerves of sight, namely those between $.0000689 \mathrm{~cm}$. and $.0000397 \mathrm{~cm}$. in length. There are many ether waves both longer and shorter than these, but they are not -visible. White light contains all the colors of the spectrum from red to violet inclusive. Most artificial light is deficient in some of the colors of the spectrum and is therefore not white light. For example, the mercury vapor electric lamp gives a light which is deficient in red waves. When objects are viewed in such a light, they have a ghostly appearance. Why?

If a piece of red glass is held in the path of the spectrum, all the colors except the red will disappear, showing that all the wave lengths, except the wave length which produced red, have been absorbed by the glass. Try glass of other colors in the same way. It will be found that glass of certain colors has greater power of absorbing light than glass of other colors. Red is one of the best absorbers of other colors.

Most substances absorb light to some degree. The absorbed light reappears as heat energy or chemical energy. The light that falls on an object and is not absorbed is reflected. The color which a body has in ordinary daylight is determined by wave lengths which are not absorbed by the body. If a body appears white in daylight, it is because it reflects or disperses all wave lengths and does not absorb one kind of wave length more than the other. If a body appears red in daylight, it is because it absorbs other rays more readily than it does red rays, so that the light which is reflected contains a large proportion of red waves. A body appears blue or 
green or yellow in daylight when it absorbs less of one of these colors than it does of the other colors contained in white light. So we see that color is really not a property of bodies, but is the sensation that a body produces when we view it in daylight. If viewed in light other than daylight, objects will often produce different color sensations. For example, a green ribbon will not appear green unless viewed in light containing green rays.

Sometimes a certain combination of colors will produce white light even when part of the spectrum is lacking. Two colors that will produce white light when mixed are called complementary colors. Red and bluish green, orange and light blue, light green and violet are such combinations.

\section{QUESTIONS}

1. Name six luminous bodies.

2. Name six non-luminous bodies.

3. Is the earth luminous or non-luminous? The moon? What reasons have you for thinking so?

4. How may we change a piece of iron from a non-luminous to a luminous body?

5. If the sun is $92,000,000$ miles from the earth, how long does it take light to travel from the sun to the earth?

6. The report of a gun is heard two seconds after the flash is seen. How far away is the gun?

7. A flash of lightning is seen five seconds before the thunder is heard. How far away is the cloud?

8. Name four transparent substances. Four translucent substances. Four opaque bodies.

9. Gold is ordinarily opaque. May it be hammered thin enough to be translucent?

10. One book is held three feet and another book is held nine feet away from the lamp. How many times as much light does the first book receive as the second book?

11. What are the positions of the sun, moon, and earth when we have an eclipse of the sun? An eclipse of the moon? 
12. Lay a triangular prism over and parallel to a pencil mark. How many marks appear? Explain.

13. Why do we not see rainbows near noon?

14. In problems 6 and 7 of this list why may we neglect the speed of light?

The Sight. - The eyes are the very complex external organs in which the fibers of the optic nerve terminate, and by means of which sight impulses are produced. The optic nerve is stimulated by light; but the structure of the eye is such that there is not a flood of light falling upon the termini of the nerve fibers but a perfect image of the lighted objects in the field of vision.

Protection of the Eyes. - The eyeballs are two nearly spherical bodies. They are admirably protected by being set into deep sockets. These sockets are lined with fatty tissue in such a way as to protect the eyes from shocks. The eyeballs are further protected by movable folds of the skin called eyelids, and the eyebrows. The eyelids are fringed by a row of stiff hairs which keep out dust particles and help to shade the eye from irritating lights. They are lined with a mucous membrane called the conjunctiva and fit the front of the eyeball perfectly. Friction between the eyelids and the eyeball is prevented by mucous secreted by the conjunctiva and tears or lachrymal fluid secreted by the lachrymal gland. Excess lachrymal fluid is drained into the nose through a small duct called the lachrymal duct. The flow of the lachrymal fluid is increased by irritations of the covering of the eye or by strong emotion. In such cases the lachrymal duct is unable to drain the fluid off fast enough, and tears overflow on to the face.

The movements of each eye are controlled by six muscles so arranged that the eyeball may be turned in any direction. 
Structure of the Eyeball. - The eyeball has a diameter of about one inch. Except at the front and at the place where the optic nerve enters, the eye is covered with an opaque, white coat called the sclerotic coat (Figure 212). This coat forms the white of the eye and the transparent

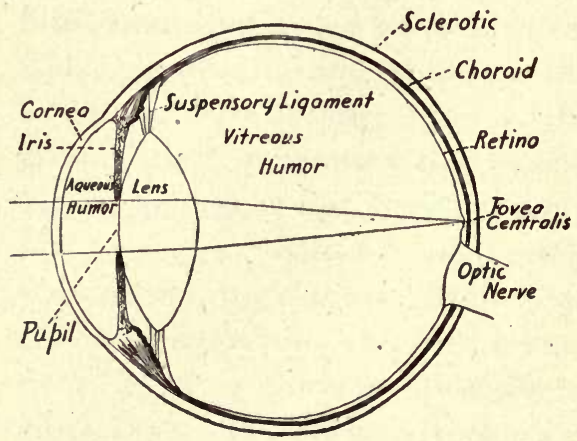

FIG. 212. - Showing a Section of the Eyeball. modification of it at the front of the eye is called the cornea. Inside the sclerotic coat is a second layer called the choroid. This coat contains a network of blood vessels and is colored black by pigment cells so that it appears like the inside of a dark grape skin. The dark surface of this coat absorbs the rays of light which would otherwise be reflected and interfere with perfect vision. In albinos this coat has no dark pigment cells, and the vision is therefore imperfect in strong light.

At the front of the eye the choroid coat is continued as a muscular curtain known as the iris. At the center of the iris is a round opening (the pupil) through which the light enters the eye. The size of the pupil is regulated by the involuntary muscles of the iris, of which there are two sets, one circular, and the other radiating. The contraction of the circular muscle fibers decreases the size of the pupil, and the contraction of the radial fibers increases the size of the pupil. The iris is colored by pigment. When we speak of a person's eyes as blue or gray we simply mean that these colors are the predomi- 
nating colors in the iris. The outer edge of the iris is fastened to the sclerotic coat by the ciliary ligament and to the choroid coat by folds known as ciliary processes.

The third layer of the eye or retina is a delicate transparent membrane of connective tissue containing an expansion of the optic nerve. It lines the interior of the eyeball with the exception of the front, where it stops with the ciliary processes and at the entrance of the optic nerve. The retina is the only part of the eye that is sensitive to light, and it is the part of the eye in which visual impulses originate.

In addition to the transparent parts of the eye already mentioned there are three other important media. The main part of the interior of the eyeball is filled with a colorless, transparent, jelly-like substance called the vitreous humor. It aids in preserving the form of the eyeball. In front of the vitreous humor and just behind the iris is the crystalline lens. It is a biconvex, transparent body which focuses the rays of light as they enter the eye, so that a clear image of the reflecting object is produced: Between the crystalline lens and the cornea is a small space which is filled with a clear, watery liquid called the aqueous humor.

The place where the optic nerve enters the eye is called the "blind spot," since the eye at that point is not sensitive to light.

How the Eye Does its Work. - The human eye resembles a camera in many essential respects. It contains a focusing lens, the crystalline lens, the iris, which acts as a shutter, and the retina, which takes the place of the camera film or sensitive plate (Figure 213). The eye, however, is vastly superior to the camera. The focusing organs of the eye possess the power of accommodating 
their shapes so that objects at different distances may be properly focused. The crystalline lens is elastic and would become more convex if it were not held under
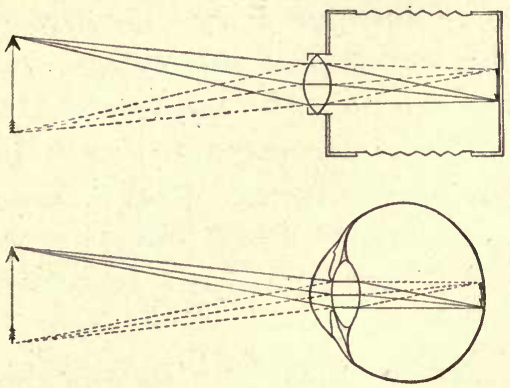

FIG. 213. - Two Cameras.

A diagram showing the similarity in the structure of a photographic camera and the eye. tension by the suspensory ligament which is attached to the choroid coat. When the distance of the object requires a change of focus, the ciliary muscles draw the choroid coat forward, thus reducing the tension on the crystalline lens. The lens immediately becomes more convex and shortens its focus. When the ciliary muscles are relaxed the crystalline lens is pulled back to its original position.

The shutter (iris) in the eye works automatically, allowing the proper amount of light to enter the eye at all times. The retina is also superior to the camera. While the photographic plate can be used but once, the retina is able to receive any number of impressions and send them to the brain for record. In other words, the camera of the eye is always "loaded."

The image formed on the retina is always inverted (Figure

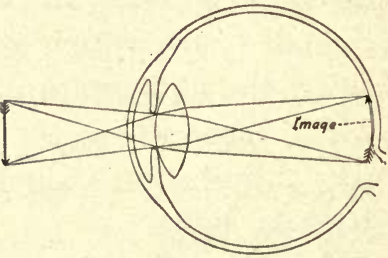

FIG. 214. - Showing the Formation of an Image upon the Retina.

214), but this does not prevent our seeing the object in its correct position; since it is the brain which translates the visual impulse of the retina into the sensation of actual sight, 
Most objects are non-luminous, every point sending out rays of reflected light. The rays that enter the eye are focused in proper scale by the cornea and crystalline lens. If for any reason these rays are not properly focused, defective vision results. For example, "near sight" results when the focus is too short and the rays meet and cross in front of the retina. "Near sight" may be corrected by the use of a concave lens in front of the eye. "Far sight" is due to the focus falling at a point behind the retina. Convex lenses are used to remedy this defect. Astigmatism is due to imperfections in the curvature of the cornea. The light which falls on some particular spot of the cornea is not properly refracted. A good oculist usually can correct these defects in vision and should be consulted whenever they appear.

\section{QUESTIONS}

1. How are the eyes protected?

2. Of what value are tears?

3. Where are the lachrymal glands situated?

4. Through what eye media does the light pass on its way to the retina?

5. Why is the inside of a camera black?

6. What coat of the eye may be compared to the black interior of the camera?

7. To what is the color of the eye due?

8. What is the use of the vitreous humor of the eye?

9. What is meant by the power of "accommodation" as applied to the eye?

10. What causes the iris to dilate and contract?

11. Why can we not see the spokes of a rapidly moving wheel?

12. The film of a moving-picture machine is really standing still when the picture is thrown on the screen. Why does it seem to. be in motion?

13. Name some cautions to be observed in the care of the eyes.

14. What is "color blindness"? 
Light in the House. - In recent years a great deal of attention has been given to the problem of proper lighting for different kinds of buildings. The manufacture of cheap glass has made it possible to have sunlight in abundance in all rooms with an outside exposure. It is quite important from the standpoint of health that we have plenty of sunlight in our homes and working places,

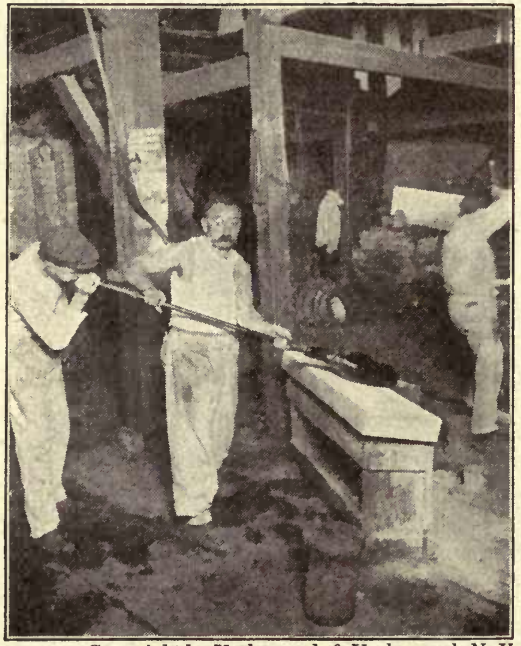

Copyright by Underwood \& Underwood, N.Y.

Fig. 215. - Glass Bottle Making. Blowing a bottle and shaping bottom of carboy base. since darkness fosters the collection of dirt and filth and the growth of disease germs.

A few generations ago sunlight in the home was a luxury because of the scarcity of transparent materials which could be used in windows.

\section{How Glass is Made. -} It is quite certain that the Egyptians and Phœnicians knew how to make glass long before the Christian Era, but it was not until the sixteenth century that window glass was first used; and then for many years it was used only by the most wealthy.

Glass commonly is made by melting together the silicates of calcium and sodium, or we may say by melting together a mixture of sand, limestone, and soda. The mixture is melted in pots of fire clay. This is the soft glass which is used in making windows and ordinary glass dishes. If potassium silicate is used instead of 
sodium silicate, a harder glass results. This glass is known by such names as hard glass, Bohemian glass, and crown glass (Figures 215, 216).

Artificial Lighting. - A history of artificial lighting includes stories of the use of many different materials.

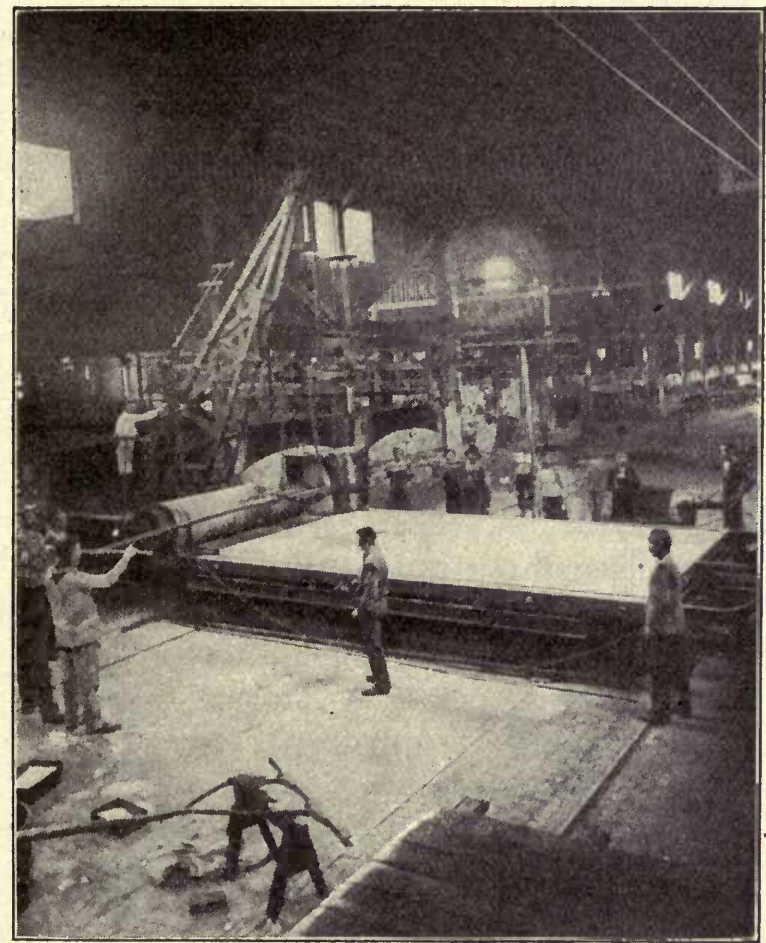

Copyright by Underwood \& Underwood, N.Y.

Frg. 216. - Glass Making.

Casting and rolling sheets of plate glass, $12 \frac{1}{2} \times 21$ feet, Tarantum, $\mathrm{Pa}$.

Prior to 1860 the common means of lighting were the burning of wood in the fireplace - pine knots gave the most light because of the turpentine they contained-- 
and the burning of heavy oils either in open dishes or in the form of candles. Lard, olive oil, and whale oil were

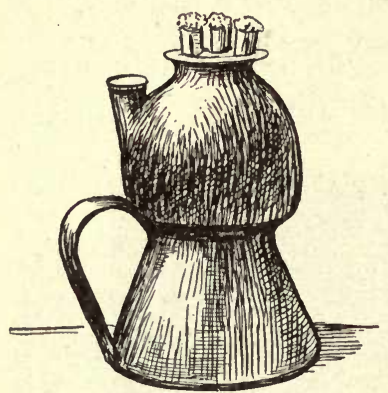
the oils commonly used (Figure 217).

Candles were originally made by dipping a wick into melted fat and allowing it to cool, repeating the operation until the candle was of the desired thickness. Candles are now made of fats and different waxes. The wick is set in a mold Frg. 217. - A Whale-oil Lamp. and the melted material is poured around it. When it has solidified, it is removed from the mold and is ready for use.

The Kerosene Lamp. - The discovery of petroleum gave a distinct impetus to lighting problems. Many forms of the kerosene lamp were placed on the market. The success of the kerosene lamp requires that the kerosene be burned in a good supply of air; otherwise the lamp will smoke (Figure 218).

Gases for Lighting. - There are a number of gases which may be used for lighting purposes with proper burners. When soft coal is heated in a retort, illuminating gas is formed. However, most of the gas which is sold as illuminating gas is formed by passing steam through a

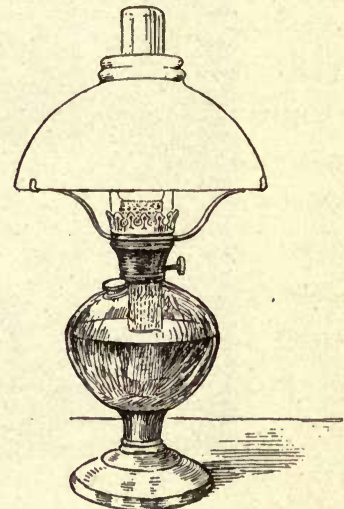

Fig. 218. - A Kerosene Lamp. hot bed of coke or hard coal. This water gas has a high fuel value but burns with an almost colorless flame, giving little or no light. In order that it may be used for 
illuminating purposes petroleum is sprayed into the hot carburetor of the gas-producing apparatus. The heat breaks the oil into gases which give illuminating power to the water gas.

As illuminating gas reaches the consumer, it burns with a bright yellow flame. To prevent smoking, it is burned from a narrow slit burner in order that more of its surface will come in contact with the air.

This gas is stored by the gas companies in large tanks inverted in water. The tanks are quite heavy and cause the gas pressure in the distributing lines. As gas is used the tanks sink into the water, and as more gas is pumped into them they rise again.

Natural Gas. - In some regions large pools of natural gas are found. So far as is known the United States has a greater supply of this gas than any other nation. It is a most perfect fuel and may be used for lighting purposes if an incandescent mantle is used. To use a mantle we simply burn the gas in a Bunsen burner and suspend the mantle over the colorless flame. The light comes from the materials of the mantle which are heated to incandescence (Figure 219).

The best mantles are made by soaking the little knitted sack in a solution of cerium and thorium salts. When the sack is dry, the solid salts fill the spaces between

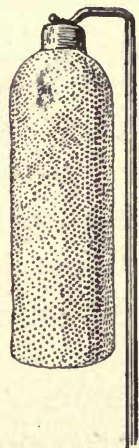

Fig. 219. - A Gas Mantle. the threads. The mantle is now set on fire. The thread burns, leaving a thin shell of the oxides of the metals. Other gases besides natural gas may be used with incandescent mantles. 
Acetylene. - The gas is made by the action of calcium carbide and water. When thoroughly mixed with air (Figure 220), it burns with a smokeless flame of brilliant whiteness.
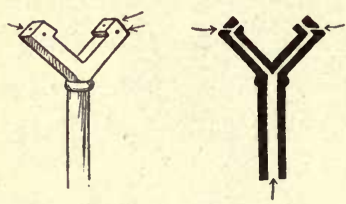

Fig. 220. - An Acetylene Burner and Flame.

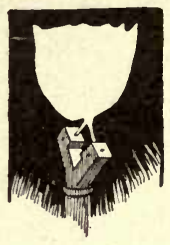

Electric Lighting. Themostcommon method of lighting in cities at the present time is by electricity, using the incandescent electric lamp, the principle of which has already been given (cf. p. 202).

Lighting Fixtures. - The last few years have produced many changes in the prevailing types of lighting fixtures. Some of the shades and globes are very wasteful of light without possessing much compensating beauty. Indirect lighting is very wasteful of light, and it is also tiring on the eyes. The best light for the eyes will be a well-diffused light with areas of light and shadow as is found in the sunlight. Indirect lighting is well diffused but throws no well-defined shadows, which are so essential to normal sight. Probably the light bowl which best meets the requirements of beauty and hygiene is the translucent bowl, which while it reflects some of the light to the ceiling also diffuses much of it directly into the room. This is called semi-indirect lighting.

For factories direct lighting will be the best and most economical.

\section{QUESTIONS}

1. What materials were used in windows before glass came into use?

2. What is "Jena" glass?

3. How was the whale-oil lamp made? 
4. When was petroleum discovered? By whom? Where?

5. Why do kerosene lamps sometimes smoke?

6. What kind of incandescent bulbs are most economical?

7. Why does a carbon filament light bulb become hotter in use than a Tungsten light bulb?

8. How did the manufacture of cheap glass advance civilization?

9. What is a fuse plug?

10. Explain how to read your gas meter. Your electric meter. 


\section{CHAPTER XIV}

\section{ELEMENTS, COMPOUNDS, AND MIXTURES}

Interrelation of the Sciences. - The different branches of science, such as physics, chemistry, biology, physical geography, and botany, are not separated by any very definite boundaries, as we shall see. In the study of physical geography we must know something of the other sciences in order to understand the changes that have occurred and are occurring in the life of the earth. Weathering depends upon both physical and chemical changes. Winds are caused by heat, a physical agent. A study of plants and animals involves the study of numerous physical and chemical changes. So we could go on citing example after example of the ways in which the sciences interlock and are dependent upon one another.

How matter is changed. - We may change most substances in many ways. If we apply heat to a piece of iron, it becomes hot; as more heat is applied it becomes red hot and gives off light. If the heating continues, the iron finally melts and may be poured into a different form. As the liquid iron cools it passes back through these various changes and finally becomes a cold, black piece of iron again. A piece of iron, when rubbed with a magnet, itself becomes a magnet and exhibits the property of attracting pieces of iron and steel. After a time the magnetism is lost. Through these various changes the iron has never ceased to be iron. 
A piece of ice when heated changes to water. If the heating is continued, the water is changed to an invisible gas and passes into the air. By cooling the air the water may be recovered. It has never ceased to be water in some form. Are these changes physical or chemical changes?

When a piece of iron wire is burned in oxygen, a new substance, iron oxide, is formed. The red rust that forms on iron when it is left out of doors or in a damp place is this same new substance. When iron and hydrochloric acid are put into a flask together, they react to form a new substance, iron chloride, and hydrogen is liberated. These new substances have properties which are quite different from those of iron.

What kind of changes are these?

Oxidation. - The union of any other element with oxygen is called oxidation, and the new substance is called an oxide.

Oxygen is the most abundant of the elements, and oxidation is the most common chemical change.

The rusting of iron and the rotting of wood are examples of slow oxidation. When we say that a substance oxidizes, we mean that one or more of its elements combines with the oxygen of the air. If the oxidation takes place rapidly, it is called combustion or burning. In such cases measurable quantities of light and heat are given off. Heat is developed in the decay of wood, but the process is so slow that the change in temperature is too small to be noticeable.

Elements, Compounds, and Mixtures. - Many substances are the result of a combination of two or more other substances. For example, hydrogen and oxygen combine to form water; iron and oxygen combine to 
form iron oxide; zinc and sulphur when heated combine to form a white powder, zinc sulphide. Substances which have been formed by the union of two or more substances are called compounds. By proper means these compounds may be separated again into the substances which were used in their production. The question of course arises as to whether these substances - oxygen, hydrogen, iron, zinc, sulphur - may be further divided into other substances. All we can say is that they have never been divided in any of the numerous attempts that have been made, and we therefore assume that they cannot be divided. Substances which we have never been able to decompose into other substances are called elements. If powdered zine and sulphur are placed in a dish and stirred, they do not unite but remain as small particles of zine and sulphur. This is called a mixture. When a flame is brought near this mixture, there is a flash of light and a cloud of white smoke. The white powder that is left in the dish is no longer a mixture of zine and sulphur but a new substance, zinc sulphide, having new properties. Sulphur will dissolve in carbon disulphide. Zinc sulphide will not. Sulphur is yellow and zinc is gray. The new substance is white.

There are numerous mixtures about us. Air is a mixture of the gases nitrogen, oxygen, carbon dioxide, and water vapor. Most rocks are mixtures of different substances. A mixture of oxygen and hydrogen does not produce water until the temperature is raised to about $620^{\circ}$ Centigrade.

The Common Elements. - There are about eighty different elementary substances or elements, but many of these are found in very small quantities. In our daily life we are not commonly concerned with more than 
twenty of the elements. Twelve of these are always found in living matter, and six more are sometimes found in living matter. The twelve which are always found are : phosphorus, sulphur; carbon, oxygen, hydrogen, nitrogen, chlorine, potassium, sodium, calcium, magnesium, and iron.

Only about one fourth of the elements are found in uncombined state. The others are found only as compounds.

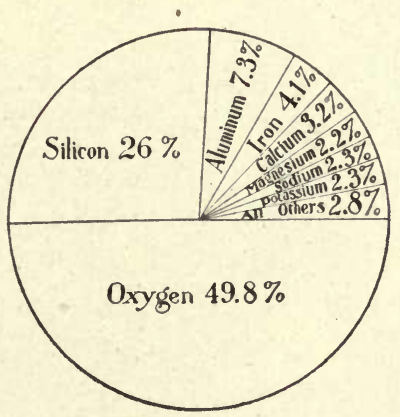

FIG. 221.

The following table is an estimate of the plentifulness of the elements. Twelve of them make up 99 per cent of the earth (Figure 221).

\section{Per Cent}

Oxygen $\quad 50.00$

Silicon . . 26.00

Aluminum . $\quad 7.25$

Iron . 4.10

Calcium . 3.15

Sodium . 2.30
Per Cent

Potassium . 2.30

Magnesium . 2.10

Hydrogen . 1.00

Titanium. . .40

Chlorine . . .20

Carbon . . .20

All the other elements, including gold, silver, sulphur, and mercury, make up the remaining one per cent of the earth. 


\section{PARTIAL LIST OF ELEMENTS}

\begin{tabular}{|c|c|c|c|c|c|c|c|c|c|c|}
\hline & NAME & & & & & & & & Symbol & State \\
\hline Metals & $\begin{array}{l}\text { Aluminum } \\
\text { Antimony } \\
\text { Arsenic } \\
\text { Barium } \\
\text { Bismuth } \\
\text { Cadmium } \\
\text { Calcium } \\
\text { Chromium } \\
\text { Cobalt. } \\
\text { Copper. } \\
\text { Gold . } \\
\text { Iron. } \\
\text { Lead } \\
\text { Magnesium } \\
\text { Manganese } \\
\text { Mercury } \\
\text { Nickel . } \\
\text { Platinum . } \\
\text { Silver . } \\
\text { Sodium } \\
\text { Tin . } \\
\text { Zinc. } \\
\text { Boron . } \\
\text { Carbon } \\
\text { Iodine . } \\
\text { Phosphorus } \\
\text { Silicon . } \\
\text { Sulphur } \\
\text { Bromine . } \\
\text { Chlorine . } \\
\text { Fluorine } \\
\text { Hydrogen . } \\
\text { Nitrogen . } \\
\text { Oxygen }\end{array}$ & • & & 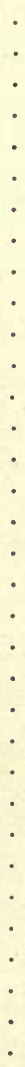 & - & & & 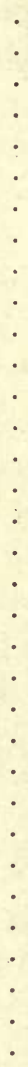 & $\begin{array}{l}\mathrm{Al} \\
\mathrm{Sb} \\
\mathrm{As} \\
\mathrm{Ba} \\
\mathrm{Bi} \\
\mathrm{Cd} \\
\mathrm{Ca} \\
\mathrm{Cr} \\
\mathrm{Co} \\
\mathrm{Cu} \\
\mathrm{Au} \\
\mathrm{Fe} \\
\mathrm{Pb} \\
\mathrm{Mg} \\
\mathrm{Mn} \\
\mathrm{Hg} \\
\mathrm{Ni} \\
\mathrm{Pt} \\
\mathrm{Ag} \\
\mathrm{Na} \\
\mathrm{Sn} \\
\mathrm{Zn} \\
\mathrm{B} \\
\mathrm{C} \\
\mathrm{I} \\
\mathrm{P} \\
\mathrm{Si} \\
\mathrm{S} \\
\mathrm{Br} \\
\mathrm{Cl} \\
\mathrm{Fl} \\
\mathrm{H} \\
\mathrm{N} \\
\mathrm{O}\end{array}$ & 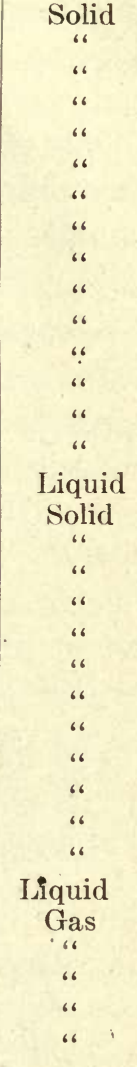 \\
\hline
\end{tabular}

A few of the elements in the above list (oxygen, nitrogen, and hydrogen) have been studied in the chapters on air and water.

Metals. - A number of metals are familiar to every one. However, a few, such as sodium and potassium, 
are seen only in the laboratory. Each metal is different from the others in certain characteristic properties. Metals have their own special properties which enable us to distinguish them as a class quite readily. For example, metals may be melted, fusibility; they conduct

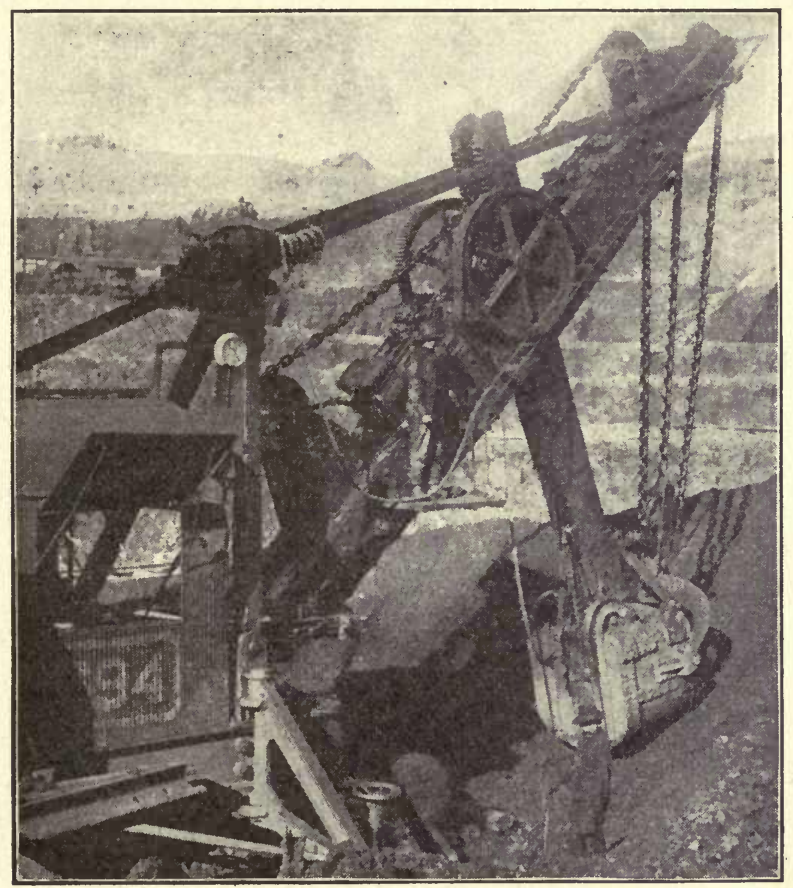

Copyright, 1915, by Keystore View Company.

FIG. 222.- Surface Mining, Mesaba Range, Minnesota.

heat and electricity, conductivity; they may be hammered into thin sheets, malleability; and the fresh surfaces of metals have a peculiar luster.

Iron. - Iron is the most important of all the metals. It is found in a number of ores and is sometimes found in a free state in igneous rocks. Its abundance and its 
properties make it the best metal for a large variety of uses. (Figure 222.)

Cast iron contains from four to five per cent of carbon and other impurities. These impurities lower the melting point of cast iron to about $1200^{\circ} \mathrm{C}$., and they also make the iron very hard and brittle.

Wrought iron is nearly pure iron. To make wrought iron, cast iron is remelted with another iron ore, hematite, and stirred until the impurities have been removed. Because of its extreme toughness, wrought iron is quite valuable. It is used for chains, wire, bolts, etc. It melts at a much higher temperature than cast iron, and for this reason is used for fire bars.

Steel has some carbon in it, but not so much as cast iron. It can be tempered to different degrees of hardness.

Copper. - Copper has a characteristic dull red color when exposed to the air. It melts at about $1050^{\circ} \mathrm{C}$., is a good conductor of heat and electricity, and is quite malleable. It was one of the first metals used by man. This was because it was found in a free state and did not need to be separated from ore and also because it is so easily hammered into desired shapes. (Figure 223.)

Copper is used for electric wiring, for evaporating pans and some cooking utensils, for sheathing ships, and for places on buildings where a metal is needed that will resist weathering. Brass is an alloy of copper and zine, and bronze is an alloy of copper and tin.

Mercury. - Most of the mercury of commerce comes from California and Spain. At ordinary temperatures it is a liquid. It solidifies at $-40^{\circ} \mathrm{C}$. and boils at $357^{\circ} \mathrm{C}$. It has a silvery, metallic luster which is not affected by air and water. Mercury has a number of commercial uses. It is used in thermometers and barometers. In 
the stamp mills, pulverized ores of gold and silver are mixed with water and allowed to pass over layers of mercury. The mercury dissolves the particles, of gold and silver. Later they are separated by distillation.

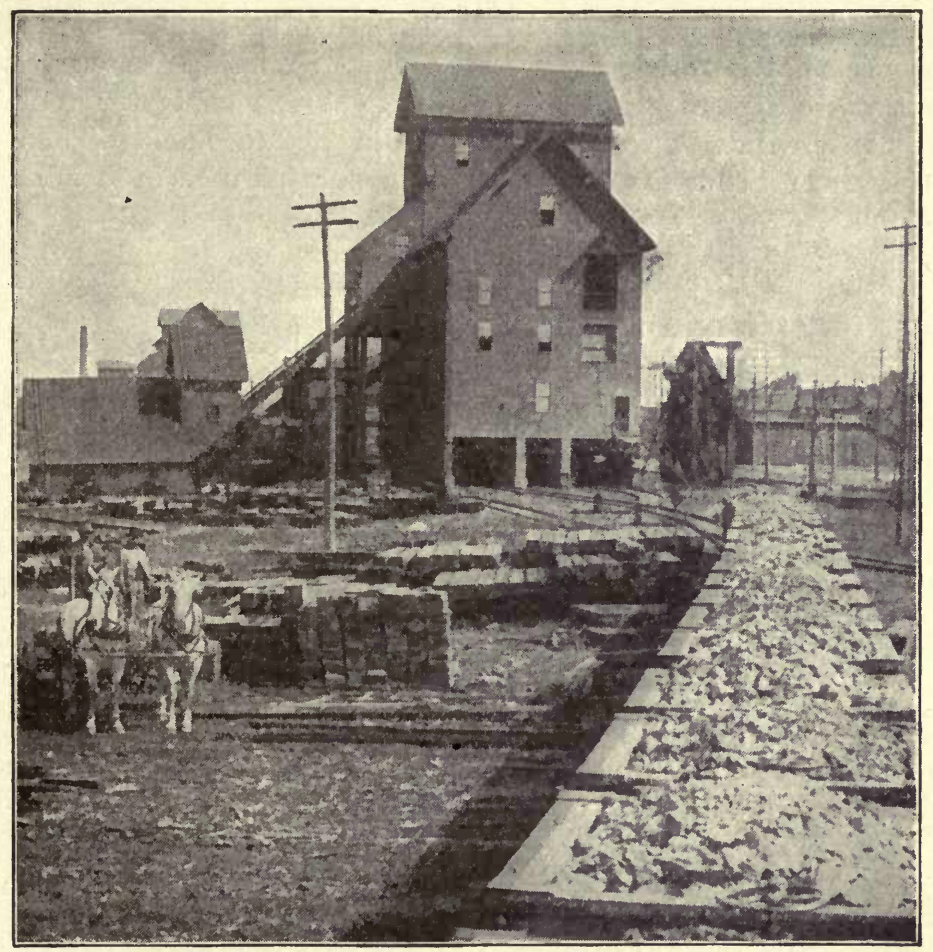

Copyright by Underwood \& Underwood, N.Y.

Fig. 223. - Copper Ore Just Hoisted from the Shaft, Calumet and Hecla Mine, Michigan.

Sodium. - Sodium is a very soft metal and may be easily cut with a knife. The surface of freshly cut sodium has a luster resembling that of silver, but it soon tarnishes when exposed to air or moisture. To keep sodium we cover it with kerosene, or some oil which contains no 
oxygen. Sodium reacts vigorously with water to form sodium hydroxide and hydrogen. The heat evolved melts the sodium which forms into a ball and floats on the water.

Experiment 69. - Place a small piece of sodium in a widemouthed bottle which is two thirds full of water and cover the bottle with a piece of glass. Test the gas formed in the top of the bottle with a flame. In this experiment care should be taken to have the hands dry as well as all apparatus used in handling sodium.

Salt, a compound of sodium, sodium chloride, is found in sea water and in extensive deposits at Stassfurt and Reichenhall in Germany, in Cheshire, England, and in several parts of the United States. It is a necessary article of diet. Salt may be obtained from salt water by evaporating the water. It crystallizes in white cubes. Other useful compounds of sodium are sodium nitrate (Chile saltpeter) and sodium bicarbonate, which is commonly known as baking soda.

Silver. - The chief supply of silver is obtained from ores of lead and copper. Silver is mined extensively in the western part of the United States, Mexico, and Australia. For silverware and coins, silver is alloyed with 10 per cent of copper ; that is, they are " 900 fine." Sterling silver is 925 fine or it is $92 \frac{1}{2}$ per cent pure silver. Articles may be plated with silver by the method described under electroplating in Chapter XI. Mirrors are silvered by cleaning the surface of the glass and pouring over it a solution of silver nitrate, ammonium hydroxide, and some reducing agent, such as formaldehyde. The film of silver which adheres to the glass is dried and varnished to protect it. Photographic plates and films are made by washing them with an emulsion of silver 


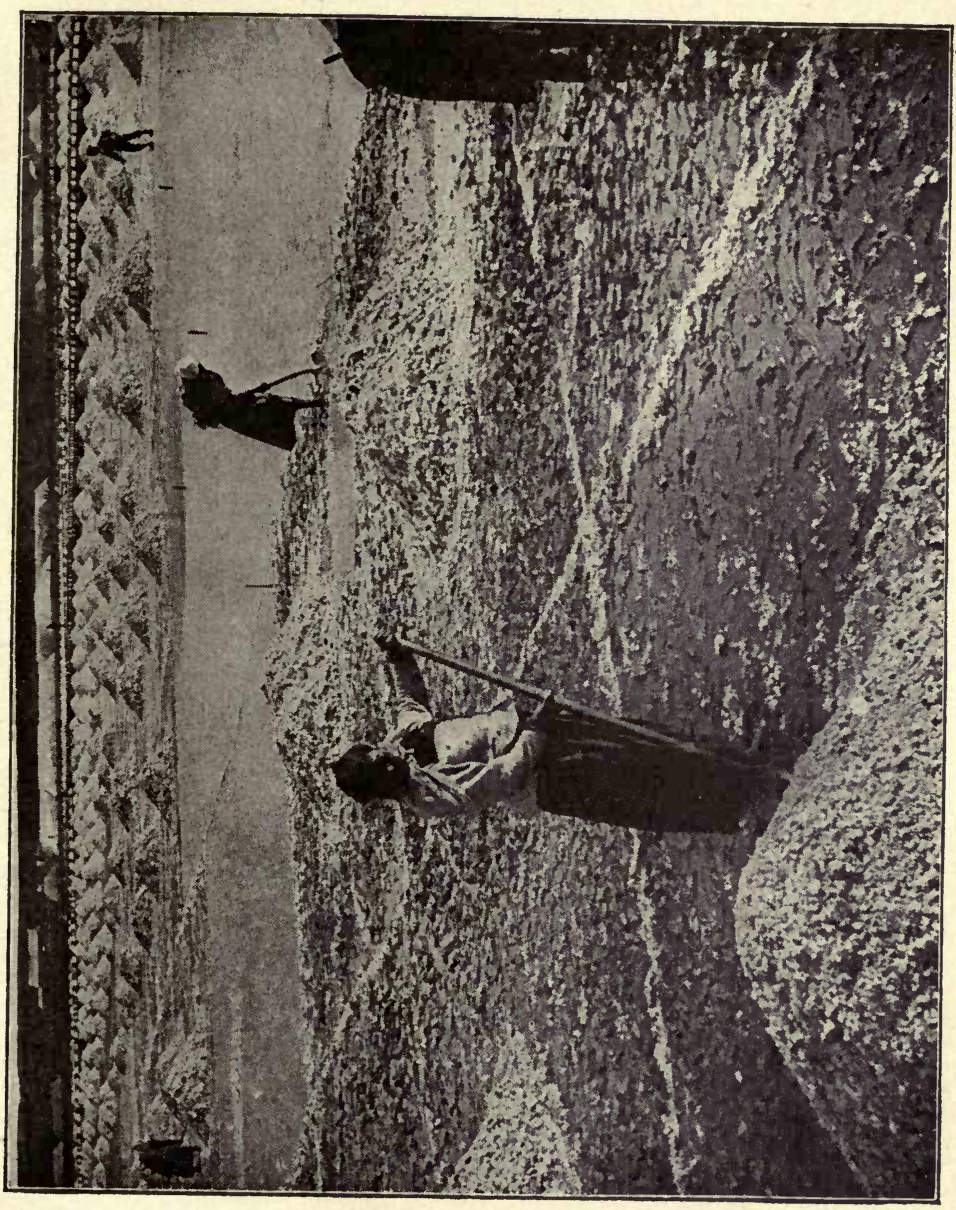



bromide and gelatine. The silver bromide is quite sensitive to light, and when the plate is exposed and properly developed a silver image of the object photographed is left upon it. The "printing paper" is essentially like the plates. Some papers are washed with a silver chloride and white of egg emulsion, and others like "velox" are rendered sensitive to lignt by being washed with a silver bromide emulsion.

Gold. - Gold is found in a pure state in veins of quartz in almost every part of the world. Occasionally large nuggets of gold are found.

Since gold is little affected by the chemical action of the air, water, and other substances, it has come into common use for coins and jewelry. It is alloyed with silver and copper to give it hardness. Twenty-four carat gold is pure gold; eighteen carat gold is three fourths pure; and fourteen carat gold is $\frac{14}{24}$ pure. The gold coins of the United States are ninety per cent gold and ten per cent copper. Gold is the most ductile and malleable of metals. It melts at $1075^{\circ} \mathrm{C}$. It is not dissolved by any single acid, but a mixture of hydrochloric and nitric acids will dissolve it.

Chlorine. - The gases, oxygen, hydrogen, and nitrogen, which we have studied previously are colorless, odorless, and tasteless; but chlorine is a green gas having a disagreeable suffocating odor. When breathed, it irritates the lining of the nose and throat. Chlorine occurs in a great many compounds, but it is found most abundantly in common salt. It is named from chloros, a Greek word meaning green.

Chlorine is a powerful bleaching agent and has a wide commercial use for this purpose. Large quantities of it are used in making bleaching powder, chloride of lime. It 
is the bleaching agent used to bleach rags in the paper mills, and to whiten cotton cloth in the cotton mill.

Chlorine may be obtained by passing a current of electricity through an aqueous solution of common salt.

Sulphur. - A large part of the sulphur used in the world comes from Sicily and Louisiana. It occurs in Louisiana in a deposit about one half mile in diameter

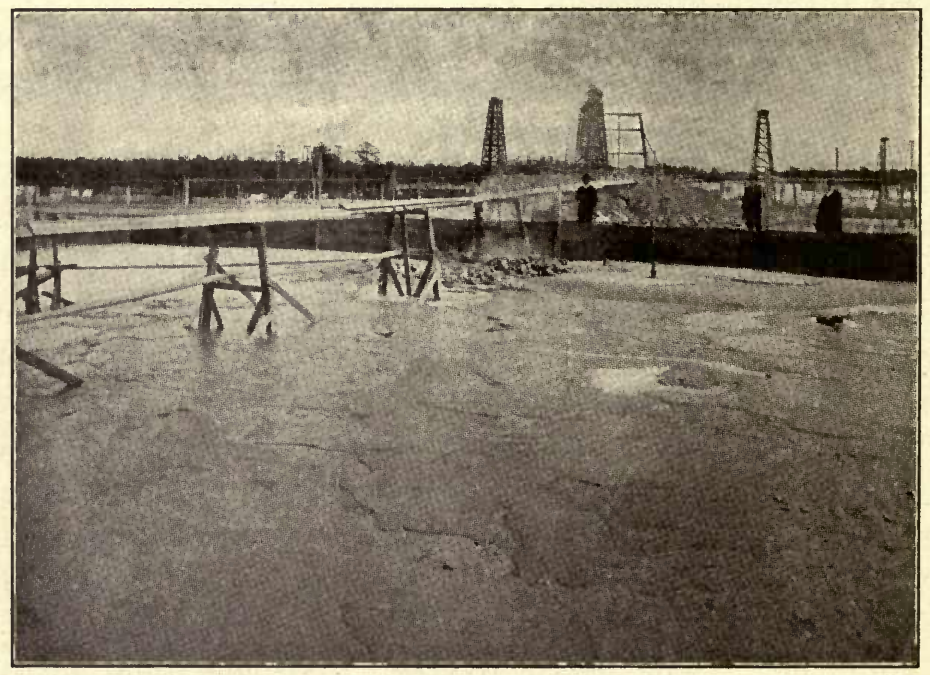

Fig. 224. - A Well Pumping Sulphur.

and at a depth of 900 feet (Figure 224). It is a yellow solid not soluble in water but soluble in carbon disulphide. If a solution of sulphur in carbon disulphide is evaporated, sulphur crystals will be formed. The evaporation must be carried on over a steam bath, as carbon disulphide is very inflammable.

Sulphur or brimstone has a number of uses. Sulphur oxide, which is formed when sulphur is burned in the air, will destroy disease germs and vermin. To fumi- 
gate a room with sulphur, it is only necessary to close the room and burn sulphur in it. Sulphur is also used as a bleaching agent for those fabrics and materials which would be injured by chlorine. The fumes from sulphur are poisonous and should not be breathed in large quantities.

Carbon. - Of all the non-metallic elements carbon is the most abundant. There are only three non-metallic elements which are solids at ordinary temperatures carbon, phosphorus, and sulphur.

Carbon appears in many common forms in the impure state, as coal, soot, or lampblack, charcoal and graphite, or the black lead of our lead pencils. It is found in considerable quantities in all vegetable and animal substances. When such substances are burned, the black carbon is exposed to view. When wood is charred, when toast is burned, or when meat is scorched, the black which appears is carbon. If the combustion is complete, the carbon disappears and only the mineral ash is left.

Except in the case of the diamond, carbon is a black solid. The diamond is so different in appearance and properties from the other forms of carbon - graphite, charcoal, soot, and coke - that it is hard to believe that it is the same element. However, if these substances are burned in pure oxygen, they all form the same product, carbon dioxide. This proves conclusively that they are the same element.

\section{QUESTIONS}

1. Give four examples of physical change.

Give four examples of chemical change.

2. What is oxidation?

3. What is an element? A compound? A mixture?

4. How do you know iron to be an element? 
5. Why do we paint iron building frames?

6. Name ten of the most useful elements.

7. Name four uses of cast iron. Three uses of wrought iron.

8. How is chlorine used in warfare?

9. Arrange iron, lead, zinc, sodium, gold, aluminum, copper, silver, and platinum in order of their densities.

10. What are some of the commercial uses of gold? Lead? Tin? Zinc? Copper? Sulphur? Aluminum?

11. What causes the hardness of razor steel?

12. What kind of iron would you use for bicycle pedals? Why? 


\section{CHAPTER XV}

\section{FUELS AND CARBON COMPOUNDS}

Fuels. - Fuels are materials used for producing heat. They must be capable of uniting with oxygen under easily obtainable conditions, with sufficient rapidity to insure the evolution of considerable heat energy. These conditions are largely filled by carbon and its compounds, occurring as gases, liquids, and solids.

Impure fuels - solids

$$
\left\{\begin{array}{l}
\text { wood } \\
\text { peat } \\
\text { soft coal }
\end{array}\right.
$$

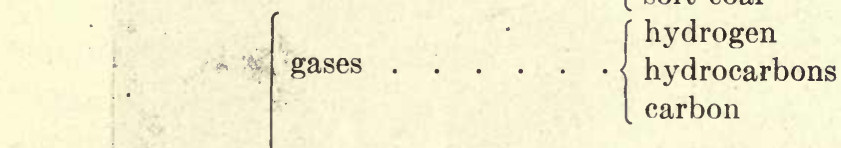

Nearly pure fuels $\left\{\right.$ liquids..$\cdot \cdot\left\{\begin{array}{l}\text { alcohols } \\ \text { hydrocarbons }\end{array}\right.$

$$
\text { solids . . }\left\{\begin{array}{l}
\text { anthracite (hard coal) } \\
\text { coke } \\
\text { charcoal }
\end{array}\right.
$$

Wood was probably the first fuel used by man. Peat came into quite general use in Europe during the middle ages and is still used to some extent (Figure 225). Soft coal was first used during the fifteenth century, while gas and hard coal were not used until the first part of the nineteenth century. Hydrocarbons in the form of gasoline and kerosene and certain artificial gases were not used for fuel until the middle of the nineteenth century, 
and alcohols are just now in the process of being developed as fuels.

Wood. - Wood is a very impure form of fuel. It contains water, resin, starch, cèllulose, gum, oil, and mineral

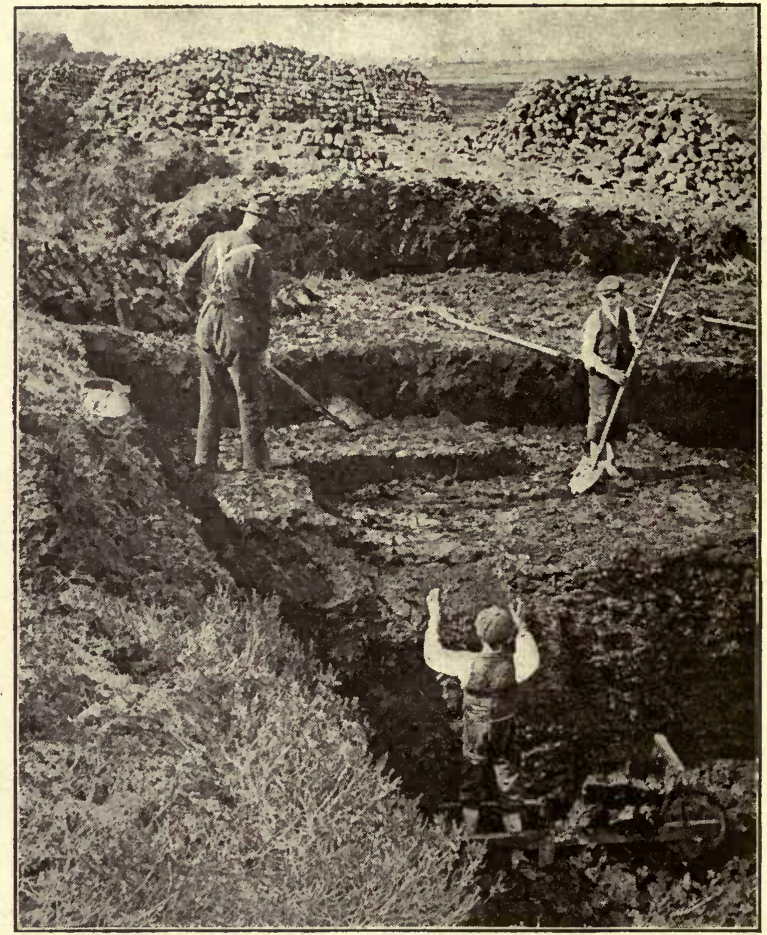

Copyright by Underwood \& Underwood, $N . Y$.

Fig. 225. - Cutting Peat, the Vegetable Substitute for Coal, Kiltoom, Roscommon, Ireland.

matter (ash). Before the wood can burn, the water must be evaporated, and the other substances contained in the wood must be raised to the temperatures at which they decompose, yielding gases of a combustible nature and charcoal. A large amount of heat is required to do 
this. The fuel efficiency of wood depends upon the relative amounts of combustible gas and carbon or charcoal furnished. Hardwood furnishes the most carbon and is therefore better for fuel than soft wood.

Coal. - If we look at soft coal through a microscope we find that it consists of a black mass of vegetable matter, such as grass, leaves, shrubs, trunks and roots of trees. Because of some change in the earth's surface, this material was covered with water and mud and thus prevented from decaying. In this condition the mass slowly changes from the carbon compounds to pure carbon. The fuel value of the coal depends upon the extent to which this change has taken place. Peat must be dried before it will burn. Bituminous, or soft coal still contains many carbon compounds and burns with a long yellow flame. Hard coal, or anthracite, in which the carbon compounds have been changed to nearly pure carbon, has no flame and can be burned only in a strong draft.

Experiment 70. - Charcoal and Coke. - Fill a long test tube one third full of dry sawdust and heat it slowly. Test the gases evolved by bringing a lighted splinter to the mouth of the test tube.

When wood is heated in a retort from which the air is excluded, several products result: inflammable gases are driven off, tarry liquids appear in

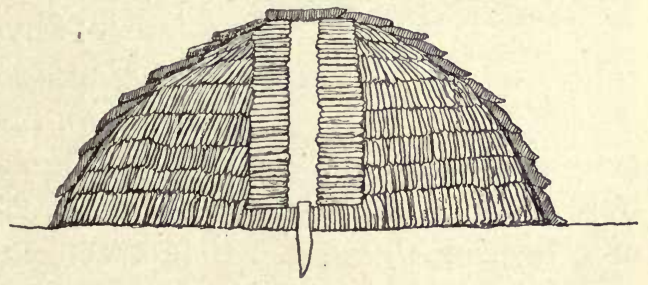

Fig. 226. - Wood Arranged for Burning into Charcoal.

the retort, and the wood changes to a porous black solid (Figure 226). In the manufacture of charcoal on a large scale the wood is heated in a large iron cylinder. The valuable liquid products are led away by tubes. Among 
these products are wood alcohol and acetic acid. Charcoal made at a low temperature is very inflammable and burns with an intense heat. It is very porous and is able to absorb many times its own volume of certain gases.

Coke bears the same relation to soft coal that charcoal does to wood. Coal is heated in a retort from which air has been excluded, until everything that has been driven off by heat has escaped. Other valuable products of the process are illuminating gas, ammonia water, benzene,

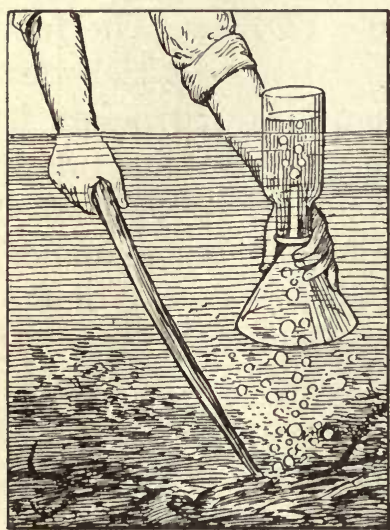

FrG. 227. - Collecting Marsh Gas. creosote, carbolic acid, pitch, and tar-camphor.

Charcoal and coke are used as fuels and in the reduction of the ores of iron and other metals. The oxide of a metal when heated with carbon decomposes; the oxygen unites with the carbon and releases the metal.

Hydrocarbons. - A compound of carbon and hydrogen is called a hydrocarbon. There are a number of hydrocarbons. Marsh gas, composed of one atom of carbon and four atoms of hydrogen, is formed in the rotting vegetable matter at the bottom of a marshy pool. Figure 227 shows a method of collecting this gas. It is a colorless, combustible gas and burns with a pale blue flame. In mines this gas is called fire damp.

In the manufacture of coke a number of gases are produced. The coal gas which we use for heating and lighting is about one third marsh gas (methane), fortyfive per cent hydrogen, some carbon monoxide, and nitro- 


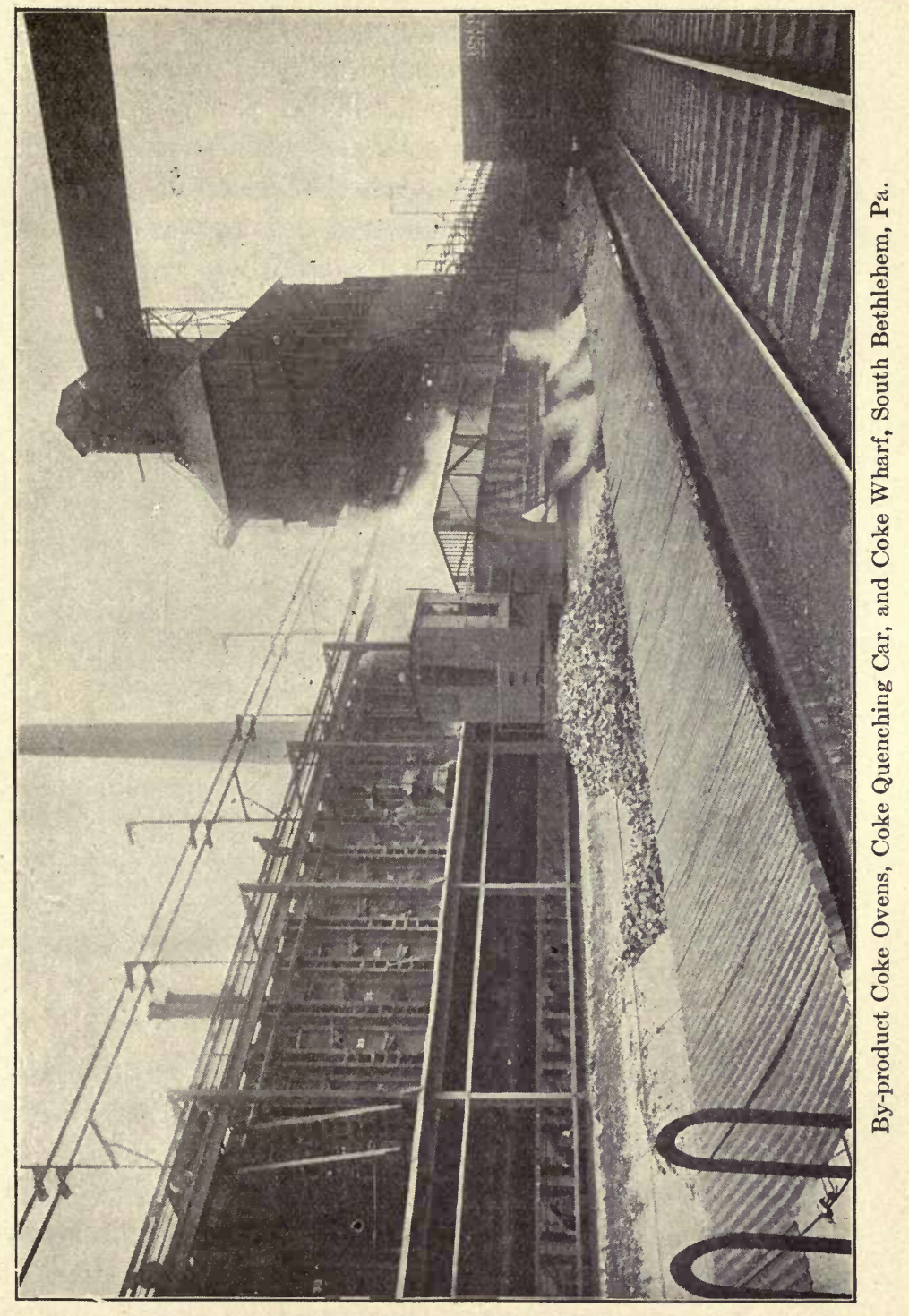



gen, and a small percentage of other hydrocarbons: acetylene, ethylene, and benzene.

Natural gas is largely used for heating and lighting purposes in certain localities and is reached by drilling. Natural gas consists mainly of hydrogen and marsh gas and is excellent for heating purposes. Since it burns with almost no flame, it has little illuminating power.

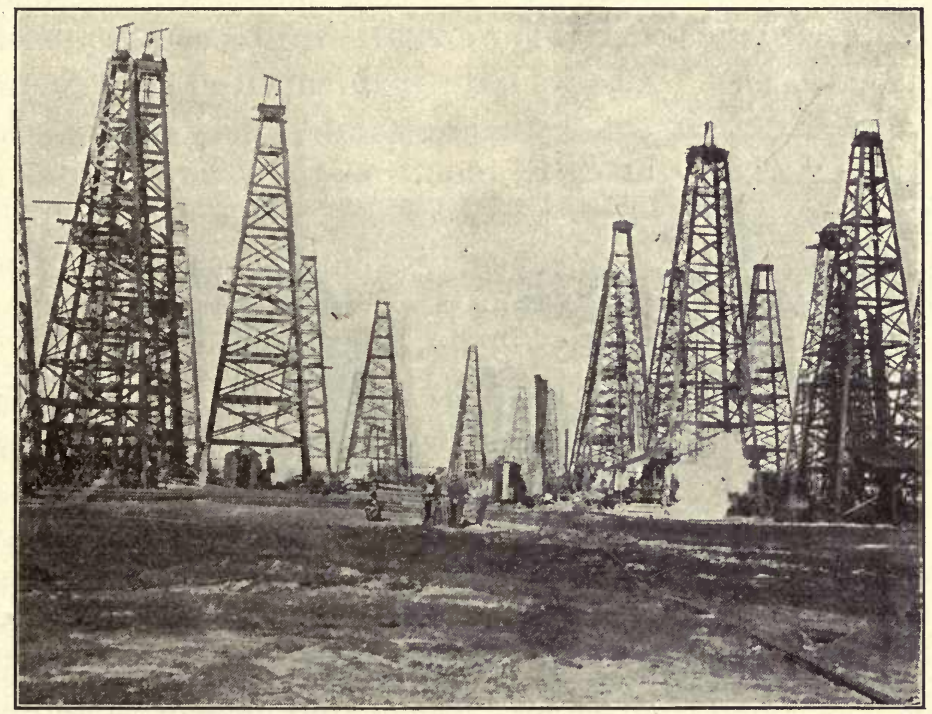

U. S. Gealogical Survey.

Fig. 228. - Oil Derricks, Beaumont, Texas.

For lighting purposes it is used to heat a mantle to incandescence.

Petroleum. - Petroleum is a dark, oily liquid obtained from oil wells in certain localities, especially in Pennsylvania, Ohio, Texas, California, and near Baku in Russia (Figure 228). All petroleums are mixtures of hydrocarbons. Nearly all the petroleum produced is refined. The 
refining of petroleum is essentially a process of distillation in which the crude oil is separated into a number of fractions which have different boiling points and compositions. Some of the products obtained are petroleum, ether, gasoline, naphtha, benzene, kerosene, paraffine, vaseline, and petroleum jelly. Of this list gasoline, naphtha, benzene, and kerosene are quite commonly used as fuels.

Flash Test. - Half fill a 200 cc. beaker with kerosene, and place over water (Figure 229). Stir constantly with

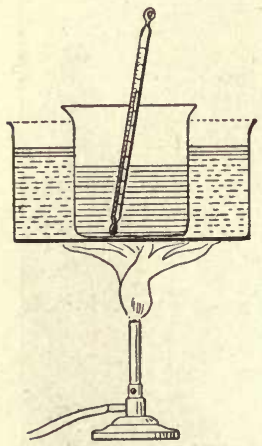

Fig. 229. - Method of Obtaining the Flashing Point of Kerosene. an accurate Fahrenheit thermometer and heat slowly until a small flame held over the mouth of the beaker causes a slight explosion and a blue flame. This is the flashing point and should not be lower than $150^{\circ}$ Fahrenheit. A lower flashing point indicates the presence of hydrocarbons which have a low boiling point, such as gasoline, and that the kerosene is not safe to use in lamps.

Alcohols. - It is quite probable that alcohol will come into common use as a fuel in the event of a scarcity of petroleum. It is composed of carbon, hydrogen, and oxygen, and has a high fuel value. Denatured alcohol is simply grain alcohol to which has been added a small quantity of wood alcohol and benzene.

Sources of Fire. - The origin of fire furnished the basis of a number of mythological tales. Just when fire was discovered is not known, for it was long before the period of authentic history. Probably friction was the first method used by primitive peoples to raise the temperature of dry wood to the kindling temperature. A hundred years ago a spark of fire was obtained by striking a 
piece of flint with steel. This spark was caught on some tinder and carefully fanned into a blaze.

The friction match, which came into use about 1827 , was a very crude invention if compared with the modern safety match, but it was far superior to the flint and steel. The first matches were made of sulphur mixed with a little potassium chlorate and antimony sulphide. This mixture was used to coat the end of a small wooden stick. When the match was rubbed over a rough surface, the

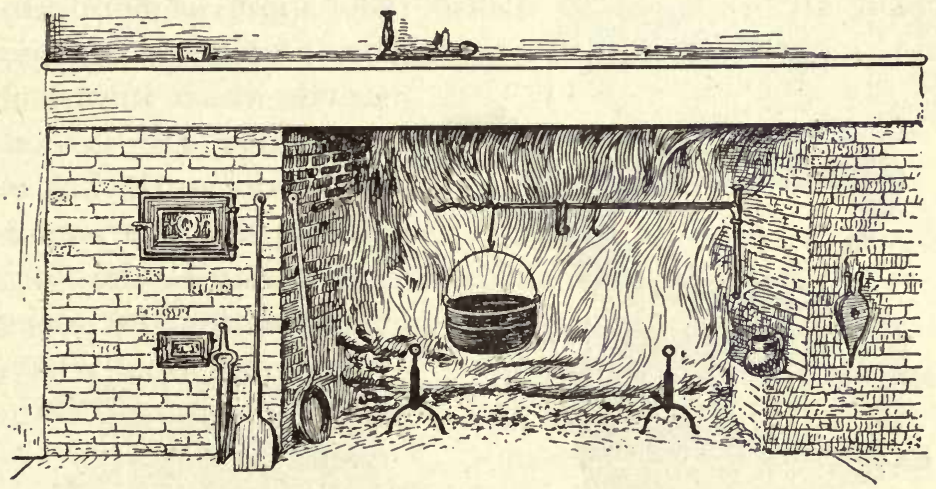

Fig. 230.-An Old-fashioned Fireplace.

friction produced enough heat to cause the sulphur to unite with the oxygen of the potassium chlorate, and the burning sulphur would ignite the wooden stick. These matches burned with a very bad odor. The match has been much improved in recent years. The safety match is now in quite common use, because it is less dangerous to use and to manufacture. Red phosphorus is used on the safety match instead of the more active yellow phosphorus and is also placed on the striking surface. The tip of the match contains antimony sulphide and some oxidizing substance, such as potassium chlorate or oxide of lead. 
Apparatus for Utilizing Fuels. - Most of the modern fireplaces are built more for ornament than for use, but it has been less than a hundred years, since the fireplace was the means of heating the home and cooking the food (Figure 230). Of course the waste of heat in the fireplace was large, but it was an excellent ventilator and furnished a cheer that is absent in the modern methods of heating.

Stoves. - There have been invented numerous stoves which are designed to utilize fuels more economically and more conveniently than the fireplace. Cooking

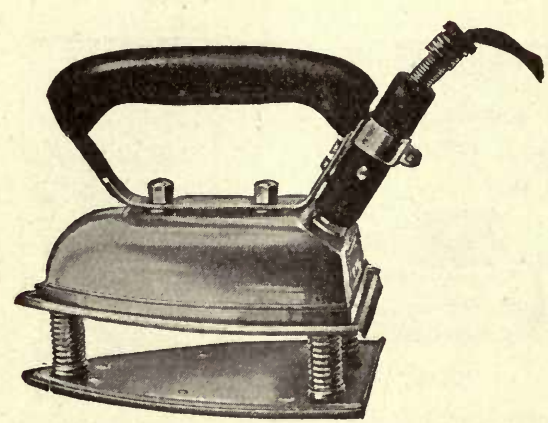

Fig. 231. - Electric Flatiron. stoves which burn coal and wood are so constructed that the heated gases pass in a roundabout way to the flues, thus heating the ovens. Gas stoves usually have special heaters for the ovens.

Gasoline stoves are really gas stoves. To

light a gasoline stove we must heat the vaporizer. This is done by burning some gasoline in a cup beneath it. When the gasoline is allowed to enter the heated vaporizer, it is changed to the gaseous form and mixed with the air which is drawn in by the force of the current of gasoline vapor. This mixture burns with a very hot flame and is much used for cooking purposes.

Electric stoves are now being used in many ways for heating and cooking and have many advantages. We now have electric toasters, coffee percolators, flatirons, 
hot-water bags, and numerous other appliances which utilize a small amount of current to apply heat exactly where it is needed (Figures 231, 232, 233).

Carbon Dioxide. - Carbon dioxide is occasionally found issuing from the ground, especially in volcanic regions, and dissolved in the water of certain springs. The charged water of the soda fountains is simply water in which a large amount of carbon dioxide has been

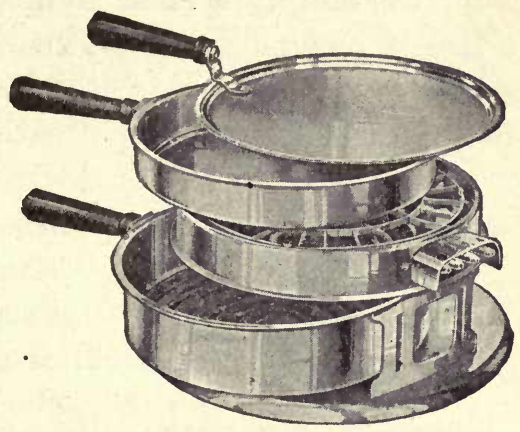

FIG. 232. - Electric Grill. dissolved under pressure. Carbon dioxide is always formed when carbon or carbon compounds burn in air or oxygen. A small amount of carbon dioxide is always

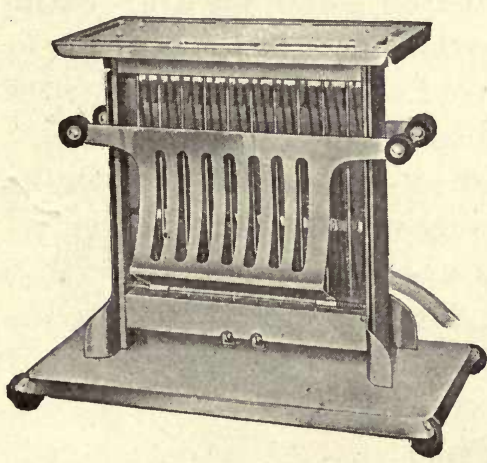

FIG. 233. - Electric Toaster. present in the air, since it is produced when any substance is burned, by the decay of all vegetable and animal matter, and by the breathing of plants and animals.

\section{Preparation of Carbon} Dioxide. - Place a few pieces of marble or limestone in a bottle (Figure 234) and pour over them a dilute solution of hydrochloric acid. The gas formed is carbon dioxide. The gas may be collected by downward displacement of air, or over water as we collected oxygen. 
Test the carbon dioxide as you tested oxygen and hydrogen to determine whether or not it will burn and support combustion. Pass some of the gas through limewater, which is simply a clear solution of slaked lime in water.

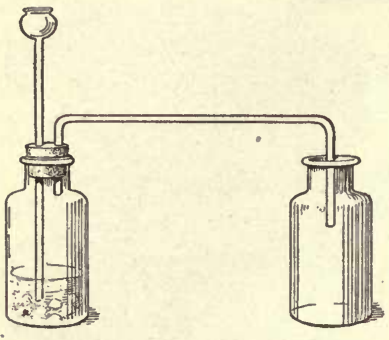

FIG. 234. - Preparation and Collection of Carbon Dioxide. The white precipitate formed is calcium carbonate. Test your breath in the same way. What does this prove about the air which is expelled from the lungs (Figure 235)?

\section{Properties and Uses of Carbon} Dioxide. - Since we can collect carbon dioxide by the downward displacement of air, we would infer that it is heavier than air and that it diffuses quite slowly. It is one and one half times as heavy as air. Since it does not support combustion, it is used in chemical fire extinguishers. Figure 236 shows such an extinguisher. The tank contains soda. When the extinguisher is inverted, the acid pours from the bottle and the large quantity of carbon dioxide made passes out through the nozzle. The heavy gas excludes the oxygen from the fire and thus extinguishes it.

Carbon dioxide does not support respiration and if breathed

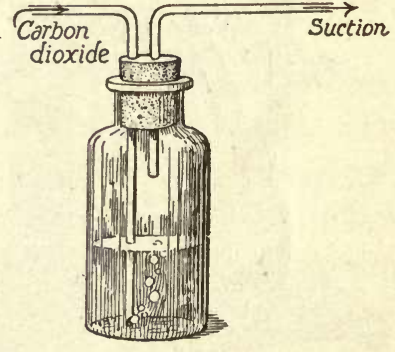

FIG. 235. in any considerable quantity will produce death, not because it is poisonous but because the necessary oxygen is excluded.

Because of its density carbon dioxide is often found at the bottom of empty wells, and in abandoned mines and 
cellars, where it is sometimes called " choke damp." Before entering such places it will be well to test for the presence of carbon dioxide by lowering a lighted candle into the cavity.

Under a pressure of 735 pounds to the square inch the gas may be liquefied at ordinary temperatures. The liquefied carbon dioxide is kept in strong cylinders from which it is taken as needed for use, as in soda fountains, by means of a valve.

Fermentation. - The fresh juice of certain fruits is sweet on account of the presence of sugar. If grape juice is heated and sealed up while hot, it will remain sweet, but when allowed to stand exposed to the air it ferments. Bubbles of gas escape and the sweet taste is replaced by the mild alcoholic taste of wine. The fermentation is caused by a plant called yeast. The spores or yeast

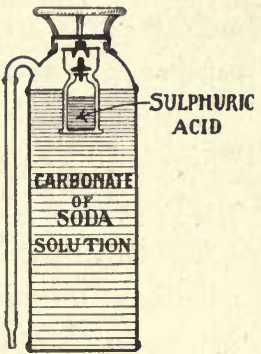

Fig. 236. - A Diagram Showing the Cross Section of a Fire Extinguisher. seeds fall into the juice and sprout, producing innumerable yeast plants. As these plants grow they change the sugar to alcohol and carbon dioxide. The gas may be tested by passing it over limewater.

When yeast is put in a mixture of flour, water, and sugar (dough), the bubbles of carbon dioxide. cause the bread to rise. The action of the yeast is arrested by the intense heat of baking.

For raising certain pastries, an artificial source of carbon dioxide is often used. This may be ordinary baking soda (sodium carbonate) used with sour milk, or baking powder. When baking soda and sour milk are used, the lactic acid of milk reacts with the baking soda in the same way that acid reacts with the marble, 
liberating carbon dioxide, which is caught by the sticky mass of dough and gives to the dough a porous structure.

Baking powder contains soda and cream of tartar. When the powder is put in moist dough, the cream of tartar acts as an acid on the soda, and carbon dioxide is formed. Acid phosphate baking powders contain acid calcium phosphate instead of cream of tartar.

Carbonates. - Other common and useful carbonates besides sodium carbonate are calcium carbonate and potassium carbonate.

Limestone is found in large quantities in the earth. When it is heated to a high temperature, it is decomposed into lime and carbon dioxide. Lime is used in many ways as the binding material in mortar and plaster.

Potassium carbonate is a white powder similar to sodium carbonate. Wood ashes were for many years the sole source of it. This is the reason it is called "potash," from which the name potassium was derived.

Potassium carbonate was once used in making soaps, but later methods use the cheaper sodium carbonate.

\section{QUESTIONS}

1. What fuel is most used in your city? Why?

2. Why is dry wood a better fuel than green wood?

3. Why is hard pine a good fuel wood?

4. The flashing point of a sample of kerosene is $150^{\circ}$ Fahrenheit. What will it be Centigrade?

5. Why does the carbon dioxide in soda water come to the surface when the water is drawn?

6. How can you prove that carbon dioxide will not support combustion?

7. Should the ventilator in a hot-air system of heating be placed at the top or bottom of a room? Why?

8. How are the flues in a cook stove arranged to provide for heating the oven? 
9. What kind of a stove does alcohol require?

10. Can alcohol be vaporized and burned like gasoline vapor?

11. What " cautions" should be observed in using a gasoline stove?

12. Is electricity an efficient source of heat? Why?

13. What is the price of artificial gas' in your city? Of electricity?

14. What are the advantages of gas over coal as a fuel for the cook stove?

15. Name three uses of carbon dioxide.

16. When acid (hydrochloric acid) is poured on calcium carbonate, what products are formed?

17. What is the purpose of raising dough?

18. When an explosion of "fire damp" occurs in a mine, "choke damp" is formed. Explain how.

19. What causes cider to "work" ? 


\section{CHAPTER XVI}

\section{COMMON COMPOUNDS OF OTHER ELEMENTS}

Classes of Compounds. - In this chapter we shall deal only with a few classes of compounds whose names are in quite common use. We have already used the terms oxide and acid a number of times in this book. These are two of the four large classes of compounds. The other two are bases and salts.

Oxides. - Because of its importance we are accustomed in our preliminary study of oxygen to think that most of it is in the air, but we soon learn that this is not the case. Eight ninths of the weight of water is oxygen, but even water contains but a small part of the total amount of oxygen in the earth. Water is an oxide as is carbon dioxide. Oxygen unites with many elements to form oxides.

Acids. - An acid is a hydrogen compound whose dilute water solution usually has a sour taste. It turns a blue litmus solution red. Litmus is a blue dye which is obtained from a kind of moss. It readily dissolves in water, and when a drop of acid is added to the resulting blue liquid, the color changes to red.

A number of acids react chemically with metals, giving off hydrogen. For example, when hydrochloric acid and zinc are brought together, zinc chloride is formed and large quantities of hydrogen are produced. Copper vessels should not be used for cooking foods which contain acids, even though they may be very inactive acids, since 
poisonous compounds of copper may be formed. The most common acids are sulphuric, hydrochloric, and nitric. These are called inorganic or mineral acids, and are manufactured in large quantities for commercial uses. Sulphuric acid is quite cheap and is used in the manufacture of other acids. Hydrochloric acid contains hydrogen and chlorine and is made by the action of sulphuric acid on salt (sodium chloride).

Another large class of acids is formed in the juices of fruit and vegetables. Among these are acetic acid, citric acid, and tartaric acid. (All of these acids contain carbon, hydrogen, and oxygen.) They are called organic acids.

When sweet fruit juices ferment, their sugar is changed to alcohol and carbon dioxide by the yeast plants. If the alcohol thus formed comes into contact with air, it changes to vinegar. While this change

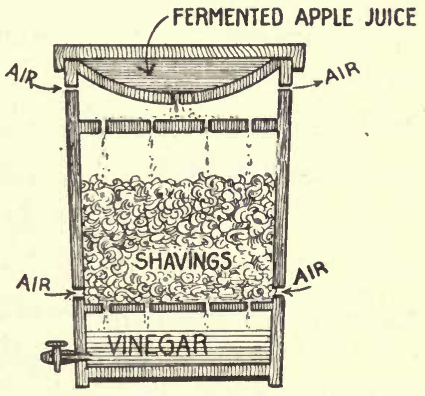

FIG. 237. from alcohol to vinegar is going on the liquid contains numerous small organisms called acetic acid bacteria. The large numbers of these bacteria form the characteristic mold known as " mother of vinegar." The sour taste of vinegar is due to the acetic acid present. In the vinegar sold for table purposes the amount of acetic acid is usually not more than four or five per cent. In the formation of vinegar the alcohol is really oxidized to acetic acid. This action is usually slow, but may be hastened by the quick - vinegar process. This is an arrangement whereby fermented apple juice is allowed to drip slowly through beechwood shavings which have been inoculated with acetic acid 
bacteria by previously wetting them with vinegar. The shavings are held in a barrel (Figure 237) having a number of holes cut in it to admit a plentiful supply of air. The change from alcohol to vinegar in such an apparatus takes place in a few minutes.

Uses of Acids. - Acids have many commercial uses. Shortly after the great European war broke out the prices of inorganic acids rose perceptibly. Both nitric and sulphuric acids are used in the manufacture of many high explosives such as nitro-glycerine, nitro-cellulose, and guncotton.

In addition to its many direct commercial uses, sulphuric acid is used in the manufacture of other acids. With the present high prices, all the old sulphuric acid plants, even those using obsolete methods of manufacture, have been placed in operation and have proved very profitable. The world's annual production of sulphuric acid is several million tons. Hydrochloric acid is now made on a large scale by the action of sulphuric acid on Chile saltpeter.

Alkalies and Bases. - A base is a substance which has a number of properties just the opposites of the properties of acids. Their solutions reverse the color changes produced by acids on sensitive dyestuffs. Litmus paper or litmus solutions which have been colored red by acids are changed back to blue by basic solutions. Soluble bases have a bitter taste quite different from the sour taste of acids.

Common bases are sodium hydroxide (caustic soda), potassium hydroxide (caustic potash), ammonium hydroxide, and calcium hydroxide. It will be noticed that the chemical term hydroxide occurs in each of the above names. This means that oxygen and hydrogen are 
combined with the different substances - sodium, potassium, ammonium, and calcium to form the base. The name alkalies is commonly applied to the first two bases mentioned above. Alkalies are simply the more soluble members of the class of substances called bases. Any basic reaction may be called an alkaline reaction. Strong alkaline solutions are "soapy" to the touch. Because of their action on grease and fats, alkalies are used in the manufacture of soaps, for cleaning, and for flushing partially clogged waste pipes leading from sinks.

Salts. - When an acid and a base are brought together in the right proportions, the characteristic properties of each disappear. The hydrogen and the oxygen of the base unite with the hydrogen of the acid to form water, while the residues of both unite to form a salt. For example:

Sodium hydroxide, a compound of sodium, hydrogen, and oxygen, reacts with

hydrochloric acid, a compound of hydrogen and chlorine, to give

water and common salt (sodium chloride).

Experiment 7I. - Add some hydrochloric acid to a water solution of litmus. Now add sodium hydroxide solution drop by drop until the red color disappears, leaving the solution white. The acid and the hydroxide have neutralized each other. Evaporate the solution to dryness and there will be a residue of white crystals, the properties of which show it to be common salt.

Uses of Salts. - There are so many different salts that a thorough discussion of their uses would involve the whole subject of chemistry. However, a few common salts and their uses may be mentioned with profit.

Copper sulphate, "blue vitriol," is a blue salt having a number of uses. It is used in the gravity batteries of the telegraph systems. The farmer has also discovered 
a number of uses for copper sulphate. For the prevention of "smut" in wheat the seeds are wet with a weak solution of it before sowing.

"Bordeaux mixture," composed of a mixture of copper sulphate and lime in water, is widely used as a fungicide; other sulphates are zinc sulphate, "white vitriol "; iron sulphate, "copperas" or "green vitriol"; potassium sulphate, used as a fertilizer for plants; magnesium sulphate, "Epsom Salt"; and ammonium sulphate, which is used in large quantities as a fertilizer to supply nitrogen to crops. The use of sodium chloride is well known.

Silver chloride and silver bromide are used in photography, since they are chemically affected by light.

Silver nitrate is used in the manufacture of photographic films and plates to produce the sensitive chloride of silver. It is also used in the indelible marking of linens, since the organic matter of cloth or skin converts the nitrate to silver and produces the black stain.

Ammonium nitrate is used in the manufacture of explosives.

Calcium phosphate, which occurs as phosphate rock in South Carolina, Tennessee, Florida, Idaho, Utah, Montana, and Wyoming, is used in the manufacture of fertilizers. More than a million tons of this rock are being used each year. To render the calcium phosphate more active it is treated with sulphuric acid, which converts the whole mass into the very soluble calcium-hydrogen phosphate, and calcium sulphate. The mixture is sold for fertilizer as "superphosphate."

Electrolytes. - The process of decomposing a compound substance by means of an electric current is called electrolysis. The substance decomposed is called an electro- 
lyte. Water solutions of acids, bases, and salts are all conductors of electricity; and in each case the substance in solution (solute) is decomposed by the electric current. The decomposition always results in two parts, one of which is carried to the positive electrode while the other is carried to the negative electrode. For example, the electrolysis of acids always gives hydrogen at the negative electrode and the remainder of the compound at the positive electrode. Acids, bases, and salts are the only substances which in water solutions are electrolytes.

A simple apparatus for showing the conductivity of electrolytes is shown in Figure 238. The lamp, which should be a small one, is placed in series with the platinum electrodes. When a conductor of electricity is placed in the jar and the connection is made with a battery of sufficient size, the lamp will glow. If only a few

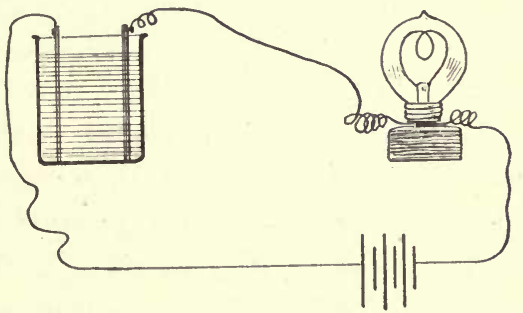

FIG. 238. cells are available, a telephone receiver may be substituted for the lamp. When the circuit is made or broken, the receiver will click. In this way test the conductivity of dry salts.

Analysis of Chemicals. - It is often quite important to determine just what elements or compounds are present in certain substances. The agricultural chemist analyzes soils to determine what crops they are best suited to, and what fertilizers are needed. Health officers analyze foods of all sorts to determine their food value and also to guard the public against harmful substances which are occasionally found in them. The geologist analyzes 
rocks and ores to determine their composition and value. To-day nearly all mining companies and manufacturing companies maintain their own laboratories for examining the materials in which they are interested.

To "test" a substance for the purpose of finding out whether a certain element or compound is present or not we simply make an examination of the substance, neglecting so far as possible other elements or compounds present. No two substances have exactly the same properties, and so "tests" may be devised for distinguishing any certain substance.

We may test for acids as a class by using litmus paper or litmus solution, but we shall not be able by this test to tell whether the acid present is hydrochloric, sulphuric, or any one of a number of other acids.

In testing a salt we need to determine: $(a)$ what metal is present and $(b)$ what acid was used in producing it. If sulphuric acid was used, we call the salt a sulphate; if hydrochloric acid was used, a chloride; if nitric acid was used, a nitrate ; if phosphoric acid was used, a phosphate ; and so on.

Iron. - If solutions containing iron salts are heated with a little concentrated nitric acid and boiled for a few minutes, and a few drops of potassium thiocyanate are added, a beautiful blood-red solution results.

Copper. - If copper is present in a solution, the addition of ammonia gives a deep blue color.

Sodium. - Sodium salts give a bright yellow color when held in a colorless gas flame.

Sulphates. - To test for sulphate salts, we add dilute nitric acid to a water solution of the salt and then add a few drops of barium chloride solution. If the salt is a sulphate, there will be a white precipitate formed. 
Chlorides. - To test for a chloride, add a few drops of nitric acid to a water solution of the salt and then add a few drops of silver nitrate solution. If the salt used is a chloride, there will be a white precipitate of silver chloride formed. When exposed to the sunlight, this precipitate turns black.

\section{QUESTIONS}

1. Name four substances which are not combustible.

2. Why does a substance which is completely oxidized not burn?

3. Which is the most important acid? Why?

4. In case an acid is spilled on clothing, what would you use to neutralize the acid?

5. Sometimes solids will show an acid reaction. What common substance would you suggest to neutralize the acid?

6. Common salt is a compound of sodium and chlorine. Suggest a way to separate the sodium from the chlorine. 


\section{CHAPTER XVII}

\section{SOILS}

The soil is not unlike many other things of this world; it needs to be understood in order to be appreciated. Contrary to the opinion quite commonly held, soil is not dirt in the sense of the word that it is something to be

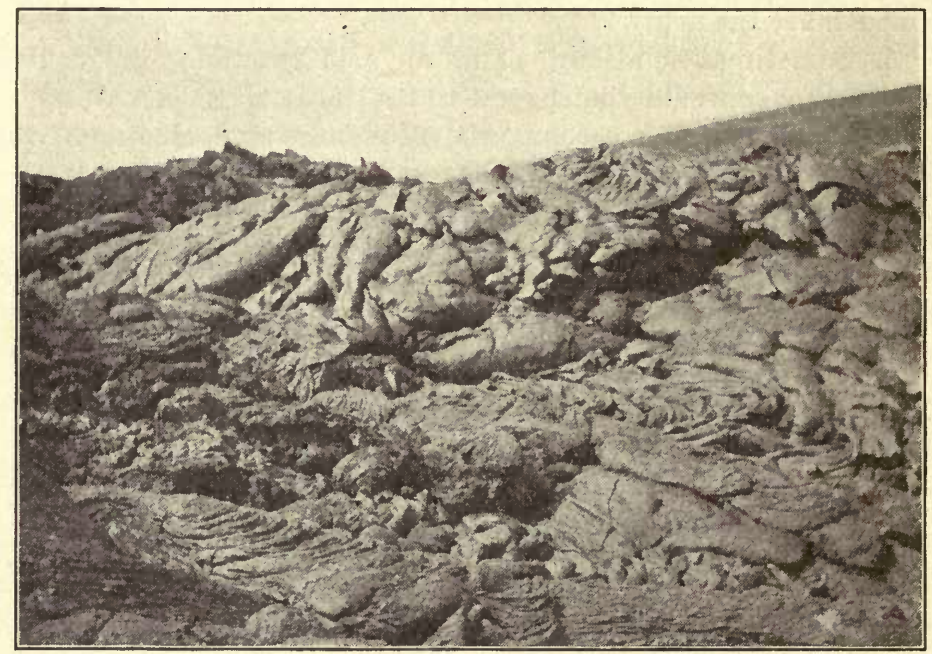

FIG. 239. - Igneous Rock.

U. S. Geological Survey.

Characteristic lava flow, Kilauea, Hawaii.

avoided. . Nearly everything that we eat and wear comes either directly or indirectly from the soil. We are dependent upon the soil for our very existence, and we should have a wholesome respect for the tillers of the soil. With 
the development of the science of agriculture a man is able to produce much more on an acre of ground than formerly. The surplus that he does not need for his own uses is exchanged for the manufactured products of his city brother, and both the manufacturer and the farmer profit thereby.

The Crust of the Earth. - The crust of the earth varies greatly in composition. Many different kinds of rocks

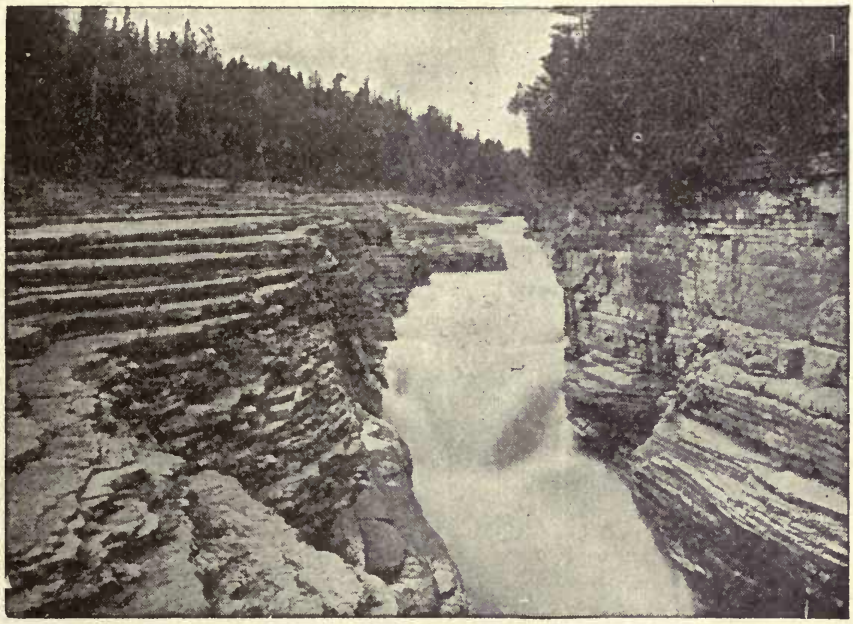

Frg. 240. - Sedimentary Rock.

Horizontal strata near Quebec, Canada, showing layers of sediment.

are found on or near the surface of the earth. These are covered with a variety of soils, the soil usually containing in each case the same elements and compounds as the rocks below it.

The rocks of the earth's crust are divided into three classes: igneous, sedimentary, and metamorphic, in accordance with the manner of their formation.

Igneous rocks are those which at some time in their 
history have been solidified from a melted condition. If on solidifying the cooling has been slow, these rocks will have a crystalline structure such as is found in marble. Lava is a form of igneous rock (Figure 239).

Sedimentary rocks are those formed by the deposition of sand, gravel, clay, and other materials, by water. The

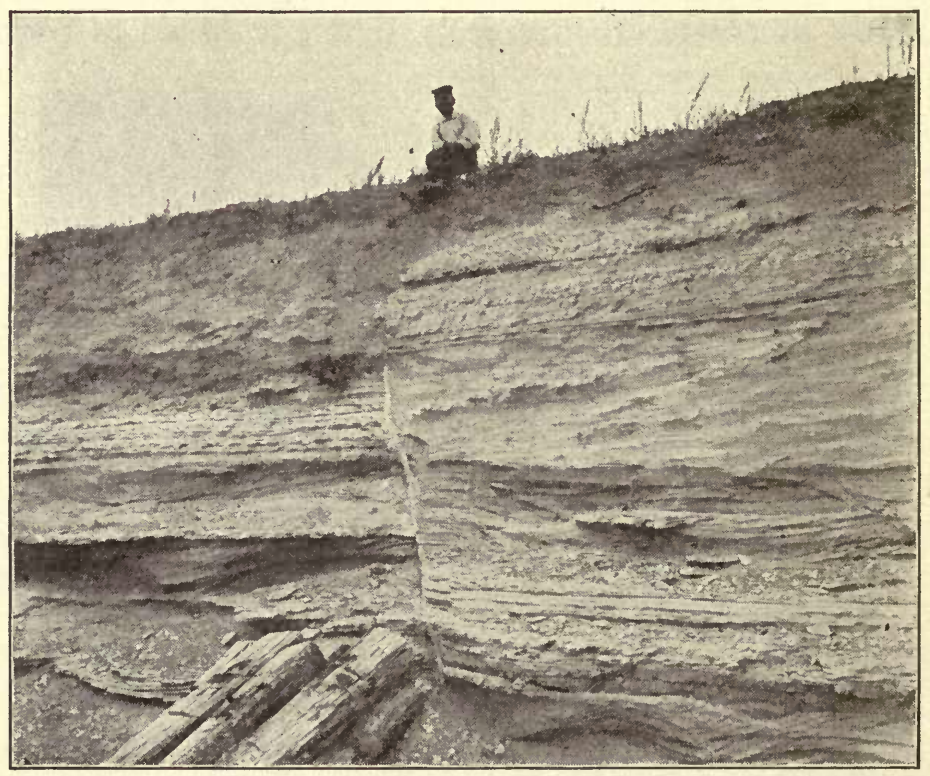

FIG. 241. - Sedimentary Rock.

U.S. Geological Survey.

Showing a fault in rock formation, Crawford, Nebraska.

gravel, sand, or clay is washed from the lands into the ocean and deposited on the ocean floor; the finest particles being carried farthest from the shore. In the course of ages these layers of sediment are changed into solid rock (Figures 240-241). Sandstone and limestone are classes of sedimentary rocks. Sandstone is formed by particles 
of sand cemented together, while limestone is made from the remains of dead sea animals (Figure 242).

Metamorphic rocks are modified forms of either igneous or sedimentary rocks. Heat and pressure are the agents which produce these changes (Figure 243).

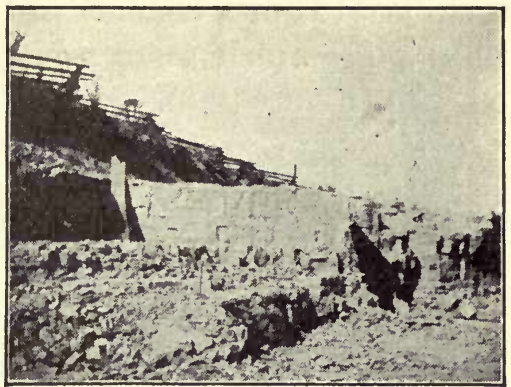

Fig. 242. - Limestone, Logan County, Ohio.

The surface of the earth differs from place to place due to the variety of positions in which these rocks are placed.

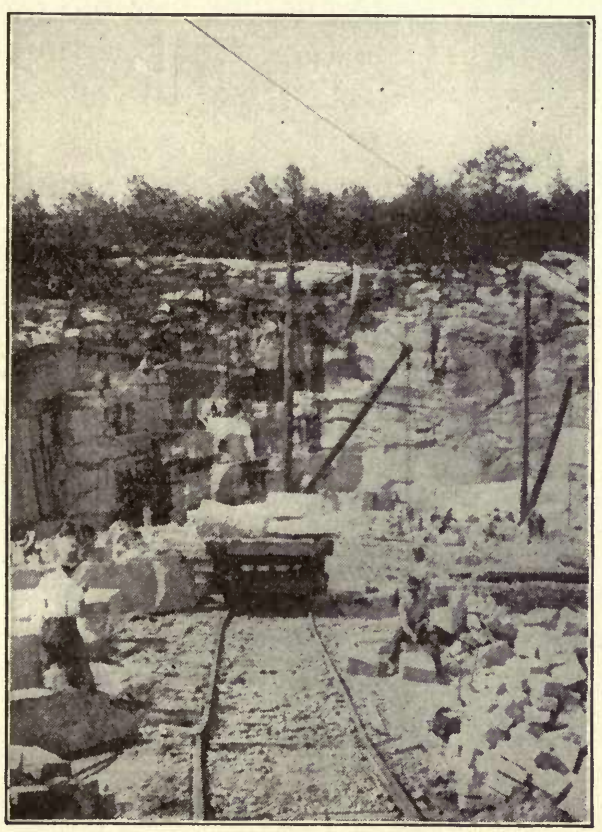

Frg. 243. - Metamorphic Rock. Granite quarries, Concord, N. H.
In some regions the surface is folded into mountains, in other regions there are deep basins filled with the water of the oceans.

Weathering. The changes in the earth's surface which are due to atmospheric conditions are called weathering. Rocks which are exposed to the atmosphere are gradually decomposed. The rate of the decomposition depends upon the climate. If the climate is moist and 
is subject to quick changes in temperature, the weathering takes place much more rapidly than in a warm and dry climate (Figure 244).

Experiment 72. - Fill a small bottle with water, cork tightly, and place in a freezing mixture. What is the effect upon the bottle? What would be the effect if water in the pores or cracks of a rock should freeze?

Some rocks weather much more rapidly than others. It is interesting to note the changes that take place on

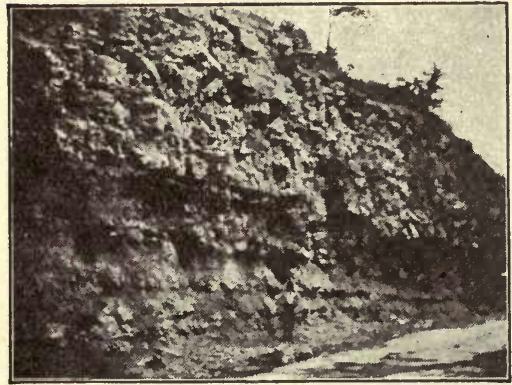

FIG. 244. - Exposed Shales, Showing Effects of Weathering. the tombstones due to weathering. In the eastern half of the United States many of the inscriptions are illegible on tombstones that have been standing for 50 or 60 years.

The uneven contraction and expansion of rocks due to the summer heat sometimes causes them to chip and crack. The roots of plants penetrate the crevices of rocks and split them apart, as they grow in size. Water at times may acquire acid properties in passing through decaying vegetable material, which makes it a solvent for some of the materials of the rock.

How Soil Is Made. - If we examine the soil in any field, we find that there is a dark layer of humus on top (Figure 245). The richness of the soil may be quite accurately estimated from the depth of this layer of dark earth. The deeper the layer of dark earth the richer the soil. The dark color of this layer is caused by the decayed plants it contains. Plants turn black as they rot. As we go 


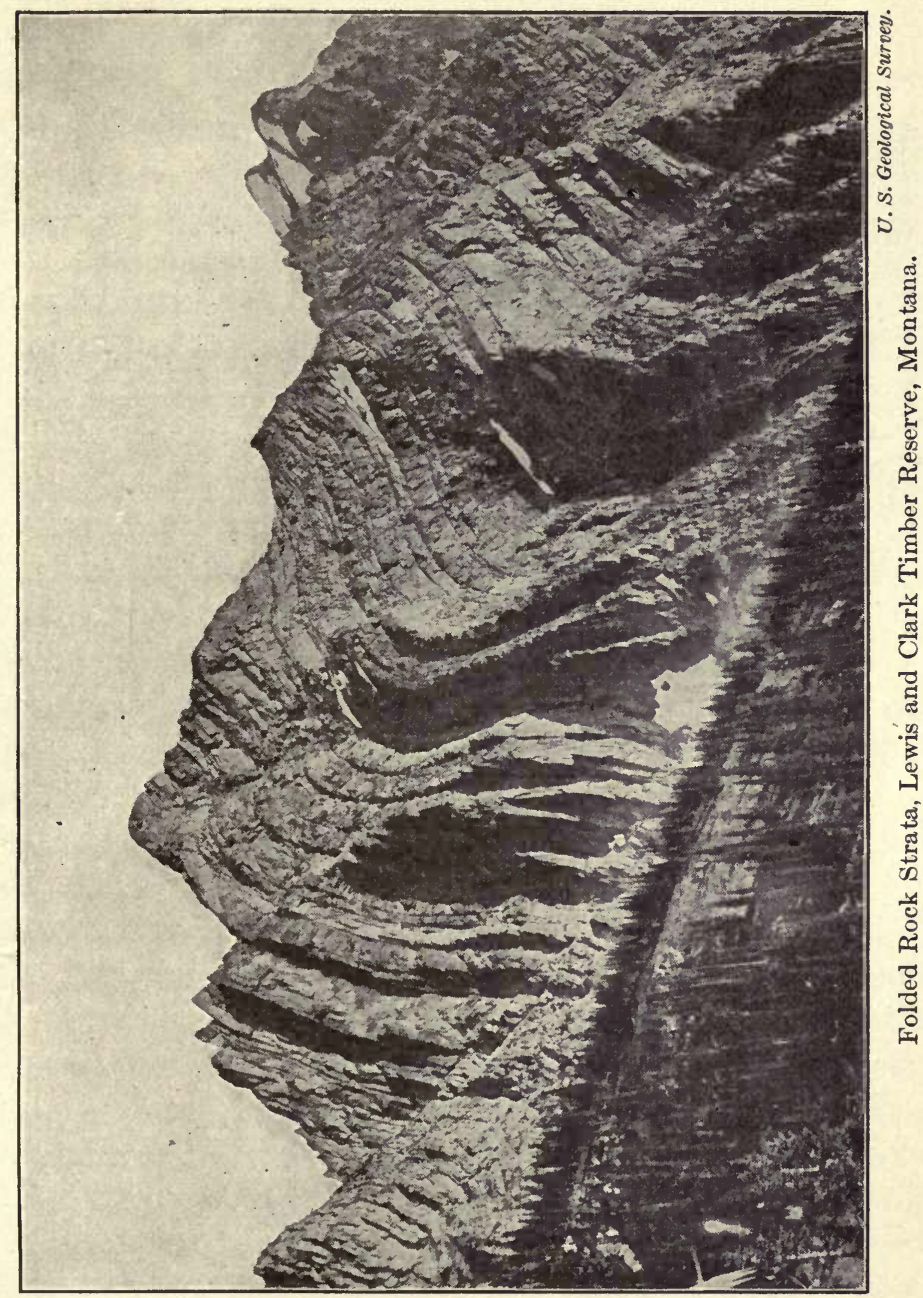



downward the color gradually changes to a lighter brown, and still deeper the stain of the decaying plants is no longer seen; the soil becomes harder and harder until we finally reach the solid rock.

This is the common sort of soil found all over the world. It is made by the gradual decay of the rock (Figure 246). If we should remove all the soil from an area of rock, it would immediately

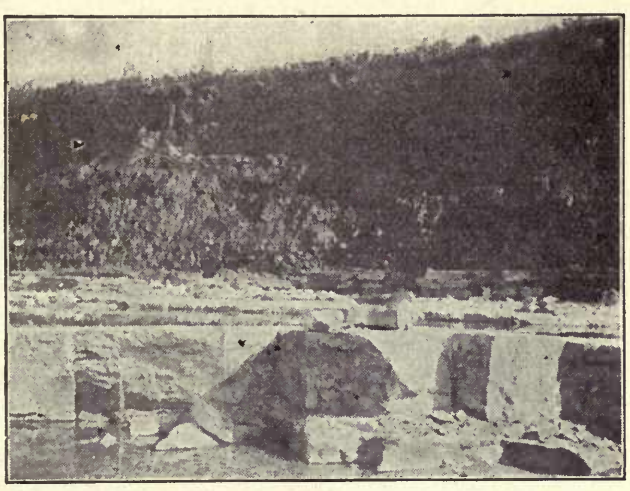

FIG. 245. - Showing Soil Above the Shale, then Solid Rock Below.

begin to form again. After a few years' exposure to the atmosphere, the rock would be decayed enough so that lichens and certain mosses would find a place to grow. They would aid in keeping the surface of the rock moist and thus aid weathering. The decay of these simple plants would produce a little humus, and soon the seeds of other larger plants would germinate and grow there. The decay of these plants gradually. adds to the layer of mold on the surface of the rock until the largest trees may find a place to grow. The atmosphere, the water, the decay of plants, the roots of the plants, and other minor agencies are all at work now deepening and enriching the soil. Of course this change may take centuries, but when compared with the enormous age of the earth it seems a very short time.

Glaciated Soil. - In the northern portions of our conti- 
nent the glaciers of the ice age had considerable to do with the soils of certain sections. As these great fields of ice moved south they carried great quantities of material from the old mountain chains of Southern Canada. This rich material was distributed over the northern

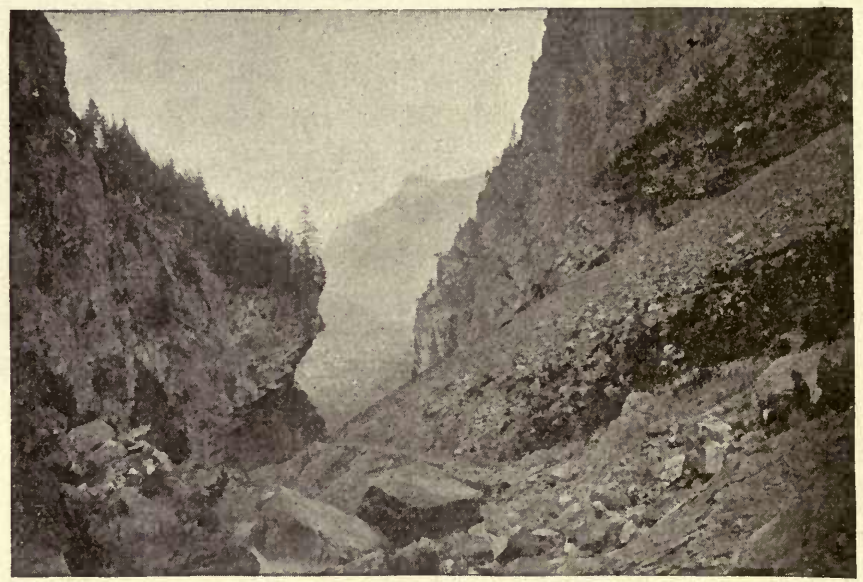

Fig. 246. - Mountain Disintegration in the Alps.

The accumulated debris on the right is rock in process of decay.

part of the United States to be ground finer and redistributed with each return of the glacier (Figure 247). Many regions owe the fertility of their soils largely to the work done by the great seas of ice.

Composition of Soils. - The wealth of our agricultural communities lies in the fertility of their soils. An agricultural soil is made of (a) decayed rock, (b) soil water, (c) soil air, $(d)$ decaying organic matter, (e) living organisms. In general we may say that fertile soils have all of the above constituents ; however, drained swamp lands (muck soils) are usually very fertile although deficient in rock particles. 
The richness of the soil will first depend upon the composition of the underlying rock and next upon the action of the plants which yield the organic matter.

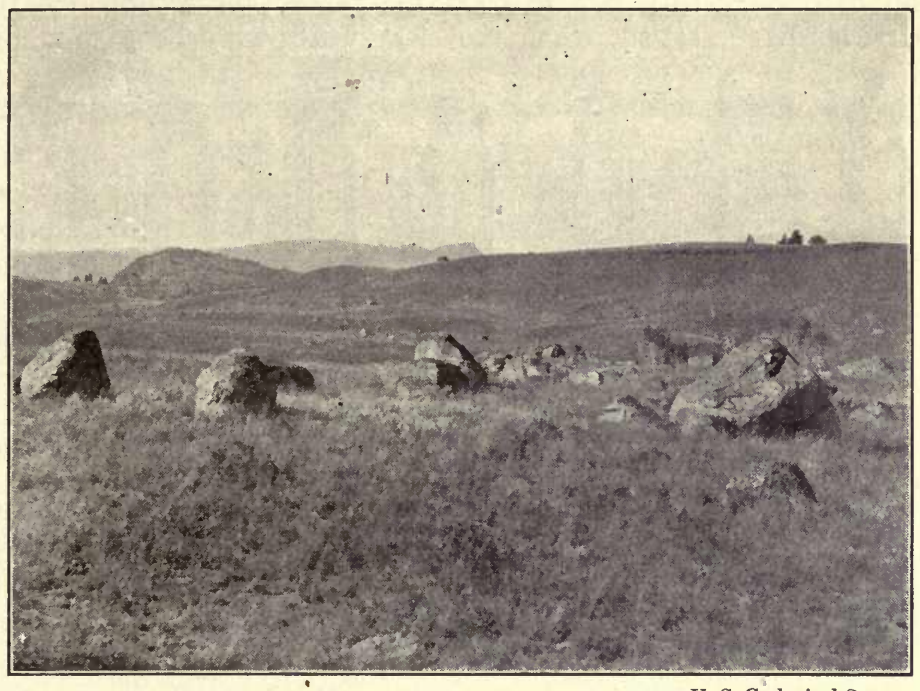

FIg. 247. - Erratic Bowlders.

U. S. Geological Surney.

These bowlders have been deposited by the glacial stream.

In most soils the rock particles predominate, making up from 60 per cent to 95 per cent of the total weight. From two to five per cent is organic matter, and most of the remainder is water.

Experiment 73. Shake a quantity of soil with water in a bottle (Figure 248), until all the particles of the soil are broken

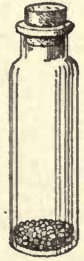

coarse sand

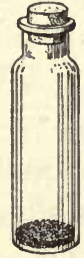

medium sand

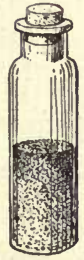

fine sand

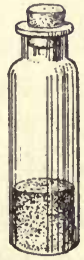

very fine sand

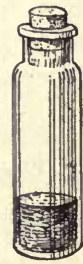

silt

FIG. 248. 
up and in a state of suspension in the water. Allow the mixture to settle for five minutes and then pour the roily water into another bottle, leaving the particles which have already settled in the first bottle. After five more minutes again pour off the roily water. Do this five times, leaving the water in the last bottle until all the particles have settled, when the clear water may be poured off.
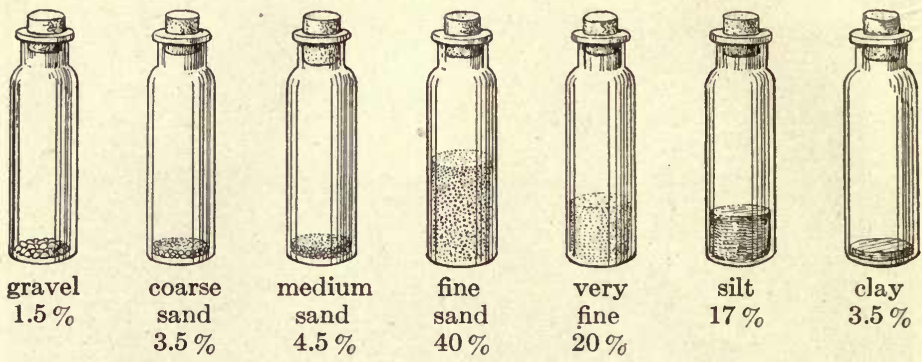

Fig. 249.

Compare the size of the particles in the different bottles. As many divisions as desired may be made in this way. Figure 249 shows the composition of sandy loam, which is an excellent soil.

\section{Table Showing Mechanical Analysis of Soils. -}

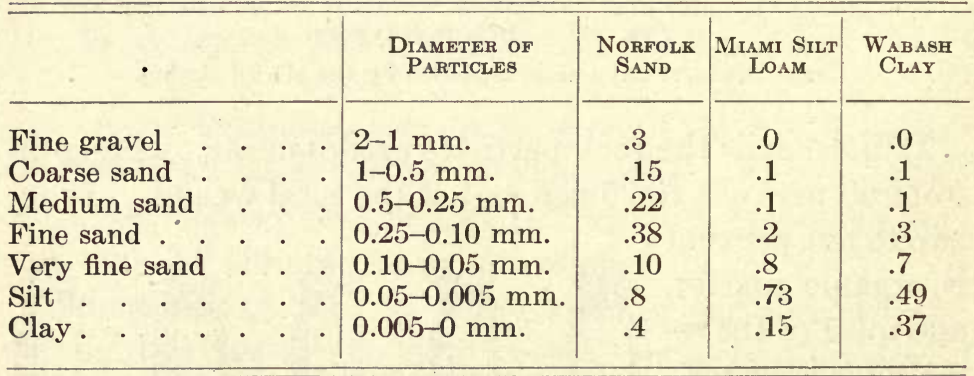

Names of Soils. - Clay soils are those which contain a large proportion of the smallest particles, while those that contain a large proportion of sand are simply called sandy soils or sands. Loams are intermediate mixtures of sand and fine particles. 
Norfolk sand is one of the common soils of the Atlantic flood plain. Miami silt loam is found in the corn belt of the central west. Wabash clay occurs along most of the river bottoms of the Mississippi valley.

Experiment 74. - Arrange several lamp chimneys as shown in Figure 250 each containing a different kind of soil. Pour water in the pan, and after allowing it to stand for an hour note the height of the water in each chimney. The capillary action of each soil may be determined in this way.

Importance of Size of Soil Particles. - The waterholding power of soils depends largely upon the

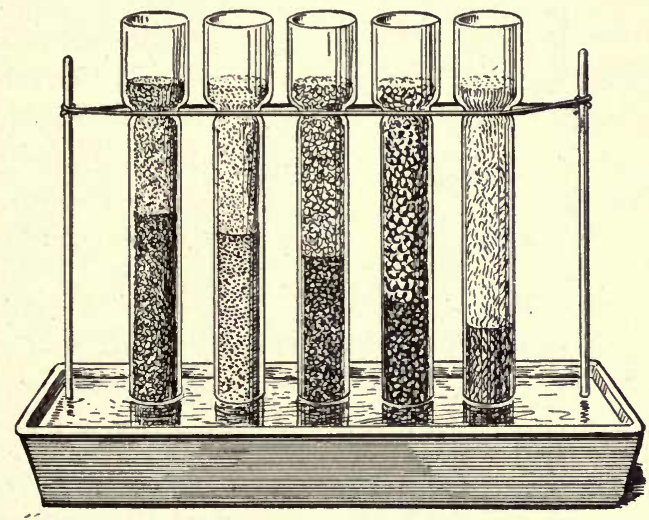

FIG. 250. surface area of the particles composing the soil. The finer the particles the greater will be the amount of water that this kind of soil will hold. The finest soil particles are less than one-thousandth of a millimeter in diameter. The total surface area of a cubic foot of such a soil would be enormous. Such soils have a high water capacity; that is, they will still hold a large amount of water after the free water has been allowed to drain out, Clay soils have the highest water capacity, sometimes as high as forty per cent; and sandy soils have the lowest water capacity: In addition to influencing the water capacity of soils, the size of the particles also affects the movements of the soil air, the amount of food that can be 
dissolved for plant use, the growth of soil organisms, and the temperature of the soil.

Experiment 75. - Close the bottom of a tall, straight lamp chimney and fill it with soil, then pour it full of water. If a hole is now made in the stopper (Figure 251), a considerable portion of the water will drain out. This is called gravitational water. Why? If the hole at the bottom is again closed and the apparatus is

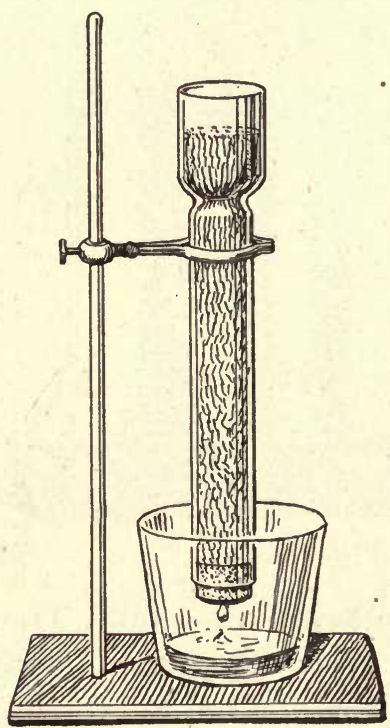

Fig. 251. allowed to stand for some time, the soil will become quite dry, since the water in the bottom will rise by the action of capillarity to replace that lost at the surface by evaporation. The water lost in this way is called capillary water. If some of this dry soil is weighed and then heated to a temperature of $100^{\circ} \mathrm{C}$., it will lose still more water. This is called hygroscopic water and is so strongly held in the soil that it is not available for plant use.

Size of Soil Particles in Relation to Temperature and Crops. - We have learned that evaporation of water lowers the temperature of the surrounding objects; therefore that soil will be coldest from which the most water evaporates. Wet soils are always cold soils. Clay soils are colder than sandy soils for this reason. They have a larger water capacity and hence furnish more water for evaporation. An additional reason for the coldness of wet soils is that the heat capacity of dry soil is but two tenths that of water; that is, it takes five times as much heat to warm a gram of water through one degree as it does to warm a gram of dry soil through one degree. Hence the sun's heat will warm the drier soil more rapidly. 
Plant food must be in the form of solutions. Since the water touches more surface in a given volume of soil when the particles are small than when they are large, it will be able to dissolve and furnish plant food more readily in the finer soils. On the other hand, the coarser soils will be better aërated, due to the fact that the spaces between the particles are larger so that the air moves more freely.

All of these things affect the crops that grow in the soils. Sandy soils which are well adapted for corn, potatoes, and market gardening are not well adapted for wheat, which may be grown to advantage on the heavier clay soil. The successful farmer will soon learn what crops are suited to his land and cease cultivating those that are not.

Conservation of the Soil. - The study of the soil and its products will soon convince us that it is quite important to care properly for this great gift to man. It has required ages for the soils to be formed and reach a certain productive stage, but it requires but a few years of careless farming to reduce them to a non-paying productive basis. The population of the United States is increasing rapidly, and this means that the demands on the soil are increasing proportionately. It is not possible to harvest crops year after year and put nothing back on the land without decreasing its fertility. Certain crops take certain kinds of food from the soil with the inevitable result that the amount of this particular food in the soil is lessened.

Measure of Soil Values. - A soil from the agricultural standpoint is measured by the abundance of its scarcest element. There are five substances in which our soils are likely to be deficient : water, nitrogen, phosphoric acid, potash, and lime. These foods are needed by different crops in varying quantities. If the soil is deficient in phosphoric acid, the addition of phosphoric acid will 
increase the crop. Suppose that the average crop of corn in a certain kind of soil is 80 bushels to the acre, but that the supply of nitrogen is so small as to limit the crop to 60 bushels to the acre, and that the supply of potash limits the crop to 50 bushels to the acre. Then of course the crop will be 50 bushels, if there are no other deficiencies. In such a case the problem is to determine what the soil lacks and then add these materials.

A soil may decrease in productivity from a number of causes.

1. It may lose its power to hold water. This may be remedied by tile drainage and the addition of humus.

2. The supply of available plant food may be exhausted by raising the same crops year after year. This may be remedied by the addition of fertilizers and by drainage.

3. The soil may become acid and unfavorable for the growth of soil organisms. In such cases an application of lime is beneficial.

The fertility of soils depends largely on the humus they contain. Humus is simply decayed organic matter. Humus in soil increases its capacity for holding water, furnishes nitrogen by its decomposition, and gives an acidity to water which makes it a better solvent for other plant foods. The farmer should never lose an opportunity to add humus to his soil, since it not only contains plant food elements but renders available much of the food that is already in the soil. Decayed vegetable matter and manures are excellent sources of humus.

Fertilizers. - It is quite a common idea that soils may be analyzed to determine what fertilizers should be used on them. In general this is true; however, the chemical analysis only shows the amounts of different substances present and not the amounts of available foods. Experi- 
ment is the most reliable way of determining what additions should be made to the land in the form of fertilizers.

Barnyard manure and growing plants plowed under are the best kinds of fertilizers (Figure 252).

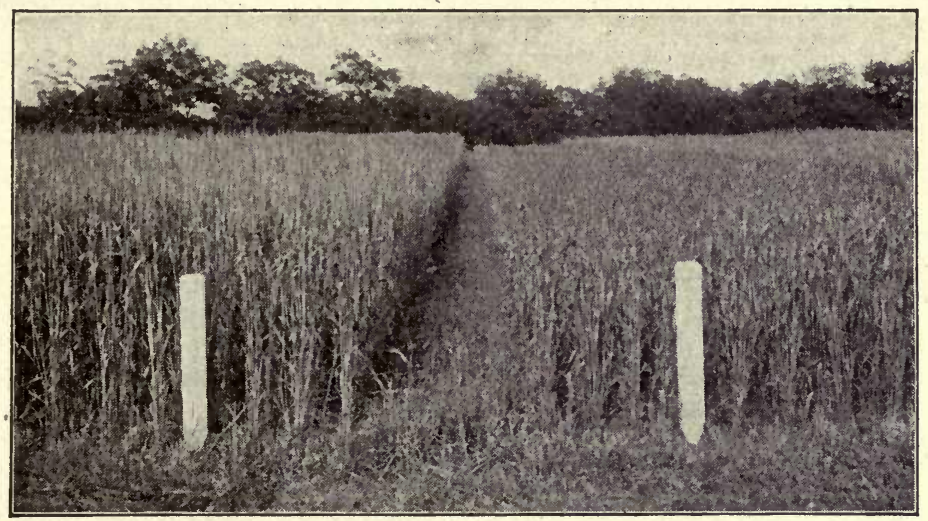

FIg. 252. - Results Obtained from Using Different Fertilizers.

Unfertilized

History tells us that the Indians used a fish in each hill of corn to fertilize it.

Commercial fertilizers, usually contain nitrogen, potash, and phosphoric acid. Lime in its ordinary form is also used.

Nitrogen as a Fertilizer. - Nitrogen is a necessary plant food. It may be applied to the soil as barnyard manure, sodium nitrate, ammonium sulphate, potassium nitrate, slaughter-house refuse, cotton-seed and linseed-oil meal, and other forms of plant refuse. The most common form in which nitrogen is sold as a commercial fertilizer is Chile saltpeter or sodium nitrate. It contains about 16 per cent of nitrogen. 
Ammonium sulphate, a by-product of the manufacture of coke, contains about 20 per cent of nitrogen.

Dried blood, tankage, and bone meal are all good fertilizers and contain considerable nitrogen which is rendered available by the action of soil bacteria. This fact makes it slower in its action and more desirable for many crops than sodium nitrate, which is very soluble and therefore likely to leach out of the soil.

Potassium as a Fertilizer. - Wood ashes contain much potash and would make an excellent fertilizer if the

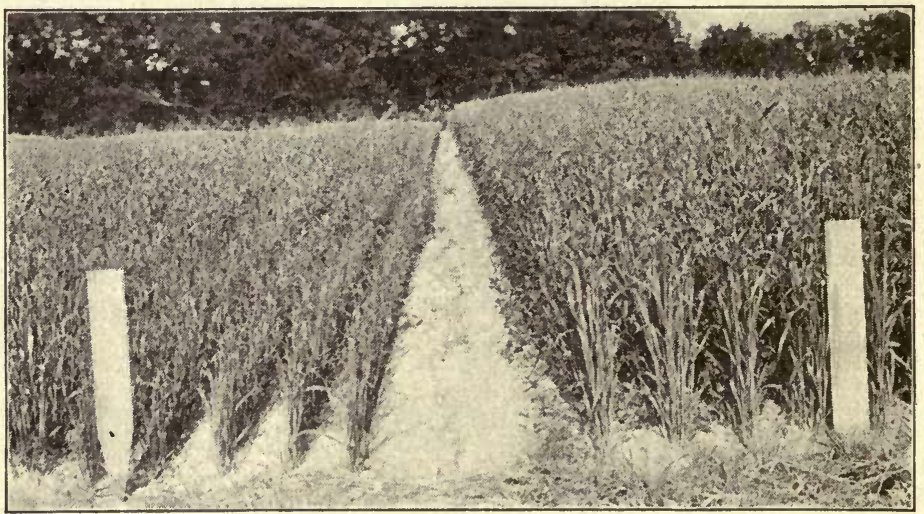

Fig. 253. - Results Obtained from Using Different Fertilizers.

Unfertilized

Nitrogen

Phosphorus

Potassium

supply were not so limited. The common commercial forms of potash are muriate of potash and sulphate of potash (Figure 253).

Nearly all of the potash used in America was formerly manufactured from Kainit from the mines of Germany in the form of muriate or sulphate of potash. 
Phosphorus as a Fertilizer. - Fertilizers containing phosphorus are barnyard manure, phosphate rock, packing house wastes, and basic slag (Figure 254).

The phosphate rock found in many parts of the United States, particularly in South Carolina, Tennessee, and Florida, furnishes most of the phosphorus used in fertilizers.

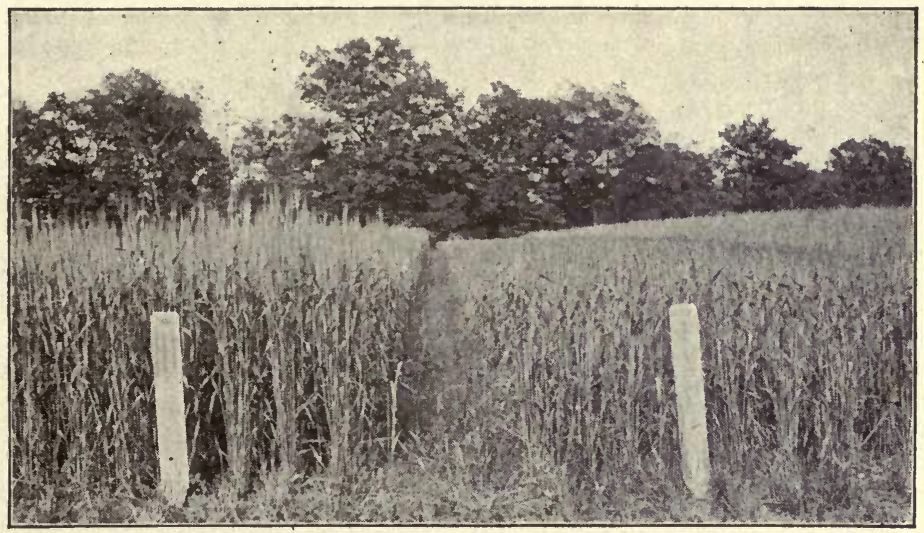

FIG. 254. - Results Obtained from Using Different Fertilizers.

Lime as a Fertilizer. - While lime is not exactly a positive plant food, it renders a valuable service to plants. It is used to correct soils that have become acid, and it assists in the liberation of other plant foods (Figure 255).

How to Experiment with Fertilizer. - The kind of fertilizer that is best suited for a particular soil may be determined easily by experiment. A small plot of land should be divided into several equal areas, or boxes may be used as shown in Figure 256 ; in one box sodium nitrate may be used, in another phosphate rock, in another potassium nitrate, in another lime, and in still other boxes combina- 
tions of different fertilizers may be used. The yields in the different boxes will give a definite measure of the relative value of the different fertilizing materials on the soil tested.

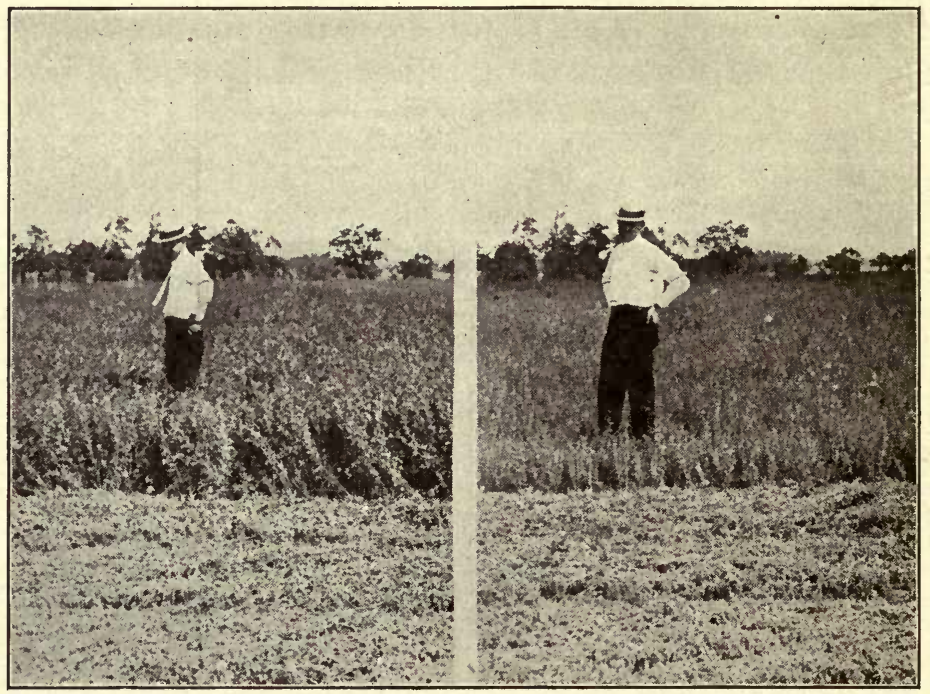

U. S. Dept. of Agriculture.

Frg. 255. - Showing the Value of Lime on Sour Soil.

The experiment station bulletins of the different states usually give quite accurate information concerning soil fertility and the materials needed in fertilizers. "On practically all Ohio soils that have been for any length of time in cultivation, possibly excepting mucks, phosphorus must be supplied before the maximum yield of any crop can be attained. The longer the land has been in cultivation, the greater the need of phosphorus." 1

Why we Cultivate. - There are a number of reasons for cultivating the soil while crops are being grown. Cultivation destroys weeds which would otherwise take the

1 Ohio Circular No. 79. 


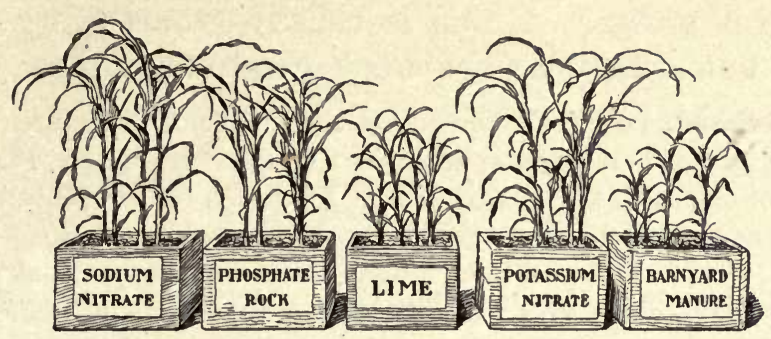

Frg. 256.

plant food which the crop needs. It conserves the soil moisture by putting the soil in a condition to absorb the rainfall and also by preventing evaporation by forming a layer of dust mulch on the surface and by breaking up capillary connections.

Since in most parts of the United States the rainfall is insufficient during the growing months for the production of maximum crops, it is quite essential that the ground be cultivated as soon as possible after rains. The first effect of cultivation is to increase evaporation, but soon the loose dry soil on the surface acts as a mulch to retard evaporation.

Dry Farming. - A large part of the United States has insufficient rainfall to raise good crops without irrigation. In some of the western states it has been possible to conserve the greater part of the rainfall for one or more years for the use of crops during the growing season. This is called dry farming and is accomplished by first plowing the ground to a depth of eight or ten inches and then cultivating continuously, so that a dry dust mulch is formed on the top of the ground. The water that falls as rain is quickly absorbed and held as capillary water by the soil. Capillary water moves very slowly through the dry dust mulch, and thus the loss by evaporation is reduced to a. minimum. When sufficient water has collected in the soil, 
a crop is grown. A crop is usually grown every other year; but sometimes two crops are grown in three years.

Experiment 76. - To determine the per cent of water, organic matter, and mineral matter in soils. Weigh a crucible. Place ten grams of the soil to be tested in it. Heat to a temperature of $110^{\circ}$ Centigrade and maintain this temperature for an hour. Weigh again. The loss shows how much water was evaporated. Now heat the remainder to a red heat and maintain for an hour. The loss this time shows the amount of organic matter in this soil, and the residue shows the amount of mineral matter. Figure the different values in per cents. Test a number of soils in this way. What kind of soils contain the most water? The most organic matter?

\section{QUESTIONS}

1. Explain how an exchange of products benefits both the farmer and the manufacturer.

2. Do field stones become larger or smaller? Why?

3. What kind of rock is slate? Marble? Sandstone? How is each kind formed?

4. What is the relative surface area of a one-foot cube of stone and the same stone divided into one-half inch cubes?

5. What climates facilitate weathering?

6. Where are the humus colored soils thickest, on the hills or in the valleys? Why?

7. Why are some soils red? Blue?

8. Are there glacial boulders in your country? From where did they come? Are there any evidences that glaciers once covered the region?

9. What are the necessary elements in an agricultural soil?

10. What kind of soil predominates in your region?

11. What is the value of air in the soil?

12. Why are sandy soils warmer than clay soils?

13. What is the advantage in mixing your own commercial fertilizers over buying them already mixed?

14. What determines the water capacity of soils?

15. What crops in your region require the least rain? Name some wet season crops.

16. Should corn cultivation be deep? Why? 


\section{CHAPTER XVIII}

\section{SURFACE WATER, DRAINAGE, AND IRRIGATION}

What Becomes of the Rainfall. - When water falls upon the earth as rain, it may do any one of three things. It may evaporate and return to the air; it may run off in little streams to join other streams and finally reach the ocean; it may sink into the ground and reach underground streams or remain in the soil as capillary water.

How the rainfall is disposed of depends upon the nature of the earth upon which it falls and also upon the intensity of the rainfall. If the rain falls gently upon loose soil or upon soil that is covered with vegetation, most of it will sink into the earth; but if it falls rapidly upon a hard, sloping surface, most of the water will run off as surface water. Many of us have noticed the little streams that so quickly assume torrential proportions after a very hard rain.

Ground Water. - Under the influence of gravity the free water that sinks into the soil percolates downward until it reaches an impervious layer of rock. It now flows over the top of that layer in the direction of its inclination until it finds an outlet as a spring or line of seepage on the side of a hill or valley. If rock layers occur in alternately porous and impervious strata and dip toward a fault fissure which appears at the surface at a lower level than the outcrop of the rock layers, water will be forced out of the fissure. This is the same principle 
as that involved in the artesian well, in which a bored hole takes the place of the fissure crack.

Caves and caverns are often formed by the dissolving of the mantle rock, such as limestone, by the ground water. The Mammoth Cave in Kentucky, Luray Cavern in Virginia, and Wyandotte Cave in Indiana are good examples of this action of ground water (Figure 257).

Work of Ground Water. - The greatest work done by ground water is due to its power of dissolving other substances. Some of these substances water dissolves easily, while others are dissolved only after the water has become charged with carbon dioxide as it does in passing through decaying vegetation. If it were not for this power that water has of dissolving all substances, life would be impossible. Plants and animals are dependent upon their circulations, which are simply currents of water in the form of sap or blood, carrying food in solution to the different parts of their being. The foods that plants take from the soil must first be dissolved. The same is true of the food of animals ; it must be dissolved before it can be used by the body.

Water is also a great distributing agent. It distributes food to plants and animals on land and sea. It aids in the distribution of heat through the agency of ocean currents, and it distributes material for the building of sedimentary rocks on the ocean floor.

River Formation. - The water that falls as rain and is neither evaporated nor absorbed by the soil collects in little streams and runs off toward the sea. Along its course it is joined by other surface streams and also by underground streams and the water from springs until it is large enough to be called a river. A river is simply a large stream of water which is bearing the run-off water 


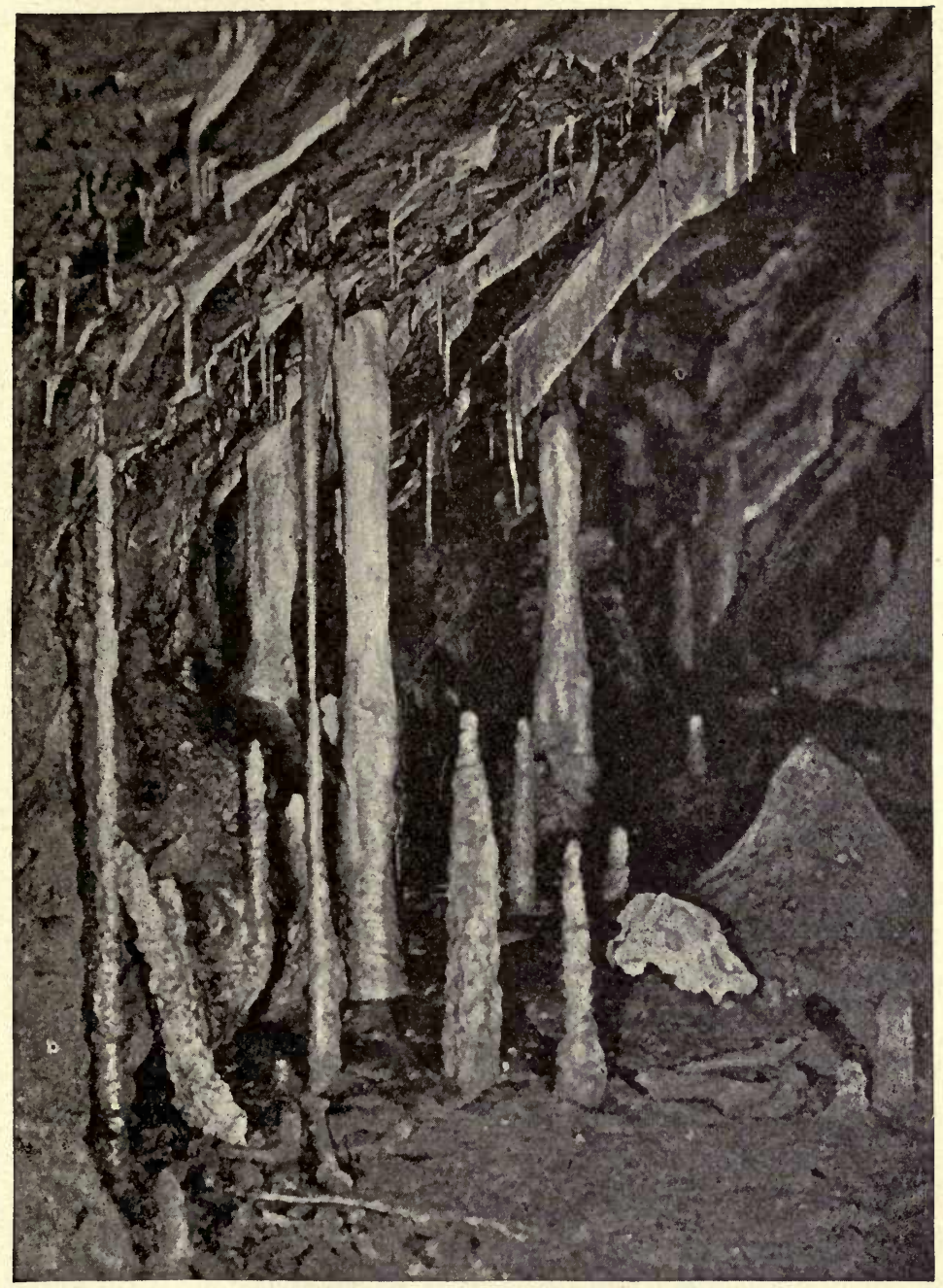

Fig. 257. - The Work of Underground Waters.

The Hermannshöhle, in the Harz Mountains, Germany, showing stalactites and stalagmites. These are deposits of calcium carbonate, formed by the waters which have passed through and partially dissolved the overlying limestone. 
and the waste of the land from higher to lower ground. Usually rivers flow to the sea. The principal stream and all the branches that flow into it constitute a river system (Figure 258). The term " river" is usually applied to the main stream or to the larger branches of a river system,

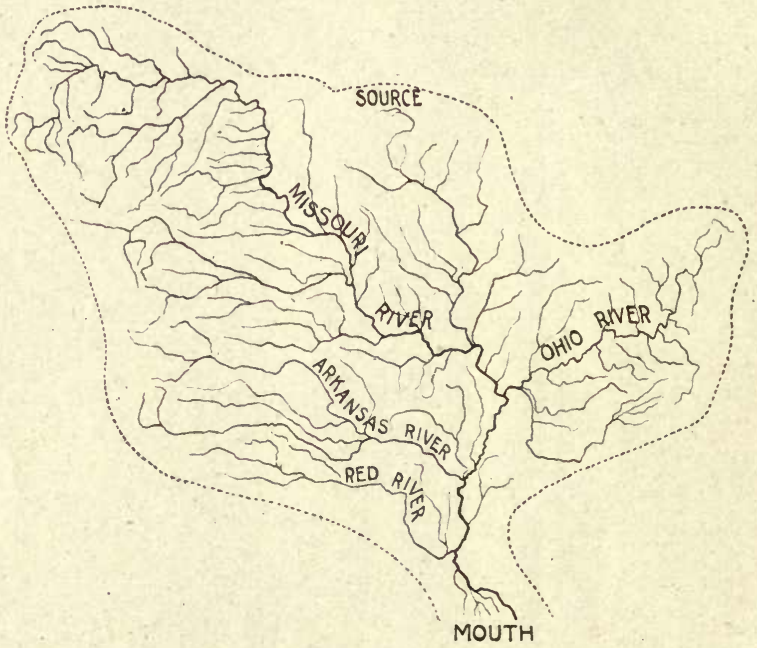

Fig. 258. - The Mississippi River System.

while the term "stream" is a general term and is used quite indiscriminately and with little reference to size.

Lakes and Inland Seas. - A great deal of the surface water in some regions drains into. depressions. When a depression is filled, the water runs over the lowest part of its rim and continues its course to the sea. If these bodies of water are small, we call them ponds; if they are large, we call them lakes. Lakes are constantly being filled by the land waste that is carried by the inflowing rivers. If the lake has an outlet, the water in it is usually very clear, due to the fact that all the sediment has been deposited on the bottom. The outlet of the lake slowly 
wears away its bed and thus lowers the level of the water behind it. Lakes may be considered as enlargements in a river's course. The river is constantly at work to remove this enlargement both by filling and by draining the lake. There are no lakes in an old river system for this reason.

Lakes are quite useful to civilized man. They often form valuable inland waterways, as in the case of the

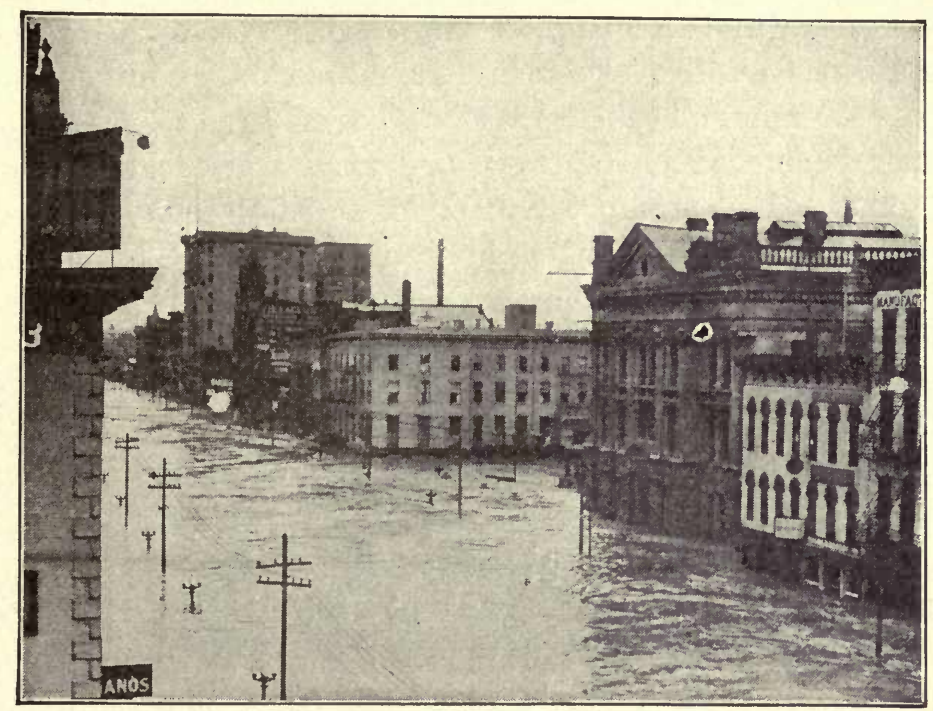

FIG. 259. - Flood, Dayton, Ohio (March, 1913).

Great Lakes of North America, the Caspian Sea, or the Lakes of Africa. Being quite large they act as reservoirs for excess water. There are no floods in the St. Lawrence River system, while in the Mississippi valley there are destructive floods almost every year (Figure 259).

When the rainfall of a region is insufficient to furnish enough water to fill depressions to overflowing, salt lakes occur. The streams which flow into the depression 
carry sediment and also various salts in solution. The evaporation of the water from the surface of the lake increases the density of these solutions. Great Salt Lake has about 15 per cent of various salts in solution, and the Dead Sea has even more.

Work of Rivers. - All streams carry sediment down their valleys. In flood times the amount of sediment is large enough to make the water muddy in quite small streams, while in many of the largest rivers the water is always muddy. Besides carrying sediment in suspension, streams roll sand, gravel, and even large stones along their beds. Much of this coarse material picked up in flood time by a stream is carried for a distance and dropped as the current of the stream becomes slower. This coarse material will be picked up again when the next high water occurs. A large part of the suspended sediment in such rivers as the Mississippi River is carried to the ocean and dropped near the shore. It is estimated that the Mississippi River carries an average of more than a million tons of suspended material into the Gulf of Mexico every day. In addition to this it carries a great deal of material in solution.

All the rivers of the world are constantly at work carrying materials from the land to the sea. The sediment carried by a stream, including that rolled along its bed, is called its load.

Erosion. - The wearing away of the materials which form the earth's crust is called erosion. It is due mainly to the mechanical action and the dissolving power of the surface water. Even the hardest rocks are decomposed by these agencies. In regions which are sufficiently cold the action of the frost also aids in wearing away the surface rock. 
SURFACE WATER, DRAINAGE, AND IRRIGATION 305

As the softer portions of the rocks are dissolved by the water, they break apart until the pieces are small enough to be moved by the running water. The larger pieces are usually started in time of high water, since the carrying power of running water increases enormously as the velocity is increased. As the loose material is rolled along, the

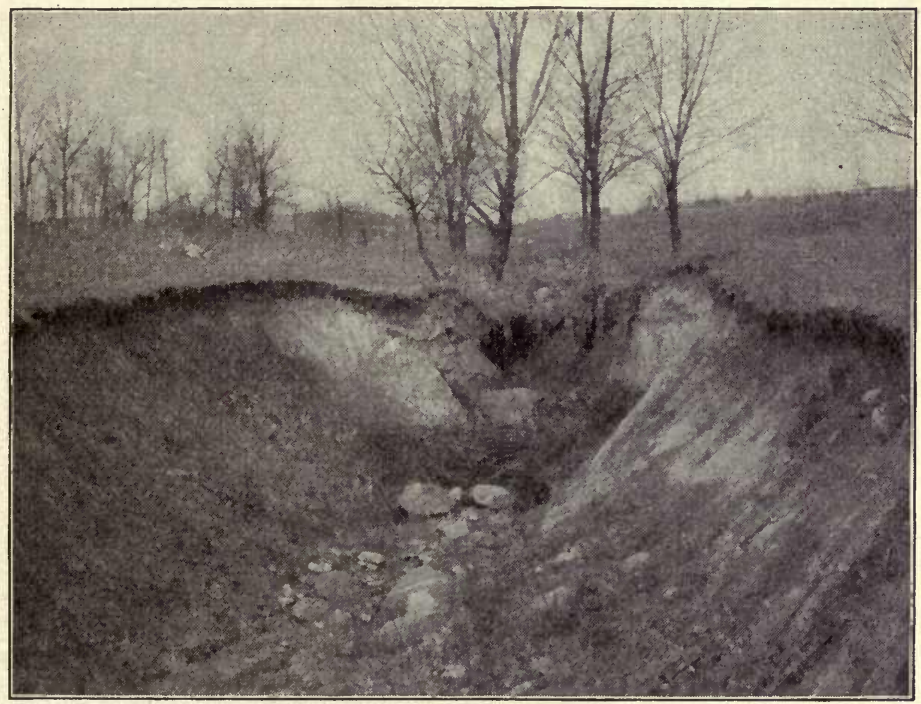

Frg. 260. - An Example of Water Erosion.

solid surfaces in the bed of the stream are cut down and the material produced is moved away by the force of the current. This work is called water erosion.

Erosion is taking place over nearly all of the exposed surface of the earth. When there is a heavy rain, many miniature streams are formed on the surface of the earth. These little streams all do their share in erosion, each one carrying away a small part of the surface material and each 
leaving its little path for another similar stream when the next rain occurs. (Figures 260, 261.)

When the surface water is concentrated into a permanent stream, the work of eroding its bed goes on rapidly. Each flood period makes many changes in the stream bed. Some streams have cut deep gullies or troughs for them-

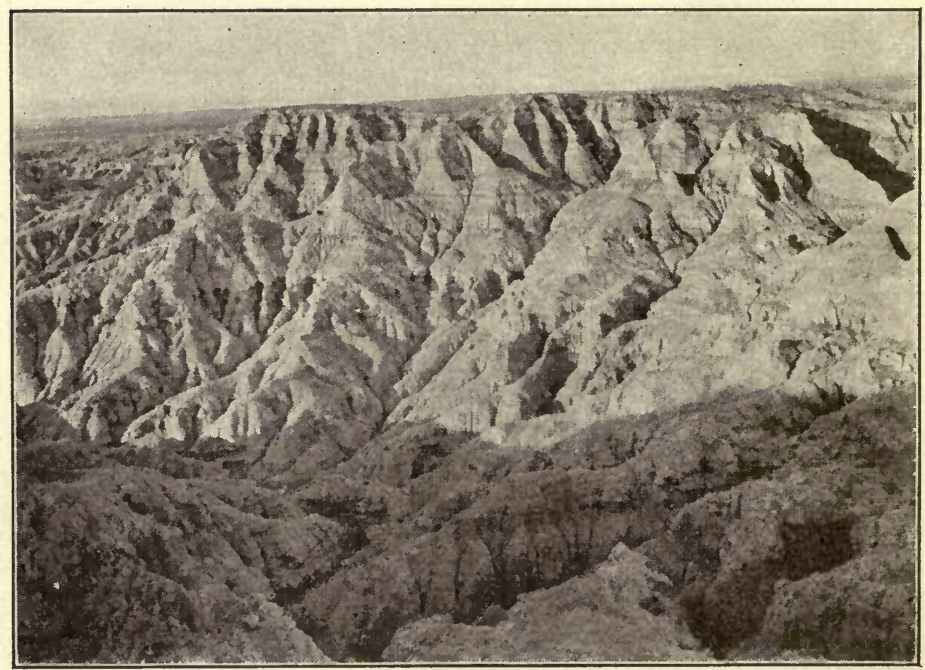

U. S. Geological Survey.

Fig. 261. - Bad Lands, Scott's Bluff, Nebraska, Showing Gullies Made by Water Erosion.

selves. The run-off water sometimes scars the slopes of hillside farms in this way, but usually such gullies are an indication of careless farming. The rapidity of the cutting by a stream depends upon the material of the stream bed, the fall of the stream, and the quantity of water.

The irregular erosion of stream beds produces the characteristic features of streams known as falls, rapids, and 
SURFACE WATER, DRAINAGE, AND IRRIGATION 307

reaches (Figure 262).

However, streams are always at work to remove such features after they are made and to reduce their beds to an even grade. Thus old rivers are devoid of rapids and falls.

In climates of mod-

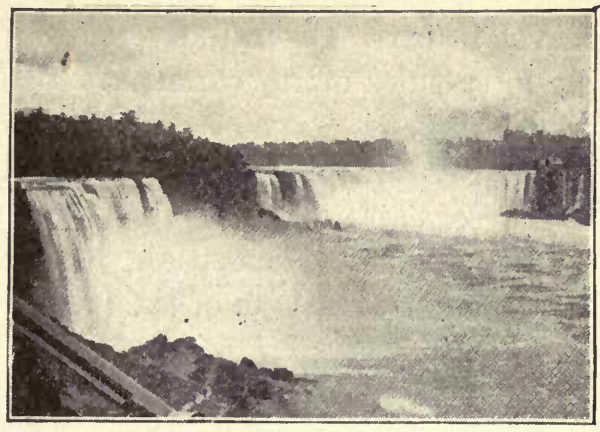

Fig. 262. - Niagara Falls.

erate or heavy rainfall, the erosion of the land is usually rapid enough to produce wide valleys; but in regions of scant rainfall, the streams cut their beds much

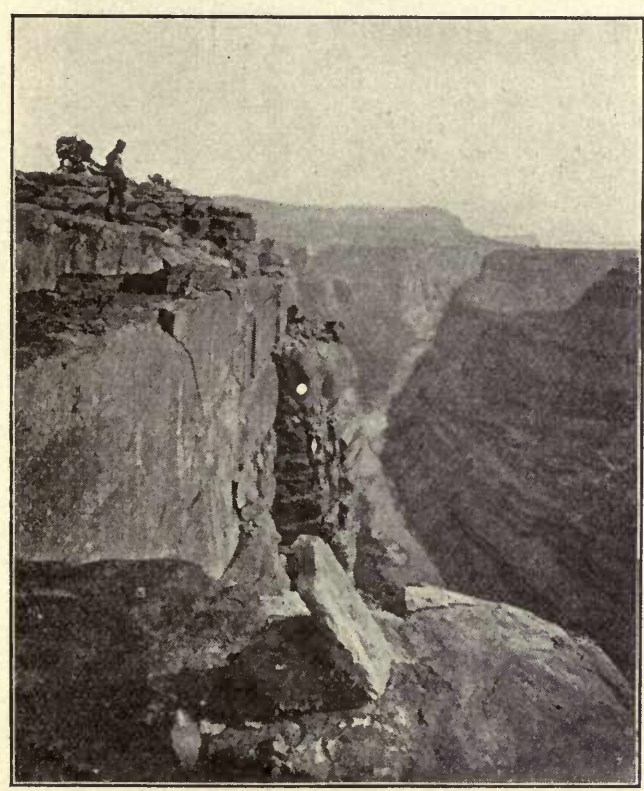

U. S. Geologiçal Survey. Fig. 263. - Grand Cañon, Arizona. faster than the adjacent land is eroded, and deep gullies or cañons result.

The Colorado is the best example of such a river (Figure 263). It flows hundreds of miles through cañons varying in depth from a few hundred feet to several thousand feet. The velocity of this river is so great that the sand and 
gravel it carries is rapidly deepening its channel, while the lofty sides of its cañon are little changed by ages of weathering.

The St. Lawrence River carries practically no sediment of any sort and so erodes its course very slowly.

Deposition. - Deposition, like erosion, depends very largely upon the slope of the river bed and the velocity of

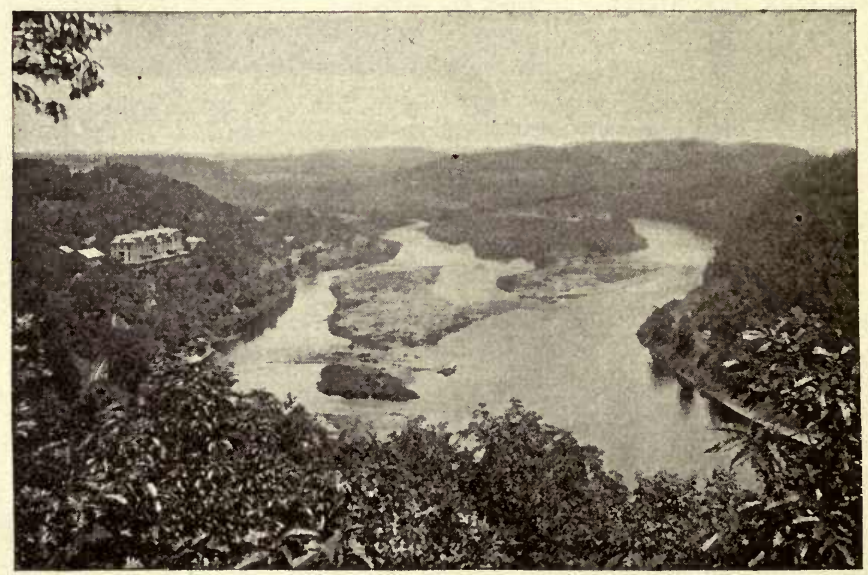

Fig. 264. - The Delaware River above Water Gap, Pa.

The deposition of materials in the bed of the river will extend to form broad, open plains.

the current. The materials carried or rolled along by rivers are deposited at various points in their channels or carried to the sea (Figure 264). The finer portions of silt may reach the ocean, where it is deposited over the ocean floor or in huge delta bars at the mouth of the river, as in the case of the Mississippi River or the Nile River (Figures 265, 266). The fine sand is deposited along the lower portion of a river's course. As we go up the river the deposit becomes coarser, until in the upper part of its course we find stones of considerable size. 
As the current of a river decreases in velocity, its carrying power is greatly lesseried, and some of its load must be deposited. The Mississippi River flows very slowly in the lower part of its course and as a result has been filling up its channel. There are many bars in the lower Mississippi which interfere with navigation. Boats require an experienced pilot because of these shifting bars. As the bed of a river has

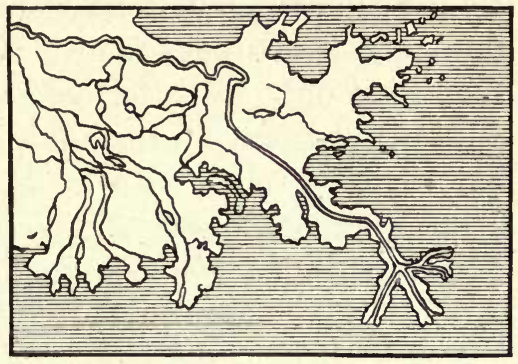

FIG. 265. - The Mississippi River Delta. been raised it has been necessary to build high artificial banks (levees) to keep the water from overflowing the agricultural land and doing great damage. The Govern-

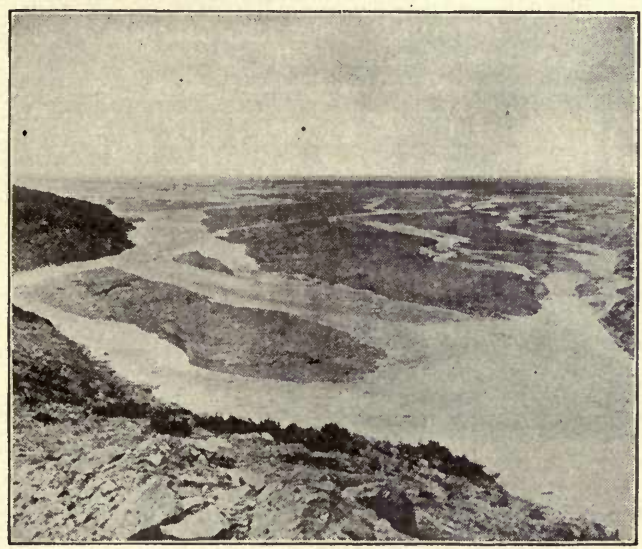

FIG. 266. - A River Delta. ment of the United States annually spends millions of dollars on these levees.

The sediment left by streams on the land in times of overflow is called alluvial deposit. The more the current is checked, the greater will be the deposit. In some places where the current is not checked the swiftly moving water may take up soil instead of depositing it. Where silt is deposited, the land will be enriched; while of course 
where surface earth is removed by running water, the soil will be impoverished.

Soil Water. - The productiveness of soil is limited by the amount of plant food that it contains. Water is the most important plant food. Since plants are able to take their food only in water solutions, the productiveness of any soil is limited by the amount of water that it can hold and by the facility with which it yields up this water to growing plants. Soil water differs from rain water in that it contains the plant foods in solution, while rain water is chemically pure.

Water exists in soil as ground water which drains away, and capillary water which varies with the kind of soil. The finer the soil particles the greater the amount of capillary water or film water.

Regulation of the Amount of Soil Water. - Soil water may limit the crop by being present in either too great or too small amounts. Dry farming and irrigation are practical ways of obtaining crops in regions where there is too small an amount of soil water. Commonly there is too much water in the soil in the early part of the season and too little as the season advances, so that the crop is injured by both extremes.

When there is too much water in the soil, air, which is essential for plant growth, is excluded, and the soil remains cold. This retards the growth of the plant and delays farm work.

Artificial Drainage. - Underground drainage of some sort is essential for the growth of ordinary crops. Where the soil is not drained, the excess of water prevents the growth of crops, and where there is no excess of water to drain away, the soil becomes either acid or alkaline due to the accumulation of salts and acids, 
which are carried in solution into the soil by the surface water.

Where the subsoil is porous, natural drainage is usually sufficient to remove the excess of water. Where the subsoil is a heavy clay, artificial drainage must be employed. Most farms can be improved by tile drains properly located, and many farms need a complete system of drains laid from two to six rods apart. (Figure 267.)

Tile drains, in addition to removing excess soil water, actually serve to protect plants in times of dry weather. When the excess soil water is quickly removed, the plant roots grow much

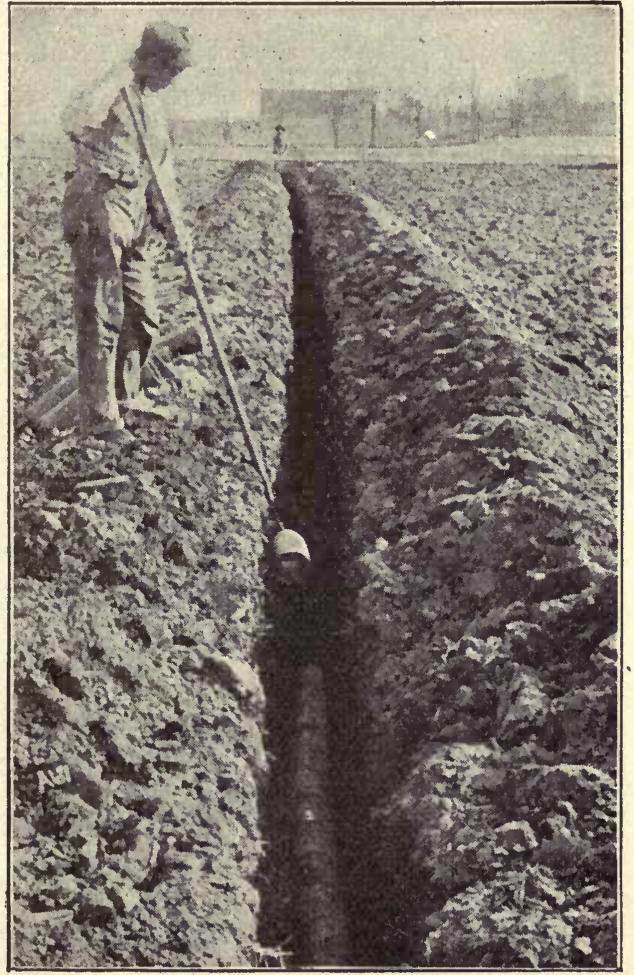

FIg. 267. - Tiling Level Lands, Illinois. deeper than in soil that is wet and cold. These plants with deep roots are then able to withstand considerable dry weather, since the roots are able to use the capillary water of the subsoil.

Irrigation. - About two fifths of the total area of the 
United States receives insufficient rainfall to produce yearly crops without irrigation. In some parts of this area occasional crops may be produced by dry farming, but this means that only a small fraction of what the land is capable of producing with adequate rainfall will be produced. The land along a stream is always more fertile than the land farther away from the stream. In some

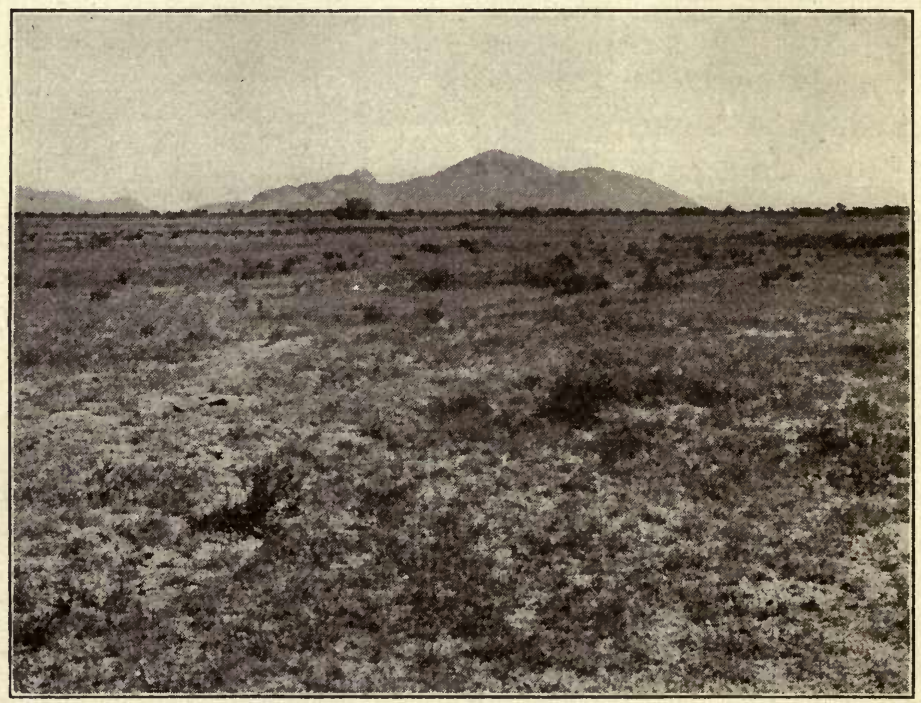

U.S. Reclamation Service.

FIG. 268. - Salt River Project, Arizona. The Desert Before Reclamation.

regions it has been possible to divert part of the water of streams through canals and ditches so that a much larger area is watered. This is called irrigation.

In the western part.of the United States, especially in the region west of the Rocky Mountains, the United States Reclamation Service and a number of private organizations are maintaining a number of irrigation systems (Figures 268, 269). The engineering problems involved 


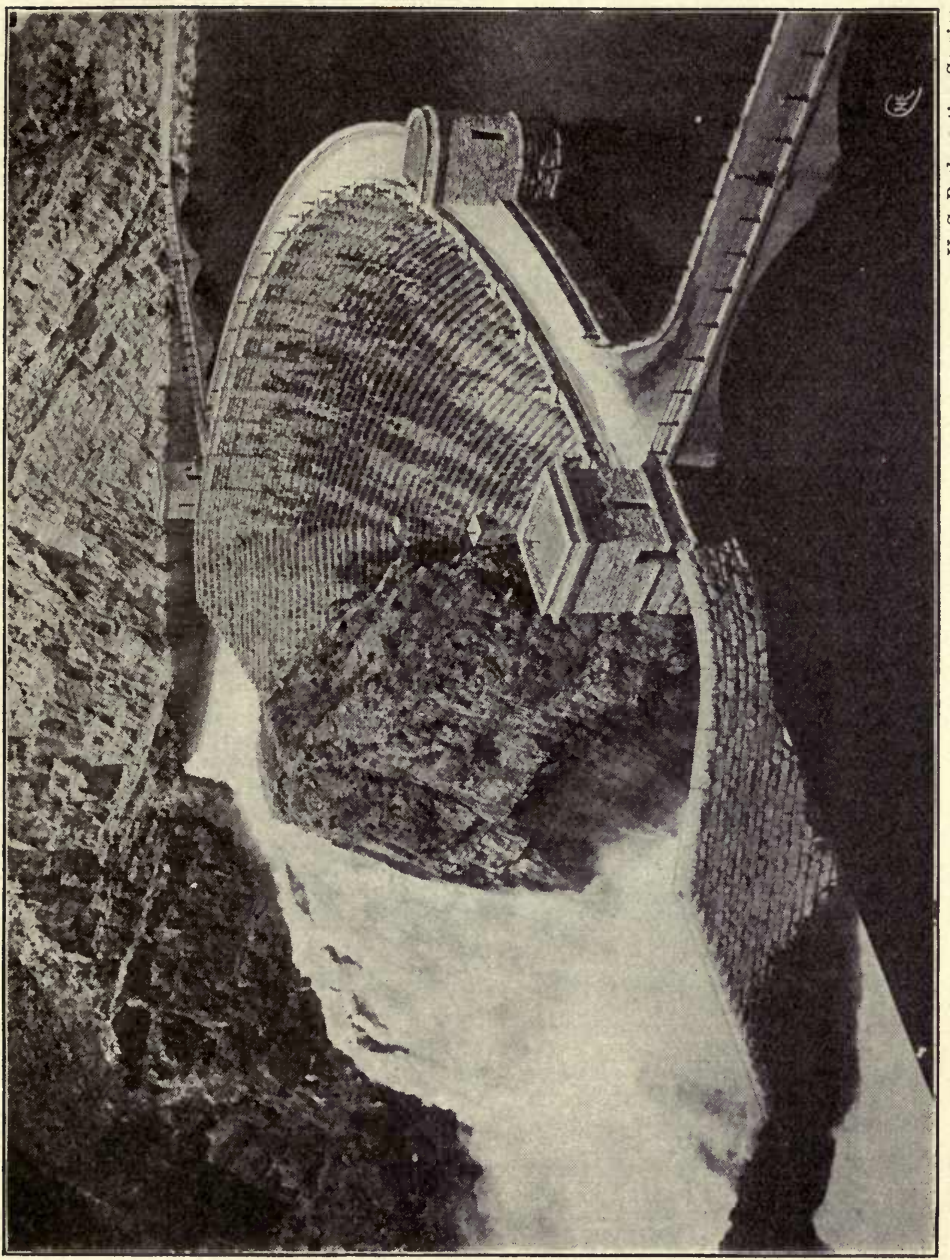

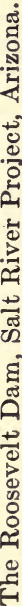



in these enterprises are enormous, but the returns have usually justified the expense. In some places immense dams are necessary. In other regions miles of concretelined canals have been built. The large storage dams retain the water that falls in certain stream basins during the winter and early spring months. As this water is

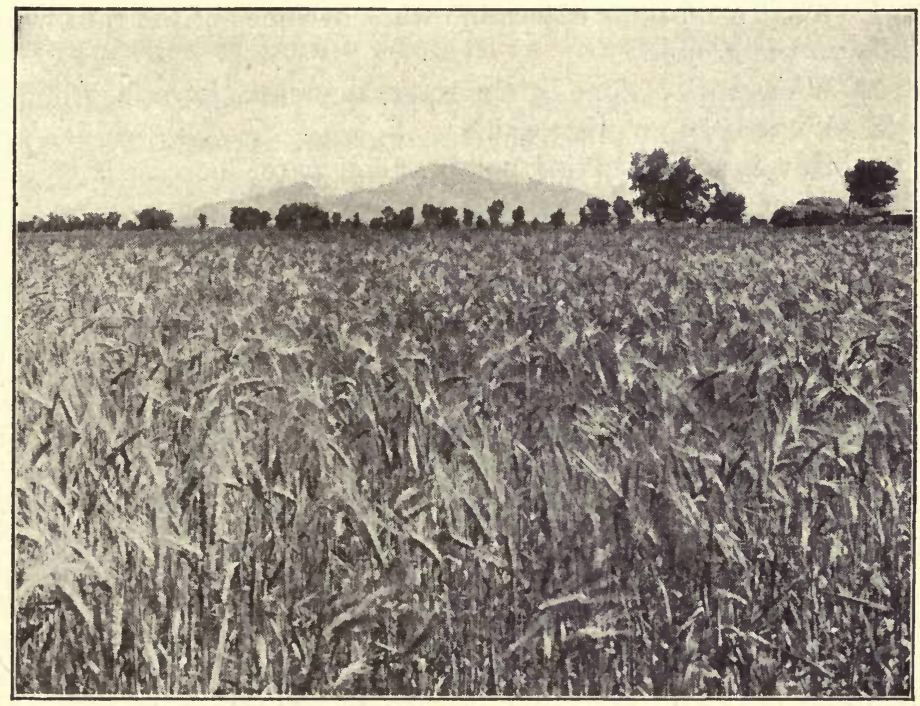

U. S. Reclamalion Service.

Fig. 269. - Salt River Project, Arizona. The Desert After Reclamation.

needed by the farmers below the dam, it is allowed to flow into canals which carry it to the tract to be watered.

It will never be possible to irrigate all the arid land in this way, since the total amount of rainfall of the dry region is only enough to water properly one tenth of it. Then too, some sections are so located that the expense of bringing water to them would be too great to make them profitable. 
In the United States Government irrigation projects the reclaimed land is sold to settlers at a price which more than pays the cost of the work. The money thus received is used in the construction of other reservoirs and canals.

Private corporations often sell the water to users.

\section{QUESTIONS}

1. What conditions determine what becomes of the rain when it falls to the ground?

2. What work is done by the water that sinks into the ground?

3. What is an artesian well?

4. How is the dissolving power of soil water increased?

5. What is a river basin? A divide?

6. What are the characteristics of a young river?

7. Why are there no floods at Detroit? Why?

8 , Is the water in a salt lake increasing or decreasing in density?

9. Why is it impossible for a person to sink in Great Salt Lake?

10. What are some of the peculiarities of rivers in dry climates?

11. How do rivers build deltas? Where?

12. If the velocity of running water is doubled, how will its carrying power be affected?

13. What kind of soil bakes hardest in the sun?

14. What crops in your region grow best on wet lands?

15. How does pressing down the soil affect the movement of soil water?

16. Is it a good thing to pack the ground over grains of corn just planted? Why?

17. What causes the alkaline soils found in some of our western states?

18. Why are wet soils cold?

19. How may the seepage in irrigation canals be lessened ?

20. After land is irrigated why should it be tilled?

21. Why do irrigated soils become alkaline? What is the remedy for this? 


\section{CHAPTER XIX}

\section{PLANTS}

Properties of Living Matter. - Living matter has quite a complex chemical composition. It contains the elements nitrogen, carbon, hydrogen, oxygen, sulphur, and phosphorus. Other elements are usually found in living matter in minute quantities. The name protoplasm

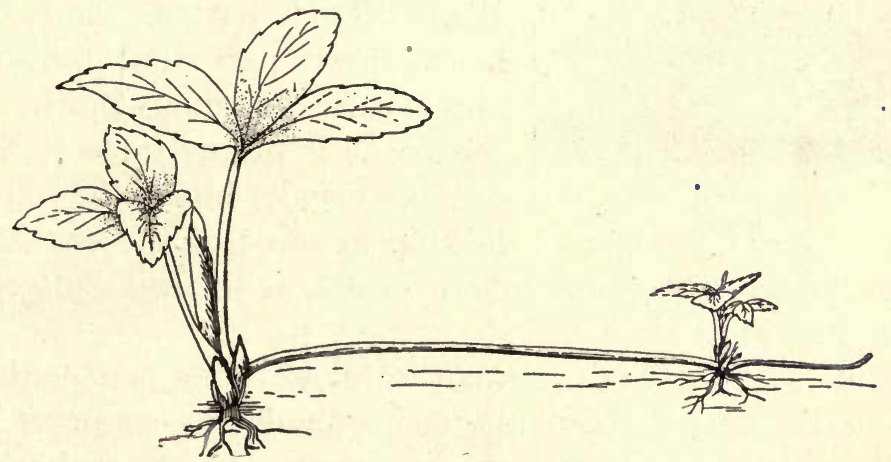

FIG. 270. - Reproduction in a Strawberry Plant by Division.

has been given to this complex combination of these elements. Life occurs only in protoplasm, and plants and animals are largely composed of it.

Protoplasm possesses the property of irritability and responds to the stimuli of light, heat, and electricity. Sprouts on tubers and plants in dark places always grow toward the light. Leaves turn toward the source of light. 
Protoplasm has the power to move by contraction. Plants move and change the position of their leaves, while the muscular action is a well-known power. Protoplasm also has the power of taking up materials that it can use as food and rejecting those materials which it cannot use. Protoplasm breathes oxygen, eliminates wastes, and has the power to reproduce its kind of plant or animal (Figure 270).

The Living Plant. - Plants are fed by the elements that they take from the soil and air. From these elements

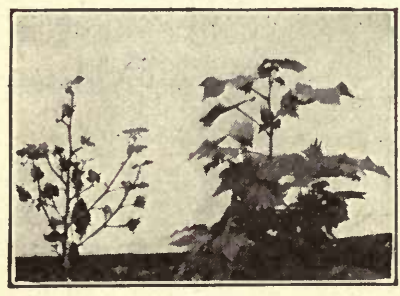

FIG. 271. - A Sick and a Well Plant. they build all the various complicated plant structures known as flowers, seeds, roots, and stems. Each part of the plant has its own particular purpose and is correspondingly built. If the plant is properly fed it will develop evenly, but if the soil is lacking in some of the essential plant foods the plant which grows on it will be weak (Figure 271).

Cells. - A cell is the smallest bit of living matter that can exist alone. All plants and animals are composed of cells which are separate masses of protoplasm, each having a nucleus and each surrounded by an envelope called the cell wall. Cells vary greatly in size. Some of them may be seen readily with the unaided eye, while others have a diameter of not more than 1/25000 of an inch. In shape they vary from long strings, such as are found in the cotton fiber, to the spherical eggs of animals.

Tissues. - Collections of similar cells, grouped to produce some particular part of a plant or animal, are tissues. Examples of tissues are woody tissue, pith 
tissue, bony tissue, and the skin covering the bodies of animals.

Organs. - Each part of a plant or animal which has a special work to do is called an organ. Tissues are combined in various ways to produce organs such as leaves; roots, and veins in plants and the many organs of the human body.

Multiplication of Cells. - When a cell reaches its maximum size, it may divide into two cells, each containing
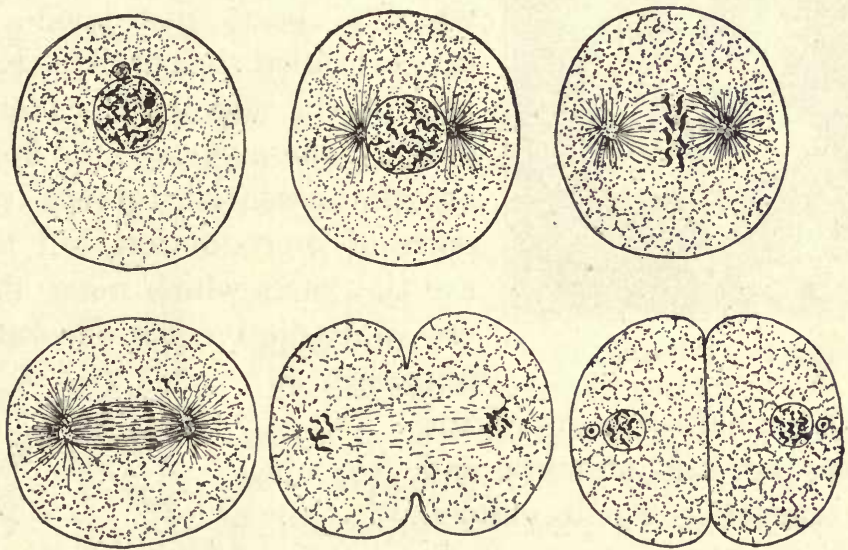

FIG. 272, - Showing the Method of Cell Division.

Note the division of the nucleus.

half of the nucleus and the protoplasm of the old cell (Figure 272). These new cells may grow to maximum size and again divide. The multiplication of cells in this way is the usual process of growth in plants and animals.

Flowers. - Flowers grow on the higher forms of plants at a comparatively mature stage in their development (Figure 273). The flower is grown for the purpose of producing other similar plants. In order to understand 


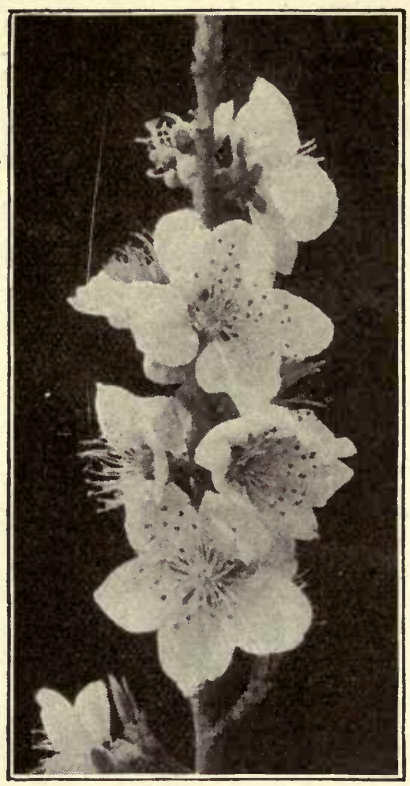

Fig. 273. - A Peach Twig.

the process of reproduction in flowering plants, it is necessary to make a study of the parts of a flower (Figure 274). The outer whorl of the leaf parts of a flower is called the calyx or cup of the flower (Figure 275). Each division of the calyx is called a sepal. These are often green in color. The next whorl of parts just inside the calyx is called the corolla. Each leaf of the corolla is called a petal. These parts of the flower are not essential for the purposes of reproduction, but they are the parts which make flowers so attractive for decorative purposes.

The essential parts of the flower are the stamens and the carpels. Each stamen consists of a supporting stalk called the filament. The anther is the enlargement at the end of the filament (Figure 276). The pollen, which resembles yellow powder, grows in the anther.

The carpels of a flower considered collectively are FIG. 274. - Diagram Showing the called the pistil (Figure 277).

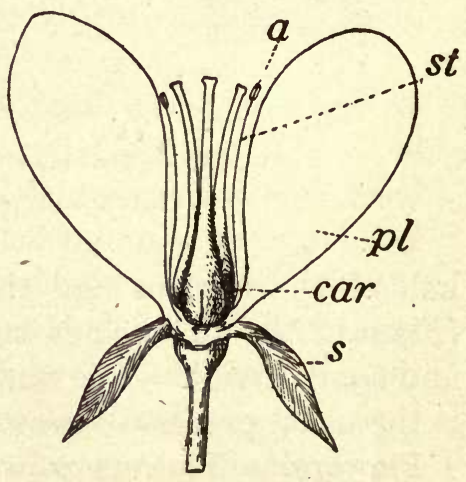
$a$, anther ; car, carpels; $p l$, petal ; A simple pistil has but one $s$, sepals; $s$, stamens. 
carpel (Figure 278); a compound pistil has more than one carpel. In the base of the pistil is a bulblike seed case called the ovary. This contains the ovules or eggs. Above the ovary is a slender stem called the style, and at the end of the style is a sticky surface called the stigma.

Pollination. - In order that seeds may be produced, it is necessary that pollen from the anthers lodge on the stigma. This may happen in several ways. The anthers of some flowers burst when ripe and scatter the pollen on the stigma. The

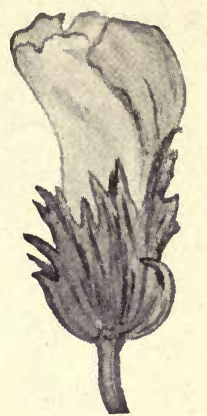

Fig. 275. - Calyx and Corolla. wind may blow the pollen so that it falls on the stigma. Insects flying from one flower to another often carry

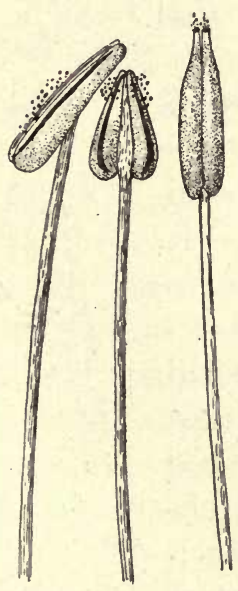

FIG. 276. - Three Stamens with Different Forms of Anther. pollen from the anthers of one flower to the stigma of another. This is crosspollination.

Fertilization. - Each pollen grain consists of a particular cell called the sperm cell. When the pollen grains fall on the stigma, they are held by the sticky surface. They immediately begin to grow, forming tubelike roots which grow downward through the stigma and the style into the ovary, where they pierce the ovule and reach the egg cell. The sperm cell of the pollen meets the egg cell of the ovule and they unite to form a single cell, which grows and forms an embryo plant (Figure 279). The parent plant stores food around this plant, with the result that fruits and seeds are formed. The fruits are simply 
produced by nature to furnish food for the new plants or to protect the seeds from climatic conditions until a favorable time for growth shall arrive.

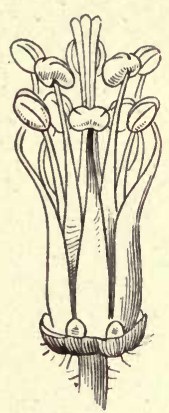

Dispersal of Seeds. - A little observation will disclose to any one a number of ways in which seeds are dispersed. Hold a maple seed high in the air and notice whether it falls directly. to the ground or not. What is the value of wings on maple seeds? Some seeds like the dandelion and thistle are provided with little parachutes (pappus) which Fig. 277. - Stamens enable the wind to carry them for long and Pistil. distances (Figure 280).

Some plants, such as tumbleweeds, break from the main stem and are then blown along the ground, scattering seeds as they go.

Squirrels, birds, and other animals are active agents in the dispersal of seeds.

Germination of Seeds. - The stages passed through by a young plant from the time it begins to sprout until it becomes an independent plant are called germination.

The process of germination may be studied by planting some seeds of various kinds. Plant some beans, squash seeds, corn, and wheat in warm, moist soil or sawdust and ob- Fic. 278. - A serve them from day to day. Some of each

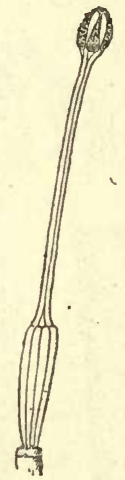
kind of seeds should be left untouched until the plant appears above the surface of the soil. Do they all come out of the soil in the same way? (Figure 281.)

As the embryo plant in the seed begins to grow, it again bursts open the seed leaves. These seed leaves contain 
starch and proteid, which are so changed by water and the digestive ferments in the seeds that they can be used as food by the growing plant until it has roots capable of taking food in solution from the ground, and leaves which take the carbon dioxide and oxygen from the air (Figure 282).

Roots. - In the germination of many plants the root grows into the soil first, to get water and food so that the top of the plant may grow. With other plants, however, the top and the root seem to grow simultaneously.

There are several factors which determine the direction taken by roots. Gravity and water are the most important of these factors (Figure 283). Water is always

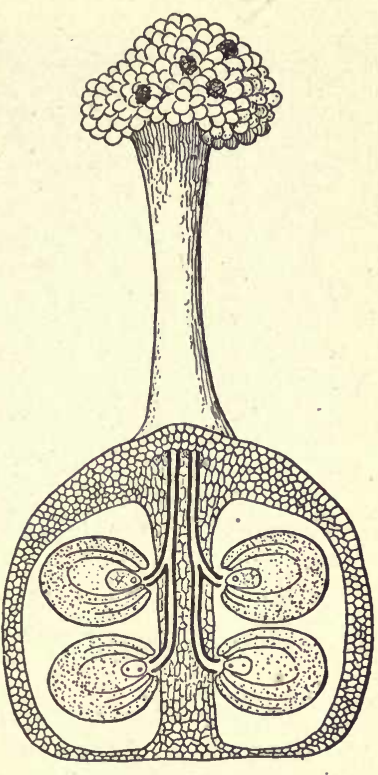
found below the surface of the FIG. 279. - Fertilization of the earth, but sometimes at such Ovule. depths that roots must go long distances to secure a supply. Many plants have a greater area of root surface than of branch surface. In arid regions tree roots have been known to penetrate the earth to a depth of sixty feet in - search of water.

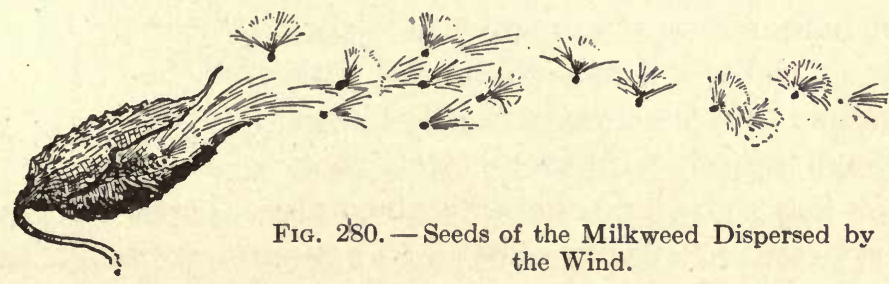


Roots serve to hold plants in an upright position and also to take food from the soil by the process of osmosis. When two fluids are separated by a porous membrane they

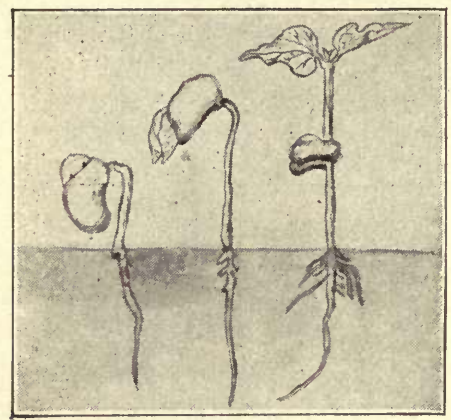

FIG. 281. - Development of the Bean.

Note how the seed is pushed out of the ground, the formation of the seed leaves, and the withering of the seed when the leaves and roots are sufficiently developed.

intermingle or diffuse, the greater flow being toward the denser medium. The fluid in the root hairs is more dense than the soil water ; therefore the soil water bearing plant food flows into the roots faster than the sap flows out, and the excess fluid is forced up into the plant.

This action takes place most rapidly near the ends of the roots where the root hairs are most numerous. A root hair is really a living plant cell with a wall so thin that water readily passes through into the interior of the root. $p$, permanent leaves. Root hairs are long, hairlike structures almost colorless in appearance (Figure 284). They are probably not 
larger than $1 / 500$ of an inch in diameter. The walls are cellulose, a substance which readily permits the passage of fluids through it. These very small roots take the

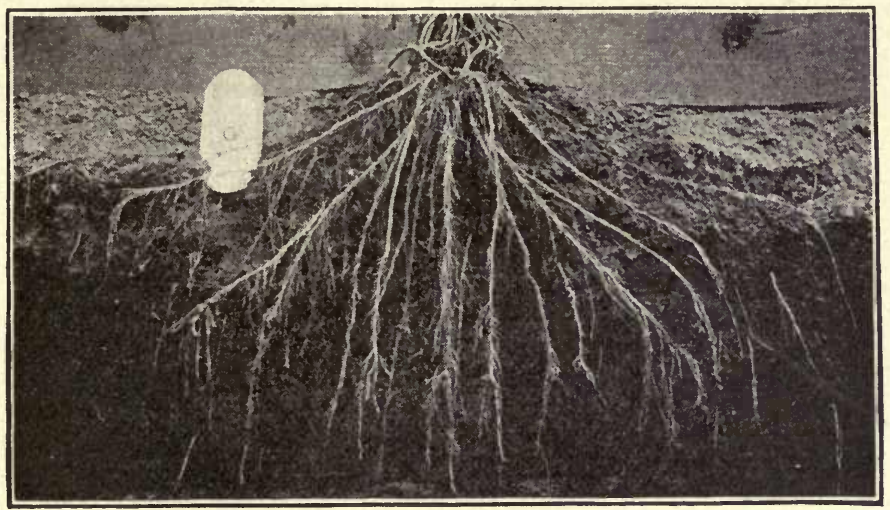

FIG. 283. - Distribution of Roots.

food from the soil, while the larger roots carry the food to the stem of the tree.

Stems. - The main stem of a plant grows in a direction opposite to that of the first root of a seed, while the

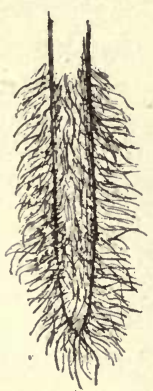
branches from the main stem grow outward in a way quite similar to the root branches underground. The roots grow in all directions to get food, while the branches from the stems grow outward to get light.

Experiment 77. - Plant some grains of wheat or oats in a box arranged so that the light is received from but one side (Figure 285). Note the direction of the seedlings as they grow.

FIa. 284.- Root Stems serve to hold the leaves in a posiHairs.

tion to receive light and air, and they also furnish a pathway for the food-bearing liquid which moves from the roots to the growing parts of the tree. 
Stems vary in structure. In corn the stem or stalk is pithy with a hard rind on the outside and numerous woody fibers running length-

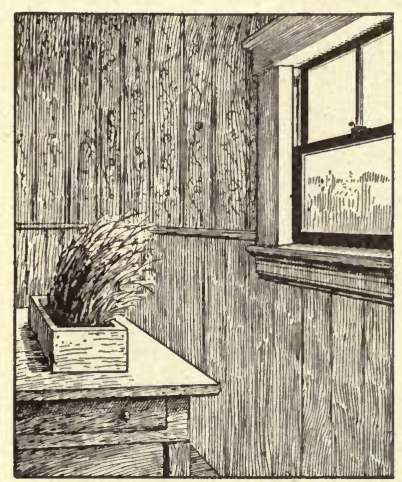

FIG. 285. - Grains of Wheat Growing Toward the Light. wise through it. These fibers and the rind are traversed by minute holes, which serve as a passageway for the sap. If a longitudinal section of the stalk is made, some of those little fibers will be found to enter the leaves at the joints. In the leaves they appear as veins and may be traced readily. The tough woody cells of the rind furnish the main support of the corn stalk as it matures.

The stem of the ordinary tree contains a very small pith center. Surrounding the pith is the woody fiber, which is composed of many little tubes and their woody walls. If the stem is several years old, we can see distinct annual rings in this woody part. The space between two consecutive rings indicates the growth during one summer (Figures 286, 287). Radiating from the center of the stem and extending to the bark are found a number of tiny lines. These lines are called the medullary rays and serve as storehouses for food and permit the flow of sap across the rings of the tree. These rays produce the beautiful effects that we see in quarter-sawed lumber.

The soil water that is taken up by the root hairs, under the influence of osmotic pressure and capillarity (root pressure), is carried by the minute tubes in the stems to all parts of the plant or tree. In a large tree most of the sap is carried by the tubes in the sapwood. This is the 
layer of white wood which lies immediately under the bark. The darker heart of the tree is composed of dead fibers and serves as a storehouse for food and as a support

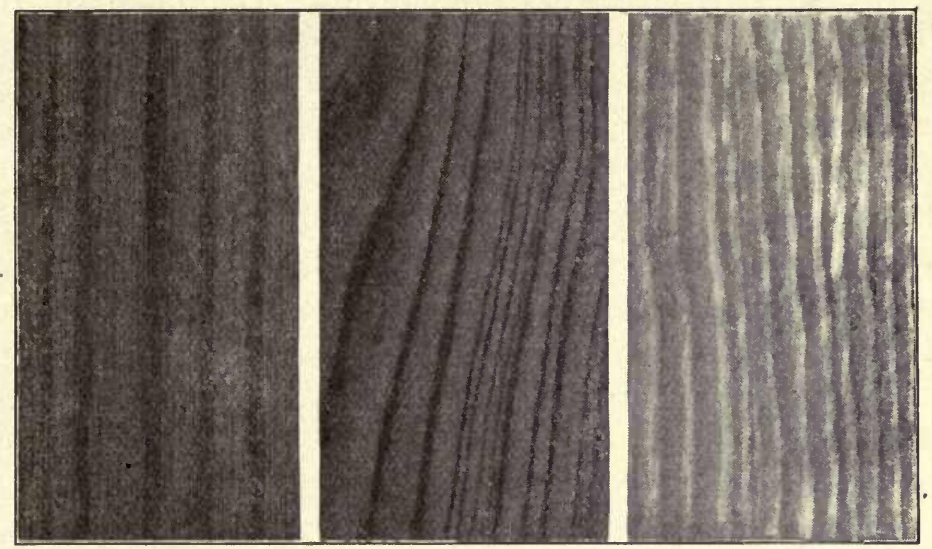

Oak.

Cypress.

Southern pine.

Fig. 286. - Wood Grains.

for the other parts of the tree. The heart of the tree is most valuable for lumber, since it has a richer color and decays slower than sapwood.

The bark is the outer portion of the woody stem. The part of the bark which is next to the sapwood is a living

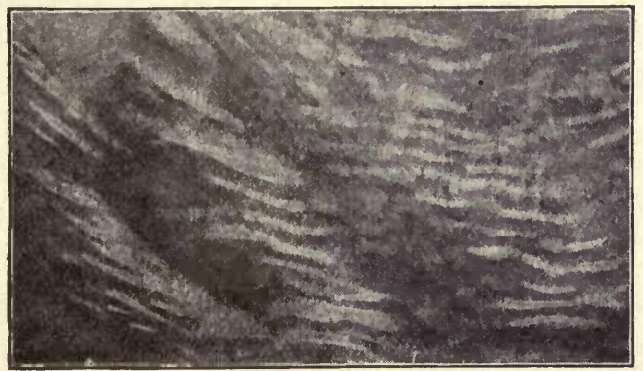

Fig. 287. - Curly Walnut, Showing Grain in the Wood. 
tissue and is called the cambium layer. All the growth of our hardy trees, such as the oak and maple, takes place from the cambium layer. It forms new wood on the inside and coarse bark on the outside. The outside bark of a tree is dead and serves only to protect the tree in various ways.

Leaves. - As soon as a seed germinates, leaves are formed on its stem. Some plants, such as the bean and the squash, have two seed leaves which appear as soon as the seed has swelled in the process of germination. Leaves are essential to plant life, as may be proved by removing the leaves from a young plant.

The broad part of a leaf is called the blade; the stem is called the petiole. Leaves may be classified according to the arrangement of the veins (Figure 288). Number 1 shows the palmate-veined leaf of the maple; number 2 , the parallel-veined leaf of the wild lily-of-the-valley; number 3, the pinnately-veined leaf of the birch; number 4, the pinnately compound leaf of the rose. Veins in leaves serve the double purpose of supporting the parts of the leaf and of furnishing tubes to carry food.

In almost every case the arrangement of the leaves on a plant is such as to secure the greatest amount of sunlight for them. In dense forests the trees grow tall with clusters of leaves near the top, while in open fields the same species of tree sends out numerous lateral branches covered with leaves. On the smaller plants the leaves arrange themselves in various ways, but always so that each leaf receives a large amount of light.

The cells of a leaf are composed of protoplasm and a green material called chlorophyll. The layer of cells forming the upper and lower part of the leaf is called the epidermis. The epidermis on the upper part of the leaf 
has slightly thicker walls for protection, while on the under side it is pierced by numerous pores called stomata. Many thousand of these openings may occur in a square

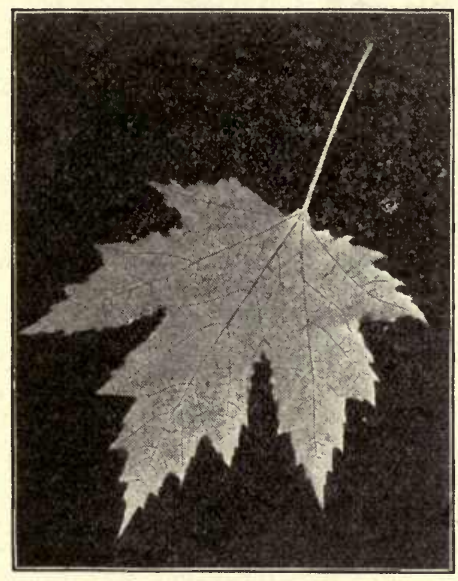

1. A Maple Leaf.

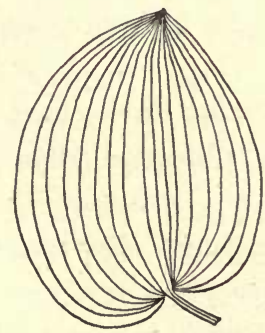

2. A Wild Lily-of-the-Valley Leaf.

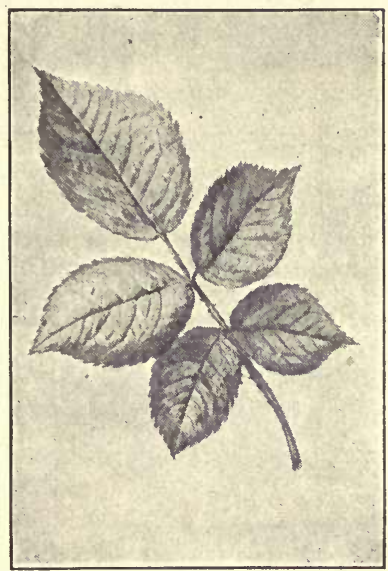

4. A Rose Leaf.

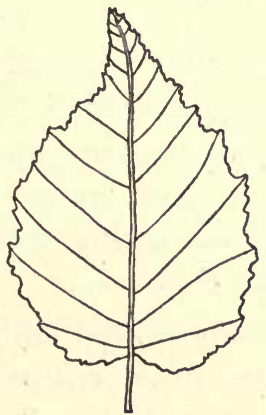

3. A Birch Leaf.

Fig. 288.

inch of leaf surface. The stomata are the lungs of the plant. They take in air and expel gases which the plant does not need. Around each stoma there are two guard 
cells, which by changing their shape control the amount of air breathed in by the plant and also the amount of evaporation of water from the leaf (Figure 289).

Starch Making by Leaves. - The leaves also serve as a factory in which starch and sugar are manufactured.

a

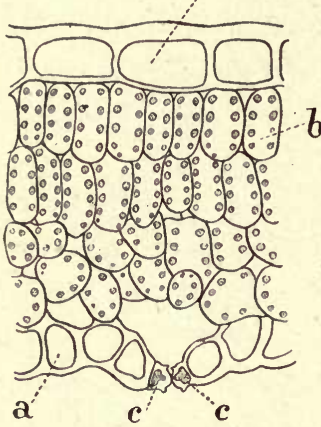

FIG. 289. - A Section of a Leaf.

$a$, epidermis ; $b$, cells ; $c$, guard cells.
To do this the leaves combine water and carbon dioxide. Since there is more oxygen in the air than is needed to produce the carbohydrates, starch and sugar, the excess oxygen is breathed out by the leaves. Thus plants tend to purify the air by exchanging oxygen for the carbon dioxide in it. The manufacture of starch and sugar in the leaf is carried on by the chlorophyll bodies within the leaves under the influence of the energy of sunlight. The sunlight and the chlorophyll bodies are the agents in the production of these carbohydrates, and the water and carbon dioxide are the raw materials from which they are made.

Plants that have been grown in the dark, and plants that lack green coloring matter (chlorophyll) contain no starch or sugar.

Digestion in Plants. - Food products in plants, as in animals, may require changes before they can be used as foods. Starch is a food for plants, but it is quite insoluble in water and must be digested before it can be used. This is accomplished by changing the starch to sugar, which is dissolved by the water and carried to the different parts of the plant.

Flowerless Plants. - The flowering plants are the 
highest class of plants. Their composition is quite complex, and they perform the functions necessary to their life, growth, and reproduction in complicated ways. There are other plants which do not have flowers and in which the function of reproduction is accomplished in other ways than by the production of seeds. Some of these plants are composed of but a single cell, which performs all the functions of the plant. Reproduction in such plants takes place when the cell separates into two cells, thus forming two plants. Higher forms of flowerless plants are reproduced by spores which contain a very small portion of protoplasm capable of reproducing its kind of plant. For convenience in study, the flowerless plants may be divided into algæ, fungi, mosses, and ferns.

Algæ. - The algæ are the lowest form of plant life, but they resemble higher plants in some respects; they all contain chlorophyll and are able to manufacture starch from water and carbon dioxide. Algæ vary in size from simple one-celled plants to the giant kelp of the Pacific Ocean, which frequently attains a length of several hundred feet. A common alga is the simple onecelled variety that is frequently found on the bark of trees and on rocks. It has a greenish color and may be found in almost any forest.

Pond scum is another alga known as spirogyra. It can be found floating on the surface of almost any stagnant pond. The cells of the spirogyra are placed end to end, so that they form long threads. It is heavier than water, but enough bubbles of oxygen cling to the masses of threads to cause them to float. The oxygen is given off by the plants as starch is formed, as in higher plants. The spirogyra grows by a division of cells and also pro- 
duces a spore, which is formed by the growing together of two cells to form one strong cell. This spore cell may remain dormant for considerable time and also be subjected to extremes of heat and cold without destroying its vitality.

Fungi. - We are probably familiar with the toadstool and the edible mushroom (Figure 290). These plants con-

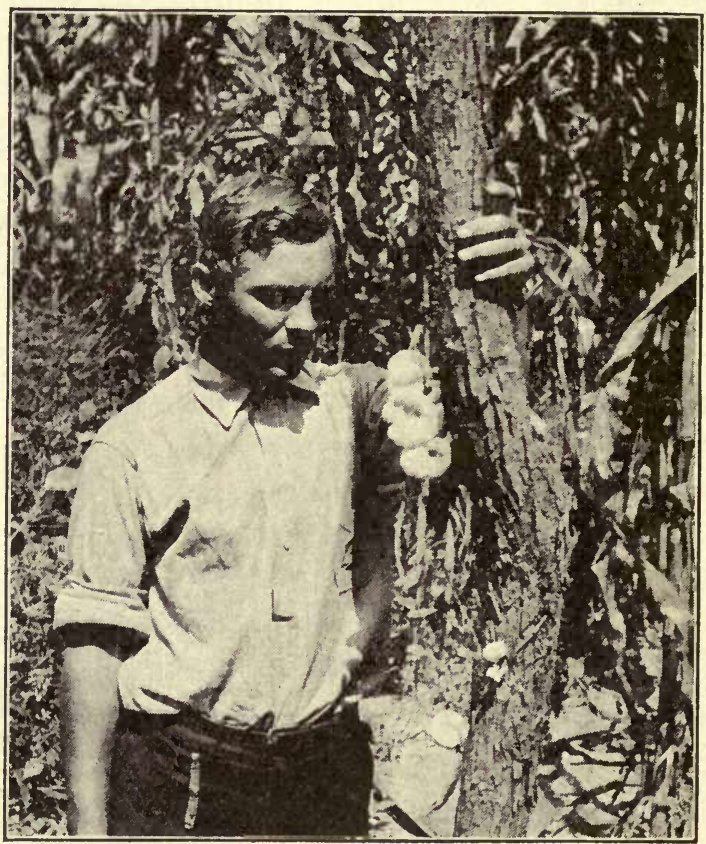

FIG. 290. - Tree Trunk Showing a Bracket Toadstool.

tain no green coloring matter or chlorophyll and so cannot manufacture starch from water and carbon dioxide. They are members of a large plant group called fungi. Such plants commonly are dependent upon decaying animal and vegetable matter for their food. However, some of 
them live on the juices of living plants. Those that feed on living plants are called parasites, while those that live on decaying matter are called saprophytes (Figure 291). Other common fungi are molds and yeasts.

Molds are little plants that grow on many organic substances. To study the growth of mold we have only to moisten some bread and cover it to prevent evaporation. The mold spores on the bread germinate and produce both root and stem threads, on which black knobs full of ripe spores soon appear. These spores are blown about by currents of air.

Yeast plants are simply one-celled fungi (Figure 292). They are useful as manufacturers of carbon dioxide, to raise bread, and in the fermentation of

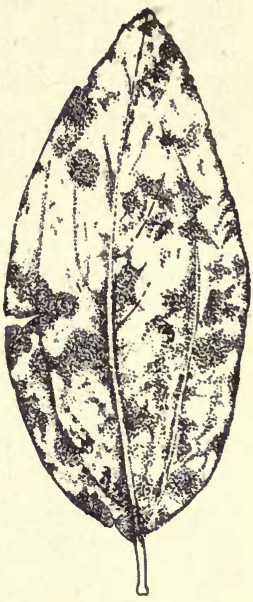

FIG. 291.-A Willow Attacked by Mildew, Caused by a Parasitic Fungus. grains and fruit juices. In the production of carbon dioxide by yeast plants, sugar is changed to alcohol.

Another group of the fungi are the bacteria. Some of these are parasitic to man and produce human diseases such

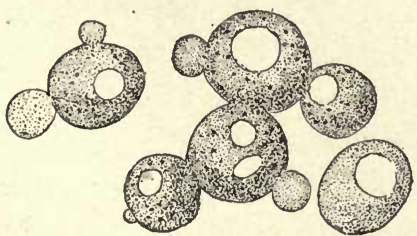

Fig. 292. - Growing Yeast Plants. as typhoid fever, diphtheria, and tuberculosis (Figures 293, 294). However, many of the bacteria are very useful (Figure 295). They cause milk to sour and enable us to make cheese and butter. They cause the decay of organic matter and so change it that it can be used as food for plants. They attach themselves to the roots of plants and help to produce proper food. 
for them. Bacteria on the roots of clover, peas, alfalfa, and similar plants take nitrogen from the air and convert it into plant food. Most plants require some particular kind of bacteria for proper growth. Food may be protected from destructive bacteria by sealing it or by using as a preservative some substance in which the bacteria

Fig. 293. Bacteria Producing Diphtheria.

\section{cannot grow.}

Mosses and Ferns. - Mosses and ferns are plants of higher development than the algx and fungi. They contain chlorophyll bodies, and therefore are able to manufacture starch.

Mosses will grow in extreme climates with little soil, and therefore have probably had considerable part in the formation of soil from rocks.

Ferns form a class of plants slightly higher than mosses. True ferns grow best in damp, shady places. They flourish in the densely wooded regions of the tropics, where they grow to immense sizes (Figure 296).

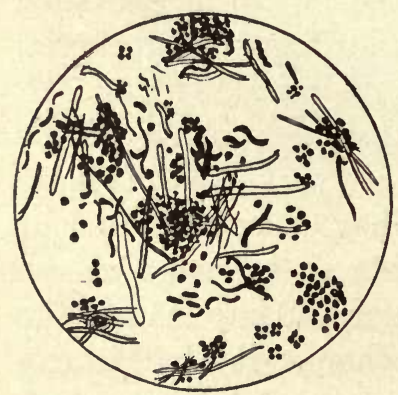

Fig. 295. - Bacteria from a Healthy Mouth Magnified.

The reproductive organs of the common ferns are called sori. They appear as little brown dots on the under surface of the leaf.

Distribution of Plants. - The distribution of plants over the earth's surface is determined by the soil and the climate. It sometimes happens that a soil is rich enough, but the climatic conditions are unfavorable to the 


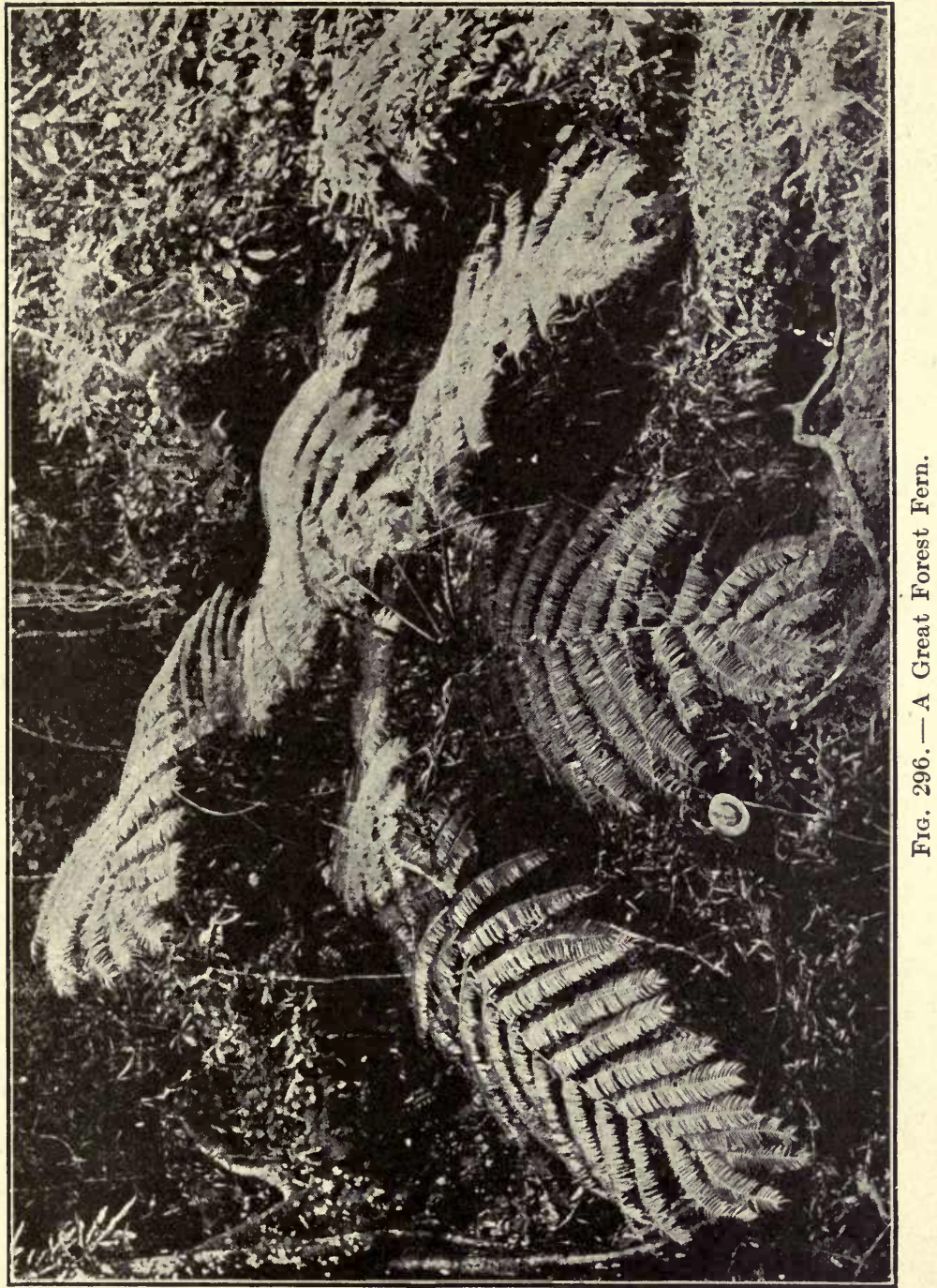


growth of certain plants. The region may be too cold, too hot, too wet, or too dry for the development of a certain plant. Some of our common grains cannot grow in the dry soil of the southwestern states where the giant cactus thrives. In the frigid zone, only mosses and lichens grow. From the Arctic circle to the equator the vegetation differs widely. In the colder part of the temperate zone, conebearing evergreen trees are found. Farther south, in the same zone, are deciduous trees such as the beech, maple, oak, and chestnut; while in the tropics are found the mahogany, cypress, and many varieties of palms.

\section{QUESTIONS}

1. Why is food essential to living matter?

2. When a plant is placed in water it will live for a while. What determines the length of time it will live?

3. Why do seeds not germinate if the ground is too wet?

4. What are the conditions necessary for seed germination?

5. Is light necessary for germination?

6. Name some plants whose seeds are dispersed by winds. By animals.

7. Which develops first, the root or the stem of a plant?

8. Why do roots grow down?

9. Name three commercial uses of bark.

10. What is meant by "quarter-sawing?"

11. From where does the water come that is evaporated from the leaves?

12. Is it essential that a plant lose water by evaporation from its leaves?

13. Name three functions of leaves.

14. Is there any starch in toadstools and mushrooms? Why?

15. Potato sprouts which grow in a dark cellar are white. Why?

16. When are molds and yeasts harmful?

17. How do yeast plants cause bread to rise?

18. How do plants prepare food? From what materials?

19. What is the effect of cultivation on plants? 


\section{CHAPTER XX}

\section{PLANTS FROM AN ECONOMIC STANDPOINT}

The Value of Trees. - Trees are valuable to mankind in many ways. They form a protective covering for a part of the earth's surface; they prevent erosion by

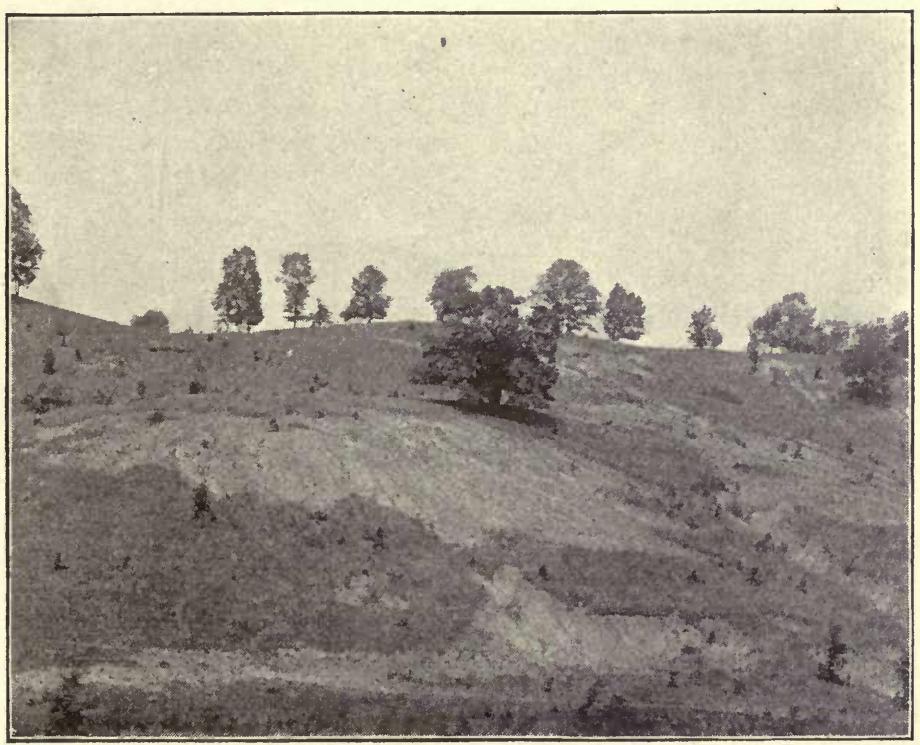

Forest Service, Washington, D. C.

FIG. 297. - Erosion of Unwisely Cleared Slope, Western North Carolina.

surface water; they help to retain the moisture in the soil by keeping the soil loose and lessening evaporation; they furnish valuable commercial and food products 
and they make city and country much more healthful and beautiful places in which to live.

Trees as a Frotective Covering for the Earth. - The soil in a forest is of such a nature that it acts like a sponge in absorbing a large amount of water. The soil is held in place by the roots of the trees, while the foliage of the trees prevents the evaporation of water from the soil. When the forests are removed, surface erosion takes place

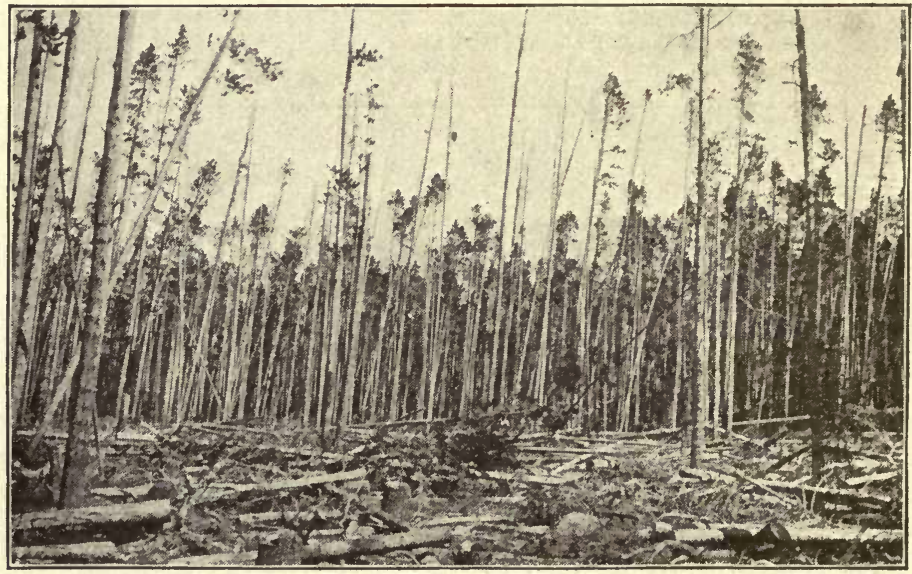

Forest Service, Washington, D. C.

Fig. 298. - A Portion of an Old Sale Area Cut too Heavily and Later Badly Windblown. The Timber is Lodgepole Pine, Medicine Bow National Forest, Wyoming.

much more rapidly (Figure 297). If observations are made while traveling in almost any part of the United States, evidences of such erosion will be seen. Sidehills will be marked with deep gullies made by the surface water as it runs off after rains. Often streams that were never dry when the region was covered with trees remain dry several months in the year after the trees have been removed. 
Uses of Wood. - The forests of the United States have an area of approximately 1,000,000 square miles, but we are rapidly decreasing this area by cutting the trees for the use of their wood. Millions of dollars worth of timber in the United States have been wasted by careless owners and wasteful methods of lumbering, and we are just beginning to take an interest in a reasonable preservation of our forests (Figure 298). In the southern states

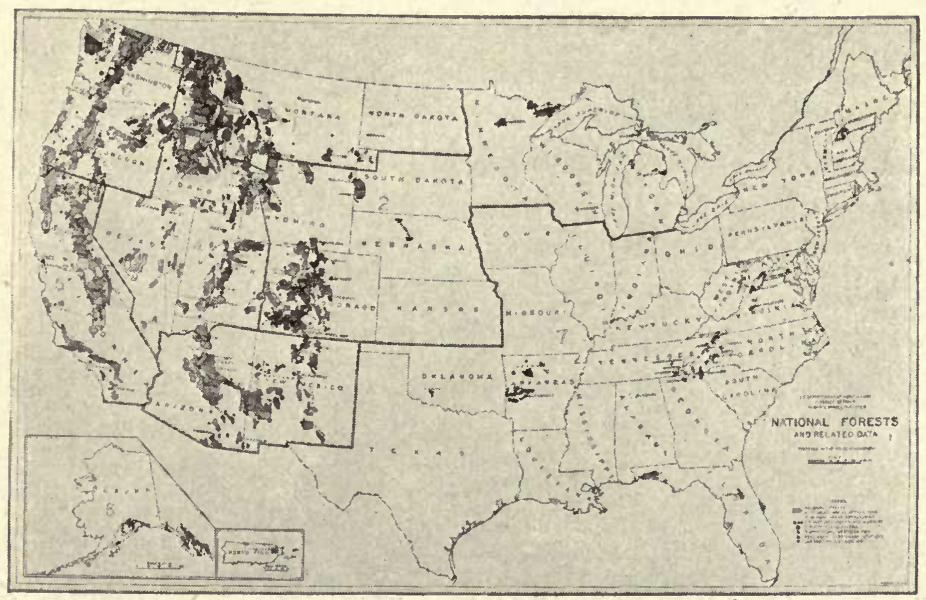

Forest Servicé, Washington, D. C.

FIg. 299. - A Forestry Map.

are vast forests of yellow pine and cypress. In Michigan, Wisconsin, Minnesota, and the northern states of New England are forests of pine and spruce. In the Appalachian region are forests of hardwood, including oak, chestnut, beech, and maple. On the Pacific slope are forests of pine, Douglas fir, spruce, and redwood (Figure 299).

In many countries of Europe the forests are a national care, and the cutting of trees is prohibited except under certain restrictions. Each year our own government 
spends an increased amount of money in Forest Service to guard against the possibility of fires and wasteful lumbering (Figure 300).

Wood is useful for fuel, for building purposes, in the production of wood alcohol, for making charcoal, for paper pulp, for furniture, and various other purposes.

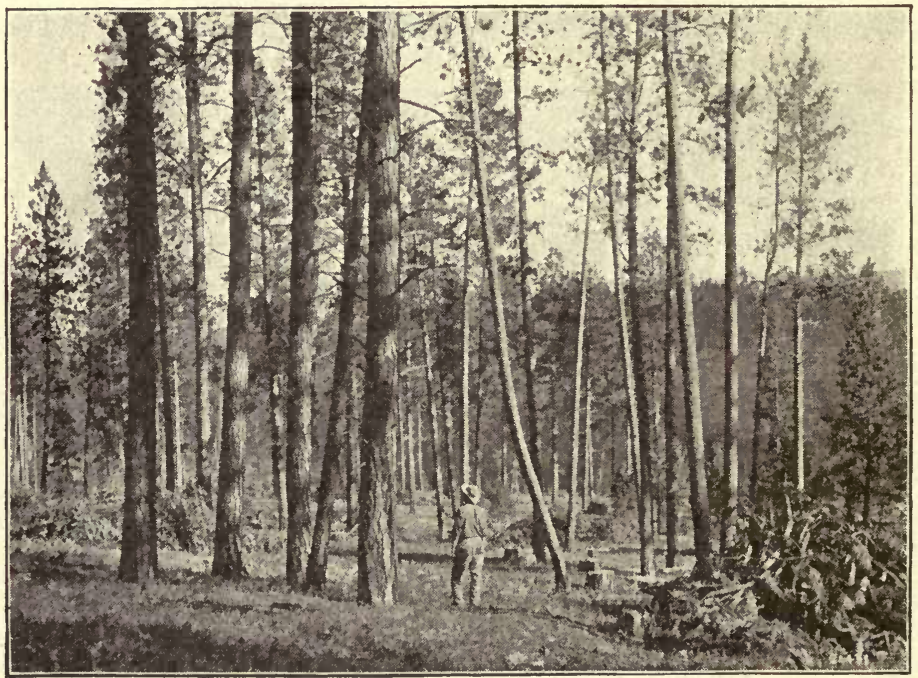

Forest Service, Washington, D.C.

Fig. 300. - Cut-over Area on which Regulated Cutting has been Observed, as Shown by the Brush Piles Ready for Burning, the Low Stumps, etc.

As a fuel it is still used in many parts of the world. Where the waste from the cutting of lumber can be utilized, it is cheaper than most fuels.

As a building material wood has an endless number of uses, ranging from heaviest construction work to the most delicate cabinet work. Some woods are particularly adapted for certain purposes. Pine is used for all building purposes ; cedar is used for shingles ; cypress, for work 
which is exposed to weather ; basswood, for work demanding lightness ; ash, where strength, lightness, and straight grain are desired; oak, cherry, mahogany, rosewood, and walnut for furniture; and so on through the large list of woods.

Trees also furnish a number of other valuable products, such as turpentine, resin, tar, creosote, cork, maple sirup, and some acids and oils.

Other Uses of Trees. - It is gratifying to note the increased attention which our cities are giving to the question of trees. In a large number of our cities we have park commissions, and in a few cities we have city foresters whose duties are to look after the planting and protecting of trees (Figure 301). Some reasons why trees should be planted in a city are: they purify the air ; they enhance property values; they make the city more beautiful; they cool the air in summer by the evaporation of moisture

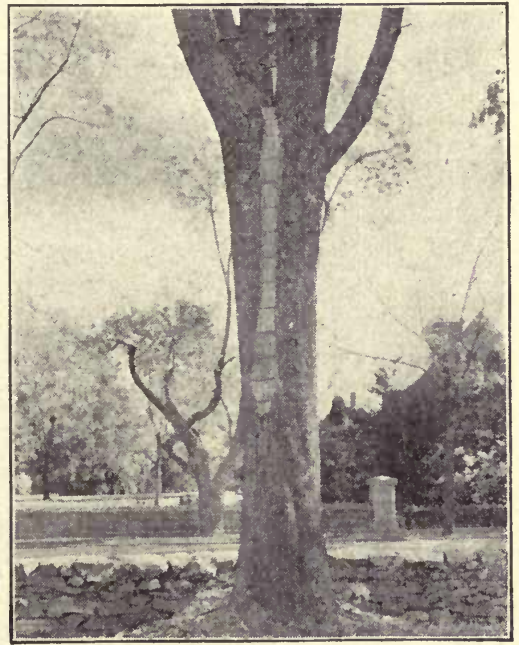

FIG. 301. - A Good Example of Tree Surgery. from their leaves ; they provide shade-for lawns and pavements ; they attract birds ; they have an educational and patriotic influence upon the citizens.

Food Plants. - Nearly all our food comes directly or indirectly from plants. Sometimes it is the root we eat, sometimes the stem, sometimes the leaves, the 
fruits, or the seeds. Even the meats we eat come indirectly from plants, for they come from animals that feed on plants.

Among the common roots that are used by man are radishes, beets, parsnips, carrots, and sweet potatoes. Celery and potatoes are stems used as foods, while cabbage, lettuce, spinach, and onions are examples of leaves used as food.

Fruits and seeds are the most important foods of man. The ease with which grains may be stored and kept for

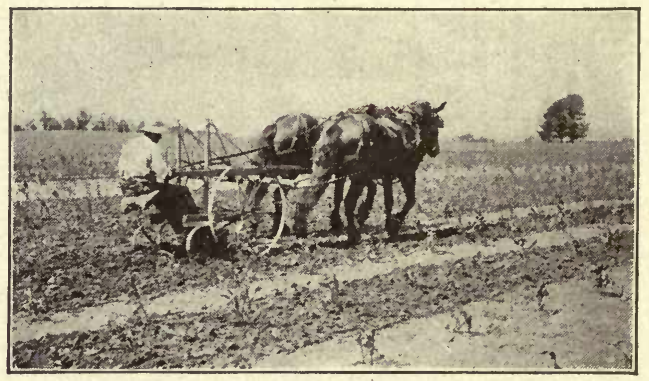

Ohio Agricultural Experiment Station.

FIG. 302. - Cultivating Corn.

future consumption adds greatly to their value as foods. Corn, wheat, and rice are the three most important grain foods of the world, while barley, rye, and oats are extensively cultivated for use as foods (Figure 302).

Textile Plants. - Cotton is the world's most important textile plant (Figure 303). It requires a long season for maturing properly and is therefore grown only in warm climates. Attached to the seeds are long, white filaments which are manufactured into threads and cloth. The cotton seeds furnish an oil which is used as a substitute for olive oil. Other plants which furnish fibers which are useful to man are flax, hemp, and jute. Linen cloth is made 


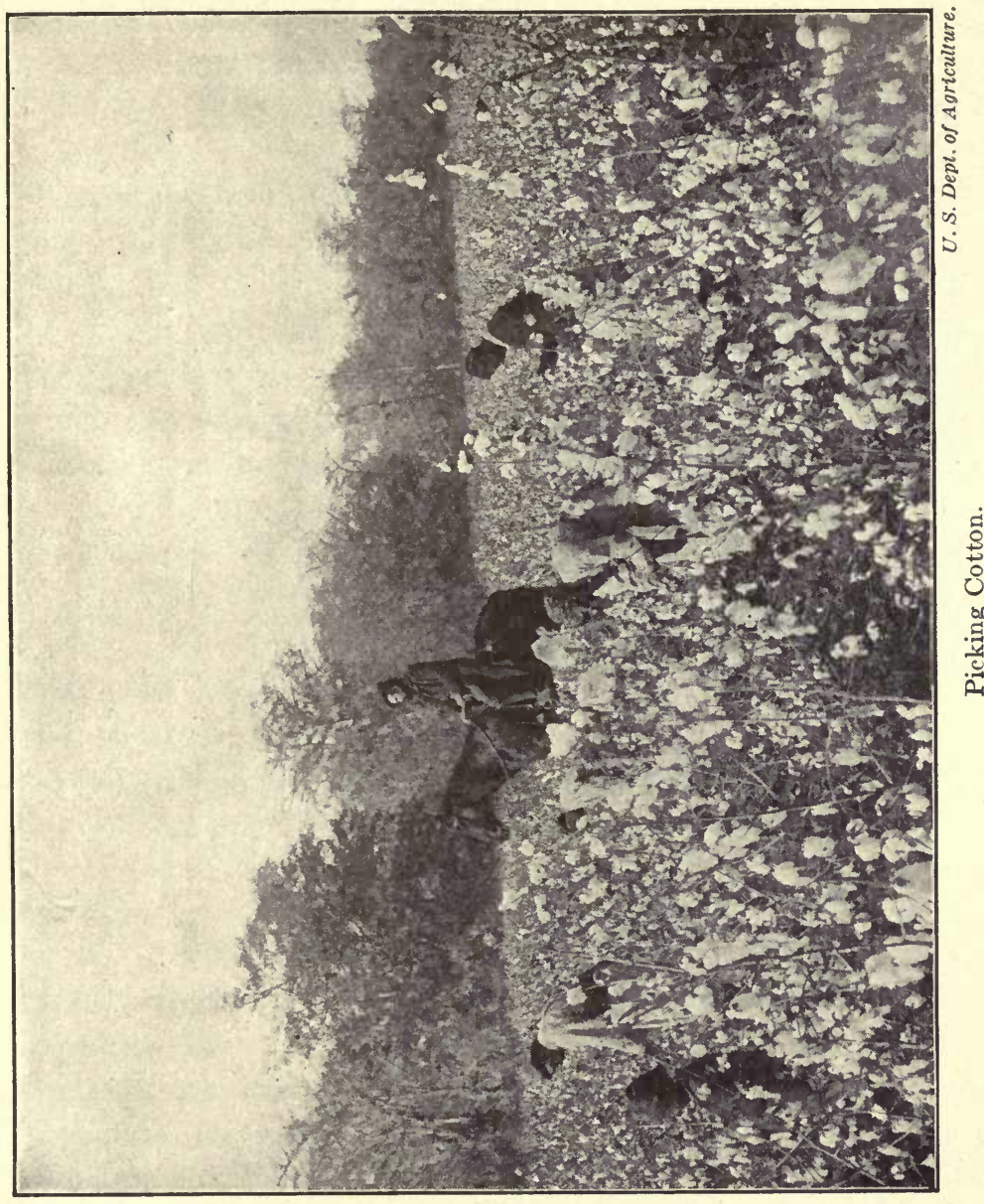



from the fibers of flax. Flax is also grown for its seed, from which linseed oil is made: Hemp has a coarse fiber not suitable for clothing. Its fibers are used for loosely woven materials such as burlap, and also for ropes.

Weeds. - Weeds are plants that have little or no economic value. They are plants that in some particular place are not wanted. In a garden all the plants which are not cultivated as flowers or vegetables are weeds. In a tennis court all plants are weeds. Most weeds seem to be unusually hardy. They possess great ability to disperse their seeds and to resist the extremes of

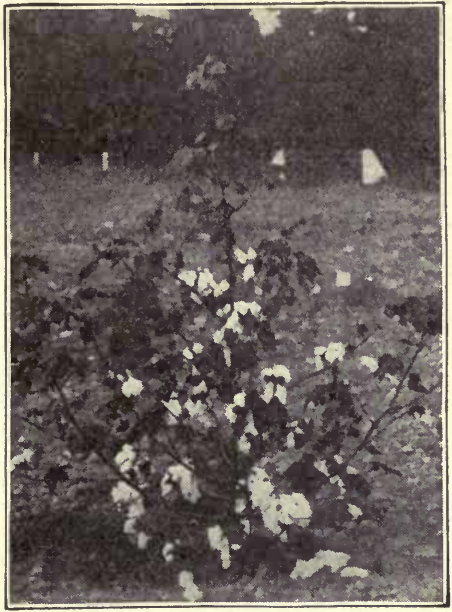

Fig. 303. - A Cotton Plant. climate and the attempts of man to eradicate them. They flourish in poor soil and in fertile soil. They preëmpt the soil and absorb the plant food so that other plants are crowded out or die from lack of food. A number of weeds are so persistent in their growth and spread so rapidly that the United States Department of Agriculture has classed them as national pests and has adopted radical measures leading to their extermination.

Weeds have some real value. They help to make soil and to renew soils in worn-out regions. When soil, through poor farming, becomes too poor to grow crops, weeds will still grow on it and build up the soil. Weeds are also valuable in that they force farmers to cultivate the land. 
Some few plants are poisonous. Probably one of the best known of these is the poison ivy, a three-leaved climbing plant which attaches itself to walls, trees, and fences by means of small roots growing from the stem. Berries from wild plants should not: be eaten until their identity is determined, since some of them are poisonous.

Plant Diseases. - Many parasitic fungi live on useful plants which are cultivated. They attack trees, grains,

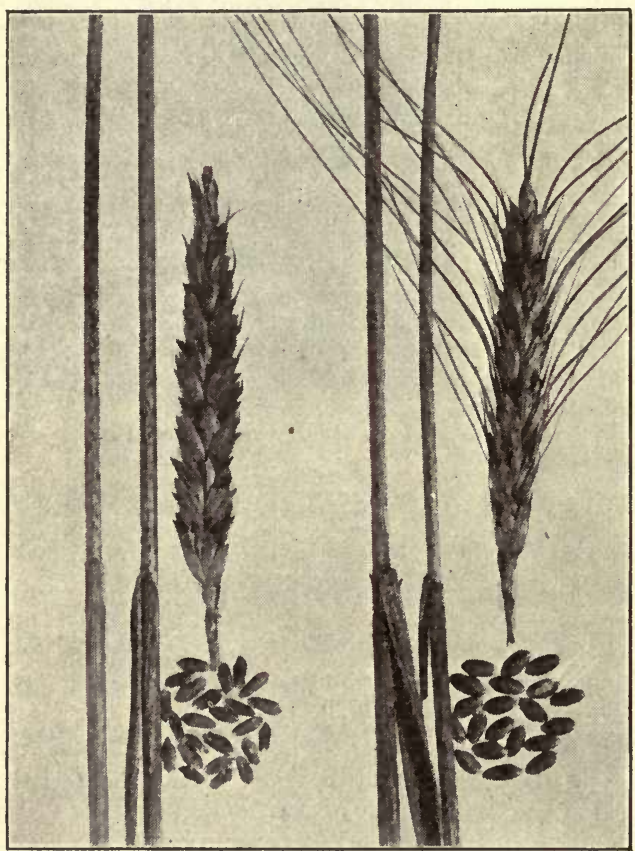

U. S. Dept. of Agriculture. Fig. 304. - Wheat Heads and Straw Showing Rust.

fruits, and vegetảbles to such an extent that the damage done by them annually amounts to millions of dollars, while probably as much more is spent in combating them. The most common parasitic plants are rusts, molds, smuts, and blight-producing fungi.

Wheat Rust. For many years wheat rust, has been one of the most destructive and most dreaded of plant diseases because it destroys a plant upon which so large a part of the civilized world is dependent for food (Figure 304). It has long been suspected but only recently determined beyond a doubt, that 
the parasite passed part of its life on barberry bushes and then transferred its place of living to the wheat plant. It appears on the wheat leaves and stalks as a collection of reddish brown spots. It extracts its food from the leaves of the wheat plant, which is so weakened that no grain is produced. Since the spores germinate readily only on the barberry, the remedy seems to be to destroy the barberry. Rotation of crops aids in controlling this disease.

Brown Rot.

A common fungous disease is the brown rot, which attacks stone fruits, particularly plums and peaches. It attacks the fruit on the tree, its appearance being marked by a brown spot. The rotted fruit falls to the ground, or shrivels and clings persis-

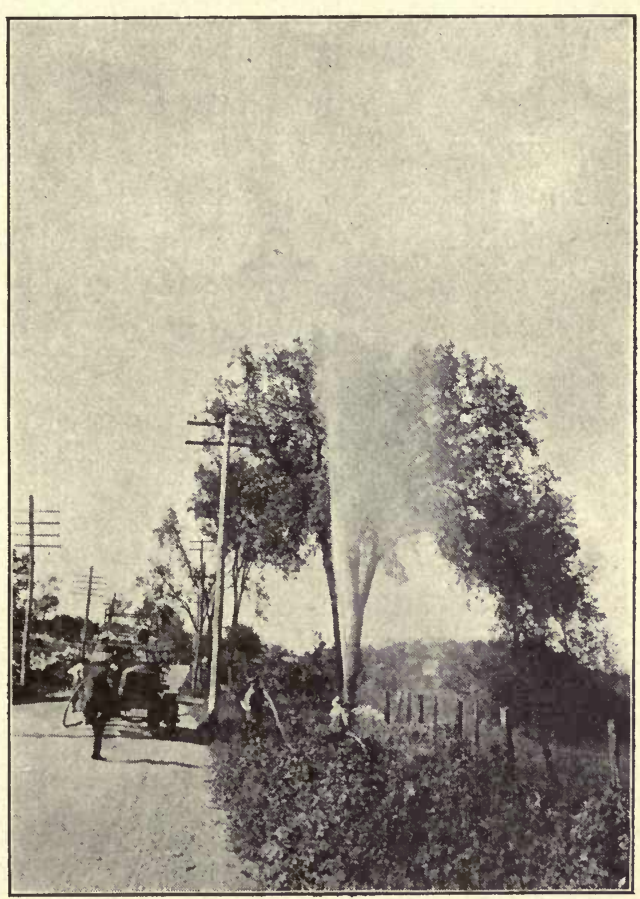

U.S. Dept. of Agriculture.

Fig. 305. - Motor Truck Sprayer in Operation. tently to the tree to form mummies that carry the disease over the winter. In the spring the spores which develop in countless numbers in these mummies are carried by the wind to the tree blossoms, where they begin their work again. The disease may be partially controlled by 
burning the diseased fruit as soon as it appears and by spraying early with Bordeaux mixture and later with lime-sulphur (Figure 305).

Pear Blight (Fire Blight). - Pear blight attacks pear trees, apple trees, and occasionally plum trees. It is caused by bacteria which live in the cambium layer just under the coarse bark. The first symptom of the disease is the death of the tips of the tender twigs. The leaves turn yellow and then dark brown, and the tree seems to be dying from the top down. In driving along the road one may often see orchards affected by this disease. If neglected, the disease will spread down the tree and to other trees, gradually destroying the whole orchard.

The diseased limbs should be removed and burned as soon as they are detected, but something more than this is usually necessary to eradicate the disease. The bacteria move down the tree and form cankers on the large limbs and on the body of the tree. Here they pass the winter. In the spring these cankers exude a sticky liquid containing large numbers of the bacteria which are carried to the flowers and other parts of the tree by insects. The logical point of attack then is the canker during the winter months. Remove all cankers and scrape the diseased parts. Wash the wound with a weak solution of corrosive sublimate, one part to five hundred of water. In a day or two the wound should be painted with lead and oil.

Mildews. - The downy mildews comprise a group of fungi which have been very destructive to cultivated crops. Probably the most destructive mildew is that of the potato, sometimes called late blight. The mildew spores are always present in the atmosphere in the summer time, and if they alight on the potato leaves when the 
proper conditions of heat and moisture are present, they produce swarm spores that soon germinate, each one sending out a slender tube which enters a near-by breathing pore of the leaf. Once inside the leaf they grow rapidly and send out numerous branches which absorb the contents of the cells and even pass down through the stalk to the tubers below. Wherever they go they kill the plant cells quickly, and the term blight is quite applicable (Figure 306). In a few days the plants of the whole field may present a dry, parched ap- pearance, since the roots (mycelium) of the mildew in the leaves send out branches which develop millions of spores. Thesemay be carried by the

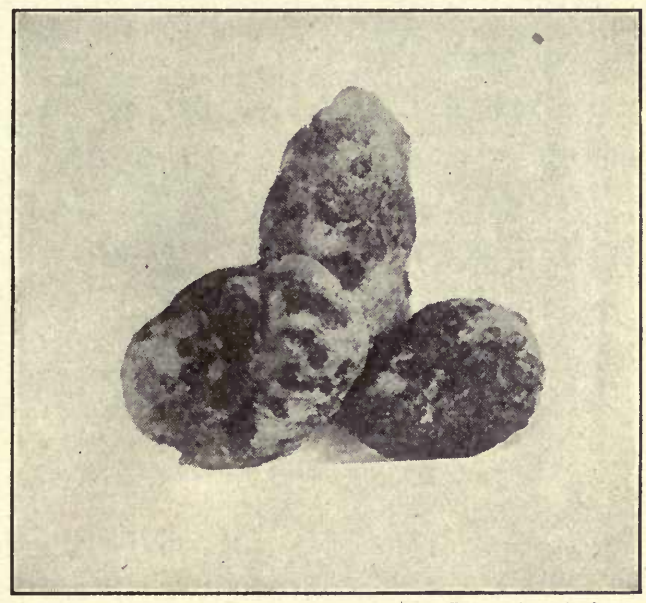

U. S. Dept. of Agriculture.

Fig. 306. - Advanced Stage of Early Blight on Potatoes.

slightest wind to other parts of the field. This fungus commonly passes the winter in diseased potatoes; hence great care should be taken to secure good seed potatoes. Spraying with Bordeaux mixture will prevent the spread of the disease.

Other mildews attack lima beans, onions, citrous fruits, grapes, and many other fruits and vegetables. The brown rot of the grape and of the lemon are downy mildews which are at times quite destructive.

Potato Scab. - This fungous disease attacks potatoes 
and may do much damage (Figure 308). It may live in the soil over winter; in such cases the only remedy is a

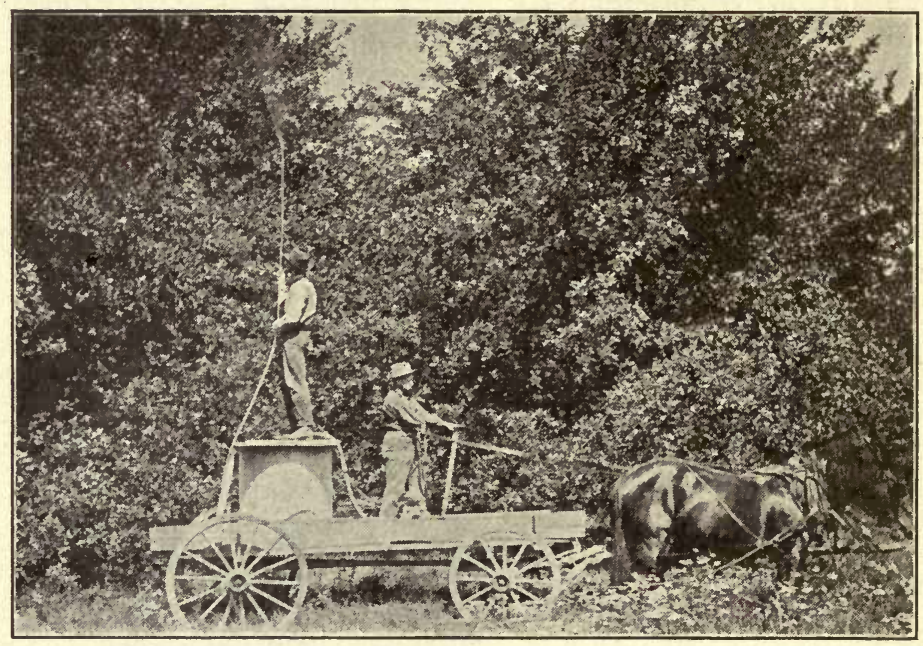

U.S. Dept. of Agriculture.

FIG. 307. - Suitable Spraying Outfit for a Small Orchard.

rotation of crops. If the soil is free from the fungi, the disease may be avoided by soaking the seed potatoes for an hour in a solution of formaldehyde, one pound of formaldehyde to

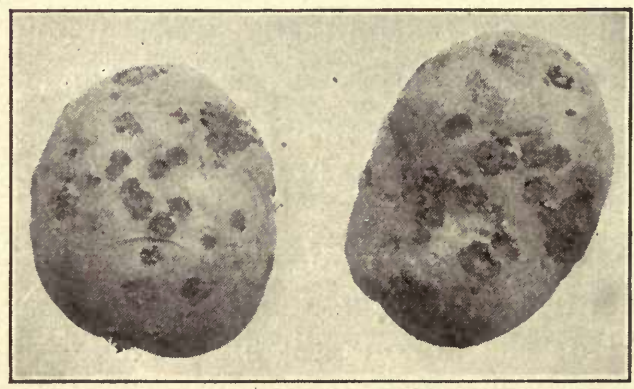

Ohio A gricultural Experiment Siation. Fig. 308. - Potato Scab. thirty gallons of water.

\section{Chestnut canker} is a fungous plant which was only recently introduced in this country, probably from Japan(Figure 309). As the plant de- 
velops, millions of spores are produced, which are blown about by the wind. When they alight on the bark of trees and sprout, they send little roots into the tree and absorb the food which is on its way to living cells. In a short time the tree dies from starvation. The only remedy seems to be the removal of infected trees.

Molds. Experiment 78. - Make some marks on a piece of fresh bread with a toothpick that has been drawn across a piece of moldy bread. Now put the piece of fresh bread in a covered can or under a dish away from the light and observe it from day to day.

Molds develop from spores which are usually present in the air. When these spores fall upon a moist substance which contains suitable food, they send out fine, rootlike threads which both cover and

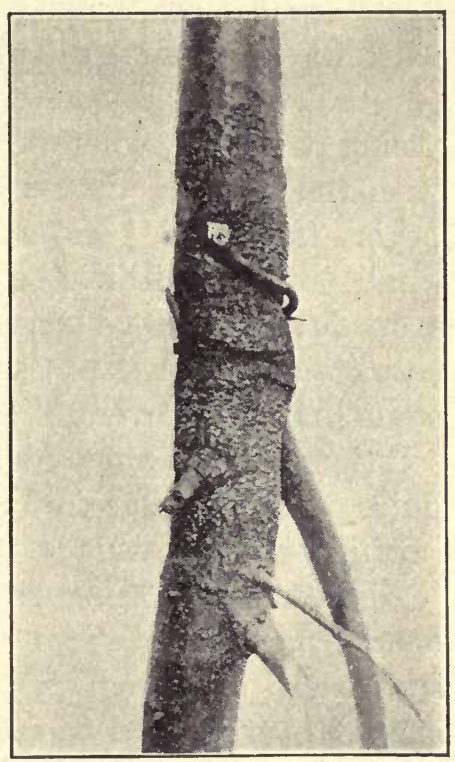

Ohio Agricultural Experiment Station.

Fig. 309. - Chestnut Canker. penetrate the surface of the attacked substance. Mold will grow on most common foods. When the spores fall on a jar of jelly, they germinate if the temperature is favorable, and soon the jelly is covered with a layer of gray mold. To kill the mold spores it is only necessary to heat the food to a temperature of $90^{\circ} \mathrm{C}$.

As a usual thing molds are destructive and render foods unfit to eat. However certain foods, as Roquefort, Camembert, and Brie cheeses depend upon the molds for their characteristic flavor. 
Smuts. - Many of us are familiar with the masses of loose black or dark brown powder which are found on the heads of oats. These black masses are composed of millions of minute spores of a fungus known as oats smut. These spores are carried through the threshing process with the chaff that is usually mixed with the grain. When planted with the oats, they germinate and enter the young oat plant, where they branch in various directions with the growing tissues of the plant. When the plant begins to "head," the smut fungus develops a mass of small threads within the tiny blossom. These threads soon develop the countless black spores that form the characteristic powder of smut as we see it on oats. Oats smut may be prevented by soaking the seed in a dilute solution of formaldehyde. Corn and onion smuts are quite common on ground where the crops are not rotated, but they are not usually very troublesome.

Black knot is a fungous disease which affects plum and cherry trees. In warm weather large numbers of the spores are produced on these knots. These spores are carried by the wind to other trees, where they are likely to germinate and start the disease.

All diseased branches and badly infected trees should be cut down and burned. Spraying diseased parts with fungicides will prevent the spread of the disease. (See Appendix II.)

Peach leaf curl is due to the growth of a parasitic fungus which enters the leaf, causing it to become enlarged and to curl. The injured leaves fall early in the summer. Winter spraying with Bordeaux mixture or with limesulphur will destroy the spores of this fungus.

There are other fungous plant diseases which are peculiar to small regions. As a usual thing they yield easily 
to treatment. When requested to do so the State Agricultural department will usually furnish pamphlets dealing with the crop pests of that state and the best methods of destroying them.

Yeast. - Man has learned how to use yeast plants so that they have an economic value. All fermentation and the process of modern bread making depend upon the yeast plant.

Experiment 79. - Add a fourth of a cake of compressed yeast cake to a pint of water containing some molasses or sugar. Now divide this mixture in three parts, placing each part in a small jar. Cover the jars and place one of them on ice. Place another in a moderately warm place, and put the third in a water bath and subject it to a boiling temperature for ten minutes, after which place it near the second jar. Two days later examine the jars. Have any bubbles appeared in the jars? In which jar or jars? What effect has heat upon yeast plants? What effect has cold? Devise an experiment to determine the kind of gas released by yeast plants. What does baking do to the yeast plants in bread?

\section{QUESTIONS}

1. In what ways do.trees cool the atmosphere?

2. What precautions are now being taken to prevent forest fires?

3. Cypress lasts better when not painted. Why?

4. Name two uses of the maple tree.

5. How is science increasing the value of our food plants?

6. How many bushels of corn were grown in the United States last year?

7. Where is hemp grown?

8. Name ten weeds.

9. How do fungi differ from common plants?

10. How do spores differ from seeds?

11. What are the worst plant diseases of your region!

12. Name some other plant rusts besides wheat rust. 


\section{CHAPTER XXI}

\section{ANIMAL LIFE}

Relation of Plants to Animals. - The simplest plants are composed of a single cell. The simplest animals also are composed of a single cell. Both plants and animals possess the power of irritability; both have the power to change their position; and both possess the power of reproduction. The elements necessary to the growth of plants and animals are all found in the soil, the air, and the water. There is a difference however between plants and animals. Animals do not have the power of making their own foods from the elements found in the soil, air, and water, but are dependent upon plants for certain essential foods. - Animals require green plants. The protoplasm of green plants reacting under the influence of sunlight manufactures food which is used by both plants and animals. Whether an animal is herbivorous, eating plants only, or carnivorous, eating flesh only, its food can be traced to the same source. Animals that eat both plant and animal flesh are called omnivorous.

In the scale of animal life we have many gradations from the simplest one-celled animal to man, the highest type of vertebrate. Between these two extremes we have such animals as worms, oysters, snails, lobsters, insects, fishes, reptiles, birds, and animals like the horse.

One-celled Animals. - Amœbæ are one-celled animals which may be obtained for purposes of study from vegetation growing near the surfaces of ponds. Unlike other 
cells we have studied they have no fixed form but have the appearance of an irregular mass of protoplasm which changes in form constantly as it moves about.

There are no separate organs in these animals. The one cell performs all the functions necessary to their

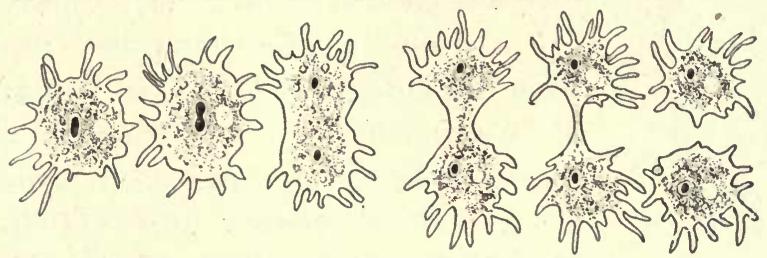

FIG. 310. - Amœba Showing Division.

life. Reproduction takes place by a division of the cell into two cells (Figure 310). In the amœba any part of the cell seems to be able to perform all its functions, such as absorbing food and oxygen and excreting waste material (Figure 311). Slightly higher forms of single-celled animals have a mouth through which food is taken.

If a small bunch of hay is placed in a glass jar nearly filled with water and allowed to stand for a few days in a warm room, certain changes will be noticed in the contents of the jar. An unpleasant odor indicates that the
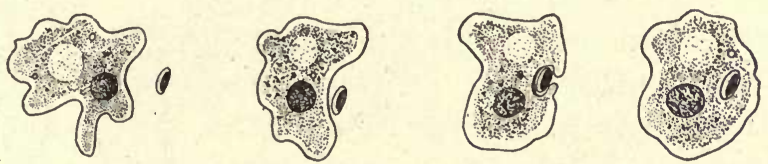

Frg. 311. - The Way an Amœba Gets its Food.

hay is decaying; a bacterial scum appears on the surface of the water; a little later small one-celled animals appear in this scum in such quantities that the surface of the water seems literally alive with them. These 
little animals must of course come from the water, the air, or the hay. They were there in a dormant state.

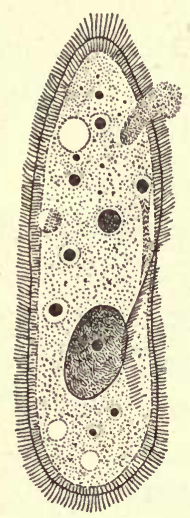

Fig. 312.- A Paramecium. We simply adjusted conditions so that these were favorable to their development. Such an animal is called a paramecium (plural paramecia) (Figures 312, 313). It is a more complex organism than the amcba. Under the microscope it is seen to have a somewhat regular shape, to have the power of moving itself by means of special parts of its cell, called cilia, and to take its food through a definite opening or mouth.

Division of Labor. - As we observe animals higher in the scale of life we find that certain parts of animals have a definite work to do. A part of a plant or animal which has a special work to do is called an organ. Thus we have organs that take food; organs that digest food; organs for the circulation of blood and other fluids; organs of breathing; organs of excretion; organs of voluntary movements; organs of special senses; nerve organs; organs of reproduction; and organs of protection. The refinements in the functions of the various organs of an animal determine its position in the scale of life, just as the degree of the development of the principle of "division of labor" determines the civilization of a people.

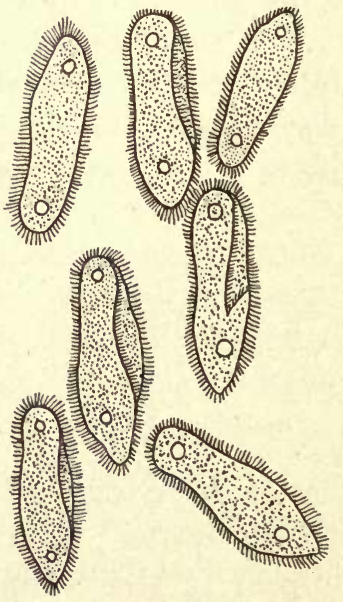

FIG. 313.-Paramecia. 
Hydra. - The hydra is a fresh-water animal which resembles a hollow bag. Food is carried into the animal by little tentacles which grasp the food and carry it toward the mouth (Figure 314). The body wall of the hydra is made up of two layers of cells. The inner layer serves to digest the food, while the outer layer serves as a protective covering for the inner layer. Between these two layers of cells are some muscular fibers and nerve cells which enable the animal to receive sensations and to move the parts of its body, or to change its position.

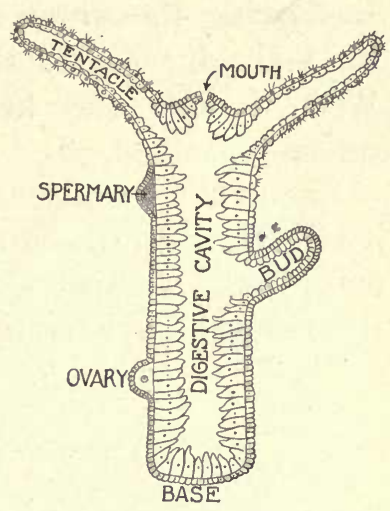

Fig. 314. - The Hydra.

Worms. - Earthworms must be classified among our most useful animals (Figure 315). They break up the soil, thus allowing water and air free passage through it. They also carry large quantities of deep soil to the surface, thus mixing the soil and making it more fertile. The damage they do to growing plants is small compared with

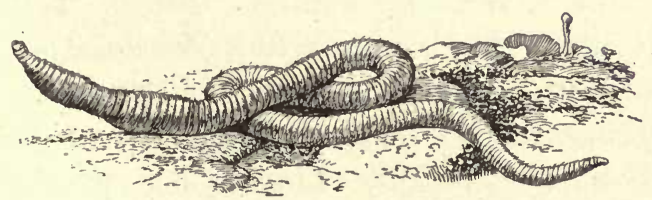

Fig. 315. - An Earthworm. the good they do in the soil.

Earthworms furnish an interesting laboratory study. They may be kept for some time in a box filled with soil. Notice how the worm moves. It has two layers of muscles which provide for its movements : an outer layer which passes in a circular direction around the body, and another longitudinal set which runs 
the length of the body. The body is shortened by the contraction of the muscles running lengthwise and lengthened by the contraction of the circular muscles. In moving the worm extends its head and then shortens its body by contraction of the longitudinal muscles. Why doesn't the head slip back as these muscles contract?

Examine the under surface of an earthworm with a good lens. How are these anchors used? The worm has no eyes, yet it is quite sensitive to light. How can you test it to determine whether it likes light or darkness?

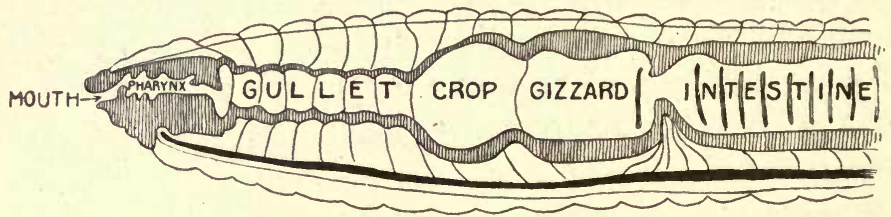

Fig. 316. - The Food-tube of an Earthworm.

These worms get their food from plants and from the soil which passes through their bodies. They are able to burrow into very hard soil. This they do by literally eating their way through (Figure 316 ).

The earthworm has a nervous system with a welldeveloped sense of touch. It also has a blood circulation. It has no lungs, the skin acting as an organ of breathing. To facilitate the passage of air (osmosis) the skin of the worm is kept moist by a secretion. During heavy rains the worms often come to the surface, because as the ground becomes soaked they are covered with water and cannot get sufficient oxygen. There are many other kinds of worms, but the earthworm is typical of the whole class.

We must be careful not to confuse caterpillars, grubs, and similar larvæ with worms. Worms retain their 
identity through life, while the larvæ simply represent a stage in the development of insects.

Insects. - All insects have six legs in their mature stage. This feature distinguishes them from crustaceans, which always have more than six legs, usually ten, the spiders, which have eight legs, and from the centipedes, which have many legs. Insects have bodies which are

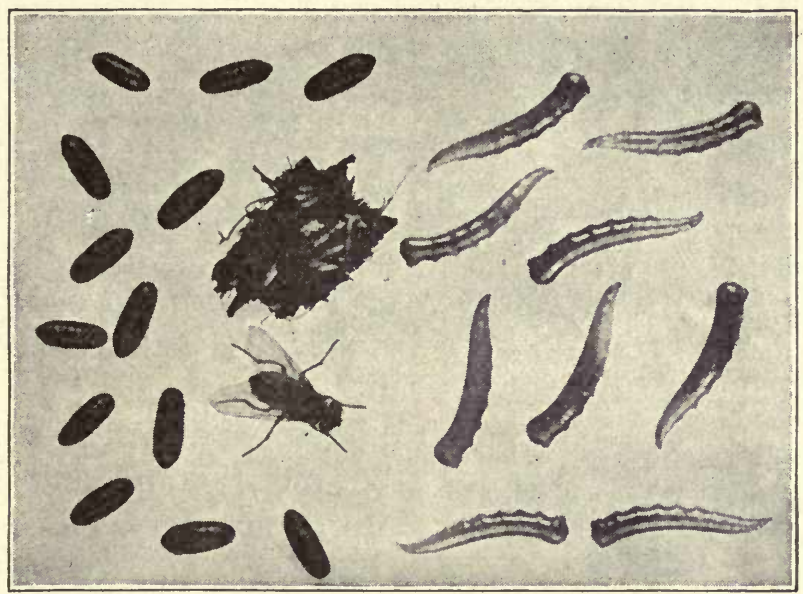

Fig. 317. - Stages in the Development of the Fly.

divided into three parts: the head, the thorax, and the abdomen. They breathe air directly by means of air tubes or trachea, which permeate all parts of the body cavity.

Insects like the common house fly pass through four different periods in their development. The female may lay from one hundred to two hundred eggs. From these eggs the maggots or larvæ hatch (Figure 317). After a few days of feeding these maggots go into the pupal stage. In about one more week the adult fly emerges 
(Figure 318). The adult fly breeds at once. Since the whole cycle from egg to fly requires but two weeks, it is easy to account for the large number of flies in late summer.

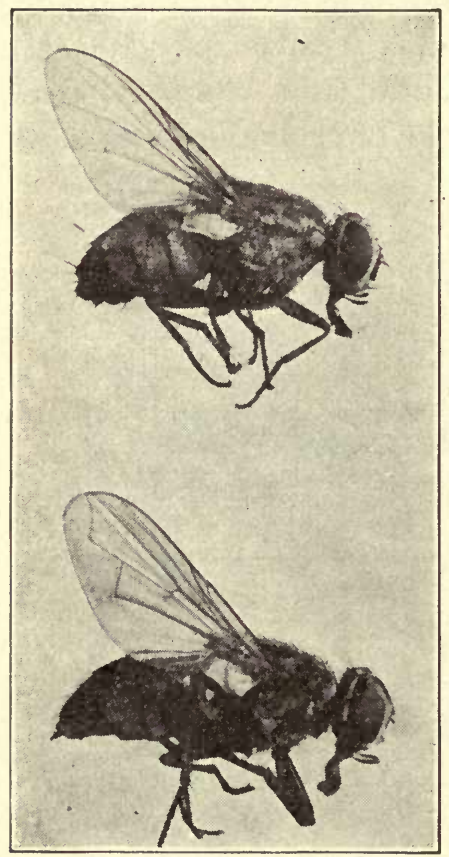

Fig. 318. - The House Fly, Male (top); Female (bottom).

Fortunately most of them are killed by the winters of the temperate regions.

Insects form by far the largest class of animals. Some of them are valuable, but many of them are destructive and live at the expense of valuable plants. Typical insects are the locusts or short-horned grasshoppers, the butterflies, the moths, the keetles, the cicadæ, the bees, ants, and wasps.

Bees and Ants. - From the social standpoint these insects are very interesting. They seem to have worked out a scheme for community life which provides for a government and a division of labor (Figure 319).

The honeybee is the most interesting individual of this class of insects. In their wild state honeybees live in colonies with a hollow tree for a home. There are three kinds of bees in a colony: the male bees or drones, the workers, and the queen or female bee (Figure 320 ). If it is possible to have a hive of bees for observation, the division of labor may be easily seen and studied. The queen's work is to lay all the eggs. This she does 
with great regularity during the warm weather, sometimes laying as many as several thousand eggs in a day. Most

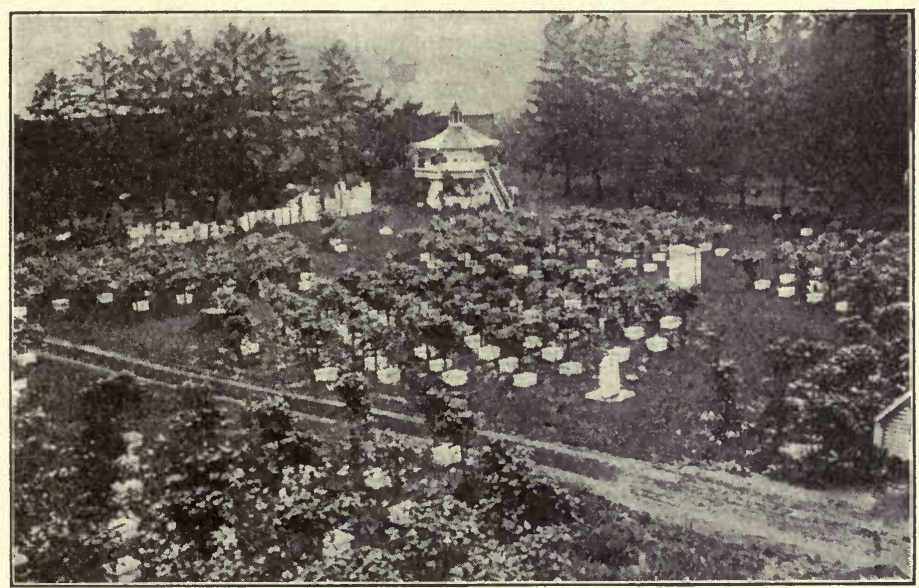

FIG. 319. - Section of a Bee Farm.

of the eggs are fertilized by the sperm cells of the males. The unfertilized eggs develop into males. The workers

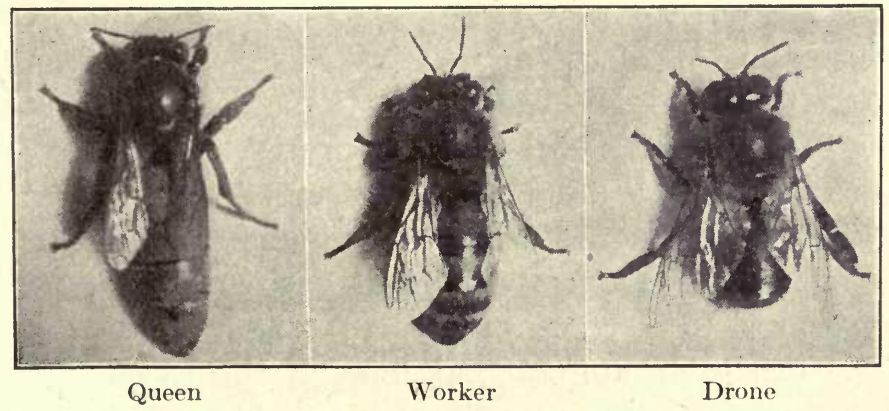

Fig. 320.

do all the work of the hive. They make the honey and the wax and feed the larvæ or grubs as they hatch, 
on food which has been partially digested in their own stomachs. After a few days the grub is given a supply of food and sealed in a cell by the workers. In about two weeks it breaks out as an adult worker.

When a new queen is to be produced, the young larva is fed during the whole pupal period upon the special food known as bee jelly and grows to a larger size than the workers. When a young queen appears, the workers usually divide. Some of them remain in the hive with the new queen, while others follow the old queen out of the hive. If the old queen alights, they settle around her, forming a mass of bees. This is called swarming. To prevent them from leaving it is necessary to provide new quarters for them.

The queen bee may live for several years, but the workers live but a few months. The drones are driven out or killed by the workers. If bees are properly cared for and provided with a source of food, they will produce enough honey to be quite profitable. Since they visit so miany flowers, they are also valuable as carriers of pollen.

Fishes. - All of the animals we have studied thus far have either had no skeleton or have had an exterior skeleton. They are called invertebrates. There are five classes of animals having flexible vertebral columns or backbones. They are fishes, amphibians, reptiles, birds, and mammals. Collectively these five classes are called vertebrates.

The fish is an animal that lives in the water. Most fish are protected by platelike scales, which overlap each other in such a way as to leave the body flexible. The tail fin of a fish is the principal organ of motion, although the other fins all have something to do. The fin on the 
back keeps the fish right side up, while the side fins are used in steering.

The specific gravity of a fish is nearly that of water. By means of an "air bladder" the fish may increase or decrease its volume enough to cause it to sink or rise in the water. The cartesian diver illustrates this principle admirably. Fishes breathe by means of gills which take oxygen from the water. The fish has a well-developed circulatory system, including a heart with two chambers.

Amphibians. - Amphibians are animals that live both on the land and in the water, as the name indicates. "Amphi " means both and "bia" means life. Common amphibians are frogs, toads, mud puppies, and salamanders. In the primary stages of their development these animals breathe in oxygen by means of gills Most of them undergo a complete change during life. Some of them develop lungs in their second stage. The circulation is of slightly higher development than in the fish, the heart having three chambers, two auricles and one ventricle.

The frog is an amphibian which may be easily studied. In the early spring the frogs lay their eggs in water. In

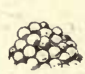

1

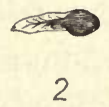

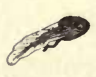

3

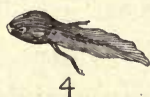

Fig. 321. - The Development of the Frog.

a short time these eggs hatch into tadpoles. Tadpoles resemble fish and breathe with gills as fish do. As the tadpole develops, legs appear on the body, and its organs of breathing change from gills to lungs. The toad tadpole develops in a month or two after hatching, but the frog tadpole does not make the complete change to a frog until the second summer (Figure 321). 
Reptiles. - Reptiles are lung-breathing vertebrates having a scaly tough skin. Common reptiles are snakes, turtles, alligators, and crocodiles. In the turtle the scaly covering has developed into a bony shell. Reptiles are related to birds. They reproduce themselves by means of eggs which they lay in the ground. A few snakes are poisonous. In the United States the most dangerous are the rattlesnake, the copperhead, and the water moccasin.

Birds. - The principal characteristics of birds are their protective covering of feathers and the presence of wings. The feathers are developed from the skin and show a variety of form and color. The bills of birds also exhibit a variety of forms adapted to the ways in which the different birds get their food. Birds of prey have a hooked beak. The woodpecker has a sharp straight bill. Birds breathe much faster than we do and have a very rapid circulation of blood. This causes a high body temperature. The body temperature of birds is often eight or ten degrees Fahrenheit higher than that of man.

Mammals. - The class of animals known as mammals includes man, a large number of quadrupeds such as the dog, cat, sheep, pig, horse, and cow, and a few animals like the whale and seal. They are called mammals because they nurse their young with milk secreted by glands known as mammary glands. Other characteristics of mammals are their covering of hair, well-developed lungs, and a highly developed nervous system.

Most of the mammals live on land. Some, like the seal and sea lion, inhabit islands and waters near the land; a few, like the whale, live in the ocean. These animals are variously adapted to their particular kind of life. Some live in trees; others burrow in the ground. Some 
eat only flesh ; while others eat nothing but plants. Some bring forth their young well developed to a form similar to their own, while others, like the kangaroo, carry their immature young in a pouch on the under side of the body until they are able to care for themselves.

Animals used as Food. - The lowest forms of animals are not used by man as food. However, these animals are eaten by higher forms of animals, and they in turn become the food of man.

Mammals, birds, fish, mollusks, crustaceans, amphibia, and reptiles are extensively used as food. Among the mammals and birds so used are many game and domestic animals. The number of edible varieties of fish is large. The different varieties of turtles are used as food by nearly all peoples of the earth.

Oysters and clams are sold in large quantities as food. The oyster industry is one of the most profitable of our fisheries and furnishes employment to a large number of men. The most productive oyster grounds are Long Island Sound, Chesapeake Bay, and Narragansett Bay.

Among the crustaceans lobsters and crabs are valuable foods. More than twenty million lobsters are taken annually along the north Atlantic coast.

In addition to the animals which are used directly as food there are many animal products which are so used, such as butter, cheese, and honey.

Animal Products used for Clothing. - Wool is a very valuable animal product (Figure 322). After the wool is cut from the sheep it is cleaned, carded, and woven into cloth.

The silkworm or caterpillar furnishes all of the real silk that we have. The worm lives upon leaves, prin- 


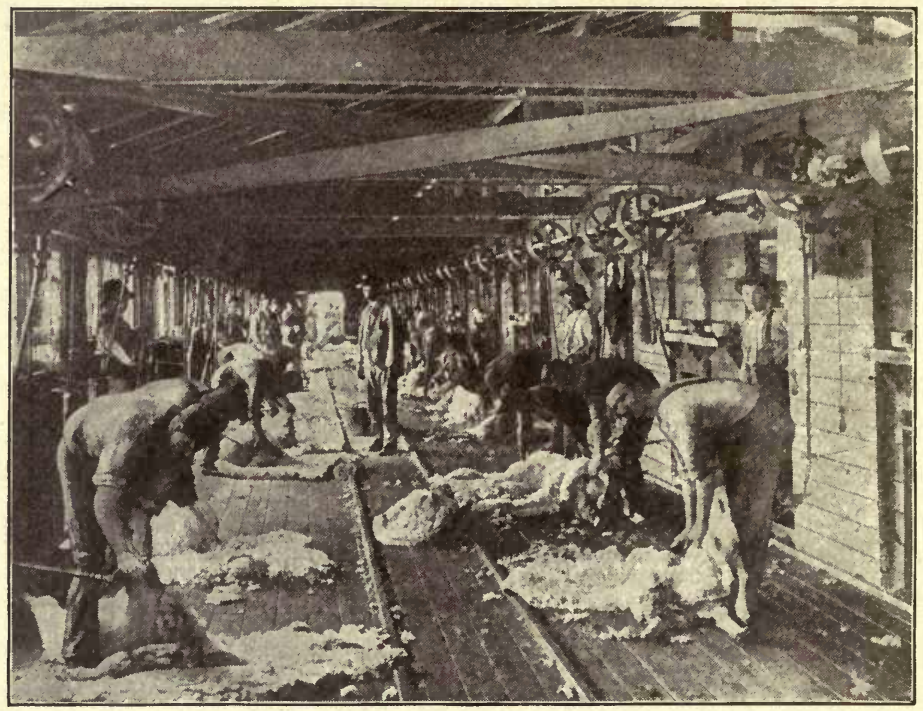

Fig. 322. - Shearing Sheep, New South Wales, Australia.

cipally those of the mulberry, and makes a cocoon from which the silk is obtained (Figure 323).

Furs from wild and domesticated animals are exten-

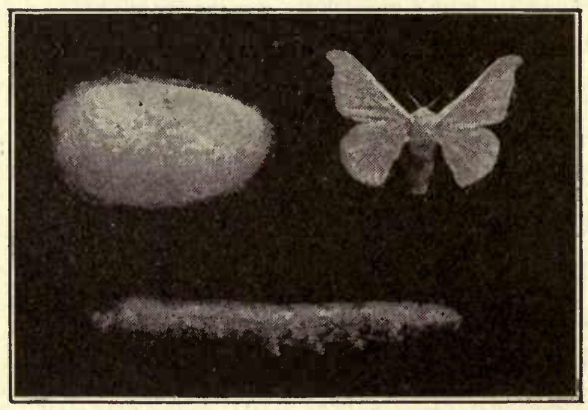

FIG. 323. - Evolution of the Silkworm. sively used for clothing. The seal is the most valuable furbearing animal.

Leather manufacture is one of the industries of any civilized country. In the United States there are a large number of manufacturing plants distributed through the states in the eastern part of our country. 
Animals which Aid Man. - A great many animals aid man by destroying harmful insects. The toad lives on insects and because of this fact is a valuable animal. It is estimated that a full-grown toad will eat from one hundred to two hundred insects daily.

Birds are also economically important for the same reason. The diet of our native birds includes many insects which are harmful to useful plants.

Certain insects do a valuable work in distributing the pollen of plants.

\section{Animal Pests.} - The cotton boll weevil has attracted much attention in recent years because it has proven so very destructive to a most important crop. It is estimated that if the pest should become generally distributed over

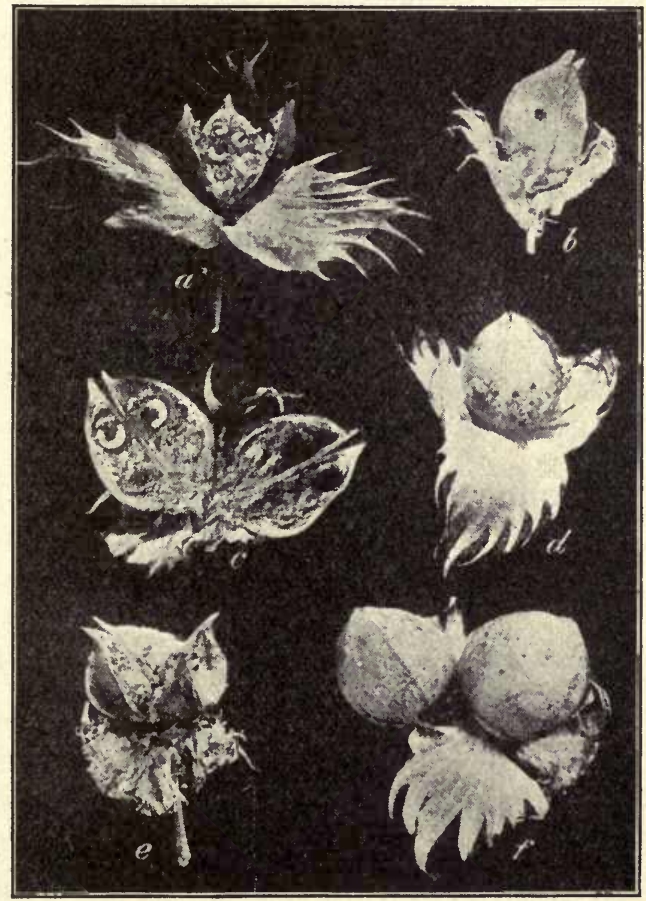

U.S. Dept. of A griculture.

FIG. 324. - Work of the Cotton Boll Weevil. the cotton belt, the possible annual loss may easily reach $\$ 250,000,000$. The adult beetles pass the winter in various places in or near the cotton fields. In the spring they attack the 
young plants, feeding upon the buds and laying eggs in some of the holes which they make in them. These eggs soon hatch into grubs that immediately begin to feed upon the interior of the buds, thus destroying the blossoms and with them the prospects of a crop (Figure 324). In a few weeks these grubs (larvæ) change to pupæ and then to adult beetles which gnaw their way out and are ready to lay eggs for a new brood of larvæ.

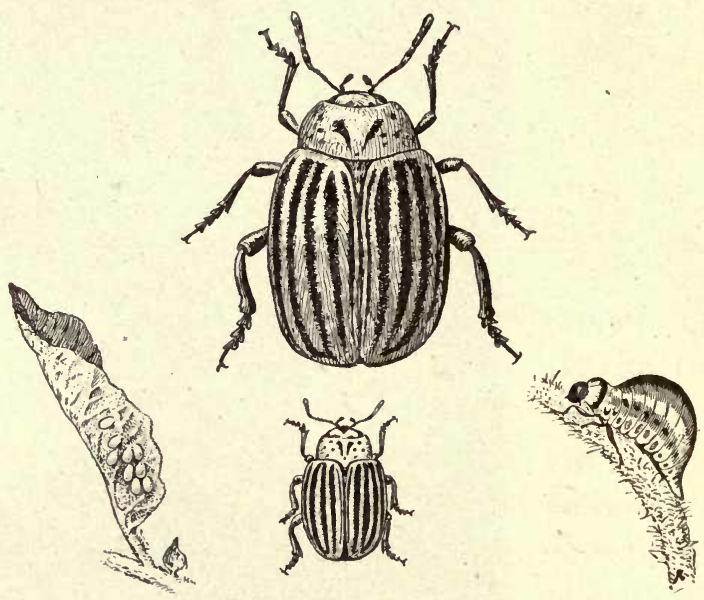

FIG. 325. - Stages in the Development of the Colorado Potato Beetle. At the left are the eggs; in the center the beetle, life-size and enlarged; and at the right the larva.

The most effective way to combat the cotton boll weevil seems to be to gather all the cotton plants late in the autumn and burn them, after which the ground should be plowed to expose the hibernating places of the bug.

Other destructive beetles are the Colorado potato bugs, the striped cucumber beetle, and curculios.

The Colorado potato beetle has made its way from the Rocky Mountain region to nearly every part of the world. 
The accompanying cut shows the various stages in its development (Figure 325). They are readily killed by the use of stomachic poisons.

The worminess of plums and cherries is largely due to the curculio (Figure 326). The plum curculio appears in fruit trees in early spring and feeds on the leaves and fruit. The females also cut crescent-shaped holes in the fruit and deposit their eggs. In from three to seven days these eggs hatch into little grubs that feed on the fruit. As a usual thing the infested fruit falls to the ground in a short time. The grub then leaves the fruit and burrows into the ground, changing later to the beetle (curculio). Spraying with arsenical

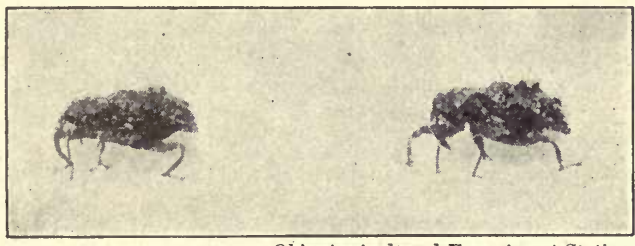

Ohio Agricultural Experiment Station. FIG. 326. - The Plum Curculio.

poison just as the blossoms are falling is an adequate remedy for curculio.

Scale Insects. - An examination of the leafy branches of the apple tree will often show what appears like miniature shells upon the bark. These are the Oyster Shell Scale. If the shell is lifted there will usually be found a number of small eggs under it. These eggs hatch early in the summer. The small insects that develop wander on the bark of the tree, finally fixing themselves in one place to feed on the sap which they suck from the bark.

The San Jose Scale. - The presence of this notoriously destructive scale at first is difficult to detect without the aid of a good magnifying glass. Figure 327 shows the appearance of the individual scales. As the scales 
increase in number they form a thick layer of scurf, which is readily removed with some sharp instrument.
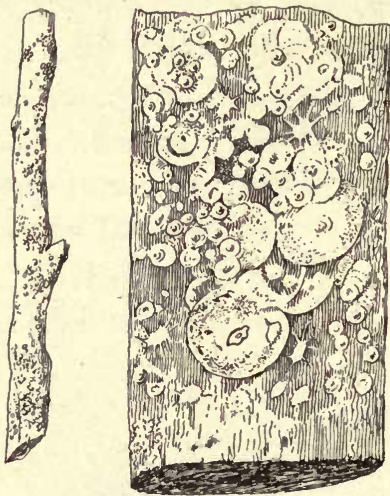

Fig. 327. - The San Jose Scale. They suck the sap and thus weaken the tree by withdrawing the food that the tree requires. In the spring the mature female scales give birth to about four hundred young. These soon fasten themselves to the bark of the tree and begin to suck the sap and grow the scale which covers them. The female scales are without wings, but the male scales develop wings and are able to fly about. The best treatment for them is lime-sulphur spray. Figure 328 shows the effect of this scale on the pear.

The Codling Moth. - Millions of dollars worth of fruit are annually destroyed by this pest. It lays its egg on the young apple. When the larva hatches, it eats its way into the apple. Young apples thus affected usually fall before they are very large. The way to. kill the codling moth is to have some poison on the apple ready for the first meal of the larva. This can be accomplished by spraying with arsenate of lead or some other poison just as the petals are falling from the blossom.

The Chinch Bug. - The central states

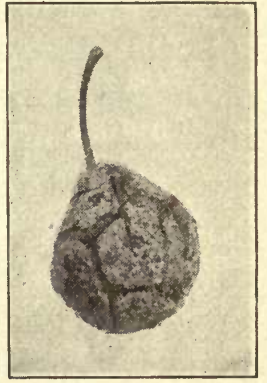

Ohio Agricultural Experiment Station.

FIG. 328. - The San Jose Scale on a Pear. of the middle West are often infested with the chinch bug (Figure 329). These insects attack the grain plants such 
as corn, wheat, and oats. They pass the winter in any field shelter such as thick grass. In the spring they lay their eggs on the young grain plants. The young bugs suck the sap of the plant, and if they are very numerous, the plant will be killed. The best method of attack on the chinch bug is to burn over places which are possible winter quarters for them.

\section{The Hessian Fly.}

These flies are mosquitolike insects which are quite destructive to growing wheat. They live in the larval state upon the growing plant. They lay their eggs on the leaves of the young plant in the fall of the year. The small larvæ which hatch work their way into the joints and by gradually absorbing the sap they greatly weaken or entirely destroy the plant. The larvæ change in the

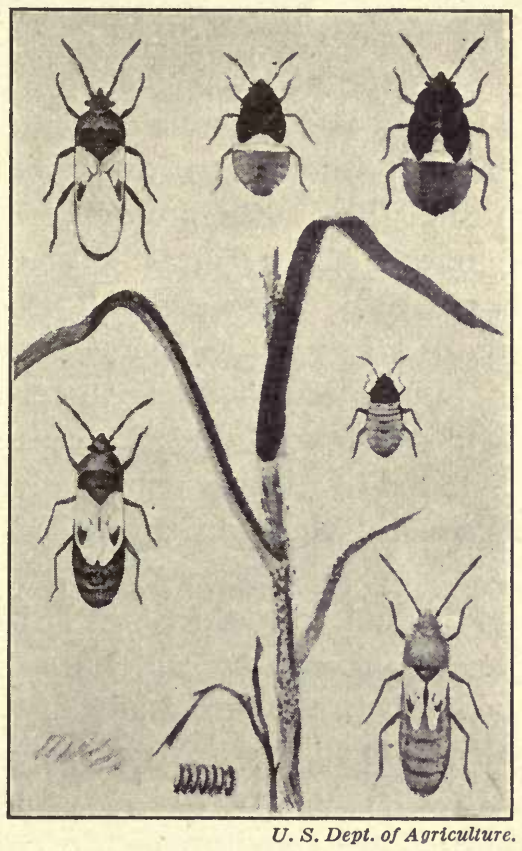

Fig. 329. - The Chinch Bug. late autumn to the pupal stage and resemble a flax seed in appearance. In this stage they pass the winter, changing in the spring to full-grown Hessian flies. These lay the eggs for another generation of larvæ which attack the wheat in the spring.

It is quite difficult to combat the Hessian fly. The most successful method has been the trap crop. Narrow 
strips of wheat are planted early to attract the fly. After the eggs are laid these strips are plowed under and the wheat planted later escapes.

There are numerous other insects, bugs, and moth larvæ which do considerable damage to plant life. The usual remedy is the destruction of the infested parts and proper spraying.

Poisons for Crop Pests. - It is estimated that insects annually destroy one tenth of the crops of the United States. In order to have strong healthy plants and sound fruits, these ever-present insect pests must be exterminated. To accomplish this result various poisons have come into quite general use. These poisons may be divided into two general classes according to the type of insect that is to be destroyed. Some pests, like the potato bug, feed directly on the leaf of the plant and may be killed by placing poison on the leaf. Poisons which are eaten by the insect are called stomachic poisons. Then there are other pests which get their nourishment by sucking the juices of the plant or the plant leaf. To kill such animals it is necessary to use a poison which acts on the body by enveloping it and drying up its tissues by cutting off its air supply or by poisoning it through absorption.

Stomachic Poisons. - Paris green is a poison which is in quite common use for those insects which swallow their food. It is prepared by combining hot solutions of arsenious acid and copper acetate. Paris green separates from this mixture as a fine, bright green powder, which is almost insoluble in water. However, it will readily dissolve in ammonia water, giving a dark blue solution. If the Paris green is adulterated with gypsum, a white powder will be suspended in the ammonia water; this will gradually settle to the bottom. 
For use in spraying mix eight ounces of Paris green with a little water and mix it into a paste, then add it to fifty gallons of water. If two pounds of lime are added to this mixture, it will serve to neutralize any free arsenious acid.

Lead arsenate has been used for a number of years as an insecticide. It is the most insoluble of the insecticides now in use. It adheres well to the leaves and is not in the least liable to scorch them as is Paris green and London purple when not used with lime.

For spraying purposes two pounds of the commercial paste in fifty gallons of water will give good results.

Contact Poisons. - Lime-sulphur is a common contact poison. It is prepared by heating together eight pounds of sulphur, four pounds of good quicklime, and five gallons of water. The lime should first be slaked, after which the sulphur mixed with water is added. The whole mixture is then boiled for an hour. More water should be added as the boiling continues, to replace that lost by evaporation. After the boiling is completed the clear liquid should be poured off and placed in closed kegs or barrels, since it oxidizes on coming into contact with the air.

For use in winter to exterminate scale insects the liquid should be diluted until it tests 5.0 on a Baumé hydrometer. For use as a summer spray it should be diluted to test 1.0 Baumé.

Since kerosene will not mix with water, an emulsion is made by dissolving one pound of soap in two gallons of water and adding two gallons of kerosene to the solution. These should be thoroughly mixed by pumping the mixture through a bucket sprayer.

When applied to the surface of pools of stagnant water, kerosene kills the mosquito pupa by suffocation. 
It cannot be applied directly to plants, but the emulsion mentioned above may be used against all sucking insects and scale when diluted to 20 gallons.

In some places the San José scale on orange trees is treated by inclosing the tree in a tent and applying hydrocyanic acid gas. This gas is very poisonous and care should be taken that none of it is breathed.

Weevils in grains may be killed by carbon disulphide placed in a dish and set on the surface of the grain. It is very volatile, the heavy vapor settling slowly through the entire bin, killing the insects as it goes. Care should be taken that no flames are brought near carbon disulphide, as its vapor is very combustible.

\section{QUESTIONS}

1. How do one-celled plants and one-celled animals differ?

2. What is the lowest form of animal life that has a nervous system?

3. Why does an earthworm die when placed in the sun?

4. How may we most easily destroy the house fly?

5. How do reptiles differ from fishes? From amphibians?

6. How do birds differ from reptiles?

7. Name five carnivorous mammals. Ten herbivorous mammals. Three omnivorous mammals.

8. What class of mammals does man use for food?

9. What amphibians are used as food by man? What mollusks? What is the lowest class of animals used as food?

10. Name five food products besides flesh that are furnished by animals.

11. Is the cat a useful or injurious animal?

12. Name five animals that are useful to man and are not used as food by him. How is each animal useful?

13. Are there birds that are classed as pests?

14. What is the source of neat's foot oil? Glue? Gelatine? Tallow? Ivory?

15. What uses are made of animal hair? 
16. What is the most serious insect pest in your county?

17. Name three poisons other than those mentioned in the text which are used to kill insects.

18. Is the United States government justified in spending money for the extermination of crop pests?

19. What other fruits do curculios attack?

20. What is a beetle? 


\section{CHAPTER XXII}

\section{MAN'S PLACE IN NATURE}

The student who has worked over the intricate problems of mensuration which seem to have little connection with his daily life will appreciate them all the more when he realizes how the carpenters and builders of centuries ago labored over these same problems in studying out the relations of distances and spaces in their work. Also when the student knows something of primitive man's crude methods of conversing by gestures and cries he will readily appreciate the advantages of articulate speech and will attack the subtleties of modern grammar with greater zest. And so it is with the various departments of science with which man is concerned. The study of man himself is a most interesting department of these sciences; but we are always studying man, since the development of all science has been man's work.

Man's Place among the Animals. - Man differs from the lower animals in many ways; but the principal differences are due to civilization. The identity of man as an animal is so completely hidden in this maze of civilization that his true position in the animal kingdom from a physiological standpoint is obscured. The anatomy of man differs very little from that of the higher mammals; but man's powers differ to an almost immeasurable extent. Man's reasoning power so far surpasses that of the highest apes that great naturalists have been at loss to account for the similarity in the 
appearance of their brains. There are many accounts of incidents which seem to indicate that reasoning power is possessed by animals; however, we are quite safe in saying that man is the only animal that has reasoning power, the power of forming abstract conclusions, and the power of articulate speech. The delicate coördination in man's brain is also reflected in the various refinements in muscular action of which he is capable.

Language. - We can learn a great deal about the different races of mankind by a careful study of their languages. Primitive peoples used various means for communicating with one another, such as making gestures,

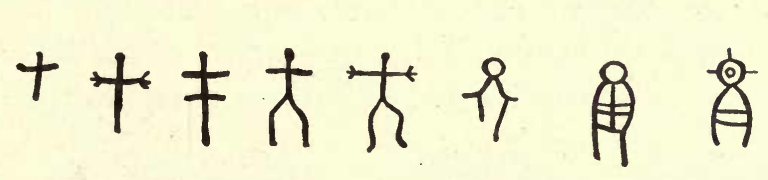

Fig. 330.- Picture Writing.

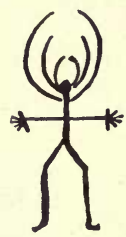

uttering cries, speaking words, drawing pictures, and writing with characters or letters (Figure 330). The language was little enriched from outside influences except in times of tribal wars, when the captives would contribute in some degree to the civilization of their captors.

Crude methods of communication among friendly tribes served to distribute bits of knowledge of general usefulness but imperfectly. Centuries sometimes added but little to the total sum of human attainments. The slowly moving story of discovery and invention is necessarily intricately interwoven with the story of the development of language. Modern methods of rapid communication and travel will serve to bring the peoples 
of the world to a better understanding of each other and to minimize the differences due to racial tendencies and environment.

Man's Tools and Weapons. - The arts which man has used to defend and maintain himself depend so much

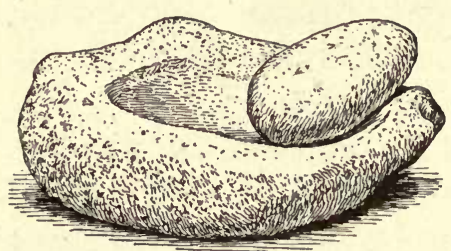

Fig. 331. - Ancient Grinding Stones. on the use of tools and weapons that some attention may well be paid to them. Man is distinctly a toolmaking animal.

The club was probably the first tool used by man, and it was used principally as a weapon of defense. For centuries he delighted in clubs ranging from the formidable looking knotty ones to the intricately carved clubs which we
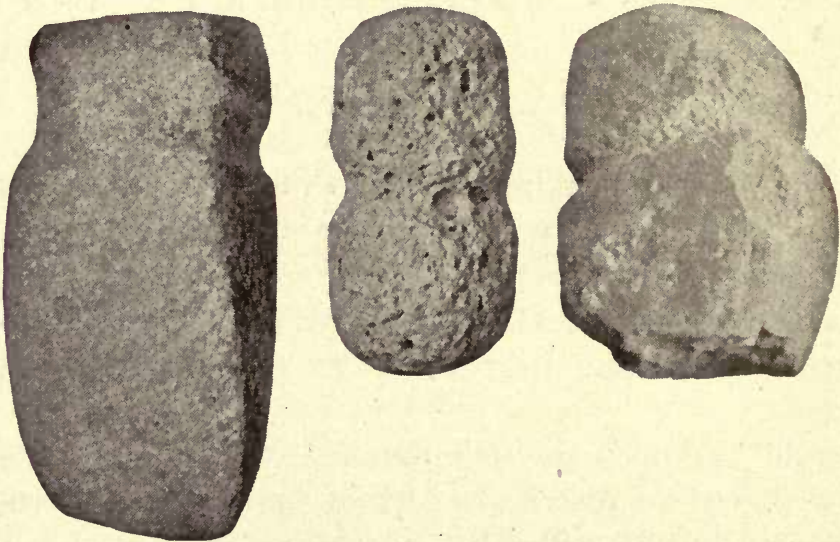

Frg. 332.- Stone Hatchet, Stone Hammer, Stone Maul.

see in the museums to-day. The hammer was a club modified for use as an implement of manufacture. It was a great step in advance when a handle was attached to a rounded stone to make a hammer (Figure 332). Later 
the stone was ground to an edge for a cutting instrument (Figures 333, 334).

It is not possible to know just when a knowledge of the mechanical principles was attained. It is quite probable that such knowledge developed slowly for centuries.

The use of metal gave certain tribes a distinct advantage over their adversaries. The use of the wheel was an-

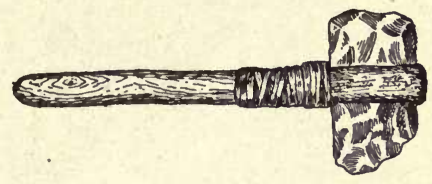

FIG. 333. - A Rude Axe, Showing other important discovery.

And so we might enumerate hundreds of inventions which we use to-day with little thought of the ages that were consumed in their development.

This age in which we are living is distinctly the age of wonderful inventions and achievements in all branches of science. We no sooner see the completion of a wonderful mechanical conception than another more marvelous attracts our attention.

The Home. - The animals inferior to

FIG. 334. - An Indian Knife with Handle. man build their homes by instinct. They have little ability to modify them to varying conditions of climate and environment. Man, however, has never been hampered by any such restrictions,
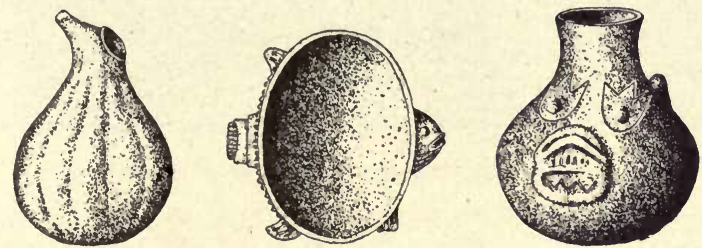

Fig. 335. - Pottery from Ancient Burial Mounds. 


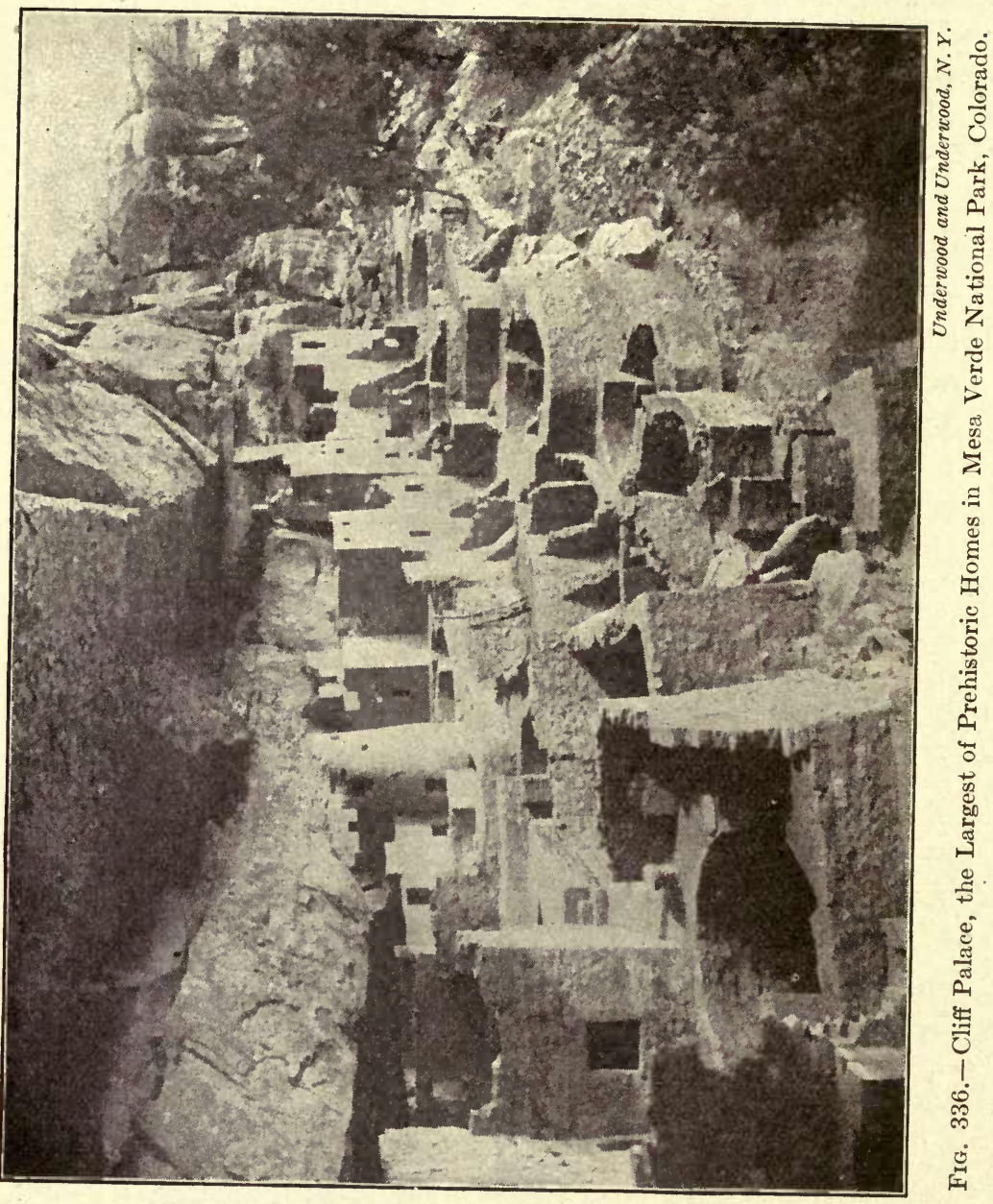


but has adapted his dwellings to suit his environment. (Figure 336). He has lived in almost every conceivable type of house, from caves and huts of skin to the magnificent structures which he is fond of building to-day; but in every case the influence of man's thought is evident in these structures as they have been varied to fit the conditions under which he lives and has lived.

In the conveniences of the home just as great changes are noticeable. Man has always had fire and water, but their uses have been greatly increased in the last century.

There are many mythological tales concerning the gift of fire, but so far as we are able to ascertain no savage tribe has been discovered that was without this great biessing (Figure

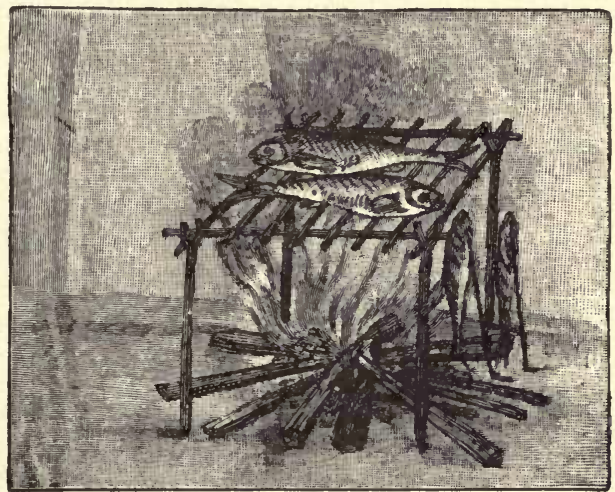

FIg. 337. - Indian Method of Broiling.

337). Among the relics of remotest antiquity pieces of charcoal and burnt bones are found. In his knowledge of fire man differs from all other creatures, since he alone has known how to manage and produce it.

In our homes the luxuries of a few years ago are necessities of to-day. The modern house is equipped with water, gas, electricity, baths, stoves, heating systems, and other contrivances which add greatly to our enjoyment of life; and the coming years will no doubt add many more useful inventions to our already enormous list. 


\section{QUESTIONS}

1. Can you give illustrations where animals seem to think?

2. Can animals be taught to use tools?

3. Name the races of man.

4. How did the Indian language compare with ours at the time America was discovered?

5. What can you tell of Indian writing?

6. How were messages sent from one tribe to another?

7. What proofs can you collect that birds build their nests by instinct? 


\section{CHAPTER XXIII}

\section{FOODS AND NUTRITION}

Need of Food. - One of the most elementary sensations of the human body is that of hunger. It is simply a provision of nature to tell us that the body needs food. As we grow older we learn that the food must be chosen with some care to enable the body to grow and maintain itself in a state of health. All living matter requires food, and food may be defined as anything which a plant or animal takes into its body as nourishment. Anything that we eat or drink which nourishes our bodies is food for us.

Bodily Energy. - We have learned that a machine which does work or produces energy must have energy expended upon it. Our civilization at all times demands supplies of food energy and supplies of mechanical energy. Energy of one kind may be changed into energy of another kind, but it is never created or destroyed. This is known as the Law of the Conservation of Energy. The body is often compared to a steam engine. When fuel is oxidized (burned) in the fire box of a steam engine, the stored energy in the fuel is released as heat energy. This heat acts upon the water in the boiler and changes it to steam, which has the power of expansion. This power may then be used in doing mechanical work or in producing heat again. The potential energy of the coal is changed to kinetic energy.

As in the steam engine, the source of energy in our 
bodies is in the oxidation of the fuel or food we eat. All the various manifestations of energy, such as heat and action in the body, have the same source. Since some parts of the body are always in action and since the body must be kept at constant temperature, there must always be a supply of food present in the body to supply this energy.

Measurement of Food Values. - By careful experiments it has been found that the work equal to lifting 427 grams a distance of one meter will produce a calorie of heat or enough heat to raise the temperature of one gram of water one degree Centigrade. Since this relation between heat energy and work exists, it is usual to give the values of different foods in terms of the calories they are capable of producing. Fuel values of foods are usually given in large calories, or a calorie equal to 1000 of the small calories mentioned above and written Calories. Food is actually fuel for the human organism, and the consideration of food on this rational and intelligible plan has furnished a logical basis for a constructive study of practical dietetics and the nutrition of the individual. There are some essential food factors whose value cannot be accurately shown in terms of calories, but they are like the lubricants for a great machine; they are essential, but they do not furnish the energy to run the machine.

Source of Food. - Agriculture is the great source of food supply. The rapidly developing transportation facilities have made it possible to utilize profitably an increasing amount of the earth's surface for agriculture. A hundred years ago it would have been a waste of energy to raise an enormous crop of wheat in the inland portions of the middle West, since there was no adequate way to 
market the crop (Figure 338). Now our transportation facilities are so highly organized that it is possible to dine on various perishable foods in places far removed from the food sources (Figure 339).

The study of our food supply involves a study not only of foad production, but a study of food preservation,

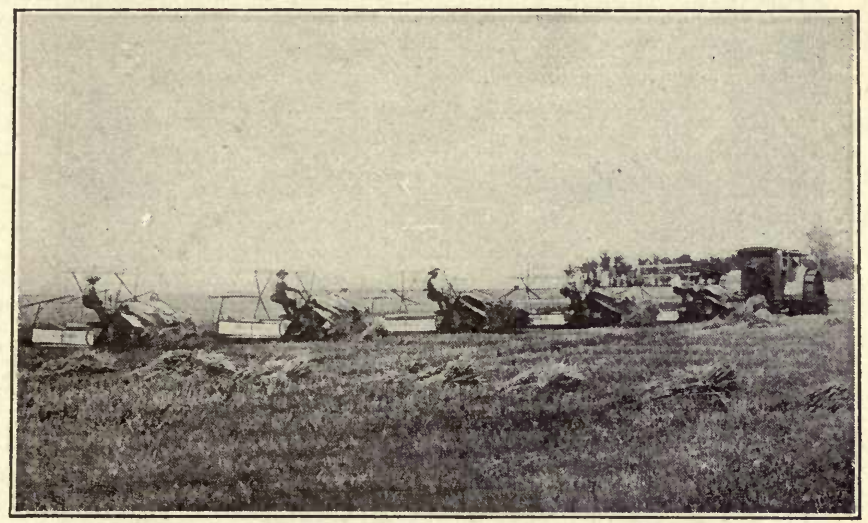

Frg. 338. - Wheat Harvesting.

A modern oil tractor operating five binders.

of food conservation, of transportation, and of industrial conditions.

Food Preservation as Related to Food Supply. Modern methods of preserving foods make it easy to maintain a dietary in times and places of relative scarcity which otherwise would be impossible. Some of the methods of preserving foods have been in use for a long time. These include drying, canning, salting, pickling, and smoking (Figure 340). The use of low temperatures and certain chemicals other than salt for preserving foods is of comparatively recent development. Cold storage methods have the great advantage of preserving 
fresh foods in their natural state for an almost indefinite length of time (Figure 341). According to Mr. Sherman, ${ }^{1}$ "Three American dairy farms exhibited raw milk at the Paris Exposition 1900, one of them sending weekly shipments throughout the summer, each of which was

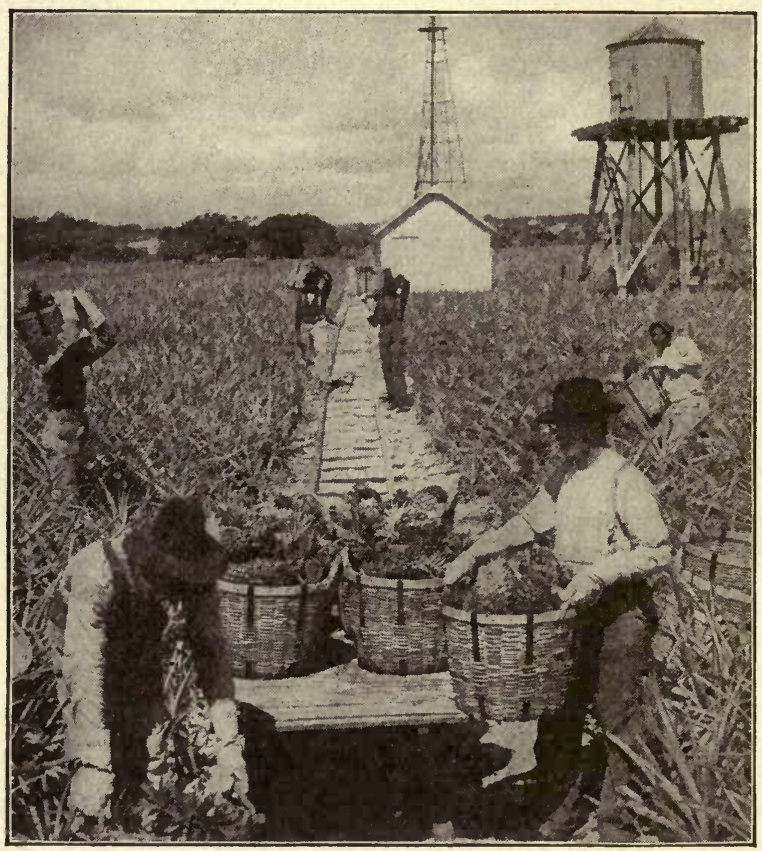

Copyright, 1915, by Keystone Vtew Company.

Frg. 339. - Harvesting Pineapples in Florida.

kept on exhibition in the raw state without spoilage until the next shipment arrived. It was difficult to convince the jury of European experts of the fact that cleanliness and cold were the only preservatives needed to accomplish the keeping of raw milk in a fresh, sweet condition for

${ }^{1}$ Professor H. C. Sherman: Food Products. 1914. 
two to four weeks in midsummer." By the use of these methods it is possible to supply the inhabitants of a large city like New York with fresh milk at a reasonable price.

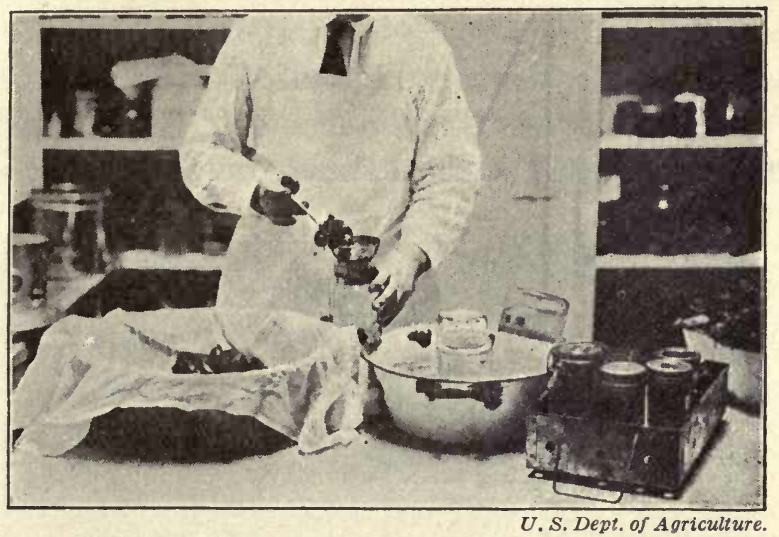

Fig. 340. - Packing Blanched and Cold-dipped Product into Jars.

Note the empty jars to be packed inverted in a pan of hot water. They are thus kept clean and hot.

Drying, which has been used for many years as a method of preserving certain foods, such as meats and fruits, has some advantages over cold (Figures 342,343$)$. After the food is thoroughly dried it may be transported easily over long distances. Early explorers usually carried large quantities of dried meats. As

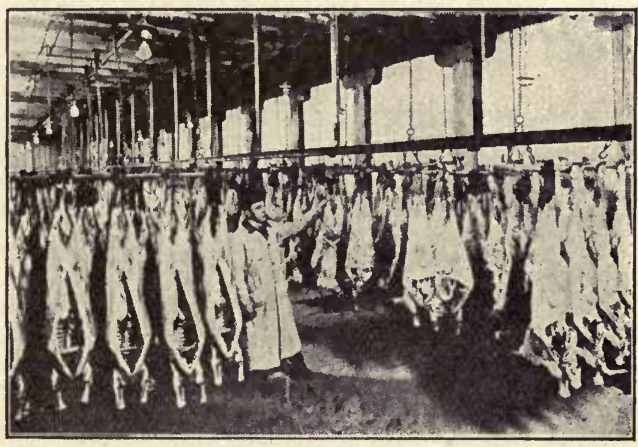

Fig. 341.-Cooling Room for Carcasses. 
a usual thing, however, some of the desirable flavors are lost in the drying process, and the food is less palatable than fresh food. Modern methods of drying in vacuum pans at lower temperatures have been quite successful in overcoming this objection, and we shall probably see an extension of the use of desiccation as a preservative. Milk furnishes a good example of this. We have had partially dried milk as condensed milk for some time;

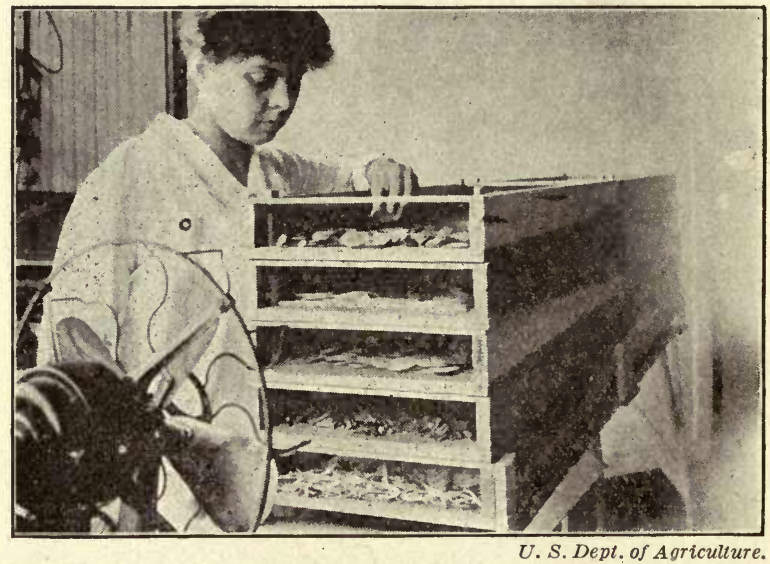

Fig. 342. - Drying Fruits and Vegetables on Homemade Racks by Forcing Air at Room Temperature across Them by Means of an Electric Fan.

but dried milk is now a reality, and it is quite likely to supersede other prepared milks if its nutritive values are not diminished in the process of drying.

Before the distribution of fresh meat was so well organized, large quantities of pork were preserved by smoking and salting. The hams and shoulders were smoked during the winter months and thus kept for use during the following summer. The sides of the hog were placed in dense brine and kept in a cool place. Meat 
could be kept in this way as long as desired. These methods are still used in rural communities with good results.

All foods are subject to the attack of destructive bacteria. The changes produced in food by these bacteria usually render them less palatable and often destroy their value as foods. The various methods of preserving foods may be classed under the general heads of physical

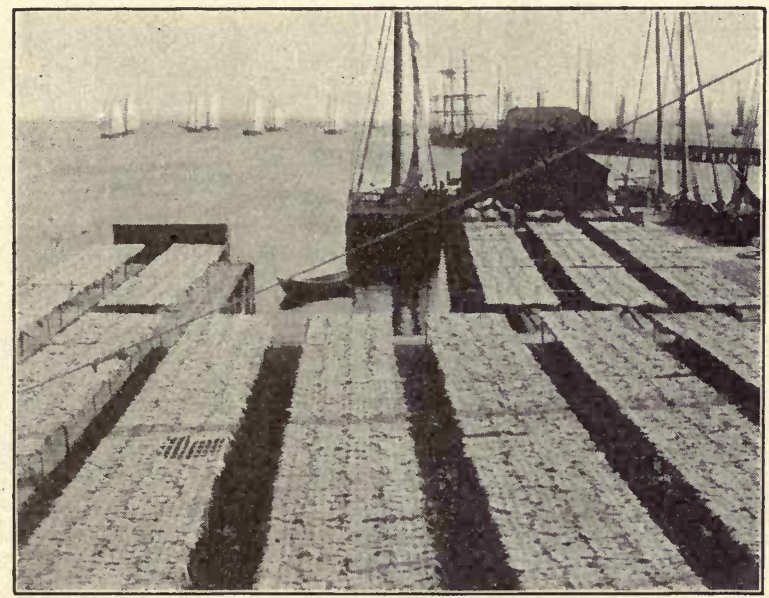

Frg. 343. - Drying Codfish, Provincetown, Mass.

and chemical methods. To the first class belong such methods as canning, refrigerating or cooling, and drying or evaporating. These methods are applicable to all kinds of foods and are highly efficient, since they make very slight changes in the flavor, appearance, and composition of the foods. The chemical methods of preserving foods involve a change of chemical conditions in the foods of such a character that decomposition takes place very slowly. Chemicals used as preservatives include 
alcohol, vinegar, sugar, salt, borates, benzoates, salicylates, and formaldehyde.

Transportation as Related to Food Supply. - The wonderful growth of the cities in the last few decades has increased the problems of food distribution. Managers of transportation facilities have been alert, and keen competition has effected many interesting developments in the exchange of com-

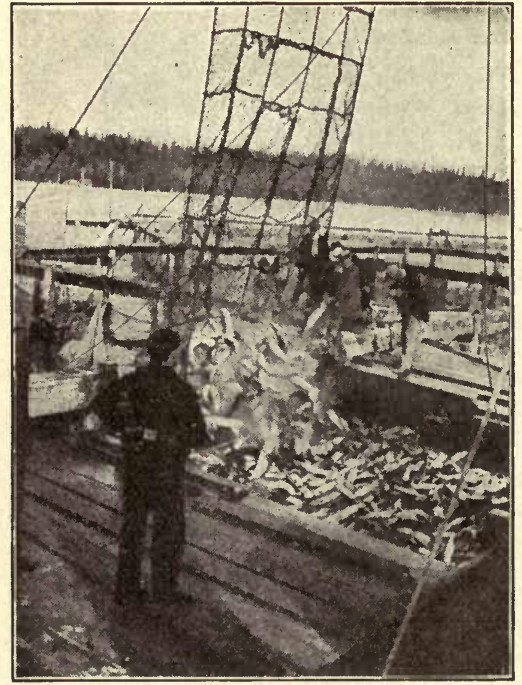

Copyright by Underwood \& Underwood, N.Y.

FIG. 344. - Taking Salmon from Trap, Puget Sound, Washington. modities. We are no longer confined to local products in our dietary, but may satisfy our taste with the foods of every climate and region. Fresh fruits may be had at all times and in most places of the world, and their wide use is fully justified by the better average health of the users. Better methods of preserving foods have made better organized transportation possible. Conversely, better transportation facilities have made the preservation of certain foods profitable to both the producer and the consumer (Figure 345 ).

The Manufacturer's Place in Food Supply. - A few minutes' time spent in studying the food advertisements in the current magazines and newspapers will convince the most skeptical that the manufacturer has a very important part in the distribution of certain foods. There 
are attractive advertisements of "breakfast foods," prepared meats, and various other foods. Of course these foods cost more than food in the bulk; but their increased use from year to year indicates that people are willing to pay more in the interest of food hygiene and their own convenience. These manufactured foods are perhaps slowly changing our eating habits. Breakfast is becoming a meal of fruits and cereals instead of the heavy meal of fifty years ago. Bread making in

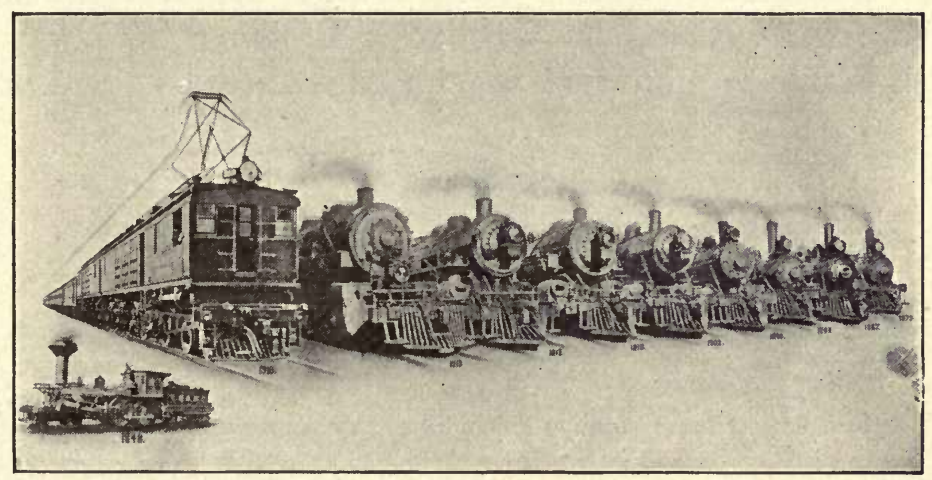

Frg. 345.- Development of the Locomotive.

some localities is already a factory problem, and with better methods of manufacture and distribution the use of baker's bread is certain to increase.

Nature's Food Factories. - Man's food must contain whatever is needed to build and repair the body, to give it fuel for heat and to regulate certain processes of the body. Plants derive their nourishment largely from the mineral kingdom, and animals derive their nourishment from plants and animals that feed upon plants. It is true that animals require certain inorganic materials such as water and salt, but the sources of real foods are plants 
and other animals. Plants make the foods which they need and also all the food for animals. They are the only real food factories.

Kinds of Foods. - The number of substances used as food is large, but these substances may be conveniently grouped according to their chemical composition and sources, as protein, carbohydrates, fats, vitamines, ash constituents, and water.

Proteins. - The proteins are frequently spoken of as the nitrogenous foods. They always contain carbon, hydrogen, oxygen, and nitrogen. Proteins are always rich in one or more such organic substances as albumen,

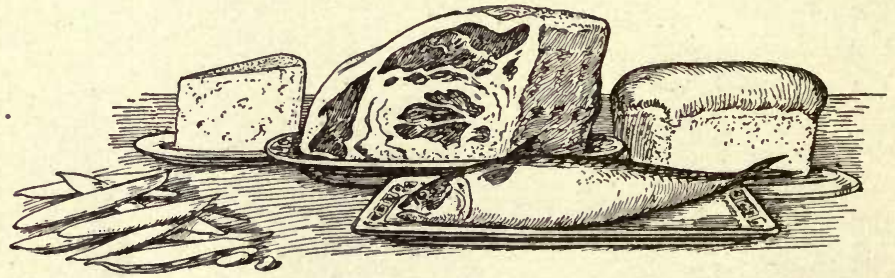

FIG. 346. - Some Building Foods that Make Bones and Muscles.

casein, fibrin, gelatin, gluten, legumin, and myosin. The principal protein food materials are milk, eggs, flesh foods, legumes, and cereals (Figures 346, 347). They are the foods which serve to build up the body and keep it in repair.

The presence of protein in a food may be detected by the following simple experiments.

Nitric Acid Test. - Boil the food to be tested in nitric acid. When cool add enough ammonia to neutralize the acid. If the yellow nitric acid solution turns to a deep orange when the ammonia is added, protein is present.

Biuret Test. - To a ten per cent solution of caustic soda add a dilute solution of copper sulphate, drop by 
drop, until a faint blue color appears, but no precipitate. Now add the solution to be tested. A violet color indicates protein.

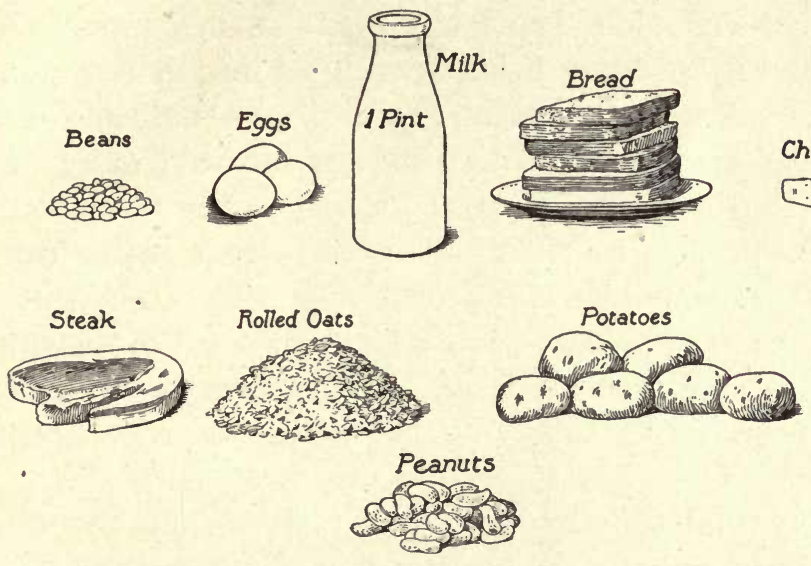

Fig. 347.- Foods Containing Equal Amounts of Proteins.

Carbohydrates. - This class of foods is made up of the starches, sugars, and glucoses, and enters largely into the composition of foods of vegetable origin. The carbohydrates contain carbon, hydrogen, and oxygen,

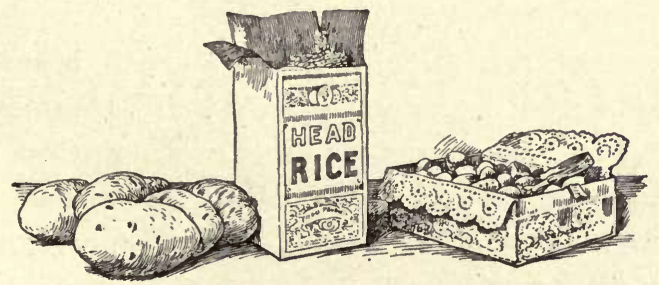

Fig. 348. - Sugar and Starch are Foods that Produce Power and Heat.

as the name indicates. They are essentially energyproducing foods or fuel foods (Figure 348).

Green plants make all of the carbohydrates which we use as food, except milk sugar. In the presence of sun- 
light and chlorophyll, plants make glucose from the carbon dioxide and water which are taken in through their leaves and roots. This form of carbohydrate the plant uses as food. But the plant makes much more food than it needs, and this is changed into more compact forms of sugar and starch and stored in its roots and seeds.

Sugar and starch are very similar substances. In fact nature is constantly at work making sugar from starch. The reason that ripe fruit is sweeter than green fruit is because in ripening much of the starch is changed to sugar. Similar changes occur in the process of digestion. When starchy foods are eaten, the starch is changed $t_{i}$ a usable form of sugar by the action of the digestive fluids.

The normal functioning of the body requires so much power that much more fuel food is required than tissuebuilding food; that is, more carbohydrates and fats are needed than proteins. Protein is both a tissue builder and a fuel food, while carbohydrates when taken into the body unmixed with other foods are usable only as fuel foods; however, they may combine with nitrogen-bearing foods and aid in the repair of tissues.

Starch. - Starch is found in all green plants, and in tubers, seeds, and tapioca (Figure 349). Its presence may be detected by the action of iodine. A solution of iodine, in potassium iodide and water, colors raw or cooked starch blue. The starch to be tested should be cool and either neutral or acid to litmus.

Test a freshly cut piece of potato for the presence of starch by putting a drop of iodine solution on it. Other vegetables and grains may be tested in the same way. Celluloses and glycogen are related to starch. Cellulose is found in the cell walls of plants and is almost insoluble; 


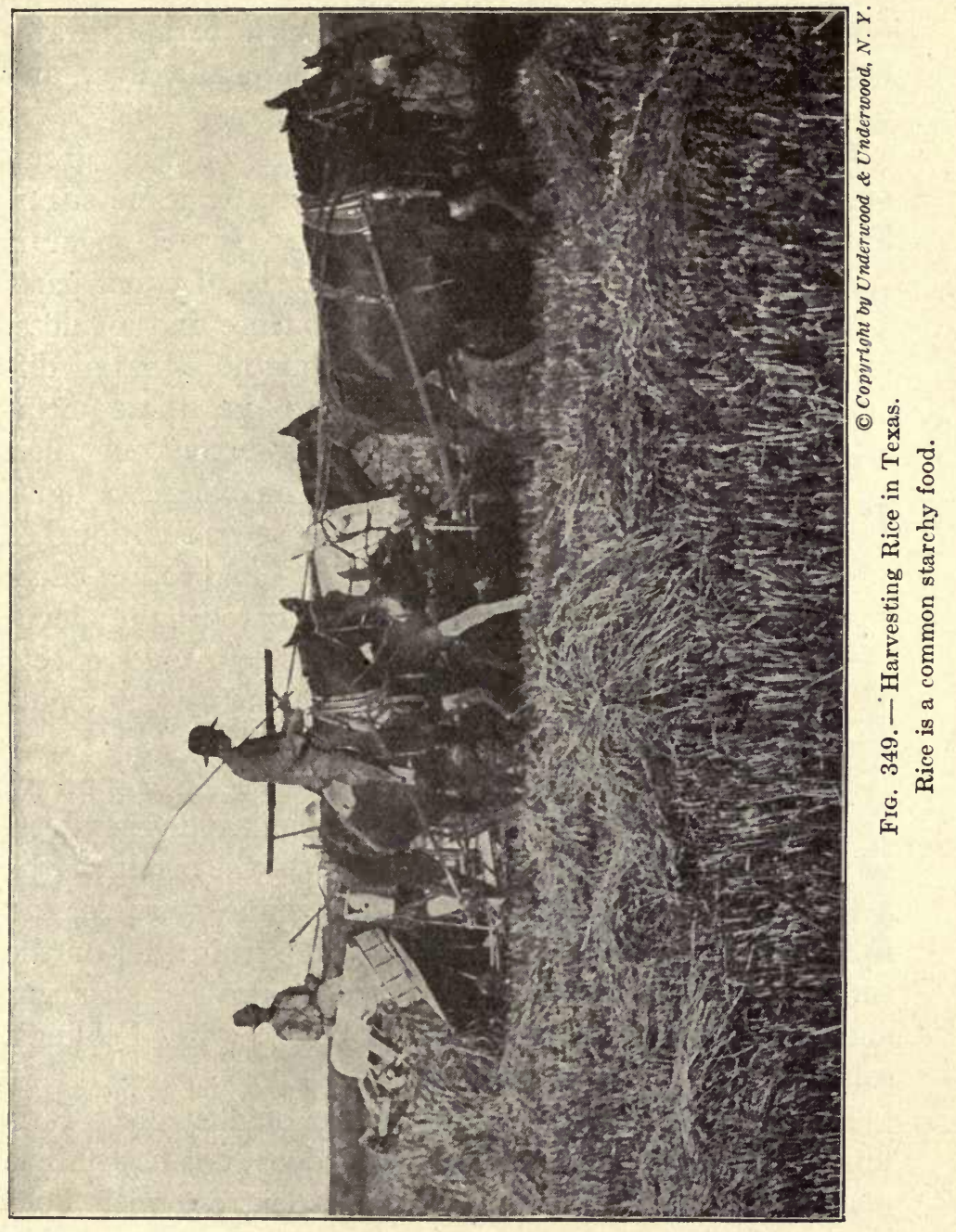


i.e., indigestible. It is needed, however, to give bulk to foods. Glycogen is the form in which carbohydrates are normally found after absorption in the liver and muscles.

Glucoses. - Common representatives of this group of carbohydrates are known as dextrose or grape sugar, levulose or fruit sugar, and galactose. Invert sugar is

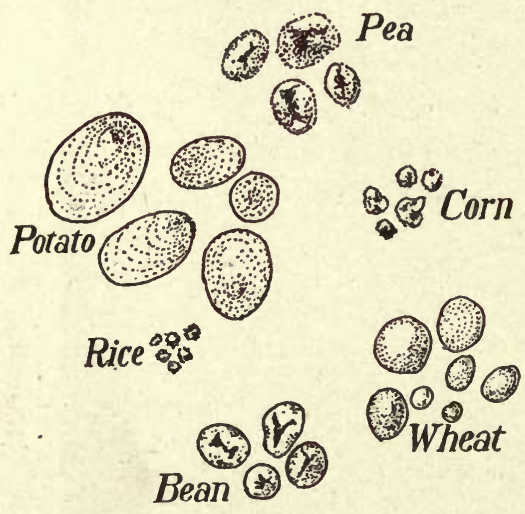

FIG. 350. - Starch Grains from Various Foods.

a mixture of equal parts of dextrose and levulose. There is no essential difference in these compounds except in their effect on polarized light. Dextrose in solution has a right-hand rotary effect on polarized light; levulose, a left-hand effect; and galactose, a righthand effect.

Dextrose is found in ripe fruits and vegetables, in corn sirup, in the digestive tract as the result of the action of the digestive juices on sugars and starches, and in cooked fruits, due to the action of the fruit acids on the sugar used for sweetening. It is less sweet than sugar and gives a reddish color when tested with Fehling's solution.

Levulose is found as a companion of dextrose. It is much sweeter than dextrose and does not crystallize readily. It gives the same test as dextrose with Fehling's solution.

Fehling's solution test. - Prepare Fehling's solution by dissolving 6.2 grams of copper sulphate, 3.5 grams of 
Rochelle salts, and 2 grams of potassium hydroxide in 100 grams of water.

Cover some chopped raisins with water and after allowing them to stand for a few minutes test the water for the presence of grape sugar by heating a little of it with about $10 \mathrm{cc}$. of the Fehling's solution. A reddish precipitate indicates dextrose or grape sugar.

Test honey, corn sirup, and cane sugar in the same way.

Contrary to a current opinion much advertised by the newspapers a few years ago, glucose is a wholesome food. It is not so sweet as cane sugar, with which it is frequently mixed because of its cheapness. We buy sugar chiefly for its sweetness, and a pound of the mixture will be worth less as a sweetening agent than the pure cane sugar.

Sugars. - The ordinary sugar of commerce is

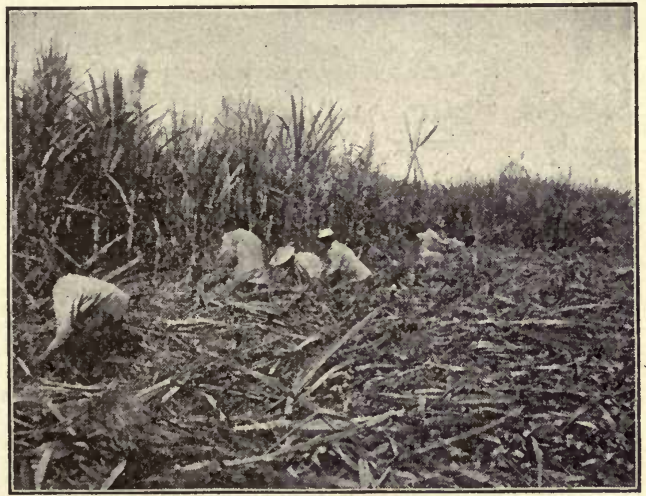

Fig. 351. - Cutting a Crop of Sugar Cane.

manufactured from sugar cane and sugar beets (Figure 351). The sugars include sucrose, maltose, and lactose. Sucrose is the sugar obtained from cane, beets, and maple sap. Maltose does not occur in nature, but is produced by a fermentation of the starch of barley and other cereals. Lactose is the sugar in milk.

Can the presence of cane sugar be determined with Fehling's solution? 
Add a few drops of vinegar to a solution of cane sugar and then test with Fehling's solution.

Add a drop of hydrochloric acid to a few cubic centimeters of cane sugar solution and boil. When cool, add enough sodium carbonate solution to neutralize, and test with Fehling's solution. What change has the hydrochloric acid produced?

Fats. - These include ordinary meat fats and all vegetable and animal oils. Fats contain the same elements as the carbohydrates, but in different proportions. The principal kinds of fats used as food are the fats of

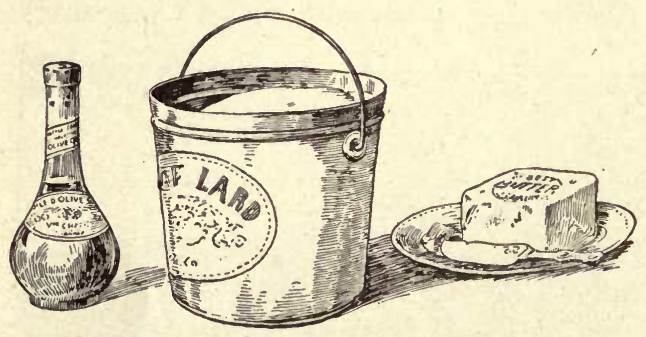

FIG. 352. - Fats are the Best Warming Foods. meats, butter, olive oil, palm oil, cottonseed oil, and almond oil (Figure 352). They are insoluble in water but are readily soluble in ether, chloroform, and gasoline.

Fatty foods are high in heat value and form the principal diet of the inhabitants of cold climates. When more fat is digested than is required for the present uses of the body, it is stored in various parts of the body as a reserve which may be used when needed.

The vegetable fats of commerce are in liquid form, while the animal fats are usually solids. This, however, is not true of the animal fats in their original state, where they too are liquids. Fat in animals occurs as minute drops inclosed in tiny sacs. When fresh milk is allowed to stand, the millions of little fat drops rise to the surface of the milk, as cream.

Vitamines. - In addition to the well-known classes of 
foocis there are substances occurring in minute quantities in certain food materials, which are essential to growth and complete nutrition. These substances are called vitamines. If they are lacking in sufficient quantities normal growth is impeded and certain diseases result.

Ash Constituents. - Mineral salts and water belong in this class. The principal minerals which enter into the composition of the body are salt, lime, iron, magnesia, phosphorus, and potash. With the exception of common salt, these substances are usually taken into the body only in combination with other plant and animal foods.

Water. - While water may not properly be called a food, it is true that a large amount of water is needed daily, since it enters into the composition of every tissue in the body and is constantly being removed by the organs of excretion. No solid matter can be absorbed and pass into the blood. All food must be dissolved in order to pass through the walls of the intestine, and, large quantities of water are needed for the process of digesting the food. "Drink plenty of water every day" is an excellent health rule.

Beverages. - Tea and coffee are in no sense foods, since what proteins and carbohydrates they contain are not capable of being dissolved and used by the body. Chocolate and cocoa have real food value. All of these drinks are stimulants of varying strengths and serve to increase mental and physical alertness. The stimulating effects of chocolate and cocoa, however, are small as compared with the effects of tea and coffee. The stimulant in tea is called theine; in coffee, caffein; in chocolate and cocoa, theobromine. Tea also contains another harmful substance called tannin, which inter- 
feres with the secretion of the digestive fluids and hence with the digestion of certain foods.

Alcohol. - The action of the governments of several of the countries of Europe during the great war gives us a good idea of the trend of civilization with respect to the use of alcohol. The question of the use of alcohol has been a much discussed one of late years among physiologists. Alcohol is composed of the elements carbon, oxygen, and hydrogen and will to some extent satisfy the requirements of the body for heat and muscular energy; yet its use is attended with dangerous results wholly lacking in the use of carbohydrates and fats. It obstructs the normal action of the liver and other organs, and loads the circulation with impurities which are quite harmful and dangerous to health.

Effects of Alcohol. - If we pour some 95 per cent alcohol on the white of an egg, the albumen will immediately coagulate and present an appearance similar to that of the cooked white of egg. This is because the alcohol takes the water from the albumen. Strong alcohol taken into the body acts in the same way and draws the water from the living protoplasm, thus hardening it.

Alcohol taken into the body in very small quantities apparently does no harm and is oxidized at the cells, but if we examine the records of the life insurance companies whose results are obtained by the averaging of thousands of cases, we find that the expectation of life is greatly decreased by even the moderate use of alcohol. When we consider its ultimate effects we must classify alcohol as poison, and not as a food. Hardening of the arteries, cirrhosis of the liver, and various nerve disorders are traceable to the use of alcohol. In addition the 
body is weakened so that it becomes an easy prey to disease germs.

How Alcohol is Made. - Alcohol is made by the action of yeasts on fruit or grain juices. Fermentation results in changing the grape sugar to alcohol, the carbon dioxide passing into the air.

Patent Medicines. - Many patent medicines contain alcohol and other harmful constituents such as opium,

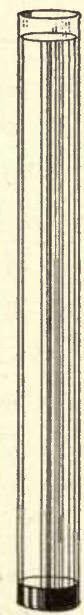

Cider

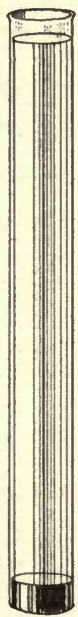

Beer
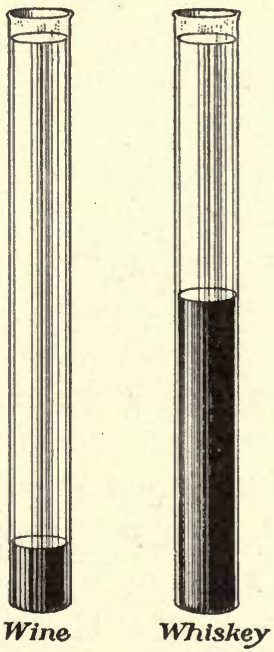

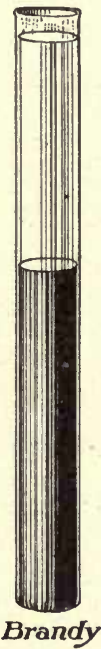

Brandy

Fig. 353. - Relative Amounts of Alcohol.

morphine, and cocaine. Bitters and tonics which are so widely advertised frequently contain alcohol in quantities ranging from 15 per cent to 45 per cent. Whiskey is 50 per cent alcohol, wines about 10 per cent, and beer 5 per cent (Figure 353). By comparing the percentage of alcohol in patent medicines with the percentages of alcohol in the ordinary alcoholic drinks, some idea of the dangerous effects of such medicines may be easily obtained. 
People who are sick or who imagine an ailment are usually quite gullible in the matter of patent medicines. A collection of articles published under the title of The Great American Fraud, by the American Medical Association, which deals with this subject, may be read with profit by any one.

Tobacco. - A narcotic has been defined as a substance "which directly induces sleep, blunts the senses, and, in large amounts, produces complete insensibility." Tobacco, opium, and cocaine are narcotics. Tobacco contains a strong poison known as nicotine. A few drops of pure nicotine would be sufficient to cause the death of an adult by its action upon the nervous system. The effects of tobacco are more marked on young people than on adults. The evidence is quite conclusive that the use of tobacco affects the heart action and retards muscular development: The boys who are habitual smokers of cigarettes average smaller in size than the non-smokers, and they average lower in their studies. The cigarette habit seriously handicaps a boy in his search for honors in scholarship, athletics, or business.

Purchase of Food. - When we consider that in the United States we annually spend about $\$ 15,000,000,000$ for food, the importance of proper marketing is impressed upon us. Every one should know something of the science of purchasing food, since such a large proportion of the total income is expended for food and since so much of health and happiness depends upon a proper dietary.

It is a great mistake to suppose that you always get the best when you pay the highest price. Fruits are best and cheapest in season. A little study of different fruits will enable one to have a variety of the best avail- 
able fruits at all times and at reasonable prices. Inexpensive cuts of meat when properly cooked often have higher food values than more expensive cuts. Adulterated foods are always expensive, but this does not mean that certain substitutes may not be used to good advantage. For example oleomargarine is quite wholesome and if purchased at the proper price may represent food values relatively as high as the food values of more expensive butter.

The better grades of prepared foods and canned foods are usually more wholesome and freer from harmful and expensive adulterations than the cheaper grades. It is quite fortunate for the majority of people that expensive foods are really no more nutritious than cheaper foods; quite often expensive foods are rich foods whose continued use produces digestive troubles.

The Dietary. - Good health is absolutely essential to our happiness and general efficiency. The dietary is so intimately connected with the subject of health and length of life of the individual that it should be studied with greatest care. The average length of human life has increased in the last quarter of a century because of the intelligent study we are giving to hygiene and to nutrition.

Taste alone should not control our selection of food. We can educate our tastes so that we can enjoy all wholesome foods. However, in selecting our foods there are some actual requirements to be considered. We must have some proteins and we must have some carbohydrates and fats to furnish the fuel food. The ideal ration is the one which gives us as nearly as possible the proper chemical elements in the proportion that they are contained in the body (Figure 354). The 
results obtained by specialists in nutrition indicate that a man who does average muscular work requires 3.7 ounces

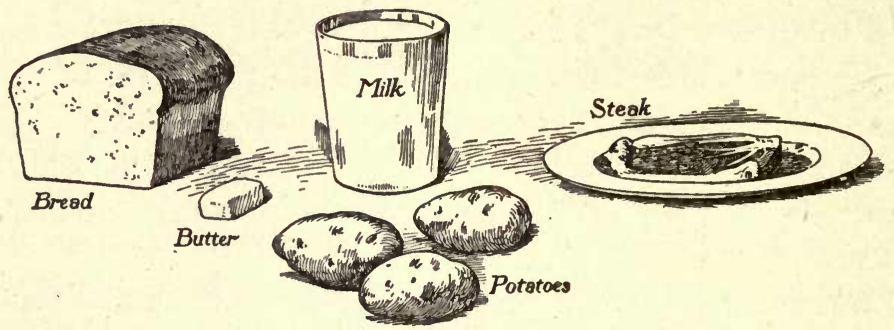

Fig. 354. - A Day's Ration.

of protein, an equal amount of fats, and 13 ounces of carbohydrates to provide the energy used up in one day and for the repair of the wasted tissues.

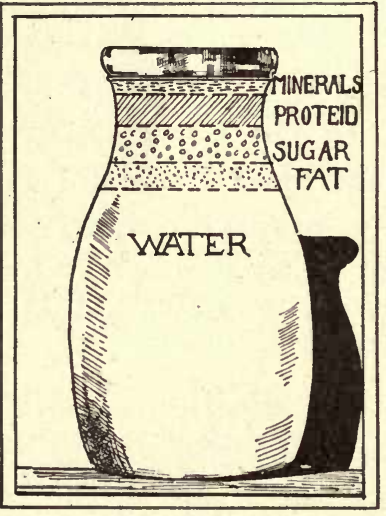

Fig. 355. - Elements Contained in Milk.

In terms of fuel values Atwater's ${ }^{1}$ standard for a man at light exercise is 2816 Calories; 410 Calories from protein, 930 Calories from fat, and 1476 Calories from carbohydrates. Professor Chittenden of Yale University gives as his standard for the same man, food to yield 2360 Calories, of which protein is to furnish 236 Calories, fat 708 Calories, and carbohydrates 1416 Calories. Professor Chittenden's diet would contain about 2.2 ounces of protein, 2.8 ounces of fat, and 13 ounces of carbohydrates. Food.

${ }^{1}$ W. O. Atwater: Principles of Nutrition and Nutritive Value of 
For the person who has a general idea of the amount and proportion of the food substances required for his daily use it will be quite easy to select a good mixed diet from the table given on page 403. A mixed diet is quite necessary, since milk is the only food that contains protein, fat, and carbohydrate in a proportion approximating that of protoplasm (Figure 355). Milk can be used as the sole food of children for this reason. By a careful selection of foods it is possible to obtain the

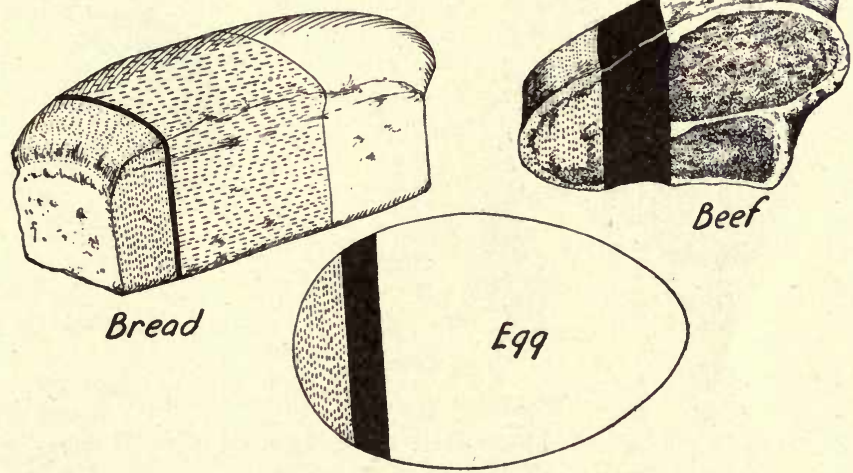

Fig. 356. - Nutritive Values of Bread, Beef, and Eggs.

Fats, black; carbohydrates, horizontal lines; proteins, vertical lines; other parts, water.

proper proportion of essentials with a minimum of waste products (Figures 356, 357). A vegetable diet contains a great deal of waste materials and for this reason is less healthy than a mixed diet containing meats. The vegetarian is correct in his contention that vegetables contain everything necessary to life, but it is quite easy to select foods which contain these same essentials and which require much less work of the digestive organs in their assimilation. Some animals live on purely vegetable 
diets, but their digestive organs are so modified that they easily take care of large quantities of waste materials, such as cellulose, which forms the indigestible walls of plant cells. The digestive organs are intended for work,

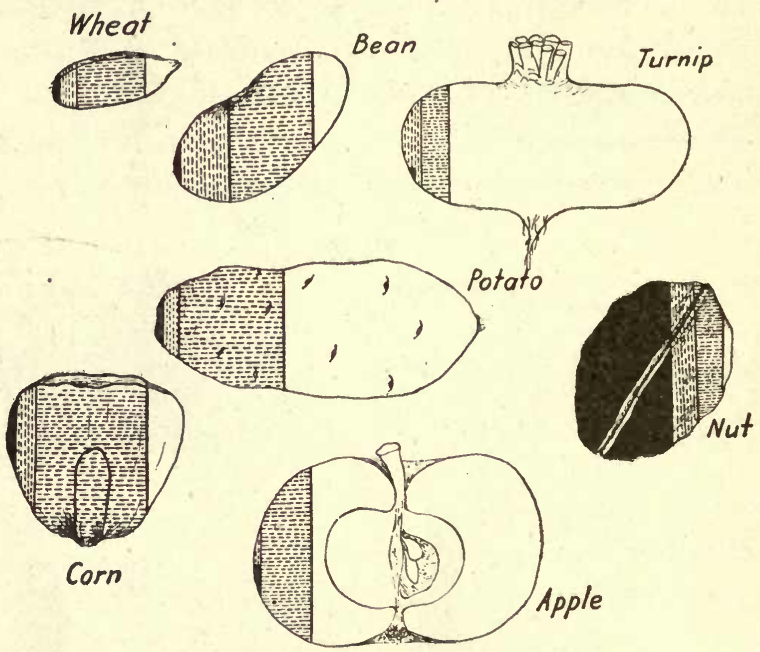

Fig. 357. - Nutritive Values of Different Foods.

Fats, black; carbohydrates, horizontal lines; proteins, vertical lines; other parts, water.

but they should not be overworked in separating from food an excess of poisonous wastes.

Principles of Cooking. - Man is the only animal that cooks his food, although a number of animals will eat cooked food. Cooking renders food more digestible or more palatable; and either is a sufficient reason for the extra trouble. With the exception of fruits only a few articles of food are eaten in their natural state.

Cooking has a marked effect on meats. Raw meat is tough and not easily pulled apart. Cooking breaks up the connective tissue and causes the muscular fibers 
Table of Food Values of Edible Portions of Some Common Foods

\begin{tabular}{|c|c|c|c|c|c|c|c|c|c|c|}
\hline & & & & & $\begin{array}{c}\text { Per Cent } \\
\text { Protein }\end{array}$ & $\begin{array}{c}\text { PER } \\
\text { CENT } \\
\text { FAT }\end{array}$ & $\begin{array}{c}\text { Per Cent } \\
\text { Carbo- } \\
\text { Hydrates }\end{array}$ & $\begin{array}{c}\text { Per } \\
\text { CENT } \\
\text { Ash }\end{array}$ & $\begin{array}{c}\text { Per. } \\
\text { CENT } \\
\text { Water }\end{array}$ & $\begin{array}{c}\text { FUEL } \\
\text { VALUE PER } \\
\text { POUND }\end{array}$ \\
\hline \multicolumn{5}{|c|}{ Cheese, American . } & 29. & 36. & .3 & 3.2 & 31.5 & 1990 \\
\hline Peanuts & 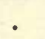 & 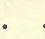 & . & & 26. & 38.5 & 24.4 & 2.0 & 9.1 & 2490 \\
\hline Leg of $\mathrm{M}$ & utt & on & & & 20. & 12.4 & 0. & 1.2 & 66.4 & 863 \\
\hline Dried Pea & & . & . & 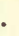 & 24.6 & 1.0 & 62.0 & 2.9 & 9.5 & 1611 \\
\hline Dried Bea & ans & & . & & 22.5 & 1.8 & 59.6 & 3.5 & 12.6 & 1565 \\
\hline Roast Be & & & . & & 22.3 & 28.6 & 0. & 1.3 & 48.2 & 1576 \\
\hline Canned S & Saln & non & & & 22. & 12.8 & 0. & 1.4 & 64.6 & 925 \\
\hline Chicken & . & . & . & & 21.5 & 2.5 & 0 . & 1.1 & 74.8 & 495 \\
\hline Veal & & & 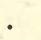 & & 20.7 & 8.3 & 0 . & 1.0 & 70.9 & 715 \\
\hline Almonds & . & & . & & 21.0 & 54.9 & 17.3 & 2.0 & 4.8 & 2940 \\
\hline Brazil Nu & ats & & . & & 17.0 & 66.8 & 7. & 3.9 & 5.3 & 3040 \\
\hline English $\mathrm{W}$ & Nal & nut & & & 18.4 & 64.4 & 13. & 1.7 & 2.5 & 3180 \\
\hline Oatmeal & . & . & . & & 16.1 & 7.2 & 67.5 & 1.9 & 7.3 & 1810 \\
\hline Wheat $\mathrm{Fl}$ & lour & & . & & 13.8 & 1.9 & 71.9 & 1.0 & 11.4 & 1630 \\
\hline Eggs . & & & . & & 13.4 & 10.5 & 0. & 1. & 73.7 & 672 \\
\hline Macaroni & & & & & 13.4 & .9 & 74.1 & 1.3 & 10.3 & 1625 \\
\hline Oyster $\mathrm{Cr}$ & $\mathrm{racl}$ & kers & & & 10.7 . & 8.8 & 71.9 & 1.8 & 6.8 & 1855 \\
\hline Bread . & & 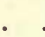 & . & & 9.1 & 1.6 & 53.3 & 1.0 & 35. & 1200 \\
\hline Rice . & . & . & . & & 8.0 & .3 & 79.0 & .5 & 12.2 & 1620 \\
\hline Green Pes & as & & & & 7.0 & .5 & 16.9 & 1.0 & 74.6 & 454 \\
\hline Sponge $\mathrm{C}$ & ake & & . & & 6.6 & 10. & 63.0 & 4. & 20.6 & 1670 \\
\hline Oysters & . & & & & 6.2 & 1.2 & 3.7 & 2.0 & 86.9 & 228 \\
\hline Gingerbre & ead & & . & & 6.0 & 10.0 & 64.0 & 3.0 & 17.0 & 1678 \\
\hline Squash $\mathrm{P}$ & & 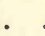 & & & 4.4 & 8.4 & 21.7 & 1.3 & 64.2 & 817 \\
\hline Tapioca & . & 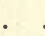 & . & & 4.1 & 3.0 & 27.1 & 1.7 & 64.1 & 687 \\
\hline Milk . & & & & & 3.4 & 4. & 5.0 & 6. & 87.0 & 314 \\
\hline Apple Pie & & & & & 3.1 & 9.8 & 42.8 & 1.8 & 42.5 & 1233 \\
\hline Green Co & $\mathrm{rn}$ & . & . & & 2.8 & 1.2 & 19.0 & .3 & 29.4 & 455 \\
\hline Potatoes & . & & & & 2.2 & .1 & 18.4 & 1.0 & 78.3 & 378 \\
\hline Dried Pru & une & & . & & 2.1 & 0. & 73.3 & .6 & 79.6 & 1368 \\
\hline Fresh Asp & par & $\mathrm{agu}$ & & - & 1.8 & .2 & 3.3 & .7 & 94.0 & 100 \\
\hline Cabbage & . & . & . & . & 1.6 & .3 & 5.6 & 1.0 & 91.5 & 143 \\
\hline Bananas & . & $\cdot$ & . & • & 1.3 & .6 & 22.0 & .8 & 75.3 & 447 \\
\hline Butter & . & . & . & $\cdot$ & 1.0 & 85. & 0. & 3.0 & 11.0 & 3491 \\
\hline
\end{tabular}

Adapted from Bulletin 28, U. S. Department of Agriculture.

1 gram of protein gives 4 Calories; 1 gram of fat gives 9 Calories; 1 gram of earbohydrates gives 4 Calories. 
to lose much of their toughness. The common methods of cooking meats are roasting, boiling, broiling, or frying. Of these methods frying is the poorest, since the particles of grease and injurious fatty acids often penetrate the food and render it indigestible in the stomach. Roasting and broiling are both good methods of cooking meats, since the albumen of the outside of the meat is coagulated before the heat penetrates to the inside, thus retaining the nutritious and palatable juices. When meat is boiled it should be plunged into very hot water at first, so that the coagulation of the surface proteid will prevent the escape of the juices.

In addition to rendering it more palatable and more digestible the cooking of meat is always advisable as a precaution against dangerous bacteria and parasites which are occasionally present.

\section{QUESTIONS}

1. Why do we require more food in winter than in summer?

2. What parts of the body are always in action?

3. Name some foods that do not come from the farm.

4. What is meant by the term "to pickle" as related to foods? "To preserve"?

5. Should formaldehyde be used to keep milk? Why?

6. How is sugar used as a preservative?

7. Name ten foods that are eaten but not produced in your community.

8. Name ten factory foods.

9. What is the objection to glucose in sugar?

10. What is the result when cane sugar is tested with Fehling's solution?

11. What is the reason for the Eskimo's peculiar diet?

12. Is alcohol a food?

13. Why does alcohol preserve foods?

14. What action did the governments of the warring countries take with reference to alcohol? 
15. What is food? Is hay food?

16. What is the advantage of a mixed diet?

17. Does occupation have anything to do with the amount of food required? Illustrate.

18. What are two uses of food?

19. Why do children become hungry more often than adults?

20. What use is made of protein in the body? Of fat? Of carbohydrate?

21. How many Calories would you consume in climbing stairs representing a vertical distance of ten feet?

.22. Which is more healthful - whole wheat flour or white flour? Why?

23. Why is the use of ice an economy in summer?

24. What causes food to spoil?

25. Why do not alcoholic drinks quench thirst?

26. What is digestion?

27. What effect has cooking upon the appearance of food?

28. What change is produced on vegetables by cooking which makes them more digestible? 


\section{CHAPTER XXIV}

\section{COMMUNITY SANITATION}

Sanitation or Sanitary Science treats of the maintenance of health and the prevention of disease. It deals mainly with (1) the factors which produce disease, and (2) the habits and means which enable mankind to resist disease.

Nearly every one recognizes the laws of personal hygiene, but the broader application of these laws to community hygiene has not yet received the attention it deserves. The subject of public health is quite modern. In fact it is only within the last fifty years that we have known anything about the power of germs as disease producers. - In 1892 a scientific cure for diphtheria was discovered. In 1901 the facts concerning the development and control of yellow fever were discovered. Scientists have learned the causes of high death rates and the way to avoid them.

The Growth of Cities. - This is a day of great cities. So rapid has been the growth of the cities in recent years that modern engineering skill has been taxed to keep pace with the problems that arise. But the problems are not merely the problems of sanitary engineers. They are the problems of all the people of the community and state and must be recognized as such if we are to have the best results.

We have had large cities for many centuries, and the occasional pestilences and scourges which partially depop- 
ulated them in earlier centuries are fearful evidence of the low community standards of those days with respect to sanitary conditions. Many of these old cities have passed out of existence or have been replaced by others.

When we contemplate the enormity of the various problems relating to food, water, sewage, and health in a large modern city like New York, we must marvel that they are so well solved. Wherever the individual is located he needs fresh air, pure water, wholesome food, shelter, a clean body, and beautiful things to look at. In the large cities his needs are emphasized by his dependence on others, and at the same time his obligations are increased because of his close association with others. The city is no longer an accumulation of human beings, but a highly organized society whose interests are the interests of the state.

Bacteria. - There are millions of tiny living organisms in the world (Figure 358). They are single-celled bodies and belong to

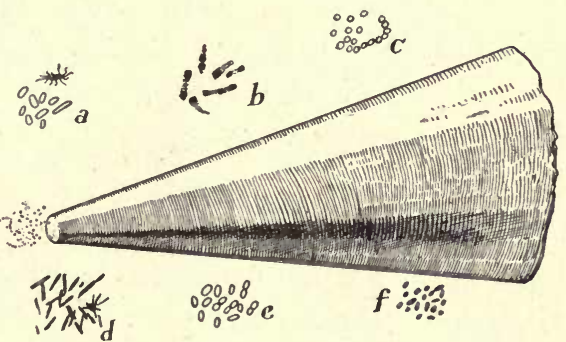

Fig. 358. - Bacteria and the Point of a Cambric Needle.

The figure shows the comparative sizes. The minute dots at the end of the needle represent the size of the bacteria. The others arranged around the needle are bacteria more highly magnified. The sources of the latter are (a) typhoid fever, (b) diphtheria, (c) boils or abscesses, (d) tuberculosis, (e) sour milk, $(f)$ grip. the divisions of plants and animals. We have already learned that many one-celled plants are known as bacteria and belong to the class of fungi.

Man divides bacteria into good and bad, according to their effect on the things which he wants to keep. Most. of the bacteria are useful and valuable - such as those 
which are active in souring milk, making vinegar, preparing plant food, and causing the decay of obnoxious dead matter (Figure 359). On the other hand, when

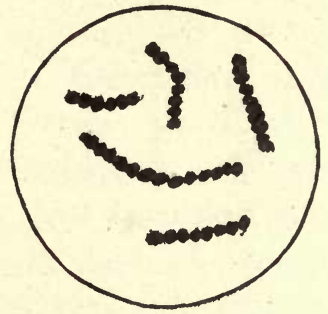

FIG. 359. - Milk-souring Bacteria. bacteria cause the decay of meats, fruits, and foods of all kinds, and the rotting of wooden appliances useful to man, we call them harmful (Figure 360 ).

If the single-celled plant or bacterium is healthy, it grows quite rapidly and divides into two cells, these two dividing in turn, and so on. We can easily see that if this division were kept up the number of bacteria would soon be overwhelming. There is not enough space or food for all, however, and each bacterium has to struggle for existence. Nevertheless, as it is, the number is countless (Figure 361).

Germs. - Certain bacteria and a few one-celled animals (protozoa) are able to live and grow as parasites in the bodies of man. These are called disease germs. Every disease is caused by a particular kind of germ. Highly magnified, showing the bacteria When bacteria or germs

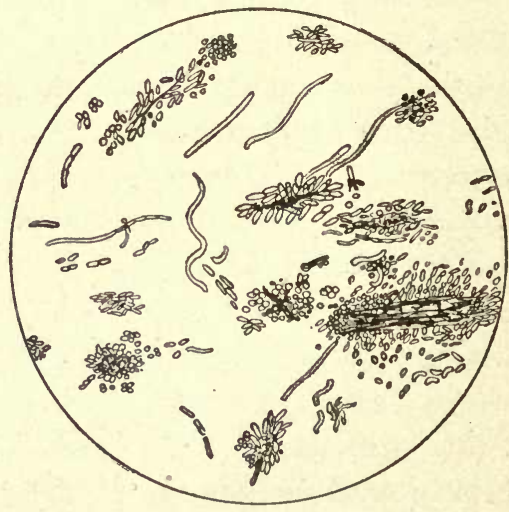

Fig. 360. - A Bit of Decaying Meat. that cause its decay.

are easily transferred from one person to another, the disease is said to be infectious.

The fact that germs cause contagious diseases was not 
proved until about 1863, although the theory had been proposed one hundred years before. Among the great names associated with the gradual development of bacteriology (the science of germs) are several of special interest. Leeuwenhoek, a Dutch lens maker, in 1683 made a lens so powerful that he could see minute living things in the scrapings from teeth. This led to the discovery and classification of bacteria. Tyndall, the noted English physicist, and the , great Louis Pasteur in 1860 a proved that air, when free

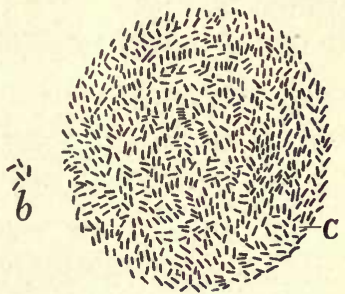
from all particles, does not Fig. 361.- Multiplication of Baccause fermentation and decay, teria in Milk.

but that these effects are produced by bacteria. In 1882 Robert Koch invented gelatin and agar culture media (nutrient substances for the growth of bacteria) so that germs could be kept separated and each kind studied by itself.

From the investigations of these men and many others we are now sure that germs are the cause of colds, tonsillitis, diphtheria, tuberculosis, pneumonia, measles, scarlet fever, typhoid fever, smallpox, blood poisoning, malaria, yellow fever, etc. If we know that all these diseases are caused by germs and can learn the means by which the germs may be destroyed, it is now only ignorance or carelessness that will allow an epidemic to spread.

To prevent the spread of an infectious disease several things about the disease and the germs which cause it must be known, namely :

(a) The source of the germs.

(b) Conditions favorable to the growth of the germs. 
(c) How germs are resisted by the body itself.

(d) How germs may be resisted by other means.

Source of Germs. - Since the air is filled with germs, many of them disease-producing, we cannot avoid receiving some of them into our systems. These are transmitted in food and drink and by contact with animals or sick persons.

Conditions Favorable to Growth. - Bacteria thrive and multiply rapidly if the surroundings into which they are introduced are favorable to their growth. Moisture, a moderate degree of warmth and darkness, and food from animal or vegetable matter are the conditions in which they thrive best. The bodies of men and animals furnish all these conditions. Food and drink are also favorable to germ growth.

Resisting Power of the Body. - The body easily receives the harmful germs through its natural openings, lined as they are with the warm, moist, mucous membrane, and through cuts and scratches. The body, however, has very efficient means of protecting itself. Some of the white corpuscles of the blood, called phagocytes, absorb disease-causing bacteria as their food, and successful resistance to infectious diseases is thought to depend largely upon the number and activity of these phagocytes. Then, too, the body produces certain other substances which are germ destroyers.

When disease germs enter the body, there are not enough of them to produce the disease at once. Usually a period of time varying with the kind of disease germ elapses, before the symptoms of the special disease appear. This time, known as the period of incubation, is occupied by the germ in multiplying and producing violent poisons called toxins. These poisons are absorbed by the blood 
and carried throughout the body, thus poisoning many other parts besides those at first affected. The cells of the body immediately begin to secrete a substance to counteract this poison, an antitoxin. If the patient is vigorous and of sufficient vitality, enough antitoxin will be secreted to overcome the disease.

The power of these natural foes of the germ depends on the healthy condition of the body. This fact and

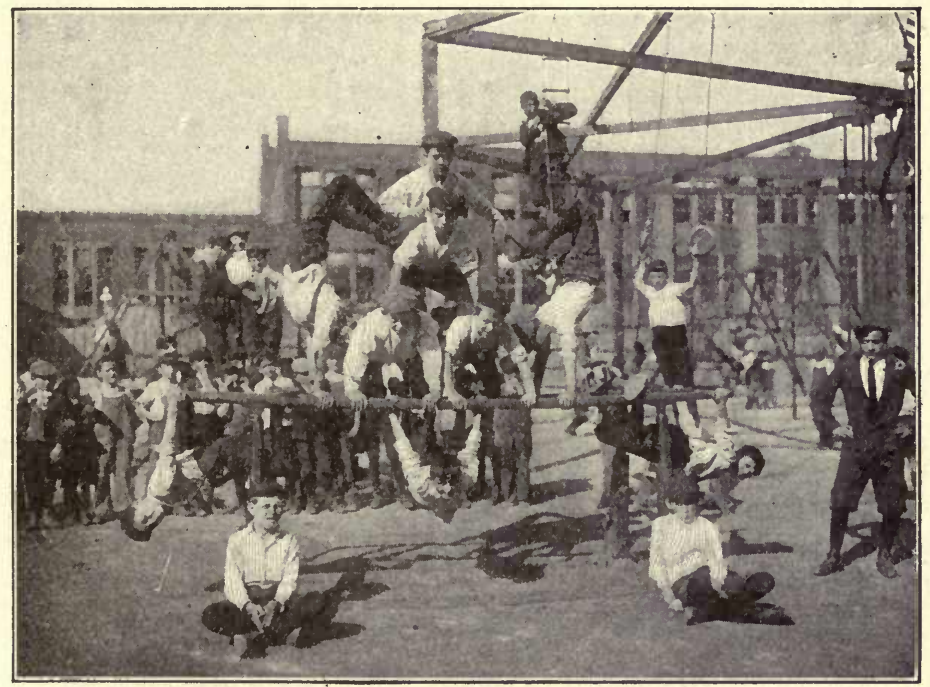

Fig. 362. - An Out-of-door Gymnasium.

others lead us to these conclusions: that we must keep the skin, all openings of the body, and all food and drink entering the body, clean; that we must keep the body well fed, rested, and vigorous that it may act naturally against disease (Figure 362). It is only when the body is weakened in some of these respects that the germ is able to thrive sufficiently to bring about disease. 
Food and Disease. - Contaminated food plays an important part in the spread of almost all kinds of disease. The body is able to cope with a few germs; but, when millions are introduced into the system in eating a small portion of impure food, the danger is increased many times. We need to be especially careful in regard to food.

When foods are handled by persons with dirty hands or clothing, stored in unprotected places, or hauled uncovered

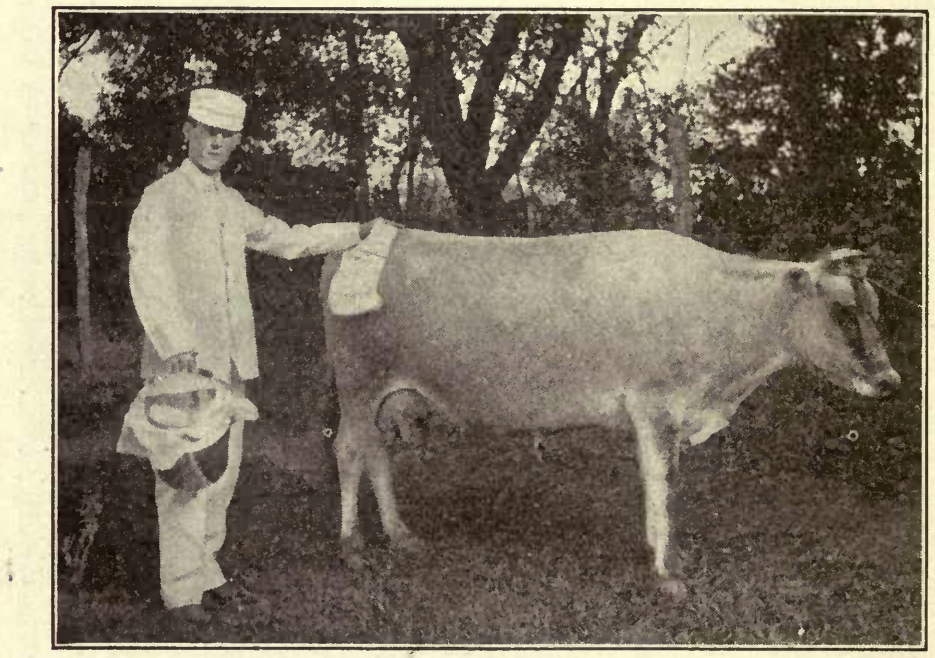

Fig. 363. - A Clean Cow and a Clean Milker.

through streets, they acquire millions of germs, rendering them unfit for food. More and more we are coming to see the necessity for pure food and are demanding of grocers, butchers, bakers, and milkmen much greater care in the handling and protection of foods (Figure 363). Since most germs cannot stand a very high temperature, foods that are well cooked are rendered free from them. 
There is much danger also in food that has reached or is approaching the spoiling point. Overripe fruit or decayed vegetables and fish are frequent causes of bowel troubles and ptomaine poisoning, ptomaines being the poisons or toxins produced in the decay of nitrogenous foodstuffs. The evils arising from tainted food are prevalent in hot weather when the housewife "economizes" by using "left overs" of various foods in which the heat and moisture have already aided in the growth of bacteria. The garbage can is the safest place for food about which there is the slightest suspicion.

Danger in Milk. - In discussing the dangers arising from impure foods, milk may be considered separately because of the great ease with which it is infected. It is classed as one of the most frequent distributors of disease germs. Many epidemics of scarlet fever, typhoid fever, and diphtheria, have been due to contaminated milk. The milk becomes infected either at the home of the dairyman, or through bottles which have

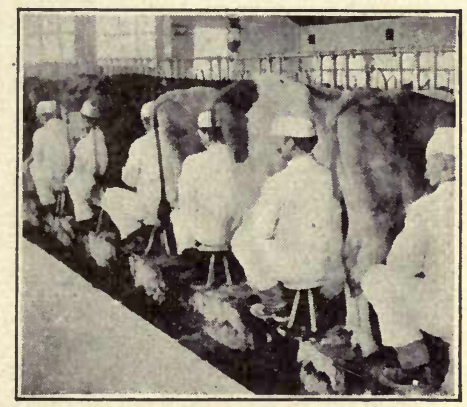

FIg. 364. - Milking Time in a Clean Barn.

been returned from homes where there is disease, and have not been thoroughly sterilized. The only safe plan in the latter case is for the dairyman to refuse to accept milk receptacles from homes where there is a contagious disease until after the quarantine is lifted. Even after that time there is danger that the proper care has not been taken to render the bottles free from germs.

The dairy from which our milk comes should be visited by a health officer, who should see that the place is 
sanitary and that the cows are in a healthy condition (Figures 364,-365). Tubercular cows mean infected milk and danger to all who drink it. In progressive communities the health authorities investigate dairy conditions and test the milk at frequent intervals to see that it is up to the proper standard of purity and richness.

It is in the hot summer months when disease germs multiply very rapidly and milk spoils quickly that the

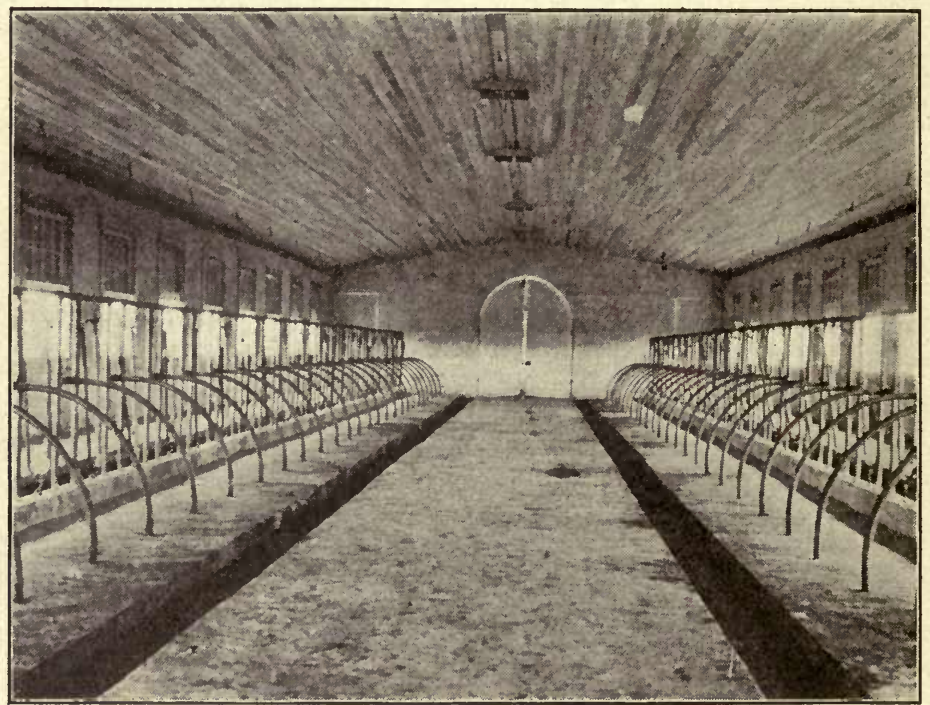

U. S. Dept. of A griculture.

Fig. 365.-A Clean, Well-lighted Dairy Barn.

danger from tainted food is greatest, especially for babies, because their food is largely milk. Steps are now being taken throughout the country to supply fresh milk and ice at either a very low cost or free, to those who are too poor to furnish these necessities themselves. Under these conditions the per cent of infant mortality is much lower. 
Milk may be pasteurized and rendered free from disease germs by heating it to a temperature of $68.3^{\circ}$. C. $\left(155^{\circ} \mathrm{F}\right.$.) for half an hour, or $77^{\circ} \mathrm{C}$. $\left(170^{\circ} \mathrm{F}\right.$.) for five minutes (Figure 366). If the milk is cooled quickly after this

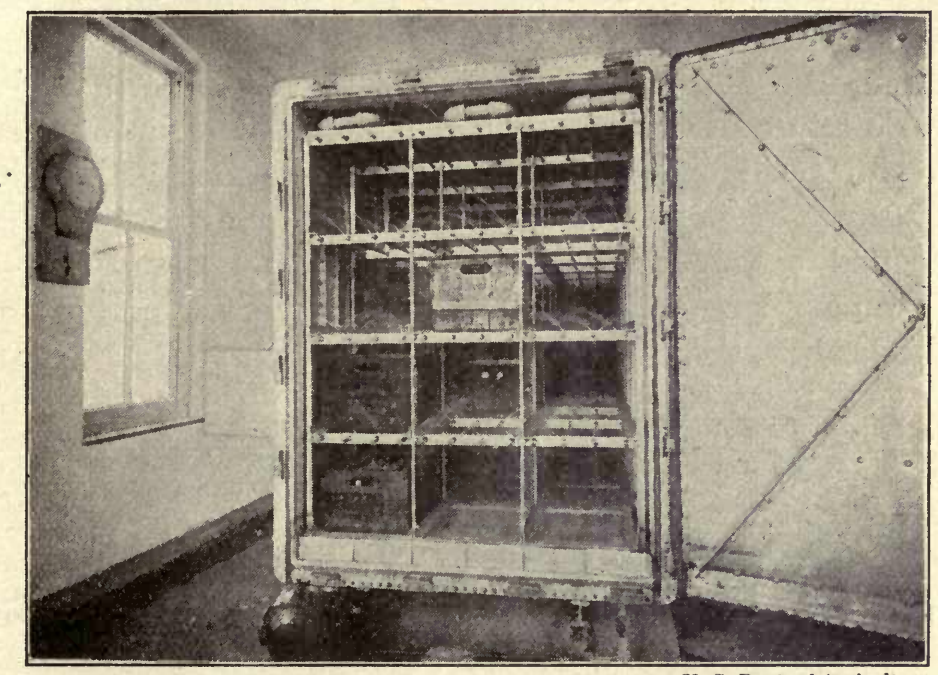

U.S. Dept. of Agriculture.

Fig. 366.-A Bottle Pasteurizer.

process and placed in a cool place it will keep longer and be a safe food for the baby.

Preservatives. - Milk and other foods are often preserved by means of chemicals which destroy the germs and prevent decay. So general has this use of chemicals as preservatives become, that stringent laws have been enacted prohibiting the practice, or forcing manufacturers to state on the labels the kind and amount of chemical used.' Many dealers in foods, and even housewives, argue that such use of chemicals is proper, as the process is cheaper than canning and it destroys germs in food. The 
danger appears, however, in the fact that these preservatives prevent food from digesting properly and, being poisonous, in time work serious injury to the body. Milk that has not been pasteurized or properly cooled will sour in a day's time in hot weather. If it does not sour it has probably been treated with formaldehyde, which is the chemical commonly used for this purpose.

Borax and boric acid will make tainted meats appear fresh. Such "doctored" meats are often made into sausage. Benzoate of soda is used in many canned and bottled foods, such as relishes, pickles, and catsups.

Danger from Water. - Nothing is more important to the health of the individual and the community than an

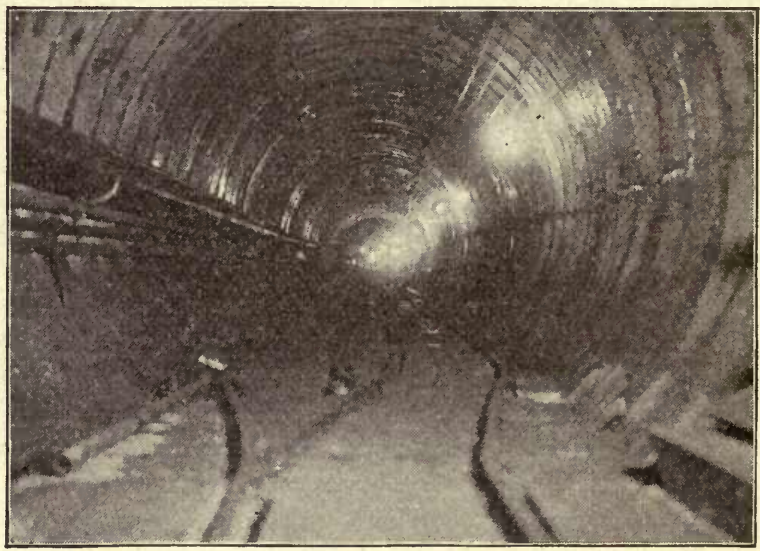

FIG. 367. - Interior of a Ten-foot Intake Water Tunnel.

abundant supply of pure water. To obtain such a supply and to see that it continues pure should be the serious concern of city and village health boards. If water from rivers or lakes must be used, all possible precaution should be taken to establish efficient filtration plants, 
or have the intakes far enough removed from any contamination to insure comparative purity of water (Figure 367). If water is obtained from wells, they should be so situated that they will not be contaminated by germ-laden seepage water.

Public Drinking Cups, Towels, etc. - No matter how many precautions are taken to provide pure water, the purpose is largely defeated if we allow the diseased and healthy to use the same cup for drinking. Nothing is more dangerous or disgusting than the public drinking cup as it has been used in many public places. Many states now have laws forbidding the use of such cups. City streets and parks, schools, public buildings, and trains are equipped largely with sani-

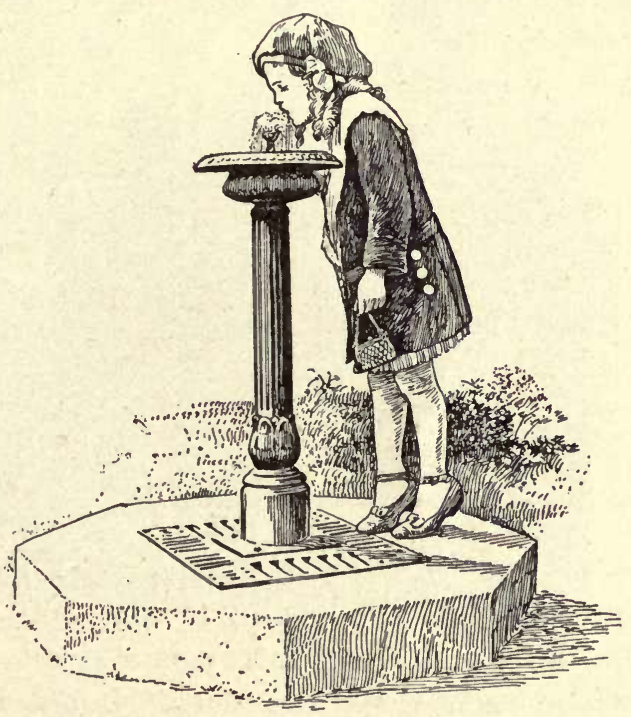

FIG. 368. - A Drinking Fountain. tary fountains and individual drinking cups (Figure 368).

Along with the drinking cup we are classing and abolishing the public towel, comb, and cake of soap. Paper towels and liquid soap are now well established public toilet commodities. Dishes and glasses used promiscuously at soda fountains and improperly washed are also coming under the ban and are being replaced by paper substitutes. 
Street Cleaning. - A number of diseases may be transmitted by means of the floating particles with which the city air is charged. Air may be tested for disease-producing bacteria by making a gelatin and agar culture of whatever things are floating in the air. There are many sources of dust in the city. The chimneys pour out some

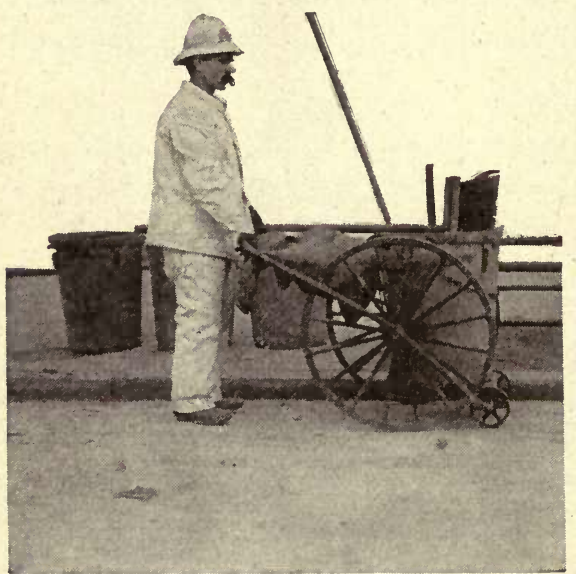

Frg. 369.-A Street Cleaner. of it; the traffic on the streets gradually grinds loose material into particles that are small enough to be carried by the wind; and the decomposition of refuse of all sorts produces dust. The best method of preventing the spread of diseases whose germs float in the air, and eliminating objectionable street odors is a thorough system of street cleaning (Figure 369). The streets should be swept at short intervals and all refuse carted away. Once each week the street should be washed clean by "flushing" it.

Garbage, Ashes, and Rubbish. - These wastes should be placed in different receptacles at the homes so that they may be collected most economically. Many modern cities are now burning their garbage and rubbish. This is an ideal way of disposing of it from a sanitary standpoint. Ashes may be used for making desired fills near the city. New York is building Rikers Island with her ashes and street sweepings. The garbage is 
carried to Barren Island, where it passes through the reduction plant. The material that arrives at Barren Island is first loaded into digesters, where it is cooked with hot steam for several hours. It is then placed in hydraulic presses which press out the grease and oil. This grease is placed in barrels and sold to the soap

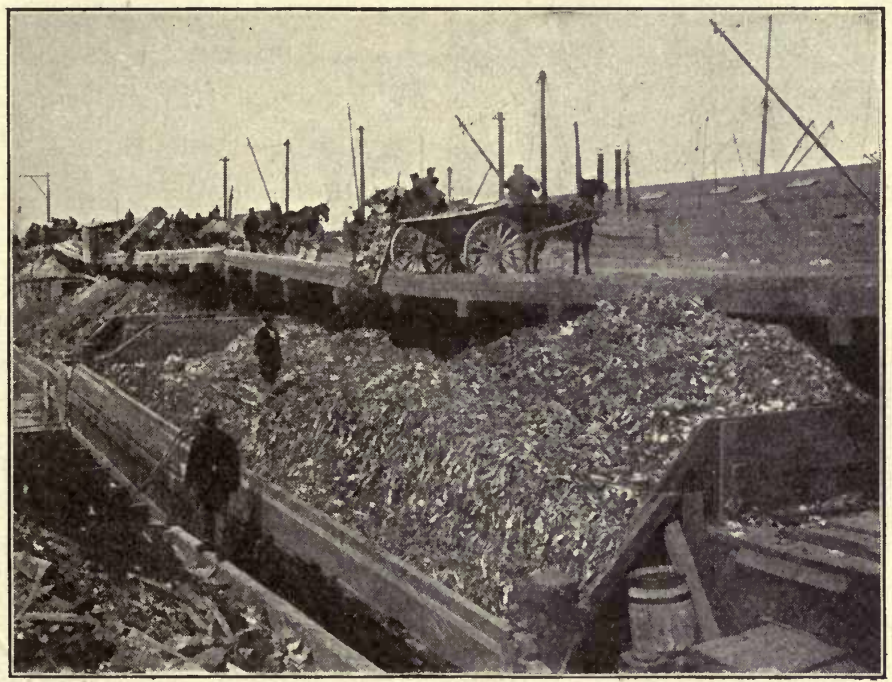

Fig. 370. - Dumping Garbage into a Scow to be Taken out to Sea.

making industries. The remainder of the digested garbage is used for fertilizer.

When garbage is permitted to accumulate, it forms a breeding place for flies and other disease-carrying insects. Flies. - "Swat the fly" is a popular and praiseworthy slogan. "Fight the Filth" is a better one, for only through the elimination of filth will the campaign against the fly be successful. Flies breed in filth, in body wastes of animals, manure piles, vaults, etc. As they grow they feed in filthy places. They enter sickrooms and gather 
germs which not only cling to their legs but pass from their bodies in the discharges known as fly specks. Flies

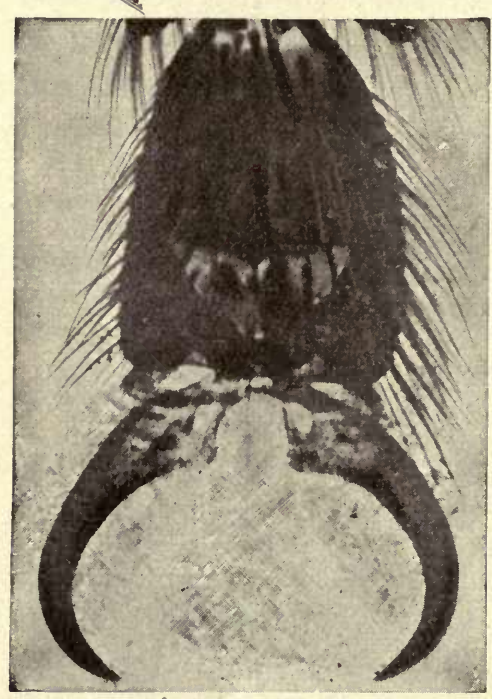

FIG. 371. - The Foot of a Fly, Magnified. are most effective spreaders of disease and must be kept screened away from sickness and away from health. It is estimated that a fly's life is spent within the radius of half a mile from its breeding place. Consequently the presence of flies indicates that there must be, in the immediate neighborhood, filth in which they can breed (Figure 371).

Keep garbage cans covered and clean; eliminate manure piles and heaps of kitchen waste; carefully screen every house. With no place to breed and little to eat, flies will cease to be a problem.

Sewage Disposal and Public Health. - We have learned by very dear experience that our drinking water must not be.polluted by sewage. The one water disease which we most fear in the United States is typhoid fever, and the only way in which a large supply of water may become contaminated with typhoid germs is through sewage wastes.

In 1903 Cleveland had an epidemic of typhoid fever as a result of contamination of the water supply by the city's sewage. Many people bought spring water while others took the precaution of boiling the city water. 
After carefully studying the problem, Cleveland built a new intake tunnel extending five miles into the lake. As soon as it was opened the doctors noticed a change for the better in the typhoid situation, and in a short time the water was considered by all as comparatively safe.

It is quite common for cities to pour their sewage into near-by waters. New York City pours its sewage into the

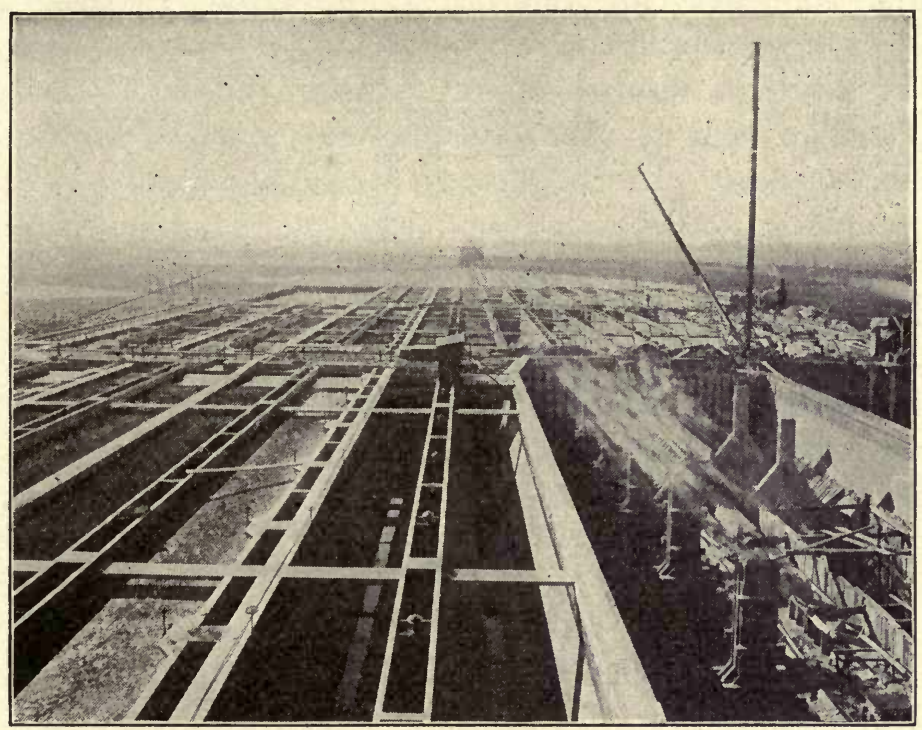

Fig. 372. - A Sewage Disposal Plant.

The Imhoff tanks, Columbus, Ohio.

harbor, Chicago into the Illinois River through the Chicago Drainage canal, Cleveland into Lake Erie. In many inland cities the problem of sewage disposal has been solved by the installation of sewage disposal plants. They have proved so satisfactory that their use undoubtedly will be greatly extended. Such plants purify the sewage by oxidation and by the aid of friendly 
bacteria which grow and prey upon the harmful bacteria of the sewage. Columbus, Ohio, recently installed a sewage disposal plant having a capacity of $20,000,000$ gallons daily.

Figure 372 shows the new Imhoff tanks that are in service at Columbus, Ohio. In the background the sprinkling filters are shown. The sewage passes through these tanks at a very low velocity, which permits the heavier solids to settle and cling to the sloping sides of the tanks. The clarified sewage passes through the tank and is sprayed over beds of broken limestone about five and one half feet deep. In the photograph these sprinkling filters are shown in the background, the round building being the place from which the liquor is distributed by means $\checkmark$ of gates to the sprinkling filters.

Typhoid Fever. - In 1900 there were recorded about 350,000 cases of typhoid fever in the United States, with more than 35,000 deaths. Typhoid germs leave the body in the wastes from intestines and kidneys, and in the sputum when pneumonia develops with typhoid. They are carried by food, water, dust, and flies; by oysters that have grown in beds near outlets to sewers; and by raw vegetables which have been watered with sewagepolluted water.

We can easily understand, then, how important a question is the disposal of sewage in considering the health of a community. Terrible epidemics of typhoid may be caused in the following ways: by throwing body waste from a patient on the ground near a stream into which it may be carried by melting snow, rain, or high water; by disposing of sewage where it may enter a well by means of seepage water (Figure 373); by turning the sewage from one city directly into a lake or river from which an- 
other community obtains its water. When the direction of the Chicago River was changed to carry Chicago's sewage into the Mississippi River system and water intakes were built four miles out in the lake, Chicago's

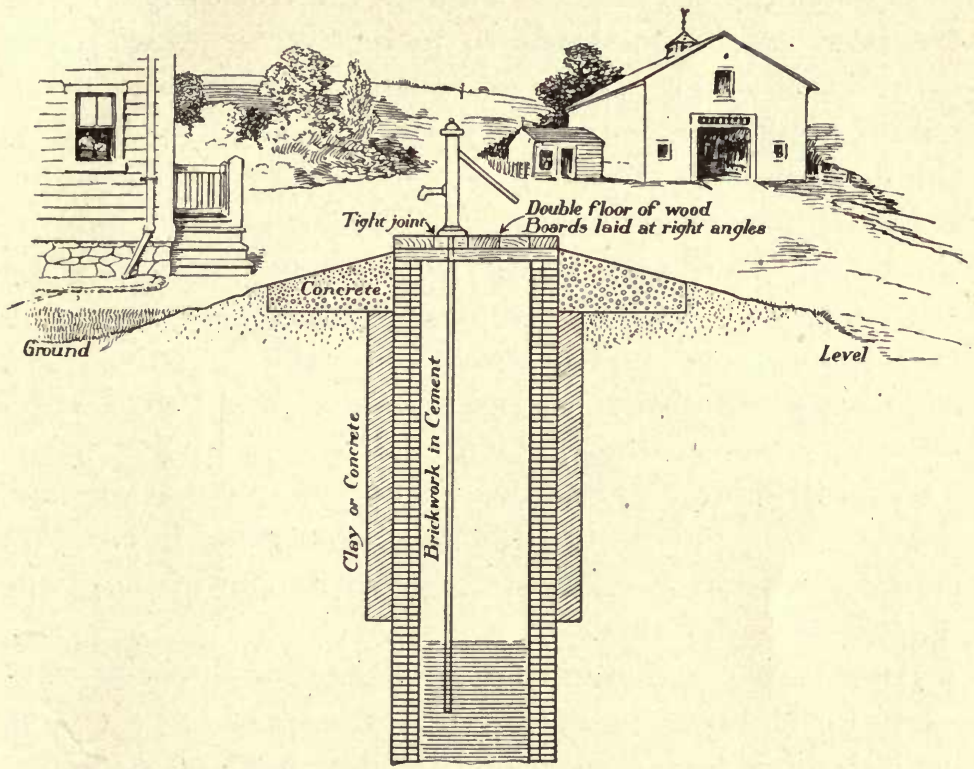

Fig. 373. - A Properly Located and Constructed Well to Prevent the Entrance of Seepage Water.

death rate was reduced from 5.97 in $1891-1900$ to 2.21 in 1901-1910.

If there is any reason to believe that your water supply has been contaminated by typhoid fever germs, do not fail to boil the water twenty minutes. It will take that long to kill the germs. Four per cent of all typhoid patients carry the germs in their bodies for from ten weeks to two years, even though they appear perfectly well. These "typhoid carriers" may give the disease to many others. 
Colds. - The spreading of infectious diseases may be prevented more easily if we understand something of the nature of each. A cold, considered quite harmless by the majority of people, is one of our serious diseases, and is brought on by bacteria. It lowers the resisting power of the body and often leads to more serious infections of throat and lungs - such as tonsillitis, bronchitis, pneumonia, diphtheria, etc. Bacteria of colds are found in the nose, mouth, throat, and eyes. They are communicated in tiny drops by coughing, sneezing, and blowing the nose. They may be carried on handkerchiefs, or on anything that comes in contact with the secretions of mouth and nose, such as towels and drinking cups. One of the most dangerous spreaders of nose and throat affections is the disgusting habit of spitting in public places. The multitude of germs thrown out in spitting, dry and become mingled with the dust and are able to live in that state for several months, to the great danger of those who must breathe the dust.

Diphtheria. - One of the most dreaded diseases, especially for children, is diphtheria. If a child has a croupy cough which persists during the day, and on examination the throat shows small grayish white patches, a physician should be summoned at once. The danger is too great for home doctoring. Membranous croup, formerly considered an entirely separate disease, is now known to be diphtheria which has invaded the larynx.

Diphtheria germs are scattered by coughing and by discharges from the nose and throat. Too great care cannot be taken in disinfecting or burning all garments and cloths used about the diphtheria patient. In the throats of patients who have recovered from diphtheria the germs may be active for months and infect food, dishes, towels, 
and clothing. In this way the disease may be passed on long after quarantine has been lifted.

When the diphtheria germ enters the body, it produces a poison (toxin) which causes the severe illness. Immediately the body begins to produce a substance which is called antitoxin. If the body is able to produce sufficient antitoxin, the patient does not succumb to the disease. In cases where the antitoxin produced by the patient is not sufficient to counteract the toxin, a liquid called diphtheria antitoxin is introduced into the blood. With this help the disease is made much less severe and if the antitoxin be taken as soon as one is exposed, the disease is often prevented entirely. Since the use of antitoxin has become general the percentage of trom diphtheria has been very much smaller.

Pneumonia. - Pneumonia, one of our most fatal diseases, spreads in practically the same way as colds and throat infections. Its action is quite rapid. Pneumonia germs may be found in the throats of almost all people; but as long as the body is strong and vigorous, it is able to resist them. It is when the system is weakened by cold or some minor disease that the germs are able to do their work. Keeping our bodies in the best possible condition is the only effective means of preventing this disease.

Tuberculosis. - "The Great White Plague" costs the United States many lives each year, and millions of dollars in caring for its patients. Tuberculosis or consumption is caused by germs which lodge in and attack any part of the body, - bony, muscular, or mucous. Those that affect the lungs probably reach them through the alimentary canal and the blood.

Most of the people in the world are attacked by the 
tuberculosis germ at some time but easily overcome it and are none the wiser. If the disease once gains a foothold on the body, however, it becomes more and more difficult to throw it off because the system continually grows weaker. We are now positive that consumption in its early stages can be wholly cured and that there

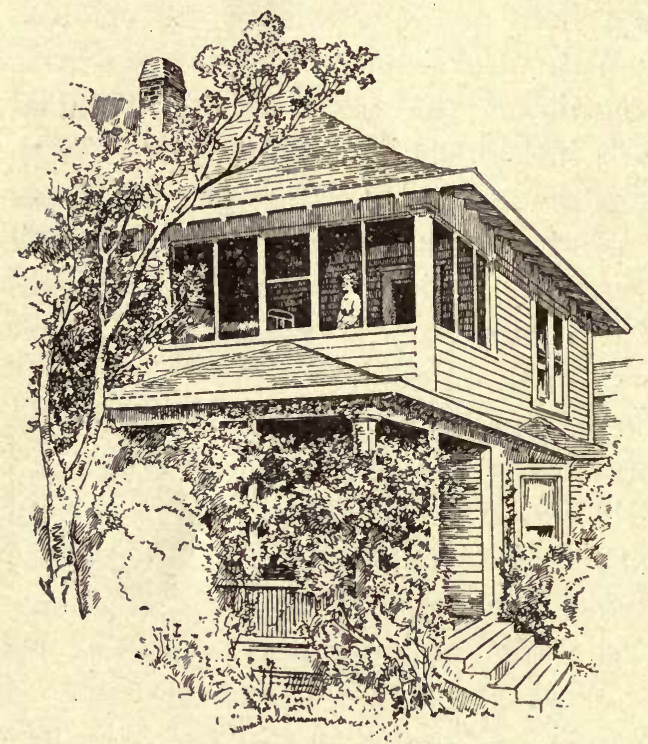

Fig. 374.-A Sleeping Porch.

is hope for those in more advanced stages if they are willing to make the effort to get well.

The remedies are simple and within the reach of almost every one. They are: to live in the open air day and night; to wear warm clothing; to eat plenty of plain, nourishing food, especially fresh milk and eggs. The old idea that people suffering from tuberculosis must live in a warm climate the year around and be protected from every breeze, has been discarded as wholly false. According to the modern theory the disease can be cured at home in any climate, though cool dry air is best. A sleeping porch, tent, or open air cottage is the main requirement, backed by determination on the part of the patient to get well (Figure 374).

Tuberculosis germs escape from the body in discharges 
from the nose, throat, and bowels. They enter other bodies on infected fingers put into the mouth; on food infected by flies and other insects; or by milk and butter from tubercular cattle; on dishes, garments, and cloths used in the care of patients. The sputum from a consumptive should never be allowed to dry. It should be received in sputum cups or on papers and cloths, and

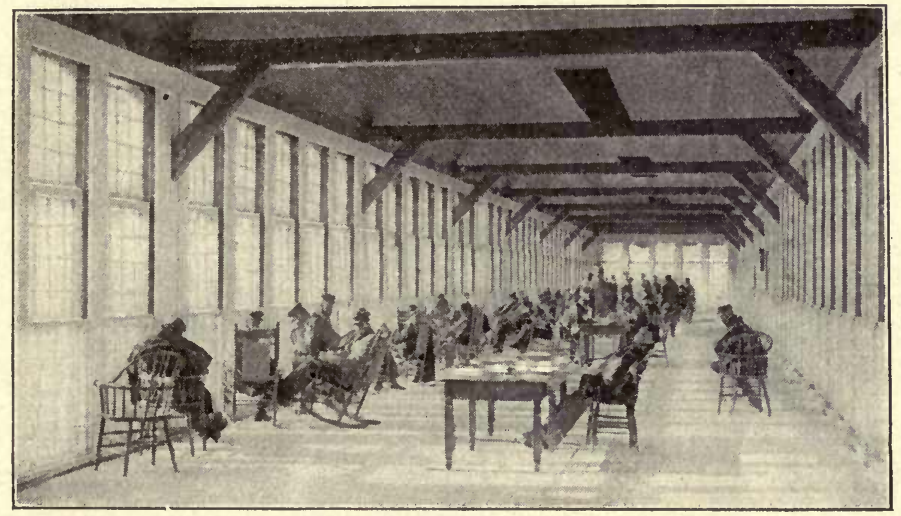

Fig. 375. - The Sun Parlor of a Large Hospital.

Sun is an excellent destroyer of germs.

these should be burned. Children do not inherit tuberculosis from their parents; but they may inherit a weak resisting power so that they succumb to the disease more easily than the children of healthy parents.

Scarlet Fever and Measles. - These diseases are characterized by eruptions and are very infectious, both occurring chiefly in childhood. The after effects of both these diseases are much more to be feared than the diseases themselves. Pneumonia and consumption, weakened sight and hearing, and other infirmities may follow if the greatest care is not taken when the patient is recov- 
ering. The danger of spreading scarlet fever lasts as long as the dead skin is " peeling off," a process which continues after the patient has otherwise recovered.

Smallpox. - Smallpox is one of the eruptive diseases, the sores being of a loathsome nature and leaving the disfiguring " pock marks " if great care is not taken. This disease was once a terrible scourge, and even up to the beginning of the nineteenth century almost everybody expected to have it.

The first attempt at preventing the disease came with the discovery that milkmaids who had become infected with cowpox (smallpox of the cow) either did not get smallpox or had very light cases. This fact in 1796 led Sir Edward Jenner to experiments which brought about the discovery of vaccination. He took the matter formed in cowpox and introduced this vaccine virus (poison from cows) into the blood of man. The germs from cowpox are so much weaker than those that come from smallpox that they cannot produce the latter disease; but they cause the body to produce the substance which kills the smallpox germ. The length of time for which vaccination renders one immune varies with the person vaccinated, lasting usually from one year to ten or twelve years.

When vaccination is attended by ill effects in other parts of the body or with very serious inflammation at the point of vaccination, we may be sure that the trouble may be laid to carelessness. The opening of the skin must be kept clean and free from other infection.

Diseases Carried by Insects. - A few diseases are caused by one-celled animals or protozoa. The protozoa do not cause disease by being carried directly from one person to another, but must live for a while in the body of some insect. The germs are sucked up in the blood 
which the insect draws when biting a diseased person. These germs live in the body of the insect for some time, multiplying rapidly. When they are ready to be injected into new victims, they pass into the salivary glands of the insect. From there they are injected into the blood of the one bitten, where they multiply and produce the disease.

Malaria. - This disease was formerly believed to be due to damp night air or swamp air. We now know that

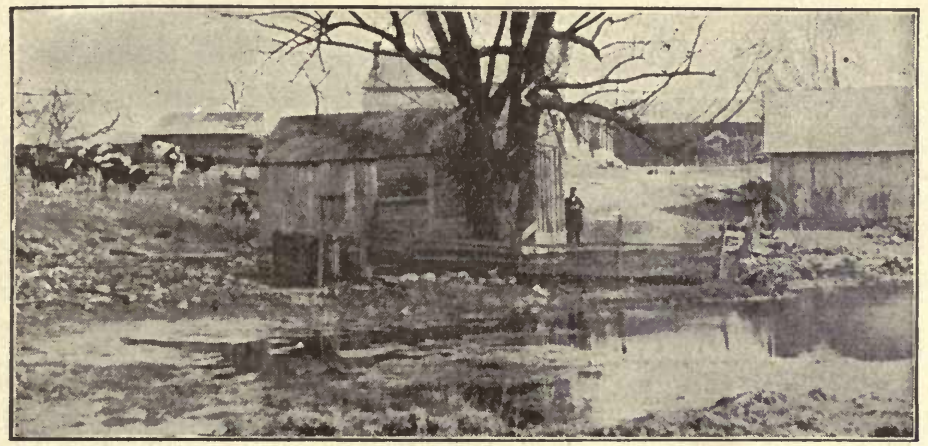

FIG. 376. - A Breeding Place for Mosquitoes.

malaria is caused by the action of small parasites, protozoa, which flourish in the body of the mosquito. The fact that mosquitoes are more active at night and breed extensively in swamps accounts for the mistaken ideas concerning malaria.

If mosquitoes can be kept from biting people who have malaria and then from biting other people, the malaria question is solved. Since mosquitoes breed in stagnant water and damp places, all puddles, ditches, and swamps should be drained, or treated with kerosene (Figure 376). The kerosene treatment is very effective where other means are not practical. The adult mosquito lays its 
eggs in little groups on the surface of stagnant water. When the eggs hatch, the larvæ (larva) come forth, little squirming insects commonly known as wrigglers. The larvæ, or wrigglers, after about seven days, change into another form called pupæ (pupa). The pupa lives in the water three days or longer and changes into the adult

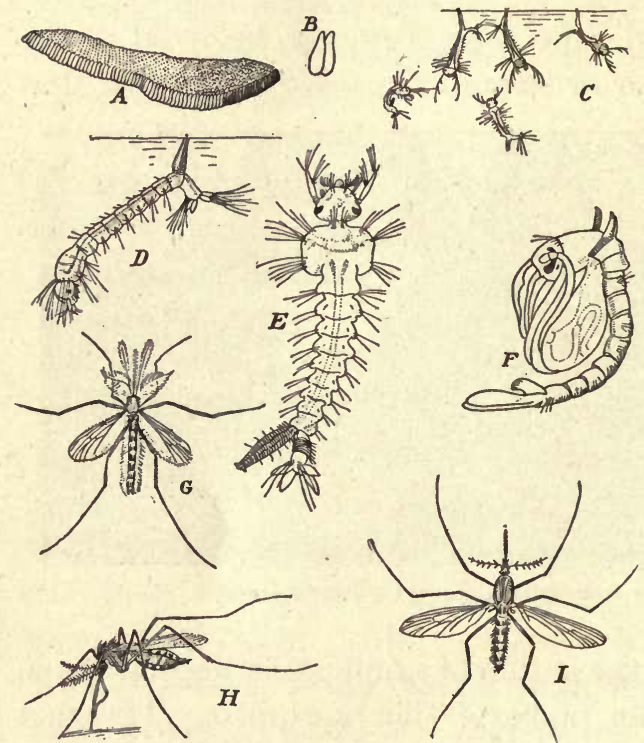

FIG. 377. - Life History of the Mosquito.

The common mosquito (Culex). A, egg raft; $B$, eggs; $C$, young "wrigglers" or larvæ; $D$ and $E$, views of larvæ; $F$, pupa; $G$, male; $H$ and $I$, females. $A, B, C, G, H$, and $I$, somewhat enlarged. $D, E$, and $F$, very much enlarged. (After Howard.) mosquito, which in turn lays eggs and begins a new cycle of life (Figure 377).

When kerosene or crude oil is poured on the water, it spreads over the surface, forming a film which prevents the larvæ and pupæ from getting air from the surface water and thus kills them. The adult mosquitoes also cannot lay their eggs on the surface of the water because they become entangled by the film of oil. The above precautions and the careful screening of houses will reduce malaria to a minimum.

Yellow Fever. - This disease is spread in the same manner as malaria with one marked difference; namely, that while malaria is carried by one variety of mosquito 
called anopheles (Figure 378), yellow fever is carried by another species called stegomyia (Figure 379 ). The name of our common mosquito, which is considered comparatively harmless, is the Culex (Figure 380).

One of the main duties of the United

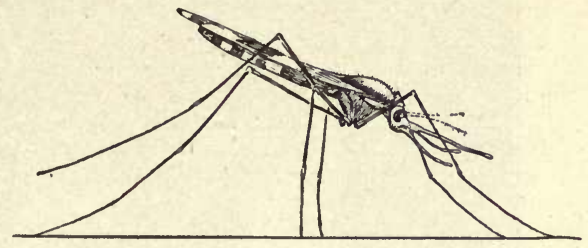
Fig. 378. - The Malarial Mosquito (anopheles). States in taking up the administration of Cuba and the work of the Panama Canal was the extermination of the yellow fever and malaria mosquitoes. This was ac-

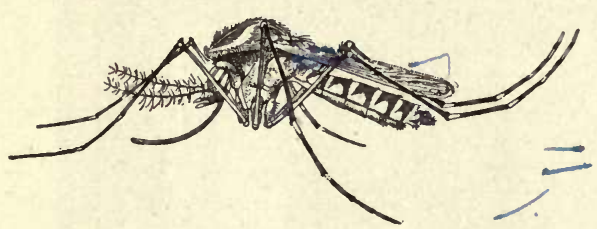

Frg. 379. - The Yellow Fever Mosquito (stegomyia). complished by draining and filling, and by a thorough clean-

- ing up of filth of various sorts (Figures $381,382)$.

Several other diseases are spread by protozoa in the bodies of insects. The terrible sleeping sickness of Africa is caused by the Tsetse fly; the bubonic plague is carried by a kind of flea which infests rats; and the spotted fever and cattle fever by species of ticks.

Quarantine.-There is one general method of preventing the spread of germs which is used in

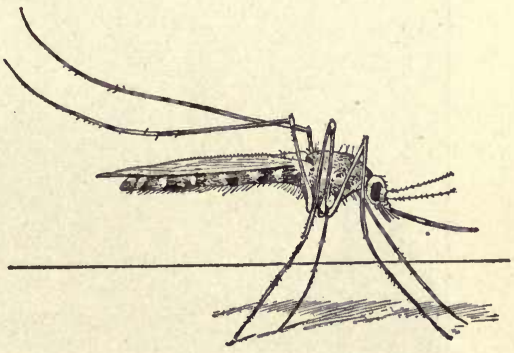

FIG. 380. - The Harmless Mosquito (Culex). dealing with all kinds of infectious diseases. This is the setting apart from the community of an individual or 


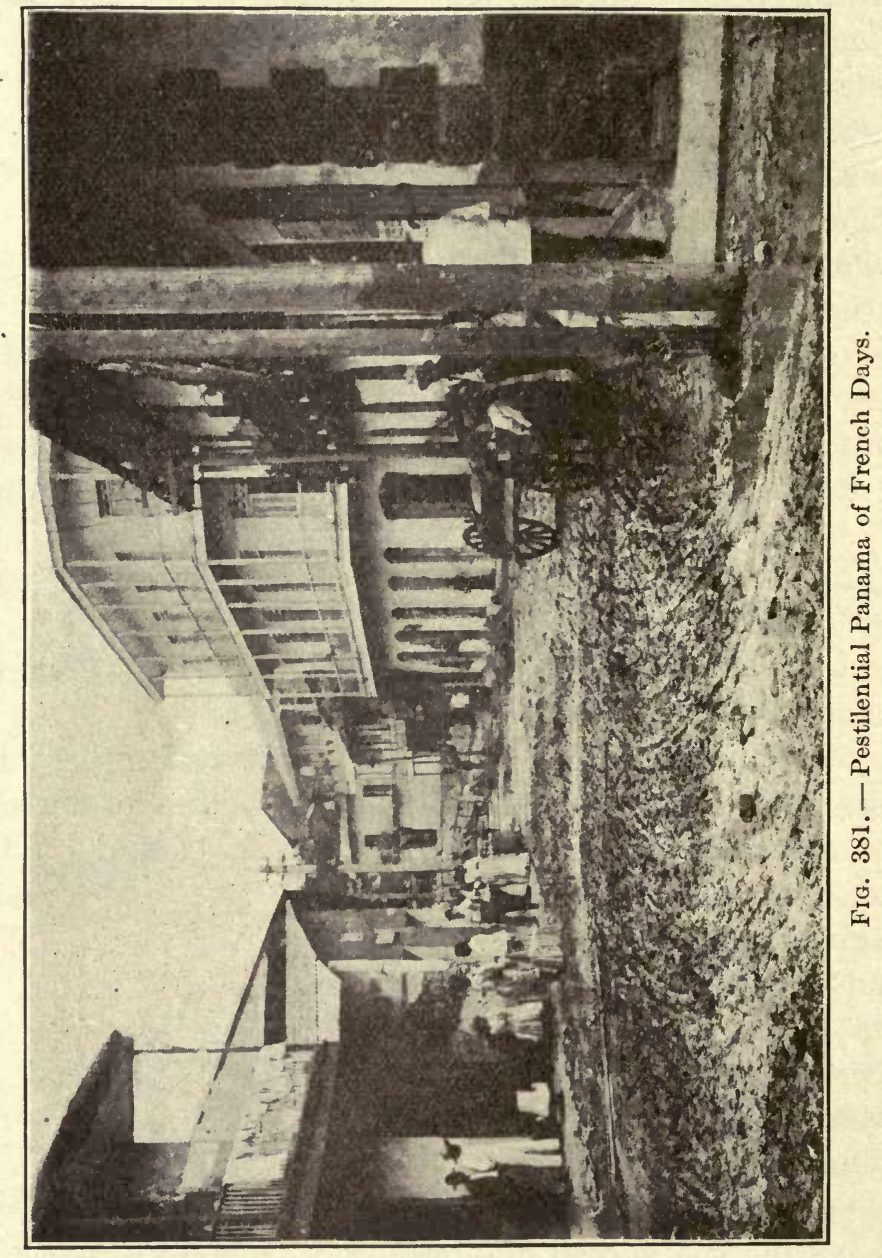




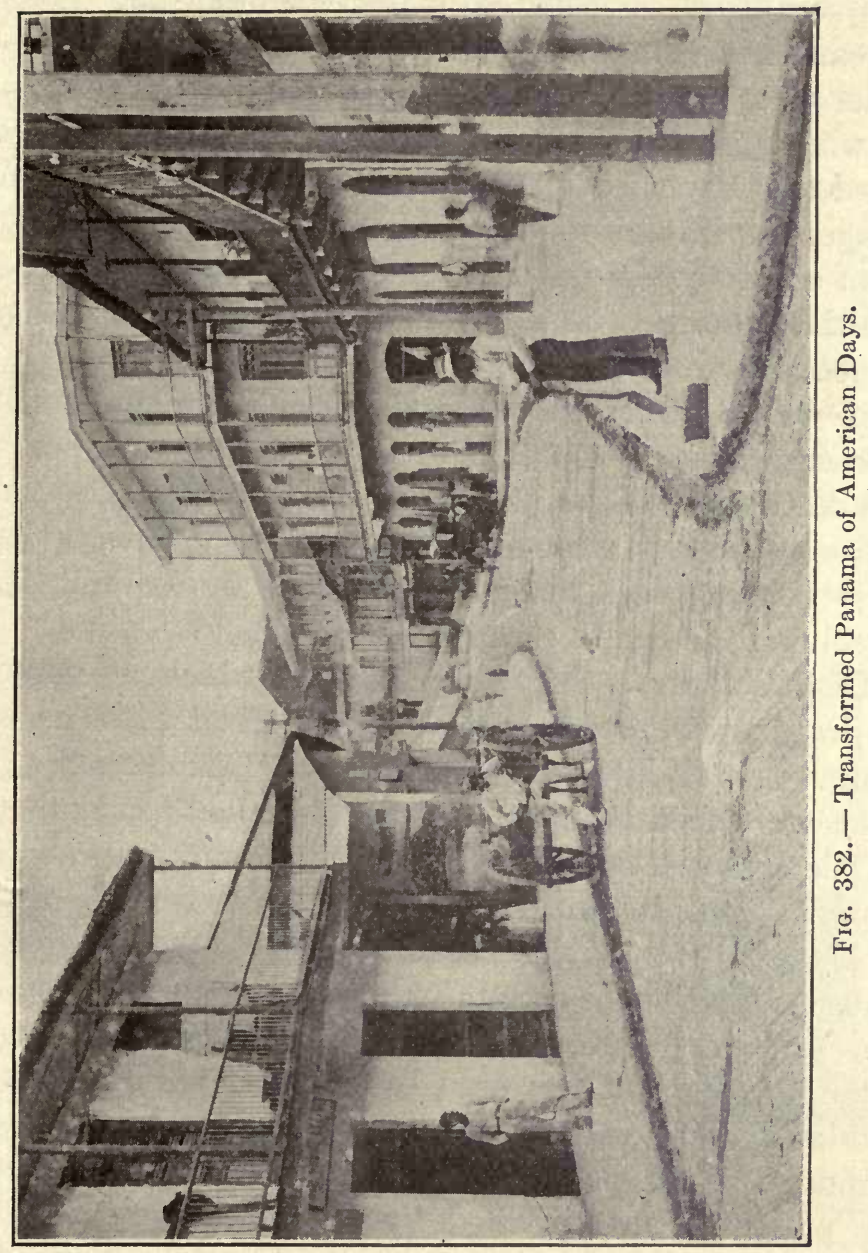


thing known or believed to be infected. Individuals may be quarantined or isolated in a single room and there all precaution taken to prevent the spread of the disease to other members of the household. Sometimes the house in which live one or more victims of disease is quarantined to prevent persons from entering, or what is more dangerous, leaving the house. Ships are often prevented from entering port for days or even for weeks because the health authorities have reason to believe that passengers or goods have been infected.

If we are truly in earnest in our campaign against disease, we must respect, and insist on others respecting, quarantines. We may suffer great inconvenience sometimes because of the restrictions which quarantine imposes, but we must remember that it is an essential factor in solving the health problem.

Disinfectants. - Any process by which germs are destroyed may be spoken of as disinfection. The substance used is called the disinfectant. Disinfection which is accomplished by means of gases is called fumigation.

The common household disinfectants are soap and hot water. Sunlight and air are great destroyers of germs. Their effectiveness has been known to generations of housewives who have hung clothes and bedding on the line to "air." Intense heat is a most efficient method of killing germs. Dishes used by diseased persons may be rendered harmless by placing in boiling water for a few minutes. Typhoid germs in water and on articles used by patients can be killed by boiling twenty minutes. Articles such as bandages and surgical dressings may be rendered sterile (free from germs) by subjecting to great heat for some time.

Many chemicals are used as disinfectants. The most 
common of these are unslaked lime, chloride of lime, carbolic acid, mercuric chloride, sulphur, and formaldehyde. Great care should be taken in using these substances because most of them are poisons. Unslaked lime may be scattered on the ground to disinfect damp, filthy places. Chloride of lime is a good disinfectant for garbage cans and closets. Carbolic acid, a very strong poison, is a general disinfectant. A solution of it may be used for many purposes: during house cleaning for wiping up floors and washing out closets and cupboards; as a disinfectant in cleansing wounds (weak solution); to mix with the water in which clothes are boiled after being used in the care of the sick. Mercuric chloride (corrosive sublimate, a deadly poison) is used in much the same way as carbolic acid. Its odor is not so strong, and it is not so hard on the skin.

Sulphur dioxide and formaldehyde are the chemicals commonly used for fumigating. Sulphur dioxide is now generally obtained by burning sulphur candles, but is not of much value unless the air of the room where it is burned is full of moisture. This may be accomplished by boiling water for some time previous in the room to be fumigated. There is on the market however a commercial fumigator which is quite satisfactory, the moisture being secured by setting the fumigator in water. Formaldehyde is a better disinfectant than

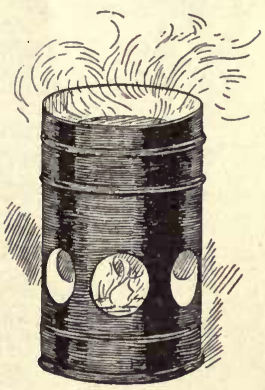

Fig. 383. - A Formaldehyde candle. sulphur dioxide. It is also used in the form of candles (Figure 383), but it is more commonly used in the form of a liquid solution. Cloths are dipped in the solution and are then hung in the room which is to be disinfected. 
In order that the gases may have time to complete their work and be most effective, rooms and houses which are being disinfected by fumigation should always be tightly closed during the process of fumigation and allowed to remain closed for several hours.

\section{QUESTIONS}

1. How does the body resist disease?

2. What are the incubation periods for scarlet fever, measles, diphtheria, hydrophobia?

3. Has your town a food inspector? Are tests of milk and ice cream made frequently?

4. Does pasteurizing milk affect its digestibility?

5. What is meant by Federal meat inspection?

6. What is the death rate for your town? How does it compare with other cities of the country?

7. How does your community obtain its water? How does it dispose of s'ewage? Garbage?

8. How do the city and country compare in healthfulness?

9. Can you name a disease which at the present time is considered incurable?

10. How are you fighting flies?

11. Why should we all be interested in community sanitation? 


\section{APPENDIX}

\section{I}

VAPOR PRESSURES OF WATER

Note. - Both the Fahrenheit (F.) and the Centigrade (C.) temperatures are given.

\begin{tabular}{|c|c|c|c|c|c|}
\hline \multicolumn{2}{|c|}{ TEMPERATURE } & \multirow{2}{*}{$\begin{array}{l}\text { Pressure iN } \\
\text { MM. }\end{array}$} & \multicolumn{2}{|c|}{ TEMPERATURE } & \multirow{2}{*}{$\begin{array}{c}\text { PRESSURE IN } \\
\text { MM. }\end{array}$} \\
\hline F. & C. & & F. & C. & \\
\hline $14^{\circ}$ & $-10^{\circ}$ & 2.2 & $62.6^{\circ}$ & $17^{\circ}$ & 14.4 \\
\hline 15.8 & -9 & 2.3 & 64.4 & 18 & 15.4 \\
\hline 17.6 & -8 & 2.5 & 66.2 & 19 & 16.3 \\
\hline 19.4 & -7 & 2.7 & 68.0 & 20 & 17.4 \\
\hline 21.2 & -6 & 2.9 & 69.8 & 21 & 18.5 \\
\hline 23.0 & -5 & 3.2 & 71.6 & 22 & 19.7 \\
\hline 24.8 & -4 & 3.4 & 73.4 & 23 & 20.9 \\
\hline 26.6 & -3 & 3.7 & 75.2 & 24 & 22.2 \\
\hline 28.4 & -2 & 3.9 & 77.0 & 25 & 23.5 \\
\hline 30.2 & -1 & 4.2 & 78.8 & 26 & 25.1 \\
\hline 32.0 & 0 & 4.6 & 80.6 & 27 & 26.5 \\
\hline 33.8 & 1 & 4.9 & 82.4 & 28 & 28.1 \\
\hline 35.6 & 2 & 5.3 & 84.2 & 29 & 29.8 \\
\hline 37.4 & 3 & 5.7 & 86.0 & 30 & 31.5 \\
\hline 39.2 & 4 & 6.1 & 87.8 & 31 & 33.4 \\
\hline 41.0 & 5 & 6.5 & 89.6 & 32 & 35.4 \\
\hline 42.8 & 6 & 7.0 & 91.4 & 33 & 37.4 \\
\hline 44.6 & 7 & 7.5 & 93.2 & 34 & 39.6 \\
\hline 46.4 & 8 & 8.0 & 95.0 & 35 & 41.8 \\
\hline 48.2 & 9 & 8.6 & 96.8 & 36 & 44.2 \\
\hline 50.0 & 10 & 9.2 & 98.6 & 37 & 46.7 \\
\hline 51.8 & 11 & 9.8 & 100.4 & 38 & 49.3 \\
\hline 53.6 & 12 & 10.5 & 102.2 & 39 & 52.0 \\
\hline 55.4 & 13 & 11.2 & 104.0 & 40 & 54.9 \\
\hline 57.2 & 14 & 11.9 & 105.8 & 41 & 57.9 \\
\hline 59.0 & 15 & 12.7 & 113.0 & 45 & 71.4 \\
\hline 60.8 & 16 & 13.5 & 212.0 & 100 & 760.0 \\
\hline
\end{tabular}




\section{II}

Fungicides. - Most of the harmful fungi may be killed by the use of Bordeaux mixture. It is made with different amounts of copper sulphate and lime according to its intended use.

\section{Bordeaux Mixture}

Copper sulphate . . . 2, 3, 4, 5, 6, pounds

Lime . . . . . . . equal to copper sulphate

Water . . . . 50 gallons

There should always be enough lime to give an alkaline reaction on litmus paper; otherwise the leaves may be scorched. It should be remembered that Bordeaux mixture is especially effective on fungi and has very little effect on plant insects. 


\section{INDEX}

Absorption of light, 233

Acetic acid, 273

Acetylene, 244, 263

Acids, 272, 273, 274

Adenoids, 91

Adhesion, 46

Adiabatic heating, 157

Aëroplane, 69

Agonic lines, 190

Air, 73, 85, 213

a mixture, 87

importance to body, 88,89

functions of, 152

movements of, 163

as conductor, 197, 199

and sound, 213

Albumen, 388

Alcohol, 264, 396-397

Algæ, 329

Alkalies, 274

Alluvial deposit, 309

Ammonium hydroxide, 274

Ammonium nitrate, 276

Amœbæ, 350

Amorphous substances, 105

Amphibians, 359

Analysis, 277

of chemicals, 278

of soils, 288

Anemometer, 164

Aneroid barometer, 77

Animals, 350, 361

one-celled, 350

as food, 361

clothing from, 361

Anode, 208

Anther, 318

Anti-toxin, 411

Anti-trade winds, 164

Ants, 356

Aqueous humor, 237

Arc lamps, 203

Archimedes' principle, 116

Aristotle, 15

Artesian wells, 115
Ash, 395

Asteroids, 2

Astigmatism, 239

Atmosphere, 73, 152

density of, 73,154

depth of, 73

composition of, 73,87

weight of, 74

pressure of, 75,157

functions of, 152

colors of, 153

temperature of, 156

movements of, 163

Atoms, 27, 194

Auditory canal, 216

Bacteria, 86, 273, 294, 331, 351, 407

Baking powders, 269

Balance, 34

Barometer, 75

Bases, 272, 274

Bees, 356

Beetles, 364

Bell, electric, 206

Beverages, 395

Big Dipper, 10

Birds, 360

Biuret test, 388

Black knot, 348

Blade, 326

Blight, pear, 344 potato, 344

Blizzard, 171

Body, resisting power of, 410

Boiling point, 100-101, 134-135

Bordeaux mixture,. 276, 344, 348, appendix

Brass, 252

Breathing, 89-90

Brickfielders, 171

Bronchial tubes, 89

Brown rot, 343

Caissons, 80

Calcium hydroxide, 274 
Calcium phosphate, 276

Calorie, 137, 380

Calyx, 318

Cambium layer, 326

Camera, 223, 229, 237

Candle power, 223

Canis major, 10

Canker, 344, 346

Cañons, 307

Capillaries, 89

Capillarity, 48

Capillary water, $289,297,310$

in soils, $289,290,297$

Carbohydrates, 328,389

Carbolic acid, 262, 435

Carbon, 203, 257

compounds of, 259

Carbonates, 270

Carbon dioxide, 73, 257, 267, 268, $321,328,331$

Carburetor, 243

Carpels, 318

Casein, 388

Cassiopeia, 10

Cathode, 208

Caves, 300

Celestial Meridian, 19

Cells, electric, 200

voltaic, 200

polarized, 201

gravity, 201

dry, 201

of living matter, $316,317,319,351$

Cellulose, 323, 390

Centigrade scale, 98, 100, 129

Center of gravity, 39

Cepheus, 10

Charcoal, 84, 260, 261

Chemical changes, 95, 222, 246, 247 source of heat, 127

Chile saltpeter, 87, 254, 274, 293

Chinch bug, 366

Chloride of lime, 255

Chlorine, 255

Chlorophyll, 326, 390

Choroid coat, 236

Cilia, 352

Ciliary ligament, 237

Ciliary processes, 237

Cinematograph, 41

Citric acid, 273

Cities, growth of, 406

problems of, 407

cleaning streets of, 418
Climate, 152, 283

Clothing, 361

Clouds, 175

Coal, 259, 261

Cochlea, 217

Cocoa, 395

Codling Moth, 366

Coffee, 395

Cohesion, 46

Coke, 84, 261

Colds, 91, 424

Cold storage, 110, 381

Color, 233

Combustion, 83, 247

Comets, 2, 5

Compounds, 27, 247, 272

of carbon, 259

classes of, 272

Compressed air, 80

Compression, 126, 127

Concave lens, 228

Condensers, 197

Conduction of heat, 140, 141

Conductivity, 140

of earth, 142

and sensation, 142

of metals, 251

Conductors, 193, 197, 277

Conjunctiva, 235

Conservation, 291

of soil, 291

of rainfall, 297

of energy, 397

Constellations, 8, 9

Convection, 143-145

Convex lens, 228

Copper, 252

Copper sulphate, 275

Cornea, 236

Corolla, 318

Cotton, 340

Cotton boll weevil, 363

Crystalline lens, 230

Crystallization, 104

Crystals, 104

Cultivation, soil, 296

Curculio, 365

Cyclones, 165

Cyclonic winds, 165

Dairy, model, 413

Davy safety lamp, 143

Decay, 284, 285, 413

Declination, 190 
Deltas, 308, 309

Density, 35 of solids, 118

Deposition, by streams, 308-309 *

Dew, 175

Dew point, 174

Dextrose, 392

Dialysis, 51

Dietary, 399, 402

Diffusion, 49, 226

Digestion, 328, 401 in plants, 328

Diphtheria, 424

Dipping needle, 191

Diseases, 342

in plants, 342 infectious, 408 .

Disinfectants, 434

Distillation, 122, 253

Dog Star, 11

Doldrums, 164

Draco, 10

Drainage, 299, 310, 311

Dry farming, 297, 310

Drying of foods, 383

Dynamo, 208

Ear, 214, 216

Earth, a planet, 1, 14-18, 23

Ebullition, 134

Echoes, 214

Eclipse, 222

Elasticity, 45, 46

Electric arc, 203 currents, 200, 202

lighting, 202, 244 .

heating, 202

bell, 206

power, 209

motors, 211

Electrical charges, 193, 195, 197

Electricity, 191 by friction, 191

positive, 192

negative, 192

theory of, 194

atmospheric, 199

Electrodes, 93

Electrolysis, 93, 208, 276

Electrolytes, 276

Electromagnets, 205

Electrons, 194, 220

Electrophorus, 198

Electroplating, 208
Elements, 27, 247-248

Energy, 37, 41, 222

kinetic, 41

potential, 41

of body, 379

conservation of, 379

Engines, steam, 44, 70-71

Epidermis, 326

Epiglottis, 89

Equatorial calms, 164

Equilibrium, 40

Erosion, 304, 306-307, 335, 336

Ether, 220

Eustachian tube, 217

Evaporation, 105-106, 297, 336

Expansion, in solids, 130 in liquids, 131

in gases, 132

Experiment stations, 296

Explosives, 45

Eyes, 230, 235-237

Fahrenheit scale, 98, 100, 129

Fats, 394

Fehling's solution, 392

Fermentation, 269

Ferns, 329

Fertility, 284, 286, 291

Fertilization, 319

Fertilizers, 292-295

Fibrin, 388

Filament, 318

Filtration, 122, 422

Fire, origin, 264

Fire damp, 262

Fire extinguisher, 268

Fireless cooker, 142

Fishes, 358

Flashing point, 264

Flies, 355, 419

Flowers, 317 parts of, 318-319

Focal length, 228

Focus, 228

Food, 379

as fuel, 380

values, 380

sources of, 380,387

preservation of, 381,415

transportation of, 386

classes of, 388

purchase of, 398

and disease, 412

Foot, 30 
Foot pound, 44, 55

Force, 37

centrifugal, 43

muscular, 43

gravitational, 43

of expanding gases, 44

of wind, 43,68

molecular, 45

Forests, 337

Formaldehyde, 346, 348, 435

Friction, 63

a source of heat, 126

Frogs, 359

Frost, 175

Fruits as food, 340

Fuels, 259, 266, 338

Fulcrum, 55

Fumigation, 434

Fungi, 329-330, 342, 345, 348, 407

Fungicides, 348, appendix

Fusibility, 251

Fusion, 133

Galactose, 392

Galileo, 37

Galvani, 200

Garbage, 418

Gas, 242

artificial, 243, 262

natural, 243,263

acetylene, 244

Gases, 26, 255

Gelatin, 388

Gelatin cultures, 409

Germination, 320

Germs, 408, 410

Glaciated soil, 285

Glass, 240

Glucoses, 392

Gluten, 388

Glycogen, 392

Gold, 255

Gram, 39

Gravitation, 17, 37

Ground water, 299-300, 310

Guncotton, 274

Hail, 178

Health, 240, $4.06-436$

Hearing, 216, 218

Heat, 126, 202, 220, 247

a form of energy, 126

sources of, 126, 259

effects of, 130
Heat - Cont.

quantity of, 137

capacity, 137

of fusion, 138

conduction of, 140

from electric current, 202

Heating systems, 147-148, 202, 266

Hercules, 10

Hessian fly, 367

Horse latitudes, 164

Horse power, 44, 65

Humidity, 151, 173

Humus, 284, 292

Hurricanes, 166

Hydra, 353

Hydraulic press, 114

Hydrocarbons, 259, 262

Hydrochloric acid, 273

Hydrogen, 94-97, 272, 274

Ice, 100, 108-109

Igneous rock, 281

Incandescent bodies, 220

Incandescent lamps, 203

Incandescent mantles, 243, 263

Incidence, angle of, 225

Inclined plane, 53, 61

Incubation, period of, 410

Inertia, 42

Inland seas, 302

Insects, 355, 356, 365, 368, 428

Insulators, 193

Invertebrates, 358

Iris, 236

Iron, 251

Irrigation, 297, 310-314

Irritability, 315

Isobars, 154

Isogonic lines, 190

Isotherms, 158

Jack screw, 62

Jupiter, 2, 3

Kerosene, 242, 264

Kilogram, 32

Kindling point, 264

Kinetic energy, 41

Koch, Robert, 409

Lachrymal fluid, 235

Lactose, 393

Lakes, 302, 303

Lamps, 143, 242 
Language, development, 373

Larvæ, 355

Larynx, 89

Latent heat, 138

Latitude, 21

Lavoisier, 83

Leaves, 317,326

parts of, 326

as food, 340

Leeuwenhoek, 409

Legumin, 388

Lens, 227, 228, 239

Levees, 309

Levers, 53-56

Levulose, 392

Leyden jar, 197

Light, 220-234

properties of, 220

as energy, 222

intensity of, 223

reflection of, 224

diffused, 226

refraction of, 226

composition of, 231

absorption of, 233

Lighting, electric, 202, 244

in the house, 240

artificial, 241-244

Lightning, 182

Lime, 295

Limestone, 270, 282

Liquids, 26

shape of free, 47

capillary action in, 49

transmission of pressure, 112

Liter, 32

Litmus, 272, 274

Little Dipper, 10

Living matter, 315

Longitude, 21

Lumbering, 337

Luminous bodies, 221

Lungs, 88-89

Machines, 53-54, 63

Magnetism, 186-188

Magnets, 186

earth as, 190

Malaria, 429

Malleability, 251

Maltose, 393

Mammals, 360

Man, 372-375

Mantle, incandescent, 243
Mars, 2, 3

Marsh gas, 262

Mass, 34

Matches, 265

Matter, 26, 246

forms of, 26

properties of, 28

changes in, 246

Measles, 427

Measurement, 29

length, 33

volume, 34

mass, 34

temperature, 128

light, 223

Mechanical advantage, 63

Medullary rays, 324

Melting point, 133

in metals, 252, 255

Mercury, planet, 2, 3

Mercury, 252

Meridians, 20

Metals, 250-251

Metamorphic rock, 281

Meter, 31

Metric system, 31

Microscope, 229

Mildews, 344

Milk, 384, 388 danger from, 413 pasteurized, 415

Mixtures, 247

Moisture, need of, in the air, 151

Molds, 331, 347

Molecules, 28, 126 relation to heat, 137 relation to light, 220

Monsoons, 172

Moon, 11, 12

Mosquitoes, 429-431

Mosses, 329

Motors, electric, 211

Mushrooms, 330

Myosin, 388

Neptune, 2, 3

Newton, Sir Isaac, 37

Nimbus clouds, 177

Nitric acid, 273, 274

Nitrogen, 85

use in explosives, 86

importance to plants, $86,291,293$, 332

Nitroglycerine, 274 
Noise, 214

North Star, 8

Nucleus; 316

Nutrition, 379

Opaque bodies, 221

Organs, 317, 352

Orion, 10

Osmosis, 50, 322, 354

Oxidation, 83, 247

Oxides, 83, 247, 272

Oxygen, 81, 247, 272, 321

Parallels, 20

Paramecium, 352

Parasites, 331, 342

Pascal's law, 112

Pasteur, Louis, 409

Patent medicines, 397

Peach leaf curl, 348

Peat, 259

Penumbra, 222

Perseus, 10

Petroleum, 263

Phagocytes, 410

Pharynx, 89

Photographic plate, 223, 229, 254, 276

Photometer, 224

Physical changes, 95, 246, 247

Pitch, 215

Planetary winds, 164

Planetoids, 2, 5

Planets, 1, 2

Plants, 316

reproduction in, 319

distribution, 332

food plants, 339,387

textile plants, 340

diseases of, 342

Pleura, 88

Pneumonia, 425

Poisons, 368-369

Poles, 93, 186, 208

Pole Star, 9

Pollen, 318

Pollination, 319

Potash, 270, 274, 294

Potassium hydroxide, 274

Potato scab, 345

Potential energy, 41

Power, 44, 65 of water, 66 of wind, 68

electric, 209
Precipitation, 173, 178

Preservation, of forests, 337 of foods, 381

Preservatives, 415

Pressure, 74, 154

in liquids, 110

transmission of, 112

Prevailing westerlies, 164

Priestley, Joseph, 81

Prime meridian, 20

Prism, 231

Properties, 28 general, 28 special, 29

Protein, 87, 388

Protoplasm, 315-316, 351

Protozoa, 408

Ptomaines, 413

Pulley, 54, 58

Pumps, 77-78

Pupil, 230

Quarantine, 431

Radiation, 145

Rain, 178

Rainfall, 178, 299

Rain gauge, 180

Rainbow, 232

Reflected light, 224

Refraction of light, 226, 231

Reproduction in plants, 318,319

Reptiles, 360

Resistance, 40, 41

Respiration, 84,88

Retina, 230

Revolution, 23

Rivers, 300, 306

Rocks, 281, 283

relation to soils, 287

Rœmer, 220

Roots, 317, 321, 340

Rotation, 18

Rust, iron, 83

wheat, 342

Salt, 254

as preservative, 384

Salts, 272, 275

Sanitation, 406

San José scale, 365

Saprophytes, 331

Sapwood, 324

Satellites, 2, 3

Saturation, 102, 173 
Saturn, 2, 5

Scale, insects, 365

Scarlet fever, 427

Scheele, Carl W., 81

Science, 246

Sclerotic coat, 236

Scorpius, 10

Screw, 53, 62

Seasons, 23

Sedimentary rock, 281

Seeds, 319, 340

Separator, 43

Sewage, disposal of, 420

Shadows, 222

Sight, 235

Silt, 308

Silver, 254

Silver nitrate, 276

Siphon, 79

Sirius, 11

Smallpox, 428

Smut, 348

Snow, 178

Soaps, 270, 275

Sodium, 253

Sodium hydroxide, 274

Soil, 280, 288, 341.

formation of, 284

composition of, 286

particles, 288

conservation of, 291

Soil water, 310

Solar system, 2

Solids, 26

Solutions, 101, 102

Sori, 332

Sound, 212, 214, 216

reflection of, 214

voice and hearing, 216

Specific gravity, 35

Specific heat, 138

Spectacles, 228

Spectrum, solar, 231, 233

Sperm cell, 319

Spirogyra, 329

Spores, 330, 343, 345

Spraying, 344-348, 365-369, appendix

Starch, 328

in foods, 390

Stars, 2, 6, 8, 9

Steam, 41, 44, 70, 100

Steel, 252

Stems, 323, 324

as food, 340
Stoves, 266

Stratus clouds, 177

Street cleaning, 418

Submarines, 117

Substances, 26

Sucrose, 393

Sugar, 328, 390, 393

Sulphates, 276

Sulphur, 256

Sulphuric acid, 273, 274

Sun, 13, 14, 127, 221

Suspension, 102

Suspensory ligament, 238

Tartaric acid, 273

Taurus, 10

Tea, 395

Telegraph, 206

Telephone, 207

Telescope, 229

Temperature, 126

measurement of, 128

of atmosphere, 156

relation to sound, 213

Thermograph, 158

Thermometer, 128

Thermos bottle, 143

Thermostat, 149

Thunder storms, 180

Time, 21

Time belts, 22

Tinctures, 102

Tissues, 316

Toadstools, 330

Tobacco, 398

Tone, 214

Tools, development, 374

Tornadoes, 166

Torricelli, 76

Toxins, 410

Trachea, 89

Trade winds, 164

Trees, 335

value of, 335-336

uses of, 337,339

Tuberculosis, 425

Turbines, water, 68 steam, 71

Tympanic membrane, 217

Typhoid fever, 422

Umbra, 222

Universe, 1

Uranus, 2, 3 
Vacuum, 212

Vaporization, 134

Veins, 317, 326

Ventilation, 147, 149

Venus, 2, 3

Vertebrates, 358

Vibrations, of sound, 212 of light, 220

Vinegar, 273

Vitamines, 394

Vitreous humor, 237

Voice, 216

Volatile liquids, 106

Volta, 200

Voltaic cells, 200

Volume, 34

Water, 93, 290, 299, 395, 416 electrolysis of, 93 freezing of, 98 uses of, 108, 120 pressure, 110 city supply, $112,120,123$ gravitational, 290 ground water, 299 and food, 395 danger from, 416

Water power, 66,68

Waterspout, 170
Watt, James, 44
Waves, sound, 212-213
light, 220, 232
Weather, 152
weather bureau, 183
Weathering, 283, 285
erosion, 304
Weapons, 374
Wedge, 54, 62
Weeds, 341
Weight, 38
Wheat rust, 342
Wheel and axle, 54, 59
Windlass, 60
Winds, 163, 164, 171.
Wood, 259, 260
uses of, 337
Work, 37, 53, 54
Worms, 353
Yard, 30
Yeast, 269
plants, 331, 349
Yellow fever, 430
Zenith, 20

18 




